\title{
Photodissoziation von Polyhalogenmethanen in Fluiden: Kurzzeitdynamik und Mechanismen
}

\author{
Dissertation \\ zur Erlangung des Doktorgrades \\ der Mathematisch-Naturwissenschaftlichen Fakultäten \\ der Georg-August-Universität zu Göttingen
}

vorgelegt von

Philipp Wagener

aus Hameln 
D7

Referent: Prof. Dr. J. Troe

Korreferent: Prof. Dr. D. Schwarzer

Tag der mündlichen Prüfung: 29.04.2008 
Meinen Eltern gewidmet 



\section{Inhaltsverzeichnis}

\begin{tabular}{ll}
\hline Zusammenfassung & 1
\end{tabular}

\begin{tabular}{lll}
\hline 1 & Einleitung & 3 \\
\hline
\end{tabular}

2 Reaktionsdynamik in Lösung 5

2.1 Der Käfigeffekt . . . . . . . . . . . . . . . . . . . . . . . . . 7

2.2 Vom Diffusionmodell zum kinematischen Käfigeffekt . . . . . . . . . . . . 8

$2.3 \quad$ Solvation in überkritischen Lösungsmitteln . . . . . . . . . . . . . . . . . 12

3 Die Polyhalogenmethane 15

3.1 Physikalische und chemische Eigenschaften . . . . . . . . . . . . . . . . . 15

3.2 Photodissoziation der Polyhalogenmethane in der Gasphase. . . . . . . . 16

3.2 .1 Diiodmethan . . . . . . . . . . . . . . . . . . 17

3.2 .2 Chloriodmethan . . . . . . . . . . . . . . . . . . . . . . . . 18

3.2 .3 Bromiodmethan $\ldots \ldots \ldots \ldots$

3.2 .4 Iodoform . . . . . . . . . . . . . . . . . . 20

$3.2 .5 \quad$ Zwei-Photonen-Photodissoziation der Polyhalogenmethane . . . . 21

3.3 Photodissoziation von Iodmethan der Gasphase . . . . . . . . . . . . . 23

3.4 Primäre Photofragmente . . . . . . . . . . . . . . . . . . 26

$3.5 \quad$ Photodynamik und -isomerisierung in der kondensierten Phase . . . . . . 29

3.5.1 $\quad$ Photoisomerisierung in Matrixumgebung . . . . . . . . . . . . . . 29

3.5 .2 Photodynamik in fluiden Lösungsmitteln . . . . . . . . . . . . . . 30

$3.5 .3 \quad$ Zeitaufgelöste Schwingungsspektroskopie in Lösung . . . . . . . . . 34

4 Experimentelle Technik 37

4.1 Das UV/VIS-Lasersystem . . . . . . . . . . . . . . . . . . . . . . . . . . . . . . . . . 37

$4.1 .1 \quad$ Clark MXR-CPA 2001 . . . . . . . . . . . . . . . . . . . . . . . . . . . . . . . . . . . . . . .

$4.1 .2 \mathrm{NOPA} \ldots \ldots \ldots \ldots \ldots$

$4.1 .3 \quad$ Erzeugung der Dritten Harmonischen (THG) . . . . . . . . . . . . 41

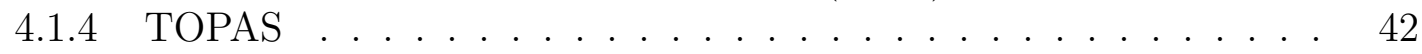

4.1 .5 Interferometer . . . . . . . . . . . . . . . . . . . . . . . . . . . . . . . . . . . . . . . . . . . . . . .

4.1 .6 Datenaufnahme . . . . . . . . . . . . . . . . . . . . . . . . . . . . . . . . . . . . . . . . . . . . .

4.1 .7 Hochdrucksystem . . . . . . . . . . . . . . . . . . . . . . . . . . . . 45

4.2 Das IR-Lasersystem $\ldots \ldots \ldots \ldots$. . . . . . . . . . . . . . . . . . . . . . 46

4.2 .1 Ti:Sa Laser . . . . . . . . . . . . . . . . . . . . . . . . . . . . . . . . . . . . . . . . . . 46

$4.2 .2 \quad$ Erzeugung der IR-Probepulse . . . . . . . . . . . . . . . . . . . . . . . . . 47

$4.2 .3 \quad$ Das IR-Spektrometer . . . . . . . . . . . . . . . . . . . . . . . . . . . 48 
4.3 Probenpräparation . . . . . . . . . . . . . . . . . . . . . . . . 50

$4.3 .1 \quad$ Messungen in überkritischen Fluiden . . . . . . . . . . . . . . . . 50

$4.3 .2 \quad$ Messungen in flüssigen Lösungsmitteln . . . . . . . . . . . . . . . 50

$4.3 .3 \quad$ Messungen von Iodmethan in flüssigen Lösungsmitteln . . . . . . 51

4.4 Anpassung der experimentellen Daten. . . . . . . . . . . . . . . . . 51

4.5 Verwendete Chemikalien . . . . . . . . . . . . . . . . . . . . . . 55

$\begin{array}{lll}5 & \text { Experimentelle Ergebnisse } & 57\end{array}$

5.1 Diiodmethan $\ldots \ldots \ldots \ldots \ldots \ldots \ldots$

5.1 .1 Druckabhängige Messungen bei $340 \mathrm{~nm}$ Anregung . . . . . . . . . 58

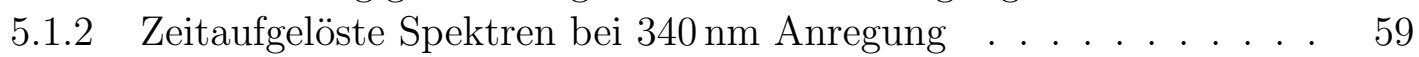

5.2 Bromiodmethan . . . . . . . . . . . . . . . . . . 61

5.2 .1 Zeitaufgelöste Spektren bei $258 \mathrm{~nm}$ Anregung . . . . . . . . . . . 64

5.2 .2 Druckabhängige Messungen bei $288 \mathrm{~nm}$ Anregung . . . . . . . . . 65

5.3 Iodoform . . . . . . . . . . . . . . . . . . 66

$5.3 .1 \quad$ Zeitaufgelöste Spektren bei $365 \mathrm{~nm}$ Anregung . . . . . . . . . . . 70

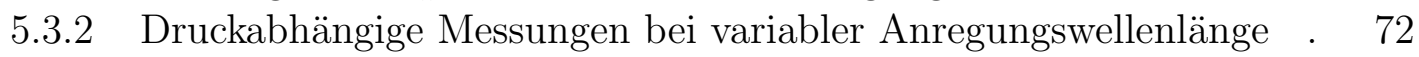

5.3 .3 Variation des Fokusvolumens . . . . . . . . . . . . . . 74

5.4 Chloriodmethan . . . . . . . . . . . . . . . . . 76

5.5 Iodmethan $\ldots \ldots \ldots \ldots \ldots$

$5.5 .1 \quad$ Stationäre Absorptionsspektren . . . . . . . . . . . . . . . 80

5.5 .2 fs-UV-Pump/UV-Probemessungen in Lösung. . . . . . . . . . . . . . . 81

5.5 .3 fs-UV-Pump/VIS-Probemessungen in Lösung . . . . . . . . . . . . 86

$5.5 .4 \quad$ fs-UV-Pump/IR-Probemessungen in Lösung . . . . . . . . . . . . 87

$\begin{array}{lll}6 & \text { Diskussion } & 91\end{array}$

6.1 Ultrakurzzeitdynamik der Photoisomerisierung von Polyhalogenmethanen 91

$6.1 .1 \quad$ Beteiligte Spezies im Ultrakurzzeitbereich . . . . . . . . . . . . . 92

6.1 .2 Bildung von Ladungstransferkomplexen . . . . . . . . . . . . . . . . . . . . . . 94

6.2 Isomerisierungsgeschwindigkeit . . . . . . . . . . . . . . . . . . . . . 99

$6.2 .1 \quad$ Spektrale Dynamik in überkritischen Lösungsmitteln . . . . . . . 100

$6.2 .2 \quad$ Anregungswellenlängenabhängigkeit der Isomerisierung . . . . . . 106

6.2 .3 Dichteabhängigkeit der Geschwindigkeitskonstante $k_{\text {iso }}$. . . . . . 107

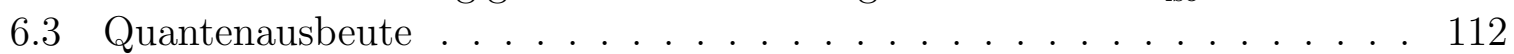

6.4 Spezielle Fälle . . . . . . . . . . . . . . . . . . . . . . . . . . . . . . . 115

6.4 .1 Isomerisierung und Zerfall von Chloriodmethan . . . . . . . . . . 115

6.4 .2 Photodynamik von Bromiodmethan nach $258 \mathrm{~nm}$ Anregung. . . . . 118

$6.4 .3 \quad$ Photoinduzierte Heterolyse von Iodoform in Lösung . . . . . . . . . 119

6.4.4 Niederdruckmessungen von Iodoform in überkritischem Kohlenstoffdioxid . . . . . . . . . . . . . . . . . . . . . . . . . . . . 124

6.5 Geminale Rekombination der Polyhalogenmethane . . . . . . . . . . . . . 127

6.6 Photodynamik von Iodmethan . . . . . . . . . . . . . . . . . . . . . . . . 129

6.6 .1 Bildung von CTTS-Komplexen . . . . . . . . . . . . . . . . . . . 129

6.6 .2 Quantenausbeuten der CTTS-Komplexe . . . . . . . . . . . . . . 132

6.6.3 $\quad$ Dynamik nach Zwei-Photonen-Absorption . . . . . . . . . . . . . 133 
6.6 .4 Dynamik des solvatisierten Elektrons . . . . . . . . . . . . . . . . 138

6.6.5 Kurzzeitdynamik von Iodmethan nach Photoionisation in Lösung 140

6.6.6 Diffusive und nicht-diffusive Rekombination solvatisierter Elektronen und Kationen . . . . . . . . . . . . . . . . . . . . . . . . . . . 145

6.6 .7 Geminale Rekombination von Iodmethan in Lösung . . . . . . . . 153

6.6.8 Zusammenfassung der photoinduzierten Dynamik von Iodmethan in Lösung . . . . . . . . . . . . . . . . . . . . . . . . 155

$\begin{array}{llr}7 & \text { Ausblick } & 159\end{array}$

7.1 Theoretische Modellierung durch quantenchemische Rechnungen . . . . . 159

7.2 $\quad$ Zeitaufgelöste Raman-Spektroskopie . . . . . . . . . . . . . . . . . . . . 160

\begin{tabular}{|ll}
\hline A Anpassungsparameter der Meßdaten & 163
\end{tabular}

\begin{tabular}{|ll|}
\hline B Physikalische Eigenschaften der verwendeten Chemikalien & 171
\end{tabular}

\begin{tabular}{ll}
\hline Abbildungsverzeichnis & 173
\end{tabular}

\begin{tabular}{ll}
\hline Tabellenverzeichnis & 177
\end{tabular}

\begin{tabular}{ll}
\hline Literaturverzeichnis & 179
\end{tabular} 



\section{Zusammenfassung}

In dieser Arbeit wurde mit Hilfe der Pump/Probe-Femtosekundenspektroskopie der molekulare Mechanismus der photoinduzierten Dynamik und Isomerisierung von Polyhalogenmethanen in überkritischer und flüssiger Lösung untersucht. Dazu wurde die transiente Absorption verschiedener halogenierter Methanverbindungen $\left(\mathrm{CH}_{2} \mathrm{I}_{2}, \mathrm{CHI}_{3}, \mathrm{CH}_{2} \mathrm{BrI}\right.$, $\mathrm{CH}_{2} \mathrm{ClI}$ sowie $\mathrm{CH}_{3} \mathrm{I}$ ) unter variablen Bedingungen in Lösung gemessen. Durch die Verwendung überkritischer Lösungsmittel konnte dabei ein weiter Dichtebereich zwischen verdichteter Gasphase und Flüssigkeit abgedeckt werden.

Eine Anregung in die erste Absorptionsbande der untersuchten Polyhalogenmethane $\left(\mathrm{CH}_{2} \mathrm{I}_{2}, \mathrm{CH}_{2} \mathrm{BrI}, \mathrm{CH}_{2} \mathrm{ClI}\right.$ und $\left.\mathrm{CHI}_{3}\right)$ führt zu einer Fragmentierung in ein Iodatom und ein $\mathrm{CH}_{2} \mathrm{X}$ bzw. $\mathrm{CHI}_{2}$-Radikal. Im Gegensatz zur Gasphase können die Photofragmente im Lösungsmittelkäfig lange genug zusammengehalten werden, um eine X-I-Bindung und somit ein CHYX-I-Isomer ( $\mathrm{Y}=\mathrm{H}, \mathrm{I}, \mathrm{X}=\mathrm{I}, \mathrm{Br}, \mathrm{Cl}$ ) zu bilden. Dazu bilden die im Lösungsmittelkäfig gefangenen Photofragmente sehr schnell ( $<200 \mathrm{fs}$ ) einen CT-Komplex (engl. charge-transfer-Komplex), der aufgrund seiner charakteristischen, extrem breitbandigen Absorption identifiziert wurde. Die Rekonstruktion zeitaufgelöster Spektren aus transienten Absorptionsmessungen zeigt anschließend das charakteristische Verhalten der Relaxation einer schwingungsangeregten Spezies. Diese Schwingungsenergierelaxation konnte als geschwindigkeitsbestimmender Schritt bei der Isomerbildung identifiziert werden, da die primären Photofragmente mit einer hohen Überschußenergie gebildet werden und die Dissoziationsenthalpie des resultierenden CHYX-I-Isomers nur gering ist. Eine Anregung mit kürzerer Wellenlänge und somit höherer Überschußenergie der Photofragmente bewirkte eine langsamere Bildungsgeschwindigkeit des Isomers, wobei der Effekt beim $\mathrm{CH}_{2} \mathrm{I}_{2}$ im Vergleich zum $\mathrm{CHI}_{3}$ wesentlich ausgeprägter ist. Diese Beobachtung konnte mit der Postulierung eines schnellen Vorgleichgewichts zwischen CT-Komplex und lösungsmittelseparierten Photofragmenten zufriedenstellend erklärt werden. Die nichtlineare Dichteabhängigkeit der Geschwindigskeitskonstante der Isomerbildung setzt sich dabei aus der Dichteabhängigkeit ihrer Teilschritte zusammen.

Die Quantenausbeute der Isomerbildung verläuft bei ausreichend hohen Dichten linear zur Lösungsmitteldichte. Dieses deutet auf einen rein kinematischen Käfigausbruch der Photofragmente direkt nach der Photodissoziation hin. Eine spätere, diffusionskontrollierte Separation der Photofragmente spielt nur bei niedrigen Dichten eine Rolle.

Die Photodynamik von $\mathrm{CH}_{3} \mathrm{I}$ in Lösung unterscheidet sich stark von der der anderen untersuchten Polyhalogenmethane. Im Vergleich zu diesen findet keine Isomerisierung 
innerhalb des Lösungsmittelkäfigs statt, da im Molekül lediglich ein Iodatom zur Verfügung steht. Im Falle des $\mathrm{CH}_{3} \mathrm{I}$ muß zwischen einer Ein-Photonen- und einer ZweiPhotonen-Absorption unterschieden werden. Die Ein-Photonen-Absorption führt zu einer Dissoziation in ein $\mathrm{CH}_{3}$-Radikal und ein Iodatom, welches sehr schnell $(<200 \mathrm{fs})$ einen CT-Komplex mit einem Lösungsmittelmolekül bildet. Diese Bildung eines CTTSKomplexes (engl. charge transfer to solvent complex) konnte in Methanol, Acetonitril und Methylcyclohexan mit einer Quantenausbeute von $\Phi \sim 0,1$ nachgewiesen werden. In $n$-Perfluorhexan findet aufgrund des hohen Ionisierungspotentials des Lösungsmittels keine CTTS-Bildung statt. Die Aufnahme zeitaufgelöster IR-Spektren in $\mathrm{CD}_{3} \mathrm{CN}$ zeigte, daß ein kleiner Anteil der primären Photofragmente innerhalb von etwa 40 ps geminal rekombiniert.

Die Zwei-Photonen-Absorption von $\mathrm{CH}_{3} \mathrm{I}$ bewirkt eine Photoionisierung, dabei wird ein freies Elektron in Lösung abgeben, welches innerhalb einiger Pikosekunden solvatisiert wird. Die Solvatationsdynamik des freien Elektrons ließ sich dabei durch Messung der transienten Absorption beobachten. Nach thermischer Äquilibrierung wird das Elektron sehr schnell ( $<200 \mathrm{fs})$ in einer „Falle“ aus elektrostatisch günstig ausgerichteten Lösungsmittelmolekülen im angeregten Zustand $p^{\prime}$ gefangen. Dieser relaxiert mit einer lösungsmittelabhängigen Zeitkonstante von etwa 2 ps in den modifizierten Grundzustand $s^{\prime \prime}$. Dabei konnte eine Emissionsbande um $400 \mathrm{~nm}$ durch stimulierte Emission zwischen dem $p^{\prime}$ - und $s^{\prime}$-Zustand nachgewiesen werden. Nach Abschluß der Solvatation im $s^{\prime \prime}$-Zustand findet eine diffusiv gesteuerte nicht-geminale Rekombination der solvatisierten Elektronen und Kationen in unpolaren Lösungsmitteln wie Methylcyclohexan statt. Eine Simulation der diffusiven Rekombination mit Hilfe der Smulochowski-Gleichung zeigt eine gute Übereinstimmung mit den experimentellen Daten.

In polaren Lösungsmitteln wie Methanol und Acetonitril konnte aufgrund der eingeschränkten Beweglichkeit der Ladungsträger keine diffusive Rekombination beobachtet werden. Stattdessen findet innerhalb von etwa 30 ps teilweise eine geminale Rekombination der primären Ladungsträger statt. 


\section{Einleitung}

Zum Verständnis chemischer Reaktionen ist eine Untersuchung der Reaktionsdynamik auf der Ebene von Elementarreaktionen (wie z. B. Dissoziationen, Rekombinationen oder Isomerisierungen) unerläßlich. Die Untersuchung der beteiligten Elementarreaktionen liefert erst ein genaues Bild über den Ablauf einer chemischen Reaktion, eine Beeinflußung und Steuerung ist nur durch genaue Kenntnis des vorherrschenden molekularen Mechanismus möglich. Die Erforschung der Reaktionsdynamik ist somit ein Grundstein der chemischen Forschung.

Der überwiegende Teil der chemischen Reaktionen findet in Lösung statt, die Spanne reicht dabei von biochemischen Prozessen in Lebewesen bis hin zur großtechnischen Synthese von chemischen Produkten aller Art. Der Einfluß eines Lösungsmittels auf eine chemische Reaktion, insbesondere auf Elementarreaktionen, ist somit von fundamentalem Interesse für die chemische Forschung und deren praktische Anwendung. Im Gegensatz zur Gasphase ist die Reaktionsdynamik in Lösung jedoch ungleich komplizierter, die vielseitigen Wechselwirkungen zwischen Gelöstem und Lösungsmittel führen zu einem wesentlich höheren Grad an Komplexität und zu sehr viel schnelleren Prozessen. Die Verfügbarkeit größerer Rechenkapazitäten für computergestützte Untersuchungen und Spektroskopiemethoden mit ultraschneller Zeitauflösung haben in diesem Bereich in den vergangen Jahrzehnten jedoch zu großen Fortschritten geführt. Insbesondere die PumpProbe-Spektroskopie in Femtosekundenauflösung kann hier einen wesentlichen Beitrag liefern, da die schnellen Prozesse in Lösung auf dieser Zeitskala stattfinden und somit eine chemische Reaktion in „Echtzeit“ beobachtet werden kann.

Der Fokus dieser Arbeit liegt in der Untersuchung des photolytischen Käfigeffekts mit Hilfe von Pump-Probe-Absorptionsspektroskopie in Femtosekundenauflösung, als Modellsystem wurde hierzu die Photodissoziation und -isomerisierung von Polyhalogenmethanen in flüssigen und überkritischen Lösungsmitteln gewählt. Dabei soll der Einfluß des Lösungsmittels, insbesondere der Käfigeffekt, untersucht und der genaue Mechanismus aufgeklärt werden, um ein möglichst detailliertes Modell für die Photoisomerisierung in Lösung aufzustellen. Frühere Untersuchungen haben gezeigt, daß die Wirkung des Käfigeffekts auf einer sehr schnellen Femto- bis Pikosekundenzeitskala stattfindet, die Anwendung einer Untersuchungsmethode mit sehr hoher Zeitauflösung ist also essentiell. Weiterhin bringt die Verwendung überkritischer Lösungsmittel große Vorteile, da dadurch die Dichte des Lösungsmittels und somit die Käfigeigenschaften über einen großen Bereich variiert werden können.

Grundlegende Betrachtungen zur Reaktionsdynamik in Lösung sowie der aktuelle Stand der Forschung zum Käfigeffekt werden im folgenden Kapitel 2 dargestellt. In Kapitel 
3 werden die als Modellsysteme verwendeten Polyhalogenmethane $\mathrm{CH}_{3} \mathrm{I}, \mathrm{CH}_{2} \mathrm{I}_{2}, \mathrm{CHI}_{3}$, $\mathrm{CH}_{2} \mathrm{BrI}$ und $\mathrm{CH}_{2} \mathrm{ClI}$ vorgestellt, während Kapitel 4 dem experimentellen Aufbau und der Spektroskopie in Femtosekundenauflösung gewidmet ist. Die gemessenen Daten sind in Kapitel 5 zusammengestellt und werden in Kapitel 6 ausführlich diskutiert. Auf ihrer Basis wird ein Modell für die Photoisomerisierung von Polyhalogenmethanen sowie die photoinduzierte Dynamik von $\mathrm{CH}_{3} \mathrm{I}$ in Lösung vorgestellt. Kapitel 7 faßt noch offene Fragen zusammen und gibt einen kurzen Überblick über mögliche weiterführende Arbeiten. 


\section{Reaktionsdynamik in Lösung}

Der Übergang von der isolierten Gasphase zu einer dichten Lösungsmittelumgebung führt zu sehr unterschiedlichen Reaktionsbedingungen, welche Auswirkungen sowohl auf die Kinetik als auch auf die Thermodynamik einer Reaktion haben. Einen Überblick über chemische Reaktionsdynamik in Lösung geben beispielsweise Schroeder [1], Hynes [2] oder auch Harris [3]. Die wichtigsten Punkte sollen hier in aller Kürze zusammengetragen werden.

Der Hauptunterschied zwischen Gasphase und Lösung liegt in erster Linie in ihrer Dichte, in Lösung nimmt die Anzahl der Stöße mit dem Solvens stark zu und isolierte Stöße sind aufgrund der hohen Dichte an Lösungsmittelmolekülen praktisch bedeutungslos. Für druckabhängige Reaktionen wie unimolekulare Reaktionen bedeutet dies, daß sie normalerweise ihren Hochdruckgrenzwert erreicht haben; der Einfluß des Lösungsmittels ist jedoch nicht allein auf die Stoßaktivierung und -desaktivierung begrenzt. So spielt beim Durchlaufen des Übergangszustands viskositätsabhängige Reibung mit dem Solvens zunehmend eine Rolle, welches in einer Verringerung der Geschwindigkeitskonstante mündet (s. Kramers-Theorie [4]), weiterhin kann das Lösungsmittel in bestimmten Fällen Einfluß auf die Barriere nehmen und die Reaktion z. B. durch Absenkung der Barriere beschleunigen [5]. Auch der Verlauf von bimolekularen Reaktion in Lösung ist im Vergleich zur Gasphase Änderungen unterworfen. Ist in der Gasphase die Stoßsequenz von zwei Reaktanten noch ein reiner Markow-Prozeß, also rein statistisch und unabhängig von den vorherigen Ereignissen, ändert sich dieser Prozeß in Lösung durch Ausbildung einer Lösungsmittelstruktur. Nach dem ersten Stoß zweier Reaktanten sind weitere Stöße sehr wahrscheinlich, da diese Reaktanten durch die Lösungsmittelumgebung räumlich zusammengehalten werden. Dieses geschieht durch die Ausbildung eines Lösungsmittelkäfigs um die Reaktanten herum (s. Abschnitt 2.1). Innerhalb des Lösungsmittels sind einzelne Moleküle zudem nicht mehr frei beweglich, neben Transportphänomenen wie Diffusion kommt mikroskopische Reibung zunehmend zum Tragen und erschwert z. B. Translation oder Rotation eines Moleküls im Solvens (vergl. Stokes-Einstein-Reibung).

Die Solvatation innerhalb eines Lösungsmittels bedeutet in erster Linie einen elektrostatischen Effekt auf das Gelöste, durch Wechselwirkung zwischen einem polaren Lösungsmittel mit Ladungen, Dipolen, Quadrupolen bzw. fluktuierenden Dipolen bei unpolaren Lösungsmitteln wird das Gelöste durch die freie Gibbs-Energie $\Delta G_{\text {Solv }}$ stabilisiert. Nach dem klassischen Modell von Onsager [6] und Kirkwood [7] auf der Grundlage des BornFormalismus für Wechselwirkungen von Ladungen mit einer dielektrischen Umgebung [8] befindet sich das Gelöste innerhalb einer sphärischen Kavität aus Lösungsmittel. Dieses 
Lösungsmittel wird im Onsager-Modell durch ein homogenes Dielektrikum mit der Dielektrizitätszahl $\epsilon_{r}$ repräsentiert, welches mit dem Gelöstem, dargestellt als ein Dipol $\mu_{G}$, wechselwirkt. Die freie Gibbsenergie $\Delta G_{\text {Solv }}$ beträgt somit:

$$
\Delta G_{\text {Solv }}=-N_{A} \frac{\epsilon_{r}-1}{2 \epsilon_{r}+1} \frac{\mu_{G}^{2}}{4 \pi \epsilon_{0} r^{3}}
$$

Im Falle von geladenen Molekülen kommt noch der entsprechende Born-Term dazu. Ein Lösungsmittel kann so Reaktionsprodukte und Übergangszustände und somit auch Reaktionsbarrieren durch elektrostatische Wechselwirkungen energetisch beeinflussen und zu essentiellen Änderungen in Produktspektrum, Thermodynamik und Kinetik im Vergleich zur Gasphase führen. Das einfache auf Dipolmomente beschränkte Onsager-Modell wurde durch Kirkwood [7] durch Einführung von Multipolen verbessert, auch heute sind noch Kontinuums-Modelle zur Beschreibung von Solvatationseffekten in Gebrauch (vergl. Übersichtsartikel von Cramer und Truhlar [9]). Weitergehende Modelle fassen das Lösungsmittel nicht als Kontinuum, sondern als explizite Lösungsmittelmoleküle auf ( $\mathrm{Su}-$ permolekülansatz). Wechselwirkungen wie Wasserstoffbrückenbindungen werden in diesem Ansatz wesentlich besser erfaßt, dieses ist insbesondere für sehr große Moleküle wie Proteine wichtig.

Weiterhin dient das Lösungsmittel als eine Art Wärmebad, durch Stöße zwischen Gelöstem und Lösungsmittel kann Überschußenergie abgeführt werden und instabile Reaktionsprodukte werden so stabilisiert. Eine Möglichkeit zur Beschreibung des Energieflusses zwischen Lösungsmittel und Gelöstem ist die Übertragung des Modells der isolierten, binären Stöße (engl. isolated binary collisions, IBC-Modell) von der Gasphase auf die flüssige Phase [10,11. Dabei wird angenommen, daß zwischen Gelöstem und Solvens von einander unabhängige, binäre Stöße stattfinden, bei denen jeweils ein dichteunabhängiger Energiebetrag übertragen wird. In der Gasphase ist die Anzahl der Stöße direkt proportional zur Dichte, in Lösung verliert dieser lineare Zusammenhang durch die komplexe Solvenstruktur seine Bedeutung und muß durch Radialverteilungsfunktionen modelliert werden [12, 13]. Die bedeutendste Art der Energieübertragung ist der Schwingungsenergietransfer (engl. vibrational energy transfer, VET), dabei wird Schwingungsenergie vom Gelöstem auf die Translations- und Schwingungsfreiheitsgrade des Lösungsmittel umverteilt (V-V bzw. V-T Energietransfer). Ein anderer Ansatz zur Modellierung des Energieflusses ins Lösungsmittel sind Langevin-Simulationen, die Langevin-Gleichung beschreibt dabei die Schwingungsbewegung des Gelösten in der Lösungsmittelumgebung. Die Relaxation einer Schwingungsmode erfolgt in der generalisierten Langevin-Gleichung durch Verwendung eines frequenzabhängigen Reibungskoeffizienten [14], Langevin-Simulationen ermöglichen dabei eine gute Beschreibung der Korrelation zwischen den Schwingungsmoden des Gelösten und dem Solvens. 


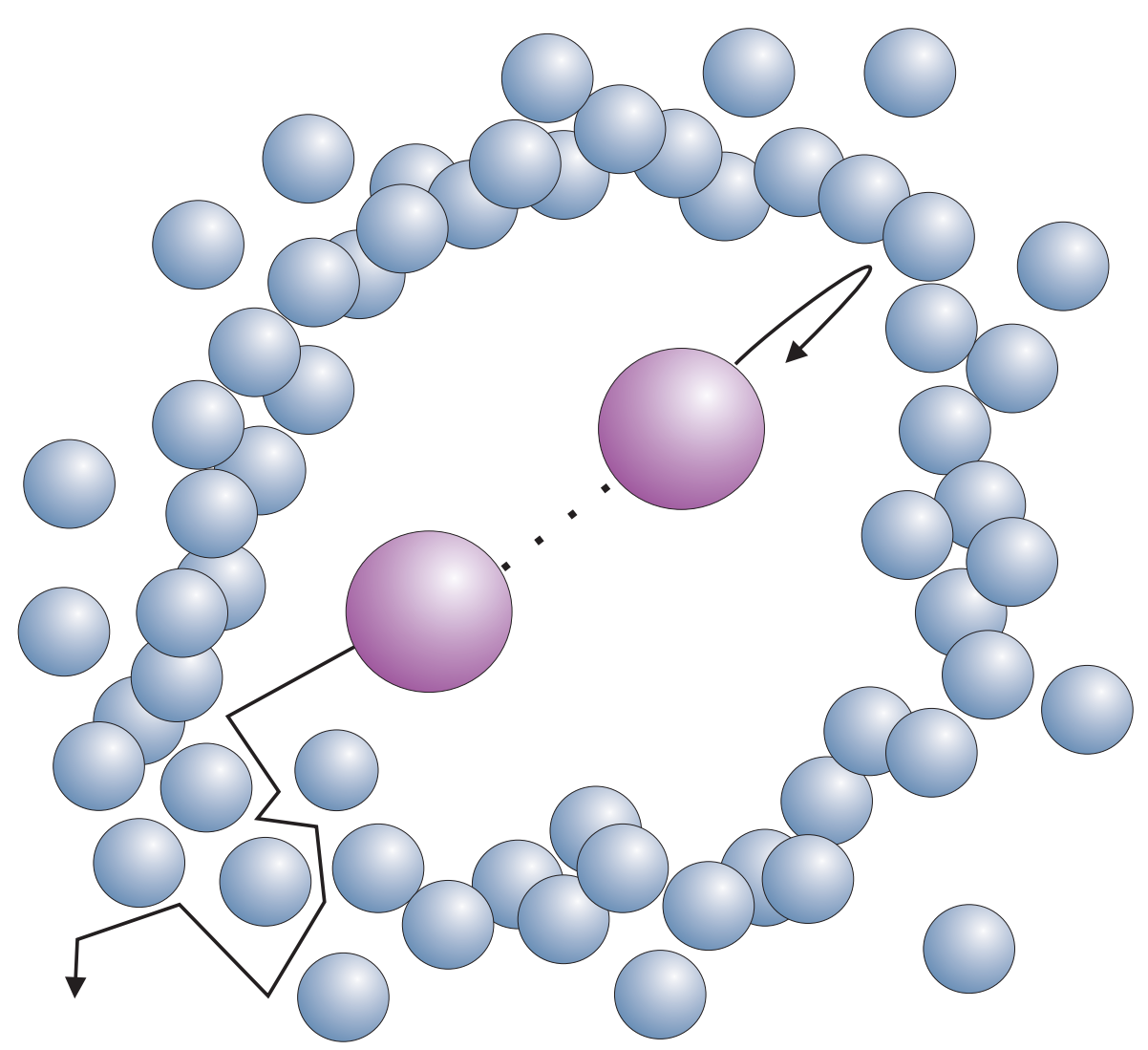

Abbildung 2.1: Iodatome nach Photodissoziation im Lösungsmittelkäfig

\subsection{Der Käfigeffekt}

In Lösung bildet sich um das Gelöste ein „Käfig“ aus Lösungsmittelmolekülen. Bimolekulare Reaktion können innerhalb des Käfigs einen völligen anderen Verlauf als in der Gasphase nehmen.

Das Konzept des Käfigeffekts wurde 1934 von Franck und Rabinowitch [15-17] eingeführt, um die Abnahme der Quantenausbeute der $\mathrm{I}_{2}$-Photodissoziation in Lösung im Vergleich zur Gasphase zu erklären. Sie postulierten, daß nach der Photodissoziation die Iodatome in einem Käfig aus Lösungsmittelmolekülen gefangen sind, welcher sie räumlich und zeitlich ausreichend lang zusammenhält, um eine geminale Rekombination zu ermöglichen (s. Abbildung 2.1). In Konkurrenz zur geminalen Rekombination steht der Käfigausbruch der Photofragmente, eine nicht-geminale Rekombination erfolgt durch einen diffusiv gesteuerten Prozeß.

Der Käfigeffekt spielt eine bedeutsame Rolle in nahezu allen Gebieten der Reaktionsdynamik in Lösung. Neben der geminalen Rekombination kleinerer Moleküle wie $\mathrm{I}_{2}$ und $\mathrm{Br}_{2}$ [18 21], I $\mathrm{I}_{3}^{-}$[22, 24] oder $\mathrm{CS}_{2}$ [25, 26] wurden auch Photoisomerisierungen von Halogenoxiden und Nitrosylhalogenen wie $\mathrm{OClO}$ oder ClNO [27, 29], $\mathrm{HOCl}$ [30, 31], ICN [32,33] oder verschiedener Polyhalogenmethane (s. Kapitel 3) als Modellsysteme für den Käfigeffekt untersucht. 
Neben der Untersuchung solcher Modellsysteme wird das Konzept des Käfigeffekts vielseitig zum Verständnis und zur Modellierung von Reaktionsdynamiken jeglicher Art angewendet. Exemplarisch genannt seien die Photochemie von Coenzym $\mathrm{B}_{12}$ [34, 35, die Reaktion von Häm mit $\mathrm{O}_{2}$ und $\mathrm{CO}$ [36, 37], Polymerisationsreaktionen bezüglich den Schritten der Initiation, Propagation und Terminierung [38], Oberflächenchemie [39], Photolyse und Produktspektrum arylsubstituierter Ketone in überkritischen Lösungsmitteln [40], Photodissoziation und insbesondere Elektrontransferreaktionen von Carbonylmetallkomplexen in Lösung [41-43].

\subsection{Vom Diffusionmodell zum kinematischen Käfigeffekt}

Das erste Modell für eine diffusionsbestimmte Reaktion in Lösung stammt von Smoluchowski [44. Dabei reagieren zwei Moleküle A und B, welche sich durch Diffusion in verdünnter Lösung bewegen. Die Teilchen der Sorte B sind dabei im Überschuß vorhanden und es wird die zeitliche Entwicklung der Konzentration von B um A herum betrachtet, welches der Verteilungsfunktion $c(r, t)$ entspricht. Die Annäherung ist dabei der geschwindigkeitsbestimmende Schritt, die eigentliche Reaktion findet beim Stoß statt und ist wesentlich schneller $\left(k_{\mathrm{r}} \gg k_{\text {diff }}\right)$ :

$$
\mathrm{A}+\mathrm{B} \rightleftharpoons[\mathrm{A} \cdots \mathrm{B}] \stackrel{\mathrm{k}_{\mathrm{r}}}{\longrightarrow} \text { Produkte }
$$

Die Geschwindigkeitskonstante $k_{\text {diff }}$, welche die Begegnung der Reaktanten in Lösung steuert, kann aus der Fick'schen Diffusionsgleichung

$$
\frac{\delta c(r, t)}{\delta t}=D\left(\frac{\delta^{2} c(r, t)}{\delta r^{2}}\right)
$$

abgeleitet werden. In der einfachen Smoluchowski-Näherung für die Verteilungsfunktion $c(r, t)$ gilt zum einen die Startbedingung $c(r, t=0)=c_{0}$ und desweiteren die Smoluchowski-Annahme, daß die Reaktionsgeschwindigkeit viel schneller als die Diffusion ist, also jeder Stoß reaktiv ist [45]. Als ein Stoß wird dabei die Annäherung auf den Kontaktabstand $R$ betrachtet.

Als stationäre Lösung für Gleichung 2.3 ergibt sich unter diesen Bedingungen die Lösung:

$$
k_{\text {Smoluchowski }}=4 \pi R D_{A B}
$$


Dabei ist $D_{A B}$ der wechselseitige Diffusionskoeffizient von A zu B. Für den zeitabhängigen Geschwindigkeitskoeffizienten $k(t)$ bedeutet die Smoluchowski-Annahme jedoch, daß $k(t)$ bei $t=0$ unendlich wird. Um diese unphysikalische Lösung zu vermeiden, nahmen Collins und Kimball [46] an, daß jeder Stoß nur mit einer gewissen Wahrscheinlichkeit zur Reaktion führt. Dazu führten sie in die Smoluchowski-Theorie eine entsprechende mathematische Randbedingung ein, diese Collins-Kimball-Randbedingung ist allgemein unter der englischen Bezeichnung radiation boundary condition bekannt geworden.

Obwohl das erweiterte Smoluchoswski-Modell für viele diffusionsgesteuerte Reaktionen gute Übereinstimmung ergibt (vergl. mit Übersichtsartikeln [47 49]) lassen sich nicht alle Reaktion dieser Art so beschreiben, da der Käfigeffekt des Solvens nicht berücksichtigt wird. Im Smulochowski-Modell sind die Stöße auch mit Collins-Kimball-Randbedingung ein Markow-Prozeß, also rein statistischer Natur. Nach einem nicht-reaktiven Stoß besitzen die Stoßpartner kein „Gedächtnis“, also der nächste findet völlig unabhängig vom vorherigen statt. Innerhalb eines Lösungsmittelkäfigs treten Stöße jedoch gehäuft auf, da die Reaktanten räumlich durch die umgebenen Lösungsmittelmoleküle zusammengehalten werden. Sobald also zwei Reaktanten innerhalb eines Käfigs gefangen sind, steigt die Wahrscheinlichkeit, daß auf einen Stoß weitere folgen. Im Vergleich zur Gasphase mit einer statistischen Gleichverteilung von Stößen kommt es in Lösung also zu diffusionskontrollierten Begegnungen, während denen viele Stöße stattfinden.

Die Untersuchung einer Käfigreaktion unter Anwendung der Smoluchowski-Theorie erfolgte durch Noyes et al.. Dabei führten sie zur Photodissoziation und Rekombination von Iod eingehende experimentelle Untersuchungen durch [50 55]. Noyes entwickelte dazu eine Theorie [56], in welcher er das Konzept eines Lösungsmittelkäfigs wie es von Franck und Rabinowitsch postuliert worden war, einzuarbeiten versuchte. Dazu führte er eine Wahrscheinlichkeitsfunktion $f(t)$ ein, welche die zeitabhängige Wahrscheinlichkeit eines weiteren Stoßes der Reaktanten nach einem nicht reaktiven Stoß bei $t=0$ angibt. $f(t)$ ist dabei unabhängig von der vorhergehenden Anzahl von Stößen, liegt also zwischen den beiden Grenzfällen sehr hoher Reaktivität (jeder Stoß führt zur Reaktion) und sehr niedriger Reaktivität (der zeitabhängige Anteil $f(t)$ spielt keine Rolle). Wie Noyes feststellte, ist die Funktion $f(t)$ nicht experimentell zugänglich, ein Versuch der Berechnung mit Hilfe von Diffusiongleichungen führte lediglich zu einer asymptotischen Abschätzung von $f(t)$ bei späten Reaktionszeiten. Somit waren Noyes et al. bei der Interpretation ihrer Daten auf das einfache Smoluchowski-Modell beschränkt. Einen Versuch zur Berechnung der Wahrscheinlichkeitsfunktion $f(t)$ mit Hilfe von modernen Molekular-DynamischenSimulationen (engl. Molecular Dynamics (MD-Simulationen)) unternahmen van Beijeren et al. [57].

So wird in Noyes' Interpretationsansatz die Lösungsmittelumgebung als ein homogenes, viskoses Kontinuum aufgefaßt. Die Iodatome als primäre Photofragmente geben ihre kinetische Überschußenergie aus der Photodissoziation durch viskositätsabhängige Stokes-Reibung an das Lösungsmittel ab. Die anschließende Rekombination erfolgt als ein rein diffusiver Prozeß. Dieses vereinfachte Diffusionsmodell von Noyes et al. konnte jedoch die experimentellen Daten nur bedingt beschreiben, so daß der Käfigeffekt der Photodissoziation von Iod weiterhin im Fokus verschiedenster Untersuchungen blieb. 
Luther und Troe verwendeten anstelle von flüssigen Lösungsmitteln komprimierte Gase [58 und konnten zeigen, daß eine lösungsmittelinduzierte geminale Rekombination bereits weit vor Dichten der flüssigen Phase stattfindet. Darüber hinaus zeigten Messungen zur Photodissoziation von Iod bzw. Brom im Molekularstrahl [59 61, daß eine Rekombination bereits durch einfache Komplexierung des Halogenmoleküls mit einzelnen Solvensmolekülen möglich ist. Ein umfassendes Modell zur Photodissoziation von Iod über einen weiten Dichtebereich in verschiedenen Lösungsmittelumgebungen wurde schließlich von Otto, Schroeder und Troe [18] aufgestellt. Das Otto-Schroeder-Troe-Modell (OSTModell) geht dabei von unterschiedlichen, dichteabhängigen Mechanismen der Iodrekombination aus. Bei niedrigen Dichten kommt es zu einer Komplexierung der primären Photofragmente ähnlich dem in Molekularstrahlexperimenten beobachteten „Ein-AtomKäfigeffekt", das angeregte $\mathrm{I}_{2}$ wird durch Solvensmoleküle stabilisiert und gibt seine Überschußenergie durch Dissoziation in $\mathrm{I}_{2}$ und Solvensmolekül ab, nicht komplexierte Iodmoleküle dissoziieren. Bei höheren Dichten erfolgt die Beschreibung durch ein Diffusionsmodell, die Iodatome geben ihre Überschußenergie aus der Dissoziation durch Reibung mit dem Lösungsmittel ab und werden beim Abstand $r_{0}$ auf thermische Geschwindigkeit abgebremst. Von da an setzt eine diffusiv gesteuerte Bewegung ein, die Quantenausbeute ergibt sich aus der Konkurrenz zwischen Energierelaxation der Photofragmente im Käfig und diffusivem Käfigausbruch. Ein direkter, nicht diffusiver Käfigausbruch findet in diesem Modell nicht statt. Messungen von Eisenthal et al. 62 zur Rekombination von Iod mit Pikosekundenauflösung zeigten Dynamiken in der Größenordnung von $\sim 100 \mathrm{ps,}$ welche sie ebenfalls auf diffusiv gesteuerte geminale Rekombination zurückführten.

Theoretische Untersuchungen zum Lösungsmittelkäfig konnte eine andere Sichtweise als die eines rein diffusiven Prozesses etablieren. Northrup und Hynes [63, 64] modellierten den Lösungsmittelkäfig mit Hilfe von Lennard-Jones-Potentialen und der SmoluchowskiGleichung. Sie fanden abweichend zur Beschreibung des Lösungsmittels durch ein viskoses Kontinuum für kurze Abstände einen Lösungsmitteleinfluß auf das Potential zwischen den Reaktanten sowie einen dynamischen, separationsabhängigen Diffusionskoeffizienten. Die direkte Rekombination innerhalb des Käfigs vor Einsetzen eines diffusiven Prozesses wurde durch weitere Rechnungen bestätigt, Bunker und Jakobsen [65] sagten mit Hilfe von Monte-Carlo-Experimenten dafür eine Zeitskala von $\lesssim 10$ ps vorraus. Die von Eisenthal et al. 62 gemessene Dynamik von $\sim 100$ ps wurde deshalb entweder auf sekundäre, diffusiv gesteuerte Rekombination oder Schwingungsenergietransfer (VER) des nach Rekombination schwingungsangeregten Iods [66, 67] zurückgeführt. Weitere theoretische Untersuchungen zur Iodrekombination in komprimierten Edelgasen [68-71] unterstützen das Bild einer primären Rekombination auf einer Femtosekundenzeitskala, die sekundäre diffusiv gesteuerte Rekombination ist von untergeordneter Bedeutung.

Die Verfügbarkeit von Spektroskopiemethoden in Piko- bzw. Femtosekundenauflösung half schließlich, das Bild des Käfigeffekts der Ioddissoziation zu vervollständigen. Hierbei seien beispielsweise die Arbeiten von Zewail et al. [20, 72, 75, Scherer et al. [76, 77] oder Zadoyan et al. [78, genannt. Die genaue Kenntnis der beteiligten Potentialzustände des $\mathrm{I}_{2}$ (s. Abbildung 2.2) ermöglicht eine zuverlässige Zuordnung der bei verschiedenen Wellenlängen gemessenen Dynamiken. 


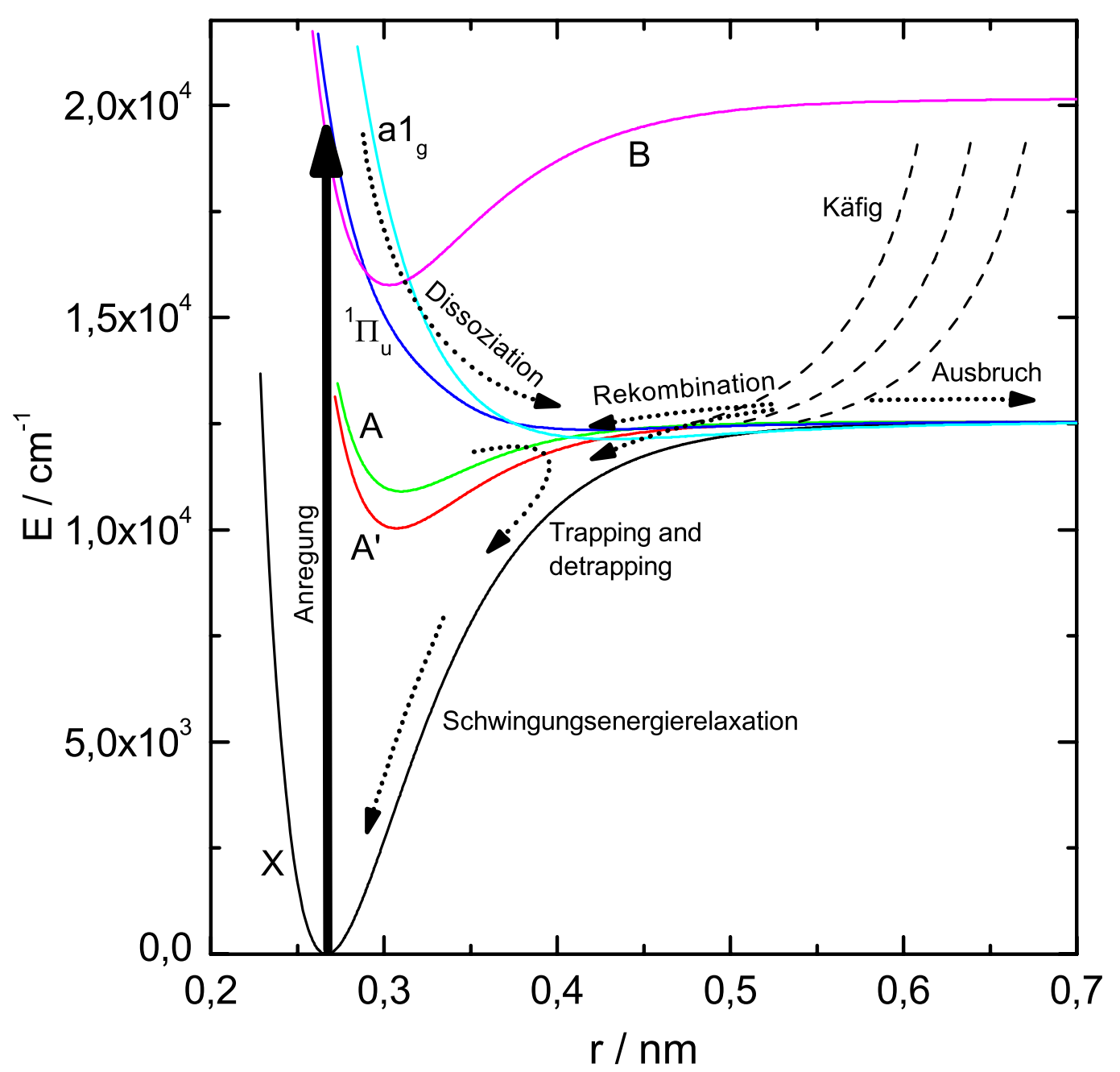

Abbildung 2.2: Relevante Potentialkurven von $\mathrm{I}_{2}$ zur Photodissoziation und rekombination [19]

Den Prozeß der Photodissoziation und -rekombination kann man vereinfacht wie folgt zusammenfassen: Nach der Anregung in einen dissoziativen oder prädissoziativen $B$ Zustand, (s. Potentialdiagramm 2.2 kommt es zu einer schnellen Dissoziation, das generierte Wellenpaket läuft aus der ursprünglich angeregten Franck-Condon-Region heraus und führt zu einer Separierung der Iodatome. Diese können nun entweder aus dem Käfig ausbrechen oder werden von der Käfigwand reflektiert, was zu einer Rekombination auf dem Grundzustandspotential oder einem schwach gebundenen A/A'-Potential führt (engl. trapping). Dieser Prozeß der Dissoziation und Käfigrekombination ist innerhalb von 1-2 ps beendet, Diffusion spielt auf dieser Zeitskala keine Rolle. Der Käfigausbruch ist dabei ein rein kinematischer Prozeß, also nur von der kinetischen Energie der Photofragmente und der Packungsdichte des Lösungsmittels abhängig. Messungen der Ioddissoziationsquantenausbeute in überkritischen Lösungsmitteln von Schwarzer etal. [19] konnten einen direkten linearen Zusammenhang zwischen Quantenausbeute und reduzierter 
Dichte als Maß für die Packungsdichte des Lösungsmittels zeigen. Anschließend wird das schwingungsangeregte Rekombinationsprodukt durch nachfolgende Schwingungsenergierelaxation (VER) auf einer Pikosekundenzeitskala (Relaxationszeiten sind lösungsmittelabhängig) stabilisiert. Die im A/A'-Zustand „gefangenen“ Iodmoleküle können durch strahlungslosen oder strahlenden Übergang in den Grundzustand übergehen, die Relaxationszeiten sind dabei vom Lösungsmittel abhängig und reichen von 60 ps in Alkanen bis hin $\mathrm{zu} 2,7 \mathrm{~ns}$ in $\mathrm{CCl}_{4}$.

Die wichtigsten Aspekte des Käfigeffekts lassen sich also wie folgt zusammenfassen:

1. Kinematischer Käfigeinfang

Nach der Dissoziation brechen die primären Photofragmente entweder auf einer Femtosekundenzeitskala aus dem Käfig aus oder bleiben darin gefangen und können rekombinieren. Die Konkurrenz dieser beiden Prozesse wird durch die Packungsdichte des Lösungsmittels gesteuert, es handelt sich um einen rein kinematischen Effekt.

2. Schwingungsenergierelaxation

Die Überschußenergie, welche die Photofragmente und insbesondere die Rekombinationsprodukte in sich tragen, wird über Stöße durch Energietransfer ins Lösungsmittel abgeben. Dadurch können die Rekombinationsprodukte stabilisiert werden.

3. Diffusion

Erst auf einer viel späteren Zeitskala (Piko-/Nanosekundenbereich) setzt eine diffusive Bewegung ein, die zu sekundären geminalen Rekombinationen oder nichtgeminaler Rekombination führen kann.

Im Falle der Ioddissoziation ergibt sich für den Käfigeffekt ein genaues und umfassendes Bild. Deswegen ist es möglich und wünschenswert die Untersuchung des Käfigeffekts auf komplexere Moleküle zu übertragen. Insbesondere zur Untersuchung des Einflusses von Schwingungsenergierelaxation sind größere Moleküle von Interesse, die in polyatomare Photofragmente zerfallen, welche neben der Translation auch innere Freiheitsgrade wie Rotation und Schwingung besitzen. Als nächstkomplexere Modellsysteme für den Käfigeffekt bieten sich nach $\mathrm{I}_{2}$ die Polyhalogenmethane an. Im folgenen Kapitel 3 sollen daher verschiedene Polyhalogenmethane mit ihren chemischen und physikalischen Eigenschaften in Bezug auf den Käfigeffekt vorgestellt werden.

\subsection{Solvation in überkritischen Lösungsmitteln}

Ein Fluid ist dann überkritisch, wenn seine Temperatur und Druck über dem stoffspezifischen kritischen Punkt liegen. Überkritische Lösungsmittel besitzen einige interessante Eigenschaften, welche sie oft zu einer Alternative herkömmlicher Lösungsmittel machen. Das Hauptaugenmerk liegt dabei darauf, daß überkritische Lösungsmittel im Gegensatz 


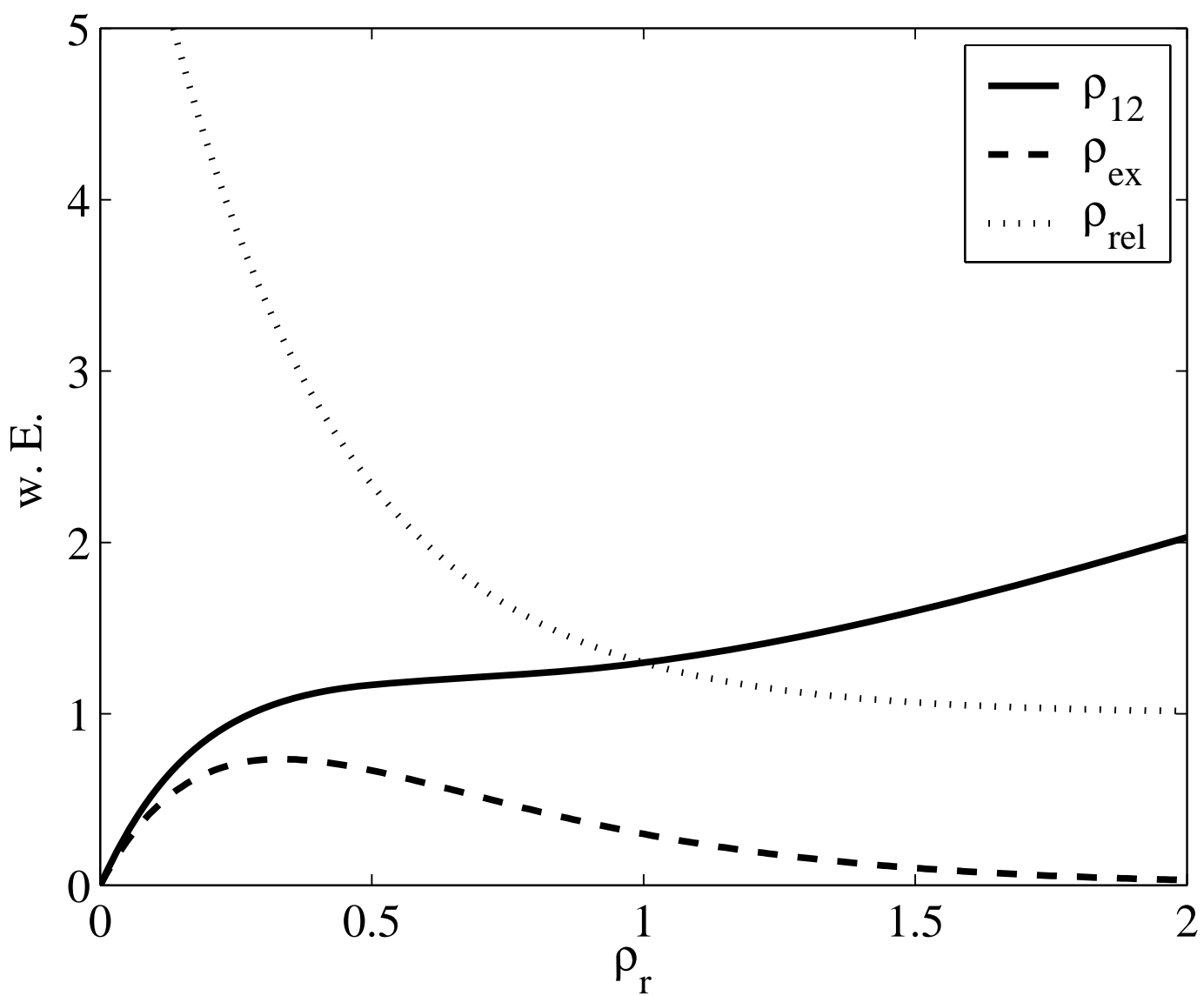

Abbildung 2.3: Auftragung der lokalen Dichte $\rho_{12}$, der lokalen Dichtevergrößerung $\rho_{\text {ex }}$ und der relativen lokalen Dichte $\rho_{\text {rel }}$ in überkritischen Fluiden nahe des kritischen Punkts

zu Flüssigkeiten gut komprimierbar sind, durch Variation des Druckes können Eigenschaften wie Dichte, Polarisierbarkeit, Viskosität u.ä. geändert werden. Die Lösungseigenschaften eines überkritischen Gases (z. B. $\mathrm{CO}_{2}$ ) können dabei annähernd so gut wie die eines organischen Lösungsmittels sein.

Eine weitere besondere Eigenschaft eines überkritischen Fluids ist die Inhomogenität insbesondere im Bereich des kritischen Punktes. Aufgrund attraktiver oder repulsiver Wechselwirkungen bilden sich fluktuierende Bereiche höherer bzw. niedriger Dichte heraus [79], dabei kommt es in dem System zu einem Entropieverlust, welcher jedoch durch den Enthalpiegewinn (Annäherung der Teilchen in den Potentialtopf der attraktiven Wechselwirkung) der Clusterbildung kompensiert wird. Im Falle einer attraktiven Mischung aus Gelöstem und überkritischen Fluid ist das Gelöste in einen Cluster aus Solvensmolekülen eingelagert, die lokale Dichte um das Gelöste $\rho_{12}$ ist somit größer als die homogene Dichte des Lösungsmittels: $\rho_{12}>\rho_{11}$ (Indizes: $1=$ Lösungsmittel, $2=$ Gelöstes). Die lokale Dichtevergrößerung $\rho_{\mathrm{ex}}$ (eng. local density augmentation, $\rho_{\mathrm{ex}}=\rho_{12}-\rho$ ) steigt bei niedrigen Dichten stark an, erreicht bei etwa einem Drittel der kritischen Dichte $\rho_{\mathrm{k}}$ ein Maximum und fällt danach wieder ab (s. Abbildung 2.3). Dadurch ergibt sich für den 
dichteabhängigen Verlauf der lokalen Dichte $\rho_{12}=\rho+\rho_{\text {ex }}$ ein Plateau im Bereich der kritischen Dichte anstelle eines linearen Anstiegs. Die relative Dichte $\rho_{\text {rel }}=\rho_{12} / \rho$ fällt von niedrigen Dichten monoton ab und läuft schließlich gegen 1 [80. Bei Temperaturen von $T_{r}=T / T_{k} \geq 1,05$ sind in der Regel keine lokalen Dichtevergrößerungseffekte mehr zu beobachten, die Cluster um das Gelöste werden mit zunehmender Temperatur kleiner und nähern sich der homogenen Dichte an. [81].

Durch Messung einer von der lokalen Dichte linear abhängigen Größe kann man indirekt den Verlauf der lokalen Dichte $\rho_{12}$ bestimmen, in diesem Fall erhält man für die Observable charakteristische Kurven, welche bei niedrigen Dichten stark ansteigen, anschließend ein Plateau im Bereich der kritischen Dichte bilden und danach proportional zur homogenen Dichte des Lösungsmittels laufen. Messungen solcher Größen wurden Ende der 90er Jahre beispielweise in den Arbeitsgruppen von Fayer [82,83], Schwarzer [13, 84] oder auch Brennecke [85] vorgenommen. Weiterhin kann man die Solvatationsstruktur durch Modellierung mit Monte-Carlo- oder MD-Simulationen bestimmen [86]. 


\section{Die Polyhalogenmethane}

In dieser Arbeit wurden die Dihalogenmethane $\mathrm{CH}_{2} \mathrm{BrI}$ und $\mathrm{CH}_{2} \mathrm{ClI}$ und die Reihe der Polyiodmethane $\mathrm{CH}_{3} \mathrm{I}, \mathrm{CH}_{2} \mathrm{I}_{2}, \mathrm{CHI}_{3}$ als Modellsysteme für den photolytischen Käfigeffekt untersucht.

\subsection{Physikalische und chemische Eigenschaften}

Zur Struktur und den Bindungsverhältnissen der für diese Arbeit relevanten Polyhalogenmethane (s. Abbildung 3.1) existieren viele experimentelle Untersuchungen z. B. durch Mikrowellen- und Infrarotspektroskopie, Elektronenbeugung in der Gasphase und am Kristallgitter 87 93 sowie theoretische Berechnungen 94. Marshall et al. 94 haben in einer Arbeit über die Thermochemie von brom- und iodhaltigen Methanen sowie Methylradikalen die Strukuren verschiedener Polyhalogenmethane durch ab-initio-Rechnungen auf QCISD/6-311G(d,p)-Niveau berechnet. Ihre Ergebnisse stehen in guter Übereinstimmung mit experimentellen Daten und sind in Tabelle 3.1 zusammengefaßt. So unterscheiden sich die untersuchten Polyhalogenmethane nur wenig in ihrer Struktur, annähernd gleich ist sowohl die $\mathrm{C}-\mathrm{H}$-Bindungslänge (um 1,08 $\mathrm{A}$ ) als auch die $\mathrm{C}$-X-Bindungslänge (um 2,1 $\AA$ ). Die Bindungswinkel weichen je nach Art der Substituierung teils deutlich vom Tetraederwinkel $\left(109,47^{\circ}\right)$ ab.

Polyhalogenmethane sind die wichtigste Quelle halogenhaltiger Kohlenwasserstoffe und spielen als Vorstufe reaktiver Halogenverbindungen und -radikale eine wichtige Rolle beim stratosphärischen Ozonabbau. Diese flüchtigen Polyhalogen-Verbindungen werden aus Meeressalzen freigesetzt oder biogen in den Ozeanen gebildet und gelangen von dort in die unterste Atmosphärenschicht, der maritimen Grenzschicht (engl. marine-boundarylayer, MBL). Durch Photolyse gebildete Abbauprodukte (insbesondere Iodatome) spielen eine wichtige Rolle in der Atmosphärenchemie, sie tragen in besonderem Maße zum Ozonabbau bei [95, 96.

\begin{tabular}{|lllllll|}
\hline Molekül & $R(\mathrm{C}-\mathrm{H}) / \AA$ & $R(\mathrm{C}-\mathrm{X}) / \AA$ & $\Theta_{\mathrm{HCH}}$ & $\Theta_{\mathrm{HCX}}$ & $\Theta_{\mathrm{XCX}}$ & $I_{a} I_{b} I_{c} / 10^{-135} \mathrm{~kg}^{3} \mathrm{~m}^{6}$ \\
\hline $\mathrm{CH}_{3} \mathrm{I}$ & 1,088 & 2,146 & $110,9^{\circ}$ & $108,0^{\circ}$ & & $6,79 \times 10^{-2}$ \\
$\mathrm{CH}_{2} \mathrm{I}_{2}$ & 1,086 & 2,143 & $111,2^{\circ}$ & $107,5^{\circ}$ & $115,9^{\circ}$ & 73,3 \\
$\mathrm{CHI}_{3}$ & 1,084 & 2,155 & & $105,7^{\circ}$ & $113,0^{\circ}$ & $5,11 \times 10^{3}$ \\
$\mathrm{CH}_{2} \mathrm{BrI}$ & 1,086 & 1,945 & $111,3^{\circ}$ & $107,9^{\circ}$ & $114,9^{\circ}$ & 33,1 \\
\hline
\end{tabular}

Tabelle 3.1: Strukturparameter Polyhalogenmethane nach Marshall [94] 

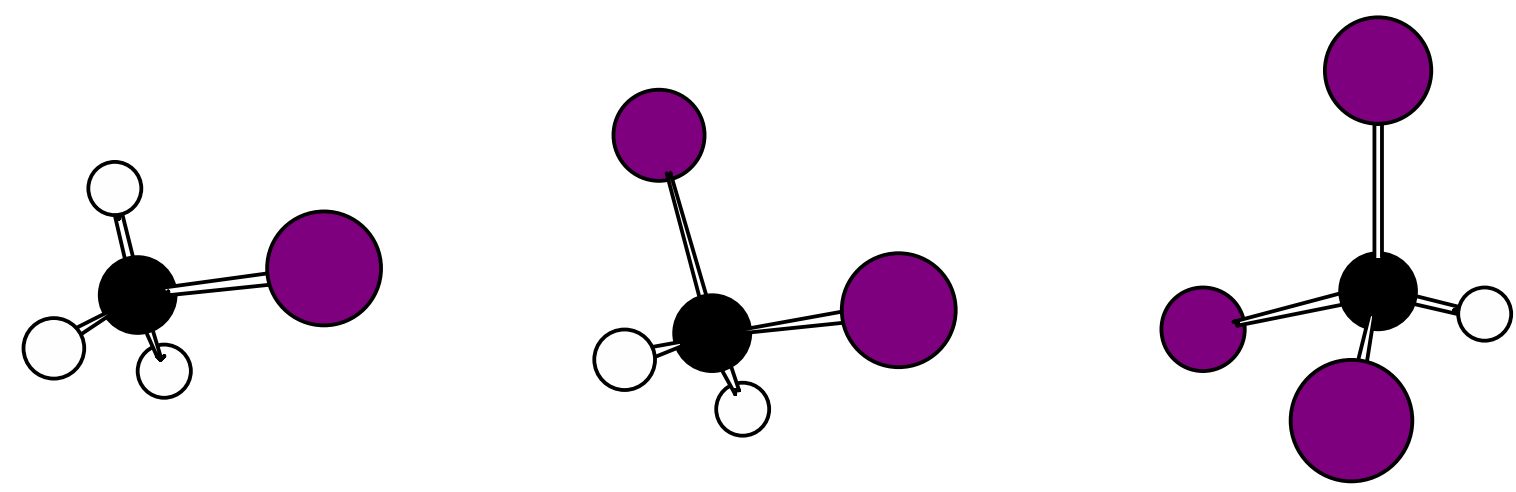

Abbildung 3.1: Strukturformeln von Iodmethan, Diiodmethan und Triiodmethan (Iodoform)

Für die Menge an freigesetzten Polyhalogenmethanen ist entscheidend in welchem Maße sie bereits durch Photolyse im Ozean abgebaut wurden. Die bisherigen Untersuchungen des Mechanismus der photoinduzierten Dissoziationsdynamik dieser Verbindungen in fluider Phase werden in Abschnitt 3.5 behandelt.

Wichtige Bedeutung in der organischen Synthese kommt $\mathrm{CH}_{2} \mathrm{I}_{2}$ z. B. in der SimmonsSmith-Synthese zu [97 99], es dient dabei als Reaganz zur Cyclopropanierung von Olefinen.

\subsection{Photodissoziation der Polyhalogenmethane in der Gasphase}

Die Photodissoziation von Polyhalogenmethanen in der Gasphase insbesondere von $\mathrm{CH}_{2} \mathrm{I}_{2}$ und $\mathrm{CH}_{3} \mathrm{I}$ ist bis heute oftmals Gegenstand sowohl theoretischer als auch experimenteller Untersuchungen gewesen. So veröffentlichte Mulliken bereits 1940 eine Arbeit über die Photoanregung von Alkylhalogeniden [100], in der er die unterschiedlich stark ausgeprägten UV-Absorptionsbanden verschiedener Alkylhalogenide einem elektronischen $n \rightarrow \sigma^{*}$-Übergang zuordnet. Dabei wird ein nicht-bindendes Elektron eines am Halogen lokalisierten Orbitals mit $p$-Charakter in ein anti-bindendes $\sigma^{*}$-Orbital der C-X-Bindung angeregt. Kimura und Nagakura [101] ordnen die Absorptionsbanden längerer Wellenlängen als $200 \mathrm{~nm}$ ebenfalls einem $n \rightarrow \sigma^{*}$-Übergang zu, charakteristisch für diesen $n \rightarrow \sigma^{*}$-Übergang ist spektrale Verschiebung der Absorptionsbande zu kürzeren Wellenlängen bei polaren Lösungsmitteln. Dabei wird der Grundzustand durch das polare Lösungsmittel stabilisiert und energetisch abgesenkt. Die bei allen Alkylhalogeniden vorhandene starke Absorptionsbande bei $194 \mathrm{~nm}$ ist nach Kimura dem ersten RydbergÜbergang $n \mathrm{p}(\mathrm{I}) \rightarrow n \mathrm{~s}(\mathrm{I})$ zuzuordnen.

Eine Anregung in der ersten Absorptionsbande $(\lambda>200 \mathrm{~nm})$ der untersuchten Polyhalogenmethane führt somit zu einer Dissoziation in ein Iodatom und ein $\mathrm{CH}_{2} \mathrm{X}$ - bzw. 


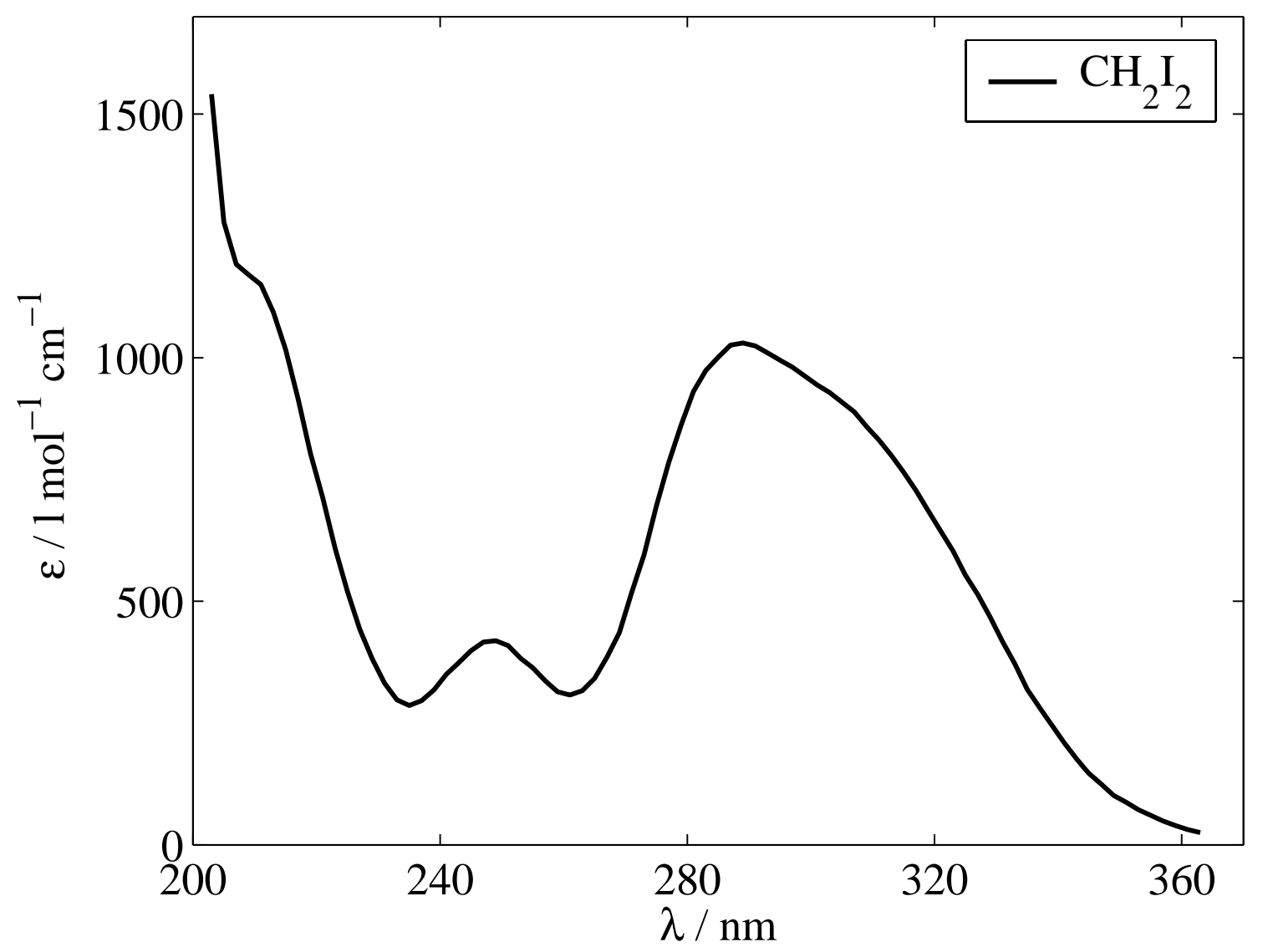

Abbildung 3.2: Gasphasen-Absorptionsspektrum von Diiodmethan [102]

$\mathrm{CHI}_{2}$-Radikal. Eine Abspaltung von molekularem Iod (bei $\mathrm{CH}_{2} \mathrm{I}_{2}$ und $\mathrm{CHI}_{3}$ ) bzw. einem I-X-Molekül (bei $\mathrm{CH}_{2} \mathrm{BrI}$ und $\mathrm{CH}_{2} \mathrm{ClI}$ ) ist nur durch eine Zwei-Photonen-Anregung möglich, diese wird in Abschnitt 3.2.5 behandelt.

\subsubsection{Diiodmethan}

Eine ausführliche Beschreibung der Photodissoziation von Diiodmethan in der Gasphase findet sich in meiner Diplomarbeit [103] sowie bei Grimm [104] und Zerbs [86]. Deswegen sollen hier nur die wichtigsten Aspekte in Kürze zusammengefaßt werden.

In Abbildung 3.2 ist das UV-Absorptionsspektrum von $\mathrm{CH}_{2} \mathrm{I}_{2}$ dargestellt. Für die erste Absorptionsbande ergeben sich in der Betrachtungweise eines einfachen Exciton-Modells nach Kawasaki [105] 5 erlaubte Übergänge aus Zuständen in der energetischen Reihenfolge $1 B_{1}, 2 B_{1}, B_{2}, 1 A_{1}$ und $2 A_{1}$. Dabei korrelieren die Zustände $1 B_{1}$ und $2 A_{1}$ mit der Bildung von einem $\mathrm{CH}_{2} \mathrm{I}$ und einem Iodatom im Grundzustand $\left({ }^{2} \mathrm{P}_{3 / 2}\right)$, während die Übergänge aus $2 B_{1}, B_{2}$ und $1 A_{1}$ zu angeregten Iodatomen $\left({ }^{2} \mathrm{P}_{1 / 2}\right)$ führen. Im Absorptionspektrum von $\mathrm{CH}_{2} \mathrm{I}_{2}$ lassen sich allerdings nur vier gaußförmige Banden zentriert bei 209, 249, 284 und $312 \mathrm{~nm}$ auflösen, die Übergange aus dem $B_{2^{-}}$sowie $1 A_{1}$-Zustand 
bei $249 \mathrm{~nm}$ lassen sich experimentell nicht auflösen. Neue theoretische Untersuchungen der Photodissoziation von $\mathrm{CH}_{2} \mathrm{I}_{2}$ unter Berücksichtigung relativistischer Effekte und der Spin-Bahndrehimpuls-Kopplung finden sich bei Liu et al. 106. Für die beiden tiefsten Singulett-Zustände errechneten Liu etal. im Gegensatz zu Kawasaki unterschiedliche Symmetrien $B_{1}$ und $B_{2}$, welcher beide $B_{1}$ zuordnet. Beide Zustände sind repulsiv und führen zu schnellen Dissoziationen in Grundzustandsprodukte. Die Spin-BahndrehimpulsKopplung des tiefsten Singulett-Zustands $2^{1} A$ bzw. $1^{1} B_{2}$ in $C_{2 v}$-Symmetrie führt zu einer Aufspaltung in zwei Dissoziationskanäle, einer korreliert mit der Bildung von I $\left({ }^{2} \mathrm{P}_{3 / 2}\right)$, der andere mit I* $\left({ }^{2} \mathrm{P}_{1 / 2}\right)$. Der nächsthöhere Singulett-Zustand $3^{1} A\left(1^{1} B_{2}\right.$ in $C_{2 v}$-Symmetrie) führt nur zur Bildung von angeregtem Iod. Dieses steht in guter Übereinstimmung mit experimentellen Daten, daß das Verhältnis von angeregtem Iod I* zu Grundzustandsiod I abhängig von der verwendeten Anregungswellenlänge ist. Bei langwelliger Anregung wird nur der $1^{1} B_{2}$-Zustand besetzt, bei kurzwelligerer Anregung spielt der energetisch höhere $1^{1} B_{1}$-Zustand zunehmend eine Rolle und das I*/I-Verhältnis wird größer. Messungen von Hunter [107] sowie Koffend [108] zeigen dafür eine annähernd lineare Abhängigkeit, das Verhältnis I*/I fällt von ca. 50 v. H. bei $240 \mathrm{~nm}$ auf nahezu Null bei $340 \mathrm{~nm}$ Anregungswellenlänge ab [86, 103].

\subsubsection{Chloriodmethan}

Das Absorptionspektrum von $\mathrm{CH}_{2} \mathrm{ClI}$ ist in Abbildung 3.3 dargestellt, es ähnelt dem von $\mathrm{CH}_{3} \mathrm{I}$ bestehend aus einer breiten Bande zentriert bei $271 \mathrm{~nm}$, welche mit einem $n \rightarrow \sigma^{*}$-Übergang an der C-I-Bindung verknüpft ist [110]. Die leichte Rotverschiebung im Vergleich zu $\mathrm{CH}_{3} \mathrm{I}$ erklärt sich durch die Anwesenheit des Chloratoms, welches das anti-bindende $\sigma^{*}(\mathrm{C}-\mathrm{I})$-Orbital stärker als das nicht-bindende Grundzustandsorbital n(I) stabilisiert. An diese $A$-Bande schließt sich bei $225 \mathrm{~nm}$ die sehr intensive $B$-Bande zentriert um $192 \mathrm{~nm}$ an, sie ist dem ersten Rydberg-Übergang am Iodatom zuzuordnen. Die $C$-Bande, welche ihren Ursprung im $\mathrm{C}$-Cl-Chromophor des Moleküls hat, liegt bei $173 \mathrm{~nm}$ und ist im dargestellten Spektrum nicht mehr abgebildet. Für die Photodissoziation von $\mathrm{CH}_{2} \mathrm{ClI}$ mit Anregungswellenlänge $>250 \mathrm{~nm}$ ist somit nur die $A$-Bande von Belang, welche zur Dissoziation in ein $\mathrm{CH}_{2} \mathrm{Cl}$-Radikal und ein Iodatom führt. Wie im Falle des $\mathrm{CH}_{2} \mathrm{I}_{2}$ kann dabei entweder ein Iodatom im angeregten Zustand $\left({ }^{2} \mathrm{P}_{1 / 2}\right)$ oder im Grundzustand $\left({ }^{2} \mathrm{P}_{3 / 2}\right)$ gebildet werden. Untersuchungen von Senapati et al. [110] zeigen keine eindeutige Abhängigkeit des I*/I-Verhältnisses von der Anregungswellenlänge, im Wellenlängenbereich von 220-280 nm gibt es einen leichten Anstieg von 0,45 auf 0,55, um bei Anregungswellenlängen $>300 \mathrm{~nm}$ auf etwa 0,3 abzufallen. In ihrem Modell zur Photodissoziation von $\mathrm{CH}_{2} \mathrm{ClI}$ korreliert der $3 A^{\prime}$ - sowie der $5 A^{\prime}$-Zustand mit der Bildung von I-Atomen, während der $4 A^{\prime}$-Zustand zu angeregtem I* führt. Weiterhin ist es möglich, daß auch bei Anregung in die $A$-Bande ein geringer Anteil an Chloratomen durch Energietransfer zwischen dem C-I-Chromphor und C-Cl-Chromophor gebildet wird [111]. Diese Kopplung ist jedoch sehr schwach [109]. 


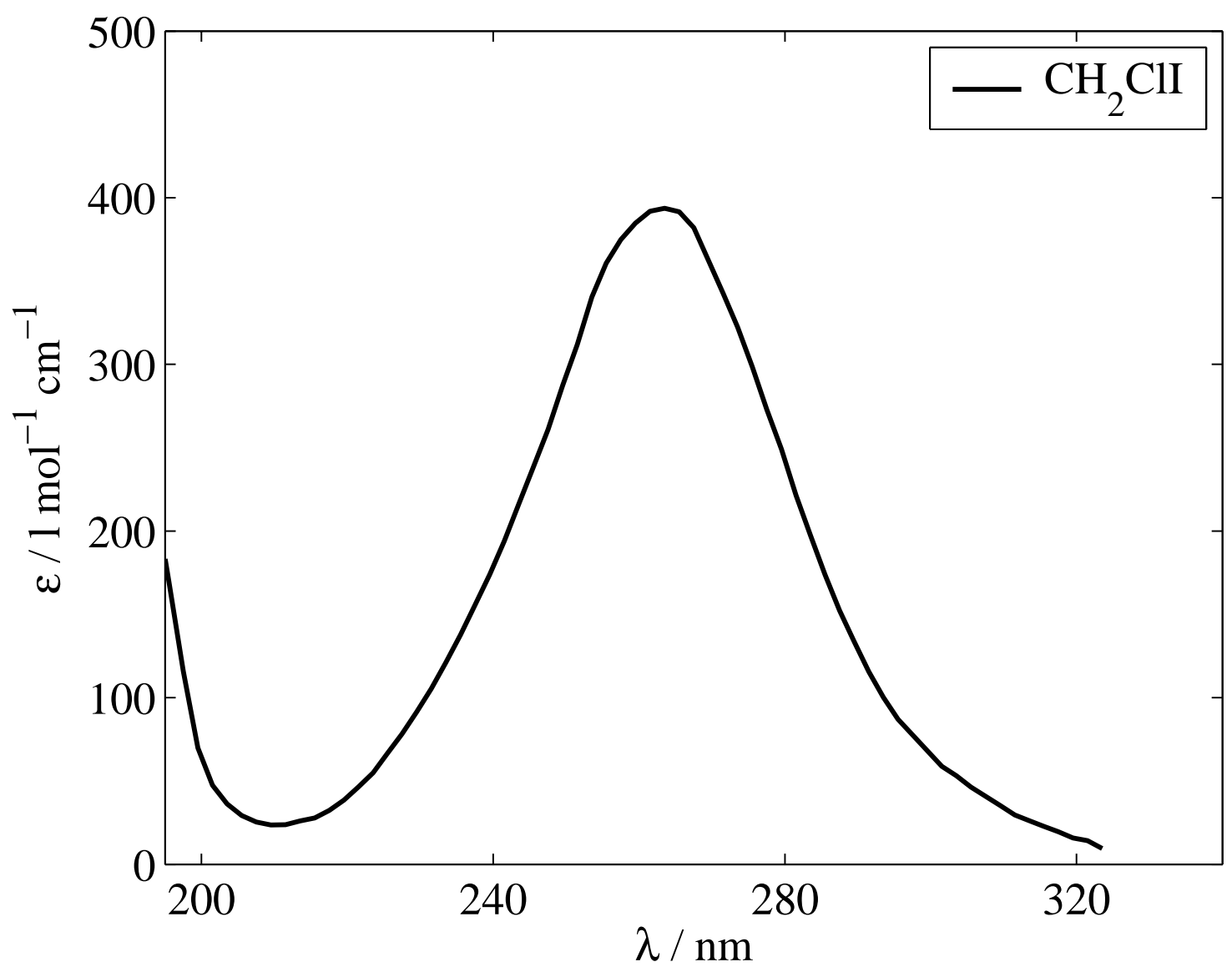

Abbildung 3.3: Gasphasen-Absorptionsspektrum von Chloriodmethan 109]

\subsubsection{Bromiodmethan}

Das Absorptionsspektrum von $\mathrm{CH}_{2} \mathrm{BrI}$ (s. Abbildung 3.4) besteht aus zwei Banden, welche einem $n(\mathrm{I}) \rightarrow \sigma^{*}(\mathrm{C}-\mathrm{I})$-Übergang bei $268 \mathrm{~nm}$ und einem $n(\mathrm{Br}) \rightarrow \sigma^{*}(\mathrm{C}$-Br)-Übergang bei $213 \mathrm{~nm}$ zugeordnet werden können [113, 114]. Im Vergleich zum $\mathrm{CH}_{2} \mathrm{ClI}$ kommt neben dem C-I-Chromophor auch dem C-Br-Chromophor eine verstärkte Bedeutung zu, so daß sich bei einer Anregungswellenlänge > $200 \mathrm{~nm}$ zwei Dissoziationskanäle öffnen:

$$
\begin{aligned}
& \mathrm{CH}_{2} \mathrm{BrI} \stackrel{\mathrm{h} \nu}{\longrightarrow} \mathrm{CH}_{2} \mathrm{Br}+\mathrm{I} \\
& \mathrm{CH}_{2} \mathrm{BrI} \stackrel{\mathrm{h} \nu}{\longrightarrow} \mathrm{CH}_{2} \mathrm{I}+\mathrm{Br}
\end{aligned}
$$

Untersuchungen zur Anregungswellenlängenabhängigkeit wurden von Butler et al. [114] und Lee 113 durchgeführt. Bei einer Anregung in die erste Absorptionsbande, dem C-I-Chromophor, bei $258 \mathrm{~nm}$ ergab die massenspektrometrische Analyse der Photofragmente einen Anteil von 86 v. H. Iodatomen und 14 v. H. Bromatomen. Eine Anregung im 


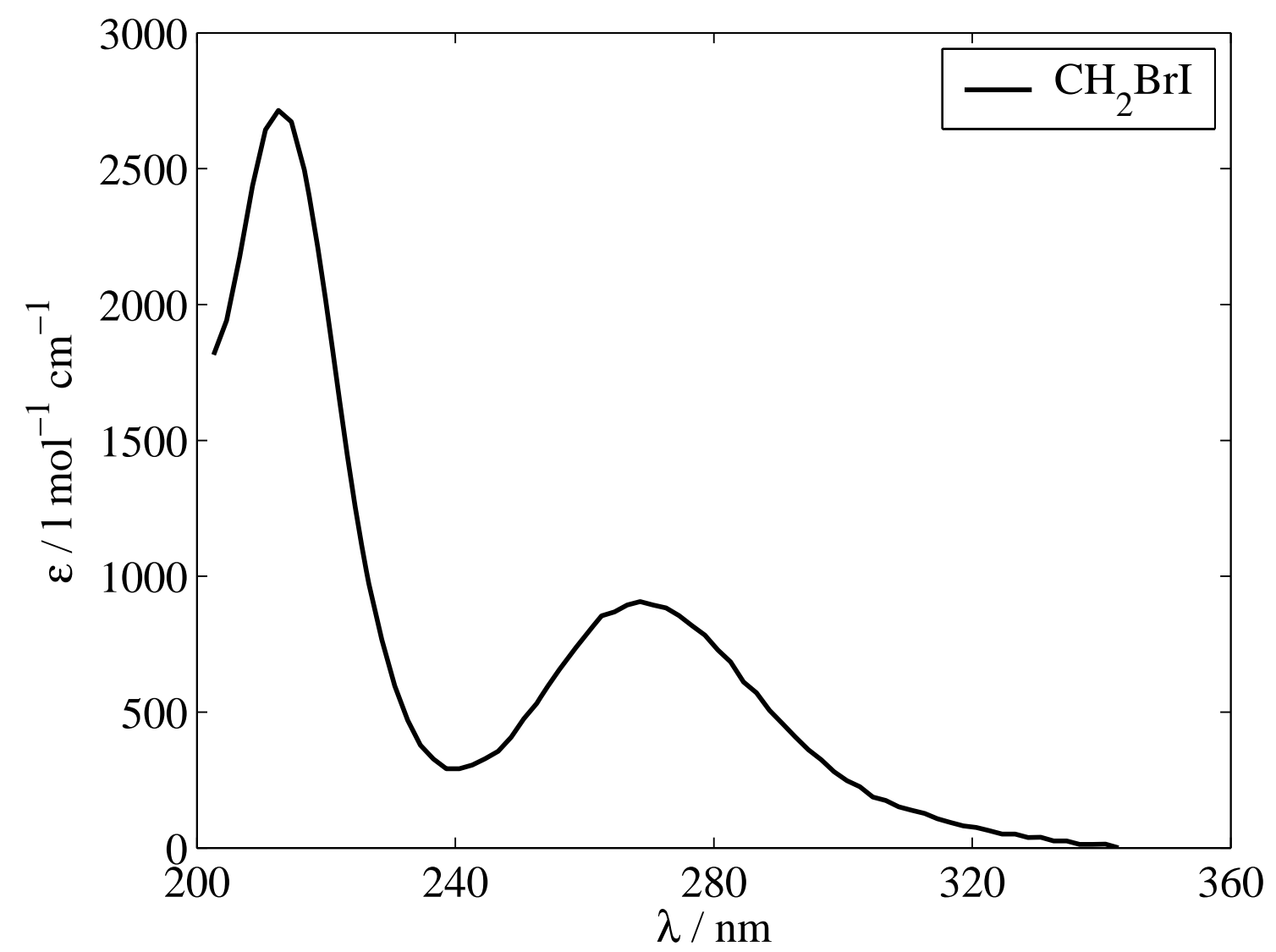

Abbildung 3.4: Gasphasen-Absorptionsspektrum von Bromiodmethan [112]

Überlappungsbereich der beiden Banden (248 nm) führt zu einem Verhältnis von gebildeten Iodatomen zu Bromatomen von 1,2. Der Reaktionskanal R1 dominiert in diesem Wellenlängenbereich nur noch schwach, dabei werden Iodatome im Grundzustand und angeregte Iodatome etwa im Verhältnis 1:0,75 gebildet. Bei einer Anregung von $210 \mathrm{~nm}$ wird kein Iod mehr abgespalten, nur Bromatome über Kanal R2. Weiterhin zeigten Abrashkevich et al. [115], daß sich die Selektivität der gespaltenen C-X-Bindung nicht nur über die Anregungswellenlänge, sondern auch durch kohärente Kontrolle des Anregungslaserpulses bei fester Wellenlänge steuern läßt.

\subsection{4 lodoform}

Beim Absorptionsspektrum des Iodoforms (s. Abbildung 3.5 zeigt sich die Aufspaltung in vier verschiedene Banden (bei 233, 274, 306 und $350 \mathrm{~nm}$ ) vergleichbar zum $\mathrm{CH}_{2} \mathrm{I}_{2}$ besonders deutlich. Bei Anregung mit $\lambda>200 \mathrm{~nm}$ findet ausschließlich eine Fragmentierung in ein Iodatom und ein $\mathrm{CHI}_{2}$-Radikal statt.

In der Betrachtungsweise des Exciton-Modells von Kawasaki [105] ergeben sich 5 Energieniveaus $E \sigma, E \pi, E \pi^{\prime}, A_{1} \pi$ und $A 1_{\sigma}$, wobei $E \sigma$ und $A 1_{\sigma}$ mit der Bildung von Iodatomen 


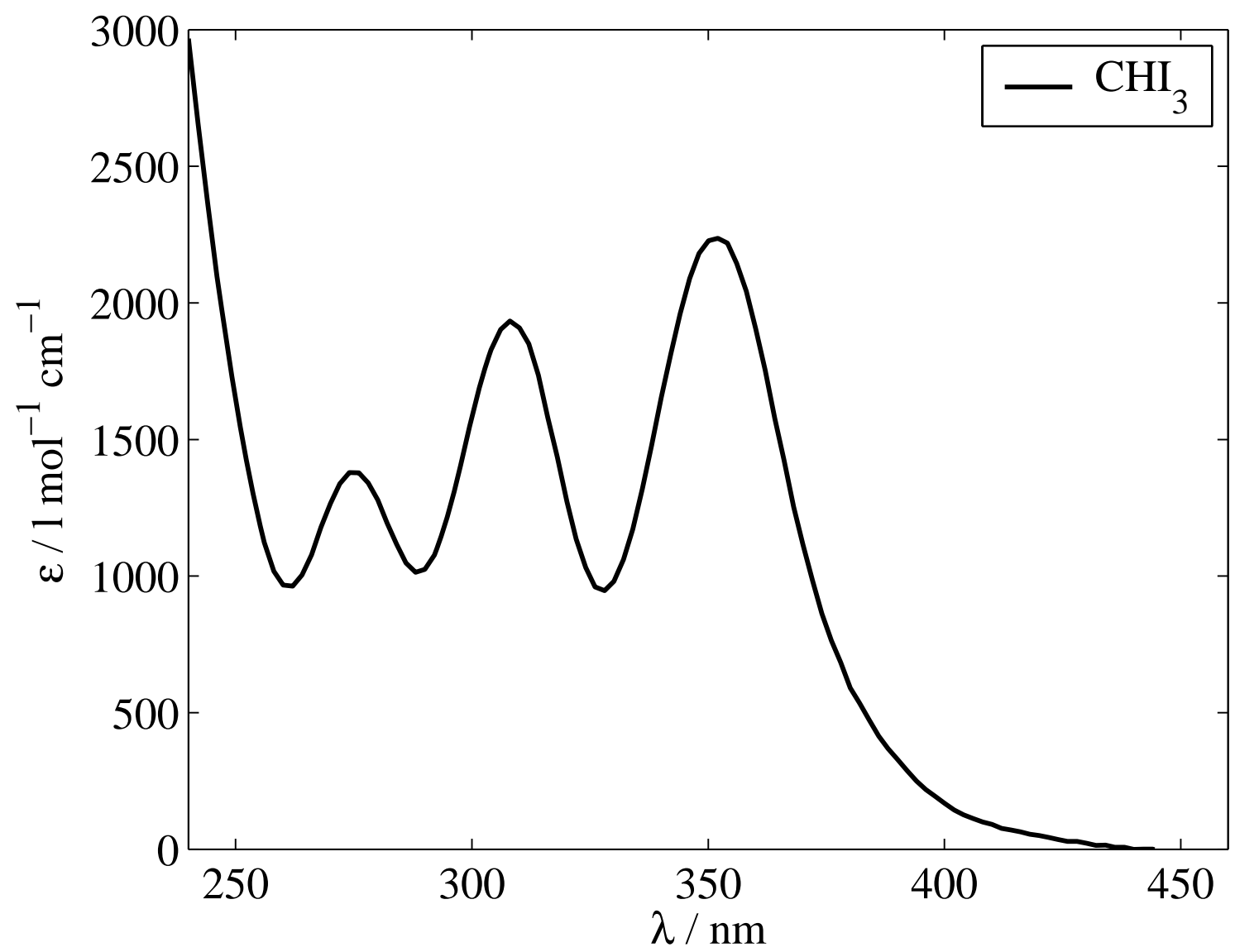

Abbildung 3.5: Absorptionsspektrum von Iodoform in Cyclohexan [116]

im Grundzustand $\left({ }^{2} \mathrm{P}_{3 / 2}\right)$ korrelieren. Übergänge aus den anderen Energieniveaus führen zum angeregten $\mathrm{I}^{*}\left({ }^{2} \mathrm{P}_{1 / 2}\right)$, wobei die beiden Zustände $E \pi, E \pi^{\prime}$ energetisch so nah beisammen liegen, daß im Spektrum nur 4 Banden aufgelöst werden können. Die spektrale Blauverschiebung des Spektrums in polaren Lösungsmitteln ist bei Iodoform besonders ausgeprägt.

\subsubsection{Zwei-Photonen-Photodissoziation der Polyhalogenmethane}

Eine Anregung in die erste Absorptionsbande der Polyhalogenmethane führt in allen bisher untersuchten Fällen zu einer schnellen Abspaltung eines Iodatoms. Andererseits ist z. B. im Falle der $\mathrm{CH}_{2} \mathrm{I}_{2}$-Dissoziation bereits bei Anregungswellenlängen $<333 \mathrm{~nm}$ die Dissoziation in $\mathrm{I}_{2}$ und ein $\mathrm{CH}_{2}$-Carben (s. R3) thermodynamisch möglich, wird jedoch experimentell nicht beobachtet 105.

$$
\mathrm{CH}_{2} \mathrm{I}_{2} \stackrel{\mathrm{h} \nu}{\longrightarrow} \mathrm{CH}_{2}+\mathrm{I}_{2}
$$


Kawasaki führt dieses darauf zurück, daß die Potentialflächen der $B$-Zustände von $\mathrm{CH}_{2} \mathrm{I}_{2}$ eine Knotenebene zwischen den Iodatomen besitzen. Dadurch wird eine abstoßende Wechselwirkung zwischen den Iodatomen bewirkt und somit kann unter Beibehaltung der $C_{2 v}$-Symmetrie die Potentialfläche der $B$-Zustände nicht zu einer Dissoziation in $\mathrm{I}_{2}$-Moleküle führen. Dieses ist nur durch eine Anregung in einen energetisch höheren $A_{1}$ Zustand möglich. Die direkte Bildung von $\mathrm{I}_{2}$ durch Vakuum-UV-Photolyse von $\mathrm{CH}_{2} \mathrm{I}_{2}$ wurde ohne Zeitauflösung bereits in den 50er Jahren durch Style et al. [117, 118] sowie Okabe et al. [119] nachgewiesen, die Ausbeuten sind jedoch verschwindend gering. Analog dazu zeigten Fotakis et al. [120], daß die $\mathrm{I}_{2}$-Abspaltung aus $\mathrm{CH}_{2} \mathrm{I}_{2}$ nicht allein durch VUV-Anregung, sondern auch durch Zwei-Photonen-Anregung bei $248 \mathrm{~nm}$ möglich ist.

Im Falle des $\mathrm{CH}_{2} \mathrm{ClI}$ sind die Ausbeuten des $\mathrm{CH}_{2}$-Dissoziationskanals signifikant größer, Untersuchungen von Schmitt etal. [109] zeigen eine starke quadratische Abhängigkeit zwischen Anregungsintensität und Folgeprodukten wie $\mathrm{C}_{2} \mathrm{H}_{4}$ oder $\mathrm{C}_{2} \mathrm{H}_{2}$, welche aus den primär gebildeten $\mathrm{CH}_{2}$ entstehen (s. R4, R5, R6).

$$
\begin{aligned}
& \mathrm{CH}_{2} \mathrm{ClI} \stackrel{2 \mathrm{~h} \nu}{\longrightarrow} \mathrm{CH}_{2}+\mathrm{ICl} \\
& \mathrm{CH}_{2}+\mathrm{CH}_{2} \mathrm{ClI} \longrightarrow \mathrm{C}_{2} \mathrm{H}_{4}+\mathrm{ICl} \\
& \mathrm{C}_{2} \mathrm{H}_{4} \longrightarrow \mathrm{C}_{2} \mathrm{H}_{2}+\mathrm{H}_{2}
\end{aligned}
$$

Der endgültige Nachweis, daß das $\mathrm{I}_{2}$ konzertiert während der Photodissoziation gebildet wird und nicht erst später durch nicht-geminale Rekombination entsteht, wurde von Marvet und Dantus erbracht [121]. Sie konnten eine kohärente Modulation der Iodfluoreszenz nachweisen, welche nur durch konzertierte Iodabspaltung aus dem Muttermolekül erklärt werden kann.

Weitere Untersuchungen zur konzertierten Abspaltung von $\mathrm{I}_{2}$ bzw. gemischten Dihalogenen (wie ICl oder IBr) aus Polyhalogenverbindungen vervollständigen das Bild dieses Dissoziationskanals [122-125]. Eine Betrachtung der Dissoziation von Dihalogenmethanen $\left(\mathrm{CX}_{2} \mathrm{Y}_{2}\right)$ mit Hilfe der Hückel-Grenzorbitale von Cain et al. [122] zeigt, daß der synchrone, konzertierte Reaktionskanal, also der gleichzeitige Bruch beider $\mathrm{C}$-X-Bindungen unter Beibehaltung der $C_{2 v}$-Symmetrie, eine Barriere besitzt. Diese wird jedoch bei Bruch der $C_{2 v}$-Symmetrie durch Rotation der $\mathrm{CH}_{2}$-Gruppe von der $\mathrm{X}$-X-Achse (X = Halogen) weg abgesenkt. Während dieses asynchronen, jedoch weiterhin konzertierten Mechanismus werden die $\mathrm{C}-\mathrm{X}$-Bindungen nacheinander gebrochen, das resultierende Halogenmolekül wird dabei rotationsangeregt gebildet. Die Anwesenheit der Barriere erklärt die geringen Ausbeuten, welche bei der Zwei-Photonen- bzw. VUV-Anregung von $\mathrm{CH}_{2} \mathrm{I}_{2}$ beobachtet werden. Im Falle von gemischten Dihalogenmethanen wie $\mathrm{CH}_{2} \mathrm{ClI}$ oder $\mathrm{CH}_{2} \mathrm{BrI}$, welche keine $C_{2 v}$-Symmetrie besitzen, ist die Barriere für die Abspaltung ICl oder IBr nicht vorhanden [124]. Die Dissoziation findet also nach dem konzertierten, synchronen Mechanismus statt. In Übereinstimmung zu diesem barrierelosen Vorgang zeigt sich, daß 


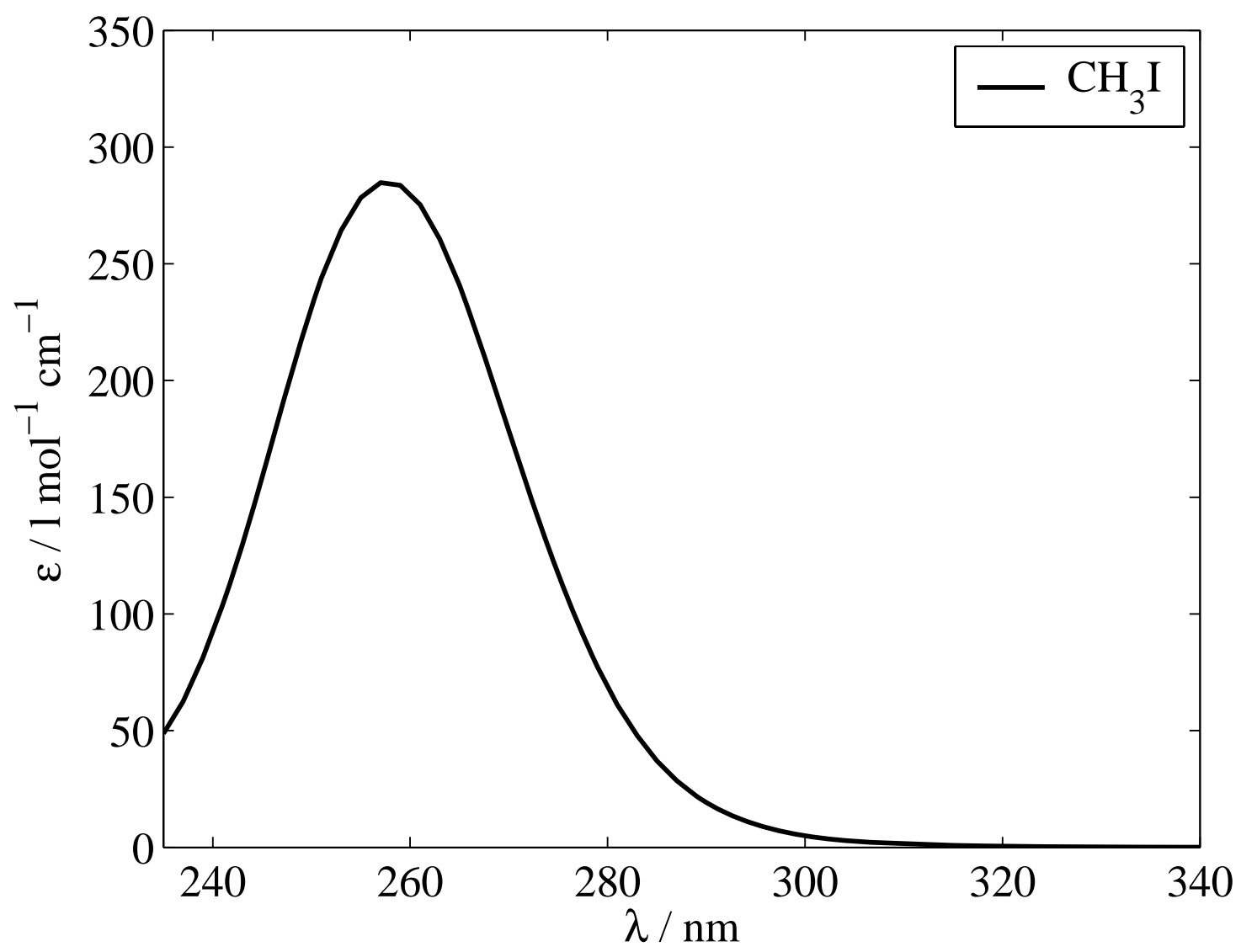

Abbildung 3.6: Gasphasen-Absorptionsspektrum von Iodmethan [126]

die Photoprodukte der Zwei-Photonen-Dissoziation von $\mathrm{CH}_{2} \mathrm{ClI}$ nach Schmitt [109] (s. R4, R5, R6) einen deutlich meßbaren Beitrag zur Photolyse von $\mathrm{CH}_{2} \mathrm{ClI}$ liefern.

Für die Zwei-Photonen-Photolyse von $\mathrm{CHI}_{3}$ wurden von Tweeten etal. [125] LIFUntersuchungen und ab-initio-Berechnungen vorgenommen. Dabei besitzt $\mathrm{CHI}_{3}$ im Übergangszustand ebenfalls keine $C_{2 v^{-}}$, sondern $C_{3 v^{-}}$Symmetrie, welche durch die Dissoziation in ein $\mathrm{I}_{2}$-Molekül ohnehin gebrochen wird. Dadurch besitzt der molekulare Dissoziationskanal von $\mathrm{CHI}_{3}$ ebenfalls eine wesentlich kleinerer Barierre als $\mathrm{CH}_{2} \mathrm{I}_{2}$.

\subsection{Photodissoziation von lodmethan der Gasphase}

Die Photodissoziation von Iodmethan gehört wohl zu den am besten untersuchten Modellsystemen für die Photodissoziationsdynamik kleiner Moleküle in der Gasphase. In den vergangenen 30 Jahren wurde sowohl eine Vielzahl experimenteller als auch theoretischer Untersuchungen durchgeführt, so daß sich für die Photodissoziation und die beteiligten Zustände und Photofragmente ein detailliertes Bild ergibt. 


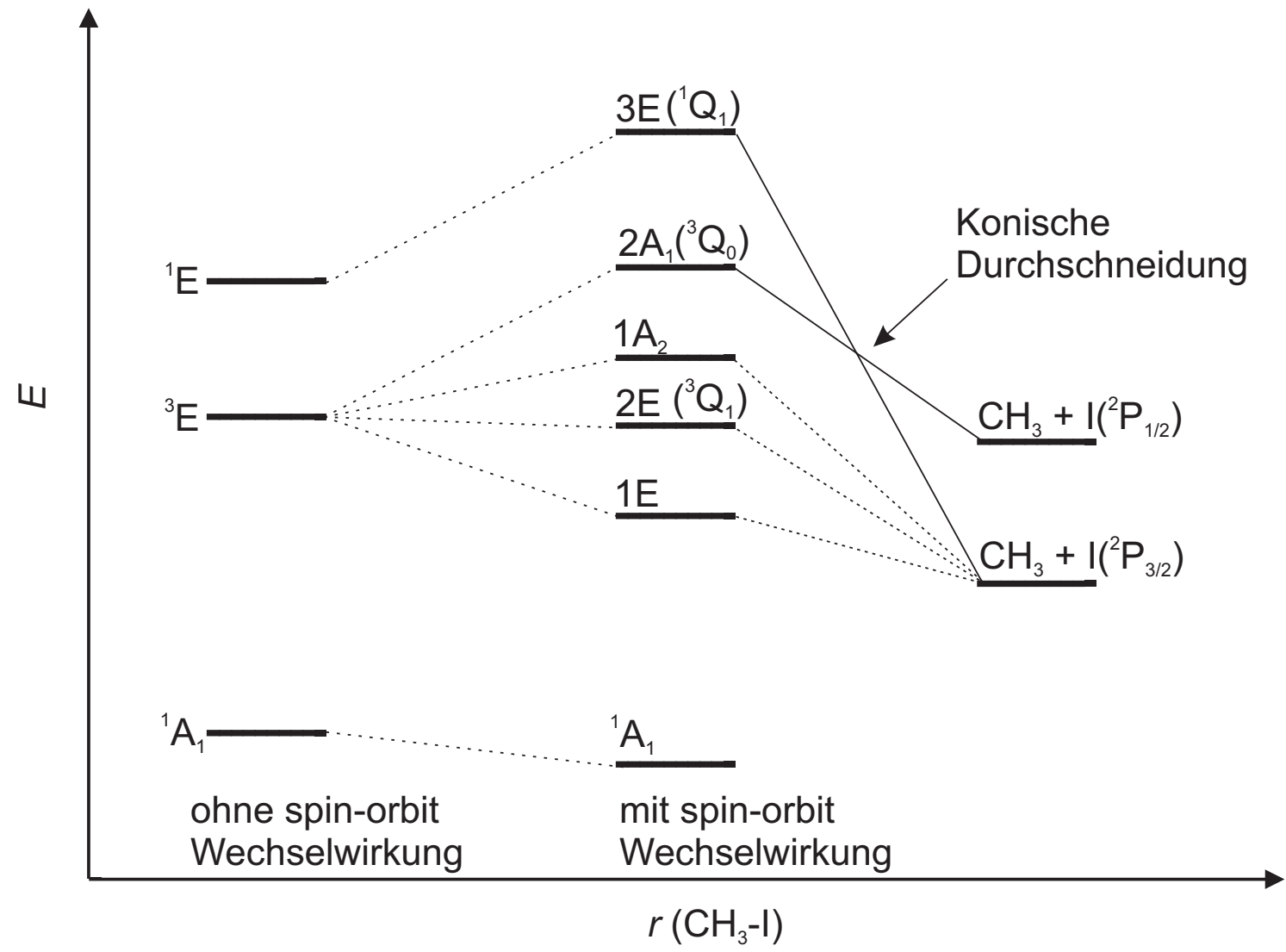

Abbildung 3.7: Korrelationsdiagramm von Iodmethan der Potentialflächen der Dissoziation mit und ohne spin-orbit-Wechselwirkung [127]

Das Absorptionsspektrum von $\mathrm{CH}_{3} \mathrm{I}$ (s. Abbildung 3.6) besteht im Wellenlängenbereich von 220-350 nm aus einer breiten Bande, der $A$-Bande, welcher verschiedene dissoziative Zustände zugeordnet werden [100]. Die 5 dissoziativen angeregte Zuständen korrelieren $\mathrm{zu} \mathrm{CH} \mathrm{CH}_{3}+\mathrm{I}\left({ }^{2} \mathrm{P}_{3 / 2}\right)$ und $\mathrm{CH}_{3}+\mathrm{I}^{*}\left({ }^{2} \mathrm{P}_{1 / 2}\right)$ (s. Abbildung 3.7). Drei dieser Zustände $\left({ }^{3} \mathrm{Q}_{1}\right.$, ${ }^{3} \mathrm{Q}_{0}$ und ${ }^{1} \mathrm{Q}_{1}$ in der Notation von Mulliken [100]) sind durch einen erlaubten optischen Übergang zugänglich [127, 128], welcher im Fall des ${ }^{3} \mathrm{Q}_{1}$ und ${ }^{1} \mathrm{Q}_{1}$-Zustands senkrecht und bei ${ }^{3} \mathrm{Q}_{0}$ parallel polarisiert ist. Gedanken und Rowe [129] entfalteten die $A$-Bande in der Form, daß die Anregung in den ${ }^{3} \mathrm{Q}_{0}$-Zustand dominiert und nur ein kleinerer Beitrag dem ${ }^{1} \mathrm{Q}_{1}$-Zustand ( $\sim 20 \mathrm{v}$. H.) bzw. dem ${ }^{3} \mathrm{Q}_{1}$-Zustand ( $\sim 1 \mathrm{v}$. H.) zuzuordnen ist. Dabei korreliert der ${ }^{3} \mathrm{Q}_{0}$-Zustand mit der Bildung von $\mathrm{I}^{*}$, während ${ }^{3} \mathrm{Q}_{1}$ und ${ }^{1} \mathrm{Q}_{1}$ zu Iod im Grundzustand führen (s. Korrelationsdiagramm Abbildung 3.7). Weitere Untersuchungen bei variabler Anregungswellenlänge zeigen jedoch keine Abhängigkeit des $\mathrm{I} / \mathrm{I}^{*}-$ Verhältnisses [130, 131, welche sich anhand einer Superposition des ${ }^{3} \mathrm{Q}_{0^{-}}$und ${ }^{1} \mathrm{Q}_{1^{-}}$ Zustands erklären ließe, bei $266 \mathrm{~nm}$ Anregung wird der ${ }^{3} \mathrm{Q}_{0}$-Zustand nahezu als einziger besetzt (<98 v. H.) [132]. Die Bildung von Iodatomen im Grundzustand erfolgt somit nicht durch eine gleichzeitige Anregung von ${ }^{3} \mathrm{Q}_{0^{-}}$und ${ }^{1} \mathrm{Q}_{1}$-Zustand, sondern wird durch eine konische Durchschneidung der beiden Zustände bewirkt, wodurch der ${ }^{3} \mathrm{Q}_{0}$-Zustand teilweise in den ${ }^{1} \mathrm{Q}_{1}$-Zustand entleert wird. Das $\mathrm{I} / \mathrm{I}^{*}$-Verhältnis ist somit ein direktes 


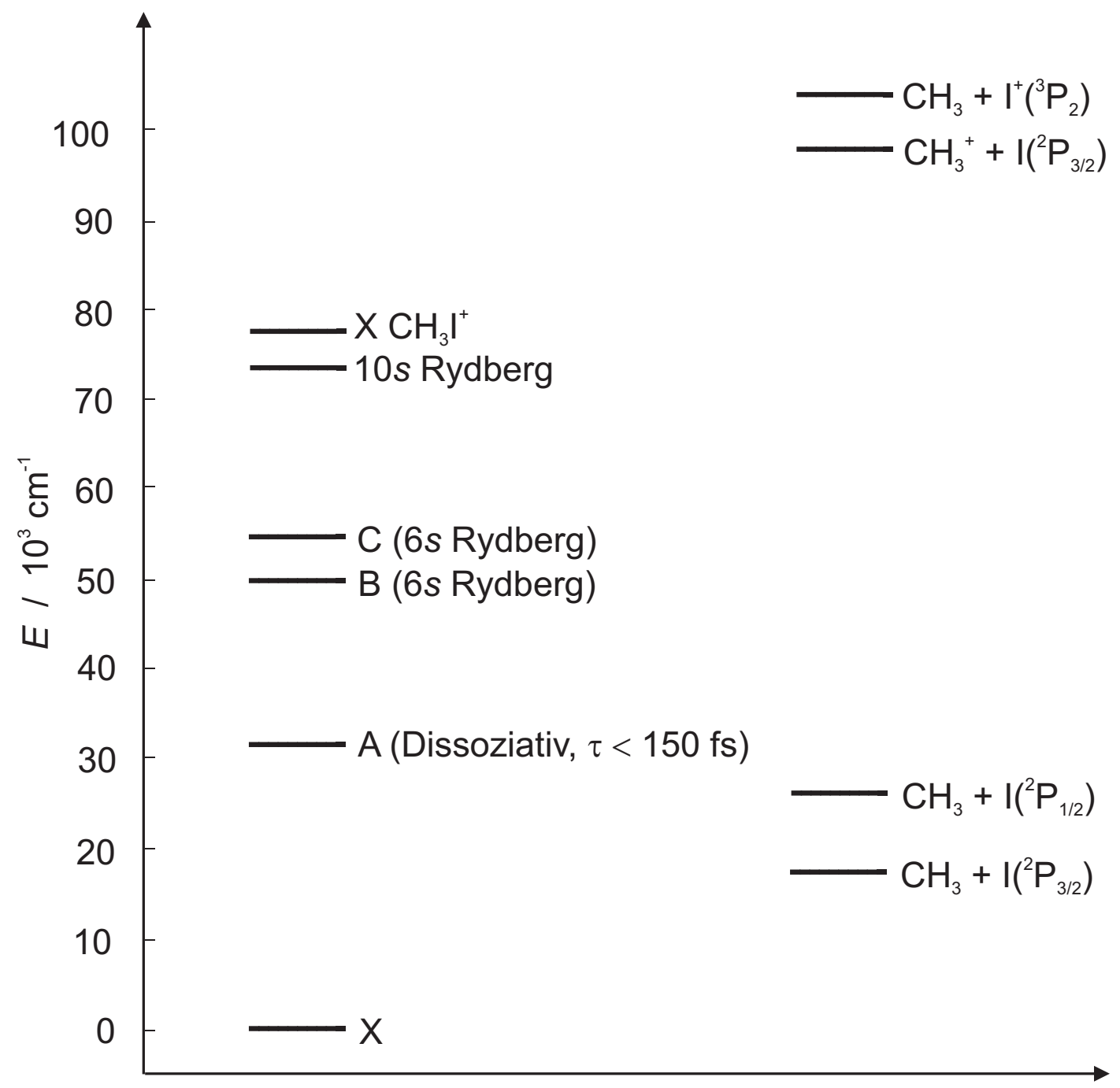

Abbildung 3.8: Energiezustandsdiagramm von Iodmethan und Photoprodukte in der Gasphase

Maß für die nicht-adiabatischen Wechselwirkungen zwischen den beiden Potentialflächen. Nach Messungen von Parker et al. [133] ergibt sich bei einer Anregung von 240-280 nm für die Quantenausbeute an I* etwa $\Phi^{*}=0,7$, bei längerer Anregungswellenlänge fällt $\Phi^{*}$ auf etwa 0,1 bei $333 \mathrm{~nm}$ ab.

Neben der $A$-Bande besitzt $\mathrm{CH}_{3} \mathrm{I}$ weitere höhere Energiezustände, welche insbesondere durch Multi-Photonen-Absorption besetzt werden können und in einer sehr unterschiedlichen Photochemie münden, ein Überblick über die Energiezustände findet sich in Diagramm 3.8. So schließen sich an die $A$-Bande die $B$-Bande bei $200 \mathrm{~nm}$ und die $C$-Bande bei $187 \mathrm{~nm}$ an, diesen Banden liegt ein Rydberg-Übergang von einem $5 p$-Iodorbital in einen Rydbergzustand wie den $6 s$ - oder den $6 p$-Zustand des Iodatoms zugrunde [134. Höhere Rydberg-Übergänge $5 p \pi \rightarrow 6 p$ bei $170 \mathrm{~nm}$ und $150 \mathrm{~nm}$ wurden von Dobber et al. 135. mit REMPI-Spektroskopie und Tsukiyama et al. [136] mit VUV-Fluoreszenspektroskopie 
untersucht. Die Lebenszeiten der Rydbergzustände wurden von Syage [137] durch Messung der spektralen Bandbreite für den $B$-Zustand auf $0,87 \mathrm{ps}$ und $0,25 \mathrm{ps}$ für den $C$ Zustand bestimmt. Zeitaufgelöste Messungen von Zewail et al. ergaben Lebenszeiten von 115-183 fs für diese Rydberg-Zustände. Die kurze Lebenszeit der Zustände ist durch eine Überschneidung mit der stark repulsiven Potentialkurve des $A$-Zustands zu erklären. $B$ - und $C$-Zustand unterliegen also demselben Prädissoziationsmechanismus, in dem sie schnell in den $A$-Zustand entleert werden, welcher sehr schnell zur Photodissoziation führt $\left(\tau_{\text {diss }}<150 \mathrm{fs}\right)$ [138].

Durch diese, insbesondere durch Zwei-Photonenanregung gut zugänglichen, RydbergZustände neigt $\mathrm{CH}_{3} \mathrm{I}$ zur Multi-Photonen-Ionisation (MPI). Die Ionisierungenergie von $\mathrm{CH}_{3} \mathrm{I}$ liegt bei $9,54 \mathrm{eV}$ und ist somit bei Wellenlängen $<130 \mathrm{~nm}$ oder durch eine MPI zu erwarten. Wenn durch eine Zwei-Photonen-Absorption ein Rydbergzustand wie der 6s-Zustand besetzt wird, ist die Wahrscheinlichkeit zur Absorption eines dritten Photons, welches zur Photoionisation führt, hoch. Diese resonanz-verstärkte Multi-PhotonenIonisation (engl. resonance enhanced multi photon ionisation, REMPI) ist eine für $\mathrm{CH}_{3} \mathrm{I}$ oft benutzte Methode zur Erzeugung und Detektion von Ionen. Der erste angeregte Zustand des $\mathrm{CH}_{3} \mathrm{I}^{+}$führt zu einer direkten Fragmentierung in $\mathrm{CH}_{3}^{+}$und I [139]. Neben der Ionisation sind jedoch auch neutrale Kanäle der Multi-Photonen-Absorption zugänglich. Tsukiyama etal. [136] konnten durch VUV-Fluoreszenzmessung die Bildung hoch angeregter Iodatome nach Drei-Photonen-Absorption von $\mathrm{CH}_{3} \mathrm{I}$ nachweisen:

$$
\mathrm{CH}_{3} \mathrm{I} \stackrel{3 \mathrm{~h} \nu}{\longrightarrow} \mathrm{CH}_{3}+\mathrm{I}\left(6^{2} \mathrm{P}_{3 / 2} \text { oder } 6{ }^{4} \mathrm{P}_{5 / 2}\right)
$$

\subsection{Primäre Photofragmente}

Nach der photolytischen Abspaltung eines Halogen-, vornehmlich eines Iodatoms, ensteht als zweites primäres Photofragment ein $\mathrm{CH}_{2} \mathrm{X}-/ \mathrm{CHI}_{2}$-Radikal oder im Fall des Iodmethans das $\mathrm{CH}_{3}$-Radikal. Strukturdaten dieser Radikale wurden von Marshall et al. berechnet 94] (s. Tabelle 3.2). Die Radikalspektren (s. Abbildung 3.9) von $\mathrm{CH}_{2} \mathrm{ClI}$ und $\mathrm{CH}_{2} \mathrm{BrI}$ ähneln einander stark, sie bestehen aus einer einzigen Bande zentriert bei $200 \mathrm{~nm}$ $\left(\mathrm{CH}_{2} \mathrm{ClI}\right)$ bzw. $230 \mathrm{~nm}\left(\mathrm{CH}_{2} \mathrm{BrI}\right)$. Das Absorptionsspektrum des $\mathrm{CH}_{2}$ I-Radikals weist zwei unterschiedliche Banden bei 280 und $340 \mathrm{~nm}$ auf.

Durch die Photodissoziation werden jedoch keine thermisch äquilibrierten Photoprodukte gebildet, sondern teilweise hoch (schwingungs)angeregte Radikalfragmente. Die Energieverteilung auf die verschiedenen Freiheitsgrade (Translation, Rotation und die verschiedenen Molekülschwingungen) sind eine direkte Folge der Photodissoziationsdynamik im angeregten Zustand. Untersuchungen dazu wurden insbesondere für $\mathrm{CH}_{2} \mathrm{I}_{2}$ und $\mathrm{CH}_{3} \mathrm{I}$ ausführlich durchgeführt. 


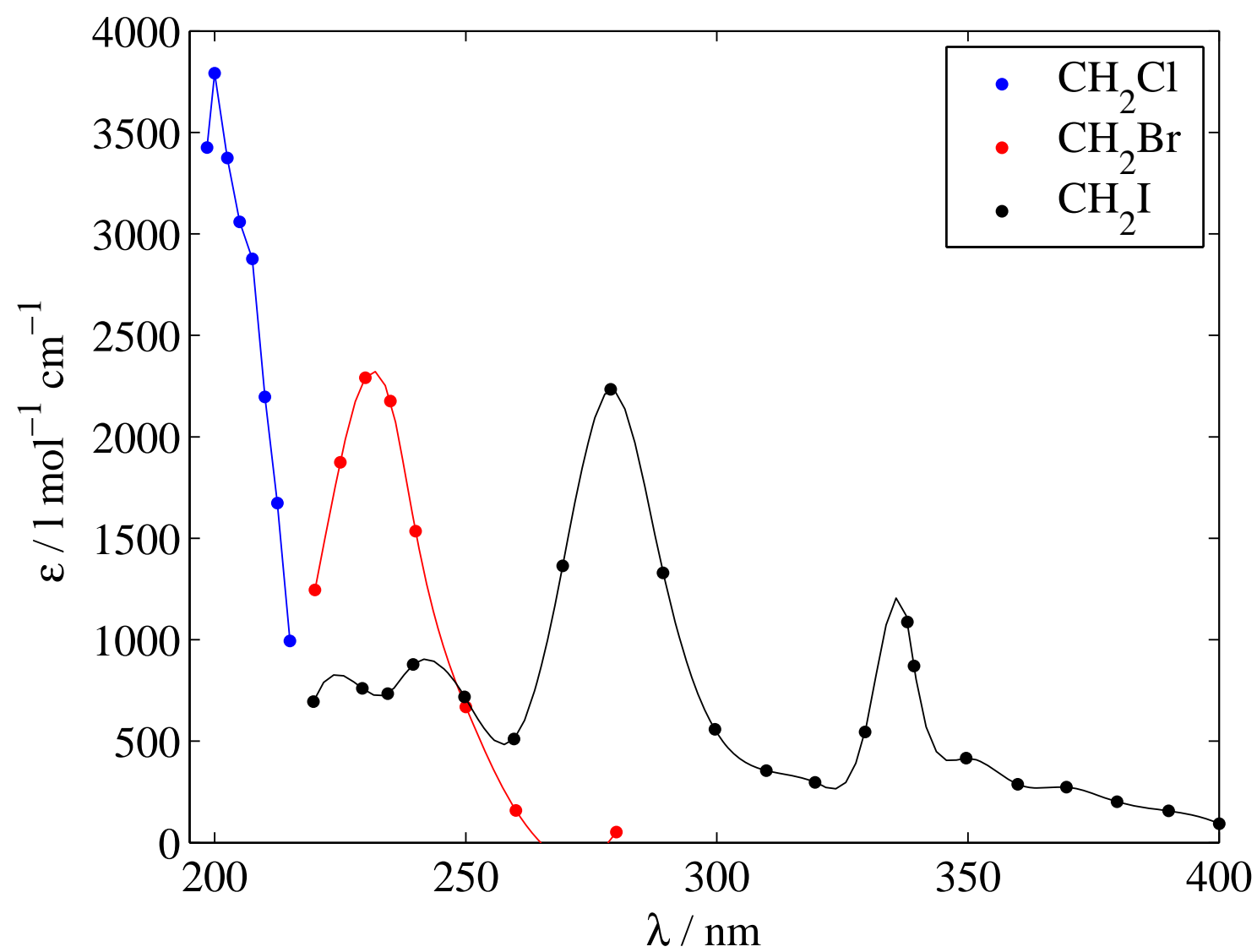

Abbildung 3.9: Gasphasen-Absorptionsspektrum vom $\mathrm{CH}_{2} \mathrm{X}$-Radikalen in der Gasphase: $\mathrm{CH}_{2} \mathrm{Cl}$ [140], $\mathrm{CH}_{2} \mathrm{Br}$ [141] und $\mathrm{CH}_{2} \mathrm{I}[142$

Im Falle des $\mathrm{CH}_{2} \mathrm{I}_{2}$ wurde die Dynamik des angeregten Zustands z. B. von Zhang et al. durch laserinduzierte Fluoreszenz (LIF) untersucht [143]. Dabei zeigt sich nach $355 \mathrm{~nm}$ Anregung eine starke Anregung der symmetrischen C-I-Streckschwingung $\left(\nu_{3}\right.$, s. Tabelle 3.3) sowie der asymmetrischen C-I-Streckschwingung $\left(\nu_{9}\right)$. Da von der asymmetrischen Streckschwingung nur der erste Oberton zu sehen ist, ist ein stark repulsives Potential anzunehmen, welches zur Dissoziation führt. Weiterhin führt die starke Anregung von symmetrischer und asymmetrischer $\mathrm{C}$-I-Streckschwingung zu einem Anschwingen der I-C-I-Streckschwingung $\left(\nu_{4}\right)$. Die Moden der C-H-Streckschwingung sind von den angeregten Moden entkoppelt, sie werden im Laufe der Photodissoziation nicht angeregt.

Die Energieverteilung auf die verschiedenen Freiheitsgrade des $\mathrm{CH}_{2}$ I-Radikals wurde sowohl von Kroger et al. [144] als auch von Xu et al. [102] durch massenspektrometrische Untersuchungen am Molekularstrahl studiert. Bei $304 \mathrm{~nm}$ Anregung zeigt sich, daß nach einer sehr schnellen Dissoziation (schneller als eine Rotationsperiode, $\tau_{\text {diss }}<100 \mathrm{fs}$ ) 80 90 v. H. der Überschußenergie aus der Photodissoziation in die inneren Freiheitsgrade des $\mathrm{CH}_{2} \mathrm{I}$ übergehen. Unabhängig von der Anregungswellenlänge sind etwa $40 \mathrm{v}$. H. der Überschußenergie in den Rotationsfreiheitsgraden und 60 v. H. als Schwingungsenergie im Radikal gespeichert, vornehmlich in der C-I-Streckschwingung, welche nahezu bis 


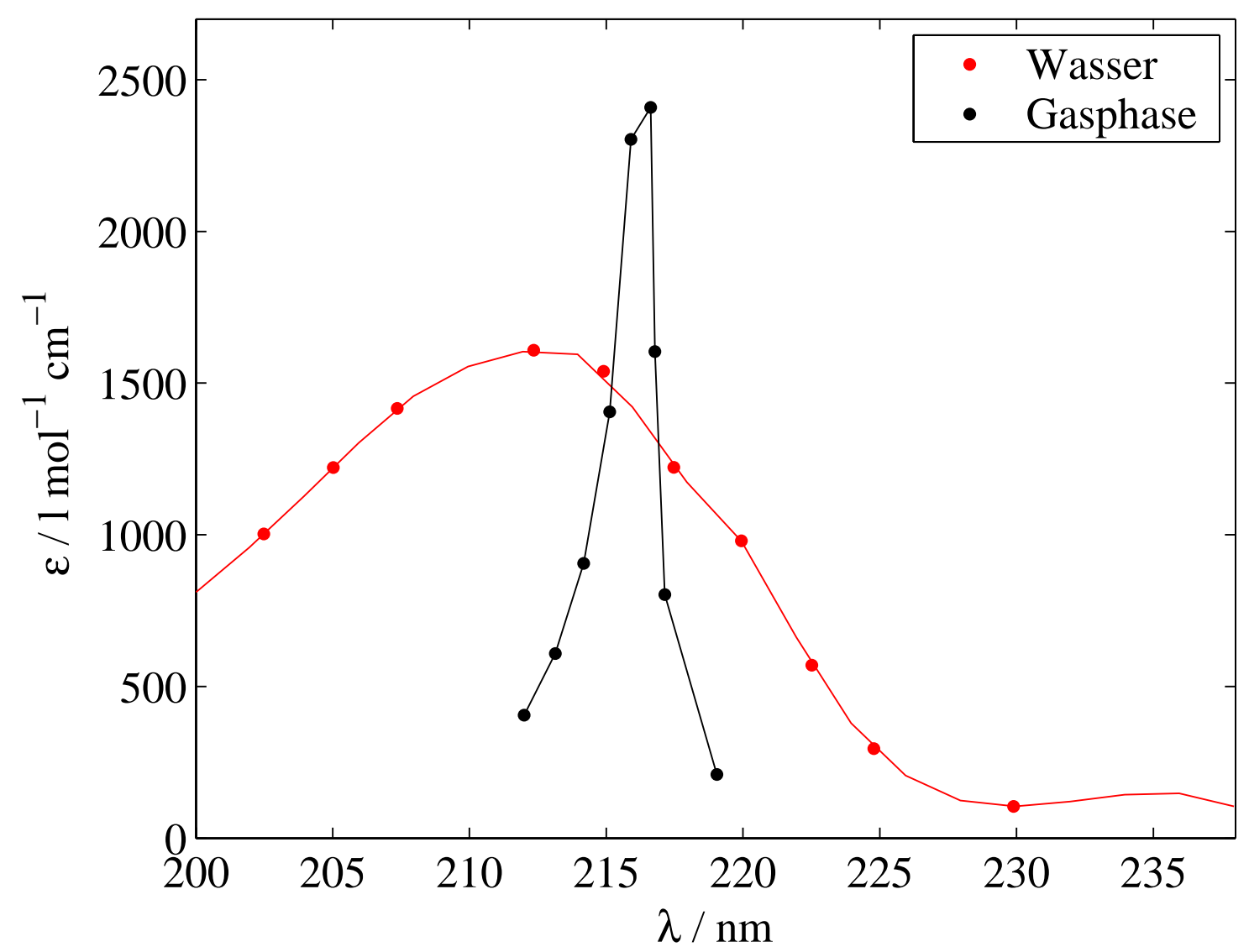

Abbildung 3.10: Absorptionsspektrum des $\mathrm{CH}_{3}$-Radikals in der Gasphase und in Wasser 145

ins Quasikontinuum angeregt ist; die Schwingungstemperatur des Radikals beträgt etwa 3000 K. Die Überschußenergie läßt sich aus den Standardbildungsenthalpien gemäß $E_{\mathrm{Exc}}=h \nu-D_{0}^{0}-E_{\text {int }}^{P}\left(\right.$ mit $D_{0}^{0}=$ Dissoziationsenthalpie und $E_{\text {int }}^{P}=$ innere Energie Muttermolekül) zu etwa $15800 \mathrm{~cm}^{-1}$ abschätzen. Für die anderen Halogenradikale $\mathrm{CH}_{2} \mathrm{Cl}$, $\mathrm{CH}_{2} \mathrm{Br}$ sowie $\mathrm{CHI}_{2}$ gibt es keine analogen Untersuchungen zur Photodissoziation, jedoch ist anzunehmen, daß Mechanismus und die Menge an Überschußenergie in den Photofragmenten ähnlich sind.

Bei der Photodissoziation von $\mathrm{CH}_{3}$ I entsteht ein $\mathrm{CH}_{3}$-Radikal. Da es kein halogenhaltiges Chromophor besitzt, ist dessen Absorptionsspektrum weit ins UV verschoben (s. Abbildung 3.10). In wäßriger Lösung zeigt sich eine Bande zentriert bei $213 \mathrm{~nm}$ [145], in der Gasphase ist diese Bande viel schmaler und etwas zu $216 \mathrm{~nm}$ verschoben. Die Dynamik der Dissoziation und insbesondere die Energieverteilung der Photofragmente ist im Laufe der letzten Jahre ausgiebig untersucht worden [128, 132, 133, 146].

Durch die Absorption eines Photons wird beim $\mathrm{CH}_{3} \mathrm{I}$ ein sehr hoher Oberton der $\mathrm{C}-$ I-Streckschwingung angeregt $\left(\nu_{3} \sim 30\right)$, durch das Herauslaufen des Wellenpakets aus dem repulsiven Potential wird die C-I-Bindung solange gestreckt, bis es zur Dissoziation kommt. Da die Dissoziation sehr schnell zustande kommt ( $\tau_{\text {diss }}<150$ fs), bleibt durch die 
axiale Rückstoßbewegung die $\mathrm{C}_{3 \mathrm{v}}$-Symmetrie des Muttermoleküls vorerst gewahrt, das $\mathrm{CH}_{3}$-Radikal liegt somit in einer pyramidalen Anordnung vor. Im elektronischen Grundzustand ist $\mathrm{CH}_{3}$ jedoch planar (s. Tabelle 3.2) ( $\mathrm{D}_{3 \mathrm{~h}}$-Symmetrie), somit ist ein Anschwingen der symmetrischen Deformationsschwingung ( $\nu_{2}$, engl. umbrella mode) zu erwarten. Im Vergleich zum $\mathrm{CH}_{2}$ I-Radikal besitzt $\mathrm{CH}_{3}$ wesentlich weniger Schwingungsenergie, der Großteil der Überschußenergie aus der Photodissoziation wird als Translationsenergie gespeichert. Die kinetische Energie von $\mathrm{CH}_{3}$ beträgt bei $266 \mathrm{~nm}$ Anregung für den I*-Kanal $9525 \mathrm{~cm}^{-1}$, auf die inneren Freiheitsgrade entfallen $1386 \mathrm{~cm}^{-1}$ [128], dabei wird neben dem rotatorischen Anteil in erster Linie die symmetrische $\mathrm{CH}_{3}$-Deformationschwingung $\left(\nu_{2}\right)$ und in geringem Maße die symmetrische $\mathrm{C}-\mathrm{H}$-Streckschwingung $\left(\nu_{1}\right)$ angeregt. Im Falle der Abspaltung eines Grundzustandsiodatoms, steht die Energiedifferenz zum I* dem $\mathrm{CH}_{3}$-Radikal als zusätzliche kinetische Energie zur Verfügung, sie beträgt in diesem Fall $17107 \mathrm{~cm}^{-1}$ [133]. Anhand dieser Daten konnte die Dissoziationsenergie von Parker et al. zu $19438 \mathrm{~cm}^{-1}$ berechnet werden.

\subsection{Photodynamik und -isomerisierung in der kondensierten Phase}

\subsubsection{Photoisomerisierung in Matrixumgebung}

Die ersten Untersuchungen zur Photochemie von Polyhalogenmethanen in einer Solvensumgebung wurden durch Einlagerungen in Matrixumgebung vorgenommen. So beobachteten Simons und Tattam [147] bereits 1966 die Änderung der Absorptionsspektren von Polyhalogenmethanen in Kohlenwasserstoffglasmatrizen nach UV-Anregung. Die Anwesenheit der neuen Banden erklärten sie durch Absorption gefangener Elektronen, welche via Rydberg-Orbitale zum Nachbarmolekül tunneln. Ähnliche Beobachtungen machten Mohan et al. für $\mathrm{CH}_{2} \mathrm{I}_{2}$ und $\mathrm{CHI}_{3}$ in gefrorenen Glasmatrizen [148], die neuen Absorptionsbanden nach UV-Einstrahlung ordneten sie allerdings dem ionisierten Muttermolekül und dem Radikal nach Abspaltung eines Iodatoms zu. Maier et al. untersuchten eine ganze Reihe Polyhalogenmethane in Argonmatrix bei $77 \mathrm{~K}$ [149, 150], sie postulierten allerdings eine Photoisomerisierung als Ursache für die Änderungen im Absorptionsspektrum. Dabei dissoziiert das Polyhalogenmethan in ein Iodatom und das entsprechende Radikal (s. Abschnitt 3.2), diese rekombinieren (wie in Abbildung 3.11 für $\mathrm{CHI}_{3}$ dargestellt) innerhalb der Matrix zu einem Isomer mit einem überbrückten Iodatom. Das Absorptionsspektrum

\begin{tabular}{|lllllll|}
\hline Radikal & $R(\mathrm{C}-\mathrm{H}) / \AA$ & $R(\mathrm{C}-\mathrm{X}) / \AA$ & $\Theta_{\mathrm{HCH}}$ & $\Theta_{\mathrm{HCX}}$ & $\Theta_{\mathrm{XCX}}$ & $I_{a} I_{b} I_{c} / 10^{-135} \mathrm{~kg}^{3} \mathrm{~m}^{6}$ \\
\hline $\mathrm{CH}_{3}$ & 1,083 & & $120^{\circ}$ & & & $5,11 \times 10^{-5}$ \\
$\mathrm{CH}_{2} \mathrm{I}$ & 1,081 & 2,049 & $122,9^{\circ}$ & $118,6^{\circ}$ & $115,9^{\circ}$ & 0,0283 \\
$\mathrm{CH}_{2} \mathrm{Br}$ & 1,081 & 1,867 & & $123,4^{\circ}$ & $117,6^{\circ}$ & 0,0179 \\
$\mathrm{CHI}_{2}$ & 1,082 & 2,062 & & $116,8^{\circ}$ & $123,7^{\circ}$ & 48,6 \\
\hline
\end{tabular}

Tabelle 3.2: Strukturparameter $\mathrm{CH}_{\mathrm{x}} \mathrm{I}_{\mathrm{z}} \mathrm{X}$-Radikale nach Marshall [94] 

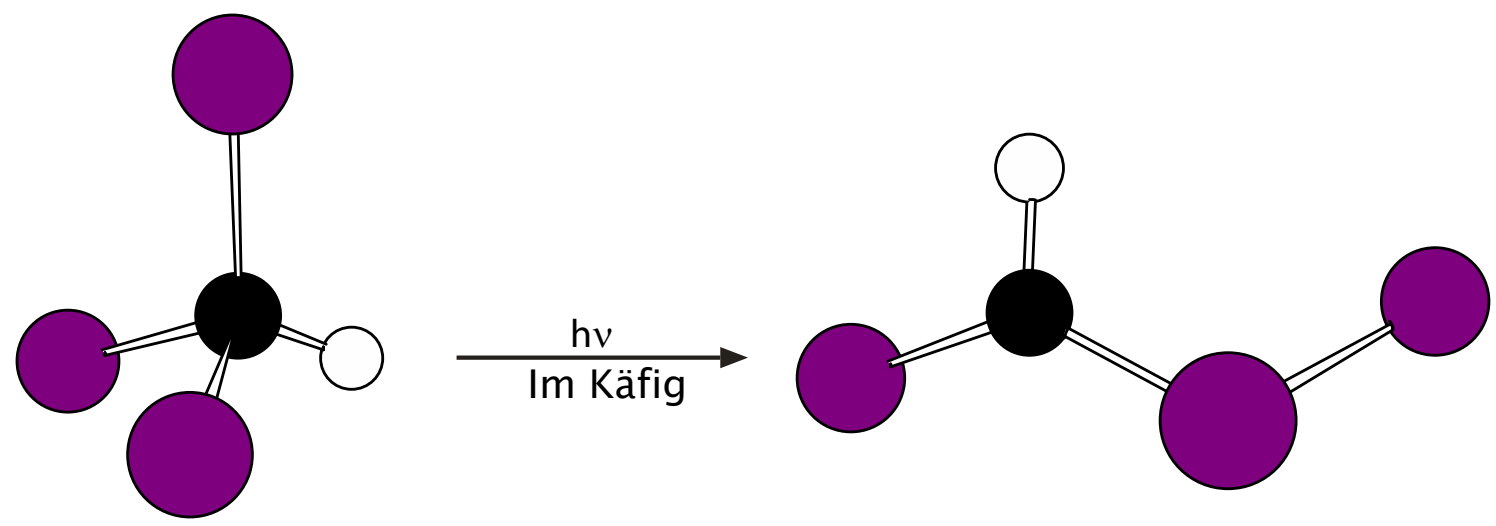

Abbildung 3.11: Photoinduzierte Isomerisierung von $\mathrm{CHI}_{3}$ innerhalb eines Lösungsmittelkäfigs

ändert sich dabei signifikant, es entstehen neue Banden sowohl im VIS- als auch im UVBereich (s. Abbildung 3.12, im Falle des $\mathrm{CH}_{2} \mathrm{I}_{2}$ eine starke Bande bei etwa 370 und eine schwächere bei etwa $550 \mathrm{~nm}$ ), für eine Isomerisierungsreaktion spricht dabei die Tatsache, daß sich durch Anregung des Produktspektrums wieder quantitativ das Eduktspektrum einstellt. Die Struktur des Isomers wurde durch Vergleich der gemessenen IR-Frequenzen mit durch ab-initio-Methoden berechneten Frequenzen bestimmt.

\subsubsection{Photodynamik in fluiden Lösungsmitteln}

Untersuchungen zur Photodynamik von $\mathrm{CH}_{2} \mathrm{I}_{2}$ in Lösung wurden unter anderem von Harris et al. [151] und Yoshihara et al. [152] mit Hilfe von zeitaufgelösten Absorptionsmessungen im Piko- bzw. Femtosekundenbereich vorgenommen, sie schlagen als Reaktionsprodukte allerdings eine geminale Rekombination zum Muttermolekül bzw. die konzentrationsabhängige Bildung eines CT-Komplexes vor. Zu dem von Maier et al. als Kontakt-Ionenpärchen postulierten $\mathrm{CH}_{2} \mathrm{I}$-I-Isomer wurden von Glukhovtsev und Bach [153] quantenchemische Rechnungen durchgeführt, diese zeigen für das Isomer ein energetisches Minimum mit einer Barriere von $134,6 \mathrm{~kJ} \mathrm{~mol}^{-1}$ zum $\mathrm{CH}_{2} \mathrm{I}_{2}$ und somit kovalent bindenden Charakter. Weitergehende quantenchemische Untersuchungen der entsprechenden Reaktionspotentialfläche von Orel und Kühn [154] schließen eine Isomerisierung im Grundzustand aus und legen den Schluß nahe, daß nach der Photodissoziation mit schneller Rekombination zum Isomer zu rechnen ist.

Ausführliche Untersuchungen zur Photodynamik von $\mathrm{CH}_{2} \mathrm{I}_{2}$ in Lösung nahmen schließlich Tarnovsky et al. 99, 155] vor. Die Aufnahme transienter Spektren mit Femtosekundenauflösung nach $266 \mathrm{~nm}$ Anregung zeigt zu frühen Reaktionszeiten (0,2-3 ps) eine breitbandige Absorption von 300-800 nm mit schwachen Banden bei 430 und $350 \mathrm{~nm}$. Im Zeitraum von 3 bis 100 ps wachsen zwei ausgeprägte Banden bei 390 und $560 \mathrm{~nm}$ heraus. Aufgrund der Ähnlichkeit der transienten Absorptionsspektren mit den Matrixsspektrum des 


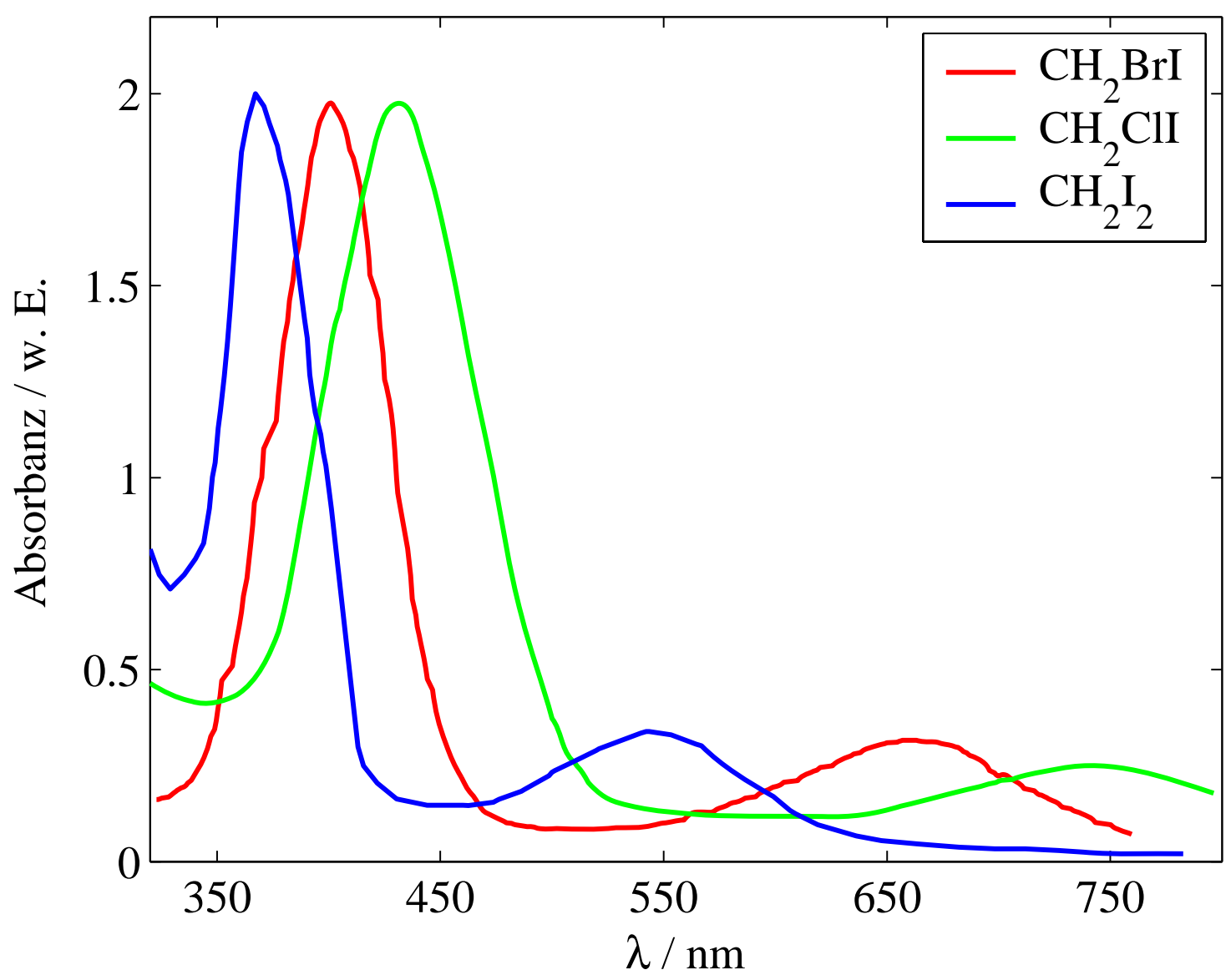

Abbildung 3.12: Absorptionsspektren der Isomere von Dihalogenmethanen in Matrix $(77 \mathrm{~K})$ [150]

$\mathrm{CH}_{2} \mathrm{I}-\mathrm{I}$ folgen sie der Interpretation von Maier, daß nach Photodissoziation die primären Photofragmente innerhalb des Lösungsmittelkäfigs zum $\mathrm{CH}_{2} \mathrm{I}$-I-Isomer rekombinieren, ein Beitrag von $\mathrm{CH}_{2} \mathrm{I}, \mathrm{CH}_{2} \mathrm{I}_{2}$ oder $\mathrm{CH}_{2} \mathrm{I}_{2}^{+}$zu den neuen beiden Banden wird ausgeschlossen. Bei ihren transienten Pump/Probeabsorptionszeitprofilen beobachten sie analog zu Harris etal. und Yoshihara etal. drei verschiedene Zeitkomponenten, eine anfängliche zeitlich von der Pulsbreite limitierte Spitze (engl. peak) bestehend aus einem schnellen Anstieg und schnellen Abfall, welcher von einen Anstieg auf einer Pikosekundenzeitskala gefolgt wird. In ihrer Interpretation wird dabei ein Wellenpaket nach UV-Anregung im repulsiven $S_{1}$-Zustand generiert, welches zu einer transienten Absorption des Probepulses in $S_{n}$-Zustände führt. Die ursprünglich angeregte Franck-Condon-Region verläßt das Wellenpaket auf einer sehr schnellen Zeitskala ( $>100 \mathrm{fs}$ ) und führt zur Dissoziation, wobei der Franck-Condon-Faktor und somit die transiente Absorption immer kleiner wird. Die sehr schnellen Prozesse der Anregung und das Verlassen des repulsiven $S_{1^{-}}$ Zustands sind somit für den anfänglichen schnellen Anstieg und Abfall der transienten Absorption verantwortlich. Die im Käfig gefangenen Photofragmente können nach Tarnovsky et al. sehr schnell ( $\sim 1 \mathrm{ps})$ zum Isomer rekombinieren, die Überschußenergie des nur schwach gebundenen Isomers wird anschließend über Schwingungsenergierelaxation 
(engl. vibrational energy relaxation, VER) an das Lösungsmittel abgegeben. Neben der schnellen Isomerbildung kann das schwingungsheiß gebildete $\mathrm{CH}_{2}$ I-Radikal nach VER auch erst später mit dem Iodatom zum Isomer rekombinieren und danach weiter abkühlen, so daß die Isomerbildung nach Tarnovsky et al. auf zwei unterschiedlichen Zeitskalen erfolgt. Das transiente Spektrum zeigt dabei die typische Dynamik der Relaxation schwingungsangeregter Moleküle, ein Anstieg der Bandenmitte und eine Verschmälerung der spektralen Flanken. Anhand einer Extrapolation der aus Matrixspektren bestimmten Extinktionskoeffizienten des Isomers wurde von Tarnovsky et al. eine Abschätzung der Quantenausbeute der Isomerisierungsreaktion vorgenommen, diese ist vom verwendeten Lösungsmittel abhängig und reicht von etwa $70 \mathrm{v}$. H. in Acetonitril bis zu etwa $90 \mathrm{v}$. H. in unpolaren Lösungsmitteln wie Dichlormethan.

Den wohl endgültigen Nachweis, daß das $\mathrm{CH}_{2} \mathrm{I}$-I-Isomer als Reaktionsprodukt nach Photodissoziation von $\mathrm{CH}_{2} \mathrm{I}_{2}$ in Lösung entsteht, erbrachten Davidsson et al. [156] durch Röntgenbeugungsexperimente mit Pikosekundenauflösung. Dabei wurde nach Anregung von $\mathrm{CH}_{2} \mathrm{I}_{2}$ bei $267 \mathrm{~nm}$ in Methanol die Intensitätsänderung der Röntgenbeugung zu verschiedenen Reaktionszeiten gemessen. Eine Simulation der Signale durch MDSimulationen zeigte eine sehr gute Übereinstimmung, wobei die Quantenausbeute der Isomerisierung etwa 38 v. H. beträgt. Der anschließende Zerfall des Isomers auf einer Nanosekundenzeitskala führt in Methanol schlußendlich zur Bildung von $\mathrm{I}_{3}^{-}$. MD-Simulationen von Odelius et al. [157] der Photodissoziationsdynamik von $\mathrm{CH}_{2} \mathrm{I}_{2}$ in Gasphase und in Lösung zeichnen ein ähnliches Bild.

Zeitaufgelöste Untersuchungen zur Photodynamik und -isomerisierung anderer Polyhalogenmethane in Lösung wurden von Tarnovsky et al. für $\mathrm{CH}_{2} \mathrm{ClI}$ [158], $\mathrm{CH}_{2} \mathrm{BrI}$ [159], $\mathrm{CHI}_{3}$ [116] analog zu denen von $\mathrm{CH}_{2} \mathrm{I}_{2}$ vorgenommen. Dabei zeigt sich für die meisten Verbindungen ein im Vergleich zu $\mathrm{CH}_{2} \mathrm{I}_{2}$ sehr ähnliches Verhalten. Im Falle des $\mathrm{CH}_{2} \mathrm{BrI}$ zeigt sich nach Anregung bei $266 \mathrm{~nm}$ in Acetonitril zu frühen Reaktionszeiten ein breites, strukturloses Spektrum, aus welchem innerhalb weniger Pikosekunden zwei neue Banden bei 445 und $640 \mathrm{~nm}$ heranwachsen. Diese lassen sich wieder dem von Maier in Matrix isolierten $\mathrm{CH}_{2} \mathrm{Br}-\mathrm{I}$-Isomer zuordnen (s. Abbildung 3.12), welches durch geminale Rekombination nach Dissoziation in ein Iodatom und ein $\mathrm{CH}_{2}$ Br-Radikal (s. Abschnitt 3.2) entsteht. Nach Tarnovsky bildet sich ein Teil des Isomers hochschwingungsangeregt innerhalb 1 ps, danach folgt wellenlängenabhängig ein weiterer Anstieg innerhalb einiger Pikosekunden, eine Integration über die beiden Isomerbanden führt zu einer Zeitkonstante von 7,5 ps. Dieser Anstieg wird mit der intramolekularen Schwingungsenergierelaxation des Isomers (engl. intramolecular internal energy-redistribution, IVR) verknüpft. Die hohe Überschußenergie aus der Photodissoziation der Isomerpopulation muß zuerst umverteilt werden, bevor eine Detektion im Beobachtungsfenster der Absorption des kalten Isomers bei 445 und $640 \mathrm{~nm}$ möglich ist. Anschließend kühlt das Isomer auf einer Skala von einigen zehn Pikosekunden ab, dabei zeigt sich wieder das typische Bild der Schwingungsrelaxation (VER), die Absorption fällt an den Flanken ab, während sie im Bandenursprung ansteigt. Die Quantenausbeute der Isomerisierung wird zu 26 v. H. abgeschätzt. Zu langen Reaktionszeiten kann ein weiterer Abfall der Absorption beobachtet werden, welcher mit dem Zerfall des $\mathrm{CH}_{2} \mathrm{Br}-\mathrm{I}$ mit einer Zeitkonstanten von $\sim 2,5 \mathrm{~ns}$ korrespondiert. Die Bildung von $\mathrm{CH}_{2} \mathrm{I}-\mathrm{Br}$ wird von Tarnovsky et al. nicht beobachtet, obwohl bei $266 \mathrm{~nm}$ Anregung 
eine Anregung des C-Br-Chromophor ebenfalls möglich ist (s. Abschnitt 3.2). Auch für die Photodynamik von $\mathrm{CHI}_{3}$ in Lösung zeigen sich große Gemeinsamkeiten, das transiente Spektrum in Acetonitril zu frühen Reaktionszeiten ist breit und strukturlos mit einer stärkeren Absorption bei $400 \mathrm{~nm}$. Anschließend wächst bei $\sim 450 \mathrm{~nm}$ die intensive und bei $\sim 600 \mathrm{~nm}$ die schwächere Isomerbande mit einer Zeitkonstanten von $\sim 7 \mathrm{ps}$ heraus. Die von Tarnovsky postulierte Bildung des Isomers auf zwei unterschiedlichen Zeitskalen mit anschließender Schwingungsrenergielaxation verläuft analog zu $\mathrm{CH}_{2} \mathrm{I}_{2}$ und $\mathrm{CH}_{2} \mathrm{BrI}$. Die in den Isomerbanden gemessenen Absorptionszeitprofile in steigen jedoch biexponentiell an, neben dem 7 ps-Anstieg ist noch eine langsame Zeitkomponente von $\sim 265 \mathrm{ps}$ vorhanden, diese wird von Tarnovsky der nicht-geminalen diffusiv gesteuerten Rekombination der primären Photofragmente zugeschrieben. Die Photodynamik von $\mathrm{CH}_{2} \mathrm{ClI}$ in Acetonitril weicht aufgrund der wesentlich geringeren thermodynamischen Stabilität des $\mathrm{CH}_{2} \mathrm{Cl}$-I-Isomers von den obengenannten Polyhalogenmethanen ab. Das von Tarnovsky et al. gemessene transiente Spektrum zeigt zu Reaktionszeiten von 20 ps die typischen Isomerbanden von $\mathrm{CH}_{2} \mathrm{Cl}-\mathrm{I}$ bei 460 und $710 \mathrm{~nm}$, welche anschließend mit einer Lebenszeit von $\sim 100$ ps zu einem Reaktionsprodukt mit einer Bande bei etwa $370 \mathrm{~nm}$ zerfallen, welche von Tarnovsky et al. der Absorption von $\mathrm{ICl}^{-}$zugeordnet wird.

Weitergehende Untersuchungen zum Zerfall der Isomere, inbesondere des $\mathrm{CH}_{2} \mathrm{I}-\mathrm{I}$, wurden ebenfalls von Tarnovsky et al. mit Hilfe von Nanosekundenblitzlichtphotolyse vorgenommen [99]. Die Reaktionsgeschwindigkeit ist dabei abhängig von der Anfangskonzentration von $\mathrm{CH}_{2} \mathrm{I}_{2}$ und somit 2. Ordnung in Bezug auf $\mathrm{CH}_{2} \mathrm{I}_{2}$ und seine Zerfallsprodukte. Die Zerfallsprodukte sind dabei abhängig von der Polarität des Lösungsmittels, in unpolaren Lösungsmitteln sind es ungeladene Verbindungen, während in polaren Lösungsmitteln auch Ionen beteiligt sind. In unpolaren Lösungsmitteln wie $\mathrm{CH}_{2} \mathrm{Cl}_{2}$ oder $n$-Hexan beträgt die Geschwindigkeitskonstante für den intramolekularen Zerfall des Isomers $k=$ $(1,8 \pm 0,4) \times 10^{5} \mathrm{~s}^{-1}(5,5 \mu \mathrm{s})$ mit einer Aktivierungsenergie von $41 \pm 2 \mathrm{~kJ} \mathrm{~mol}^{-1}$. Der genaue Zerfallsmechanismus ist dabei komplexer Natur und führt schließlich zu molekularen $\mathrm{I}_{2}$ als Endprodukt. Eine wichtige Rolle spielt dabei ein CT-Komplex aus einem Iodatom und Muttermolekül bzw. Isomer, die typische CT-Absorption (für $\mathrm{CH}_{2} \mathrm{I}_{2} \cdot \mathrm{I}$ bei $\sim 390 \mathrm{~nm}$ ) kann in den zeitaufgelösten Spektren nachgewiesen werden. Somit zerfällt das Isomer nicht direkt zum $\mathrm{I}_{2}$, sondern spaltet zuerst ein Iodatom ab, welches mit einem anderen Isomer oder auch $\mathrm{CH}_{2} \mathrm{I}_{2}$ einen CT-Komplex bildet und wieder in Iodatom und $\mathrm{CH}_{2} \mathrm{I}_{2}$ zerfallen kann. Schließlich reichert sich durch Rekombination der Iodatome $\mathrm{I}_{2}$ an. In polaren Lösungsmitteln wie Acetonitril ist der intramolekulare Zerfall des Isomers schneller $\left(k=(4,3 \pm 0,3) \times 10^{6} \mathrm{~s}^{-1}(230 \pm 20 \mathrm{~ns})\right)$, und führt zur Bildung von $\mathrm{I}_{3}^{-}$als Endprodukt. Dabei zerfällt $\mathrm{CH}_{2} \mathrm{I}-\mathrm{I}$ nicht in zwei Radikale, sondern in zwei Ionen $\mathrm{CH}_{2} \mathrm{I}^{+}$ und $\mathrm{I}^{-}$, welche sich spektrokopisch nachweisen lassen. Das $\mathrm{I}^{-}$-Anion sorgt dabei durch Reaktion mit anderen Iodatomen für die sukzessive Bildung von $\mathrm{I}_{2}^{-}$und schlußendlich $\mathrm{I}_{3}^{-}$ als Endprodukt.

Der Zerfall des $\mathrm{CH}_{2} \mathrm{I}-\mathrm{I}$ kann dabei als genereller Mechanismus auch für andere Isomere der Polyhalogenmethane wie $\mathrm{CH}_{2} \mathrm{BrI}, \mathrm{CH}_{2} \mathrm{ClI}$ oder $\mathrm{CHI}_{3}$ aufgefaßt werden. Dabei besitzen $\mathrm{CH}_{2} \mathrm{Cl}-\mathrm{I}$ und $\mathrm{CH}_{2} \mathrm{Br}-\mathrm{I}$ eine geringere thermodynamische Stabilität als das $\mathrm{CH}_{2} \mathrm{I}-\mathrm{I}$, sie zerfallen auf wesentlich schnelleren Zeitskalen $\left(\sim 0,12 \mathrm{~ns}\left(\mathrm{CH}_{2} \mathrm{ClI}\right)\right.$ bzw. 


\begin{tabular}{|llll|}
\hline Schwingungsmode & Energie $/ \mathrm{cm}^{-1}$ & Zuordnung & Symmetrie \\
\hline$\nu_{1}$ & 2968 & $\mathrm{CH}_{2}$ sym. Streckschwingung & $a_{1}$ \\
$\nu_{2}$ & 1351 & $\mathrm{CH}_{2}$ Deformationsschwingung & $a_{1}$ \\
$\nu_{3}$ & 486 & $\mathrm{CI}$ sym. Streckschwingung & $a_{1}$ \\
$\nu_{4}$ & 121 & ICI Knickschwingung & $a_{1}$ \\
$\nu_{5}$ & 1028 & $\mathrm{CH}_{2}$ twisting-Schwingung & $a_{2}$ \\
$\nu_{6}$ & 3049 & $\mathrm{CH}_{2}$ asym. Streckschwingung & $b_{1}$ \\
$\nu_{7}$ & 716 & $\mathrm{CH}_{2}$ rocking-Schwingung & $b_{1}$ \\
$\nu_{8}$ & 1105 & $\mathrm{CH}_{2}$ wagging-Schwingung & $b_{2}$ \\
$\nu_{9}$ & 570 & $\mathrm{CI}$ asym. Streckschwingung & $b_{2}$ \\
\hline
\end{tabular}

Tabelle 3.3: Grundzustandschwingungsmoden $\left(\right.$ in $\mathrm{cm}^{-1}$ ) von $\mathrm{CH}_{2} \mathrm{I}_{2}$ [164 in Lösung

$\left.\sim 2,5 \mathrm{~ns}\left(\mathrm{CH}_{2} \mathrm{BrI}\right)\right)$ [158, 159]. Für die Lebenszeit des $\mathrm{CHI}_{3}$-Isomers zeigt sich mit $\sim 1,8 \mu \mathrm{s}$ in Cyclohexan und $\sim 0,2 \mu \mathrm{s}$ in Acetonitril ein ähnlicher Trend wie bei $\mathrm{CH}_{2} \mathrm{I}_{2}$ [116].

Im Gegensatz zur Gasphase ist die Photodynamik des $\mathrm{CH}_{3} \mathrm{I}$ in Lösung nur sehr spärlich untersucht worden. Im Vergleich zu anderen Polyhalogenmethanen ist eine direkte Photoisomerisierung der primären Photoprodukte (s. Abschnitt 3.2) innerhalb des Lösungmittelkäfigs nicht möglich, da dem Molekül lediglich ein Iodatom zur Verfügung steht. Alternativ dazu ist jedoch eine geminale Rekombination zum Muttermolekül innerhalb des Käfigs analog zur Iodrekombination (s. Abschnitt 2.1) denkbar. Iodmethan kann demnach als Modellsystem für die geminale Rekombination eines polyatomaren Moleküls dienen, ohne daß ein konkurrierender Reaktionskanal wie die Isomerisierung im Falle der Polyhalogenmethane auftreten kann. Frühere Untersuchungen durch Blitzlichtphotolyse mit Zeitauflösung im Minutenbereich konnten lediglich Folgeprodukte wie $\mathrm{I}_{2}$, $\mathrm{HI}$ oder $\mathrm{CH}_{4}$ nachweisen und keinerlei Hinweise zum Ultrakurzzeitmechanismus der Reaktion liefern [160 162. Untersuchungen mit Pikosekundenzeitauflösung wurden schließlich von Hopkins et al. vorgenommen [163], sie führten transiente Absorptionsmessungen von $\mathrm{CH}_{3} \mathrm{I}$ nach Anregung bei $266 \mathrm{~nm}$ in verschiedenen Lösungsmitteln durch. Der Probepuls lag bei $213 \mathrm{~nm}$, also im Zentrum der Bande des $\mathrm{CH}_{3}$-Radikals als primärem Photoprodukt (s. Abschnitt 3.2). Die Absorptionszeitprofile zeigen nach einer anfänglichen Intensitätsspitze einen exponentiellen Abfall auf einer Pikosekundenzeitskala. Hopkins et al. führen dies auf Bildung und Schwingungsenergierelaxation (VER) des schwingungsheiß gebildeten $\mathrm{CH}_{3}$-Radikals zurück, die Zeitkonstanten für den VET liegen bei 14 ps, 25 ps und 27 ps in Cyclohexan, Heptan und Hexan.

\subsubsection{Zeitaufgelöste Schwingungsspektroskopie in Lösung}

Zur Photodynamik der Polyhalogenmethane in Lösung wurden insbesondere von Phillips etal. schwingungsspektrokopische Untersuchungen mit Hilfe von zeitaufgelöster Resonanz-Raman-Spektroskopie durchgeführt. Nach UV-Anregung des Diiodmethans in der Gasphase 164 zeigt das Resonanz-Raman-Spektrum die meiste Intensität in den Fundamentalen, Obertönen und Kombinationen verschiedener Normalmoden (s. 


\begin{tabular}{|lllll|}
\hline Schwingungsmode & $\mathrm{CH}_{2} \mathrm{ClI}$ & $\mathrm{CH}_{2} \mathrm{BrI}$ & $\mathrm{CHI}_{3}$ & $\mathrm{CH}_{3} \mathrm{I}$ \\
\hline$\nu_{1}$ & 2978 & 2978 & 3013 & 2970 \\
$\nu_{2}$ & 1392 & 1374 & 425 & 1252 \\
$\nu_{3}$ & 1183 & 1150 & 154 & 533 \\
$\nu_{4}$ & 720 & 616 & 1068 & 3060 \\
$\nu_{5}$ & 531 & 517 & 578 & 1436 \\
$\nu_{6}$ & 196 & 144 & 110 & 882 \\
$\nu_{7}$ & 3050 & 3053 & & \\
$\nu_{8}$ & 1108 & 1065 & & \\
$\nu_{9}$ & 789 & 754 & & \\
\hline
\end{tabular}

Tabelle 3.4: Grundzustandsschwingungsmoden (in $\mathrm{cm}^{-1}$ ) von $\mathrm{CH}_{2} \mathrm{ClI}$ [169], $\mathrm{CH}_{2} \mathrm{BrI}$ [170], $\mathrm{CHI}_{3}$ [171] und $\mathrm{CH}_{3} \mathrm{I}$ [170] in Lösung

Tabelle 3.3), insbesondere der symmetrischen C-I-Streckschwingung $\left(\nu_{3}\right)$, der I-C-IKnickschwingung $\left(\nu_{4}\right)$ und der antisymmetrischen C-I-Streckschwingung $\left(\nu_{9}\right)$. Dies steht in guter Übereinstimmung mit früheren Messungen von Zhang und Imre [143], in Lösung zeigt sich jedoch ein anderes Bild: Neben einer Änderung der Intensitätsverhältnisse [164166] erscheinen innerhalb weniger Pikosekunden neue Banden $(\sim 8$ ps in Cyclohexan bzw. $5 \mathrm{ps}$ in Acetonitril), insbesondere eine niederfrequente Schwingung bei $\sim 120 \mathrm{~cm}^{-1}$ bzw. ihre Obertöne und Kombinationen. Quantenmechanische Rechnungen auf B3LYP/SadlejTZVP-Niveau identifizieren diese Schwingung als die I-I-Streckschwingung des $\mathrm{CH}_{2} \mathrm{I}-\mathrm{I}$ Isomers, andere mögliche Photoprodukte wie $\mathrm{CH}_{2} \mathrm{I}$ oder $\mathrm{CH}_{2} \mathrm{I}_{2}^{+}$besitzen keine Schwingungsmoden in diesem Frequenzbereich. Weiterhin zeigt sich ein Anwachsen und Verschiebung zu höheren Frequenzen der dem Isomer zugeordneten Schwingungsmode innerhalb mehrerer zehn Pikosekunden. Dieses steht in guter Übereinstimmung mit dem bei transienten fs-Absorptionsmessungen [86, 155, 167] beobachteten Verhalten, daß das $\mathrm{CH}_{2} \mathrm{I}-\mathrm{I}$ schwingungsheiß gebildet wird und auf einer Pikosekundenzeitskala durch Schwingungsenergietransfer relaxiert. Die Photoisomerisierung des $\mathrm{CHI}_{3}$ in Lösung ana$\log$ zu der von $\mathrm{CH}_{2} \mathrm{I}_{2}$ konnte von Phillips et al. ebenfalls nachgewiesen werden [168].

Neben $\mathrm{CH}_{2} \mathrm{I}_{2}$ untersuchten Phillips et al. weitere Halogenmethane wie z.B. $\mathrm{CH}_{2} \mathrm{ClI}$, $\mathrm{CH}_{2} \mathrm{BrI}, \mathrm{CHI}_{3}, \mathrm{CH}_{3} \mathrm{I}, \mathrm{CF}_{2} \mathrm{I}_{2}, \mathrm{CHBr}_{2} \mathrm{Cl}$ oder $\mathrm{CCl}_{3} \mathrm{Br}$. Diese zeigen zumeist ein dem $\mathrm{CH}_{2} \mathrm{I}_{2}$ ähnliches Verhalten nach Photodissoziation in Lösung, auf die für diese Arbeit relevanten Verbindungen $\mathrm{CH}_{2} \mathrm{ClI}, \mathrm{CH}_{2} \mathrm{BrI}, \mathrm{CHI}_{3}$ und $\mathrm{CH}_{3} \mathrm{I}$ soll kurz näher eingegangen werden. Im Falle des $\mathrm{CH}_{2} \mathrm{BrI}$ und $\mathrm{CH}_{2} \mathrm{ClI}$ sind die Schwingungsmoden denen von $\mathrm{CH}_{2} \mathrm{I}_{2}$ ähnlich (s. Tabelle 3.4), $\mathrm{CHI}_{3}$ und $\mathrm{CH}_{3} \mathrm{I}$ besitzen aufgrund ihrer höheren Symmetrie weniger Schwingungsmoden. Für das $\mathrm{CH}_{2} \mathrm{ClI}$ existieren von Phillips et al. ähnlich ausführliche Resonanz-Raman-Messungen mit Nano- und Pikosekundenzeitauflösung wie für das $\mathrm{CH}_{2} \mathrm{I}_{2}$ [172 175]. Dabei entsteht innerhalb 3 ps nach Anregung bei $267 \mathrm{~nm}$ in Acetonitril neben der Fundamentalen, Obertönen und Kombinationen der FranckCondon-aktiven Schwingungsmoden des Muttermoleküls eine neue Schwingungsmode bei $\sim 175 \mathrm{~cm}^{-1}$. Diese kann aufgrund von quantenchemischen Rechnungen (B3LYP/SadlejPVTZ-Niveau) der Cl-I-Streckschwingung des $\mathrm{CH}_{2} \mathrm{Cl}$-I-Isomers zugeordnet werden, die neue Bande verschwindet innerhalb von 3-500 ps wieder, während im gleichen Maße eine 
weitere Schwingungsbande bei $\sim 207 \mathrm{~cm}^{-1}$ entsteht. Diesen Zerfall des $\mathrm{CH}_{2} \mathrm{Cl}-\mathrm{I}$ konnten auch Tarnovsky et al. [159] durch Messung der transienten Absorptionsspektren beobachten. Im Gegensatz zu ihrer Interpretation, welche $\mathrm{ICl}^{-}$als primäres Reaktionsprodukt vorsieht, ordnen Phillips et al. die neu entstehende Bande bei $207 \mathrm{~cm}^{-1}$ aufgrund der Ergebnisse ihrer ab-initio-Rechnungen der I-Cl-Streckschwingung des $\mathrm{CH}_{2} \mathrm{I}-\mathrm{Cl}$-Isomers zu. Somit isomerisiert das instabile $\mathrm{CH}_{2} \mathrm{Cl}-\mathrm{I}$ wahrscheinlich über einen Übergangszustand, welcher die konzertierte Ausbildung einer C-I-Bindung, Verstärkung der I-Cl-Bindung und Bruch der $\mathrm{C}-\mathrm{Cl}$-Bindung beinhaltet.

Im Falle der Photodissoziation von $\mathrm{CH}_{2} \mathrm{BrI}$ in Cyclohexan [112, 176, 177] konnten Phillips et al. ebenfalls zwei Isomere als Photoprodukte, namentlich das $\mathrm{CH}_{2} \mathrm{Br}-\mathrm{I}$ und das $\mathrm{CH}_{2} \mathrm{I}-\mathrm{Br}$, beobachten. Im Gegensatz zum $\mathrm{CH}_{2} \mathrm{ClI}$ erfolgt die Bildung des zweiten Isomers jedoch nicht durch eine konzertierte Isomerisierungsreaktion, sondern beide werden gleichzeitig nach der Photodissoziation gebildet. Da im Absorptionsspektrum des $\mathrm{CH}_{2} \mathrm{BrI}$ die Chromophore für die Abspaltung eines Iod- bzw. Bromatoms überlappen (s. Abschnitt 3.2), werden bei Anregungswellenlängen $<270 \mathrm{~nm}$, wie sie von Phillips et al. verwendet wurden, neben dem vornehmlichen Bruch der C-I-Bindung auch CBr-Bindungen gespalten. Somit können sich im Lösungsmittelkäfig nach Rekombination der primären Photoprodukte entweder $\mathrm{CH}_{2} \mathrm{Br}-\mathrm{I}$ oder $\mathrm{CH}_{2} \mathrm{I}$-Br-Isomere bilden, je nachdem welches Chromophor beim betreffenden Molekül angeregt wurde. Dabei ist das $\mathrm{CH}_{2} \mathrm{I}-\mathrm{Br}$ thermodynamisch stabiler als das $\mathrm{CH}_{2} \mathrm{Br}-\mathrm{I}$, auf einer Nanosekundenzeitskala kann $\mathrm{CH}_{2} \mathrm{I}-\mathrm{Br}$ im Gegensatz zu $\mathrm{CH}_{2} \mathrm{Br}-\mathrm{I}$ noch nachgewiesen werden. Desweiteren ergeben ab-initio-Rechnungen von Phillips et al. eine Stabilisierungsenergie von $17,2 \mathrm{~kJ} \mathrm{~mol}^{-1}$ für das $\mathrm{CH}_{2} \mathrm{I}-\mathrm{Br}$ im Vergleich zum $\mathrm{CH}_{2} \mathrm{Br}-\mathrm{I}$.

Für das $\mathrm{CH}_{3} \mathrm{I}$ ist die Situation im Vergleich zu den Polyhalogenmethanen etwas anders. Da das Molekül neben der Methylgruppe nur aus einem Iodatom besteht, ist eine direkte Rekombination zu einer Isomerspezies mit überbrückten Iodatom nicht möglich. Vergleichbar dazu haben Li et al. 178] mit Hilfe von Resonanz-Raman-Spektroskopie die Bildung von $\mathrm{CH}_{3} \mathrm{I}-\mathrm{I}$ nach Anregung bei $266 \mathrm{~nm}$ in Lösung beobachtet. Dabei bindet das nach Photodissoziation abgespaltene Iodatom nach Diffusion durch das Lösungsmittel an ein nicht zerfallendes $\mathrm{CH}_{3} \mathrm{I}$. Für diese Reaktion kommt der Käfigeffekt nicht zum Tragen, es handelt sich um einen rein diffusiven Prozeß. 


\section{Experimentelle Technik}

Die Photodynamik und -isomerisierung der Polyhalogenmethane in Lösung läuft auf einer Femto- bis Pikosekundenzeitskala ab, somit muß die verwendete Meßmethode eine extrem hohe Zeitauflösung besitzen.

Die Meßdaten wurden mit Hilfe der Pump/Probe-Spektroskopie aufgenommen, als Laserquelle diente dabei ein modengekoppelter Titan:Saphir-Laser, welcher ultrakurze Pulse auf einer Femtosekundenzeitskala erzeugt und optisch-parametrische Verstärker zur Generierung von Pump- und Probepuls speist. Dadurch kann durch gezielte Verzögerung des Probepulses die transiente Absorption des zu untersuchenden Moleküls gemessen werden. Um einen weiten Spektralbereich für den Probepuls abzudecken, standen zwei verschiedene Lasersysteme zur Verfügung. Mit dem ersten sind Probepulse im UV und VIS-Bereich zugänglich, während das zweite breitbandige IR-Pulse erzeugt. Die beiden Lasersysteme mit ihren einzelnen Komponenten sollen hier kurz vorgestellt werden.

\subsection{Das UV/VIS-Lasersystem}

Das für diese Arbeit verwendete UV/VIS-Lasersystem wurde bereits ausführlich in verschiedenen Publikationen beschrieben [86, 103, 104, 167, 179] und soll deshalb hier nur kurz vorgestellt werden. Ein schematische Zeichnung ist in Abbildung 4.1 abgebildet.

Bei der Laserquelle handelt es sich um ein kommerziell erhältliches Lasersystem Clark MXR CPA-2001. Dieses besteht aus einem regenerativen Verstärker auf Titan:SaphirBasis, welcher mit ultrakurzen Pulsen aus einem modengekoppelten Faserlaser gespeist wird. Der Laser emittiert Pulse bei einer Zentralwellenlänge von $773 \mathrm{~nm}$ mit einer Repetitionsrate von $1 \mathrm{kHz}$. Die Ausgangsintensität beträgt $900 \mathrm{~mW}$, davon werden etwa $2 / 3$ über einen Strahlteiler in den TOPAS (s. Abschnitt 4.1.4) zur Erzeugung des Probepulses eingespeist, während der Rest zum Pumpen das NOPA dient (s. Abschnitt 4.1.2), welcher den Pumppuls generiert. Alternativ dazu kann als Pumppuls die Dritte Harmonische (engl. Third Harmonic Generation, THG) des Lasersystems bei einer Zentralwellenlänge von $258 \mathrm{~nm}$ erzeugt werden. Der Pumpstrahl wird mit Hilfe einer rotierenden Chopper-Scheibe moduliert, welche jeden zweiten Puls ausblendet. Die Verzögerung des Probestrahls erfolgt über eine rechnergesteuerte Verschiebebühne (VE) der Firma Physik Instrumente (PI M-415.DG), anschließend wird der Strahl über einen Strahlteiler (ST) aufgeteilt. Ein Teil wird über eine Photodiode (PD) mit integrierter Integratorschaltung [180] detektiert, während der andere Teil mit dem Pumpstrahl über ein Interferometer 


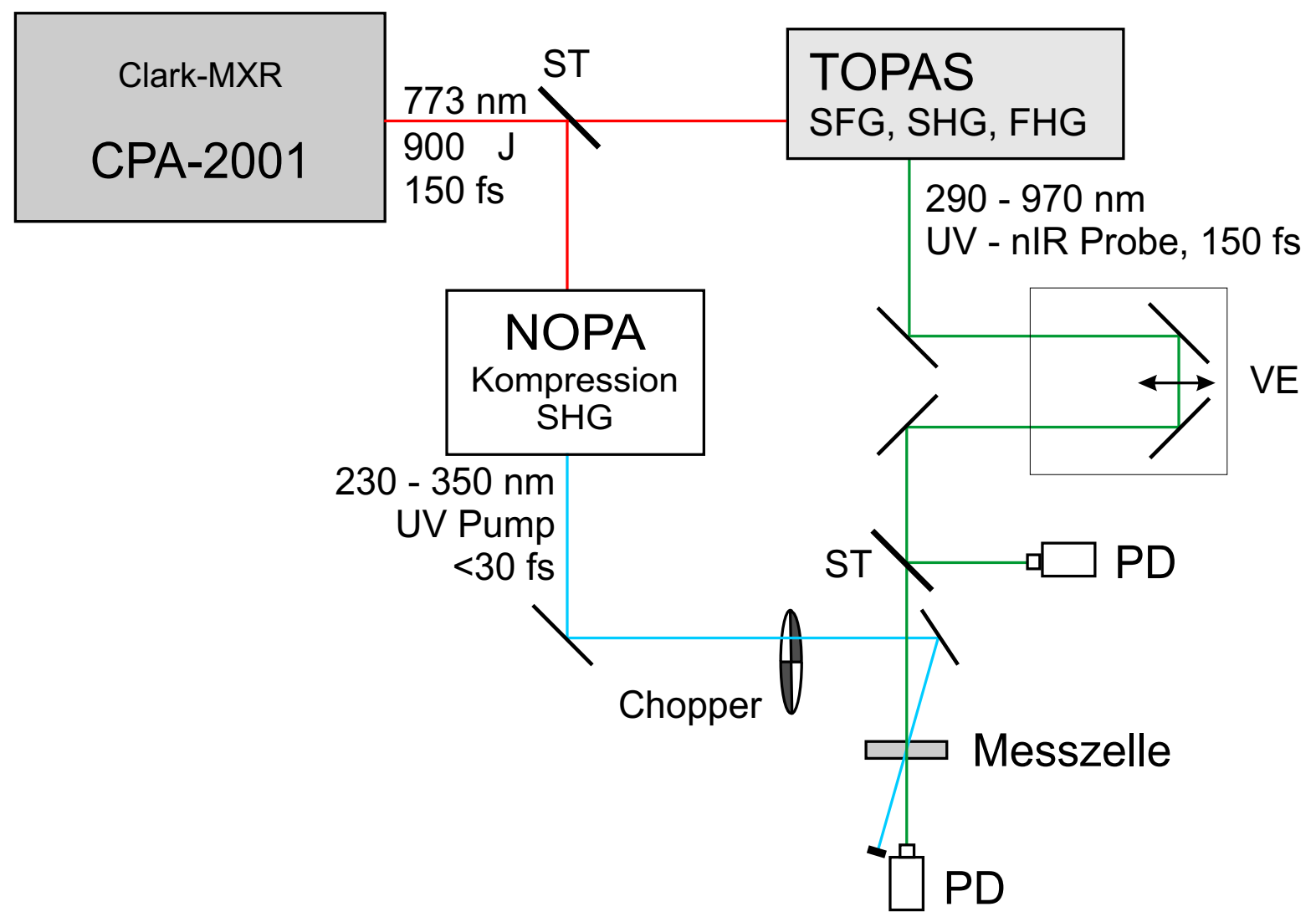

Abbildung 4.1: Aufbau des verwendeten UV/VIS-Lasersystems

in der Meßzelle zum Überlapp gebracht wird. Die Detektion des transmittierten Probestrahls erfolgt ebenfalls über eine integrierende Photodiode (PD). Die vom Experiment aufgenommene Meßgröße ist dabei die Änderung der optischen Dichte $(\triangle O D)$. Nach dem Lambert-Beer-Gesetz der Absorption gilt für diese:

$$
O D=-\lg \left(\frac{I_{0}}{I}\right)
$$

Durch die Modulation des Pumpstrahls mit der rotierenden Chopperscheibe wird jeder zweite Pumppuls ausgeblendet. Eine Differenzbildung aus einer Messung mit Pumppuls und einer ohne korrigiert die Absorptionsmessung um ihre Basislinie. Diese Meßgröße wird als die Änderung der optischen Dichte $(\Delta O D)$ bezeichnet:

$$
\Delta O D=\lg \left(\frac{I_{0}}{I}\right)_{\text {mit Pumppuls }}-\lg \left(\frac{I_{0}}{I}\right)_{\text {ohne Pumppuls }}
$$

Die einzelnen optischen Komponenten, die elektronische Steuerung, das verwendete Hochdrucksystem sowie die Anpassungsroutinen für die Meßdaten werden im folgenden kurz vorgestellt. 


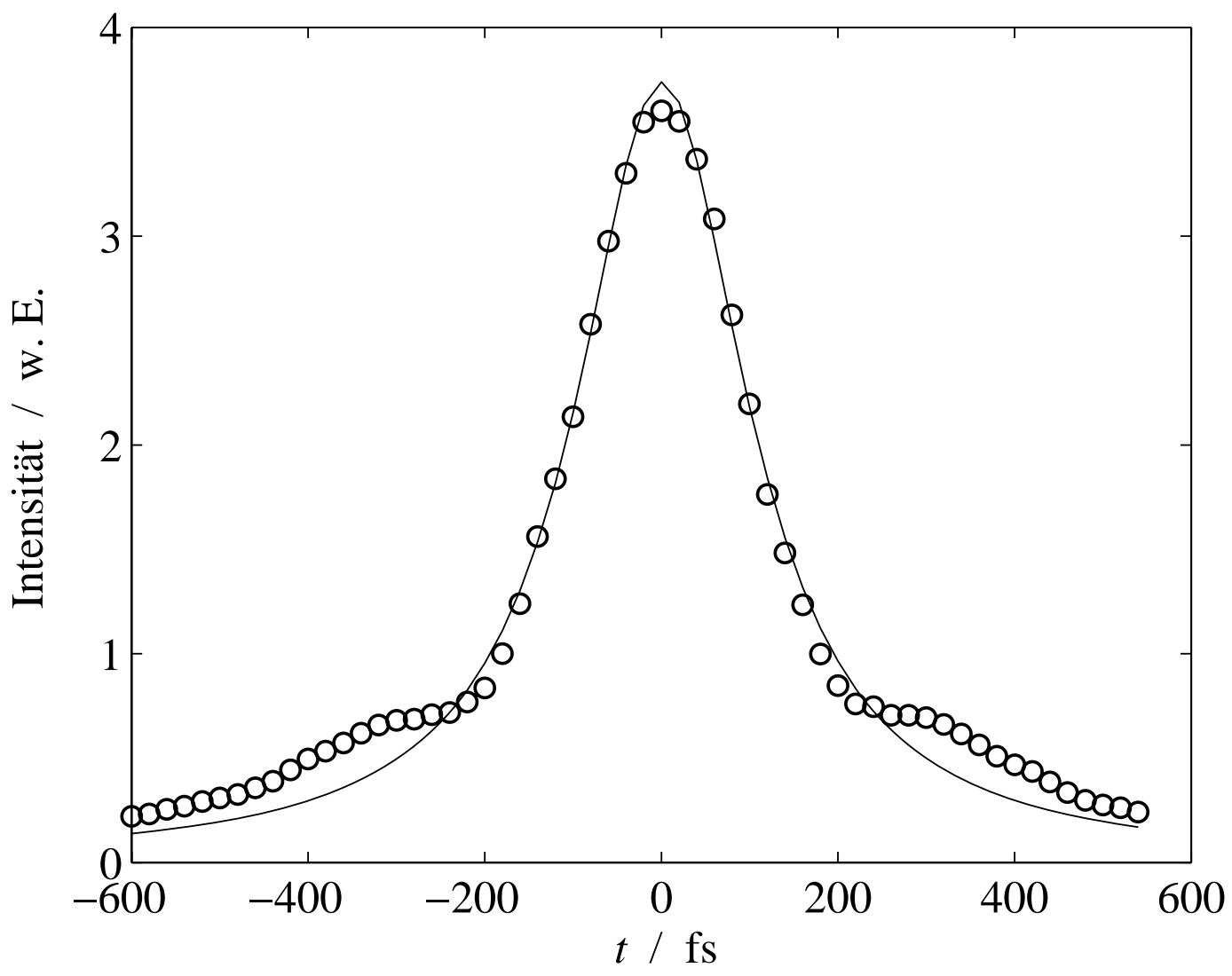

Abbildung 4.2: Autokorrelation des Clark MXR

\subsubsection{Clark MXR-CPA 2001}

Titan:Saphir basierte Lasersysteme sind zur Erzeugung ultrakurzer Pulse bei weitem die verbreitetsten. Ihre Vorteile liegen in ihrem breitbandigen Laserübergang, welcher eine hohe Zustandsdichte besitzt. Dieses ist eine Grundvoraussetzung der passiven Modenkopplung zur Erzeugung ultrakurzer Pulse. Die Funktionsweise des Clark MXR-CPA 2001 beruht auf dem Prinzip der chirped pulse amplification (CPA). Als chirp (engl. Zwitschern) wird dabei die frequenzabhängige Verzögerung der Frequenzkomponenten eines Pulses bezeichnet, der Puls wird zeitlich verbreitert. Im Rahmen des CPA-Konzepts wird somit ein ultrakurzer Puls zeitlich stark verbreitert, in einem regenerativen Verstärker verstärkt und anschließend wieder komprimiert. Für eine tiefergehende Betrachtung der chirped pulse amplification sei z. B. auf die Arbeiten von Maine et al. verwiesen [181.

Die eigentliche Quelle der ultrakurzen Pulse im Clark MXR ist ein passiv modengekoppelter Faserlaser. Im Betriebszustand wird von einem Diodenlaser eine Fundamentale von $980 \mathrm{~nm}$ über einen 980/1550 nm-WDM-Koppler (engl. wavelength division multiplexing, WDM) in eine Ringkavität eingekoppelt. Das laseraktive Medium ist eine $\mathrm{Er}^{3+}$ dotierte Glasfaser mit normaler Dispersion (GVD $=+0,075 \mathrm{ps}^{2} / \mathrm{m}$ bei $1,57 \mu \mathrm{m}$ ), welche bei $1550 \mathrm{~nm}$ emittiert. Dadurch wird eine positive Gruppengeschwindigkeitsdispersion 
(engl. group velocity dispersion, GVD) hervorgerufen, da die verschiedenen Wellenlängenanteile des Pulses unterschiedliche Geschwindigkeiten im dispersen Medium besitzen, der Puls also zeitlich verbreitert wird. Diese positive GVD wird durch eine Faser (SMF28) mit anormaler Dispersion (GVD $=-0,023 \mathrm{ps}^{2} / \mathrm{m}$ bei $\left.1,56 \mu \mathrm{m}\right)$ wieder kompensiert. Der Pulsbetrieb wird nun durch polarisations-additives Modenkoppeln (APM) erreicht, durch Verzögerungsplättchen (engl. wave plates, WP) wird ein Teil der Laserenergie (bis 50 v. H.) ausgekoppelt, die Repetitionsrate des Faserlaser beträgt dabei 27,5 MHz. Bei nicht ordnungsgemäßem Pulsbetrieb ist meist lediglich eine kleine Justage der Verzögerungsplättchen notwendig. Nach Auskopplung aus dem Faserlaser wird der Puls auf etwa $100 \mathrm{fs}$ komprimiert, mit Hilfe eines Lithiumtriborat-Kristalles (LTB) auf $775 \mathrm{~nm}$ frequenzverdoppelt und durch einen Strecker wieder zeitlich verbreitert.

Das Herzstück des regenerativen Verstärkers ist der Ti:Sa-Kristall, welcher den aus dem Faserlaser eingekoppelten Puls verstärkt. Dazu wird bei einer Repetitionsrate von $1 \mathrm{kHz}$ mit einem frequenzverdoppelten Nd:YAG-Laser (ORC-1000) eine Besetzungsinversion im Kristall erzeugt. Anschließend wird zeitverzögert der Puls aus dem Faserlaser in den Resonator eingekoppelt und dieser bei jedem Resonatorumlauf durch den Ti:Sa-Kristall verstärkt. Nach maximaler Verstärkung des Seedpulses wird dieser ausgekoppelt und durch einen Gitterkompressor auf etwa 150 fs komprimiert, die Pulsenergie beträgt etwa $900 \mu \mathrm{J}$ bei einer Zentralwellenlänge von $773 \mathrm{~nm}$. Eine Autokorrelationsmessung ist in Abbildung 4.2 wiedergegeben, die Anpassung entspricht einem Lorentz-Profil mit einer Halbwertsbreite von etwa 120 fs.

\subsubsection{NOPA}

Beim Nopa (engl. Non-Collinear Optical Parametrical Amplifier) handelt es sich um einen optisch parametrischen Verstärker mit nicht-kollinearem Aufbau nach dem Riedle-Prinzip [182, 183. In diesem wird ein Drei-Wellen-Mischverfahren angewandt, um aus der Fundamentalen des Lasersystems Signal- und Idlerpulse über einen weiten Frequenzbereich zu generieren. Für eine weitergehende Betrachtung der nicht-linearen Wellenmischverfahren sei auf weitergehende Literatur wie z. B. von Lauterborn [184] verwiesen.

Der Strahlengang ist in Abbildung 4.3 dargestellt. Über den Strahlteiler ST1 wird ein geringer Teil des Pumplichts abgetrennt und zur Kontinuumserzeugung (KE) in ein Saphirplättchen fokussiert. Der Rest wird mit Hilfe eines BBO-Kristalls (Beta-Bariumborat) $\left(8 \times 8 \times 0,7 \mathrm{~mm}^{3}, \Theta=30^{\circ}\right.$, Typ-I Phasenanpassung) zu $387 \mathrm{~nm}$ frequenzverdoppelt. Mit Hilfe eines Strahlteilers (ST2) wird das Pumplicht aufgeteilt, 20v. H. der Intensität dienen zum Pumpen der ersten Stufe, die restlichen 80 v. H. zum Betrieb der zweiten. Für den erforderlichen Drei-Wellen-Mischprozeß werden nun Weißlichtkontinuum und der Pumpstrahl nicht-kollinear in einem BBO-Kristall $\left(5 \times 5 \times 1 \mathrm{~mm}^{3}, \Theta=32,5^{\circ}\right.$, Typ-I Phasenanpassung) überlagert. Dabei entstehen neben Pumppuls ein Signal- und ein Idlerpuls, durch die nicht-lineare (der Überlagerungswinkel beträgt etwa $6^{\circ}$ ) Geometrie ist das Weißlichtkontinuum mit dem Signalpuls überlagert. Da das Weißlichtkontinuum einer starken Gruppengeschwindigkeitsdispersion (engl. group velocity dispersion, GVD) unterliegt, kann mit einer Verschiebebühne nun der zur Verstärkung gewünschte 


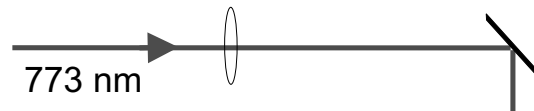

1. Stufe

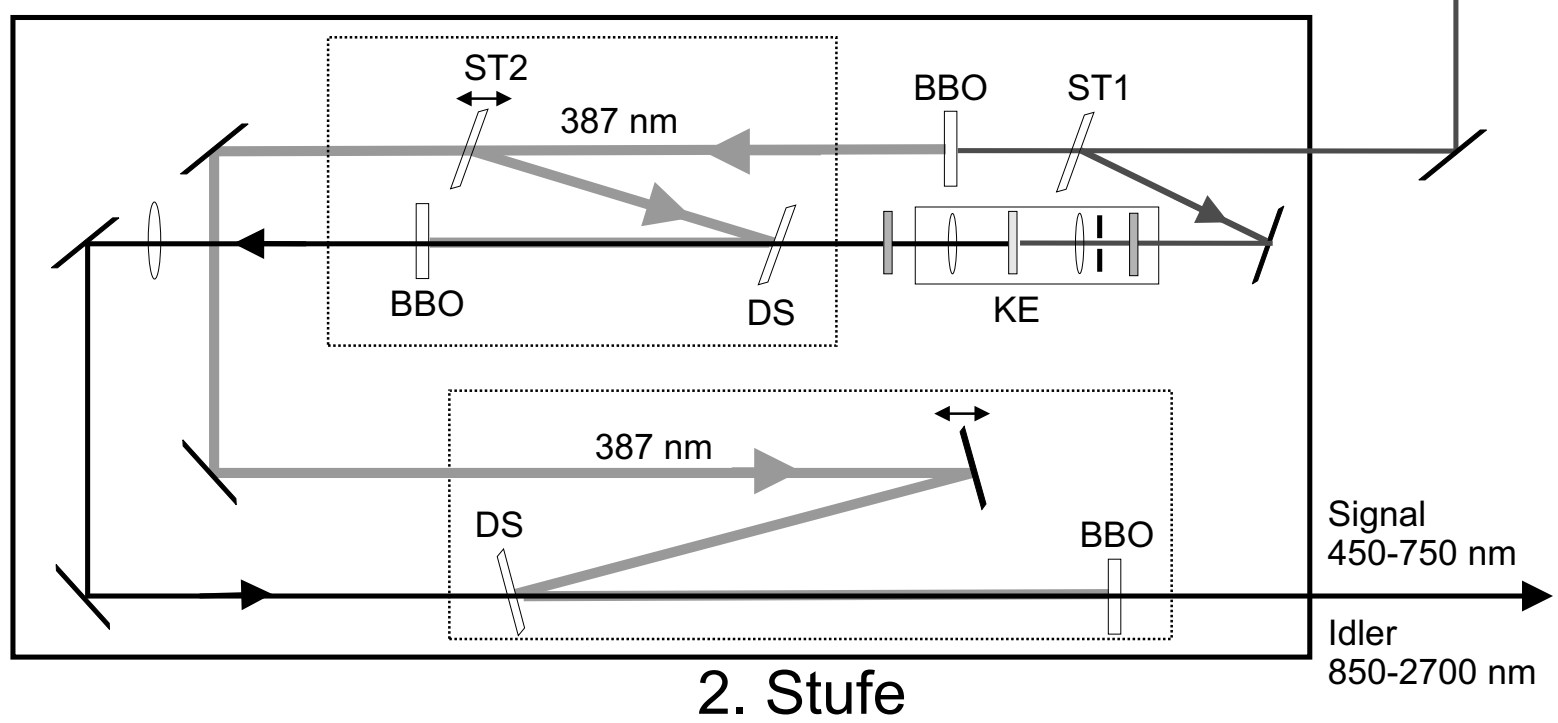

Abbildung 4.3: Aufbau und Strahlengang des NOPA [185]

spektrale Bereich des Weißlichkontinuums ausgewählt werden. Nach Erzeugen des gewünschten Signalpulses werden überschüssiges Pumplicht und Idlerpuls geblockt und der Signalpuls zur weiteren Verstärkung in die zweite Stufe eingespeist. Diese funktioniert nach dem gleichen Prinzip wie die erste, wird allerdings mit wesentlich mehr Pumpintensität (80v. H.) betrieben. Schlußendlich steht ein Signalpuls im durchstimmbaren Wellenlängenbereich von 450-750 nm zur Verfügung, die Ausgangsintensität ist wellenlängenabhängig und liegt typischerweise um $15 \mathrm{~mW}$. Anschließend wird der Ausgangspuls durch einen Prismenkompressor [185] zeitlich komprimiert und zur Erzeugung von UVPulsen frequenzverdoppelt. Dazu passiert er ein einfaches Kepler-Teleskop (2 Quarzlinsen mit $f=100 \mathrm{~mm})$ mit einem BBO-Kristall im Brennpunkt $\left(5 \times 5 \times 0,1 \mathrm{~mm}^{3}, \Theta=38^{\circ}\right.$ bzw. 51 ${ }^{\circ}$, Typ-I Phasenanpassung), die Fundamentale wird durch einen Polarisationsfilter und dielektrische Spiegel (HR 280-380 nm) abgetrennt. Anschließend durchläuft der frequenzverdoppelte Pumppuls (230-370 nm) noch ein wellenlängendurchstimmbares $\lambda / 2$-Plättchen (Fa. ALPHALas), mit welchem seine Polarisationrichtung im Vergleich zum Probepuls eingestellt werden kann. Üblicherweise wurde im „magischen“ Winkel [186] von $54,7^{\circ}$ gemessen.

\subsubsection{Erzeugung der Dritten Harmonischen (THG)}

Für die Messungen von $\mathrm{CH}_{2} \mathrm{ClI}$ und $\mathrm{CH}_{2} \mathrm{BrI}$ wurde anstelle des NOPA mit der Dritten Harmonischen (THG) des Lasersystems angeregt. Dazu wurde die Fundamentale mit einem BBO-Kristall $\left(5 \times 5 \times 0,5 \mathrm{~mm}^{3}, \Theta=30^{\circ}\right.$, Typ-I Phasenanpassung $)$ frequenzverdop- 
pelt und mit einem dielektrischen Spiegel (HR 800) in Zweite Harmonische (SHG) und Fundamentale aufgespalten. Durch ein $\lambda / 2$-Plättchen wurde die Polarisationsrichtung der Fundamentalen um $90^{\circ}$ gedreht und diese zusammen mit der SHG in einen weiteren BBO-Kristall $\left(5 \times 5 \times 1 \mathrm{~mm}^{3}, \Theta=47^{\circ}\right.$, Typ-I Phasenanpassung $)$ fokussiert. Die bestmögliche Frequenzmischung erfolgte durch Einstellung des zeitlichen Überlapps mit Hilfe einer justierbaren Verschiebebühne und durch Einstellung der Phasenanpassung durch Drehen des BBO-Kristalls. Die Ausgangsintensität lag dabei typischerweise um $10 \mathrm{~mW}$ bei $258 \mathrm{~nm}$.

\subsubsection{TOPAS}

Der Probestrahl wurde mit Hilfe eines TOPAS (engl. Traveling-Wave Optical Parametric Amplifier of Superfluorescence) der Firma Light Conversion Ltd. erzeugt. Das Prinzip des TOPAS basiert auf der Erzeugung und Verstärkung von Superfluoreszenz. Dazu wird ein Pumppuls in einen nicht-linearen optischen Kristall eingestrahlt, bei genügend hoher Intensität entsteht nun wieder ein Drei-Wellen-Mischprozeß, in welchem Signal- und Idlerpuls entstehen. Im Gegensatz zum NOPA wird jedoch kein bestimmtes Signal/IdlerPärchen durch Überlagerung mit einem Seedpuls ausgewählt. Die Wellenlängenselektivität wird in diesem Fall durch optische Beugungsgitter gesteuert.

Dazu wird die in den TOPAS eingekoppelte Fundamentale in drei Komponenten aufgeteilt, für den genauen Strahlengang siehe [187]. Mit einem Teil wird in einem dreistufigen Durchlaufen des nicht-linearen Kristalls Superfluoreszenz erzeugt (Signal- und Idlerpuls liegen dazu in einem Bereich von 1-3 $\mu \mathrm{m}$ ) und die gewünschte Wellenlänge durch ein Gitter selektiert. Der abgetrennte Teil dient als Seedpuls für die Stufen 4 und 5, welche mit dem größten Anteil der Fundamentalen gepumpt werden und den Signal/Idlerpuls auf die erforderliche Intensität verstärken. Dabei können Signalpulse im Wellenlängenbereich von 1070-1560 nm (s-polarisiert) und Idlerpulse von 1560-2790 nm (p-polarisiert) erzeugt werden. Durch nachgeschaltete Wellenmischverfahren kann ein sehr breites Spektrum an Ausgangswellenlängen im TOPAS erzeugt werden.

Mögliche Mischverfahren sind:

- SHI Zweite Harmonische des Idler-Pulses (770-1070 nm, p-polarisiert)

- SHS Zweite Harmonische des Signal-Pulses (571-770 nm, s-polarisiert)

- SFS Summenfrequenzerzeugung aus Signal-Puls und Fundamentaler (450-515 nm, s-polarisiert)

- SFI Summenfrequenzerzeugung aus Idler-Puls und Fundamentaler (515-600 nm, s-polarisiert)

- FHI Vierte Harmonische des Idler-Pulses (385-450 nm, p-polarisiert)

- FHS Vierte Harmonische des Signal-Pulses (285-385 nm, p-polarisiert) 
- SH(SFS) Zweite Harmonische aus Signal-Puls und Fundamentaler $(225-258 \mathrm{~nm}$, p-polarisiert)

- SH(SFI) Zweite Harmonische aus Idler-Puls und Fundamentaler (258-285 nm, ppolarisiert)

Noch kurzwelligere Pulse können durch Frequenzmischverfahren mit einem Teil der Fundamentalen, welcher vor Einspeisung in den TOPAS abgetrennt und durch eine Einheit mit identischem Strahlengang (Deep-UV-Einheit) gelenkt wird, erzeugt werden.

- P+FHS Summenfrequenz aus Vierter Harmonischer des Signalpulses und der Fundamentalen aus der Deep-UV-Einheit (210-250 nm, s-polarisiert)

- $\mathrm{P}+\mathrm{SH}(\mathrm{SF})$ Summenfrequenz aus Fundamentaler der Deep-UV-Einheit und $\mathrm{SH}(\mathrm{SFS})$ sowie $\mathrm{SH}(\mathrm{SFI})(190-210 \mathrm{~nm}, \mathrm{~s}$-polarisiert)

Die Steuerung des TOPAS erfolgt über den Meßrechner mit Hilfe der Software WinTOPAS Deep UV der Firma Light Conversion Ltd. Manuelle Justage ist normalerweise nicht notwendig, bei zu starkem Rauschen des Ausgangspulses kann die Einkopplung in den TOPAS nachjustiert werden.

\subsubsection{Interferometer}

Die Messung als solche erfolgt durch Überlagerung von Pump- und Probestrahl mit Hilfe eines Interferometers. Dazu wird der Probestrahl mit einem Strahlteiler in zwei Strahlengänge aufgespalten, wobei ein Teilstrahl mit einer Photodiode (Hamamatsu 1226-8BQ bzw. 1336-8BQ0A) vor der Meßzelle als Referenz PD $I_{0}$ gemessen wird. Der andere Teilstrahl wird mit einem $90^{\circ}$-off-axis-Parabolspiegel $(f=152,4 \mathrm{~mm})$ in die Meßzelle fokussiert und hinter dieser mit einer weiteren Photodiode (PD $I$ ) detektiert. Der Pumpstrahl wird mit einem zweiten Parabolspiegel $(f=203,3 \mathrm{~mm})$ unter spitzem Winkel (etwa $8^{\circ}$ ) mit dem Probestrahl in der Meßzelle überlagert. Durch diese nicht-kollineare Anordnung kann nicht absorbiertes Pumplicht hinter der Zelle durch eine Blende geblockt werden, ohne daß es auf die Photodiode PD I fällt. Die Signale der Photodioden werden mit Hilfe einer elektronischen Schaltung integriert [180] und von der A/D-Karte in den Meßrechner eingelesen. Zu Justagezwecken können diese Signale auf einem Oszilloskop (Tektronix TDS-3052) beobachtet werden, zur Verbesserung des Signal/Rausch-Verhältnisses wird das Signal der Referenzphotodiode PD $I_{0}$ durch variable Graufilter auf das der Photodiode PD I abgeglichen.

\subsubsection{Datenaufnahme}

Das Prinzip der Datenaufnahme ist in Abbildung 4.4 skizziert. Die Taktfrequenz wird über die Repetitionsrate des Lasers bestimmt, dazu wird das $1 \mathrm{kHz}$-Signal der Pockelszelle (DT) (Hochfrequenztreiber DT-505) mit einem Delaygenerator (DG) der Firma Stanford Research Systems Inc. (DG-535) aufgenommen und zeitlich verzögert als Triggersignal für 


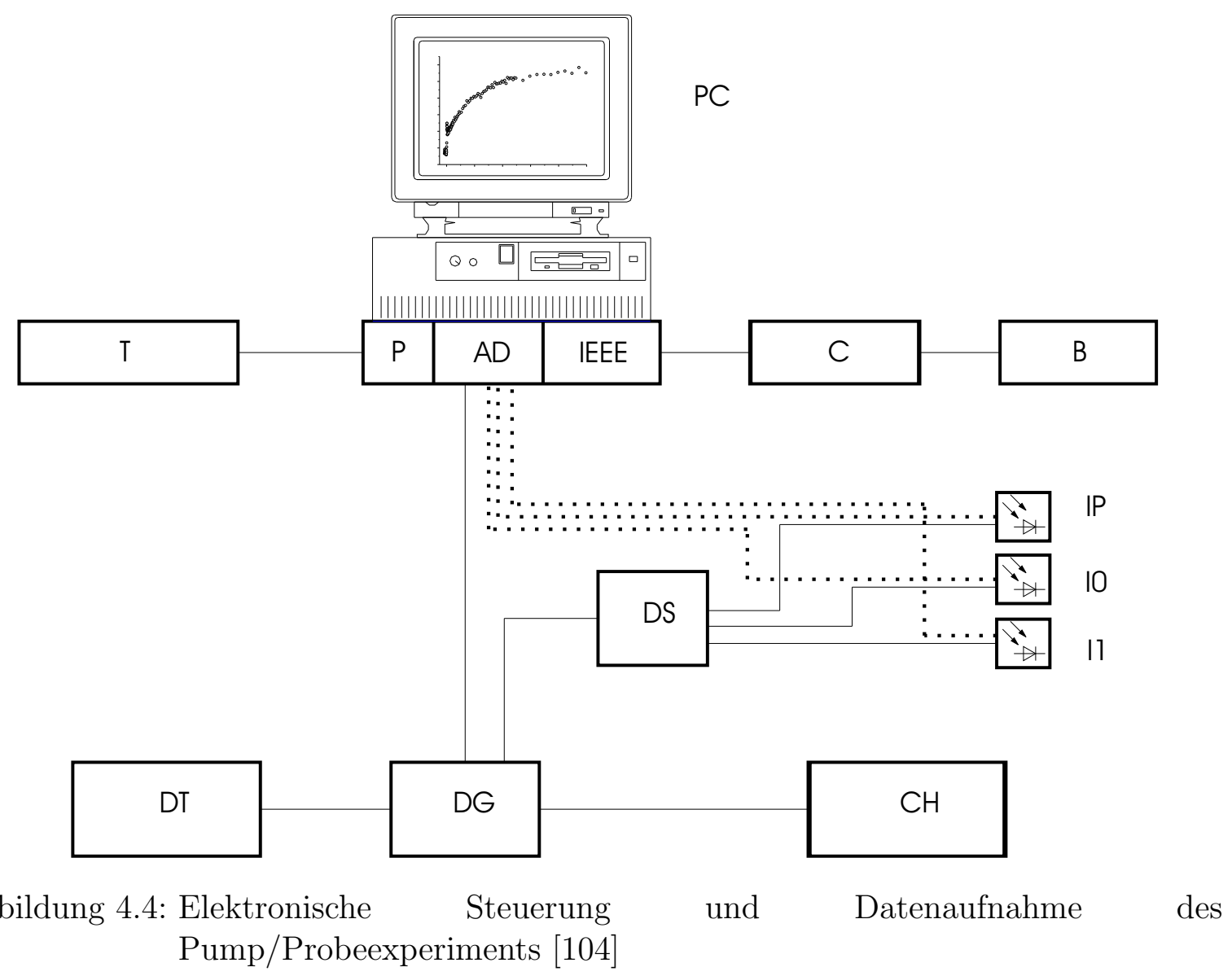

alle anderen Geräte ausgegeben. Neben der Synchronisation der Choppersteuerung (CH) startet das Triggersignal des DG-535 die Integrationsschaltung der Photodioden (DS), welche über jeden detektierten Laserpuls integrieren und das Ergebnis als Spannung zwischen 1-10 V ausgeben. Die Auslesung des Diodensignals erfolgt über eine A/D-Karte (AD) (Data Translation, DT 3010), dabei werden Referenzphotodiode $I_{0}$, die Signalphotodiode $I_{1}$ und die Photodiode $I_{P}$ ausgelesen. Die Photodiode $I_{P}$ fängt einen Reflex nach der Modulation durch den Chopper auf, sie dient zur Zuordnung, ob ein Signal bei geschlossenem oder geöffnetem Chopper gemessen wurde. Die Signale der Photodioden werden nun nach Gleichung 4.2 in $\triangle O D$ umgerechnet, dazu wird über 200 Laserpulse gemittelt und das Ergebnis anschließend graphisch am Meßrechner ausgegeben. Die Verzögerungszeit dieser Messung von Pump- und Probestrahl wird über die Position der Verschiebebühne (B) (Physik Instrumente, PI M-415.DG) eingestellt. Dazu wird diese vom Meßrechner durch eine PCI-Bus-IEEE488.2-GPIB-Interfacekarte über die Steuereinheit (C) (Physik Instrumente, PI C-844) angesteuert. Die Verzögerungszeit liegt dabei üblicherweise im Bereich von 0-500 ps, kann aber auf bis zu 1 ns ausgedehnt werden. Die Datenaufnahme und Ansteuerung aller Komponenten wird dabei über ein selbstgeschriebenes Programm [104] der Software HP VEE (Agilent HP VEE 6.2) vorgenommen. Der TOPAS (T) wird ebenfalls vom Meßrechner über eine parallele Schnittstelle (P) angesteuert. 


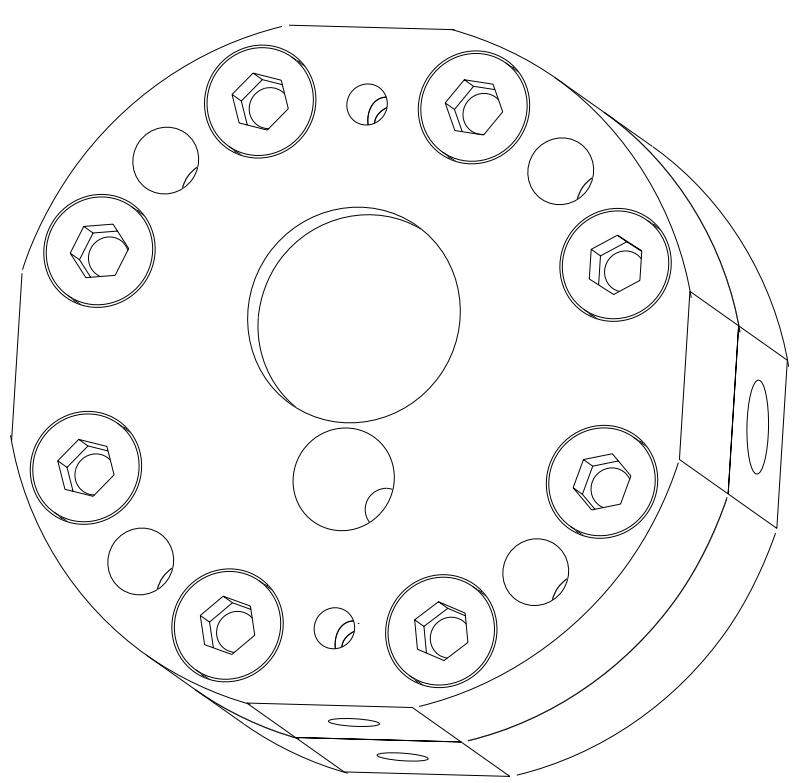

Abbildung 4.5: Hochdruckzelle zur Verwendung von überkritischen Fluiden als Lösungsmittel [104]

\subsubsection{Hochdrucksystem}

Für Messungen in überkritischen Lösungsmitteln wurde eine spezielle Hochdruckzelle (s. Abbildung 4.5) verwendet. Das Design der Hochdruckzelle ist an einen Entwurf der Arbeitsgruppe von Prof. Kajimoto aus Tokio angelehnt. Diese besteht aus hochfesten, iodresistenten Stahl (Inconel Alloy 718, Materialnummer 2.4969) und wird mit 8 M6Schrauben mit einem Teflon-O-Ring als Dichtung verschlossen. In der Zelle befindet sich ein Reservoir mit einem Rührstäbchen, welches über einen außen angebrachten Magnetrüher bewegt wird. Die Saphirfenster (Korth $\varnothing 10 \mathrm{~mm}, 2 \mathrm{~mm}$ Stärke) sind mit Zweikomponentenkleber (UHU Plus Endfest 300) in die Halterungen eingeklebt. Die optische Weglänge zwischen den Fenstern entspricht etwa 2,5 mm. Zum Befüllen sind an der Zelle Laborverschraubungen angebracht (NOVA Swiss, 3/16"-24UNF für 1/16"-Kapillaren), die über Bohrungen mit dem Innenraum verbunden sind. Desweiteren besitzt der Zellkörper 4 Bohrungen für Heizpatronen (HotSet HHP, 6,5 ×45 mm², $120 \mathrm{~W}, 230 \mathrm{~V}$ ), die Temperaturregelung wird mit Hilfe eines Heizreglers (Haake TP24) und einem Pt-100-Meßfühler vorgenommen.

Zum Befüllen der Zelle wurde ein Hochdrucksystem verwendet, dieses besteht aus einem Kompressor (NOVA Swiss Membrankompressor, 1000 bar, ölfrei), welcher das zu verwendende Gas aus der Druckgasflasche über 1/4"-Kapillaren (NOVA Swiss, 4 kbar) in eine Spindelpresse pumpt. Der Vordruck ist dabei einstellbar, üblicherweise liegt er bei 150 bar. Mit der Spindelpresse kann nun ein Druck von bis zu 1000 bar eingestellt werden und das Gas über Kapillaren (Techlab Edelstahlkapillare, $\varnothing_{\text {Innen }}$ 0,25 mm, 1000 bar) in die Meßzelle eingeleitet werden. 


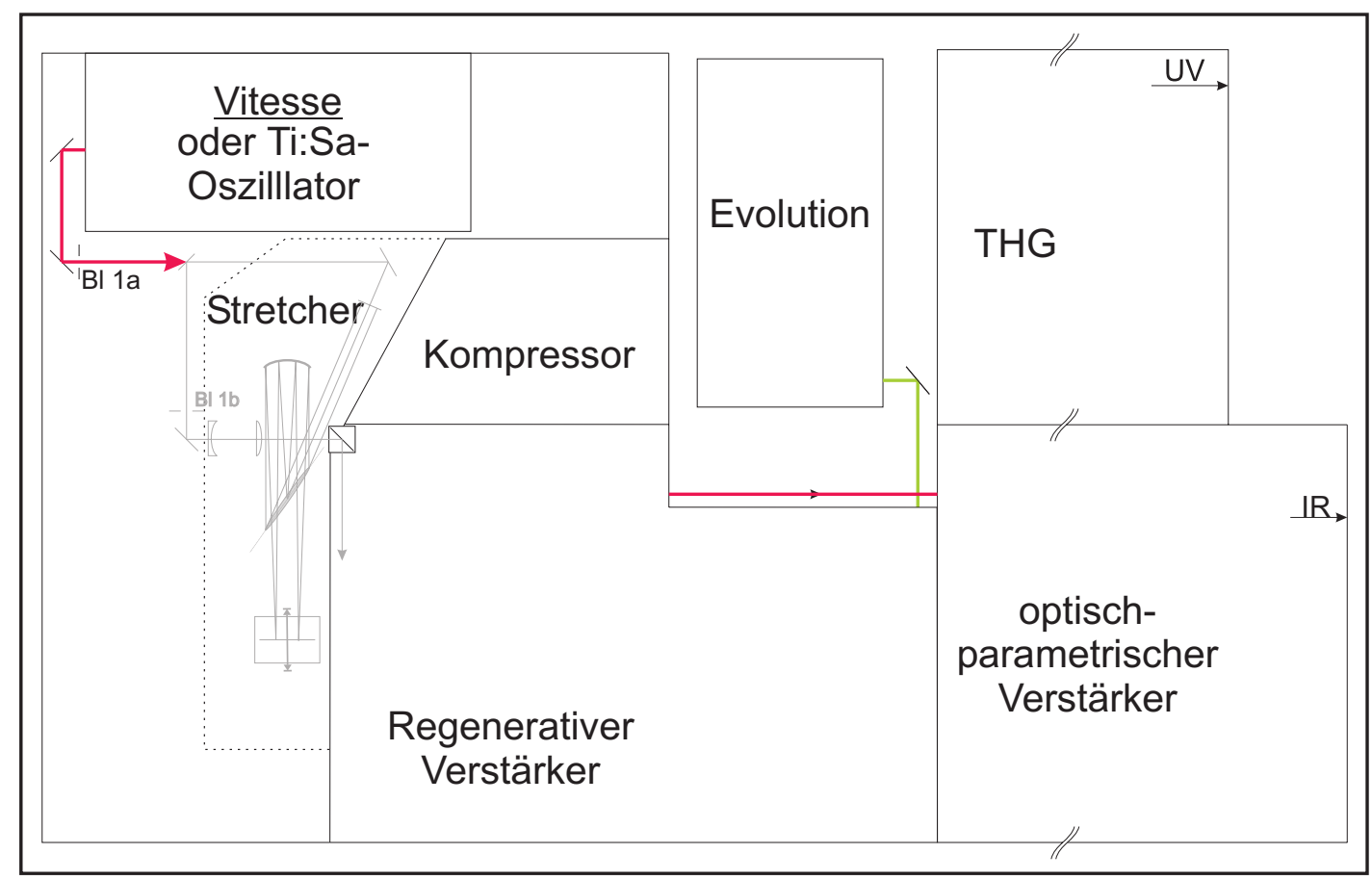

Abbildung 4.6: Komponenten und Strahlengang des IR-Lasersystems [188]

\subsection{Das IR-Lasersystem}

Die Messungen mit dem fs-UV-Pump/IR-Probelasersystem wurden in Zusammenarbeit mit der Arbeitsgruppe von Prof. Dr. D. Schwarzer am Max-Planck-Institut für Biophysikalische Chemie Göttingen durchgeführt. Herzstück dieser Anlage ist ein von Dr. Christian Reichardt konstruiertes fs-Pump/IR-Probe-Spektrometer, welches in der Lage ist, den Probestrahl im Bereich von 3-9 $\mu \mathrm{m}$ mit einer Bandbreite von etwa $250 \mathrm{~cm}^{-1}$ aufzulösen. Über das Lasersystem und seine einzelnen Komponenten soll hier nur ein sehr kurzer Überblick gegeben werden, eine ausführliche Beschreibung findet sich in der Arbeit von Dr. C. Reichardt [188].

\subsubsection{Ti:Sa Laser}

Bei der Laserquelle des IR-Lasersystems handelt es sich ebenfalls um ein Ti:SaLasersystem nach dem CPA-Konzept (chirped pulse amplification, vgl. Abschnitt 4.1.1). Der Aufbau und Strahlengang ist in Abbildung 4.6 wiedergegeben. Dabei stehen die Abkürzungen HRxxx für hochreflektierende Spiegel für die Zentralwellenlänge xxx nm und GS für goldbeschichtete Spiegel, welche sich durch eine gute IR-Reflektivität auszeichnen.

Der regenerative Verstärker wird mit Seedpulsen aus einem kommerziell erhältlichen Ti:Sa-Laser (Coherent Vitesse) gespeist, als Pumplaser dient ein synchronisierter Nd:YLF-Laser (Coherent Evolution). Der Seedpuls wird mit Hilfe zweier Gitter durch 
einen positiven linearen Chirp auf etwa 500 ps zeitlich gestreckt und in den regnerativen Verstärker eingespeist. Dabei durchläuft er mehrfach den Ti:Sa-Kristall, welcher als laseraktives Medium kontinuierlich vom Pumplaser gepumpt wird. Nach der Verstärkung und Auskopplung aus dem regenerativen Verstärker mit Hilfe einer Pockelszelle beträgt die Pulsenergie etwa $1 \mathrm{~mJ}$ bei einer Zentralwellenlänge von $800 \mathrm{~nm}$ und einer Repetitionsrate von $1 \mathrm{kHz}$. Die ausgekoppelten Pulse werden über holographische Gitter anschließend zeitlich auf etwa $100 \mathrm{fs}$ bei einer Bandbreite von ca. $250 \mathrm{~cm}^{-1}$ komprimiert, die Verluste liegen dabei etwa bei 20-30 v. H. Danach wird die Fundamentale mit einem Strahlteiler in einen Pump- und einen Probestrahl aufgeteilt. Der Pumppuls wird durch Erzeugen der Dritten Harmonischen bei $266 \mathrm{~nm}$ generiert (s. Abschnitt 4.1.3), während der breitbandige IR-Probepuls durch einen optisch parametrischen Verstärker (OPA) erzeugt wird.

\subsubsection{Erzeugung der IR-Probepulse}

Der in Abbildung 4.7 dargestellte optisch-parametrische Verstärker (OPA) ist ein zweistufiges System, welches Signalpulse von 1,20-2,47 $\mu \mathrm{m}$ und entsprechende Idlerpulse von 2,40-1,76 $\mu \mathrm{m}$ erzeugen kann. Der OPA wird dabei über einen Strahlteiler mit $40 \mathrm{v}$. H. der Fundamentalen bei $800 \mathrm{~nm}$ des regenerativen Verstärker gepumpt. Ein kleiner Teil (1 v. H.) wird mit einem Strahlteiler abgetrennt und dient zur Erzeugung eines Weißlichtkontinuums durch Fokussierung in einen Saphir-Kristall.

Der parametrische Prozeß wird durch Drei-Wellen-Mischen in einem nicht-linearen optischen BBO-Kristall ( $\beta$-Barium-Borat) erzeugt. Dabei werden für die erste Stufe mit einem Strahlteiler etwa 10 v. H. vom Pumppuls abgetrennt und über einen dichroiden Spiegel mit dem Weißlichtkontinuum im BBO-Kristall $\left(8 \times 4 \times 5 \mathrm{~mm}^{3}, \phi=0^{\circ}\right.$ und $\Theta=$ $27^{\circ}$, Typ-II Phasenanpassung) zum Überlapp gebracht. Dabei werden ein Signal- und ein Idlerpuls erzeugt, welche anschließend über einen dichroiden Spiegel voneinander abgetrennt werden. Der Idlerpuls wird mit Hilfe einer Ge-Diode (IR D1) detektiert, das Diodensignal kann dabei zur Optimierung der Justage herangezogen werden. Der Signalpuls wird über einen Goldhohlspiegel zurück in den BBO-Kristall geführt, wo er mit dem restlichen Pumplicht zum Überlapp gebracht wird und somit in der zweiten Stufe des OPA Signal- und Idlerpuls erzeugt. Signal- und Idlerpuls verlassen dabei möglichst kollinear den BBO-Kristall und werden anschließend in ein Michelson-Interferometer eingekoppelt, das aus einem dichroiden Spiegel in der Mitte und zwei Goldhohlspiegeln sowie einer Photodiode (IR D2) zur Kontrolle des zeitlichen Überlapps besteht. Danach werden beide Pulse zur Erzeugung der Differenzfrequenz in einen $\mathrm{AgGaS}_{2}$-Kristall fokussiert. Schlußendlich stehen damit Pulse im Frequenzbereich von 3000-9000 nm zur Verfügung, die Wellenlänge kann dabei über die beiden Winkel $\Theta_{1}$ und $\Theta_{2}$ der beiden nicht-linearen optischen Kristalle eingestellt werden. 


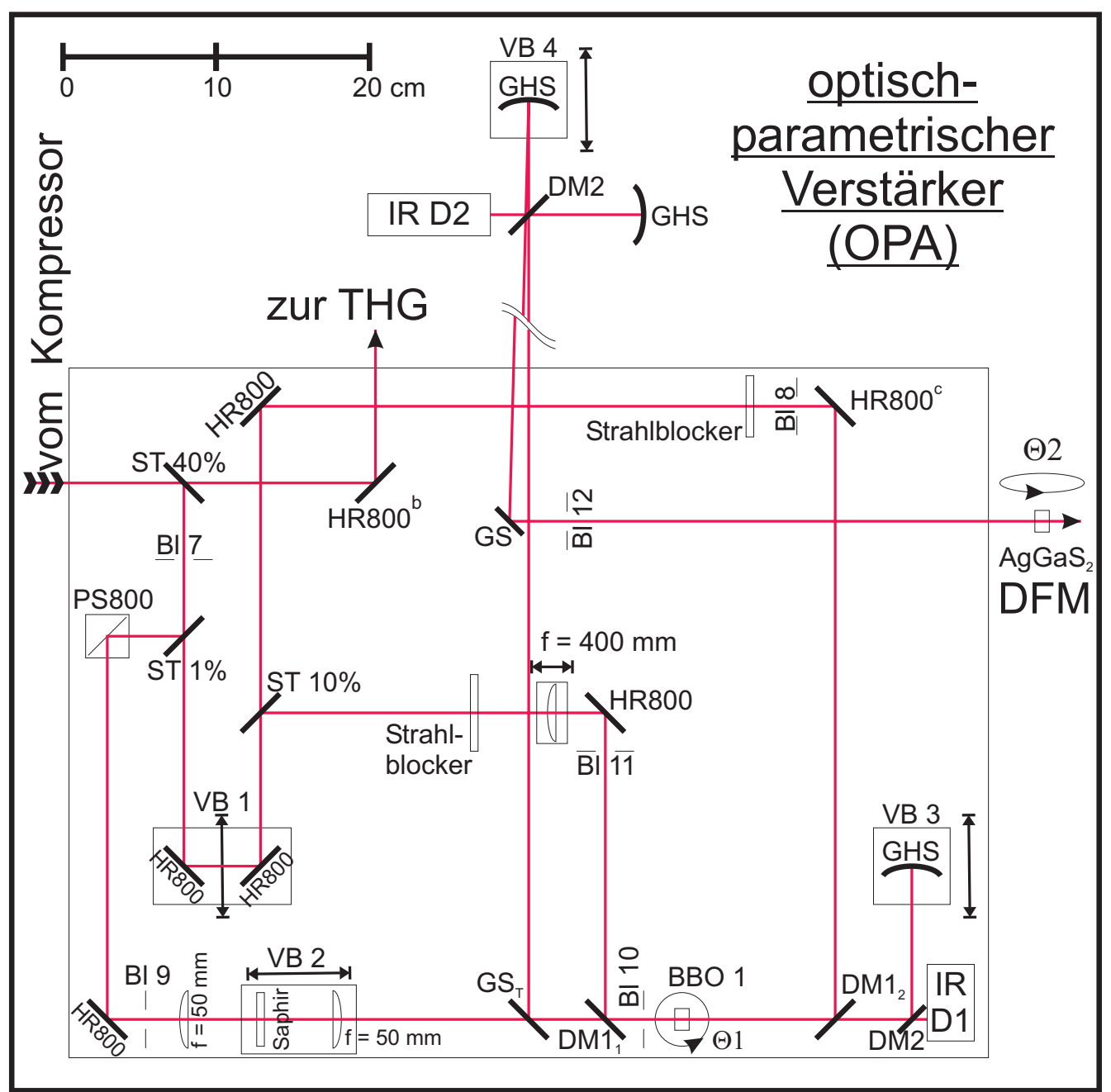

Abbildung 4.7: Aufbau und Strahlengang des IR-OPA [188]

\subsubsection{Das IR-Spektrometer}

Der im $\mathrm{AgGaS}_{2}$-Kristall erzeugte IR-Puls durchläuft anschließend zur Kollimation ein Galilei-Teleskop ( $\mathrm{CaF}_{2}$-Linsen mit $f_{1}=50 \mathrm{~mm}$ und $\left.f_{2}=100 \mathrm{~mm}\right)$ und wird mit Hilfe eines IR-Strahlteilers aus $\mathrm{CaF}_{2}$ in einen Referenzpuls $I_{0}$ und einen Probepuls $I$ aufgespalten. Nach Durchlaufen von zwei unterschiedlichen Verzögerungsstrecken passieren Referenzstrahl $I_{0}$ und Probestrahl $I$ die Meßzelle (MZ). Die Verzögerungsstrecke ist dabei so gewählt, daß der Referenzstrahl immer etwas früher als der Pumppuls durch die Meßzelle läuft. Probepuls und Pumppuls werden in der Meßzelle räumlich zum Überlapp gebracht, durch die Verschiebebühne VB7 kann die zeitliche Verzögerung variiert werden.

Nachdem Referenzstrahl $I_{0}$ und Probestrahl $I$ die Meßzelle durchlaufen haben, werden beide Strahlen durch eine Kombination aus Goldplanspiegeln (GPS1-2 und DGS) über einen Langpaßfilter (blockt Wellenlängen $<2400 \mathrm{~nm}$ ) gekreuzt auf einen Silberparabol- 


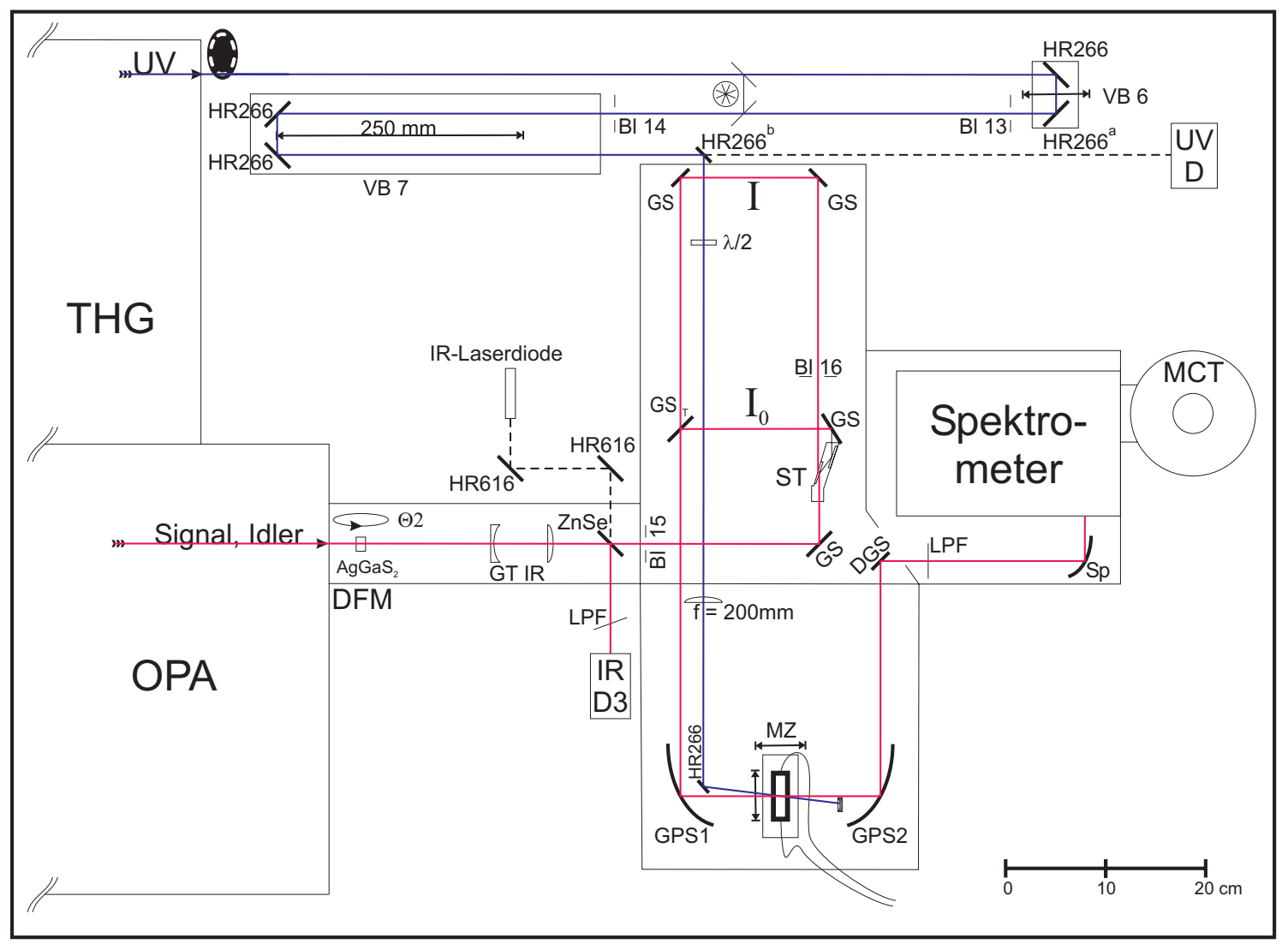

Abbildung 4.8: Aufbau und Strahlengang des IR-Spektrometers [188]

spiegel (Sp) gelenkt, welcher beide Strahlen durch einen einstellbaren Eingangsschlitz in das Spektrometer reflektiert. Das Gitterspektrometer (150 Linien $/ \mathrm{mm}$ ) bildet dabei die Spektren von Referenzstrahl $I_{0}$ und Probestrahl $I$ übereinander auf dem MCT-IRDetektor ab (Fa. Infrared Systems). Dieser besteht aus zwei übereinander angeordneten Diodenarrays mit jeweils $32 \mathrm{HgCdTe-Dioden.} \mathrm{Die} 64$ Kanäle der Photodioden sowie eine Referenzphotodiode zur Zuordnung, ob die Messung bei geöffnetem oder geschlossenem Chopper erfolgte, wurden anschließend mit Hilfe einer A/D-Wandlerkarte in den Meßrechner eingelesen.

Die so aufgenommenen Rohdaten werden anschließend durch ein in der Programmierumgebung LabView (National Instruments LabView 8.0) geschriebenes Programm aufgenommen und zur Abtrennung vom Hintergrundrauschen diskriminiert. Anschließend wird um das Hintergrundsignal korrigiert und die Daten mit Hilfe eines in MathCad (MathSoft MathCad 13) geschriebenen Programms ausgewertet. Für nähere Details dieser Datenauswertung sei hier noch einmal auf die Dissertation von Dr. C. Reichardt verwiesen [188]. 


\subsection{Probenpräparation}

\subsubsection{Messungen in überkritischen Fluiden}

Zur Aufnahme der Absorptionszeitprofile der Polyhalogenmethane in sc. $\mathrm{CO}_{2}$ wurde die in Abschnitt 4.1.7 beschriebene Hochdruckzelle verwendet. Vor Durchführung jeder Messung wurde die Zelle auseinandergeschraubt und mit Methanol (für die UVSpektroskopie, Fluka) und optischem Papier (Whatman Lens Cleaning Tissue) gereinigt. Anschließend wurden etwa 2,5 $\mu \mathrm{l}\left(\mathrm{CH}_{2} \mathrm{BrI}\right.$ und $\left.\mathrm{CH}_{2} \mathrm{I}_{2}\right)$ oder ein kleine Spatelspitze $\left(\mathrm{CHI}_{3}\right)$ des entsprechenden Polyhalogenmethan in das Reservoir der Zelle gegeben (dies entspricht bei $\mathrm{CH}_{2} \mathrm{I}_{2}$ bzw. $\mathrm{CH}_{2} \mathrm{BrI}$ einer Konzentration von etwa $12 \mathrm{mmol} \mathrm{l}^{-1}$ ), das Rührstäbchen in die entsprechenden Halterungen eingesetzt und die Zelle wieder zusammengeschraubt (Drehmoment der Schrauben $9 \mathrm{Nm}$ ). Die Zelle wurde nun im Interferomter des Lasersystems positioniert und der Magnetrührer vor der Zelle eingeschaltet. Nach Anschluß der Laborverschraubungen an das Hochdrucksystem wurde ein entsprechender Druck an flüssigem $\mathrm{CO}_{2}$ auf die Zelle gegeben, die Heizpatronen eingesetzt und anschließend die Zelle mit Hilfe der Heizsteuerung auf die gewünschte Temperatur geheizt. Diese lag üblicherweise mit $35^{\circ} \mathrm{C}$ knapp über der kritischen Temperatur $T_{k}$ von $\mathrm{CO}_{2}$ (entspricht einer reduzierten Temperatur $T_{r}=T / T_{k}$ von 1,01). Nachdem sich das Temperaturgleichgewicht eingestellt hatte, wurde anhand der Absorption des Pumpstrahls geprüft, ob das Polyhalogenmethan sich in ausreichendem Maße gelöst hatte, was bei Drücken > 100 bar jedoch kein Problem darstellte.

Mit diesem Meßaufbau konnte mehrere Stunden bei kontinuierlicher Bestrahlung mit dem Pumppuls gemessen werden, ohne daß sich eine Veränderung des Signals oder sonstige Zeichen einer Degeneration der überkritischen Lösung zeigte. Nach Abschluß der Messungen wurden die Hochdruckhähne der Zelle geschlossen, die Verschraubungen zum Hochdrucksystem gelöst und der Inhalt unter dem Abzug abgeblasen. Anschließend wurde die Zelle und das Rührstäbchen mit optischem Papier und Methanol gereinigt.

\subsubsection{Messungen in flüssigen Lösungsmitteln}

Bei Verwendung von flüssigen Lösungsmitteln wurde mit einem Durchflußsystem gearbeitet. Dieses besteht aus einer Durchflußzelle (wahlweise Schichtdicke 0,5 oder $0,7 \mathrm{~cm}$, Deckglas Quarz Suprasil), welche über Teflonschläuche mit einer modifizierten Laserfarbstoffpumpe und einem Reservoir verbunden ist. Das Reservoir faßt $100 \mathrm{ml}$ und muß zu einer Messung mit etwa $50 \mathrm{ml}$ befüllt werden. Der Flux wird über ein Regelgerät so eingestellt, daß keine Blasen durch die Zelle gepumpt und keine Vibrationen von der Pumpe auf den Lasertisch übertragen werden. Nach Beendigung einer Messung wurde das Durchflußsystem mehrmals zur Reinigung mit dem verwendeten Lösungsmittel gespült. Alle Messungen mit flüssigen Lösungsmitteln wurden bei Raumtemperatur (295 K) aufgenommen. Leermessungen der reinen Lösungsmittel wurden mit einer stationären Küvette (0,1 oder 0,2 cm, Quarz Suprasil) durchgeführt. 


\subsubsection{Messungen von lodmethan in flüssigen Lösungsmitteln}

Zur Probenpräparation wurde etwas $\mathrm{CH}_{3} \mathrm{I}$ im entsprechenden Lösungsmittel in einer stationären Küvette (Schichtdicke 0,2 cm, Quarz Suprasil) gelöst und die Konzentration mit Hilfe eines Absorptionsspektrometers (Varian CARY 5E) auf eine optische Dichte $(O D)$ von etwa 2-3 eingestellt, dies entspricht einer Konzentration von etwa $30 \mathrm{mmol}$ $1^{-1}$. Damit konnten mehrere Messungen durchgeführt werden, das Absorptionspektrum der bestrahlten unterscheidet sich nur sehr geringfügig von der unbestrahlten Lösung, so daß es in der Küvette offenkundig nicht zu einer Anreicherung von Abbauprodukten kommt.

Messungen mit der beschriebenen Durchflußzelle $(0,2 \mathrm{~cm}$, Suprasil) bei vergleichbarer Konzentration zeigen keinen signifikanten Unterschied der Absorptionszeitprofile im Vergleich zu Messungen mit der stationären Küvette. Allerdings ist das Signal/RauschVerhältnis in diesem Fall wesentlich schlechter, weswegen die stationäre Küvette insbesondere bei Messungen mit sehr teuren Lösungsmitteln, wie $n$-Perfluorhexan, der Durchflußzelle vorgezogen wurde.

\subsection{Anpassung der experimentellen Daten}

Die experimentell gemessenen Absorptionszeitprofile wurden mit Hilfe eines mit der Software Matlab (Mathworks, Matlab 6.5.1.199709 Release 13) von Dr. C. Grimm geschriebenen Programms [104 ausgewertet und angepaßt. Nach Mittelung der Meßdaten erfolgte die Anpassung der Daten mit einem Programm auf der Grundlage des LevenbergMarquardt Algorithmus in der Curve-Fitting Toolbox in der Matlab-Umgebung (Matlab Curve Fitting Toolbox 1.1, Release 13). Dabei stehen verschiedene Modellfunktionen $f(t)$ zur Verfügung:

Eine Modellfunktion $f(t)$ setzt sich aus der Faltung einer Gauß-Funktion $g(t)$ mit einer Exponentialfunktion $e(t)$ zusammen:

$$
f(t)=(g \otimes e)(t)=\int_{0}^{\infty} g(t-\tau) \cdot e(\tau) \mathrm{d} \tau,
$$

mit

$$
g(t)=\frac{1}{\sqrt{2 \pi \sigma^{2}}} \exp \left(-\frac{t^{2}}{2 \sigma^{2}}\right) .
$$

Die Exponentialfunktion $e(t)$ setzt sich aus der Summe eines monoexponentiellen Abfalls $e_{\mathrm{d}}(t)$ und eines monoexponentiellen Anstiegs $e_{\mathrm{r}}(t)$ zusammen:

$$
\begin{aligned}
& e(t)=\exp \left(-\frac{t}{\tau_{\mathrm{d}}}\right) \text { bzw. } \\
& e(t)=1-\exp \left(-\frac{t}{\tau_{\mathrm{r}}}\right)
\end{aligned}
$$


Die Faltung der Gauß-Funktion $g(t)$ mit dem exponentiellen Abfall $f_{\mathrm{d}}(t)$ führt zu:

$$
\begin{aligned}
f_{\mathrm{d}}(t) & =\int_{0}^{\infty} g(t-\tau) \cdot \exp \left(\frac{t}{\tau_{\mathrm{d}}}\right) \mathrm{d} \tau \\
& =\frac{1}{2} \exp \left(-\frac{2\left(t-t_{0}\right) \tau_{\mathrm{d}}-\sigma^{2}}{2 \tau_{\mathrm{d}}^{2}}\right)\left[\operatorname{erf}\left(\frac{\left(\left(t-t_{0}\right) \tau_{\mathrm{d}}-\sigma^{2}\right) \sqrt{2}}{2 \sigma \tau_{\mathrm{d}}}\right)+1\right] .
\end{aligned}
$$

Analog dazu ergibt sich für den exponentiellen Anstieg:

$$
\begin{aligned}
f_{\mathrm{r}}(t)= & \int_{0}^{\infty} g(t-\tau) \cdot \exp \left(1-\frac{t}{\tau_{\mathrm{r}}}\right) \mathrm{d} \tau \\
= & \frac{1}{2}\left[\operatorname{erf}\left(\frac{\sqrt{2}\left(t-t_{0}\right)}{2 \sigma}\right) \exp \left(\frac{t-t_{0}}{\tau_{r}}\right)-\exp \left(\frac{\sigma^{2}}{2 \tau_{\mathrm{r}}^{2}}\right) \operatorname{erf}\left(\frac{\left(\left(t-t_{0}\right) \tau_{\mathrm{d}}-\sigma^{2}\right) \sqrt{2}}{2 \sigma \tau_{\mathrm{r}}}\right)\right. \\
& \left.+\exp \left(\frac{t-t_{0}}{\tau_{\mathrm{r}}}\right)-\exp \left(\frac{\sigma^{2}}{2 \tau_{\mathrm{r}}^{2}}\right)\right] \exp \left(-\frac{t-t_{0}}{\tau_{\mathrm{r}}}\right) .
\end{aligned}
$$

Den End-Offset $I_{0}$ implementiert man über eine weitere Funktion $f_{0}(t)$ in die Modellfunktion:

$$
\begin{aligned}
f_{0}(t) & =I_{0} \int_{0}^{\infty} 1 \cdot g(t-\tau) \mathrm{d} \tau \\
& =\frac{1}{2} \operatorname{erf}\left(\frac{\sqrt{2}\left(t-t_{0}\right)}{2 \sigma}\right)+\frac{1}{2} I_{0} .
\end{aligned}
$$

Eine Modellfunktion $f(t)$ mit $i$ exponentiellen Anstiegen und $j$ exponentiellen Abfällen kann wie folgt berechnet werden:

$$
f(t)=\sum_{i} A_{i} f_{\mathrm{d}}\left(t, \tau_{\mathrm{d}, i}\right)+\sum_{j} B_{j} f_{\mathrm{r}}\left(t, \tau_{\mathrm{r}, j}\right)+f_{0}(t)+I_{1} .
$$

Die Parameter stehen dabei für:

- $A_{\mathrm{i}}, B_{\mathrm{j}}$ Amplituden

- $t_{0}$ Zeitnullpunkt

- $\sigma$ halbe Halbwertsbreite der Gauß-Funktion

- $\tau_{\mathrm{d}}, \tau_{\mathrm{r}}$ Lebensdauer des Abfalls/Anstiegs

- $I_{0}$ End-Offset

- $I_{1}$ Gesamt-Offset 

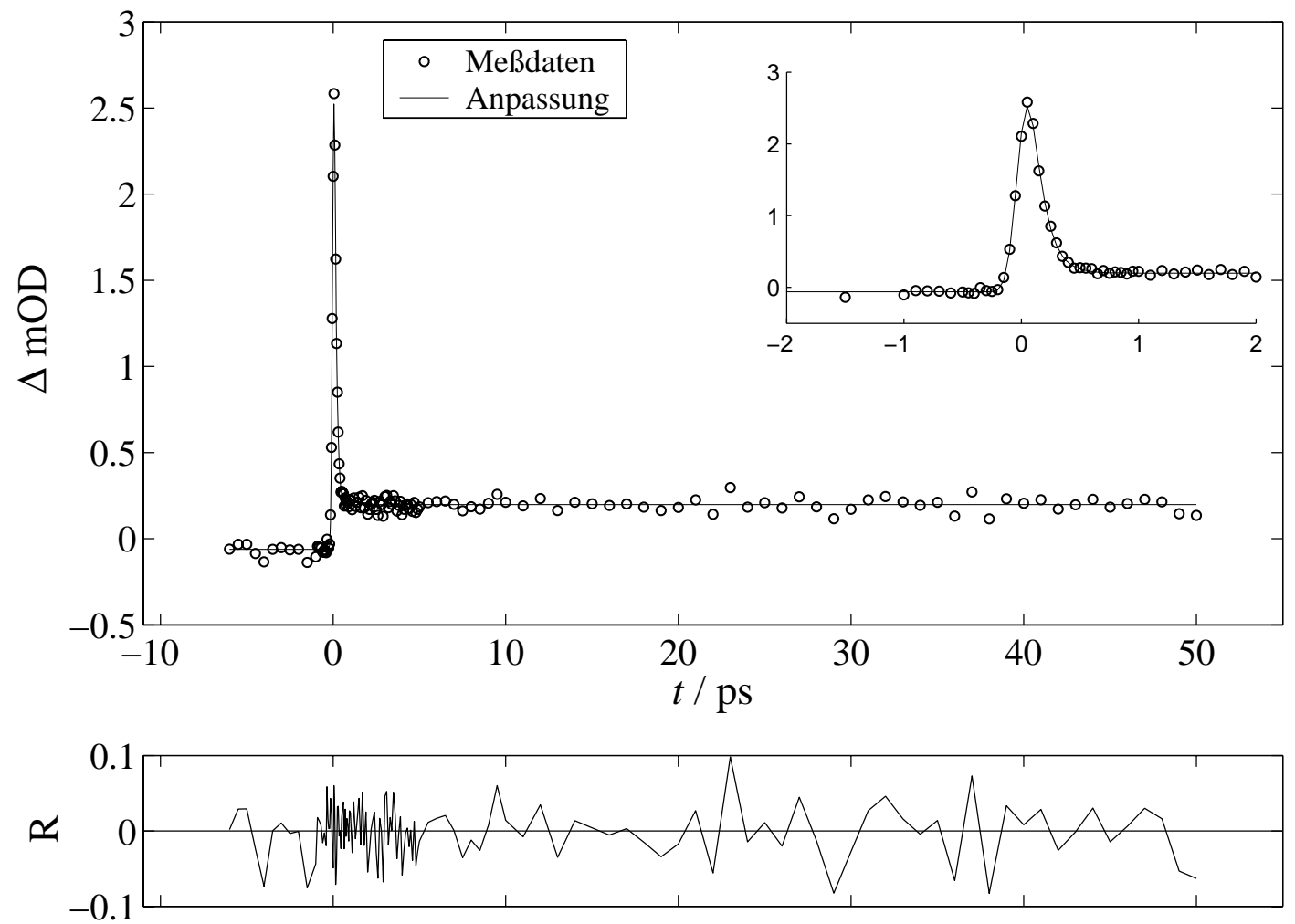

Abbildung 4.9: Messung der transienten Absorption von $\mathrm{CH}_{3} \mathrm{I}$ in Methylcyclohexan nach $260 \mathrm{~nm}$ Anregung und $330 \mathrm{~nm}$ Abfrage mit Anpassung und Residuen (R)

Die Ergebnisse der Anpassung werden als Datei im . sim-Format, die verwendeten Anpassungsparameter als Dateien im .par- und .out-Format sowie die errechneten Residuen im .res-Format abgespeichert.

In Abbildung 4.9 und 4.10 sind exemplarisch zwei Messungen der transienten Absorption von $\mathrm{CH}_{3} \mathrm{I}$ in Methylcyclohexan und $\mathrm{CHI}_{3}$ in sc. $\mathrm{CO}_{2}$ sowie ihre Anpassung und die entsprechenden Residuen wiedergegeben. Dem schnellen Anstieg liegt dabei die elektronischer Anregung des untersuchten Moleküls als ultraschneller Prozeß jenseits der verfügbaren Zeitauflösung zugrunde. Anhand der Anpassungsparameter der Gaußfunktion läßt sich somit die erreichte Zeitauflösung des Experiments bestimmen.

Die Halbwertsbreite der Gaußfunktion (engl. full width half maximum, FWHM) beträgt $2 \sigma$, dieser Wert entspricht der Messung der Kreuzkorrelation von Pump- und Probepuls mit der Zeitkonstanten $\tau_{c c}$. Zur Bestimmung der effektiven Zeitauflösung muß $\tau_{c c}=2 \sigma$ noch durch $\sqrt{2}$ dividiert werden. In der in Abbildung 4.9 dargestellten Messung der transienten Absorption von $\mathrm{CH}_{3} \mathrm{I}$ in Methylcyclohexan, konnte $\tau_{c c}$ zu $146 \mathrm{fs}$ angepaßt werden, was einer Halbwertsbreite des Pulses und somit einer Zeitauflösung des Experiments von 102 fs entspricht. Die Kreuzkorrelation der Messung von $\mathrm{CHI}_{3}$ in sc. $\mathrm{CO}_{2}$ beträgt $243 \mathrm{fs}$, was einer Zeitauflösung von 172 fs entspricht. 

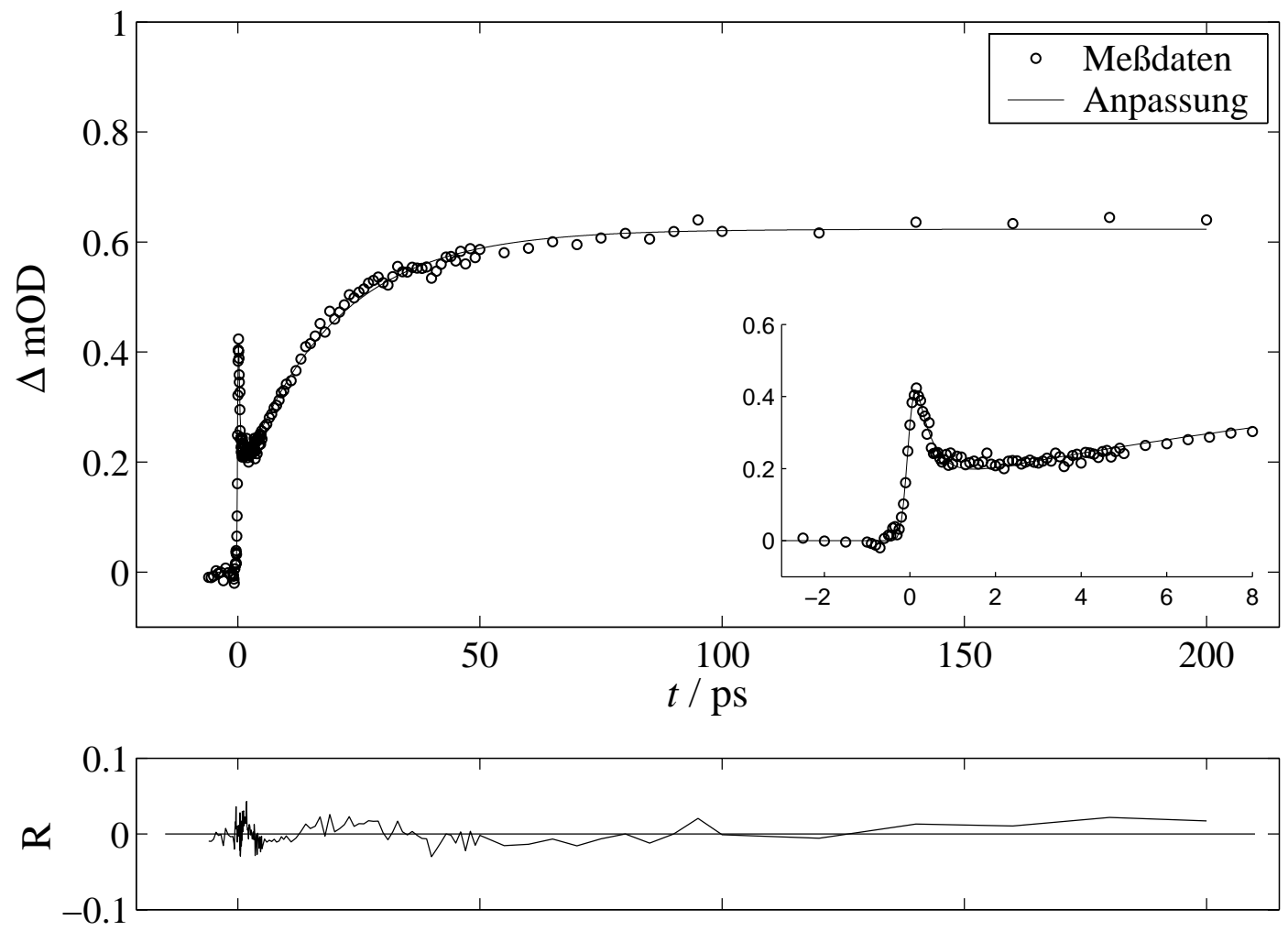

Abbildung 4.10: Messung der transienten Absorption von $\mathrm{CHI}_{3}$ in sc. $\mathrm{CO}_{2}\left(35^{\circ} \mathrm{C} 505\right.$ bar $)$ nach $267 \mathrm{~nm}$ Anregung und $600 \mathrm{~nm}$ Abfrage mit Anpassung und Residuen $(\mathrm{R})$

Die erreichte Zeitauflösung der Pump/Probe-Messungen ist dabei von der gewählten Wellenlänge des Pump- und Probepulses sowie der Güte der Justage abhängig. Deswegen wird bei der Auswertung für jede Messung die Kreuzkorrelation durch die anfangs beschriebene Faltung mit einer Gauß-Funktion jedesmal neu bestimmt. Sie liegt typischerweise zwischen 150-200 fs, wobei Messungen in flüssiger Lösung aufgrund der kürzeren optischen Weglänge der verwendeten Zellen meist eine etwas bessere Zeitauflösung besitzen. 


\subsection{Verwendete Chemikalien}

Die für diese Arbeit benötigten Chemikalien, Lösungsmittel und Gase (s. Tabelle 4.1) wurden über kommerzielle Anbieter bezogen und ohne weitere Reinigung oder Aufarbeitung verwendet.

\begin{tabular}{|llll|}
\hline Chemikalie & Hersteller & Summenformel & Reinheit \\
\hline Diiodmethan purum & Fluka & $\mathrm{CH}_{2} \mathrm{I}_{2}$ & $>98 \mathrm{v} . \mathrm{H}$. \\
Chloriodmethan purum & Fluka & $\mathrm{CH}_{2} \mathrm{ClI}$ & $>97 \mathrm{v} . \mathrm{H}$. \\
Bromiodmethan & Aldrich & $\mathrm{CH}_{2} \mathrm{BrI}$ & k. A. \\
Iodoform purum & Fluka & $\mathrm{CHI}_{3}$ & $>99,0 \mathrm{v} . \mathrm{H}$. \\
Iodmethan puriss. & Fluka & $\mathrm{CH}_{3} \mathrm{I}$ & $>99,5 \mathrm{v} . \mathrm{H}$. \\
Methanol f. UV-Spektroskopie & Fluka & $\mathrm{CH}_{2} \mathrm{OH}$ & $>99,8 \mathrm{v} . \mathrm{H}$. \\
Acetonitril f. UV-Spektroskopie & Fluka & $\mathrm{CH}_{3} \mathrm{CN}$ & $>99,8 \mathrm{v} . \mathrm{H}$. \\
Acetonitril-d & Deutero $\mathrm{GmbH}$ & $\mathrm{CD}_{3} \mathrm{CN}$ & $99,6 \mathrm{v} . \mathrm{H}$. \\
Methylcyclohexan f. UV-Spektroskopie & Riedel-de Haën & $\mathrm{C}_{6} \mathrm{H}_{11} \mathrm{CH}_{3}$ & $>99,8 \mathrm{v} . \mathrm{H}$. \\
Tetrachlorkohlenstoff Uvasol & Merck & $\mathrm{CCl}_{4}$ & $>99,9 \mathrm{v} . \mathrm{H}$. \\
$n$-Hexan purum & Fluka & $\mathrm{CH}_{3}\left(\mathrm{CH}_{2}\right)_{4} \mathrm{CH}_{3}$ & $>95,0 \mathrm{v} . \mathrm{H}$. \\
$n$-Perfluorhexan & Aldrich & $\mathrm{CF}_{3}\left(\mathrm{CF}_{2}\right)_{4} \mathrm{CF}_{3}$ & $>99,9 \mathrm{v} . \mathrm{H}$. \\
Kohlenstoffdioxid & Messer-Griesheim & $\mathrm{CO}_{2}$ & 4,5 \\
Fluoroform & Messer-Griesheim & $\mathrm{CHF}_{3}$ & 4,5 \\
Ethan & Messer-Griesheim & $\mathrm{C}_{2} \mathrm{H}_{6}$ & 2,5 \\
\hline
\end{tabular}

Tabelle 4.1: Verwendete Chemikalien 



\section{Experimentelle Ergebnisse}

In diesem Kapitel werden die unter verschiedenen Bedingungen erhaltenen Resultate der fs-Pump/Probemessungen an $\mathrm{CH}_{2} \mathrm{I}_{2}, \mathrm{CH}_{2} \mathrm{BrI}, \mathrm{CH}_{2} \mathrm{ClI}, \mathrm{CHI}_{3}$ und $\mathrm{CH}_{3} \mathrm{I}$ vorgestellt. Dabei wurden sowohl überkritische als auch flüssige Lösungsmittel verwendet und die transiente Absorption bei unterschiedlichen Anregungs- und Abfragewellenlängen gemessen.

\subsection{Diiodmethan}

Die Messungen der transienten Absorption von $\mathrm{CH}_{2} \mathrm{I}_{2}$ wurden bei einer Anregungswellänge von $340 \mathrm{~nm}$ in sc. $\mathrm{CO}_{2}\left(35^{\circ} \mathrm{C}\right)$ vorgenommen. Ein typisches Absorptionszeitprofil von $\mathrm{CH}_{2} \mathrm{I}_{2}$ bei einer Probewellenlänge von $405 \mathrm{~nm}$ ist in Abbildung 5.1 wiedergegeben. Die Probewellenlänge ist dabei so gewählt, daß sie innerhalb der isosbestischen Region (s. Abschnitt 6.2.1) liegt und somit die transiente Absorption ohne Überlagerung mit spektraler Dynamik wiedergibt.
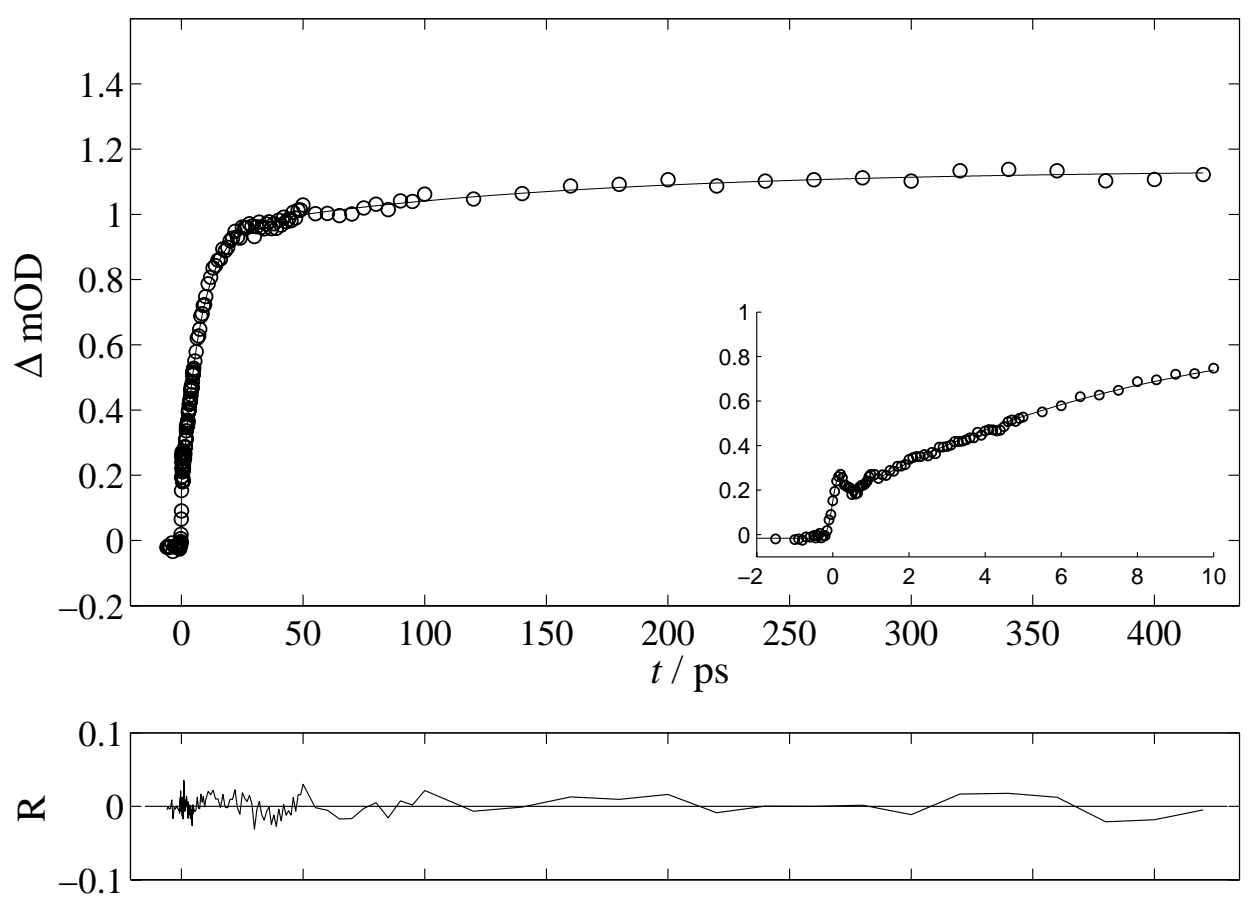

Abbildung 5.1: Typisches Absorptionszeitprofil von $\mathrm{CH}_{2} \mathrm{I}_{2}$ in sc. $\mathrm{CO}_{2}\left(35^{\circ} \mathrm{C}, 400\right.$ bar $)$ bei $340 \mathrm{~nm}$ Anregung und $405 \mathrm{~nm}$ Abfrage 


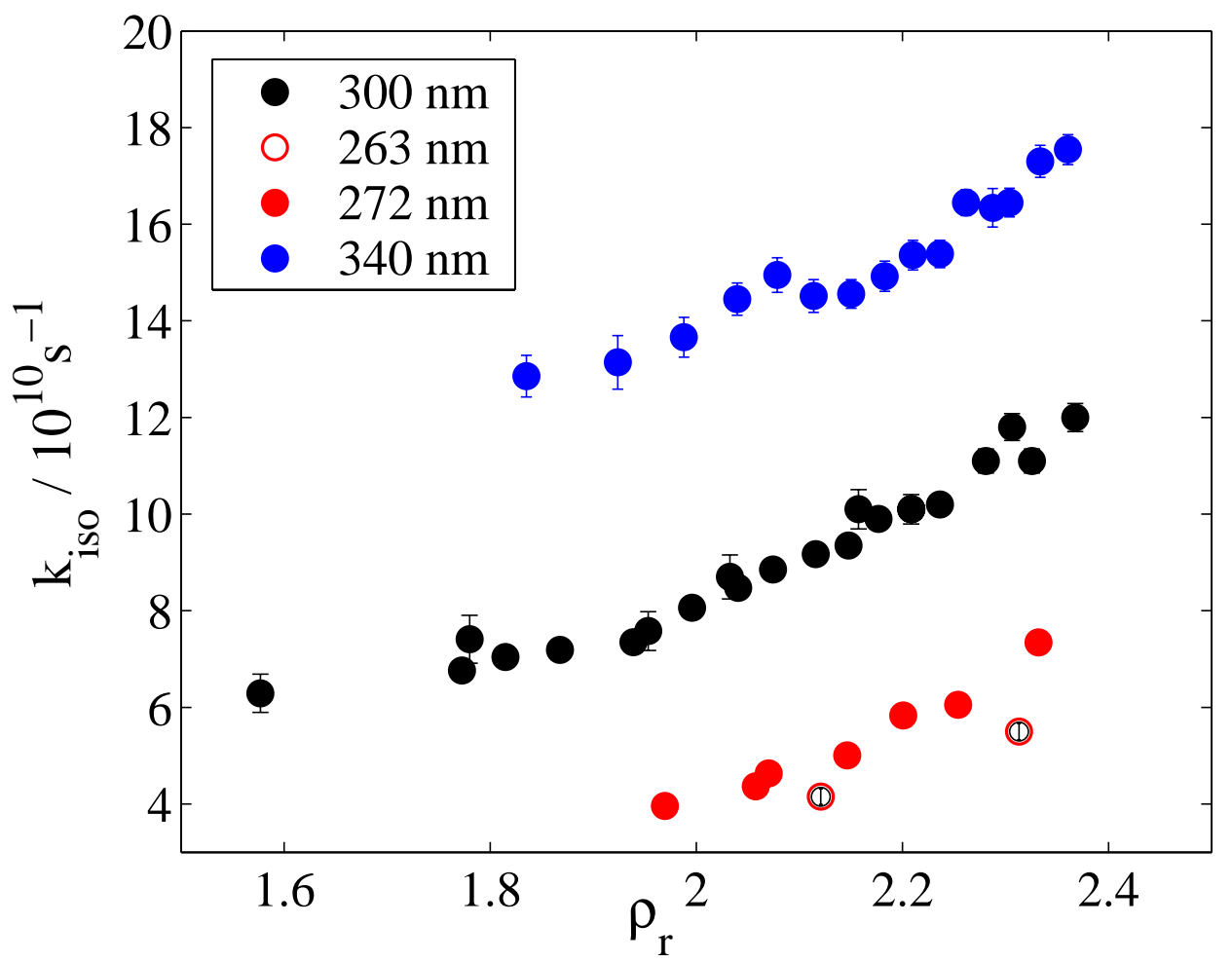

Abbildung 5.2: Dichteabhängigkeit $k_{\text {iso }}$ von $\mathrm{CH}_{2} \mathrm{I}_{2}$ bei variabler Anregungswellenlänge und Probewellenlänge von $405 \mathrm{~nm}$ in sc. $\mathrm{CO}_{2}\left(35^{\circ} \mathrm{C}\right)$

\subsubsection{Druckabhängige Messungen bei $340 \mathrm{~nm}$ Anregung}

Zur Vervollständigung früherer Messungen mit variabler Anregungswellenlänge [103], wurden druckabhängige Messungen bei $340 \mathrm{~nm}$ Anregung durchgeführt. Die Anregungswellenlänge von $340 \mathrm{~nm}$ liegt dabei auf der roten Flanke des Absorptionsspektrums von $\mathrm{CH}_{2} \mathrm{I}_{2}$ (s. Abbildung 3.2). Dazu wurden Absorptionszeitprofile mit einer Probewellenlänge von $405 \mathrm{~nm}$ bei unterschiedlichen Drücken von $200-1000$ bar in sc. $\mathrm{CO}_{2}\left(35^{\circ} \mathrm{C}\right)$ aufgenommen. Die Anstiegszeit des langsamen monoexponentiellen Anstiegs wurde dazu mit der Isomerbildung verknüpft (s. Abbildung 5.1) und über die Beziehung

$$
k_{\text {iso }}=\frac{1}{\tau_{\text {iso }}}
$$

in eine Geschwindigkeitskonstante $k_{\text {iso }}$ umgerechnet. Die Dichteabhängigkeit von $k_{\text {iso }}$ ist in Abbildung 5.2 wiedergegeben. Als Maß für die Packungsdichte des Lösungsmittels wurde dazu die reduzierte Dichte $\rho_{r}$, also der Quotient $\rho / \rho_{k}$ aus Dichte $\rho$ und kritischer Dichte $\rho_{k}$ herangezogen. Zum Vergleich sind Daten bei etwa $265 \mathrm{~nm}$ Anregung [103] und $300 \mathrm{~nm}$ Anregung [167] ebenfalls mit aufgetragen. Da die Quantenausbeute der Reaktion nicht direkt bestimmt werden kann, wird die Endamplitude des langsamen Anstiegs als Maß für die Quantenausbeute herangezogen. Die Vergleichbarkeit der Messungen ist dabei durch die Tatsache gegeben, daß alle dichteabhängigen Messungen bei $340 \mathrm{~nm}$ Anregung 


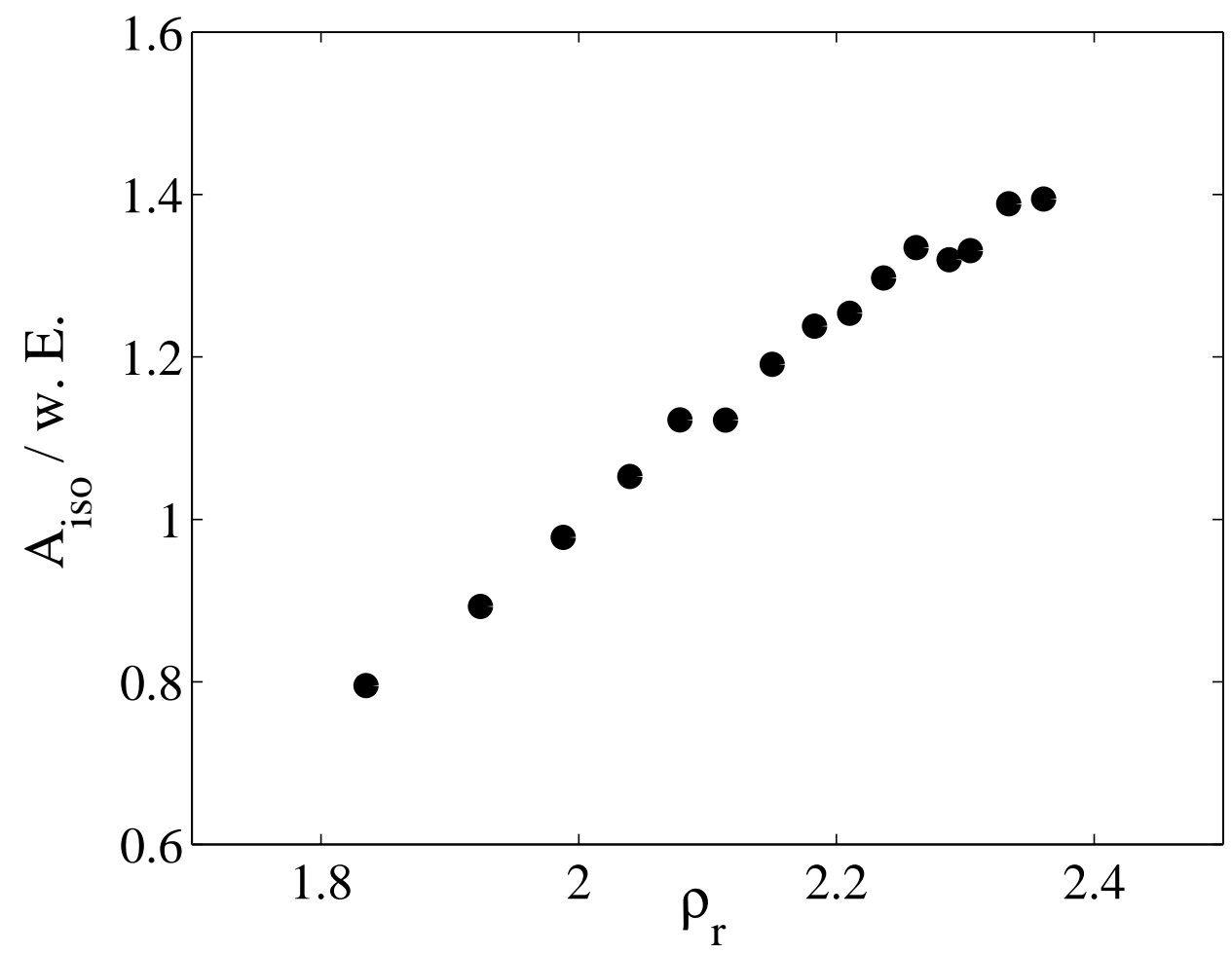

Abbildung 5.3: Dichteabhängigkeit $A_{\text {iso }}$ von $\mathrm{CH}_{2} \mathrm{I}_{2}$ in sc. $\mathrm{CO}_{2}\left(35^{\circ} \mathrm{C}\right)$ bei $340 \mathrm{~nm}$ Anregung und Probewellenlänge von $405 \mathrm{~nm}$

bei gleichbleibender Pumpintensität unmittelbar nacheinander durchgeführt wurden. Die Dichteabhängigkeit von $A_{\text {iso }}$ ist in Abbildung 5.3 dargestellt.

\subsubsection{Zeitaufgelöste Spektren bei $340 \mathrm{~nm}$ Anregung}

Zur Rekonstruktion zeitaufgelöster Spektren wurden Absorptionszeitprofile von $\mathrm{CH}_{2} \mathrm{I}_{2}$ in sc. $\mathrm{CO}_{2}\left(35^{\circ} \mathrm{C}, 400\right.$ bar $)$ nach $340 \mathrm{~nm}$ Anregung bei verschiedenen Probewellenlängen gemessen. Die Versuchsbedingungen wurden dabei konstant gelassen und der Probestrahl von 355-445 nm durchgestimmt. Der verfügbare Spektralbereich ist dabei zu kurzen Wellenlängen von der Absorption des Muttermoleküls begrenzt, bei Wellenlängen $<350 \mathrm{~nm}$ ist die Absorption des $\mathrm{CH}_{2} \mathrm{I}_{2}$ bereits so stark, daß der Probestrahl beim Durchlaufen der Meßzelle komplett absorbiert wird. Bei Wellenlängen > $445 \mathrm{~nm}$ ist das Signal/Rausch-Verhältnis der transienten Absorption des gebildeten $\mathrm{CH}_{2} \mathrm{I}-\mathrm{I}$-Isomers zu klein, um auswertbare Messungen durchzuführen.

$\mathrm{Zu}$ Beginn jeder Messung muß die Justage von Pump- und Probestrahl überprüft und das Signal optimiert werden, da ein Wechsel der Ausgangswellenlänge aufgrund der Winkelabhängigkeit der nicht-linearen Mischprozesse im TOPAS immer mit einem kleinem Strahlversatz verbunden ist. Weiterhin ist zu überprüfen, ob sich eventuell durch einen Wechsel des nicht-linearen Mischprozesses im TOPAS die Polarisation des Probestrahls 


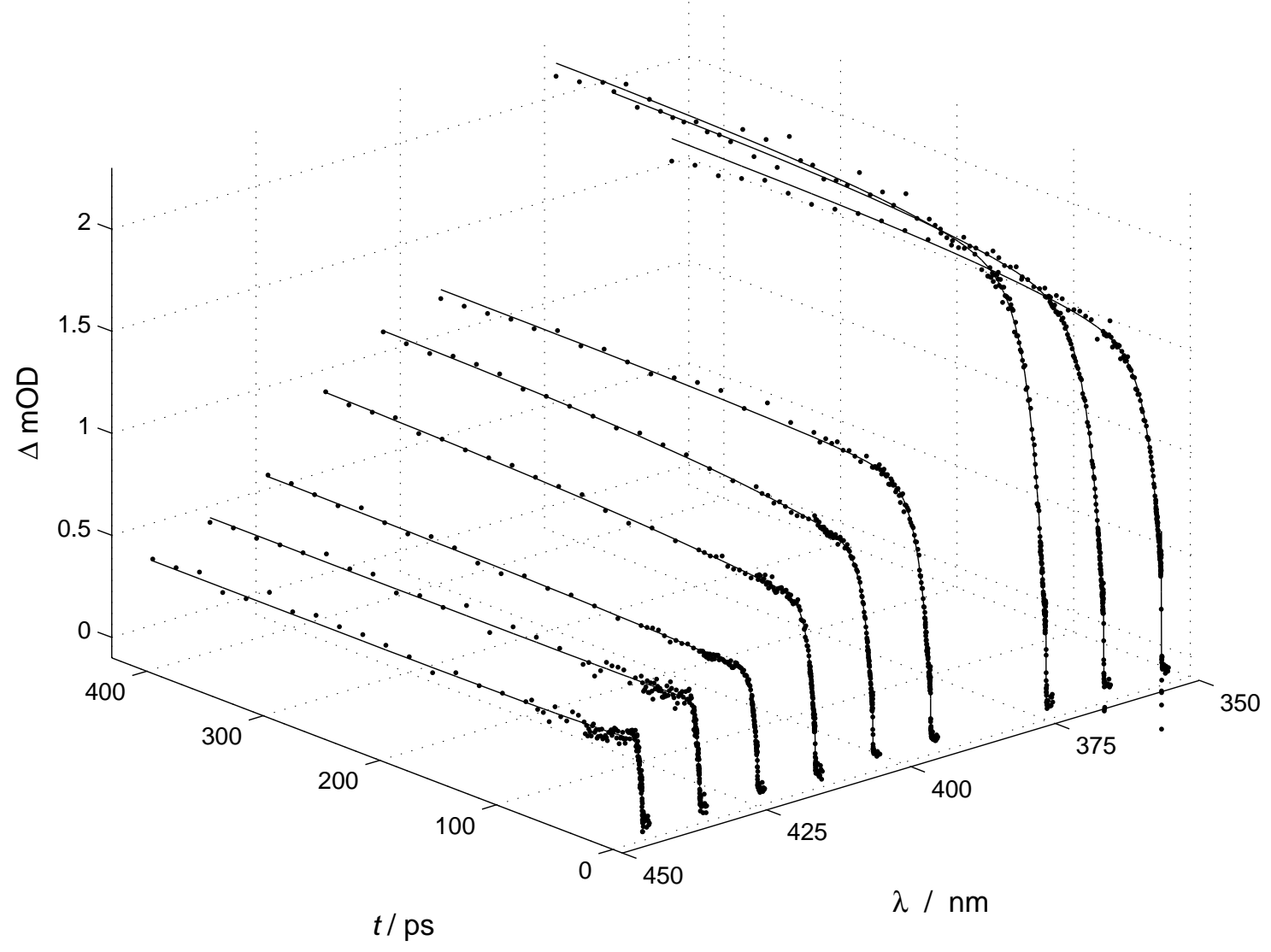

Abbildung 5.4: Transiente Absorption von $\mathrm{CH}_{2} \mathrm{I}_{2}$ in sc. $\mathrm{CO}_{2}\left(35^{\circ} \mathrm{C}, 400\right.$ bar $)$ bei $340 \mathrm{~nm}$ Anregung und Probewellenlänge von 355-445 nm

geändert hat. In einem solchen Fall muß das $\lambda / 2$-Plättchen verstellt werden, um den gewünschten Winkel zwischen der Polarisationsrichtung von Pump- und Probestrahl wieder einzustellen (wenn nicht anders vermerkt, wurde im „magischen“ Winkel von 54,7 gemessen).

Die gemessenen Absorptionszeitprofile wurden mit dem Matlab-Programm (s. Abschnitt 4.4) je nach Kurvenverlauf mehrfachexponentiell angepaßt. Die Meßdaten wurden anschließend mit dem so ermittelten Gesamt-off-set und Zeitnullpunkt $t_{0}$ der Anpassung korrigiert, indem die entsprechenden Werte von den $\triangle O D$ - bzw. Zeitwerten abgezogen wurden. Die korrigierten Absorptionszeitprofile sind in Abbildung 5.4 dargestellt. Zeitaufgelöste Spektren können nun durch Auslesen der transienten Absorption der korrigierten Anpassungen zu frei wählbaren Verzögerungszeiten rekonstruiert werden. Die zu den festgelegten Zeiten ausgelesene Absorption wird gegen die Wellenlänge aufgetragen und ergibt ein zeitaufgelöstes Spektrum, wie es in Abbildung 5.5 für $\mathrm{CH}_{2} \mathrm{I}_{2}$ in sc. $\mathrm{CO}_{2}\left(35^{\circ} \mathrm{C}\right.$, 400 bar) dargestellt ist. Die spektrale Auflösung ist dabei vom Wellenlängenabstand der durchgeführten Messungen mit unterschiedlicher Probewellenlänge abhängig. Die Auflösung ist mit üblicherweise $10 \mathrm{~nm}$ relativ gering, sollte allerdings aufgrund der großen spektralen Bandbreite der fs-Probepulse (FWHM $\sim 6 \mathrm{~nm}$ ) nicht enger gesetzt werden. 


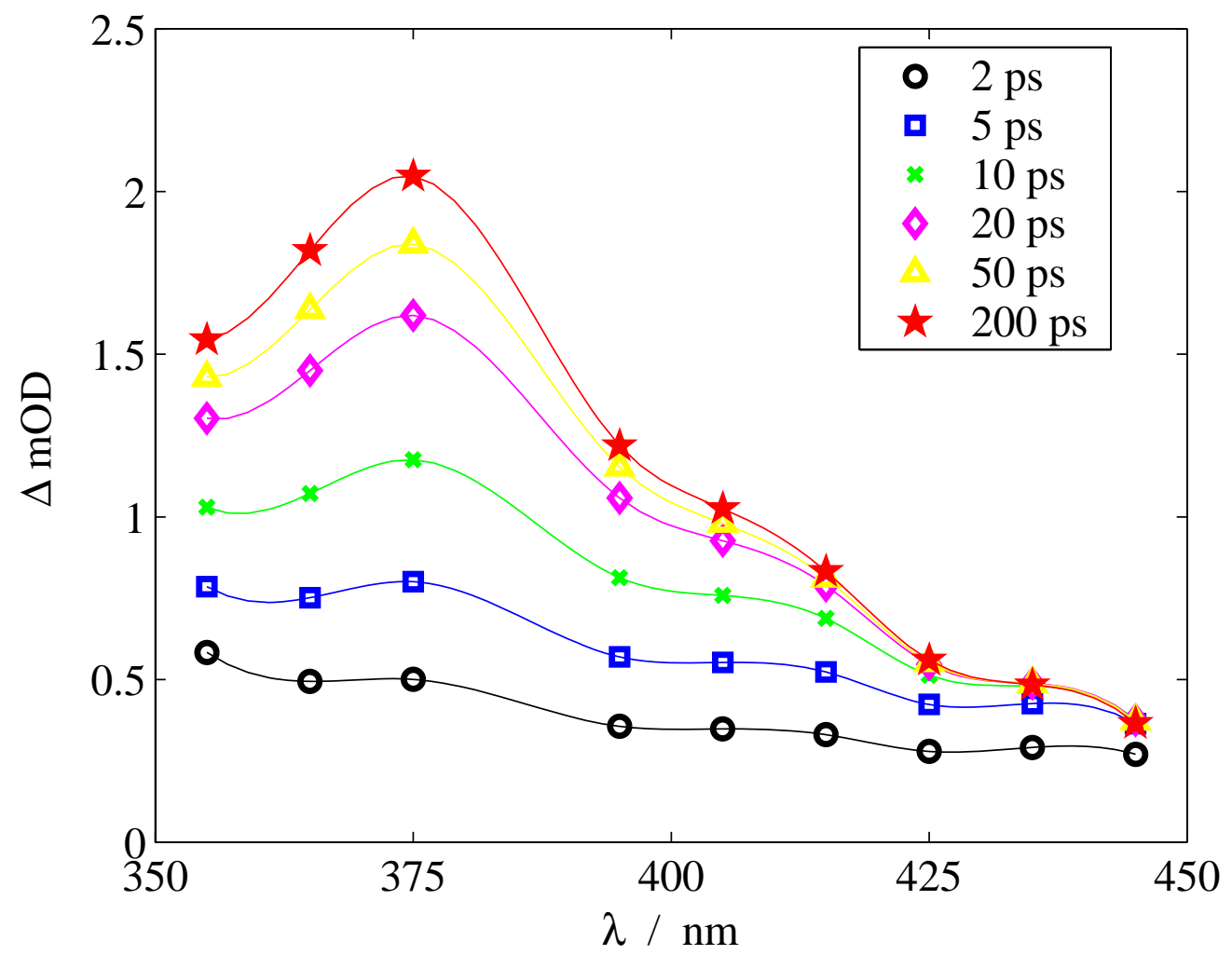

Abbildung 5.5: Zeitaufgelöstes Spektrum $\mathrm{CH}_{2} \mathrm{I}_{2}$ in sc. $\mathrm{CO}_{2}\left(35^{\circ} \mathrm{C}, 400\right.$ bar $)$ bei $340 \mathrm{~nm}$ Anregung

Das so rekonstruierte Spektrum (Abbildung 5.5) ist zu frühen Reaktionszeiten (2 ps) noch breit und strukturlos, im Laufe einer Pikosekundenzeitskala wächst anschließend eine Bande bei etwa $370 \mathrm{~nm}$ heraus, welche dem $\mathrm{CH}_{2} \mathrm{I}$-I-Isomer zugeordnet werden kann (s. Abschnitt 3.5). Die Absorption auf der roten Flanke des Spektrums fällt im Laufe der Reaktionszeit wieder ab.

\subsection{Bromiodmethan}

Die Messung von Absorptionszeitprofilen und Rekonstruktion zeitaufgelöster Spektrum von $\mathrm{CH}_{2} \mathrm{BrI}$ wurde analog zur Verfahrensweise bei $\mathrm{CH}_{2} \mathrm{I}_{2}$ durchgeführt. Dabei wurden unterschiedliche Anregungswellenlängen verwendet, zum einen die Dritte Harmonische des UV/VIS-Lasersystems bei $258 \mathrm{~nm}$ (s. Abschnitt 4.1.3) sowie der frequenzverdoppelte Ausgangpuls des NOPA bei $288 \mathrm{~nm}$. Die Dritte Harmonische wurde auf eine Pulsenergie von etwa $100 \mathrm{~nJ}$ abgeschwächt, um vergleichbare Ergebnisse zur Anregung bei $288 \mathrm{~nm}$ zu erzielen (etwa 40 nJ Pulsenergie). In Abbildung 5.6 und 5.7 sind zwei typische Absorptionszeitprofile bei 258 bzw. $288 \mathrm{~nm}$ Anregung und $400 \mathrm{~nm}$ Abfrage (transiente Bande des $\mathrm{CH}_{2} \mathrm{Br}$-I-Isomers (s. Abbildung 3.12) dargestellt. 

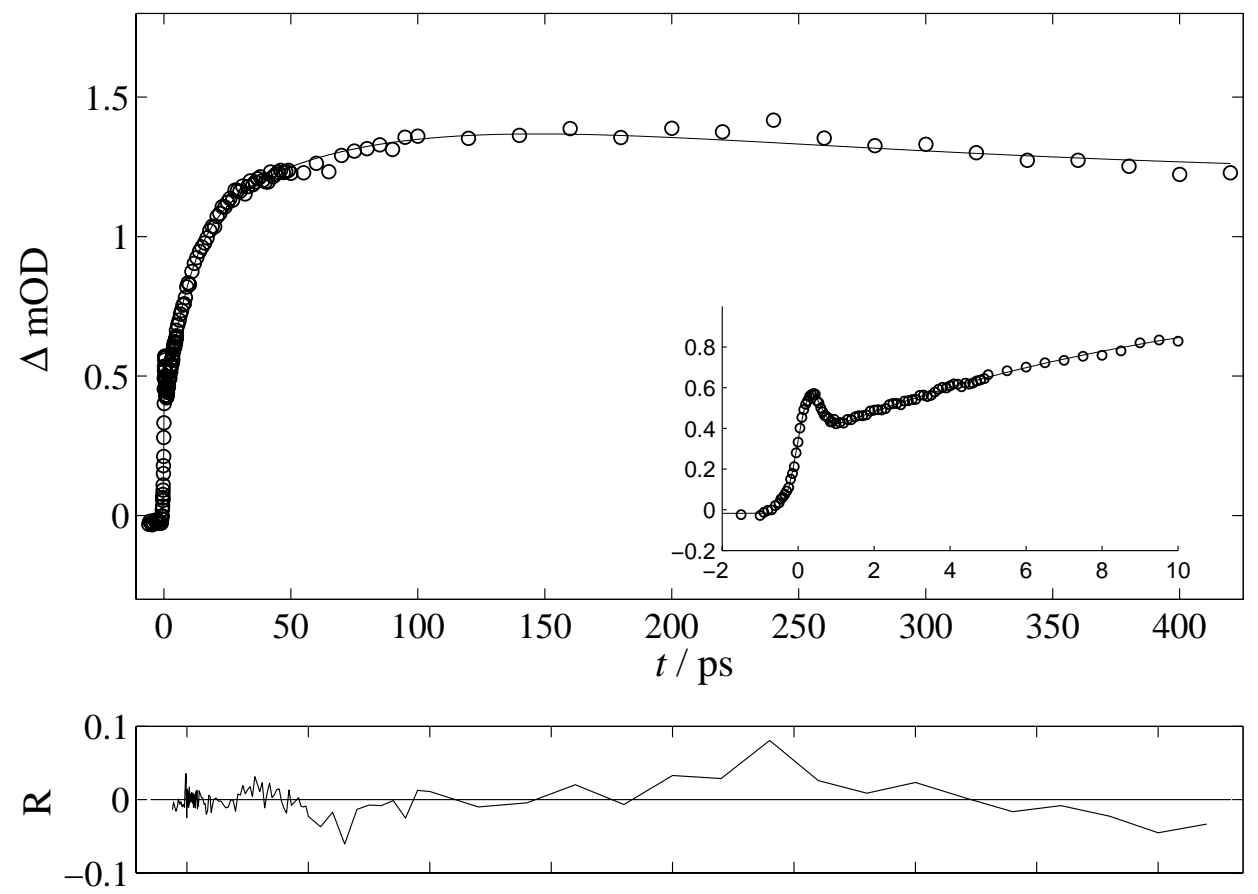

Abbildung 5.6: Absorptionszeitprofil von $\mathrm{CH}_{2} \mathrm{BrI}$ in sc. $\mathrm{CO}_{2}\left(35^{\circ} \mathrm{C}, 600\right.$ bar $)$ bei $258 \mathrm{~nm}$ Anregung und $400 \mathrm{~nm}$ Abfrage
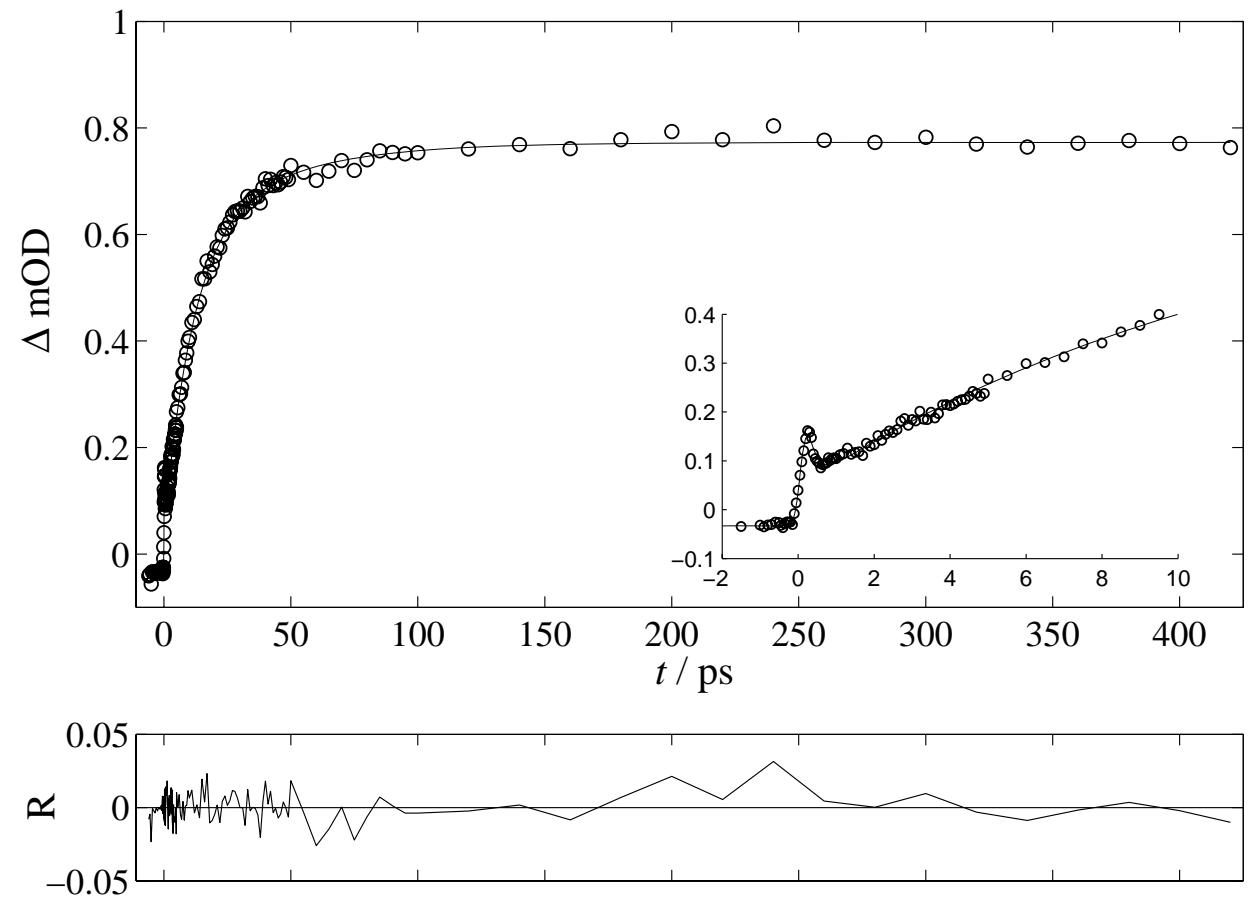

Abbildung 5.7: Absorptionszeitprofil von $\mathrm{CH}_{2} \mathrm{BrI}$ in sc. $\mathrm{CO}_{2}\left(35^{\circ} \mathrm{C}, 754\right.$ bar $)$ bei $288 \mathrm{~nm}$ Anregung und $400 \mathrm{~nm}$ Abfrage 


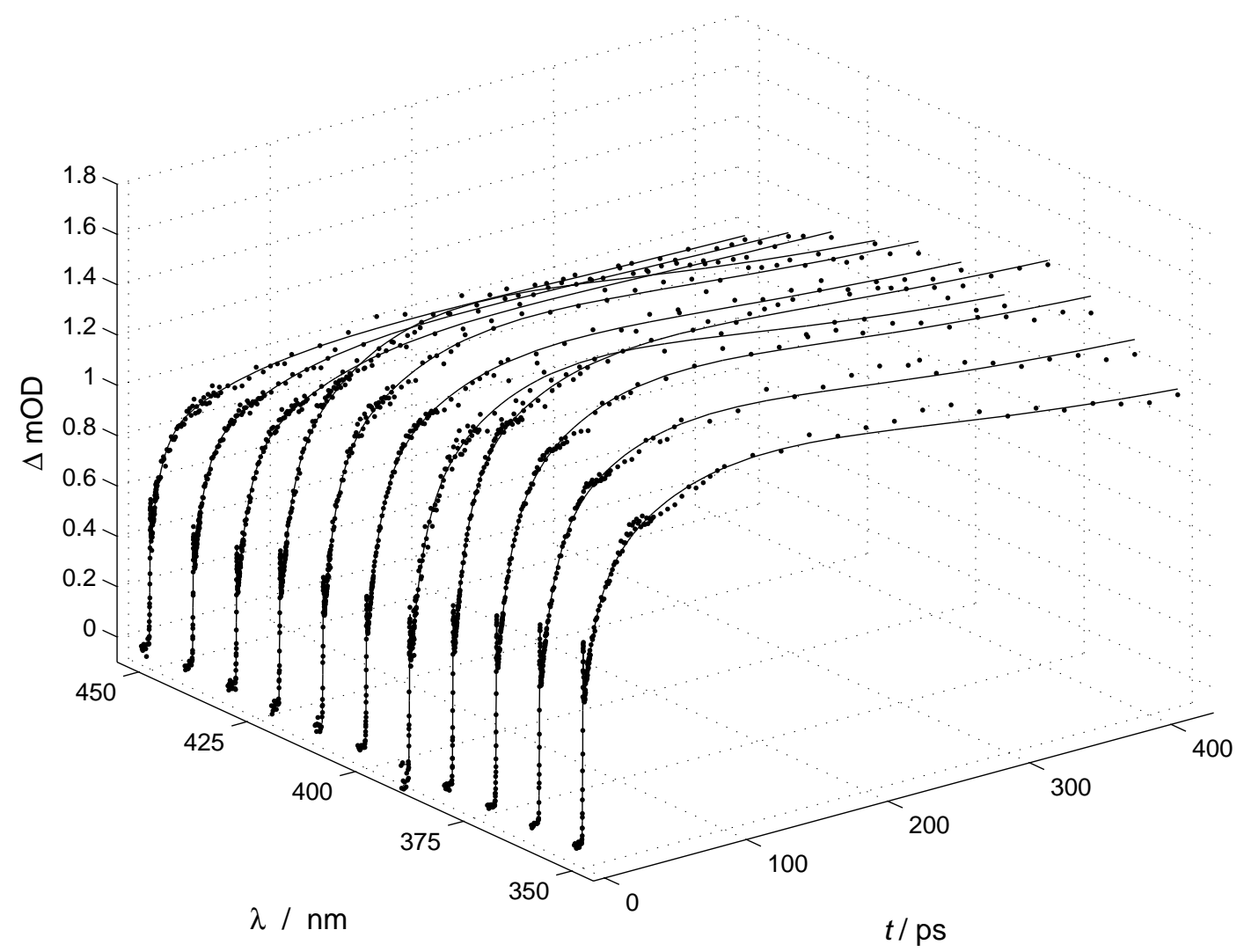

Abbildung 5.8: Transiente Absorption von $\mathrm{CH}_{2} \mathrm{BrI}$ in sc. $\mathrm{CO}_{2}\left(35^{\circ} \mathrm{C}, 600\right.$ bar $)$ nach $258 \mathrm{~nm}$ Anregung

Dabei zeigt sich für die Absorptionszeitprofile ein im Vergleich zu $\mathrm{CH}_{2} \mathrm{I}_{2}$ sehr ähnlicher Verlauf mit drei unterschiedlichen Zeitkomponenten: Einen schnellen Anstieg innerhalb der Zeitauflösung, ein nachfolgender schneller Abfall ( $200 \mathrm{fs}$ ) und anschließend ein Anstieg der Absorption im Laufe einer Pikosekundenzeitskala. Zu langen Verzögerungszeiten zeigt sich bei $258 \mathrm{~nm}$ Anregung allerdings ein leichter, monoexponentieller Abfall in der Größenordnung von etwa $200 \mathrm{ps}$, welcher bei $\mathrm{CH}_{2} \mathrm{I}_{2}$ nicht beobachtet wurde. Dieser Abfall war auch nach mehrfacher, gründlicher Justage der Verschiebebühne reproduzierbar, so daß nicht von einem Artefakt durch eine Dejustage des Strahlengangs bei verschiedenen Bühnenpositionen auszugehen ist. Bei $288 \mathrm{~nm}$ Anregung bleibt die Absorption im gemessenen Zeitbereich bis 400 ps konstant ohne abzufallen.

Die Absorptionszeitprofile von $\mathrm{CH}_{2} \mathrm{BrI}$ in sc. $\mathrm{CO}_{2}\left(35^{\circ} \mathrm{C}\right.$, 600 bar $)$ nach $258 \mathrm{~nm}$ Anregung wurden über einen Wellenlängenbereich von 350-450 nm gemessen und sind in Abbildung 5.8 dargestellt. Der bereits bei $400 \mathrm{~nm}$ Abfrage beobachtete Abfall der Absorption zu langen Reaktionszeiten, ist bei allen Wellenlängen $<420 \mathrm{~nm}$ zu beobachten. 


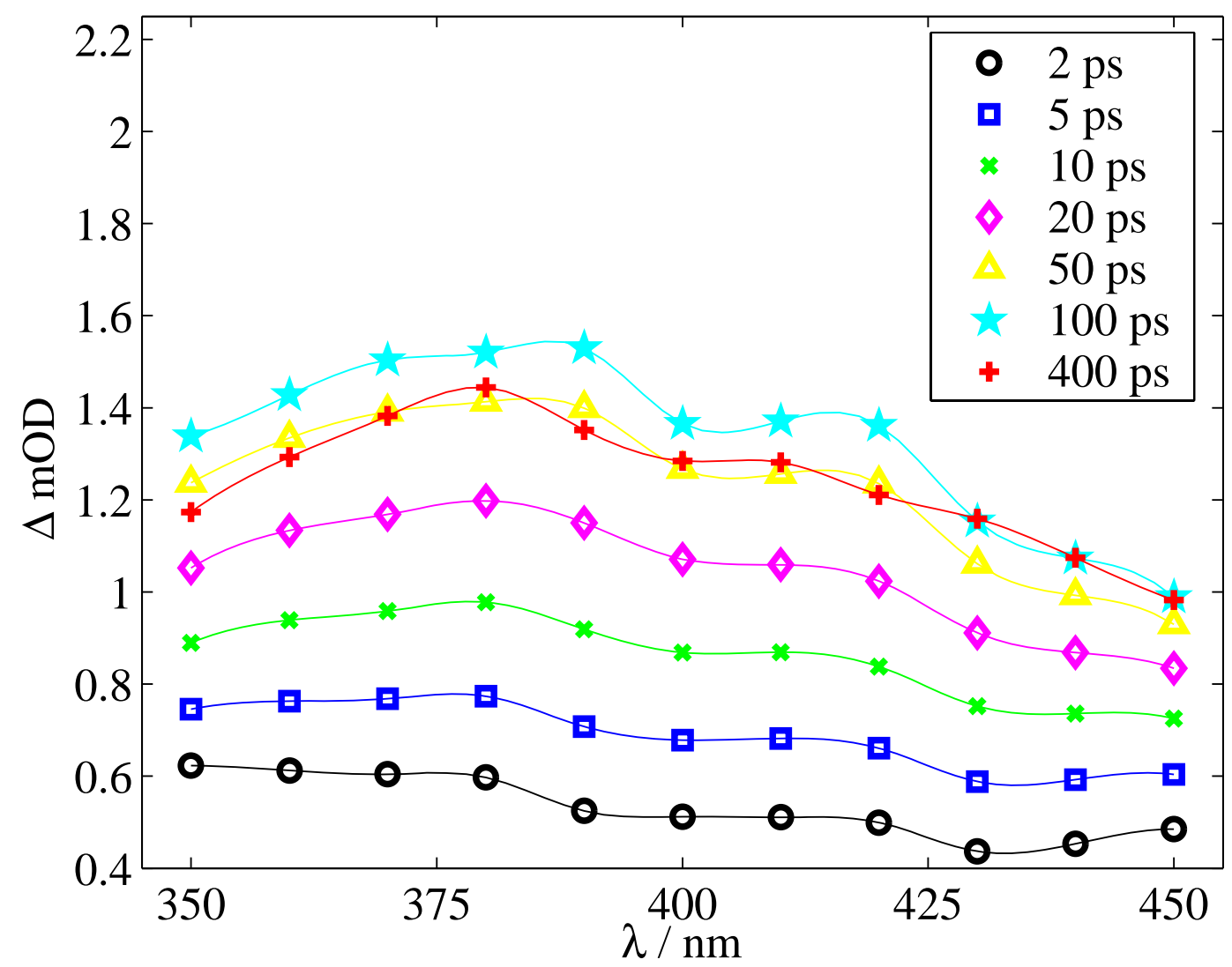

Abbildung 5.9: Zeitaufgelöstes Spektrum $\mathrm{CH}_{2} \mathrm{BrI}$ in sc. $\mathrm{CO}_{2}\left(35^{\circ} \mathrm{C}, 600\right.$ bar $)$ bei $258 \mathrm{~nm}$ Anregung

\subsubsection{Zeitaufgelöste Spektren bei $258 \mathrm{~nm}$ Anregung}

Die Aufnahme zeitaufgelöster Spektren von $\mathrm{CH}_{2} \mathrm{BrI}$ in sc. $\mathrm{CO}_{2}$ erfolgte analog zu dem bei $\mathrm{CH}_{2} \mathrm{I}_{2}$ (s. Abschnitt 5.1.2) beschriebenen Verfahren. Dazu wurden Absorptionszeitprofile von $\mathrm{CH}_{2} \mathrm{BrI}$ in sc. $\mathrm{CO}_{2}\left(35^{\circ} \mathrm{C}, 600\right.$ bar $)$ nach $258 \mathrm{~nm}$ Anregung mit der Dritten Harmonischen des UV/VIS-Lasersystems im Probewellenlängenbereich von 350-450 nm gemessen. Ein rekonstruiertes Spektrum zu verschiedenen Verzögerungszeiten ist in Abbildung 5.9 dargestellt.

Dabei zeigt sich zu frühen Reaktionszeiten (2ps) eine flache, breitbandige Absorption, aus welcher innerhalb einer Pikosekundenzeitskala eine Bande bei etwa $400 \mathrm{~nm}$ herauswächst, welche der UV-Absorptionsbande des $\mathrm{CH}_{2} \mathrm{Br}$-I-Isomers zugeordnet werden kann (s. Abbildung 3.12). Im Vergleich $\mathrm{zu} \mathrm{CH}_{2} \mathrm{I}_{2}$ ist diese Bande sehr breit und besitzt keinen eindeutigen Peak, sondern zwei lokale Maxima bei etwa 380 und $420 \mathrm{~nm}$. Nach etwa 100 ps ist die maximale Absorption erreicht und anschließend fällt die Bande bei Wellenlängen $<420 \mathrm{~nm}$ im Laufe mehrerer hundert Pikosekunden wieder ab. Dieses Verhalten konnte bei $\mathrm{CH}_{2} \mathrm{I}_{2}$ ebenfalls nicht beobachtet werden. 


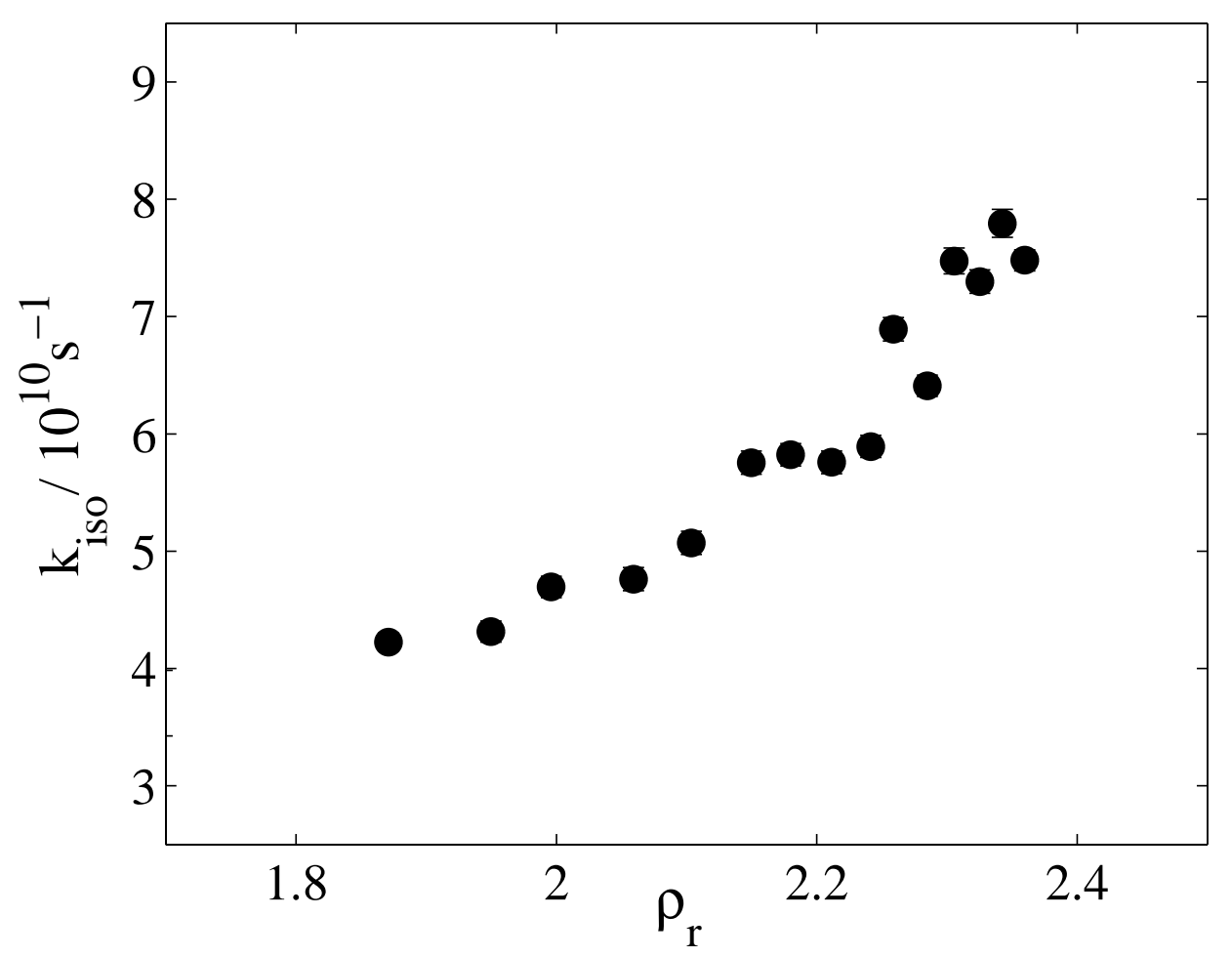

Abbildung 5.10: Dichteabhängigkeit $k_{\text {iso }}$ von $\mathrm{CH}_{2} \mathrm{BrI}$ in sc. $\mathrm{CO}_{2}\left(35^{\circ} \mathrm{C}\right)$ bei $288 \mathrm{~nm} \mathrm{An}$ regung und Probewellenlänge von $400 \mathrm{~nm}$

\subsubsection{Druckabhängige Messungen bei $288 \mathrm{~nm}$ Anregung}

Da im Falle einer Anregung bei $288 \mathrm{~nm}$ kein Abfall der Absorption zu langen Reaktionszeiten zu beobachten war, wurden druckabhängige Messungen bei dieser Wellenlänge durchgeführt. Die Probewellenlänge lag dabei zentriert auf der UV-Absorptionsbande des Isomers bei $400 \mathrm{~nm}$. Anhand der mit dem Matlab-Programm vorgenommenen Anpassungen wurden die Anstiegszeiten des langsamen Anstiegs $\tau_{\text {iso }}$ nach Gleichung 5.1 in eine Geschwindigkeitskonstante $k_{\text {iso }}$ umgerechnet und in Abbildung 5.10 gegen die reduzierte Dichte $\rho_{r}$ des Lösungsmittels $\left(\mathrm{CO}_{2}\right.$ bei $\left.35^{\circ} \mathrm{C}\right)$ aufgetragen.

Dabei zeigt sich bei steigender Dichte ein nichtlineares Anwachsen von $k_{\text {iso }}$, ähnlich den Messungen von $\mathrm{CH}_{2} \mathrm{I}_{2}$. Als Maß für die Quantenausbeute der Isomerisierung $k_{\text {iso }}$ wurde die Endamplitude des langsamen Anstiegs herangezogen. Im Gegensatz zu $\mathrm{CH}_{2} \mathrm{I}_{2}$ (s. Abschnitt 5.1.1) konnten die Messungen nicht innerhalb eines kurzen Zeitraums aufgenommen werden. Dabei ergibt sich das Problem, daß $A_{\text {iso }}$ nicht nur proportional zur Menge an gebildetem Isomer ist, sondern auch von schwankenden experimentellen Parametern wie Pumpintensität und Justage abhängt. Zur Vergleichbarkeit müssen die Messungen deswegen normiert werden; unter der Annahme, daß der transiente Anfangspeak der Absorption proportional zur Menge an angeregtem $\mathrm{CH}_{2} \mathrm{BrI}$ ist, kann die Endabsorption $A_{\text {iso }}$ auf diesen Peak normiert werden, um den Einfluß experimenteller Parameter auszuschließen. Dadurch sind auch Messungen unter verschiedenen Bedingungen, wie leicht unterschiedlicher Pumpintensität, vergleichbar. In Abbildung 5.11 sind die normierten 


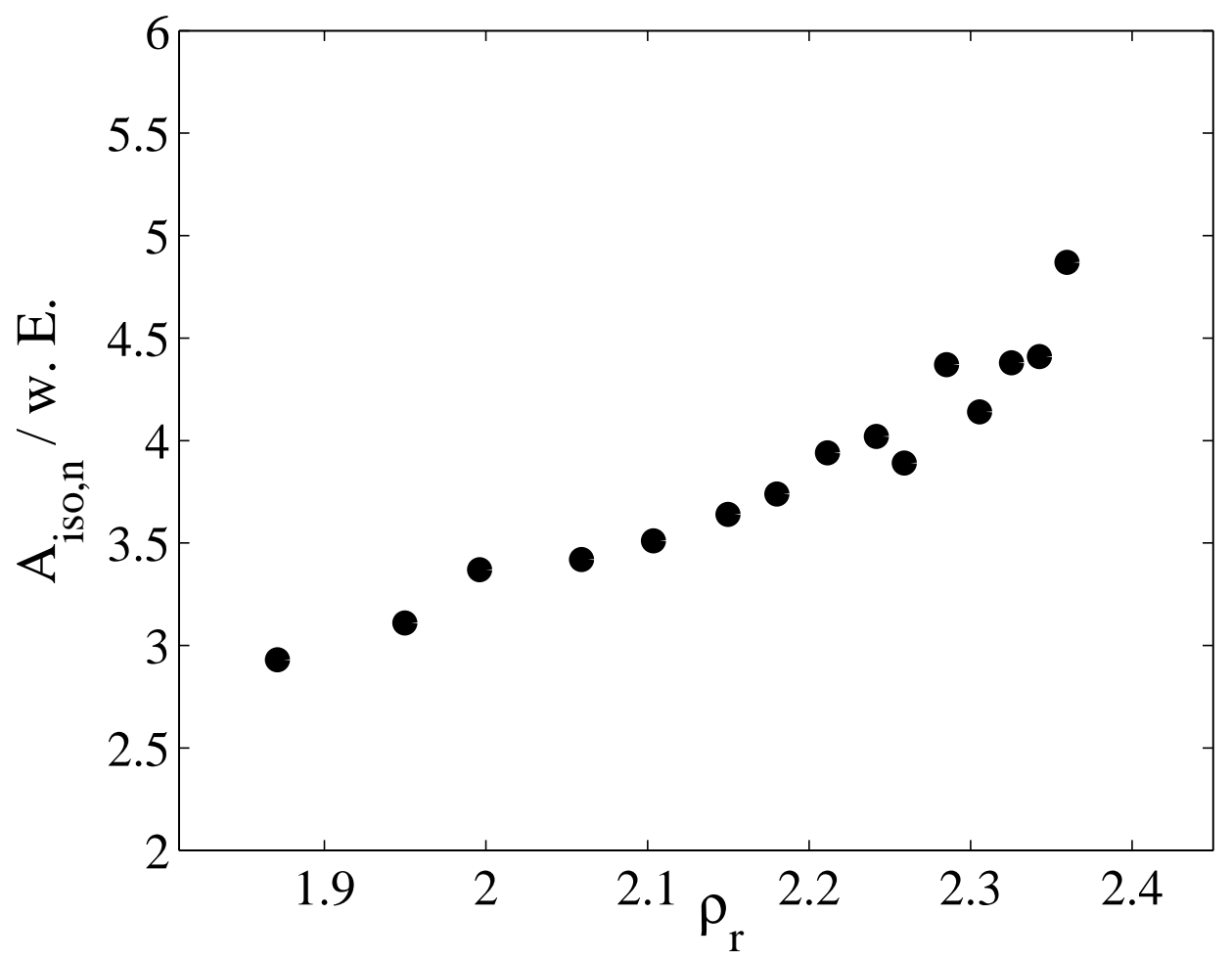

Abbildung 5.11: Dichteabhängigkeit $A_{\text {iso,n }}$ von $\mathrm{CH}_{2} \mathrm{BrI}$ in sc. $\mathrm{CO}_{2}\left(35^{\circ} \mathrm{C}\right)$ bei $288 \mathrm{~nm}$ Anregung und Probewellenlänge von $400 \mathrm{~nm}$

Endamplituden $A_{\text {iso,n }}$ gegen die reduzierte Dichte in sc. $\mathrm{CO}_{2}\left(35^{\circ} \mathrm{C}\right)$ aufgetragen. Dabei zeigt sich, daß die normierte Endamplitude $A_{\text {iso,n }}$ als Maß für die Quantenausbeute der Isomerisierungsreaktion nahezu linear von der Dichte abhängt. Dieses Verhalten konnte schon bei $\mathrm{CH}_{2} \mathrm{I}_{2}$ nach Anregung auf der roten Flanke des Absorptionsspektrums beobachtet werden $\left(340 \mathrm{~nm}\right.$ bei $\mathrm{CH}_{2} \mathrm{I}_{2}$ bzw. $288 \mathrm{~nm}$ bei $\left.\mathrm{CH}_{2} \mathrm{BrI}\right)$.

\section{3 lodoform}

Die Messung von Absorptionszeitprofilen und zeitaufgelösten Spektren von $\mathrm{CHI}_{3}$ in überkritischen und flüssigen Lösungsmitteln erfolgte analog zu $\mathrm{CH}_{2} \mathrm{I}_{2}$ und $\mathrm{CH}_{2} \mathrm{BrI}$. Die Löslichkeit von $\mathrm{CHI}_{3}$ in den verwendeten Lösungsmitteln ist etwas schlechter als die von $\mathrm{CH}_{2} \mathrm{I}_{2}$ und $\mathrm{CH}_{2} \mathrm{BrI}$, was allerdings durch den wesentlich höheren Extinktionskoeffizienten von $\mathrm{CHI}_{3}$ (s. Abbildung 3.5 kompensiert wurde. Ein typisches Absorptionszeitprofil von Iodoform in Acetonitril ist in Abbildung 5.12 wiedergegeben, dabei wurde $\mathrm{CHI}_{3}$ bei $267 \mathrm{~nm}$ angeregt und die transiente Absorption im Zentrum der stärkeren Isomerbande bei $460 \mathrm{~nm}$ abgefragt. Im Gegensatz zu den anderen Polyhalogenmethanen konnte bei $\mathrm{CHI}_{3}$ auch die schwächere Isomerbande im VIS-Bereich bei etwa $600 \mathrm{~nm}$ durch transiente Absorptionsmessungen gemessen werden. Ein typisches Absorptionszeitprofil von $\mathrm{CHI}_{3}$ nach $267 \mathrm{~nm}$ in sc. $\mathrm{CO}_{2}$ bei $600 \mathrm{~nm}$ Probewellenlänge ist in Abbildung 5.13 abgebildet. 

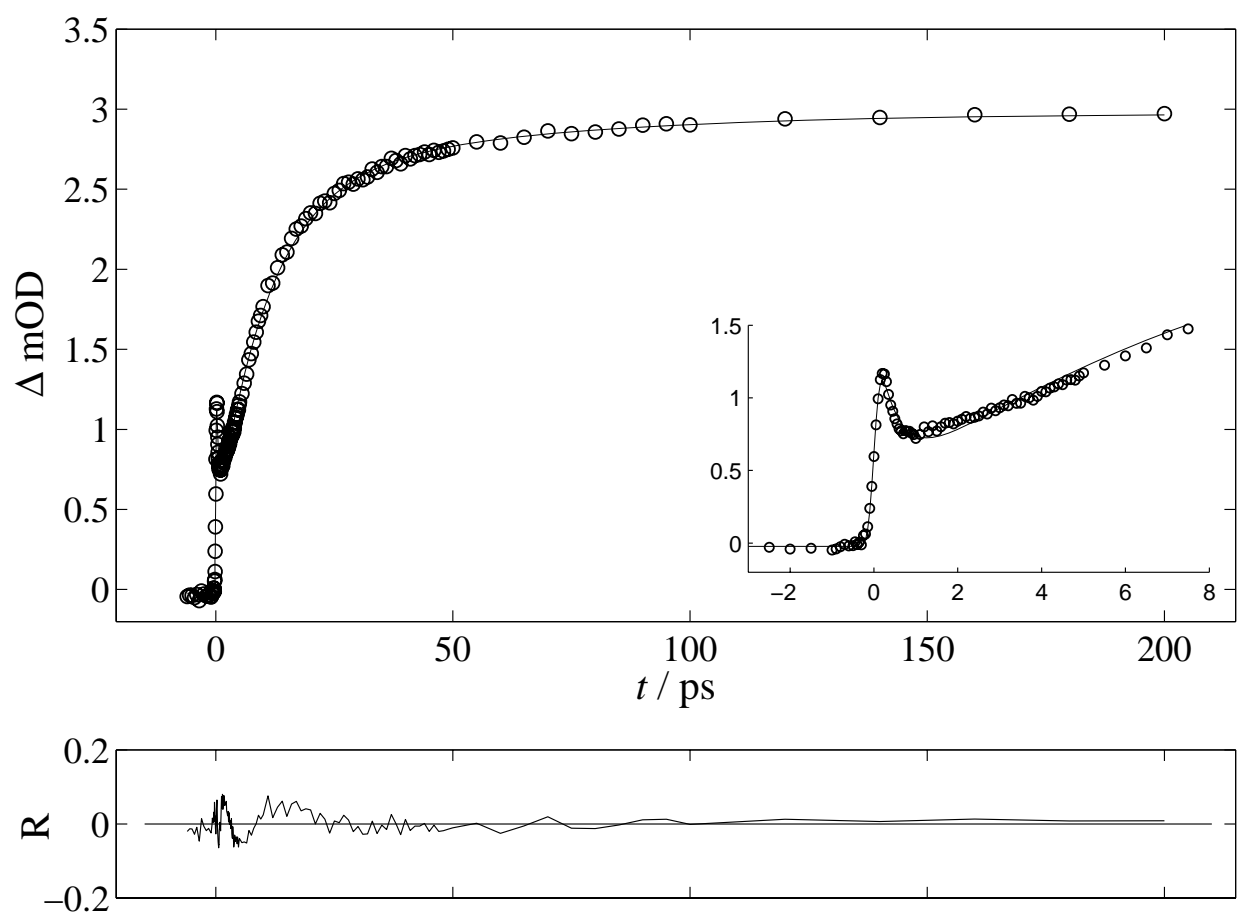

Abbildung 5.12: Absorptionszeitprofil von $\mathrm{CHI}_{3}$ in Acetonitril bei $267 \mathrm{~nm}$ Anregung und $460 \mathrm{~nm}$ Abfrage
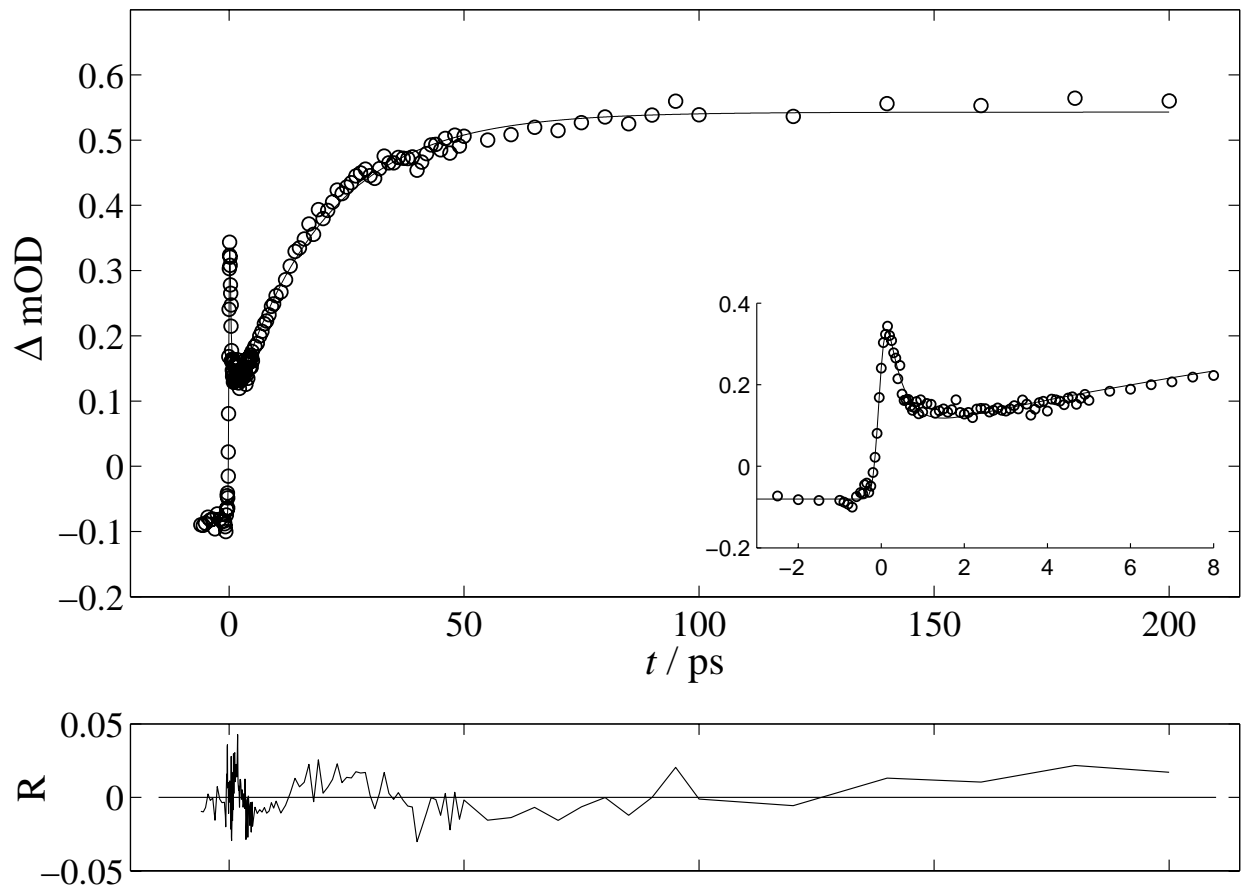

Abbildung 5.13: Absorptionszeitprofil von $\mathrm{CHI}_{3}$ in sc. $\mathrm{CO}_{2}\left(35^{\circ} \mathrm{C}, 505\right.$ bar $)$ bei $267 \mathrm{~nm}$ Anregung und $600 \mathrm{~nm}$ Abfrage 


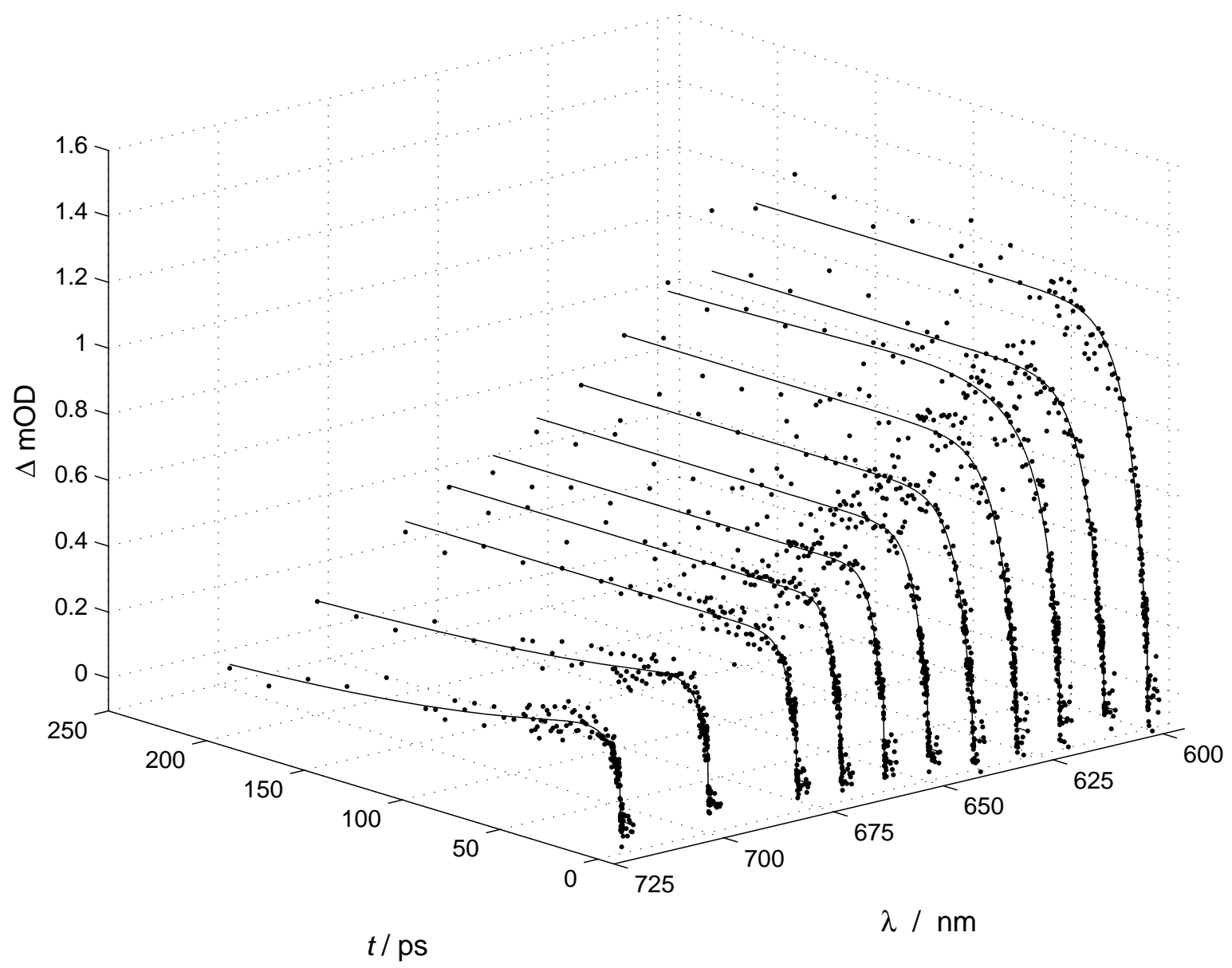

Abbildung 5.14: Transiente Absorption von $\mathrm{CHI}_{3}$ in sc. $\mathrm{CO}_{2}\left(35^{\circ} \mathrm{C}, 505\right.$ bar $)$ nach $365 \mathrm{~nm}$ Anregung

Im Bereich der VIS-Absorptionsbande des Isomers wurde die transiente Absorption bei verschiedenen Probewellenlänge aufgenommen, Probewellenlängen $<600 \mathrm{~nm}$ waren aufgrund des schlechten Signal/Rausch-Verhältnisses des TOPAS in diesem Bereich leider nicht zugänglich. Für die Spektren wurde sc. $\mathrm{CO}_{2}$ als überkritisches Lösungsmittel verwendet, in Abbildung 5.14 sind entsprechende Absorptionszeitprofile von $\mathrm{CHI}_{3}$ nach $365 \mathrm{~nm}$ Anregung in sc. $\mathrm{CO}_{2}\left(35^{\circ} \mathrm{C}\right)$ bei 505 bar dargestellt. Eine Anregung in die erste Bande des $\mathrm{CHI}_{3}$ bei $267 \mathrm{~nm}$ führte zu sehr ähnlichen Ergebnissen, die Messungen in sc. $\mathrm{CO}_{2}$ bei 505 bar sind in Abbildung 5.15 dargestellt. Da insbesondere der Niederdruckbereich für die Photoisomerisierung von besonderem Interesse ist, wurden die Messungen bei $267 \mathrm{~nm}$ Anregung zu niedrigen Drücken (<200 bar) ausgedehnt, dabei gibt es jedoch zunehmend Probleme mit der Löslichkeit des $\mathrm{CHI}_{3}$. Nichtsdestotz wurden einige Absorptionszeitprofile bei 192 und 142 bar aufgenommen, die Messungen bei 192 bar sind in Abbildung 5.16 exemplarisch dargestellt. 


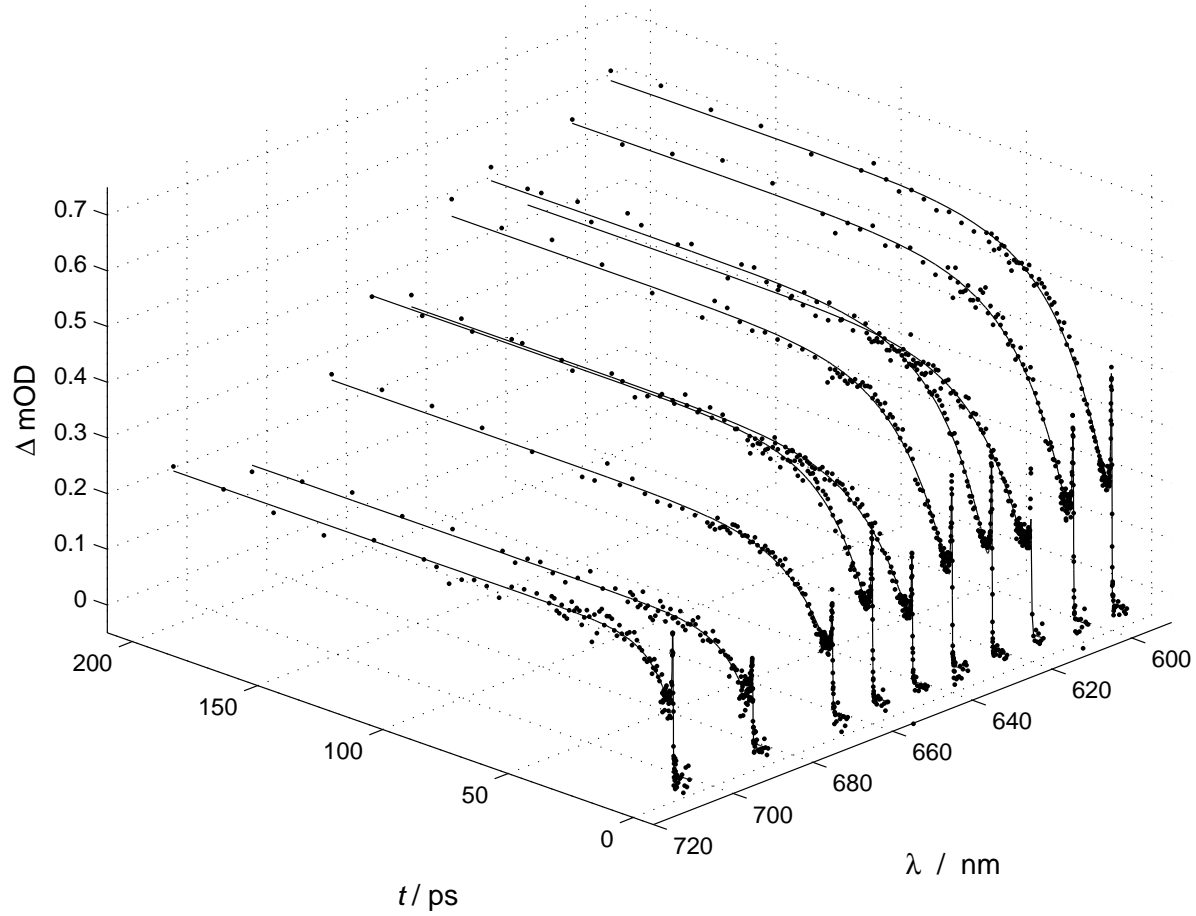

Abbildung 5.15: Transiente Absorption von $\mathrm{CHI}_{3}$ in sc. $\mathrm{CO}_{2}\left(35^{\circ} \mathrm{C}, 505\right.$ bar $)$ nach $267 \mathrm{~nm}$ Anregung

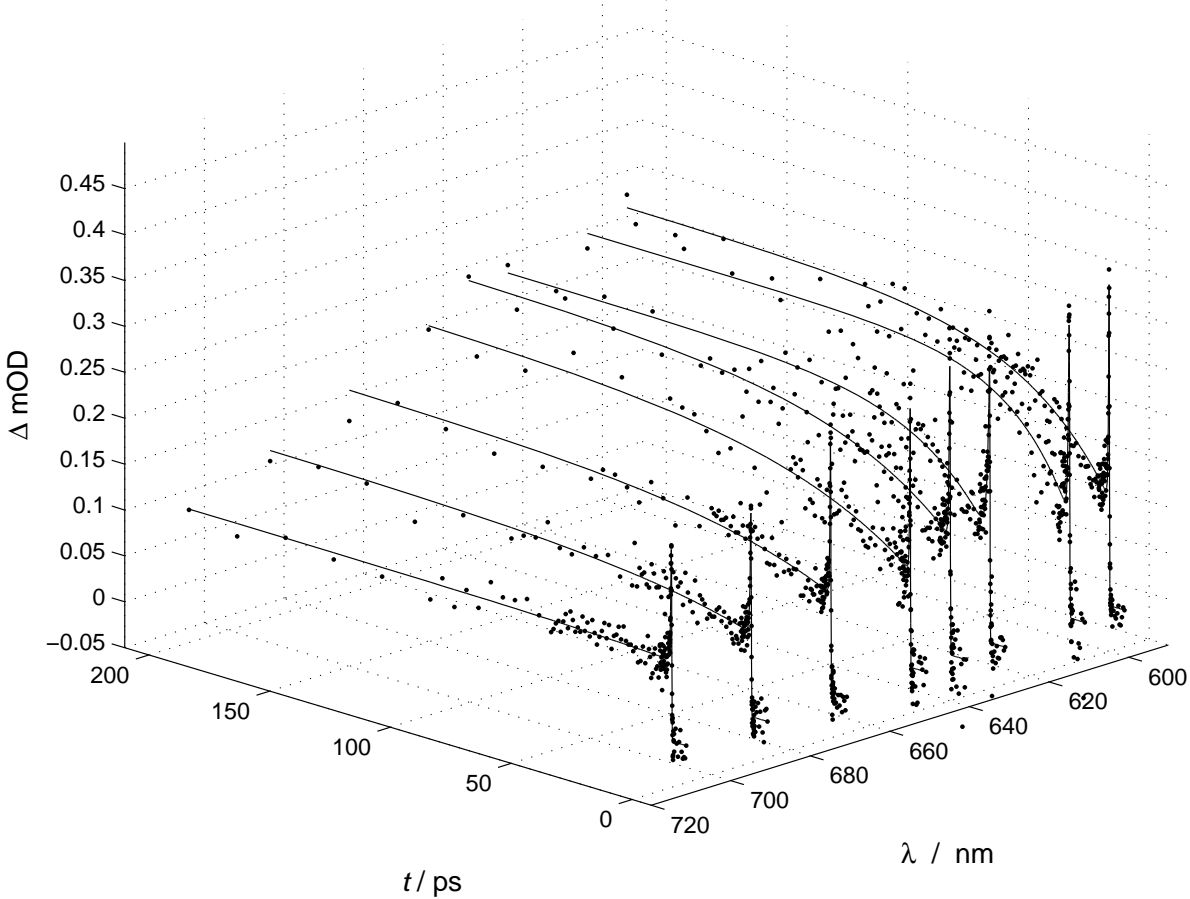

Abbildung 5.16: Transiente Absorption von $\mathrm{CHI}_{3}$ in sc. $\mathrm{CO}_{2}\left(35^{\circ} \mathrm{C}, 192\right.$ bar $)$ nach $267 \mathrm{~nm}$ Anregung 


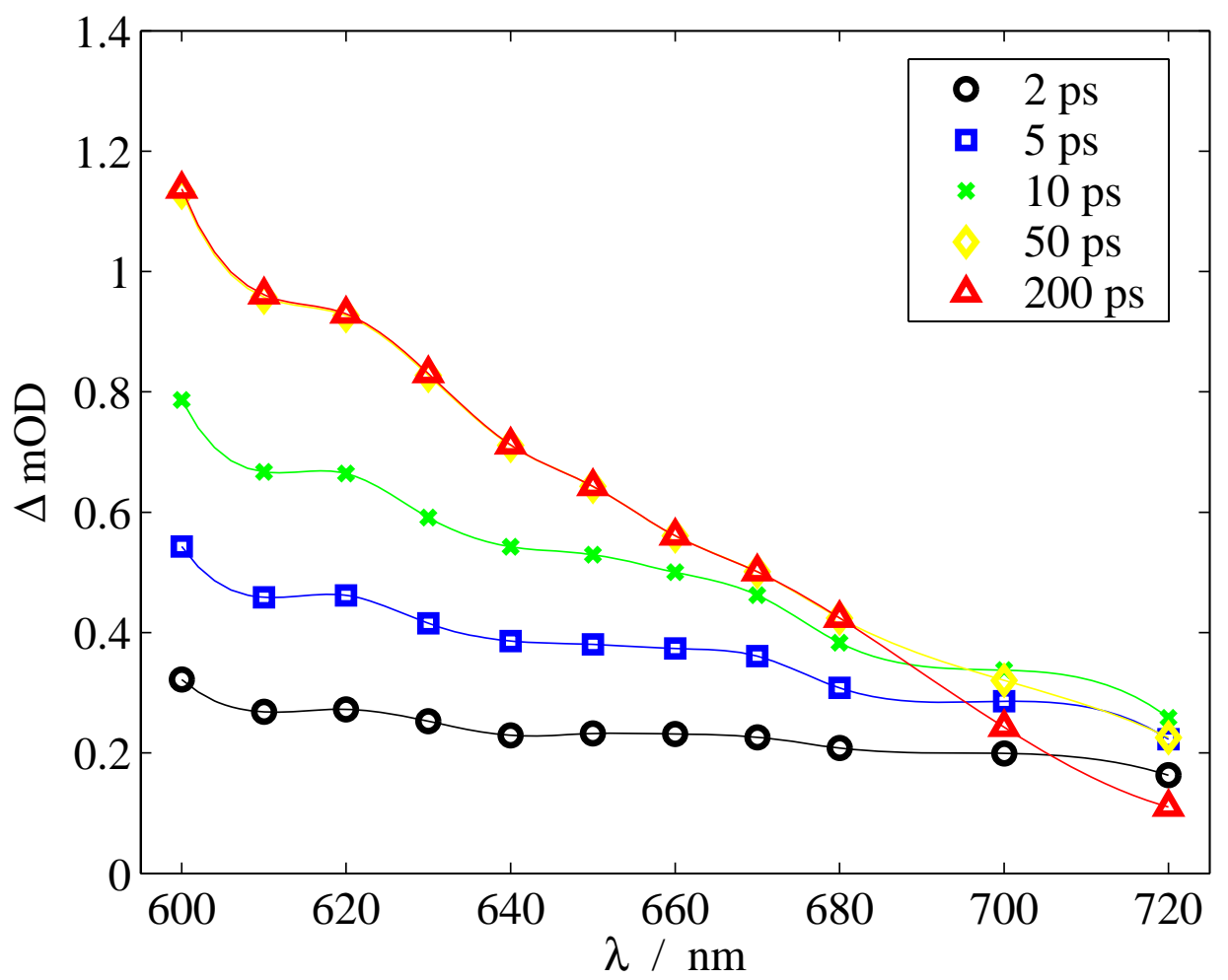

Abbildung 5.17: Zeitaufgelöstes Spektrum $\mathrm{CHI}_{3}$ in sc. $\mathrm{CO}_{2}\left(35^{\circ} \mathrm{C}, 505\right.$ bar $)$ nach $365 \mathrm{~nm}$ Anregung

\subsubsection{Zeitaufgelöste Spektren bei $365 \mathrm{~nm}$ Anregung}

Mit dem bereits beschriebenen Verfahren wurden aus den Absorptionszeitprofilen bei verschiedenen Drücken und variabler Anregungswellenlänge zeitaufgelöste Spektren rekonstruiert. Das Spektrum von $\mathrm{CHI}_{3}$ in sc. $\mathrm{CO}_{2}\left(35^{\circ} \mathrm{C}, 505\right.$ bar $)$ nach $365 \mathrm{~nm}$ Anregung ist in Abbildung 5.17 dargestellt.

Dabei zeigt sich für die VIS-Absorptionsbande des Isomers ein sehr ähnliches Bild wie im Fall der UV-Bande. Am Anfang ist das Spektrum breit und strukturlos, woraus im Anschluß im Laufe einiger Pikosekunden eine Bande zentriert bei etwa $600 \mathrm{~nm}$ herauswächst. Das Anwachsen ist nach 50 ps beendet und die Absorption ändert sich bis zu einer Verzögerungszeit von 200 ps nicht mehr. Die blaue Flanke dieser Bande konnte nicht aufgelöst werden, da in diesem Wellenlängenbereich die Ausgangspulse des TOPAS ein sehr kleines Signal/Rausch-Verhältnis erzeugen. Die rote Flanke zeigt bei Wellenlängen $>700 \mathrm{~nm}$ einen leichten Abfall der Absorption. Die Spektren von $\mathrm{CHI}_{3}$ nach $267 \mathrm{~nm}$ Anregung bei verschiedenen Drücken (505, 192 und 142 bar) sind sehr ähnlich und in Abbildung 5.18, 5.19 und 5.20 dargestellt. Dabei ist der Anstieg der Bande bei niedrigen Drücken wesentlich langsamer, sowie die Amplitude geringer. Die zu kurzen Zeiten (um 2 ps) beobachtete Anfangsabsorption ist jedoch bei allen Drücken nahezu gleich groß. 


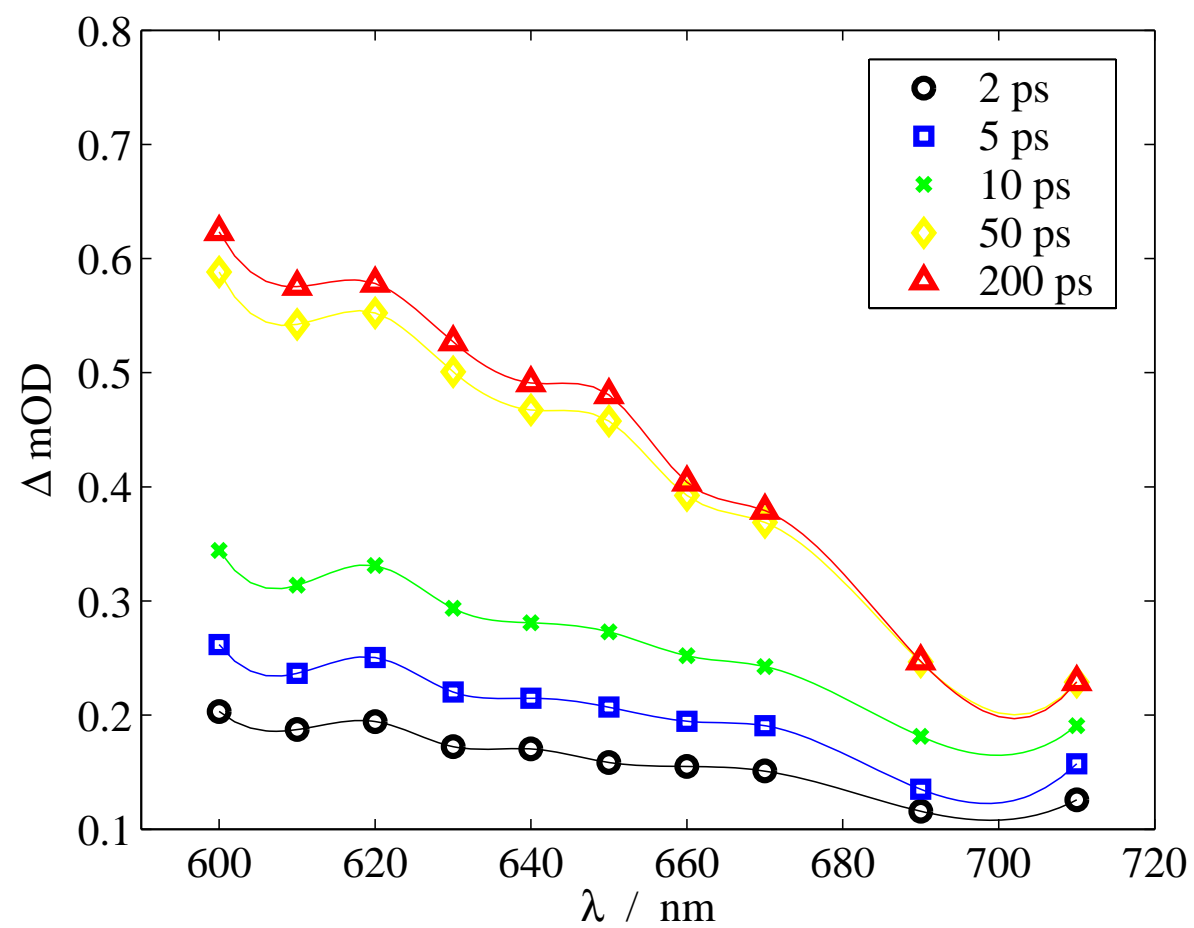

Abbildung 5.18: Zeitaufgelöstes Spektrum $\mathrm{CHI}_{3}$ in sc. $\mathrm{CO}_{2}\left(35^{\circ} \mathrm{C}, 505\right.$ bar $)$ nach $267 \mathrm{~nm}$ Anregung

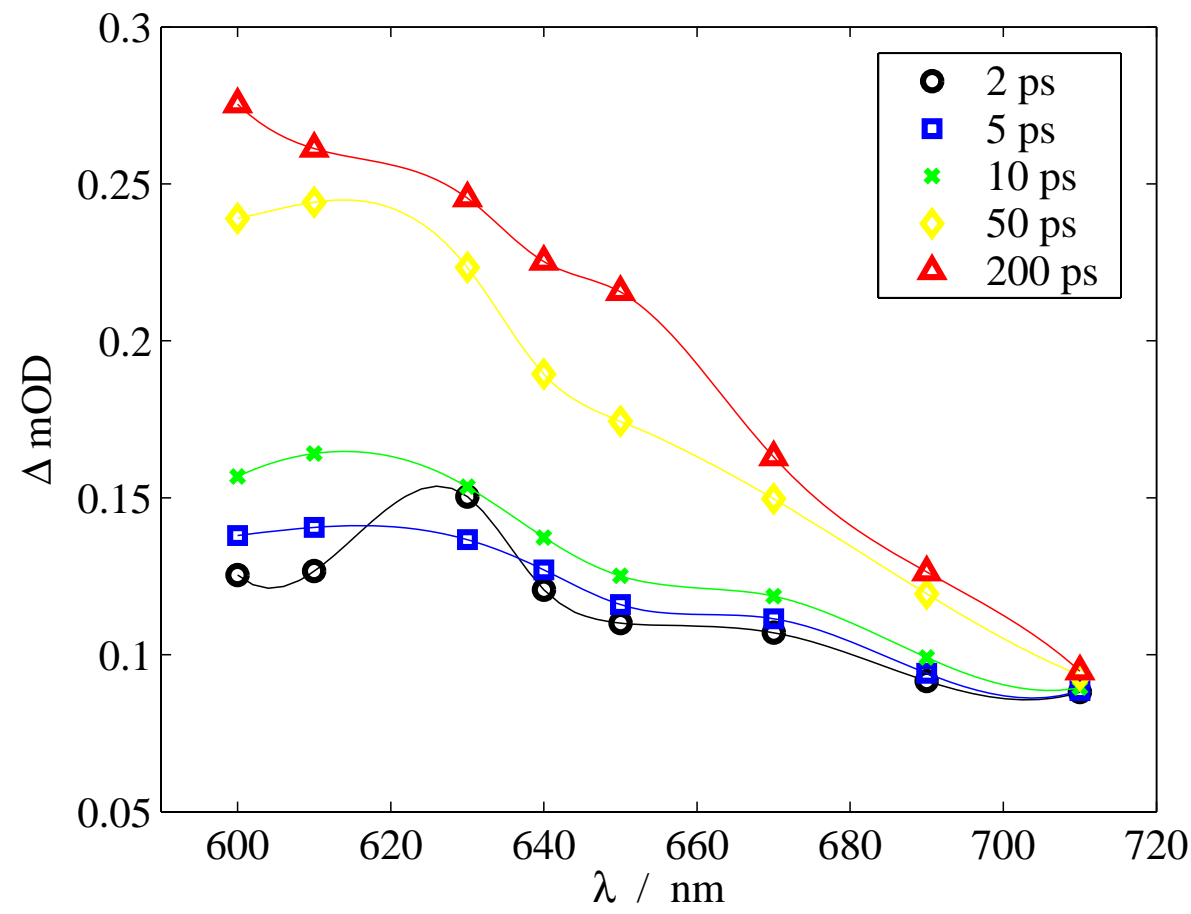

Abbildung 5.19: Zeitaufgelöstes Spektrum $\mathrm{CHI}_{3}$ in sc. $\mathrm{CO}_{2}\left(35^{\circ} \mathrm{C}, 192\right.$ bar $)$ nach $267 \mathrm{~nm}$ Anregung 


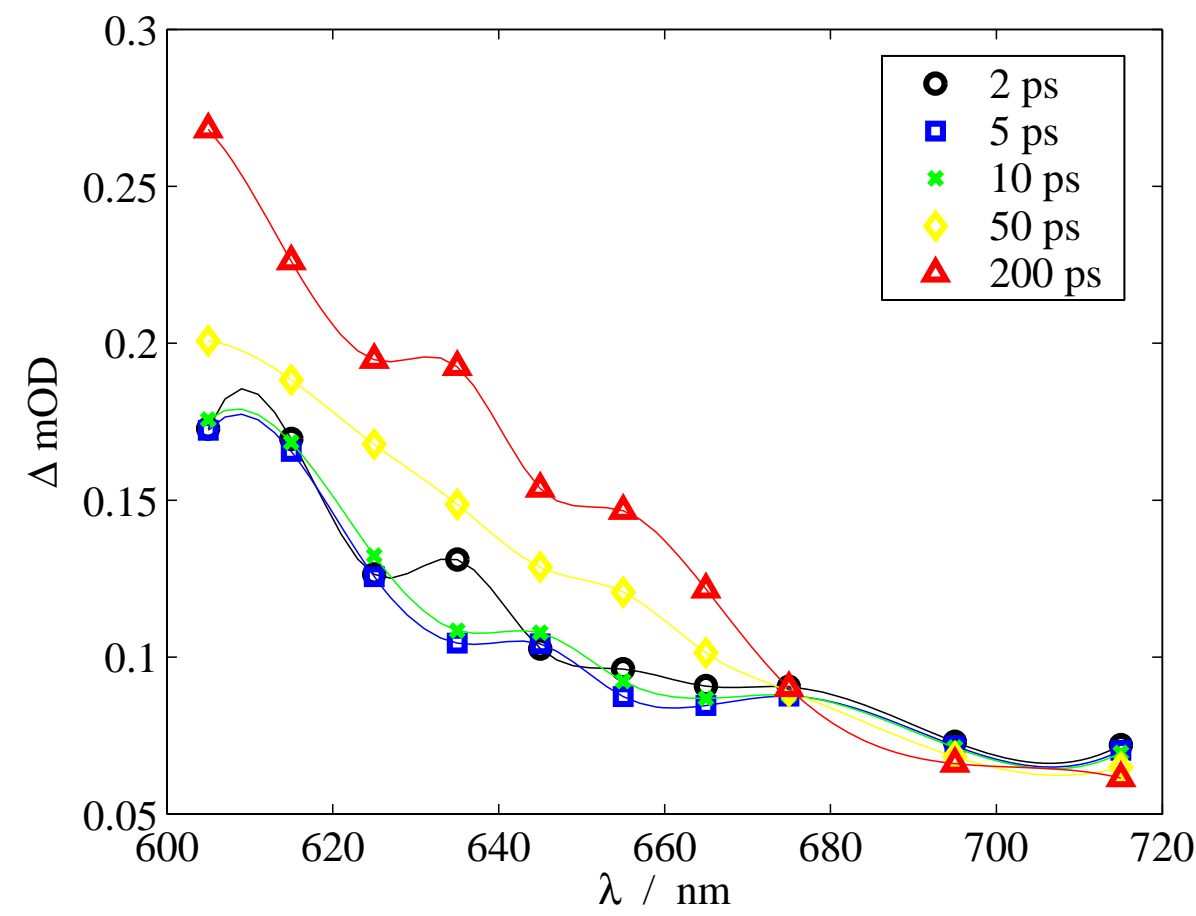

Abbildung 5.20: Zeitaufgelöstes Spektrum $\mathrm{CHI}_{3}$ in sc. $\mathrm{CO}_{2}\left(35^{\circ} \mathrm{C}, 142\right.$ bar $)$ nach $267 \mathrm{~nm}$ Anregung

\subsubsection{Druckabhängige Messungen bei variabler Anregungswellenlänge}

Analog zu den druckabhängigen Messungen von $\mathrm{CH}_{2} \mathrm{I}_{2}$ und $\mathrm{CH}_{2} \mathrm{BrI}$ wurde die Druckabhängigkeit der Absorptionszeitprofile von $\mathrm{CHI}_{3}$ in sc. $\mathrm{CO}_{2}$ bei verschiedenen Anregungswellenlängen gemessen. Dabei wurde jeweils in eine der drei Absorptionsbanden von $\mathrm{CHI}_{3}$ bei 340, 295 und $267 \mathrm{~nm}$ angeregt, die Probewellenlänge lag bei $460 \mathrm{~nm}$ (Maximum der UV-Absorptionsbande des Isomers). Die Zeitkonstante $k_{\text {iso }}$ des langsamen Anstiegs, welche mit der Isomerisierung verknüpft wird, zeigt dabei eine deutliche Dichteabhängigkeit. In Abbildung 5.21 ist $k_{\text {iso }}$ gegen die reduzierte Dichte $\rho_{r}$ des Lösungsmittels aufgetragen. Dabei zeigt sich ein dem $\mathrm{CH}_{2} \mathrm{I}_{2}$ sehr ähnliches Verhalten. Die Dichteabhängigkeit von $k_{\text {iso }}$ ist bei allen drei gemessenen Anregungswellenlängen sehr ähnlich, allerdings sind die Geschwindigkeitskonstanten bei einer langwelligen Anregung größer als bei Anregung mit einem kurzwelligeren Pumppuls. Die Reaktion wird somit nach Absorption eines Photons mit höherer Energie langsamer, dieses Verhalten konnte bereits bei $\mathrm{CH}_{2} \mathrm{I}_{2}$ beobachtet werden (s. Abbildung 5.2). Allerdings ist der Effekt bei $\mathrm{CHI}_{3}$ wesentlich kleiner als im Falle des $\mathrm{CH}_{2} \mathrm{I}_{2}$. Neben $k_{\text {iso }}$ wurde die Druckabhängigkeit von $A_{\text {iso,n }}$ als Maß für die Quantenausbeute der Isomerisierungsreaktion ausgewertet. Dazu wurde die Endamplitude $A_{\text {iso }}$, wie in Abschnitt 5.2.2 beschrieben, auf den Anfangspeak normiert. Die normierte Endamplitude $A_{\text {iso,n }}$ ist in Abbildung 5.22 gegen die reduzierte Dichte des Lösungsmittels aufgetragen. Dabei zeigt sich für die unterschiedlichen Anregungswellenlängen ein nahezu linearer Verlauf. 


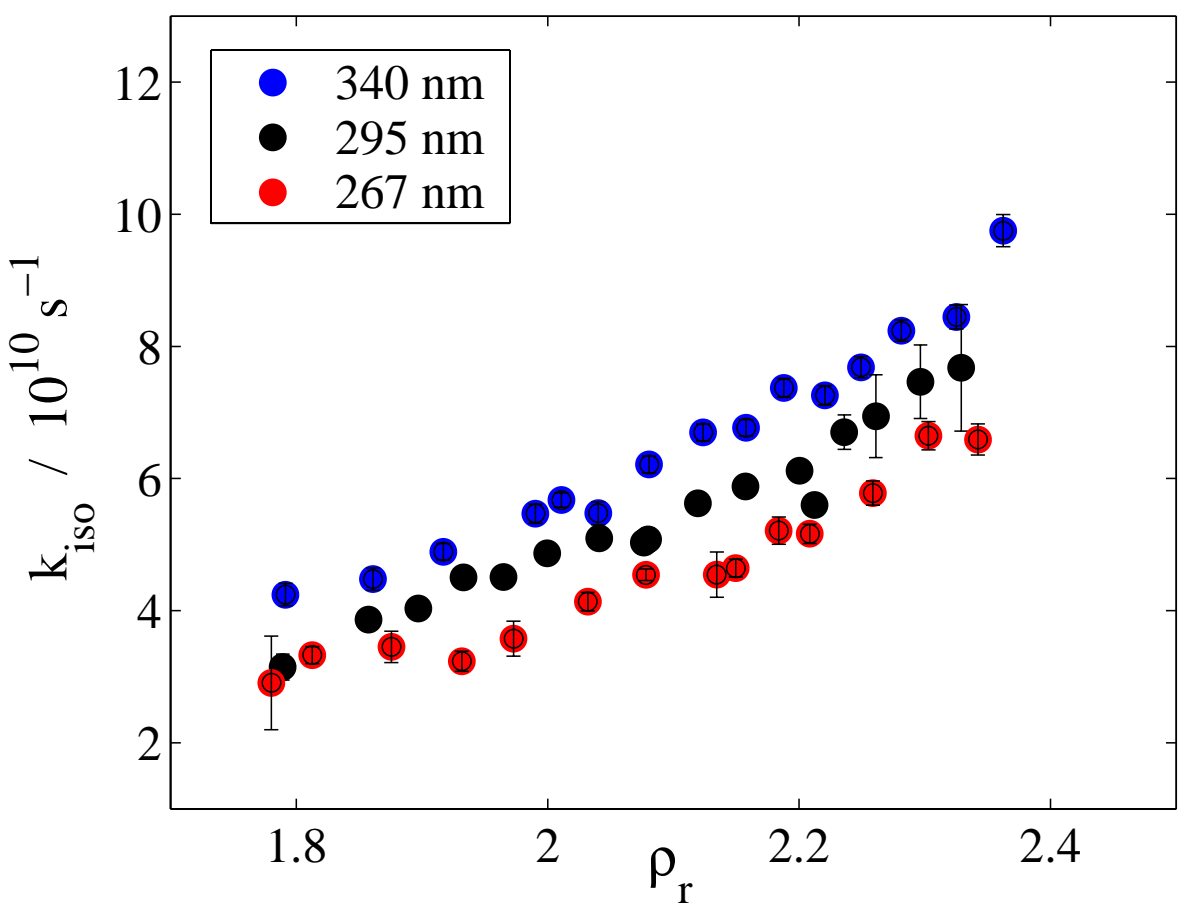

Abbildung 5.21: Dichteabhängigkeit $k_{\text {iso }}$ von $\mathrm{CHI}_{3}$ in sc. $\mathrm{CO}_{2}\left(35^{\circ} \mathrm{C}\right)$ bei 267,295 und $340 \mathrm{~nm}$ Anregung und Probewellenlänge von $460 \mathrm{~nm}$

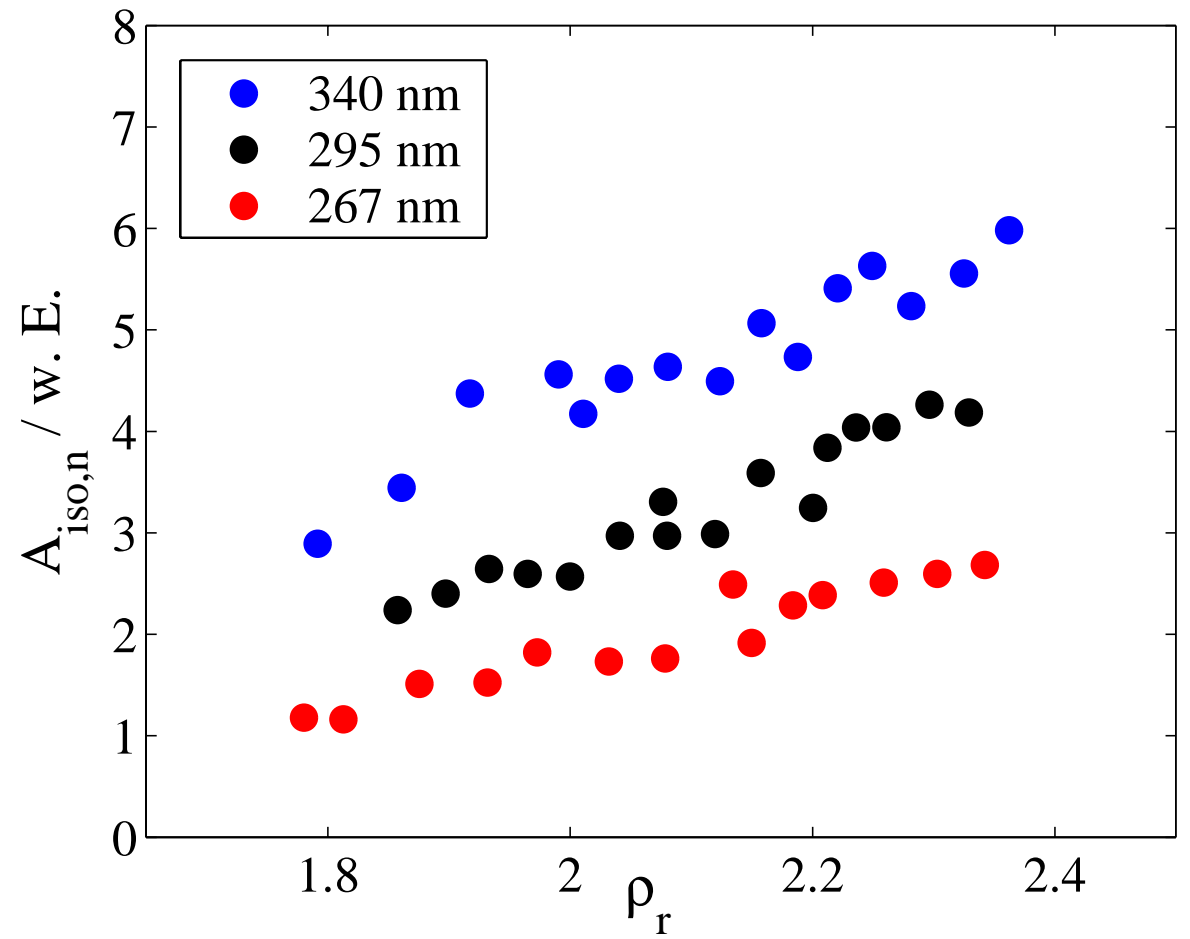

Abbildung 5.22: Dichteabhängigkeit $A_{\text {iso,n }}$ von $\mathrm{CHI}_{3}$ in sc. $\mathrm{CO}_{2}\left(35^{\circ} \mathrm{C}\right)$ bei 267,295 und $340 \mathrm{~nm}$ Anregung und Probewellenlänge von $460 \mathrm{~nm}$ 

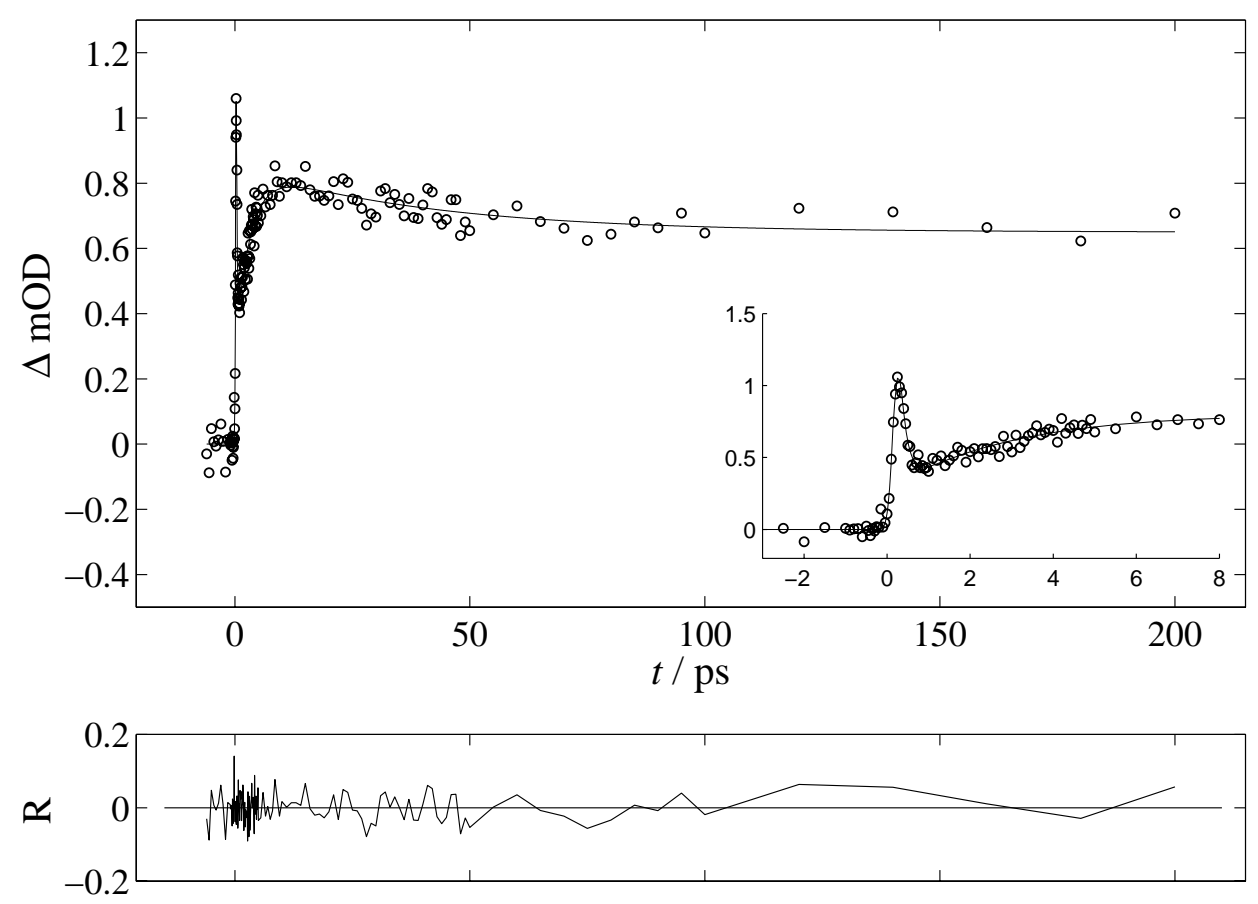

Abbildung 5.23: Absorptionszeitprofil von $\mathrm{CHI}_{3}$ in Acetonitril bei $267 \mathrm{~nm}$ Anregung und $370 \mathrm{~nm}$ Abfrage (Fokuslage vor der Zelle)

\subsubsection{Variation des Fokusvolumens}

Um die Frage nach einer möglichen geminalen Rekombination der primären Photofragmente innerhalb des Lösungsmittelkäfigs zu beantworten, wurden Ausbleichexperimente von $\mathrm{CHI}_{3}$ in Acetonitril durchgeführt. Dazu wurde in die erste Bande des $\mathrm{CHI}_{3}$ bei $267 \mathrm{~nm}$ angeregt und die transiente Absorption auf der roten Flanke des Absorptionsspektrums bei etwa $370 \mathrm{~nm}$ abgefragt. Anstelle der Hochdruckzelle wurde die Durchflußzelle für flüssige Lösungsmittel verwendet. Für eine Messung wurden etwa $0,3 \mathrm{~g} \mathrm{CHI}_{3}$ in $50 \mathrm{ml}$ Acetonitril gelöst und mit der Lösung die Zelle befüllt, das entspricht einer Konzentration von etwa $15 \mathrm{mmol} \mathrm{l}^{-1}$.

Ein Absorptionszeitprofil einer Messung von $\mathrm{CHI}_{3}$ in Acetonitril bei $267 \mathrm{~nm}$ Anregung und $370 \mathrm{~nm}$ Abfrage ist in Abbildung 5.23 dargestellt. Dabei zeigt sich das typische Isomersignal. Anschließend wurde der Fokusdurchmesser des Pumpstrahls in der Zelle verkleinert, um eine höhere Pumpintensität in der Meßzelle zu erzeugen. Dafür wurde der Fokus, welcher üblicherweise etwas vor der Zelle lag, direkt in die Zelle gelegt. Dabei ändert sich die Form des Absorptionszeitprofils signifikant, zwei Messungen mit verschieden großen Pumpfoki sind in Abbildung 5.24 und 5.25 dargestellt. Anstelle des Anfangspeaks mit anschließendem langsamen Anstieg tritt nun ein negatives Signal, welches mit einer Zeitkonstante von etwa 5 ps wieder relaxiert. 

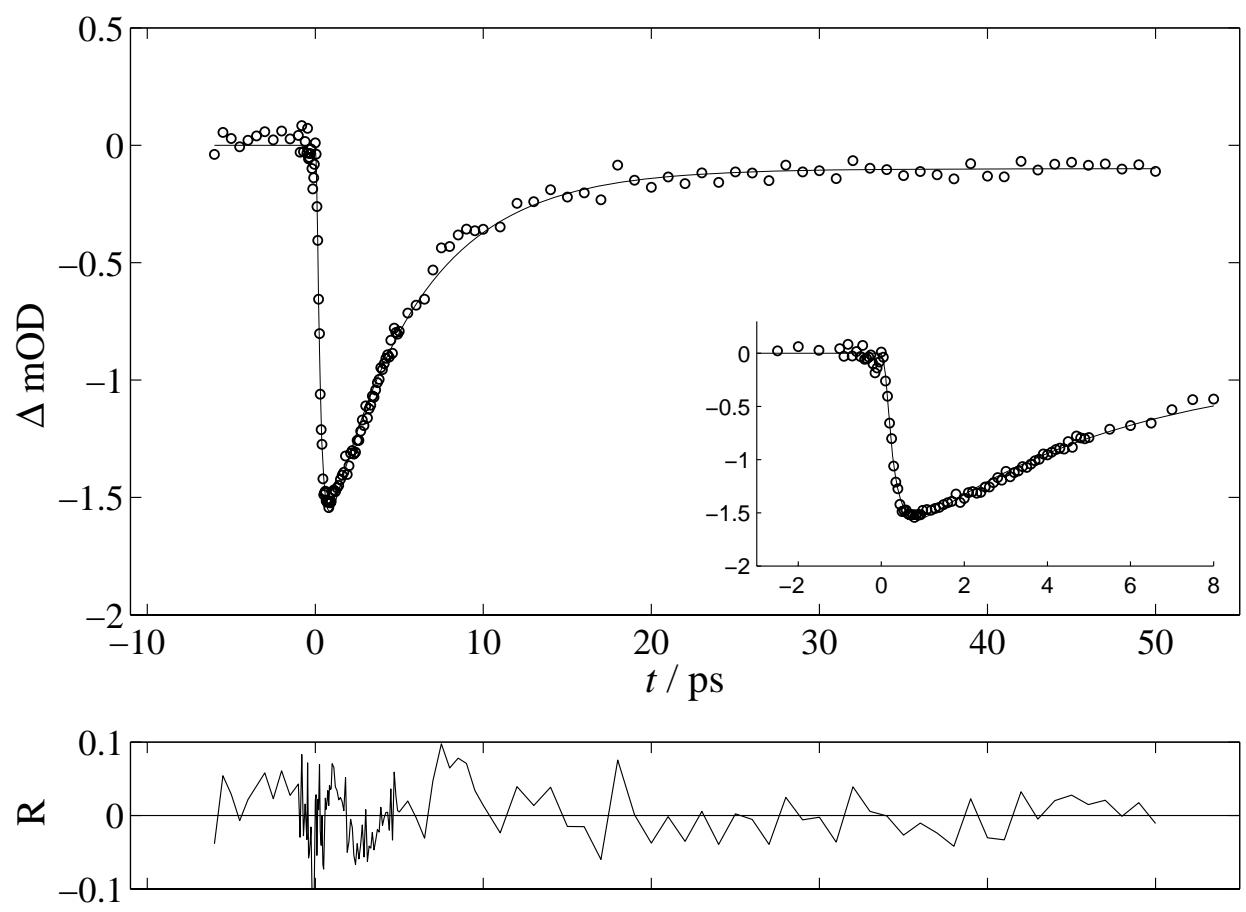

Abbildung 5.24: Absorptionszeitprofil von $\mathrm{CHI}_{3}$ in Acetonitril bei $267 \mathrm{~nm}$ Anregung und $370 \mathrm{~nm}$ Abfrage (Fokuslage direkt in der Zelle)
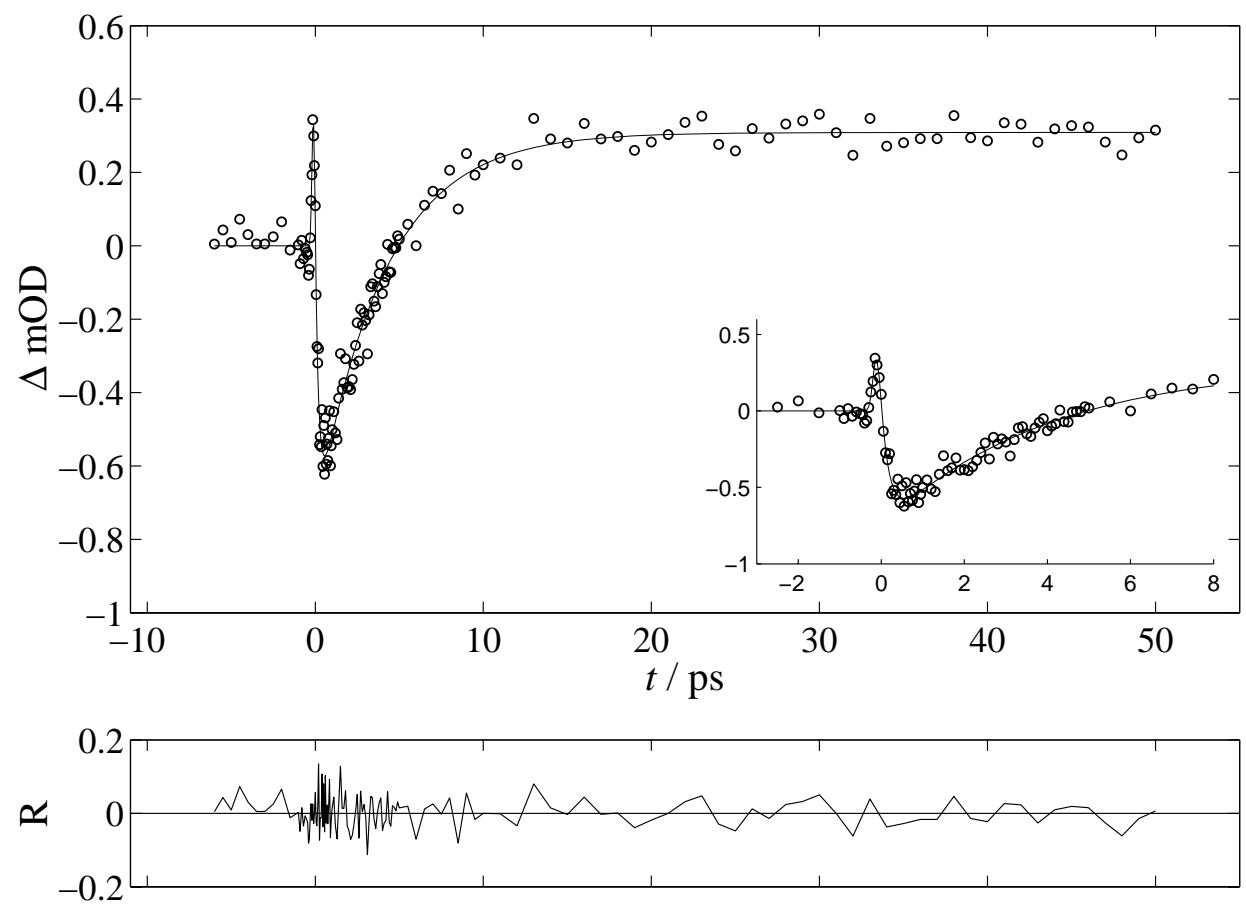

Abbildung 5.25: Absorptionszeitprofil von $\mathrm{CHI}_{3}$ in Acetonitril bei $267 \mathrm{~nm}$ Anregung und $370 \mathrm{~nm}$ Abfrage (Fokuslage knapp vor der Zelle) 

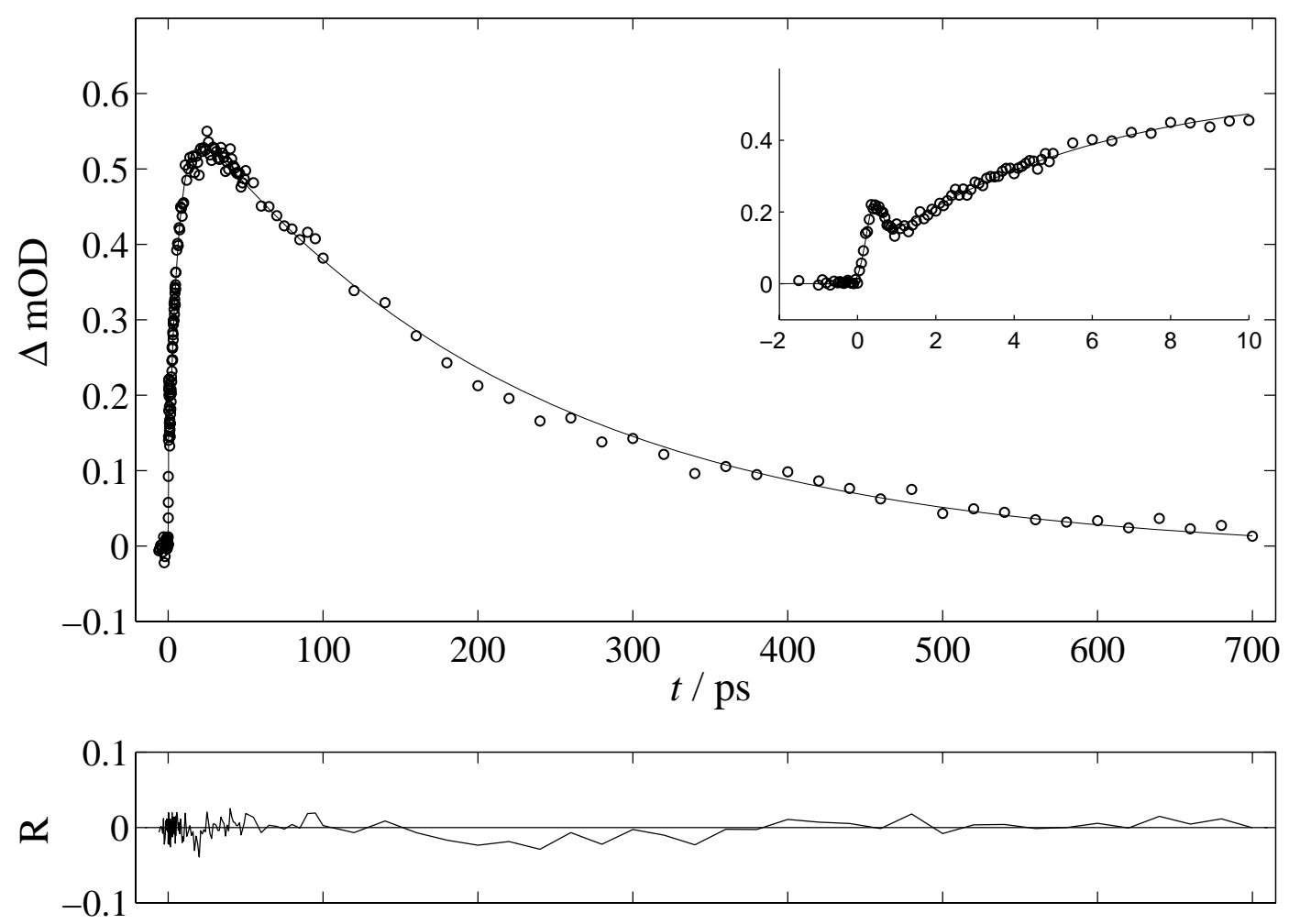

Abbildung 5.26: Absorptionszeitprofil von $\mathrm{CH}_{2} \mathrm{ClI}$ in Acetonitril nach $258 \mathrm{~nm}$ Anregung und $590 \mathrm{~nm}$ Abfrage

\subsection{Chloriodmethan}

Im Gegensatz zu den anderen Polyhalogenmethanen besitzt $\mathrm{CH}_{2} \mathrm{ClI}$ in der $A$-Bande bei $260 \mathrm{~nm}$ nur einen sehr kleinen Extinktionskoeffizienten (s. Abbildung 3.3). Für ein ausreichend großes Signal/Rausch-Verhältnis muß demnach mit einer höheren Pumpintensität angeregt werden. Dazu wurde anstelle mit der Zweiten Harmonischen des NOPAAusgangspulses mit der wesentlich intensiveren Dritten Harmonischen (THG, s. Abschnitt 4.1.3 des UV/VIS-Lasersystems bei $258 \mathrm{~nm}$ angeregt. Die Pulsenergie betrug dabei etwa $500 \mathrm{~nJ}$.

Zur Aufnahme von Absorptionszeitprofilen bei unterschiedlichen Probewellenlängen wurde die bereits beschriebene Durchflußzelle verwendet. Dazu wurde eine Lösung von $\mathrm{CH}_{2} \mathrm{ClI}$ in Acetonitril mit einer Konzentration von etwa $0,3 \mathrm{~mol} \mathrm{l}^{-1}$ verwendet. Ein typisches Absorptionszeitprofil von $\mathrm{CH}_{2} \mathrm{ClI}$ in Acetonitril bei einer Probewellenlänge von $590 \mathrm{~nm}$ ist in Abbildung 5.26 dargestellt.

Dabei ist der Verlauf der transienten Absorption bis zu einer Verzögerungszeit von etwa 100 ps denen der anderen Polyhalogenmethane sehr ähnlich. Nach einem Anfangspeak, welcher durch die Zeitauflösung begrenzt ist, steigt die Absorption im Laufe einiger Pikosekunden an, fällt danach jedoch monoexponentiell mit einer Zeitkonstante von etwa 200 ps wieder ab. Bei $\mathrm{CH}_{2} \mathrm{I}_{2}$ und $\mathrm{CHI}_{3}$ ist nach dem langsamen Anstieg kein Abfall der 


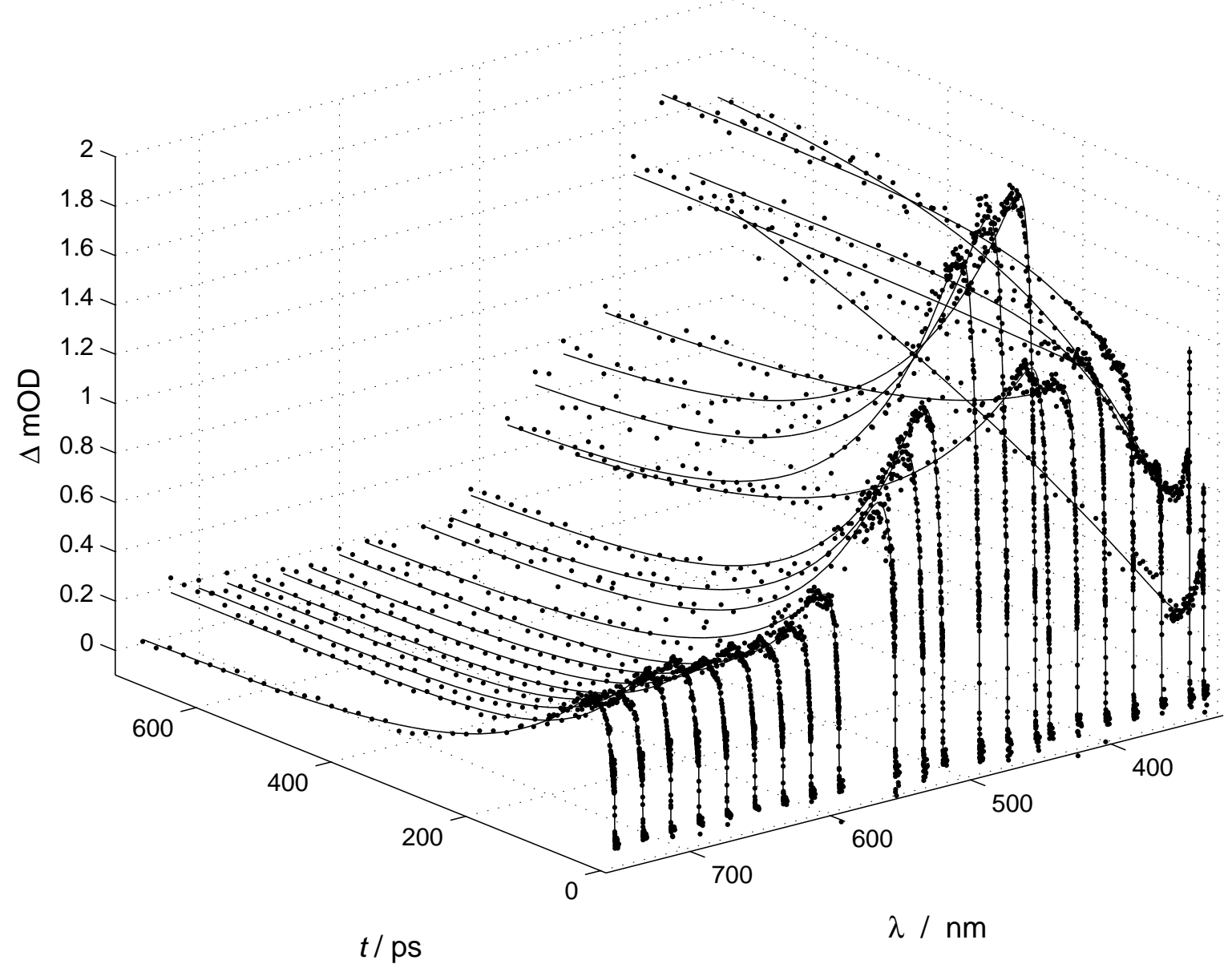

Abbildung 5.27: Transiente Absorption von $\mathrm{CH}_{2} \mathrm{ClI}$ in Acetonitril nach $258 \mathrm{~nm}$ Anregung

Absorption zu beobachten, im Falle von $\mathrm{CH}_{2} \mathrm{BrI}$ verläuft der Abfall nur mit geringer Amplitude. In Abbildung 5.27 sind die Absorptionszeitprofile von $\mathrm{CH}_{2} \mathrm{ClI}$ in Acetonitril über einen breiten Spektralbereich von 330-750 nm dargestellt. Das zeitliche Verhalten der transienten Absorption ist dabei stark von der Wellenlänge abhängig. Bei allen Wellenlängen folgt auf den Anfangspeak ein Anstieg mit einer probenwellenlängenabhängigen Zeitkonstante von 4,5-12,5 ps, anschließend fällt die Absorption innerhalb von 100-250 ps wieder ab. Bei Probewellenlängen $<400 \mathrm{~nm}$ ist kein Abfall, sondern ein weiterer Anstieg der Absorption innerhalb einiger hundert Pikosekunden zu beobachten. Neben Acetonitril wurden auch unpolare und überkritische Lösungsmittel verwendet. Typische Absorptionszeitprofile sind dazu in den Abbildungen $5.28\left(\mathrm{CCl}_{4}\right), 5.29$ ( $n$-Hexan), 5.30 (sc. $\mathrm{CHF}_{3}$ ) und 5.31 (sc. Ethan) abgebildet. In überkritischen Fluiden konnte dabei erst bei hohen Drücken (> 900 bar) eine transiente Absorption mit akzeptablem Signal/RauschVerhältnis gemessen werden. Aus diesem Grunde konnte keine druckabhängigen Messungen durchgeführt werden, da der Maximaldruck der verwendeten Meßzelle (1000 bar) schon fast erreicht war. 

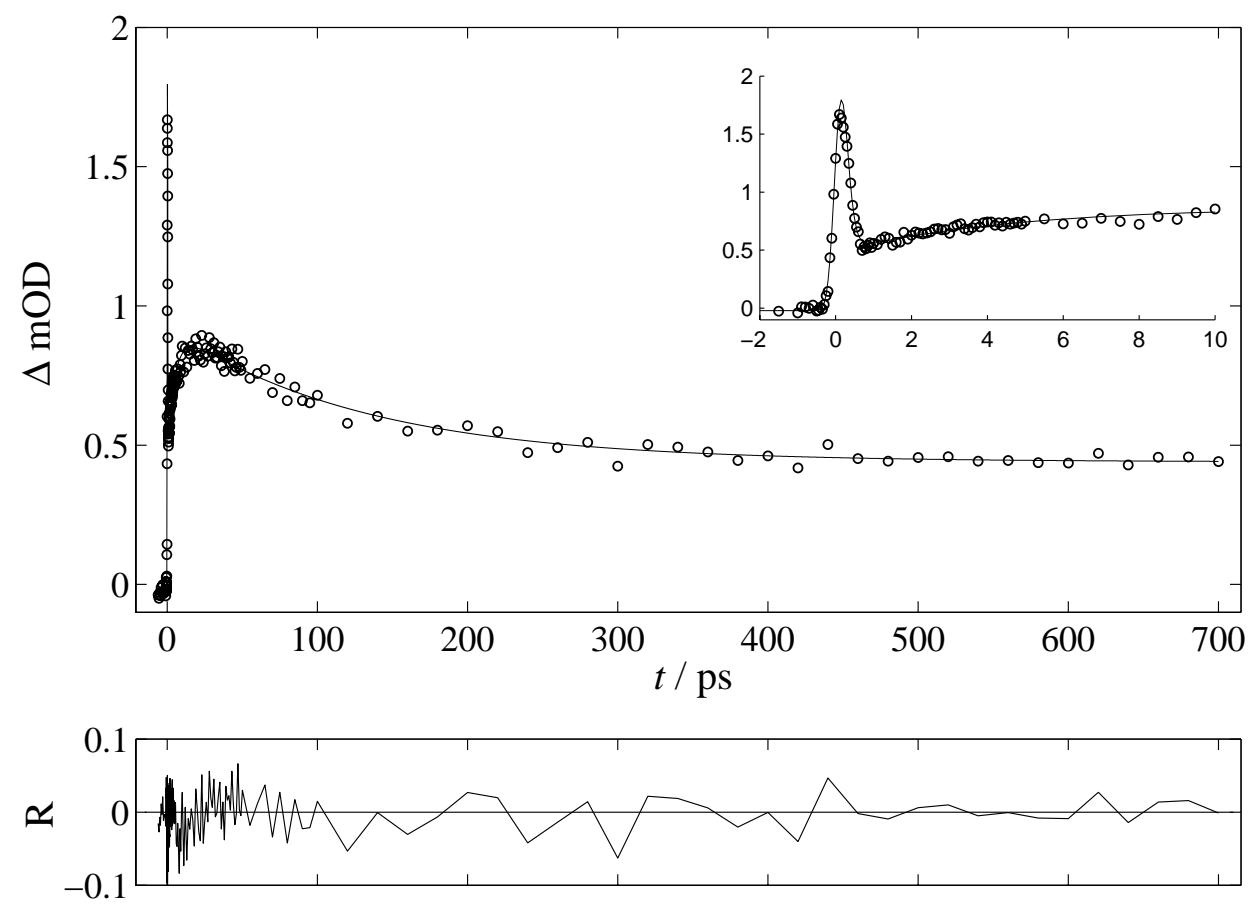

Abbildung 5.28: Absorptionszeitprofil von $\mathrm{CH}_{2} \mathrm{ClI}$ in $\mathrm{CCl}_{4}$ nach $258 \mathrm{~nm}$ Anregung und $450 \mathrm{~nm}$ Abfrage
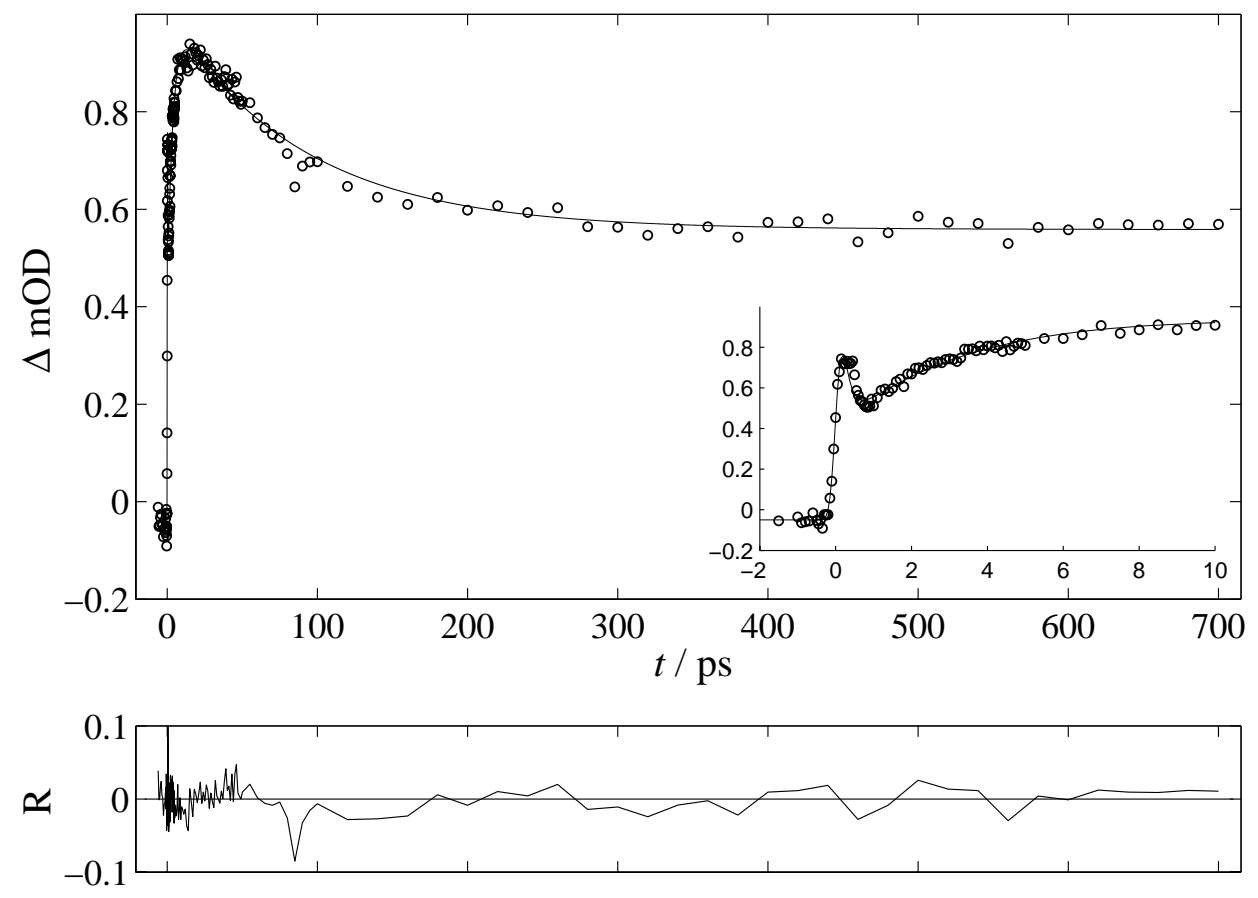

Abbildung 5.29: Absorptionszeitprofil von $\mathrm{CH}_{2} \mathrm{ClI}$ in $n$-Hexan nach $258 \mathrm{~nm}$ Anregung und $450 \mathrm{~nm}$ Abfrage 

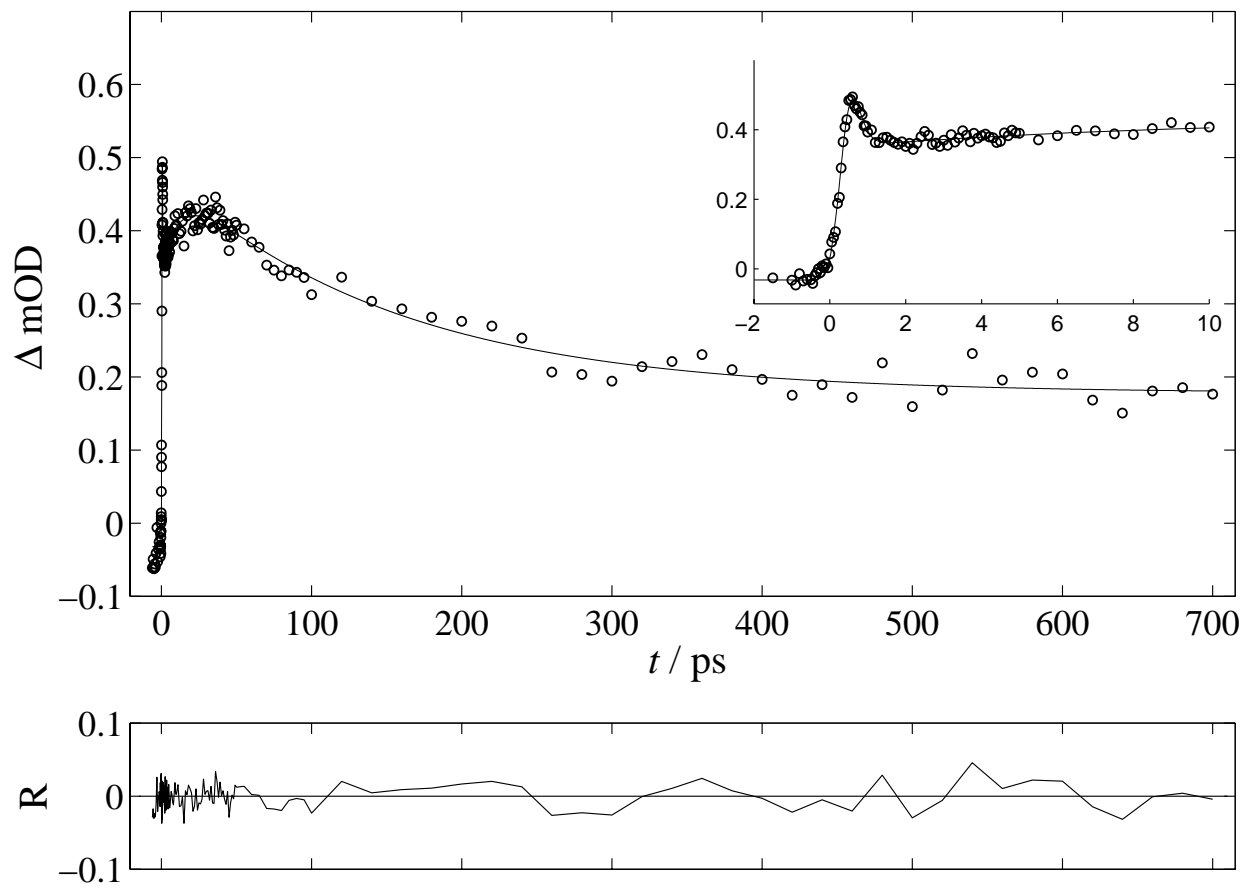

Abbildung 5.30: Absorptionszeitprofil von $\mathrm{CH}_{2} \mathrm{ClI}$ in sc. $\mathrm{CHF}_{3}\left(37^{\circ} \mathrm{C}, 951\right.$ bar $)$ nach $258 \mathrm{~nm}$ Anregung und $450 \mathrm{~nm}$ Abfrage
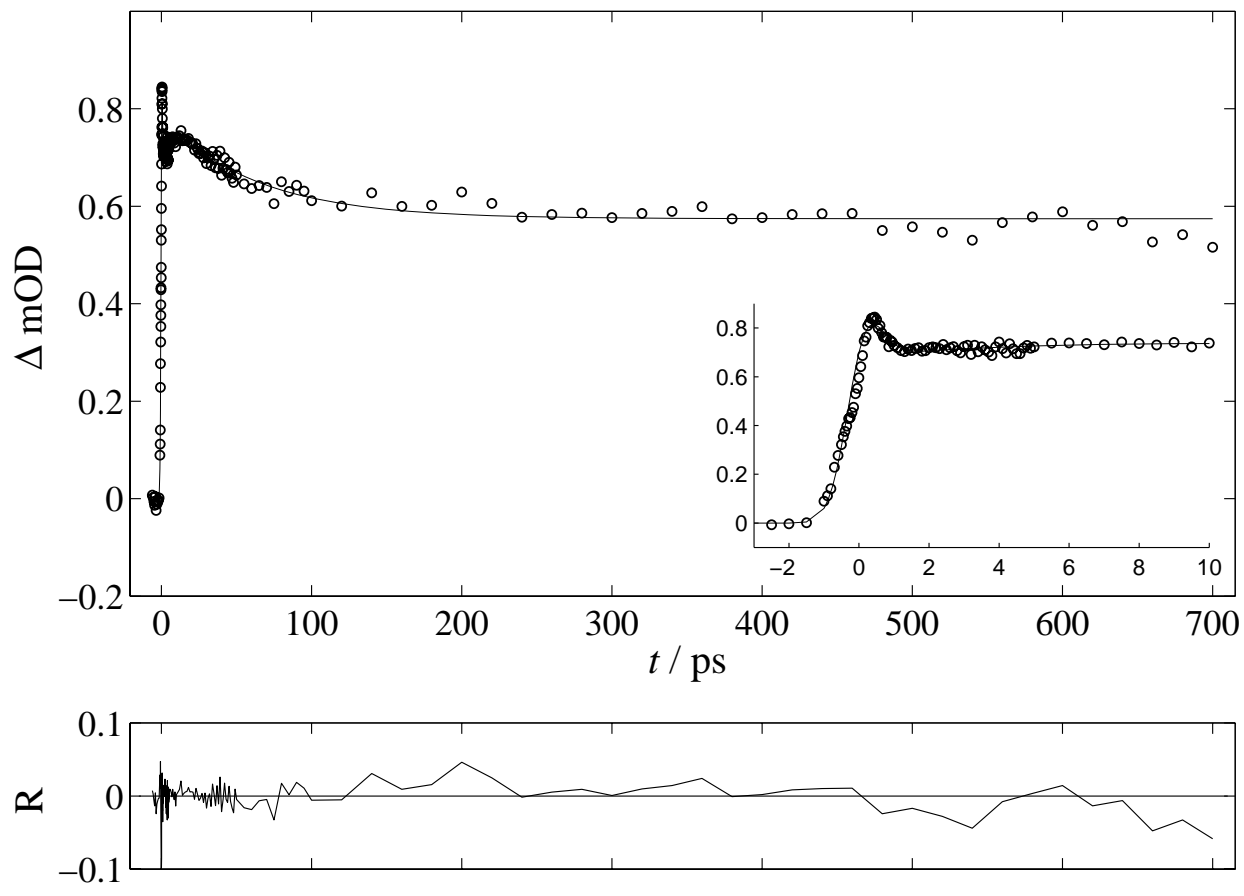

Abbildung 5.31: Absorptionszeitprofil von $\mathrm{CH}_{2} \mathrm{ClI}$ in sc. Ethan $\left(37^{\circ} \mathrm{C}, 950\right.$ bar $)$ nach $258 \mathrm{~nm}$ Anregung und $450 \mathrm{~nm}$ Abfrage 


\section{5 lodmethan}

\subsubsection{Stationäre Absorptionsspektren}

Stationäre Absorptionsspektren von $\mathrm{CH}_{3} \mathrm{I}$ in verschiedenen Lösungsmitteln wurden mit Hilfe eines Absorptionsspektrometers (Varian CARY 5E) aufgenommen. Dazu wurde etwas $\mathrm{CH}_{3} \mathrm{I}$ im entsprechenden Lösungsmittel in einer Küvette $(0,2 \mathrm{~cm}$, Quarz Suprasil) gelöst und ein Spektrum im Wellenlängenbereich von 200-800 nm aufgenommen, die Konzentration lag dabei bei etwa $50 \mathrm{mmol} \mathrm{l}^{-1}$. Anschließend wurden die Daten um die Absorption des reinen Lösungsmittels korrigiert und auf ihr Maximum normiert (s. Abbildung 5.32). Die Gestalt des Absorptionsspektrums in verschiedenen Lösungsmitteln bleibt dabei sehr ähnlich. Es findet lediglich eine geringe Blauverschiebung (s. Tabelle 5.1) in polaren Lösungsmitteln statt, welche auf eine Stabilisierung des Grundzustands in polaren Lösungsmitteln zurückzuführen ist. Das Spektrum in $n$-Perfluorhexan ist dem der Gasphase sehr ähnlich.

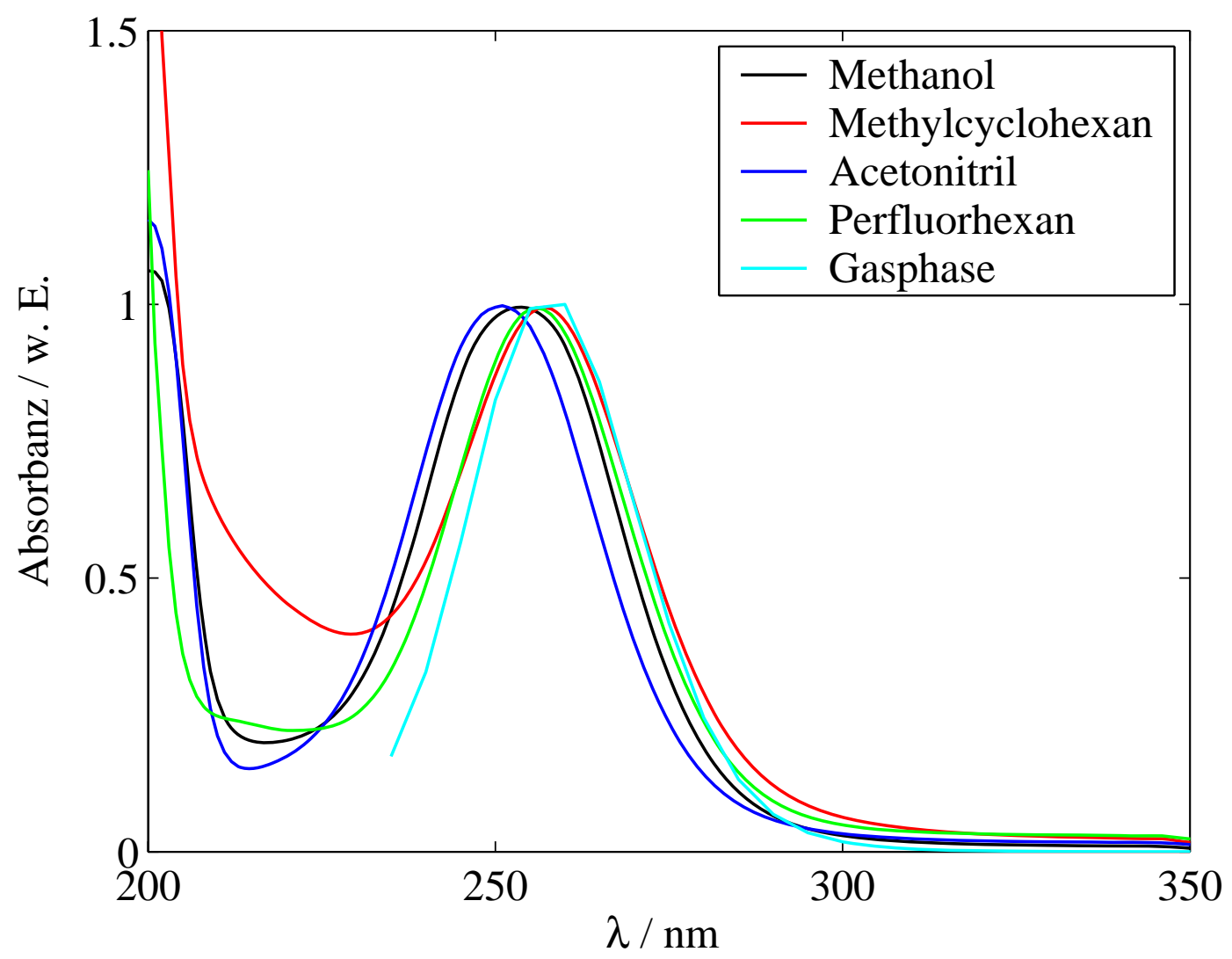

Abbildung 5.32: Stationäre Absorptionsspektren von $\mathrm{CH}_{3} \mathrm{I}$ in den verwendeten Lösungsmitteln 


\begin{tabular}{|lc|}
\hline Lösungsmittel & Absorptionsmaximum / nm \\
Acetonitril & 251,1 \\
Methanol & 253,5 \\
$n$-Perfluorhexan & 255,9 \\
Methylcyclohexan & 256,8 \\
\hline
\end{tabular}

Tabelle 5.1: Absorptionsmaximum $\mathrm{CH}_{3} \mathrm{I}$ in verschiedenen Lösungsmitteln

\subsection{2 fs-UV-Pump/UV-Probemessungen in Lösung}

Die UV-Pump/UV-Probemessungen wurden mit dem UV/VIS-Lasersystem bei einer Anregung von $255 \mathrm{~nm}$ (Methanol und Methylcyclohexan) bzw. bei $265 \mathrm{~nm}$ (Acetonitril und $n$-Perfluorhexan) vorgenommen. Durch Verwendung von hochreflektierenden UVSpiegeln und eines UV-Strahlteilers für den Probestrahl konnte dieser im Wellenlängenbereich von $280-400 \mathrm{~nm}$ durchgestimmt werden.

Typische Messung von $\mathrm{CH}_{3} \mathrm{I}$ in Methanol und Methylcyclohexan sind in Abbildung 5.33 und 5.35 dargestellt, sie bestehen aus einem kohärenten Anfangspeak mit einer anschließenden, konstanten Absorption. In Abbildung 5.34 ist eine Serie von Messungen bei durchgestimmten Probestrahl in Methanol abgebildet. Bei Wellenlängen unter $300 \mathrm{~nm}$ wird das Signal/Rauschverhältnis aufgrund der Absorption des Muttermoleküls zunehmend schlechter. Neben Methanol wurden Spektren in Methylcyclohexan (s. Abbildung 5.36), Acetonitril (s. Abbildung 5.37) und n-Perfluorhexan (s. Abbildung 5.39) aufgenommen. Die Spektren in Methanol, Acetonitril und Methylcyclohexan sind sich relativ ähnlich, während das in $n$-Perfluorhexan deutlich abweicht. Eine typische Messung in diesem Lösungsmittel ist in Abbildung 5.38 abgebildet, dabei schließt sich an den kohärenten Anfangspeak ein monoexponentieller Abfall von mehreren Pikosekunden an. Die Amplitude des monoexponentiellen Abfalls nimmt zu längeren Wellenlängen hin zu.

Um den Beitrag eines Lösungsmittelsignals zu berücksichtigen, wurden zudem Leermessungen mit dem reinem Lösungsmittel vorgenommen. Dabei zeigt sich ein von der Zeitauflösung begrenzter kohärenter Peak, welcher zu kürzeren Probewellenlängen immer intensiver wird. In Abbildung 5.40 sind diese Messungen für Methanol exemplarisch abgebildet, für die anderen Lösungsmittel ergibt sich ein sehr ähnliches Bild. 

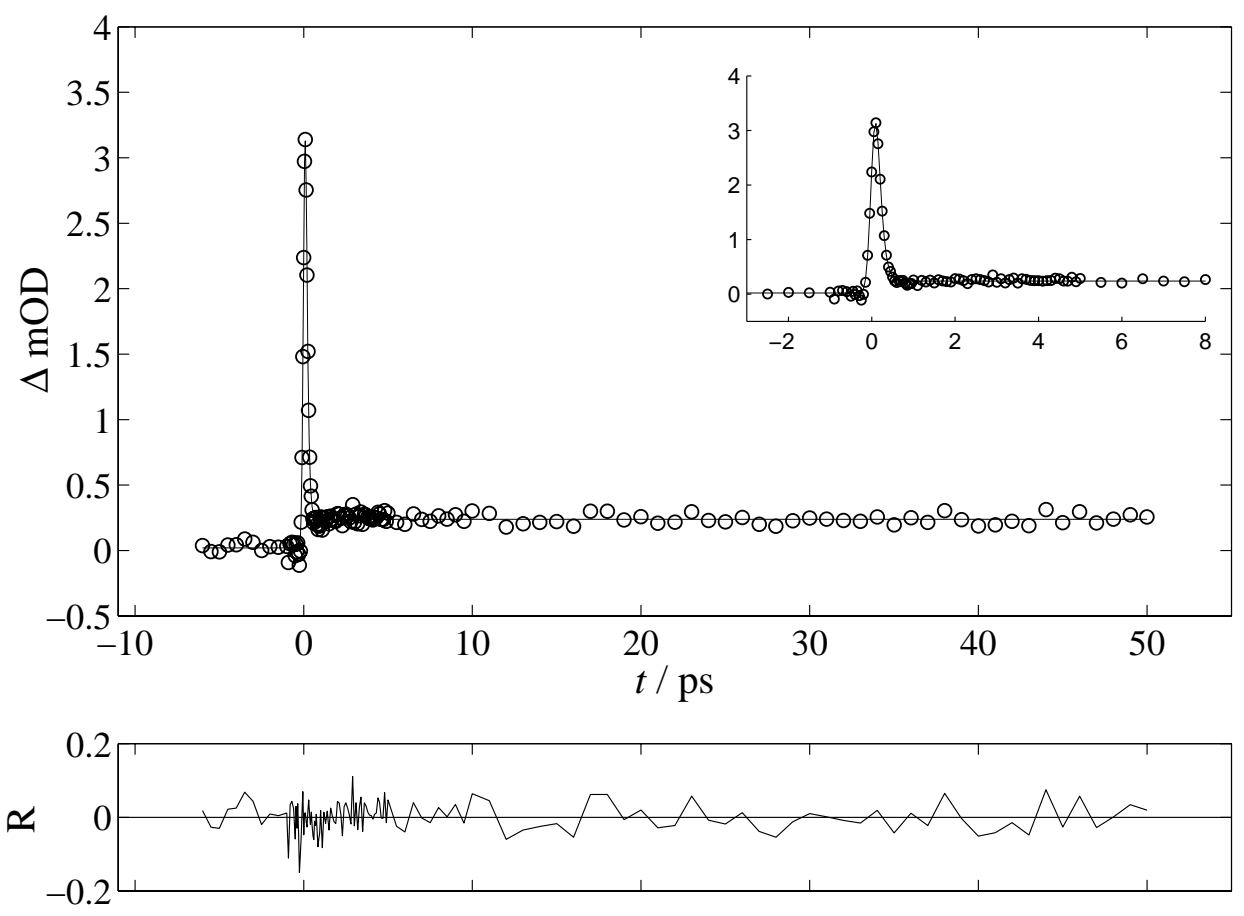

Abbildung 5.33: Absorptionszeitprofil von $\mathrm{CH}_{3} \mathrm{I}$ in $\mathrm{CH}_{3} \mathrm{OH}$ bei $255 \mathrm{~nm}$ Anregung und $320 \mathrm{~nm}$ Abfrage

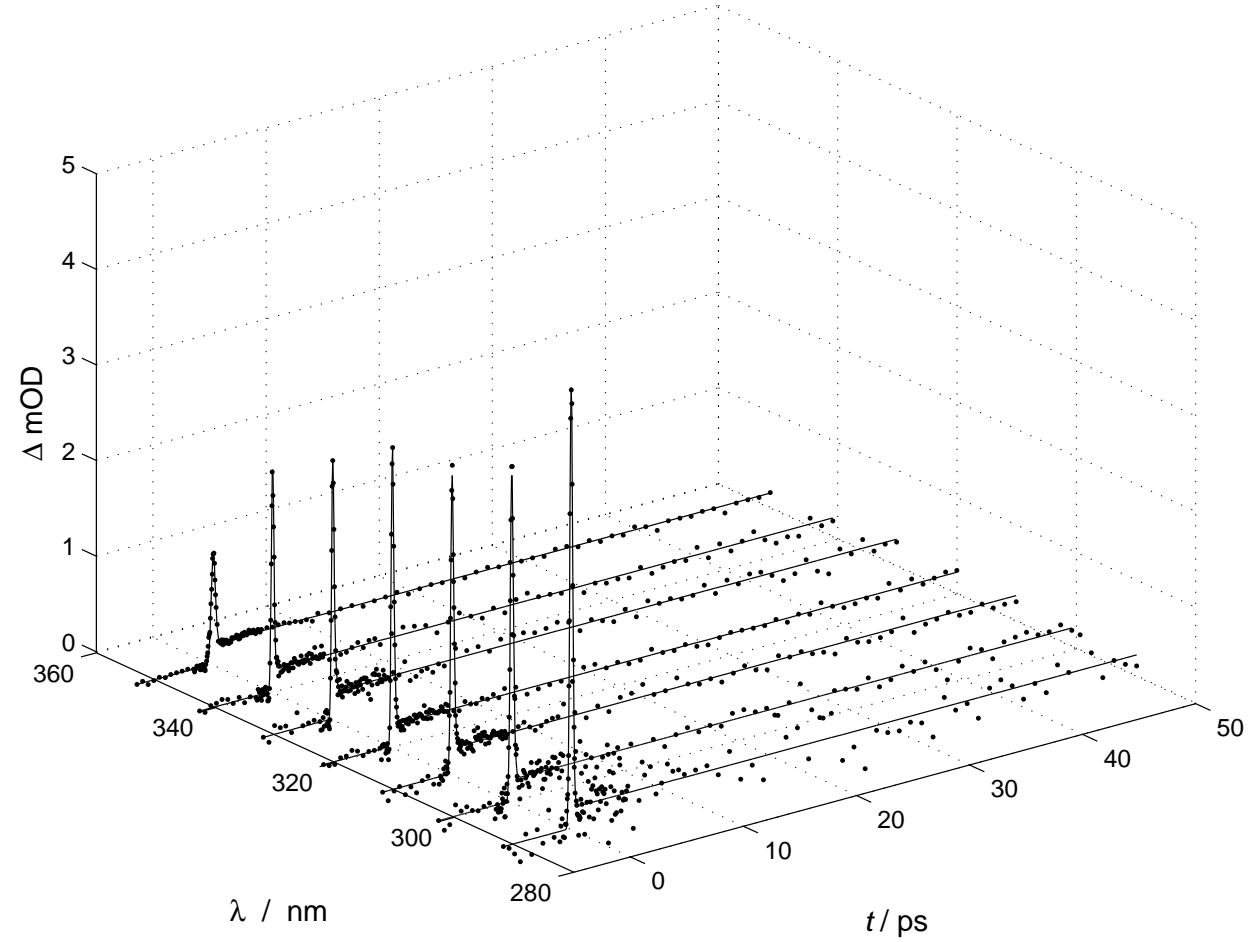

Abbildung 5.34: Transiente Absorption von $\mathrm{CH}_{3} \mathrm{I}$ in $\mathrm{CH}_{3} \mathrm{OH}$ bei $255 \mathrm{~nm}$ Anregung und UV-Abfrage 

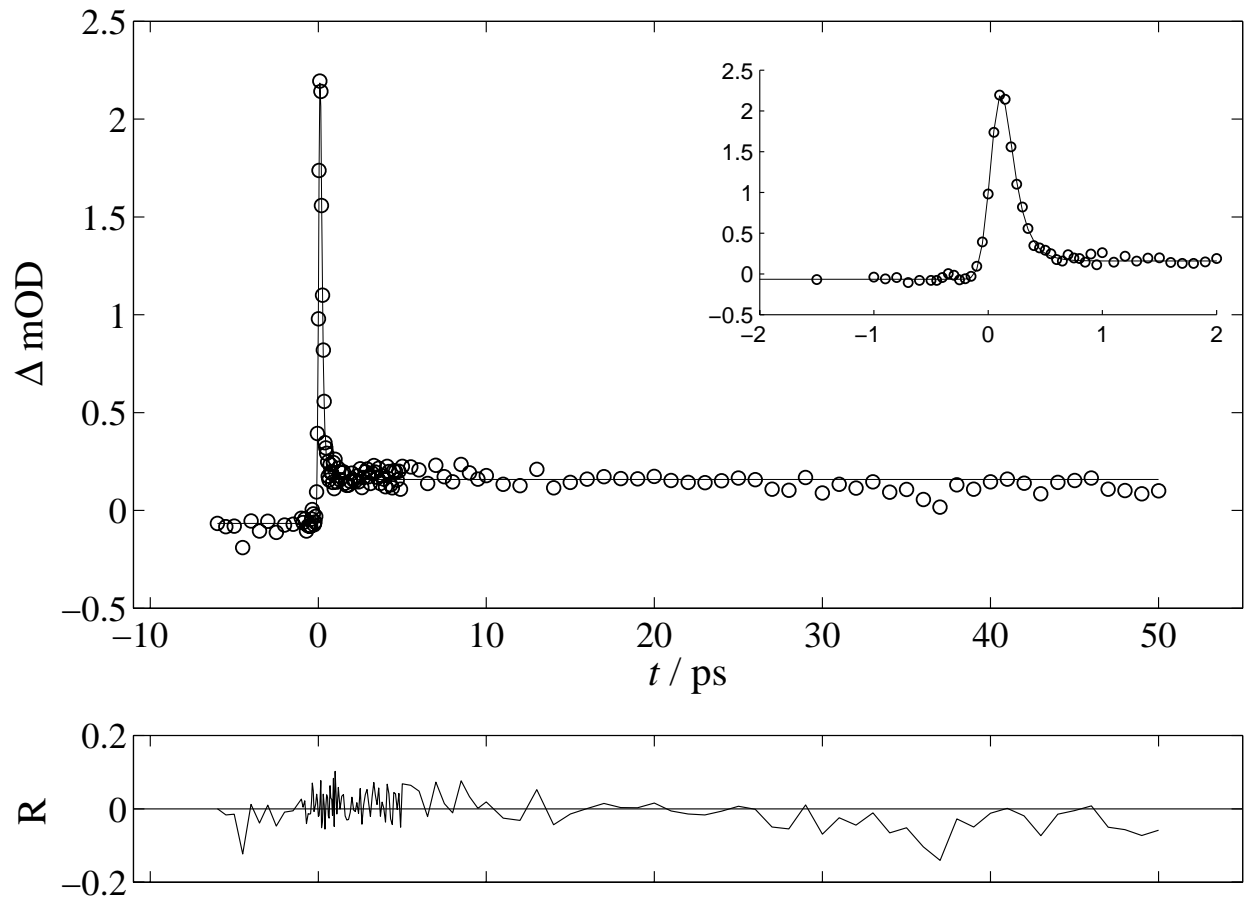

Abbildung 5.35: Absorptionszeitprofil von $\mathrm{CH}_{3} \mathrm{I}$ in Methylcyclohexan bei $265 \mathrm{~nm}$ Anregung und $360 \mathrm{~nm}$ Abfrage

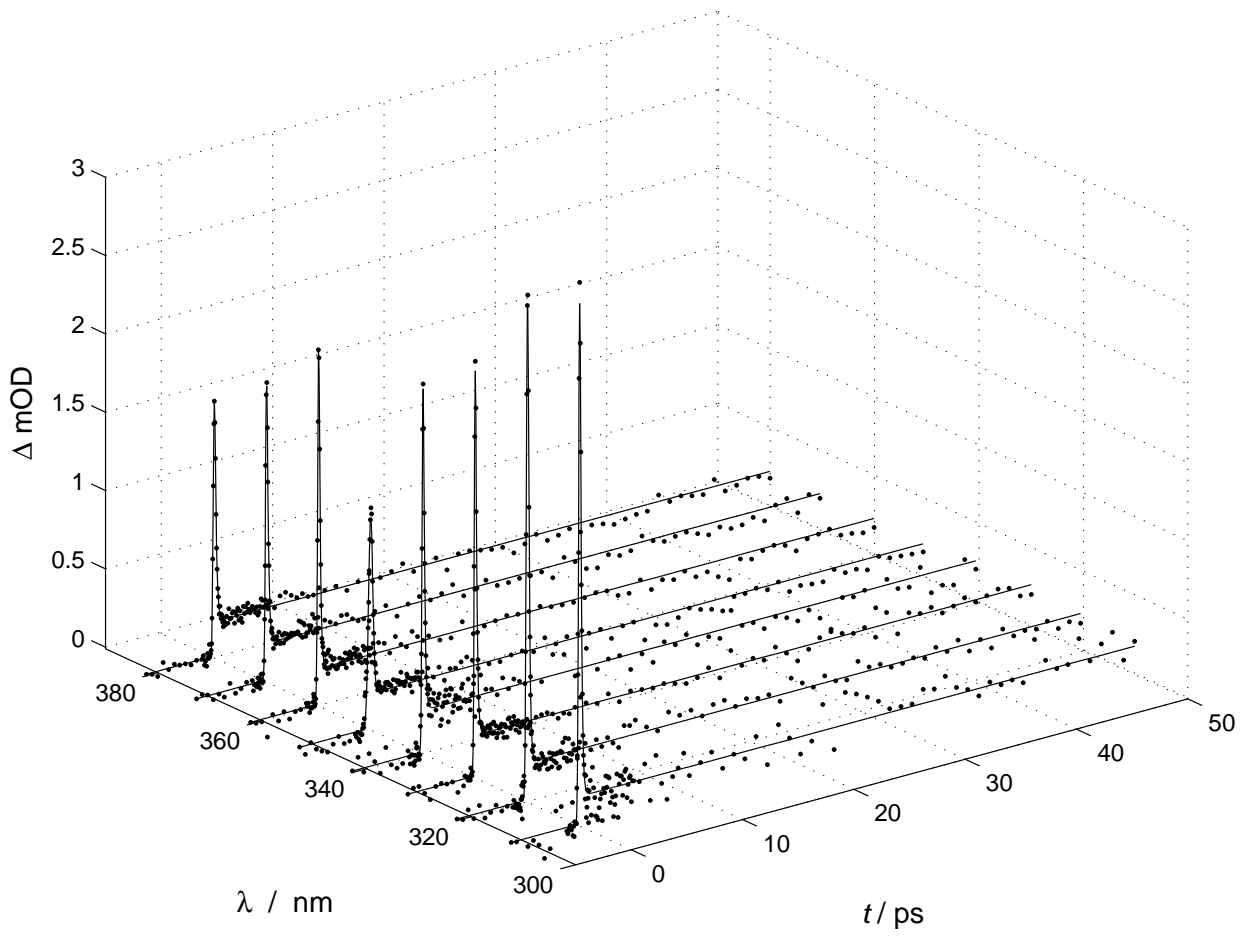

Abbildung 5.36: Transiente Absorption von $\mathrm{CH}_{3} \mathrm{I}$ in Methylcyclohexan bei $255 \mathrm{~nm}$ Anregung und UV-Abfrage 


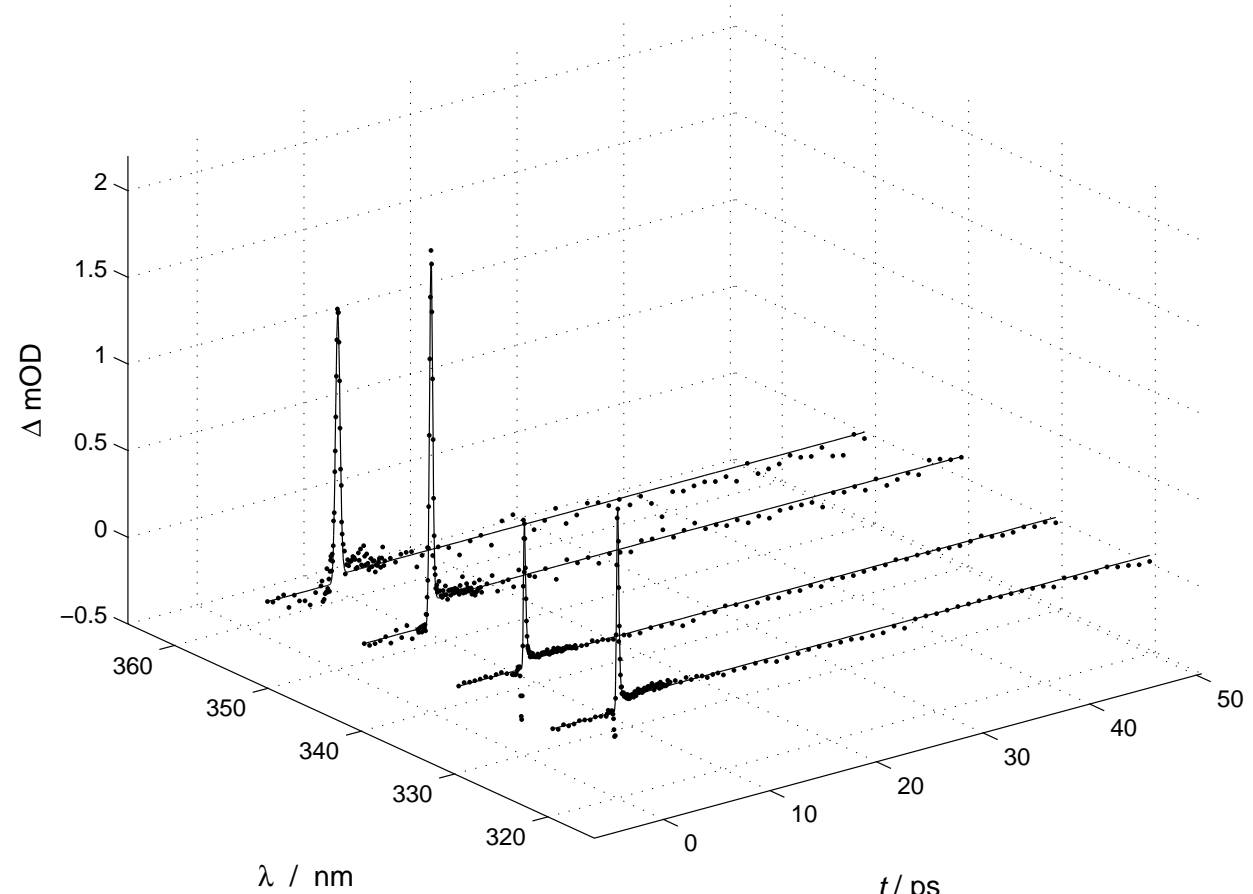

Abbildung 5.37: Transiente Absorption von $\mathrm{CH}_{3} \mathrm{I}$ in Acetonitril bei $265 \mathrm{~nm}$ Anregung und UV-Abfrage
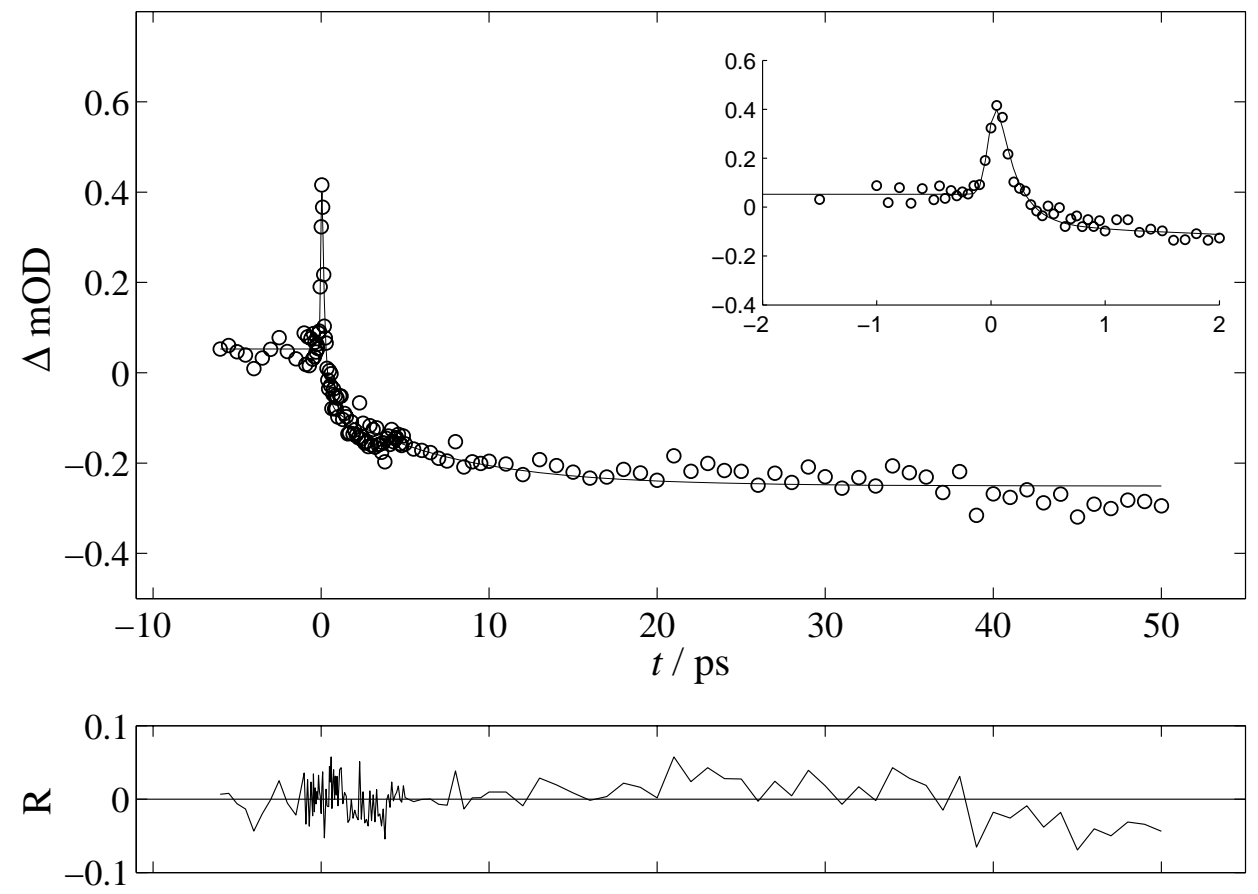

Abbildung 5.38: Absorptionszeitprofil von $\mathrm{CH}_{3} \mathrm{I}$ in $n$-Perfluorhexan bei $265 \mathrm{~nm}$ Anregung und $330 \mathrm{~nm}$ Abfrage 


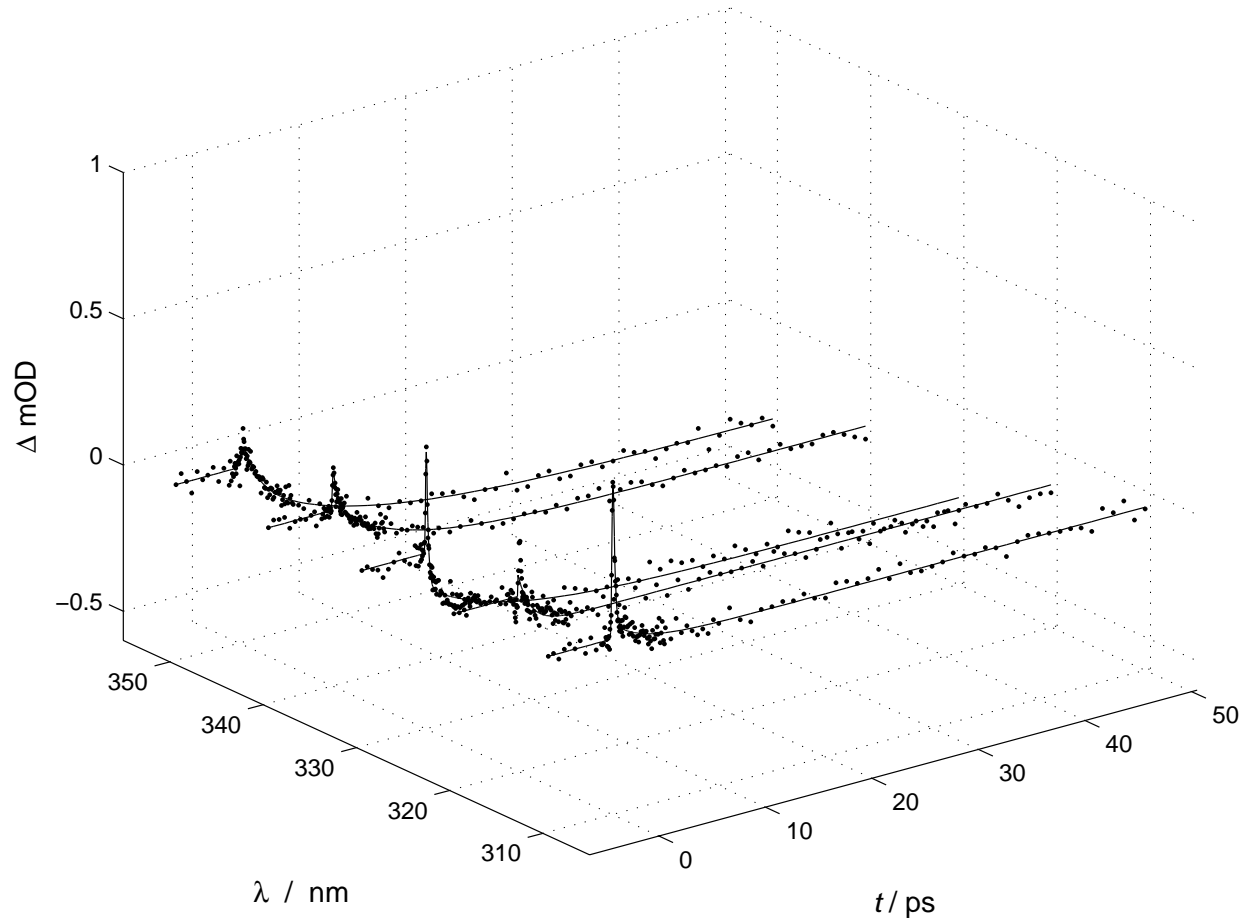

Abbildung 5.39: Transiente Absorption von $\mathrm{CH}_{3} \mathrm{I}$ in $n$-Perfluorhexan bei $265 \mathrm{~nm}$ Anregung und UV-Abfrage

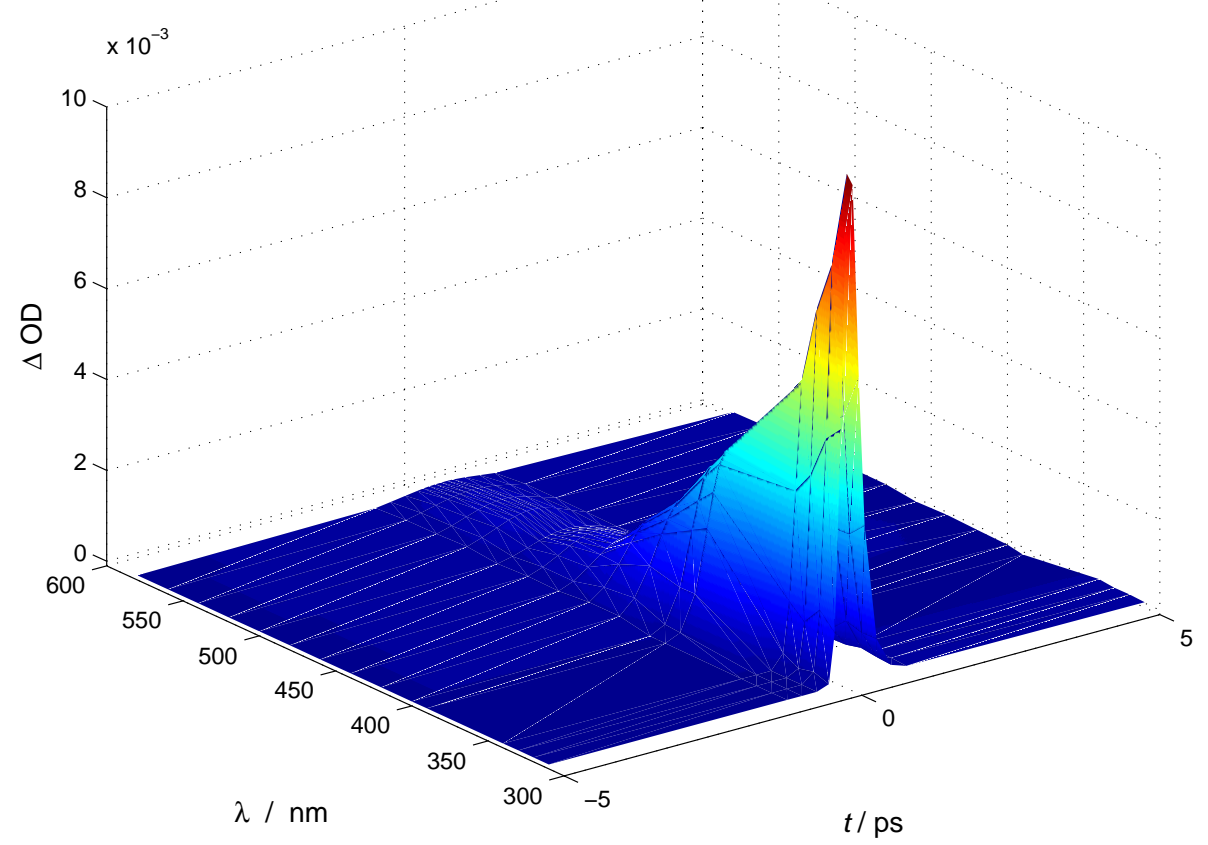

Abbildung 5.40: Lösungsmittelsignal vom Methanol bei $260 \mathrm{~nm}$ Anregung 


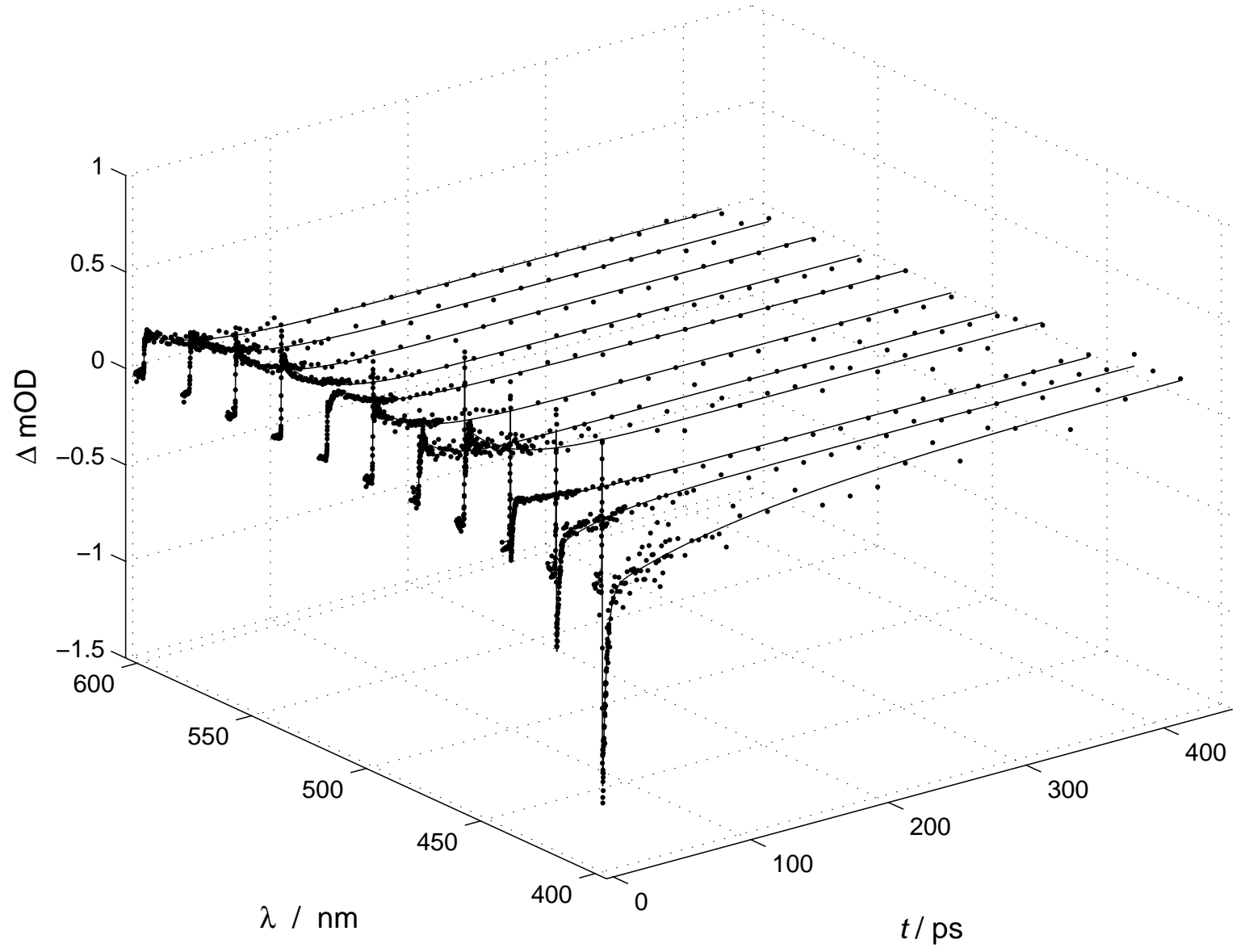

Abbildung 5.41: Transiente Absorption von $\mathrm{CH}_{3} \mathrm{I}$ in Methanol bei $255 \mathrm{~nm}$ Anregung und VIS-Abfrage

\subsection{3 fs-UV-Pump/VIS-Probemessungen in Lösung}

Weiterhin wurden Messungen mit sichtbaren Probestrahl durchgeführt. Dazu wurden der TOPAS auf sichtbare Wellenlängen umgestellt und die Spiegel des Probestrahls gegen hochreflektierende Ag-Spiegel ausgetauscht. Außerdem wurde der Strahlteiler, welcher den Probestrahl in einen Referenz- und den eigentlichen Probestrahl aufspaltet, gegen einen für VIS-Wellenlängen geeigneten ausgetauscht. Da die Intensität des Probestrahls im sichtbaren Bereich viel höher als im UV-Bereich ist, mußte der Probestrahl vor Detektion durch Graufilter abgeschwächt werden, um eine Sättigung der Photodioden zu vermeiden. Ansonsten ist die Versuchsdurchführung analog zu den Messungen im UVBereich mit einer Probenpräparation, wie in Abschnitt 4.3 beschrieben.

Die Absorptionszeitprofile im VIS-Bereich (380-600 nm) sind zu denen im UV-Bereich sehr verschieden. Die transiente Absorption zeigt unterschiedliche Gestalt, in polaren Lösungsmitteln wie Methanol (s. Abbildung 5.41) oder Acetonitril (s. Abbildung 5.42) wird zudem ein negatives Signal anstelle einer Absorption bei Wellenlängen um $400 \mathrm{~nm}$ gemessen. In Methylcyclohexan (s. Abbildung 5.43) fehlt dieses negatives Signal. 


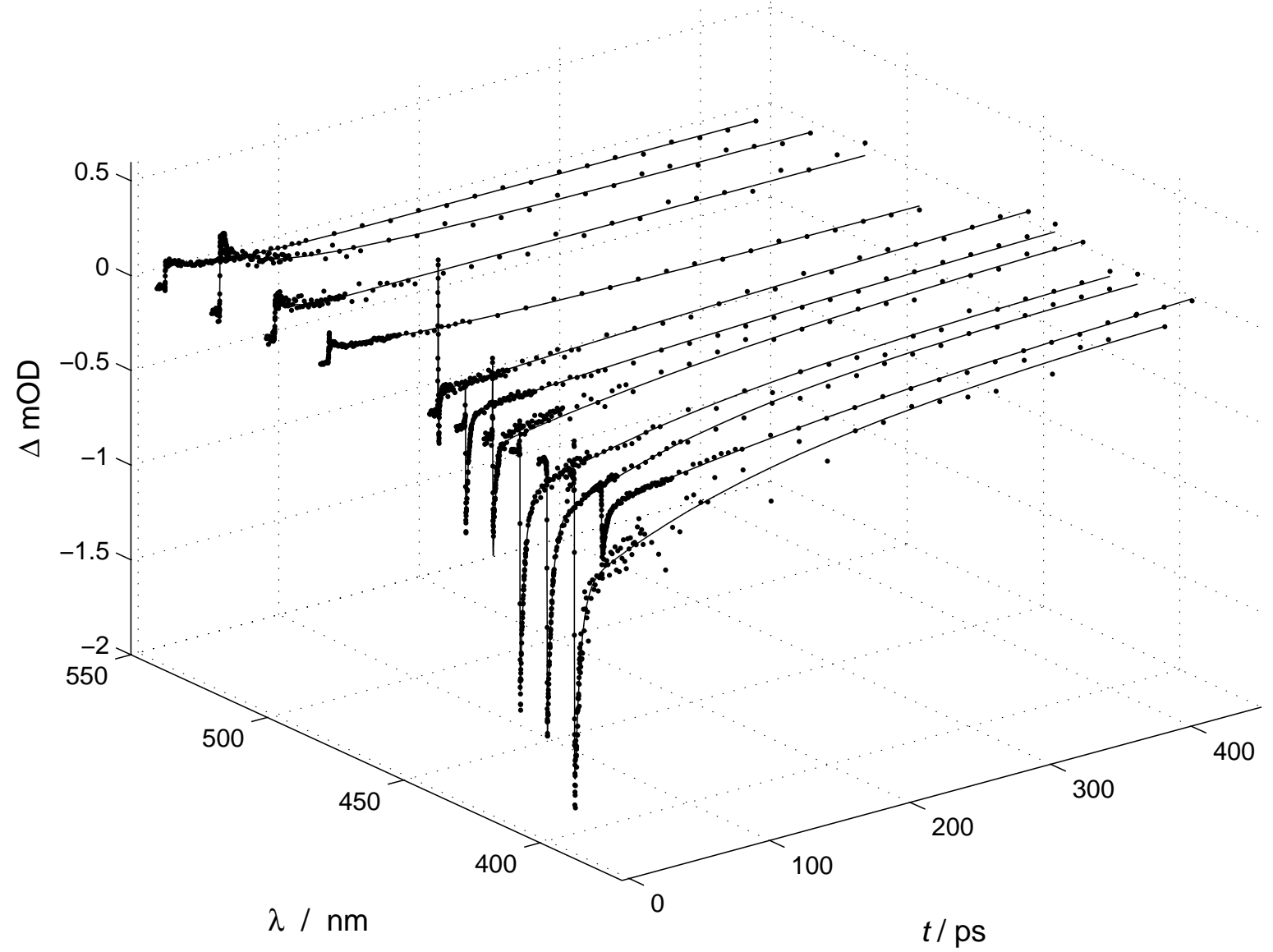

Abbildung 5.42: Transiente Absorption von $\mathrm{CH}_{3} \mathrm{I}$ in Acetonitril bei $265 \mathrm{~nm}$ Anregung und VIS-Abfrage

Desweiteren fällt die Absorption in Methylcyclohexan im Laufe der Reaktionszeit wieder komplett auf Null ab, während in Methanol und Acetonitril nach einem monoexponentiellen Abfall stets ein konstanter off-set stehen bleibt.

\subsection{4 fs-UV-Pump/IR-Probemessungen in Lösung}

Mit Hilfe des IR-Lasersystems (s. Abschnitt 4.2) wurden zeitaufgelöste Spektren von $\mathrm{CH}_{3} \mathrm{I}$ in $\mathrm{CD}_{3} \mathrm{CN}$ als Lösungsmittel aufgenommen. Zur Probenpräparation wurden wenige Tropfen $\mathrm{CH}_{3} \mathrm{I}$ in $6 \mathrm{ml} \mathrm{CD} \mathrm{CD}_{3} \mathrm{CN}$ gelöst und in den Vorratsbehälter der Durchflußpumpe gegeben. Die Meßzelle, welche über Schlauchverbindungen mit der Durchflußpumpe verbunden ist, besteht aus aus zwei $\mathrm{CaF}_{2}$-Fenstern $(\varnothing 10 \mathrm{~mm}, 2 \mathrm{~mm}$ Stärke), welche mit einer Viton-Dichtung über zwei Messingzylinder in eine Stahlfassung eingefaßt sind. Die optische Weglänge zwischen den Fenstern ist einstellbar, üblicherweise wurde ein Wert um 0,6 mm gewählt. Ein genaue Beschreibung der verwendeten Meßzelle findet sich in der Dissertation von C. Reichardt [188]. Die Fließgeschwindigkeit wurde auf etwa $7 \mathrm{ml}$ pro Minute eingestellt. 


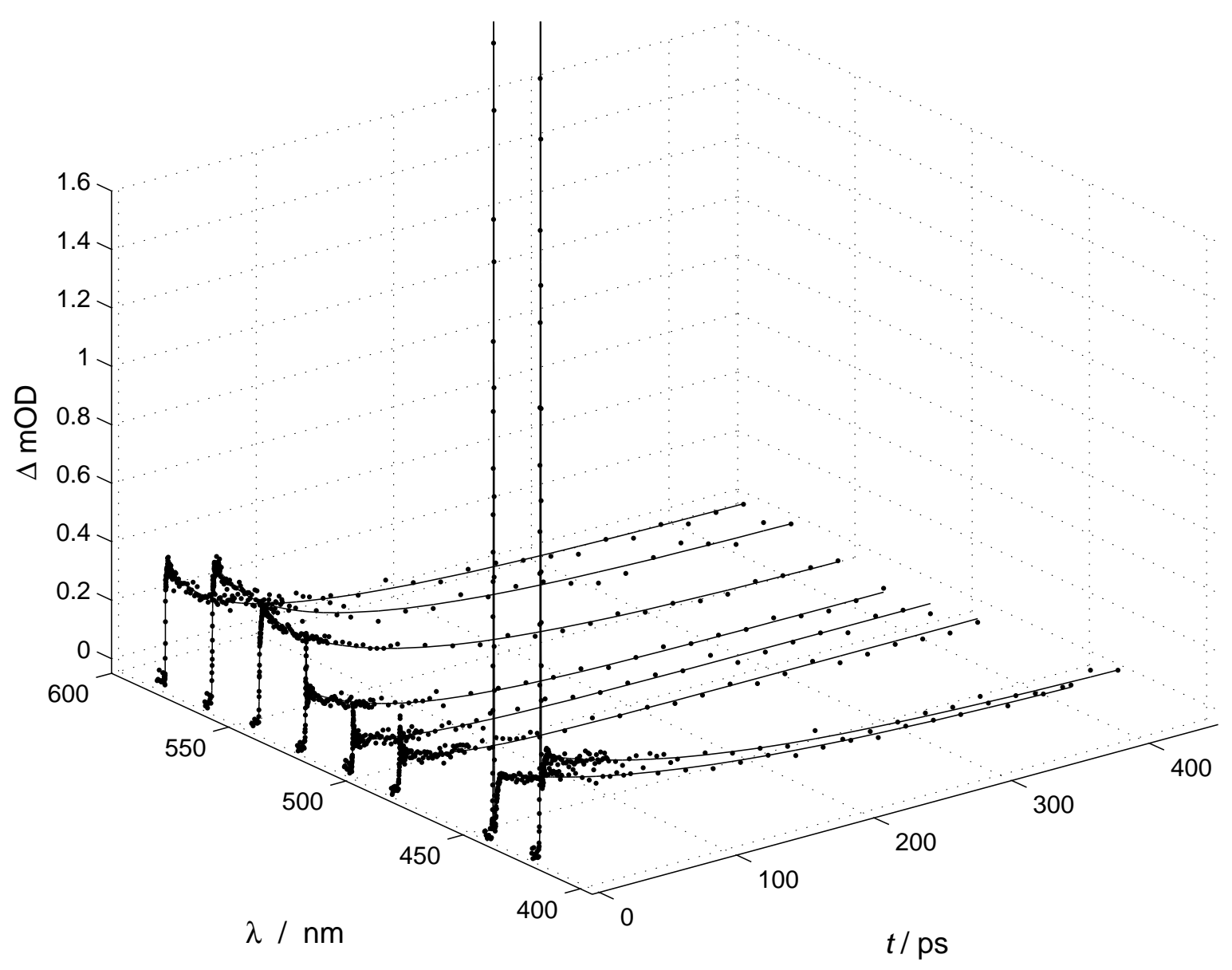

Abbildung 5.43: Transiente Absorption von $\mathrm{CH}_{3} \mathrm{I}$ in Methylcyclohexan bei $265 \mathrm{~nm}$ Anregung und VIS-Abfrage

Eine Messung bei $266 \mathrm{~nm}$ Anregung und einem IR-Probepuls zentriert bei $1250 \mathrm{~cm}^{-1}$ ist in Abbildung 5.44 dargestellt, die Pulsenergie des Pumppulses betrug dabei $E_{\text {Puls }}$ $=17 \mu \mathrm{W} . \mathrm{Zu}$ frühen Reaktionszeiten $(<3 \mathrm{ps})$ ist das Signal vom einem starken nichtlinearen Lösungsmittelsignal überlagert. Zur Vermeidung des Lösungsmittelsignals und für ein besseres Signal/Rausch-Verhältnis wurden die Messungen mit weniger Pumpleistung (Pulsenergie $E_{\text {Puls }}=5,5 \mu \mathrm{W}$ ) wiederholt (s. Abbildung 5.45). Dabei konnte das nicht-lineare Lösungsmittelsignal zu Beginn verringert werden, allerdings auf Kosten der Signalintensität.

Dabei zeigt sich bei beiden Messungen (s. Abbildung 5.44 und 5.45) ein deutliches Ausbleichen der Bande bei $1250 \mathrm{~cm}^{-1}$ innerhalb der Zeitauflösung des Experiments. Anschließend kann ein leichter Anstieg des negativen Signals innerhalb von etwa 40 ps beobachtet werden. 


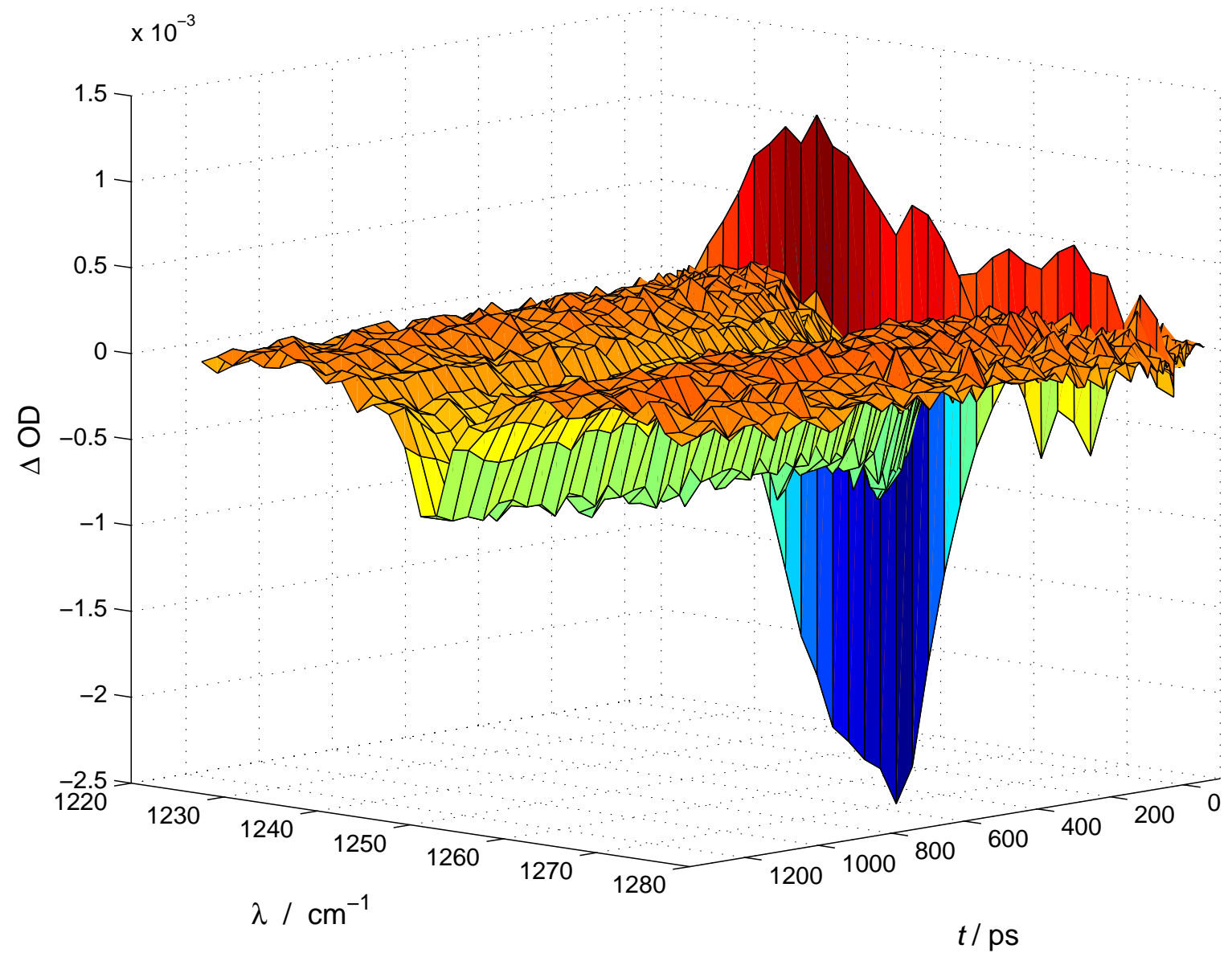

Abbildung 5.44: Zeitaufgelöstes IR-Spektrum von $\mathrm{CH}_{3} \mathrm{I}$ in $\mathrm{CD}_{3} \mathrm{CN}$ nach $266 \mathrm{~nm}$ Anregung $\left(E_{\mathrm{Puls}}=17 \mu \mathrm{W}\right)$ im Bereich von $1220-1280 \mathrm{~cm}^{-1}$ 


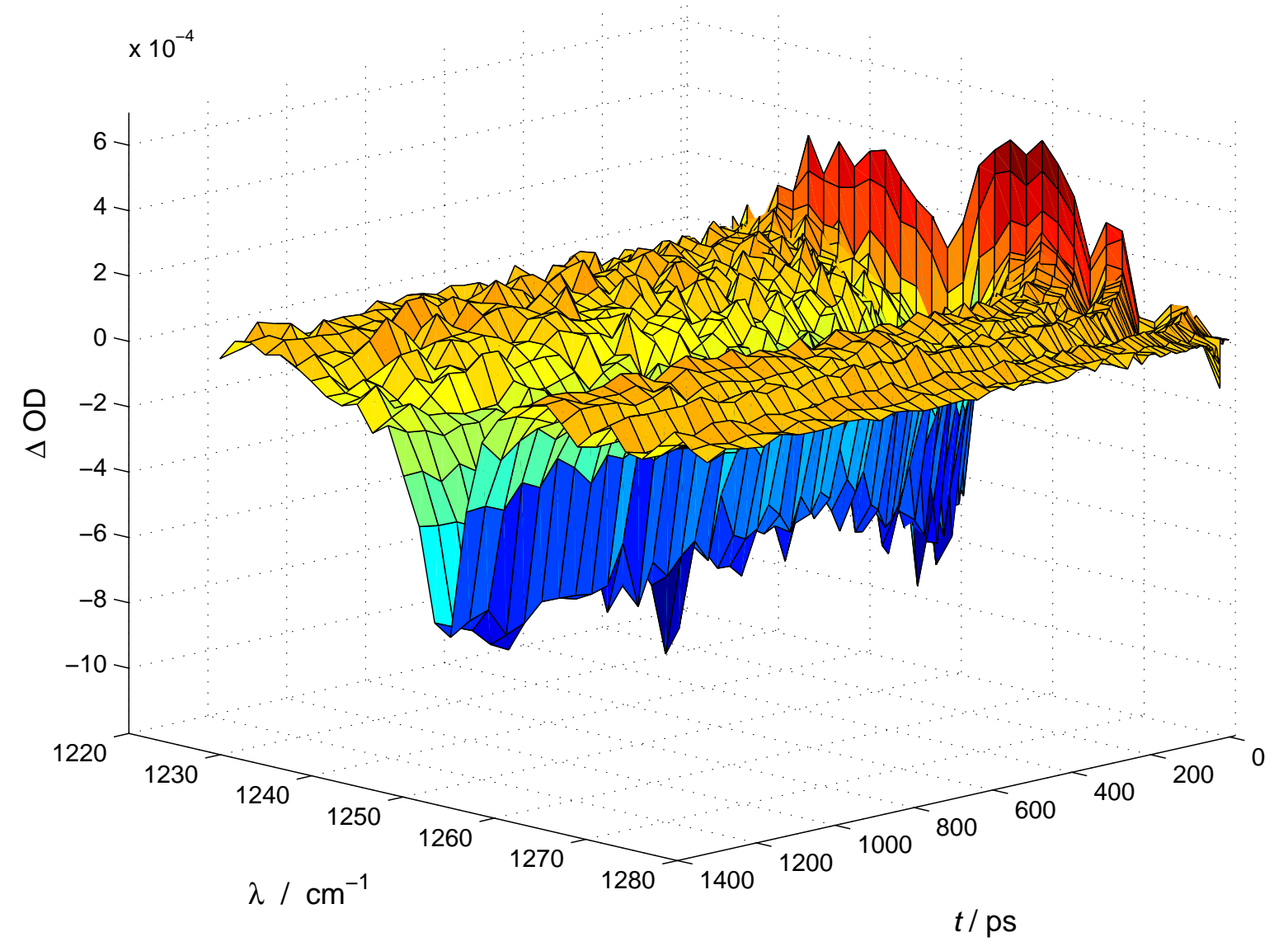

Abbildung 5.45: Zeitaufgelöstes IR-Spektrum von $\mathrm{CH}_{3} \mathrm{I}$ in $\mathrm{CD}_{3} \mathrm{CN}$ nach $266 \mathrm{~nm}$ Anregung $\left(E_{\mathrm{Puls}}=5,5 \mu \mathrm{W}\right)$ im Bereich von $1220-1280 \mathrm{~cm}^{-1}$ 


\section{Diskussion}

\subsection{Ultrakurzzeitdynamik der Photoisomerisierung von Polyhalogenmethanen}

Die Ultrakurzzeitdynamik der Polyhalogenmethane $\mathrm{CH}_{2} \mathrm{I}_{2}, \mathrm{CH}_{2} \mathrm{BrI}, \mathrm{CHI}_{3}$ und $\mathrm{CH}_{2} \mathrm{ClI}$ nach Photoanregung in die erste Absorptionsbande ist durch die Dissoziation in ein $\mathrm{CH}_{2} \mathrm{X}$ - bzw. $\mathrm{CHI}_{2}$-Radikal und ein Iodatom geprägt (s. Abschnitt 3.2). Ein typisches Absorptionszeitprofil wie z. B. von $\mathrm{CHI}_{3}$ in sc. $\mathrm{CO}_{2}$ nach $295 \mathrm{~nm}$ Anregung (s. Abbildung 6.1) zeigt zwei schnelle Zeitkomponenten auf einer Femtosekundenzeitskala und einen langsamen Anstieg auf einer Pikosekundenzeitskala. Der langsame Anstieg kann dabei der Bildung des entsprechenden Isomers zugeordnet werden.

Der erste Anstieg erfolgt innerhalb der Zeitauflösung des Experiments (um $200 \mathrm{fs}$ ) und korrespondiert mit der elektronischen Anregung des Polyhalogenmethans. Dabei wird für alle untersuchten Moleküle im dissoziativen $S_{1}$-Zustand ein Wellenpaket generiert, der Probepuls detektiert dabei die transiente Absorption in einen $S_{n}$-Zustand des Moleküls. Danach läuft das Wellenpaket aus der ursprünglich angeregten Franck-Condon-Region des repulsiven Potentials des $S_{1}$-Zustands heraus, der Franck-Condon-Faktor für die transiente Absorption in einen $S_{n}$-Zustand nimmt ab und folglich auch die Absorption, was für den schnellen Abfall ( $200 \mathrm{fs}$ ) des Absorptionszeitprofils verantwortlich ist. Die Dissoziation ist somit nach weniger als 200 fs abgeschlossen und es liegen die primären Photofragmente innerhalb des Käfigs vor. Folgende Reaktionsschritte laufen demnach ab (Y $=\mathrm{H}, \mathrm{I}$ und $\mathrm{X}=\mathrm{I}, \mathrm{Cl}, \mathrm{Br})$ :

$$
\mathrm{CHYXI} \underset{\tau_{\mathrm{cc}}}{\stackrel{\mathrm{h} \nu}{\longrightarrow}} \mathrm{CHYXI}^{*} \underset{\tau_{\text {diss }}}{\longrightarrow}[\mathrm{CHYX}+\mathrm{I}]
$$

Anhand von Abbildung 6.1 ist jedoch ersichtlich, daß die Absorption nach der Dissoziation nicht komplett auf Null abfällt, sondern im Ultrakurzzeitbereich (um 2 ps) stets eine signifikante Absorption vorhanden ist. Eine kinetische Analyse zeigt, daß dieser Absorptionsverlauf nicht alleine durch den schnellen Abfall und nachfolgenden langsamen Anstieg hervorgerufen werden kann. Extrapolatiert man den langsamen Anstieg Richtung Zeitnullpunkt, wird deutlich, daß das lokale Minimum, welches durch Anstieg und Abfall entsteht, viel höher liegt, als durch Kombination der beiden Exponentialfunktionen zu erwarten wäre. Somit muß zu frühen Zeiten ein deutlicher Anteil zur Absorption durch eine 

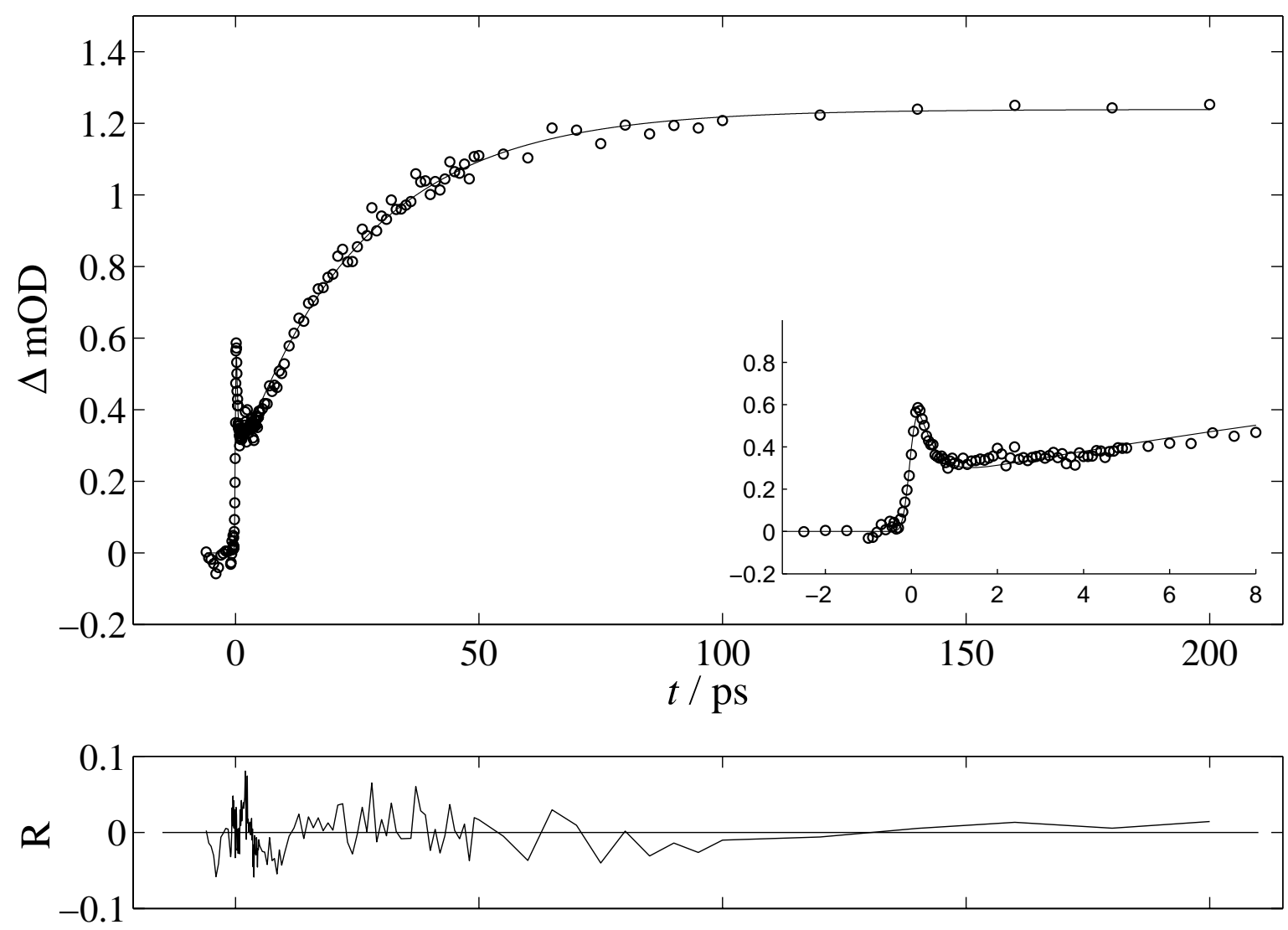

Abbildung 6.1: Absorptionszeitprofil von $\mathrm{CHI}_{3}$ in sc. $\mathrm{CO}_{2}\left(35^{\circ} \mathrm{C} 204\right.$ bar $)$ nach $295 \mathrm{~nm}$ Anregung und Abfrage bei $460 \mathrm{~nm}$

weitere Spezies beigetragen werden. Dabei ist diese Absorption eines Zwischenzustands für jedes untersuchte Polyhalogenmethan bei Probewellenlängen im Bereich von 350$750 \mathrm{~nm}$ beobachtbar. Für diese Kurzzeitabsorption zu frühen Zeiten (um 2 ps) werden in der Literatur unterschiedliche Spezies verantwortlich gemacht.

\subsubsection{Beteiligte Spezies im Ultrakurzzeitbereich}

Denkbare Spezies im Ultrakurzzeitbereich nach Photodissoziation der Polyhalogenmethane sind:

- Schnell gebildetes Isomer $\left(\tau_{\text {iso }}<1 \mathrm{ps}\right)$

- Geminal rekombiniertes Muttermolekül

- Schwingungsangeregtes $\mathrm{CH}_{2} \mathrm{X}$ bzw. $\mathrm{CHI}_{2}$-Radikal

- Ladungstransferkomplex der primären Photoprodukte 
Tarnovsky etal. postulieren [158, daß die Bildung des Isomers auf zwei unterschiedlichen Zeitskalen stattfindet. Neben der Isomerbildung auf der Pikosekundenzeitskala, was mit dem langsamen Anstieg verknüpft wird, soll sich ein Teil des Isomers sehr schnell innerhalb einer Pikosekunde bilden. Dieses aufgrund der Überschußenergie der primären Photofragmente hoch schwingungsangeregte Isomer soll für die Absorption im Ultrakurzzeitbereich verantwortlich sein und relaxiert anschließend nur noch. Gegen diese schnelle Isomerbildung spricht jedoch die große Überschußenergie, welche die primären Photofragmente nach der Dissoziation besitzen. Im Falle des $\mathrm{CH}_{2} \mathrm{I}_{2}$ nach $304 \mathrm{~nm}$ Anregung beträgt die Überschußenergie $15800 \mathrm{~cm}^{-1}$, davon sind etwa $20 \mathrm{v}$. H. als kinetische Energie und der restliche Anteil in den inneren Freiheitsgraden des $\mathrm{CH}_{2}$ I-Radikals (Rotation und Schwingung) gespeichert [102, 144]. Die Dissoziationsenthalpie des $\mathrm{CH}_{2} \mathrm{I}-\mathrm{I}$-Isomers wurde zu etwa $1600 \mathrm{~cm}^{-1}$ von Glukhovtsev und Bach 153 berechnet. Somit übersteigt die Überschußenergie des $\mathrm{CH}_{2}$ I-Radikals mit etwa $12500 \mathrm{~cm}^{-1}$ die Dissoziationsenthalpie nahezu um den Faktor 10. Es erscheint sehr unwahrscheinlich, daß es möglich ist, ein $\mathrm{CH}_{2} \mathrm{I}$-I-Isomer innerhalb einer Pikosekunde zu bilden und so zu stabilisieren, daß es nicht zu einer sofortigen Dissoziation kommt. Zwar sind für die anderen Polyhalogenmethane keine detaillierten Untersuchungen zur Überschußenergie nach Dissoziation und Dissoziationsenthalpie des entsprechenden Isomers bekannt, allerdings ist anzunehmen, daß es sich um vergleichbare Werte handelt.

Harris et al. ordnen den schnellen Abfall der Absorption einer schnellen, geminalen Rekombination zu [151. Somit könnte es sich bei der Kurzzeitabsorption auch um transiente Absorption des Muttermoleküls handeln. Allerdings sprechen ähnliche Argumente gegen einen signifikanten Anteil an geminal rekombiniertem Muttermolekül. Die Dissoziationsenthalpie des $\mathrm{CH}_{2} \mathrm{I}_{2}$ ist größer als die seines Isomers, jedoch wird die Überschußenergie der primären Photofragmente kurz nach der Dissoziation zu hoch sein, um das Rekombinationsprodukt innerhalb des Käfigs zu stabilisieren. Desweiteren können schwingungsheiße geminal rekombinierte Polyhalogenmethane nicht für die Kurzzeitabsorption bei Probewellenlängen > $400 \mathrm{~nm}$ verantwortlich gemacht werden, da sie bei Wellenlängen > $350 \mathrm{~nm}$ keine Absorptionsbanden besitzen (s. Abbildung 3.2, 3.3, 3.4 und 3.5).

Eine schnelle Isomerbildung wie von Tarnovsky et al. postuliert sowie die schnelle geminale Rekombination können somit ausgeschlossen werden, für die signifikante Absorption im Kurzzeitbereich muß demnach eine andere Spezies verantwortlich sein. Diese Spezies muß zudem ein extrem breitbandiges Absorptionsspektrum besitzen, da die Kurzzeitabsorption bei Probewellenlängen von 350-750 nm gemessen wurde. Dadurch ist ein Beitrag durch die primären Photofragmente wie $\mathrm{CH}_{2} \mathrm{X}$ bzw. $\mathrm{CHI}_{2}$-Radikal ebenfalls sehr unwahrscheinlich, da deren Absorptionsspektren (s. Abbildung 3.9p ausnahmslos im UV-Bereich liegen. Aufgrund der hohen Schwingungsanregung der Radikale kann ihr Absorptionsspektrum allerdings wesentlich breiter als das des „kalten“ Radikals sein. Transiente Absorptionsmessungen des schwingungsheißen Radikals nach Photolyse von $\mathrm{CH}_{2} \mathrm{I}_{2}$ in der Gasphase von Lenzer et al. [189] zeigen eine breite Bande von 330-400 nm. Eine Abschätzung des „heißen" $\mathrm{CH}_{2} \mathrm{I}$-Absorptionsspektrums von Zerbs auf der Grundlage eines modifizierten Sulzer-Wieland-Modells zeigt ebenfalls, daß die spektrale Verbreiterung auch von hoch schwingungsangeregtem $\mathrm{CH}_{2} \mathrm{I}$ zu gering ist, um die Kurzzeitabsorption bei Wellenlängen $>400 \mathrm{~nm}$ zu erklären [86]. 


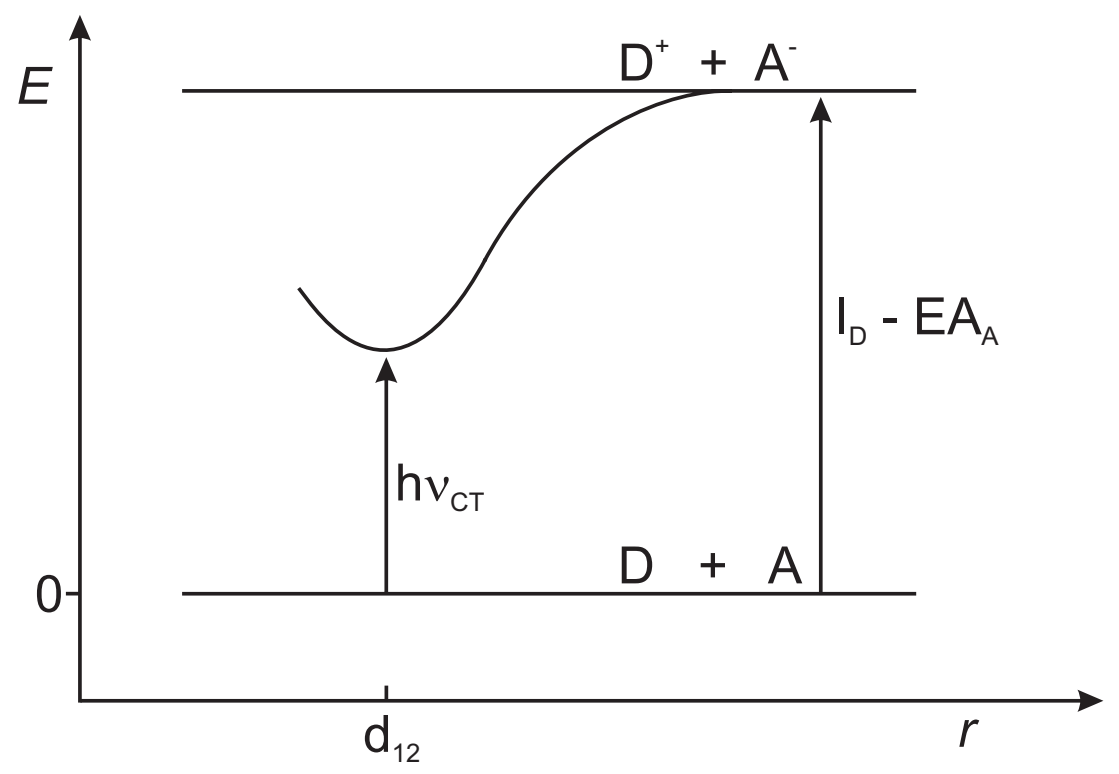

Abbildung 6.2: Potentialdiagramm und CT-Übergang eines Donor-Akzeptor-Komplexes

\subsubsection{Bildung von Ladungstransferkomplexen}

Die wahrscheinlichste Spezies für die Kurzzeitabsorption ist wohl die schnelle Bildung eines Ladungstransferkomplexes (engl. charge-transfer complex, CT-Komplex) aus Iodatom und $\mathrm{CH}_{2} \mathrm{X}$ bzw. $\mathrm{CHI}_{2}$-Radikal.

Solche CT-Komplexe bestehen aus einem Donor (in diesem Fall das Radikal) und einem Akzeptor (Iodatom). Ihre Bildung und die entsprechenden spektralen Eigenschaften sind ausführlich in der Literatur beschrieben, beispielsweise in verschiedenen Übersichtsartikeln von Tamres [190, 191].

In Abbildung 6.2 ist die Wechselwirkung zwischen einem neutralen Donor und einem neutralen Akzeptor in einem einfachen Potentialdiagramm dargestellt. Dabei wird nur die Coulombwechselwirkung zwischen Donor und Akzeptor im geladenen Zustand berücksichtigt. Energetische Effekte, welche durch Mischung der beiden Wellenfunktionen $\Psi_{D, A}$ und $\Psi_{D^{+}, A^{-}}$oder Resonanzstabilisierung zustande kommen, bleiben in diesem einfachen Modell unberücksichtigt. Dabei ist $I_{D}$ die vertikale Ionisierungsenergie des Donors und $E A_{A}$ die vertikale Elektronenaffinität des Akzeptors. Der ionische Zustand ist durch das Coulombpotential stabilisiert, welches sich aus der Annäherung der Ionen von unendlicher Separation auf den Gleichgewichtsabstand $d_{12}$ ergibt. Das Maximum des CTAbsorptionsspektrums ergibt sich anhand des CT-Übergangs $h \nu_{\mathrm{CT}}$ und läßt sich demnach nach folgender Formel berechnen:

$$
h \nu_{\mathrm{CT}}=I_{D}-E A_{A}-\frac{1}{4 \pi \epsilon_{0} \epsilon_{r}} \frac{e^{2}}{d_{12}}
$$


Dabei sind die spektralen Eigenschaften der CT-Komplexe nicht auf einen festen Gleichgewichtsabstand $d_{12}$ festgelegt, es handelt sich vielmehr um eine statistische Mittelung über alle möglichen Konfigurationen im thermischen Gleichgewicht [192]. In Lösung kommt neben dem Gleichgewichtsabstand $d_{12}$ also eine Verteilung von verschiedenen Abständen $r$ vor, somit ergibt sich ein breites Absorptionspektrum für den CT-Komplex. Diese CT-Banden sind üblicherweise sehr intensiv mit Extinktionskoeffizienten in der Größenordnung von $10^{4} \mathrm{l} \mathrm{mol}^{-1} \mathrm{~cm}^{-1}$.

Nach der Photodissoziation befinden sich die primären Photofragmente, welche nicht ausgebrochen sind, innerhalb des Lösungsmittelkäfigs. Dem Iodatom als Akzeptor stehen prinzipiell das primäre Radikal und Lösungsmittelmoleküle als Donor zur Verfügung. Die Ionisierungspotentiale möglicher Radikale $\left(\mathrm{CH}_{2} \mathrm{I}, \mathrm{CH}_{2} \mathrm{Br}\right.$ und $\left.\mathrm{CH}_{2} \mathrm{Cl}\right)$ sowie der verwendeten Lösungsmittel sind in Tabelle 6.1 zusammengefaßt. Der Vergleich zeigt dabei, daß die Radikale wesentlich geringere Ionisierungspotentiale besitzen und somit bevorzugt einen CT-Komplex mit dem Iodatom ausbilden. Ein CT-Komplex aus Iodatom und Lösungsmittel (engl. charge-transfer to solvent complex, CTTS-Komplex) spielt demnach nur eine untergeordnete Rolle. Solchen CTTS-Komplexen kommt nur im Falle der Dissoziation von $\mathrm{CH}_{3} \mathrm{I}$ eine besondere Bedeutung zu (s. Abschnitt 6.6.1).

Das spektrale Maximum der CT-Absorption hängt nach Gleichung 6.2 noch vom Gleichgewichtsabstand $d_{12}$ ab. Da keine Messungen oder Rechnungen zu den Gleichgewichtsabständen der beobachteten CT-Komplexe verfügbar waren, wurde eine empirische Formel zur Berechnung von $\lambda_{\max }$ verwendet. Eine entsprechende Abschätzung des CT-Übergangs von I . . D Donor-Komplexen in der Gasphase in Abhängigkeit des Ionisierungspotentials $I_{D}$ des Donors findet sich bei Brosseau et al. [194]:

$$
h \nu_{\mathrm{CT}}=I_{D}-C_{1}+\frac{C_{2}}{I_{D}-C_{1}}
$$

Dabei ist $I_{D}$ das Ionisierungspotential des Donors sowie $C_{1}$ und $C_{2}$ Anpassungsparameter $\left(C_{1}=6,9 \mathrm{eV}\right.$ und $\left.C_{2}=1,2 \mathrm{eV}\right)$. Damit lassen sich die Maxima der CT-Übergänge berechnen, diese sind ebenfalls in Tabelle 6.1 dargestellt. Es zeigt sich, daß die CTKomplexe aus den $\mathrm{CH}_{2} \mathrm{X}$-Radikalen und einem Iodatom ein Absorptionsmaximum $\lambda_{\mathrm{CT}}$ bei etwa $500 \mathrm{~nm}$ besitzen. Die Breite dieser Bande hängt nun von der Verteilung der Donor-Akzeptor-Abstände ab. Diese Abstandsverteilung ist in erster Linie von der Größe

\begin{tabular}{|lcc|}
\hline Molekül/Radikal & $I_{D} / \mathrm{eV}$ & $\lambda_{\mathrm{CT}, \max } / \mathrm{nm}$ \\
\hline $\mathrm{CH}_{2} \mathrm{I}$ & 8,52 & 525 \\
$\mathrm{CH}_{2} \mathrm{Br}$ & 8,61 & 514 \\
$\mathrm{CH}_{2} \mathrm{Cl}$ & 8,75 & 496 \\
$\mathrm{CO}_{2}$ & 13,77 & 176 \\
$\mathrm{CH}_{3} \mathrm{CN}$ & 12,2 & 224 \\
\hline
\end{tabular}

Tabelle 6.1: Ionisierungspotentiale der primären Radikale nach Photodissoziation und der verwendeten Lösungsmittel [193] 


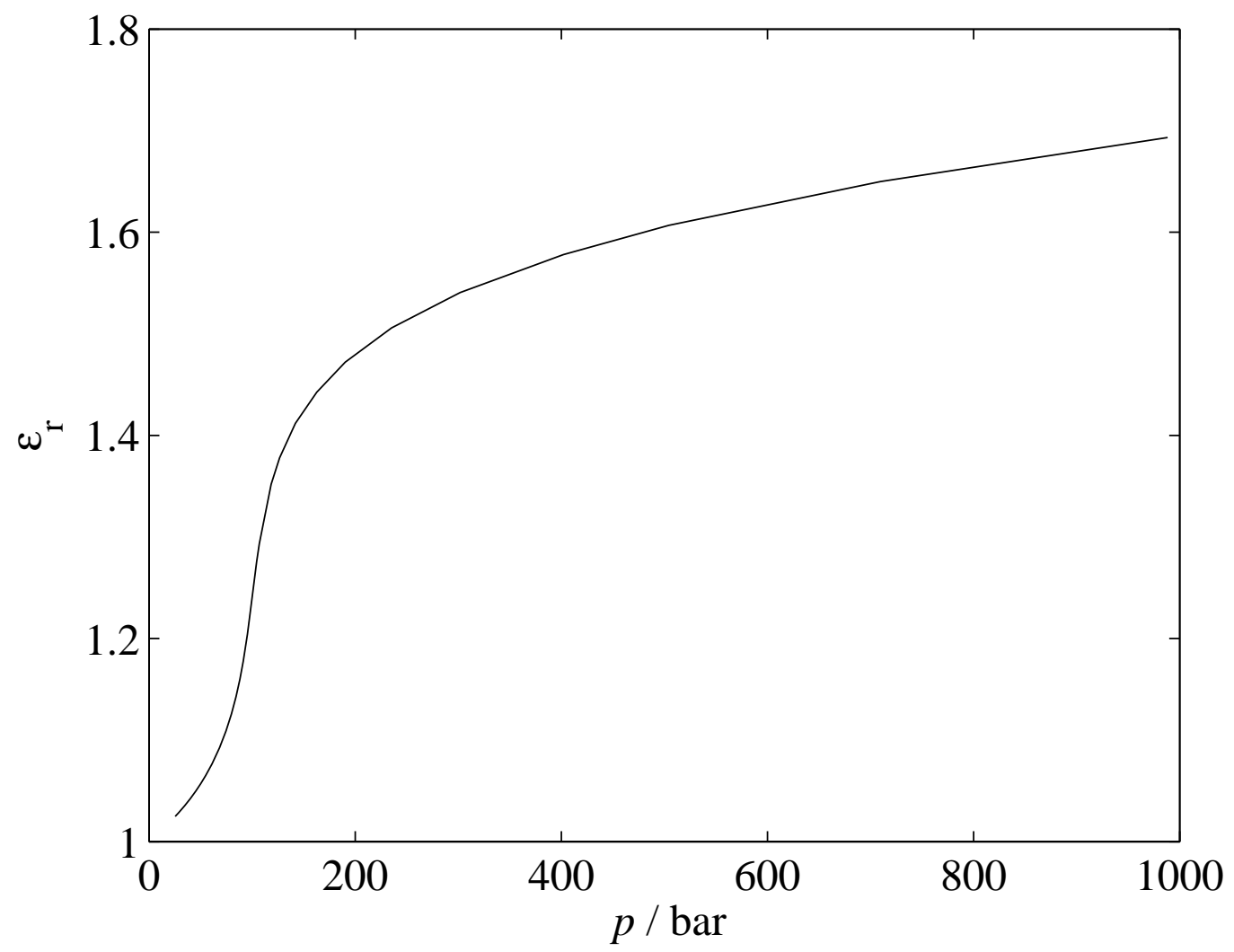

Abbildung 6.3: Druckabhängigkeit der relativen Dielektrizitätszahl $\epsilon_{r}$ von sc. $\mathrm{CO}_{2}$ bei $50{ }^{\circ} \mathrm{C}$ nach Michels und Kleerekoper [195]

des Lösungsmittelkäfigs bestimmt, in welchem die Photofragmente nach der Dissoziation gefangen sind. Die Größe dieser Kavität und somit mögliche Kontaktabstände lassen sich z. B. durch MD-Simulationen abschätzen. Solche Simulationen wurden von Zerbs und Kandratsenka [86] durchgeführt, dabei wurde die radiale Verteilungsdichte von Lösungsmittelmolekülen (z. B. $\mathrm{CO}_{2}$ ) um ein $\mathrm{CH}_{2} \mathrm{I}_{2}$-Molekül berechnet. Unter der Annahme, daß der Käfig in den ersten Pikosekunden nach der Photolyse relativ starr bleibt, sich also das $\mathrm{CH}_{2} \mathrm{X}$-Radikal und das Iodatom in einem Käfig mit konstantem Radius $r$ bewegen, kann man den CT-Übergang für alle möglichen Konfigurationen innerhalb des Lösungsmittelkäfigs berechnen. Dazu wird Gleichung 6.2 mit den in Tabelle 6.1 zusammengefaßten Ionisierungsenergien der Radikale $\mathrm{CH}_{2} \mathrm{I}, \mathrm{CH}_{2} \mathrm{Br}$ und $\mathrm{CH}_{2} \mathrm{Cl}$ verwendet. Die Elektronenaffinität eines Iodatoms beträgt $3,06 \mathrm{eV}$ [193. Die relative Dielektrizitätskonstante von $\mathrm{CO}_{2}$ ist dabei druckabhängig, in Abbildung 6.3 ist die Druckabhängigkeit von $\epsilon_{r}$ in $\mathrm{CO}_{2}$ bei $50^{\circ} \mathrm{C}$ wiedergegeben $[195$. Die Temperatur liegt dabei etwas über der experimentellen Temperatur von $35^{\circ} \mathrm{C}$, allerdings zeigt der Vergleich mit Daten für $\epsilon_{r}$ bei $35^{\circ} \mathrm{C}$ (die in der Literatur leider nur bis 100 bar verfügbar sind) keine große Abweichung. Die Dielektrizitätszahl $\epsilon_{r}$ steigt in sc. $\mathrm{CO}_{2}$ im betrachteten Druckbereich von 100-1000 bar von etwa 1,2 auf 1,7 an. Anhand dieser Daten kann eine Abschätzung zum Absorptionsspektrum des CT-Komplexes innerhalb des Lösungsmittelkäfigs am Beispiel des $\mathrm{CH}_{2} \mathrm{I}_{2}$ in sc. $\mathrm{CO}_{2}$ vorgenommen werden. Dazu werden die CT-Übergänge für verschiedene Abstände $r$ 


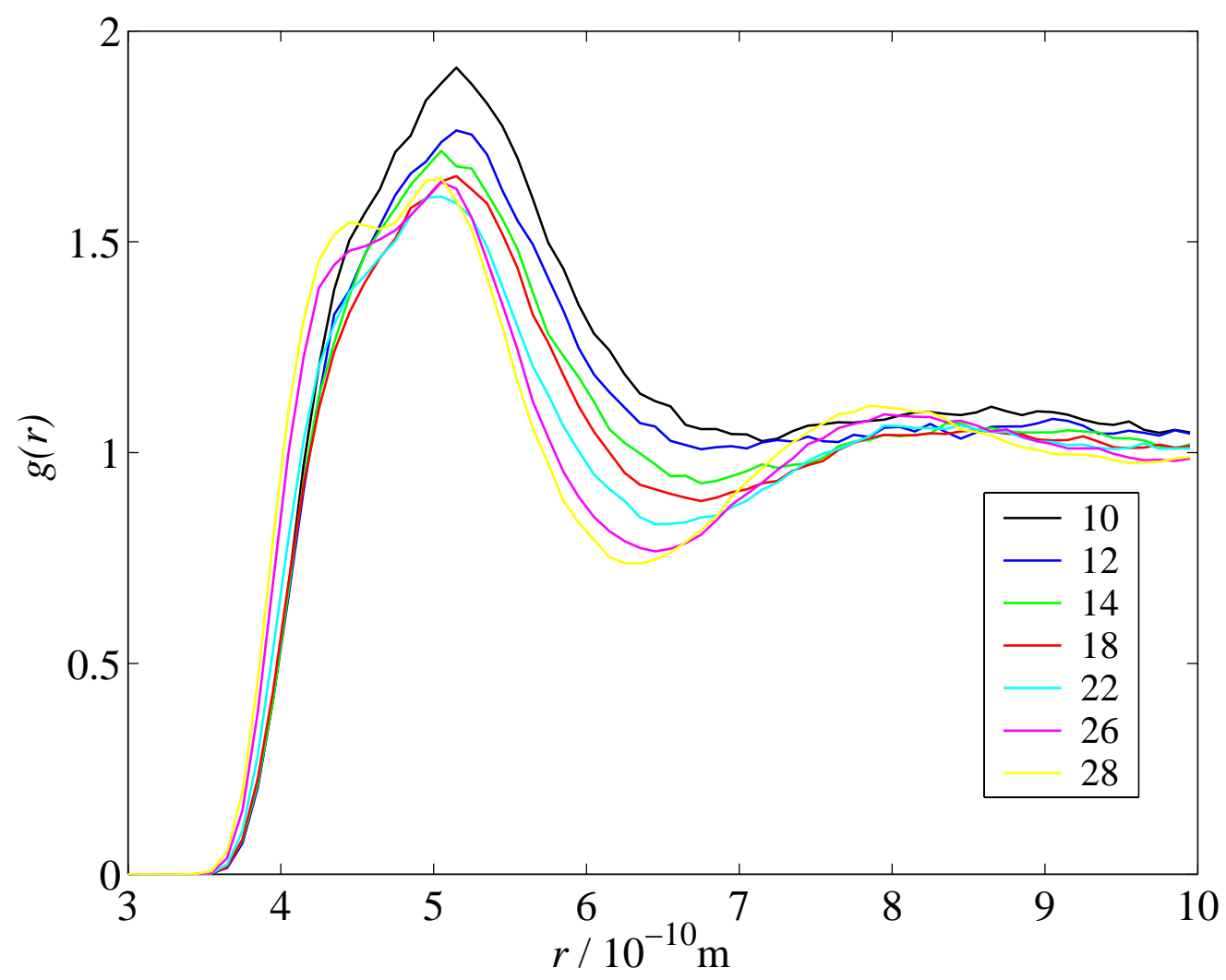

Abbildung 6.4: Radialverteilungsfunktion $g(r) \mathrm{CH}_{2} \mathrm{I}_{2}$ in sc. $\mathrm{CO}_{2}\left(35^{\circ} \mathrm{C}\right)$ bei verschiedenen Dichten (in mol $\mathrm{l}^{-1}$ )

berechnet, die untere Grenze wird durch den Kontaktabstand der beiden Iodatome (mit einem kovalenten Radius $r_{\mathrm{I}}=133 \mathrm{pm}$ ) bestimmt, liegt also bei etwa $2,6 \times 10^{-10} \mathrm{~m}$. Der Maximalabstand wird durch die Größe der Lösungsmittelkavität begrenzt, dazu werden die MD-Simulationen von Zerbs und Kandratsenka [86] herangezogen, in Abbildung 6.4 ist die radiale Verteilungsdichte von $\mathrm{CO}_{2}$ um $\mathrm{CH}_{2} \mathrm{I}_{2}$ bei verschiedenen Dichten dargestellt. Es zeigt sich, daß im Abstand von etwa $5 \times 10^{-10} \mathrm{~m}$ zum gelöstem $\mathrm{CH}_{2} \mathrm{I}_{2}$ die erste Lösungsmittelschale liegt. Unter Annahme einer sphärischen Lösungsmittelkavität beträgt der Durchmesser der Kugel $10 \times 10^{-10} \mathrm{~m}$, davon sind noch die Radien des Iodatoms sowie des $\mathrm{CH}_{2} \mathrm{I}$ abzuziehen. Der maximale Abstand innerhalb des Lösungsmittelkäfigs liegt also bei etwa $8 \times 10^{-10} \mathrm{~m}$. Für die Dielektrizitätszahl $\epsilon_{r}$ wird ein Wert von etwa 1,5 angenommen, was einem Druck von etwa 400 bar entspricht. In Abbildung 6.5 ist aufgrund dieser Daten die Wellenlänge des CT-Übergangs aller möglichen Käfigkonfigurationen des CT-Komplexes im Abstand von 2,5-8 × 10 ${ }^{-10} \mathrm{~m}$ dargestellt.

Dabei zeigt sich eine Absorption im Bereich von 300-750 nm. Über die genaue Abstandsverteilung und somit die Bandenstruktur des Spektrums kann leider keine Angabe gemacht werden. Nach der Photodissoziation besitzen die Fragmente eine Geschwindigkeitsverteilung, aus welcher eine Abstandsverteilung resultiert, die anschließend thermisch äquilibriert. Diese Dynamik kann im Experiment nicht beobachtet werden, jedoch ist erkenntlich, daß für jede Wellenlänge im Probewellenlängenbereich von 350-750 nm 


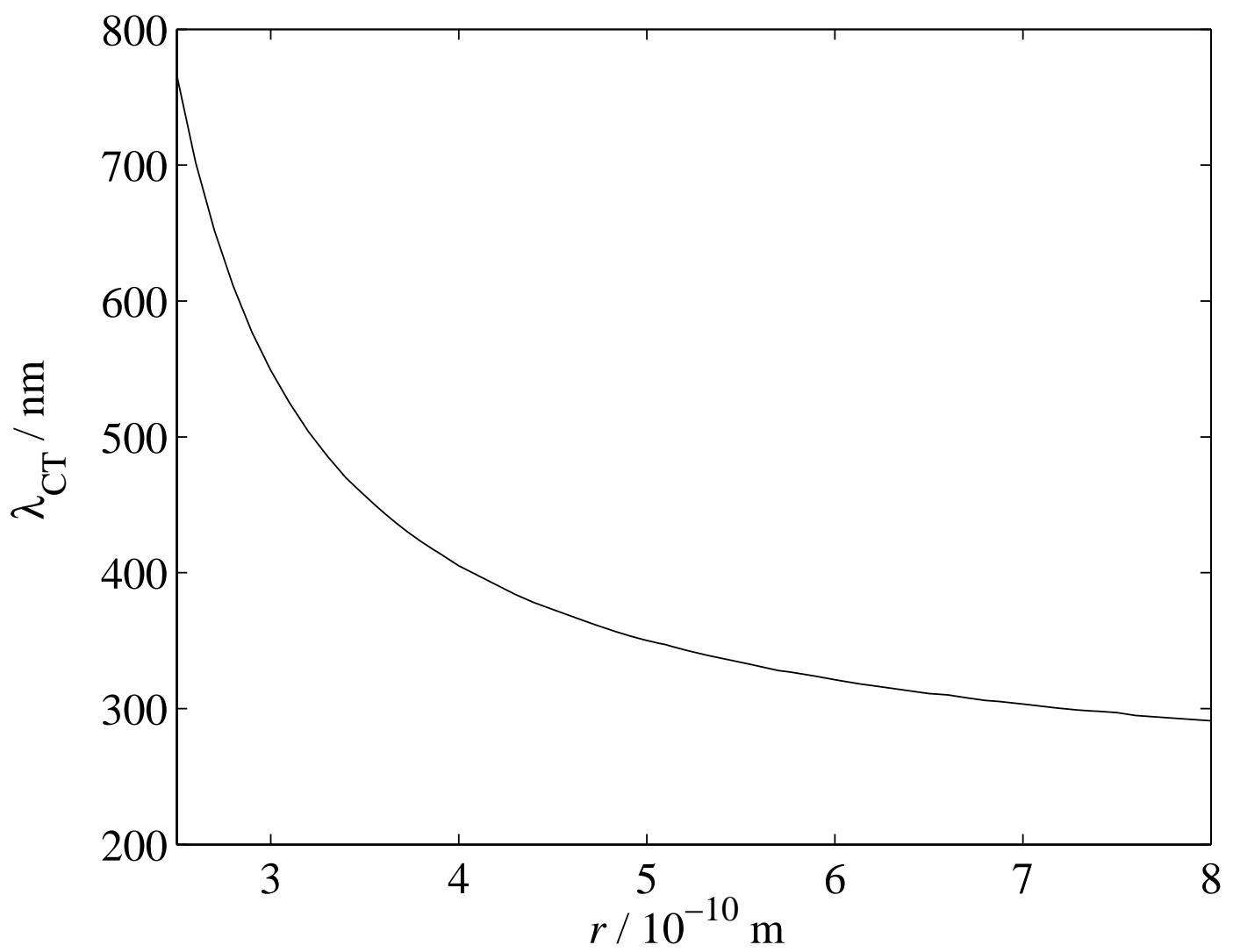

Abbildung 6.5: Abstandsabhängigkeit des CT-Übergangs innerhalb der Lösungsmittelkavität

eine mögliche CT-Konfiguration im Käfig existiert. Die transiente Kurzzeitabsorption kann somit auf die CT-Wechselwirkung der primären Photofragmente im Lösungsmittelkäfig zurückgeführt werden. Nach Ausbruch der Photofragmente bzw. wenn diese durch mindestens ein Lösungsmittelmolekül getrennt werden, findet keine CT-Wechselwirkung mehr statt.

Die Ultrakurzzeitreaktionsdynamik der Photodissoziation von Polyhalogenmethanen in Lösung läßt sich somit folgendermaßen in einem kinetischen Schema zusammen fassen $(\mathrm{Y}=\mathrm{H}, \mathrm{I}$ und $\mathrm{X}=\mathrm{I}, \mathrm{Br}, \mathrm{Cl})$ :

$$
\mathrm{CHYXI} \underset{\tau_{\mathrm{cc}}}{\stackrel{\mathrm{h} \nu}{\longrightarrow}} \mathrm{CHYXI}^{*} \underset{\tau_{\mathrm{diss}}}{\longrightarrow}[\mathrm{CHYX}+\mathrm{I}] \longrightarrow[\mathrm{CHYX} \cdots \mathrm{I}] \quad \text { CT-Komplex }
$$

Die beobachteten Absorptionszeitprofile lassen sich wie folgt interpretieren: Der Anfangspeak setzt sich aus transienter Absorption des elektronisch angeregten Muttermoleküls und anschließender Dissoziation zusammen. Nach dem Zerfall in die primären Photofragmente erfolgt innerhalb des Lösungsmittelkäfigs die schnelle Ausbildung eines CT- 

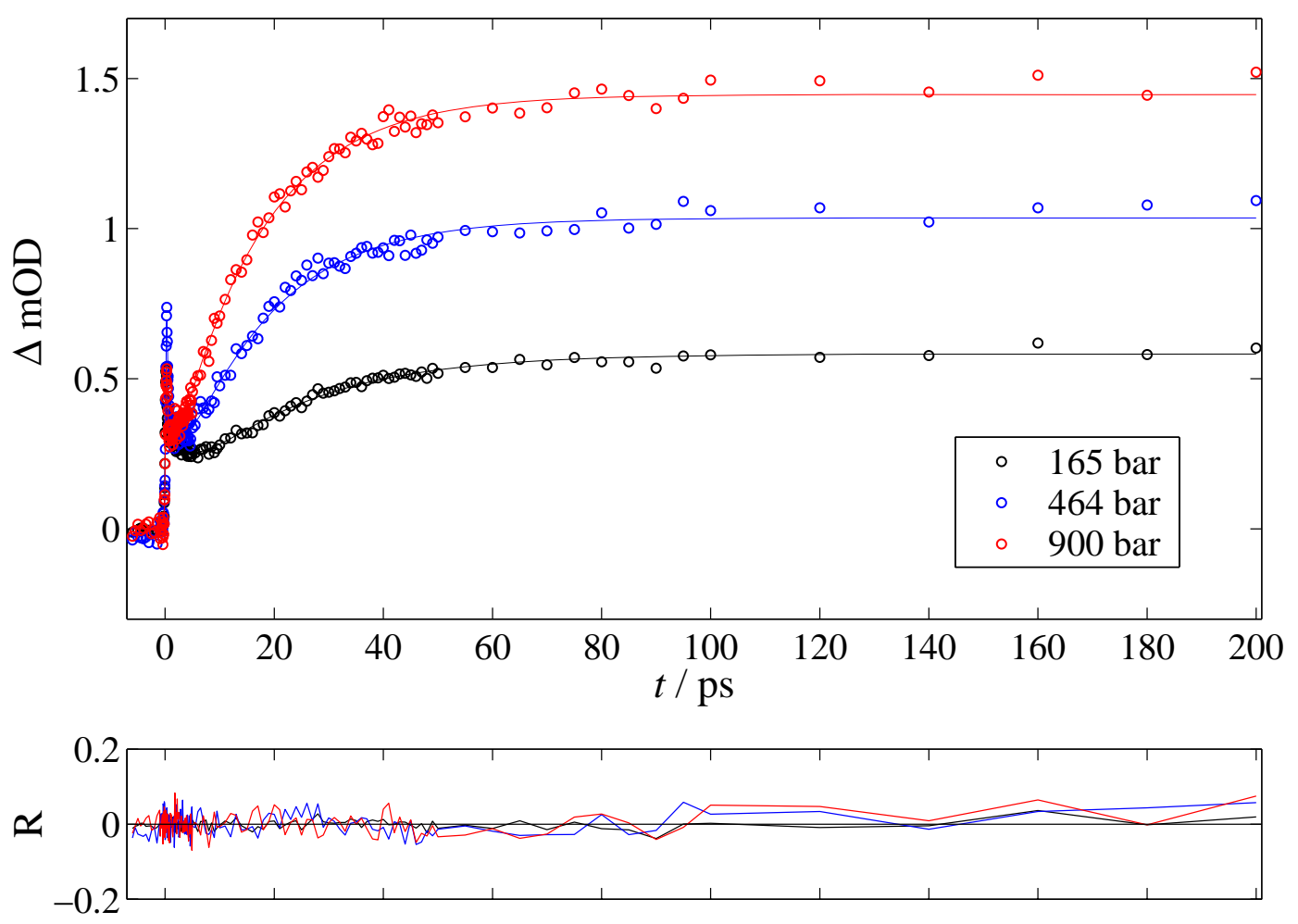

Abbildung 6.6: Druckabhängigkeit der Absorptionszeitprofile von $\mathrm{CHI}_{3}$ in sc. $\mathrm{CO}_{2}\left(35^{\circ} \mathrm{C}\right)$ nach $267 \mathrm{~nm}$ Anregung und $460 \mathrm{~nm}$ Abfrage

Komplexes. Dieser Spezies kann die signifikante Absorption zu frühen Reaktionszeiten (um 2 ps) zugeordnet werden.

\subsection{Isomerisierungsgeschwindigkeit}

Der langsame Anstieg eines Absorptionszeitprofils bei einer für das Isomer resonanten Probewellenlänge resultiert aus der Bildung des entsprechenden Isomers. Der Anstieg erfolgt dabei lösungsmittelabhängig innerhalb 5-50 ps, in überkritischen Lösungsmitteln wie $\mathrm{CO}_{2}$ zeigt sich zudem eine ausgeprägte Druckabhängigkeit. In Abbildung 6.6 sind drei Absorptionszeitprofile von $\mathrm{CHI}_{3}$ in sc. $\mathrm{CO}_{2}$ bei verschiedenen Drücken dargestellt. Dabei zeigt sich, daß der Anstieg bei höheren Drücken schneller wird, die Geschwindigkeitskonstante $k_{\text {iso }}$ nimmt also bei steigender Dichte $\rho$ des Lösungsmittels zu. Die Dichteabhängigkeit von $k_{\text {iso }}$ in sc. $\mathrm{CO}_{2}$ wurde für $\mathrm{CH}_{2} \mathrm{I}_{2}, \mathrm{CH}_{2} \mathrm{BrI}$ und $\mathrm{CHI}_{3}$ bei verschiedenen Anregungs- und Probewellenlängen gemessen. Ihr Verlauf ist in Abbildung 5.2 für $\mathrm{CH}_{2} \mathrm{I}_{2}$, in Abbildung 5.10 für $\mathrm{CH}_{2} \mathrm{BrI}$ und in Abbildung 5.21 für $\mathrm{CHI}_{3}$ dargestellt.

Dabei zeigt sich ein nicht-linearer Anstieg von $k_{\text {iso }}$ mit der reduzierten Dichte des LÖsungsmittels. Wie bereits in Abschnitt 6.1 beschrieben, trägt das $\mathrm{CH}_{2} \mathrm{X} / \mathrm{CHI}_{2}$-Radikal direkt nach der Photodissoziation noch eine zu große Überschußenergie in sich, um direkt zum Isomer rekombinieren zu können. Die Relaxation des schwingungsheißen Radikals 
ist demnach die Voraussetzung zur Bildung eines stabilen Isomers. Diese Relaxation wird durch Schwingungsenergietransfer (VET) an das Lösungsmittel vorgenommen, dabei steigt die Effizienz des VET und somit auch $k_{\text {iso }}$ mit steigender Dichte des Lösungsmittels an. Die Bedeutung der Schwingungsenergierelaxation für die Isomerbildung wurde bereits von Tarnovsky et al. [155] und unserer Arbeitsgruppe als geschwindigkeitsbestimmender Schritt erkannt [196]. Im Modell der isolierten, binären Stöße (IBC-Modell) korreliert die Schwingungsenergierelaxation mit der Anzahl der Stöße mit dem Lösungsmittel, dabei wird die Annahme gemacht, daß immer der gleiche Energiebetrag pro Stoß übertragen wird (s. Abschnitt 2). Die Anzahl der Lösungsmittelstöße verläuft linear mit der Dichte des Lösungsmittels. Die Geschwindigkeitskonstante der Schwingungsenergierelaxation $k_{\text {VER }}$ ist somit direkt proportional zur Dichte des Lösungsmittels. Wie bereits erwähnt, zeigt sich jedoch keine lineare Dichteabhängigkeit von $k_{\text {iso }}$. In meiner Diplomarbeit [103] wird dazu der Einfluß von lokalen Dichteeffekten diskutiert, aufgrund attraktiver Wechselwirkung zwischen Lösungsmittel und Gelöstem kommt es im Bereich der kritischen Dichte zu einer lokalen Dichtevergrößerung um das Gelöste herum (s. Abschnitt 2.3). Somit verläuft im Bereich der kritischen Dichte die lokale Dichte nicht mehr linear zur homogenen Dichte, sondern ist etwas erhöht, was sich in einer Art Plateaubildung niederschlägt (s. Abbildung 2.3).

Bei dem Versuch, die Nicht-Linearität von $k_{\text {iso }}$ auf lokale Dichteeffekte zurückzuführen, zeigen sich jedoch Unstimmigkeiten. So verläuft die Steigung von $k_{\text {iso }}$ in Abhängigkeit von $\rho_{r}$ bereits in einem Dichtebereich von $\rho_{r} \sim 2$ nicht mehr linear, sondern wird zunehmend flacher. Die MD-Simulationen von Zerbs zeigen [86], daß erst im Bereich einer Dichte von $\rho_{r} \leqslant 1,5$ mit einer lokalen Dichtevergrößerung und somit der Ausbildung eines Plateaus zu rechnen ist. Die Nicht-Linearität von $k_{\text {iso }}$ ist somit nicht allein auf einen lokalen Dichteeffekt, sondern auch auf den Einfluß anderer dichteabhängiger Prozesse auf die Isomerisierungsgeschwindigkeit zurückzuführen. Niederdruckmessungen von $\mathrm{CHI}_{3}$ und $\mathrm{CH}_{2} \mathrm{BrI}$ konnten aufgrund der schlechteren Löslichkeit dieser beiden Verbindungen leider nicht durchgeführt werden, so daß für Messungen bei Dichten $\rho_{r}<1,5$ lediglich die Daten von $\mathrm{CH}_{2} \mathrm{I}_{2}$ zur Verfügung stehen.

\subsubsection{Spektrale Dynamik in überkritischen Lösungsmitteln}

Um den Einfluß des Schwingungsenergietransfers auf die Reaktion zu untersuchen, wurde die spektrale Dynamik der Modellsysteme in überkritischen Lösungsmitteln gemessen. Rekonstruierte, zeitaufgelöste Spektren von $\mathrm{CH}_{2} \mathrm{I}_{2}$ (s. Abbildung 5.5), $\mathrm{CH}_{2} \mathrm{BrI}$ (s. Abbildung 5.9) und $\mathrm{CHI}_{3}$ (s. Abbildung 5.17) sind in Kapitel 5 dargestellt.

Anhand des Reaktionsschemas R4 ist ersichtlich, daß die Bildung des CT-Komplexes als Vorgängerspezies für das Isomer auf einer Femtosekundenzeitskala stattfindet. Unter der Annahme, daß nach etwa 2 ps nur noch das Isomer für die spektrale Dynamik des zeitaufgelösten Spektrums verantwortlich ist, kann man dieses populationsnormieren. Dazu wird das Flächenintegral des zeitaufgelösten Spektrums berechnet und diese Fläche für alle Verzögerungszeiten gleichgesetzt. Ein normiertes, zeitaufgelöstes Spektrum von $\mathrm{CH}_{2} \mathrm{I}_{2}$ in sc. $\mathrm{CO}_{2}$ nach $340 \mathrm{~nm}$ Anregung ist in Abbildung 6.7 dargestellt. Dabei zeigt 


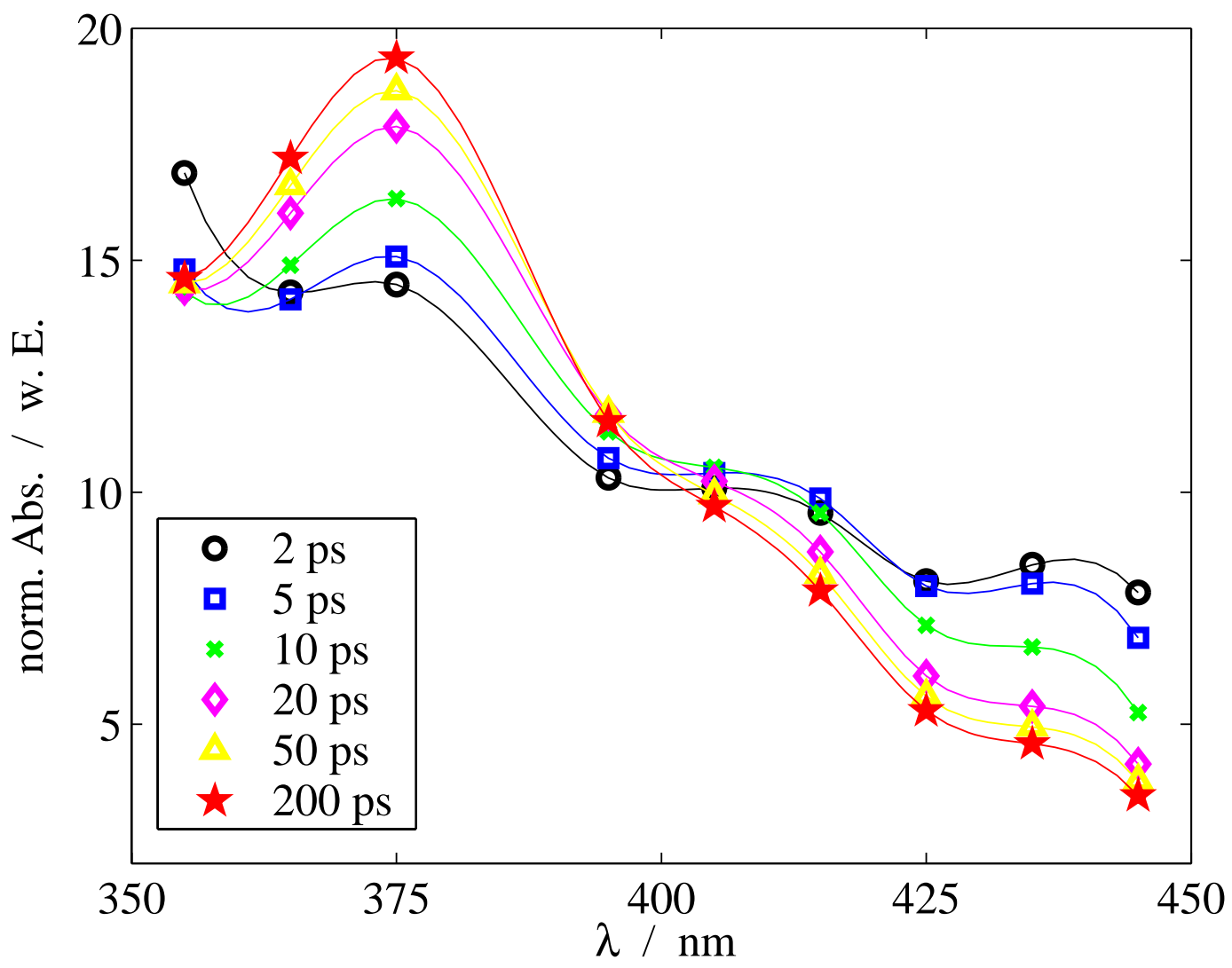

Abbildung 6.7: Normiertes zeitaufgelöstes Spektrum von $\mathrm{CH}_{2} \mathrm{I}_{2}$ in sc. $\mathrm{CO}_{2}\left(35^{\circ} \mathrm{C}\right.$, 400 bar) nach $340 \mathrm{~nm}$ Anregung

sich das typische Bild der Schwingungsenergierelaxation einer hoch schwingungsangeregten Spezies. Zuerst ist das Spektrum sehr breit und flach, was einer breiten Verteilung an angeregten Schwingungszuständen entspricht. Anschließend relaxieren die hoch angeregten Zustände am schnellsten, was einen Abfall der Absorption auf der roten Flanke des Spektrums bewirkt. Im Zentrum der Bande steigt die Absorption unterdessen an, dazwischen liegt ein isosbestischer Punkt, an welchem sich die Absorption im Laufe der Zeit nicht ändert. Diese charakteristische spektrale Entwicklung schwingungsangeregter Moleküle, welche eine Verschmälerung der Bande und die Existenz eines isosbestischen Punktes beinhaltet, können insbesondere in Stoßwellenexperimenten beobachtet werden [197.

Für $\mathrm{CHI}_{3}$ konnte im Gegensatz zu den anderen Polyhalogenmethanen auch die VISAbsorptionsbande des Isomers als zeitaufgelöstes Spektrum in überkritischer Lösung gemessen werden (s. Abbildung 5.17 (rekonstruiertes Spektrum) und 6.8 (populationsnormiertes Spektrum)). Hier zeigt sich wieder das typische Bild einer abkühlenden Spezies, das zuerst breite und strukturlose Spektrum verschmälert sich und im Laufe einer Pikosekundenzeitskala wächst die Isomerbande bei etwa $600 \mathrm{~nm}$ heraus, der isosbestische Punkt liegt bei $655 \mathrm{~nm}$. Im Fall der UV-Bande von $\mathrm{CH}_{2} \mathrm{I}_{2}$ liegt dieser isosbestische Punkt bei $405 \mathrm{~nm}$, die an Abbildung 5.2 dargestellte Dichteabhängigkeit von $k_{\text {iso }}$ wurde bei dieser 


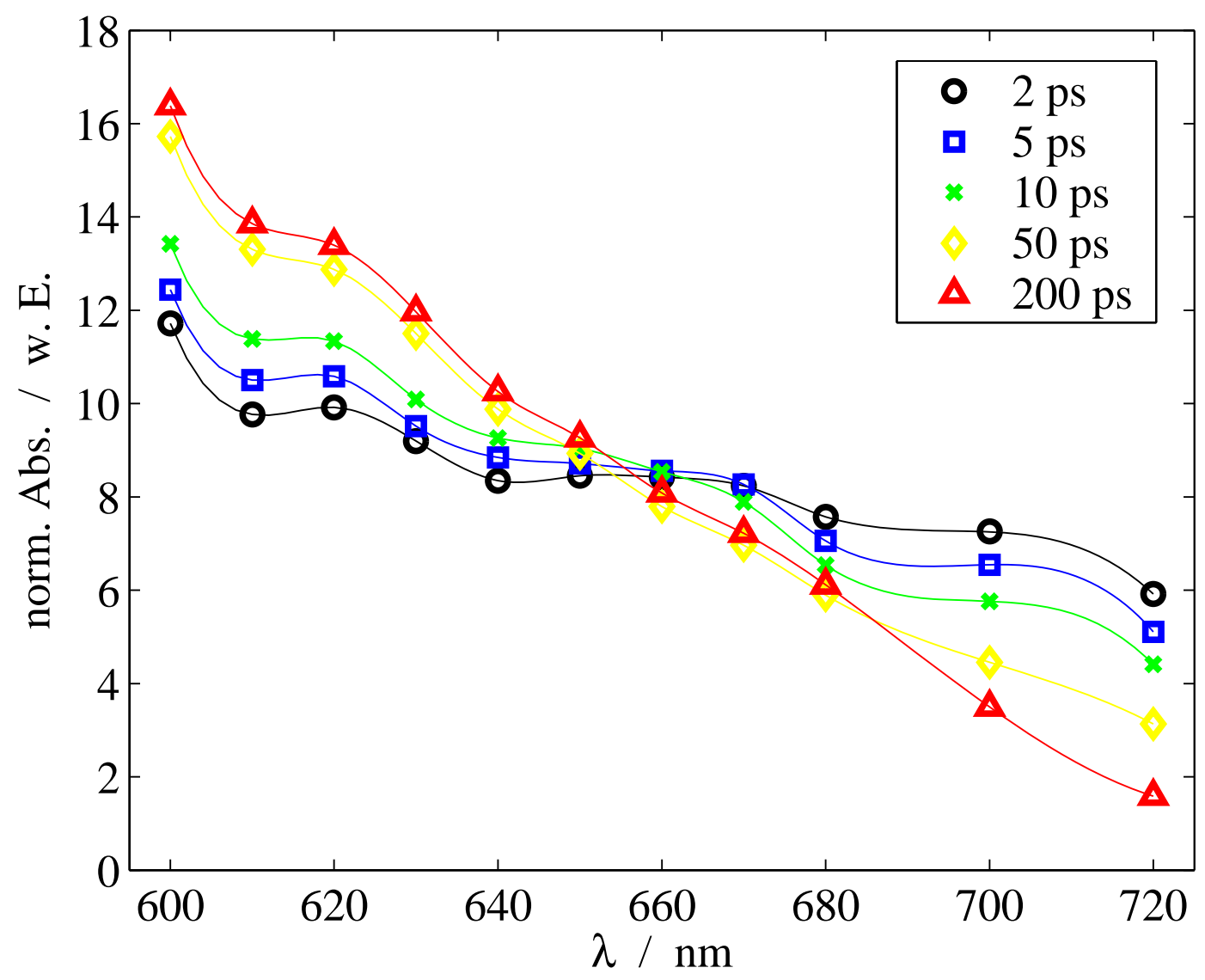

Abbildung 6.8: Normiertes zeitaufgelöstes Spektrum von $\mathrm{CHI}_{3}$ in sc. $\mathrm{CO}_{2}\left(35^{\circ} \mathrm{C}, 505\right.$ bar $)$ nach $365 \mathrm{~nm}$ Anregung

Probewellenlänge gemessen, um die reine Isomerbildung ohne Überlagerung von spektraler Dynamik zu messen.

Anhand der spektralen Dynamik lassen sich weitere Informationen über den Energietransfer zwischen hochangeregten Photoprodukten und dem Lösungsmittel gewinnen. Beispielsweise lassen sich die Zeitkonstanten des Schwingungsenergietransfers (VET) bestimmen. Dazu müssen allerdings die schwingungstemperaturabhängigen Extinktionskoeffizienten der abkühlenden Spezies bekannt sein. Im Fall der Relaxation einer schwingungsangeregten Spezies ändert sich die Besetzung der Schwingungszustände und somit auch der Extinktionskoeffizient bei einer bestimmten Wellenlänge. Die Besetzung der Schwingungszustände ist temperaturabhängig und somit kann den unterschiedlichen Besetzungen eine mikroskopische Schwingungstemperatur und eine bestimmte mittlere Energie zugeordnet werden. Die Berechnung der mittleren Energie erfolgt über alle Schwingungsfreiheitsgrade $i$ des Moleküls nach Gleichung 6.5;

$$
\langle E\rangle=\sum_{i=1}^{i} \frac{h \nu_{i}}{\exp \left(\frac{h \nu_{i}}{k_{B} T}\right)-1}+\frac{3}{2} k_{B} T
$$




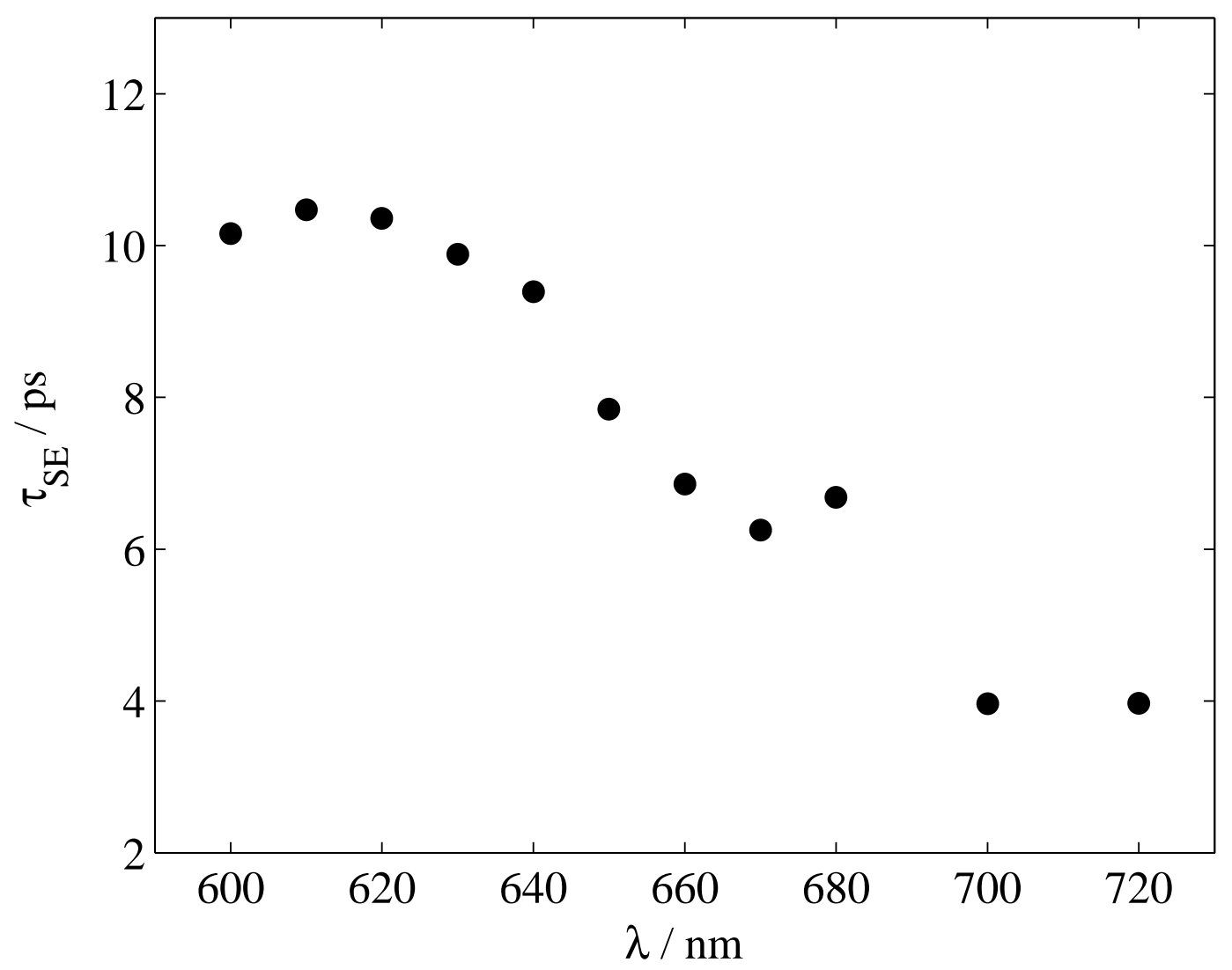

Abbildung 6.9: Zeitkonstanten der spektralen Entwicklung von $\mathrm{CHI}_{3}$ in sc. $\mathrm{CO}_{2}\left(35^{\circ} \mathrm{C}\right.$, 505 bar) nach $365 \mathrm{~nm}$ Anregung

Die Relaxation des Moleküls und somit die Änderung der Schwingungstemperatur bzw. der mittleren Energie geht nun mit einer Änderung des Extinktionskoeffizienten einher. Um die korrekten Schwingungsenergierelaxationszeiten zu berechnen, muß nun der $\mathrm{Zu}$ sammenhang zwischen mittlerer Energie des Moleküls und Extinktionskoeffizient bekannt sein. Dieser Zusammenhang kann z. B. mit Hilfe des Sulzer-Wieland-Modells berechnet werden [198, wobei das klassische Modell in seiner Anwendung allerdings auf zweiatomige Moleküle begrenzt ist. Eine Erweiterung des Sulzer-Wieland-Modells auf größere Moleküle wurde von Hippler et al. vorgenommen [199]. Eine andere Möglichkeit ist die Aufnahme von Spektren bei definierten Temperaturen wie es beispielsweise durch Stoßwellenexperimente realisiert werden kann [200,201].

Für die Polyhalogenmethane können Daten über den Zusammenhang von mittlerer Energie und Extinktionskoeffizient der Literatur entnommen werden, beispielsweise für $\mathrm{CH}_{2} \mathrm{I}_{2}$ von Charvat et al. [202]. Im Falle der Isomere der Polyhalogenmethane sind solche Daten nicht vorhanden, da deren Lebensdauer von maximal einigen Nanosekunden zur Durchführung von Stoßwellenexperimenten zu gering ist. Somit können keine direkten Schwingungsenergierelaxationszeiten berechnet werden, sondern lediglich die Zeitkonstanten der spektralen Entwicklung bei unterschiedlichen Wellenlängen miteinander verglichen werden. Dazu wurde der zeitliche Verlauf der Absorption im populationsnormierten Spek- 


\begin{tabular}{|lccccc|}
\hline Molekül & $\lambda_{\text {Exc }} / \mathrm{nm}$ & $p_{\mathrm{CO}_{2}} /$ bar & $\tau_{\text {auf }} / \mathrm{ps}$ & $\tau_{\text {ab }} / \mathrm{ps}$ & $\bar{M}_{\tau} / \mathrm{ps}$ \\
$\mathrm{CH}_{2} \mathrm{I}_{2}$ & 340 & 400 & 17,3 & 15,5 & 16,4 \\
$\mathrm{CHI}_{3}$ & 365 & 505 & 15,1 & 14,8 & 15,0 \\
$\mathrm{CHI}_{3}$ & 267 & 505 & 26,8 & 26,2 & 26,5 \\
$\mathrm{CHI}_{3}$ & 267 & 142 & 52,3 & 54,5 & 53,4 \\
\hline
\end{tabular}

Tabelle 6.2: Durch Integration der zeitaufgelösten Isomerbande bestimmte Schwingungsenergierelaxationszeiten verschiedenener Isomere unter unterschiedlichen Bedingungen in sc. $\mathrm{CO}_{2}\left(35^{\circ} \mathrm{C}\right)$

trum bei einer Wellenlänge monoexponentiell angepaßt. Diese Zeitkonstanten $\tau_{\mathrm{SE}}$ der spektralen Entwicklung sind in Abbildung 6.9 wellenlängenabhängig für $\mathrm{CHI}_{3}$ in sc. $\mathrm{CO}_{2}$ $\left(35^{\circ} \mathrm{C}, 505\right.$ bar $)$ nach $365 \mathrm{~nm}$ Anregung aufgetragen.

Dabei zeigt sich, daß die zeitliche Entwicklung der Absorption auf der roten Flanke zu längeren Wellenlängen hin immer schneller wird. Bei längeren Wellenlängen wird ein höher angeregter und somit schwächer besetzter Teil der Boltzmann-Verteilung beobachtet, welcher schneller relaxiert. Für $\mathrm{CH}_{2} \mathrm{I}_{2}$ in verschiedenen überkritischen Lösungsmitteln zeigt sich ein sehr ähnliches Bild, Untersuchungen zur Schwingungsenergierelaxation finden sich beispielsweise in der Dissertation von Dr. C. Grimm [104] und in meiner Diplomarbeit [103].

Eine bessere Möglichkeit zur Bestimmung der Zeitkonstanten der Schwingungsenergierelaxation bei unbekannten Extinktionskoeffizienten ist die Integration des normierten Spektrums zu verschiedenen Zeiten. Im normierten Spektrum fällt die Absorption auf der roten Flanke rechts des isosbestischen Punkts ab, während sie links davon anwächst. Durch Integration des Spektrums zu verschiedenen Zeiten rechts und links des isosbestischen Punkt kann die spektrale Dynamik, welche durch den Schwingungsenergietransfer verusacht wird, am besten beschrieben werden. Dieses zeitabhängige Integral wurde für verschiedene zeitaufgelöste Spektren von $\mathrm{CH}_{2} \mathrm{I}_{2}$ und $\mathrm{CHI}_{3}$ in sc. $\mathrm{CO}_{2}$ gebildet und mit einem monoexponentiellen Anstieg bzw. Abfall angepaßt. Die Ergebnisse dieser Anpassungen sind in Tabelle 6.2 zusammengestellt. Dabei zeigt sich daß die Zeitkonstanten $\tau_{\text {auf }}$ für den monoexponentiellen Anstieg im Zentrum der Bande sowie $\tau_{\mathrm{ab}}$ für den Abfall auf der roten Flanke fast identisch sind und somit den gleichen Prozeß, nämlich den Schwingungsenergietransfer, beschreiben.

Zum Vergleich mit denen durch die verschiedenen Methoden ermittelten Zeiten für den Schwingungsenergietransfer können lediglich die Relaxationszeiten der entsprechenden Muttermoleküle herangezogen werden. Für $\mathrm{CHI}_{3}$ wurde die Relaxation der $\mathrm{C}-\mathrm{H}-$ Streckschwingung nach Anregung bei $3030 \mathrm{~cm}^{-1}$ in Lösung von Crim et al. gemessen [203], die Relaxationszeiten reichen dabei von $10 \mathrm{ps}$ in $d_{6}$-Aceton bis zu $170 \mathrm{ps}$ in $\mathrm{CDCl}_{3}$. Der Wert in $d_{6}$-Aceton liegt dabei sehr nahe an den wellenlängenabhängigen Zeitkonstanten der spektralen Entwicklung (4-10 ps) sowie den durch Integration bestimmten Wert von 15,0 ps. Dabei bleibt zu beachten, daß die Messungen von Crim et al. in flüssigen Lösungsmitteln, also bei höherer Dichte als die Messung von $\mathrm{CHI}_{3}$ in sc. $\mathrm{CO}_{2}$ durchgeführt wurden. In überkritischer Lösung bei etwas niedrigerer Dichte sollte die spektrale Evolution 


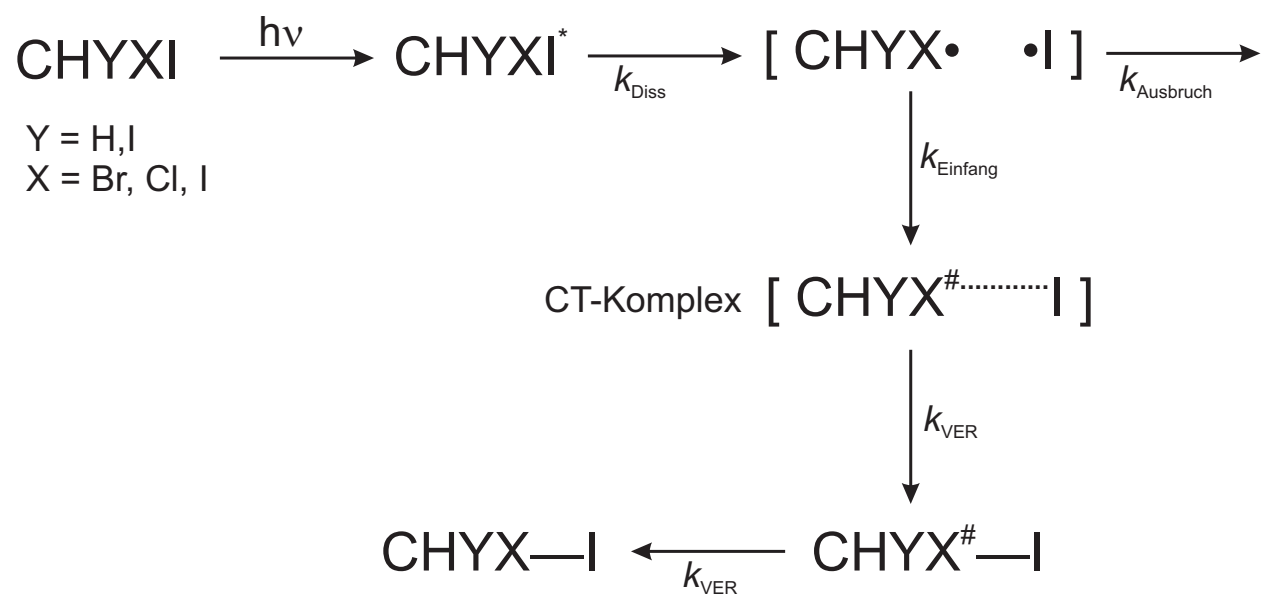

Abbildung 6.10: Vorläufiges kinetisches Schema der Käfigprozesse und Isomerbildung nach UV-Anregung von Polyhalogenmethanen in Lösung

tendenziell etwas langsamer sein. Das läßt sich auch anhand Schwingungsenergierelaxationszeiten von $\mathrm{CHI}_{3}$ in sc. $\mathrm{CO}_{2}$ bei verschiedenen Drücken zeigen; nach $267 \mathrm{~nm}$ Anregung bei 505 bar beträgt diese 26,5 ps während bei dem geringeren Druck von 142 bar diese auf 53,4 ps anwächst. Desweiteren werden hier die Zeiten der Schwingungsenergierelaxation des Muttermoleküls mit denen des Isomers verglichen. Die Energieübertragung findet nach dem IBC-Modell durch resonante Stöße zwischen Lösungsmittel und Gelöstem statt. Dabei wird die Energie entweder auf die Translationsfreiheitsgrade (V-T-Stöße) oder auf Schwingungsfreiheitsgrade des Lösungsmittels umverteilt (V-V-Stöße). Die Energieübertragung ist umso effizienter, je besser die Kopplung zwischen den Moden des Gelösten und denen des Lösungsmittels ist. Eine Aussage darüber zu machen, ob das Isomer oder das Muttermolekül die bessere Kopplung mit den Moden des Lösungsmittels besitzt, ist ohne tiefergehende Untersuchungen schwierig. Allerdings läßt sich generell feststellen, daß die Energieübertragung von niederfrequenten Moden wesentlich effizienter als die von hochfrequenten ist. In diesem Fall ist es nützlich, die Schwingungsmoden von Isomer und Muttermolekül (s. Tabelle 3.3 und 3.4 zu vergleichen. Philips et al. konnten aufgrund von $a b$ initio-Rechnungen zeigen, daß bei der Bildung des Isomers jeweils eine neue, niederfrequente Mode bei etwa $150 \mathrm{~cm}^{-1}$ entsteht (s. Abschnitt 3.5.3). Diese niederfrequente Mode kann den Schwingungsenergietransfer ins Lösungsmittel beschleunigen, so daß die Stabilisierung des Isomers schneller abgeschlossen wird. Die normierten Spektren zeigen somit, daß die Schwingungsenergierelaxation der geschwindigkeitsbestimmende Schritt bei der Isomerbildung ist. Diese findet im Anschluß an die Ultrakurzzeitdynamik im Lösungsmittelkäfig auf einer Pikosekundenzeitskala statt. Auf dieser Grundlage läßt sich ein vorläufiges kinetisches Schema zum Mechanismus der Isomerbildung aufstellen (s. Abbildung 6.10. 


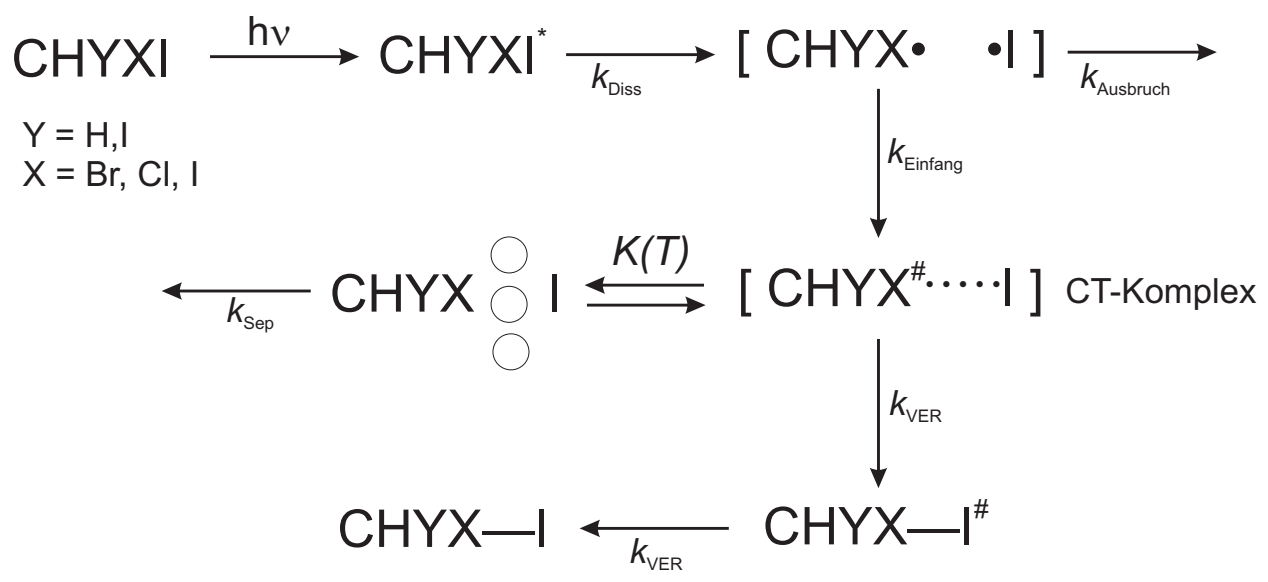

Abbildung 6.11: Um schnelles Vorgleichgewicht erweitertes kinetisches Schema der Käfigprozesse und Isomerbildung nach UV-Anregung von Polyhalogenmethanen in Lösung

\subsubsection{Anregungswellenlängenabhängigkeit der Isomerisierung}

Messungen der transienten Absorption von $\mathrm{CH}_{2} \mathrm{I}_{2}$ und $\mathrm{CHI}_{3}$ bei variabler Anregungswellenlänge zeigen, daß $k_{\text {iso }}$ eine je nach Polyhalogenverbindung unterschiedliche Abhängigkeit von der Anregungswellenlänge besitzt. Der dichteabhängige Verlauf von $k_{\text {iso }}$ ist bei unterschiedlichen Wellenlängen gleich, bei Anregung mit kürzerer Wellenlänge wird $k_{\text {iso }}$ jedoch kleiner, die Isomerisierungsgeschwindigkeit somit langsamer. Dieser Effekt ist bei $\mathrm{CH}_{2} \mathrm{I}_{2}$ (s. Abbildung 5.2) ausgeprägter als bei $\mathrm{CHI}_{3}$ (s. Abbildung 5.21). Dieses auf den ersten Blick ungewöhnliche Verhalten kann nicht mit Hilfe des bisher postulierten kinetischen Schemas erklärt werden. Dieses Modell muß deshalb erweitert werden.

Nach der Photodissoziation wird von allen Photofragmenten, die nicht aus dem Lösungsmittelkäfig ausgebrochen sind, ein CT-Komplex gebildet. Dieser liegt in unterschiedlichen Konfigurationen je nach Energie- und somit Abstandsverteilung vor. Aufgrund der signifikanten Überschußenergie, die die Photofragmente nach der Photodissoziation in sich tragen, können diese den Lösungsmittelkäfig auch nach dem primären Käfigausbruch noch verlassen. Da der kinetische Anteil der Überschußenergie jedoch bereits nach wenigen Stößen äquilibriert ist, handelt es sich dabei nicht mehr um einen kinematischen Ausbruch, der die Photofragmente ausreichend weit im Lösungsmittel separiert. Die Fragmente verlassen lediglich die erste Lösungsmittelschale; schiebt sich dabei mindestens ein Lösungsmittelmolekül zwischen Radikal und Iodatom, wird die CT-Wechselwirkung beendet. Eine Rückreaktion und Neuausbildung des CT-Komplexes ist jederzeit möglich, daher stellt sich nach kurzer Zeit ein Gleichgewicht zwischen Photofragmenten im Käfig mit CT-Wechselwirkung und den durch das Lösungsmittel separierten Fragmenten ein. Das einfache Reaktionsschema wurde dazu in Abbildung 6.11 um ein schnelles Vorgleichgewicht mit der Gleichgewichtskonstante $K(T)$ ergänzt. Dieses Gleichgewicht ist dabei von der Schwingungstemperatur der Photofragmente abhängig; je größer die Überschußenergie ist, desto mehr verschiebt es sich auf die Seite der Photofragmente ohne CT-Wechselwirkung. Dadurch verlangsamt sich die Reaktion, wenn mit mehr Überschu- 
ßenergie angeregt wird, da die Isomerbildung nur aus einem relaxierten CT-Komplex innerhalb des Käfigs erfolgen kann. Weiterhin deutet sich an, daß neben dem ersten, kinematischen Käfigausbruch noch eine diffusiv gesteuerte Separation der Photofragmente zur Minderung der Quantenausbeute der Isomerisierung beitragen kann. Sind die Photofragmente durch die erste Lösungsmittelschale separiert, können sie neben der Rückreaktion zum CT-Komplex auch diffusiv voneinander getrennt werden. Diese diffusive Separation findet mit der Geschwindigkeitskonstante $k_{\text {Sep }}$ statt.

\subsubsection{Dichteabhängigkeit der Geschwindigkeitskonstante $k_{\text {iso }}$}

Durch einen Blick auf das erweiterte Reaktionsschema in Abbildung 6.11 kann man $k_{\text {iso }}$ insbesondere auf die experimentell beobachtete Dichteabhängigkeit untersuchen. Die Geschwindigkeitskonstante der Isomerisierung $k_{\text {iso }}$ ist dabei als resultierende Gesamtgeschwindigkeitskonstante aufzufassen, welche sich aus allen Teilschritten zusammensetzt. Da die Geschwindigkeitskonstanten der primären Käfigprozesse der Dissoziation $k_{\text {Diss }}$, des Käfigeinfangs $k_{\text {Einfang }}$ und -ausbruchs $k_{\text {Ausbruch }}$ sehr viel größer als die der nachfolgenden Prozesse (Schwingungsenergierelaxation, diffusiver Käfigausbruch) sind, lassen sich die schnellen Prozesse von den langsamen separieren:

$$
k_{\text {Ausbruch }}, k_{\text {Diss }}, k_{\text {Einfang }} \gg k_{\mathrm{VER}}, k_{\text {Sep }}
$$

Somit setzt sich $k_{\text {iso }}$ aus der Schwingungsenergierelaxation $k_{\text {VER }}$, dem schnellen Vorgleichgewicht $K(T)$ und dem diffusiven Käfigausbruch $k_{\text {Sep }}$ zusammen:

$$
k_{\text {iso }}=k_{\mathrm{Sep}}+K(T) \cdot k_{\mathrm{VER}}
$$

Um die Dichteabhängigkeit von $k_{\text {iso }}$ zu bestimmen, muß man nun die Dichteabhängigkeit der einzelnen Komponenten, aus denen sich $k_{\text {iso }}$ zusammensetzt, betrachten.

\section{Schwingungsenergierelaxationsgeschwindigkeitskonstante $k_{V E R}$}

Behandelt man $k_{\text {Ver }}$ auf der Grundlage des IBC-Modells, so ist die Geschwindigkeitskonstante direkt proportional zur Anzahl der Stöße mit dem Lösungsmittel:

$$
k_{\mathrm{VER}}(\rho, T)=P(T) \cdot Z(\rho, T)
$$

Dabei ist $P(T)$ ein Maß für die Wahrscheinlichkeit der Relaxation und $Z(\rho)$ die Funktion der Stöße mit dem Lösungsmittel. Für $Z(\rho)$ kann man die Gleichung für die Stoßzahl $Z_{0}$ in der verdünnten Gasphase heranziehen: 


$$
Z_{0}=\rho \sigma^{2} \sqrt{\frac{8 \pi k_{\mathrm{B}} T}{\mu}}
$$

$\rho$ ist dabei die Dichte des Lösungsmittels, $\sigma$ der effektive Stoßquerschnitt und $\mu$ die reduzierte Masse der Stoßpartner. Die Solvensstruktur der lokalen Dichte in Lösung kann anschließend noch mit einer radialen Verteilungsfunktion $g(\rho, T, \sigma)$ implementiert werden:

$$
Z=Z_{0} \cdot g(\rho, T, \sigma)
$$

Dabei ist $k_{\text {VER }}$ direkt proportional zur lokalen Dichte $\rho_{\text {lokal }}$, welche bei sich Dichten über der kritischen Dichte $\rho_{\text {krit }}$ zunehmend der homogenen Dichte $\rho$ annähert.

\section{Gleichgewichtskonstante $\mathrm{K}(\mathrm{T})$}

Die Druckabhängigkeit der Gleichgewichtskonstante $K(T)$ beruht auf einer Solvensstabilisierung des CT-Komplexes in der Lösungsmittelkavität. Der CT-Komplex besitzt durch die Ladungstrennung zwischen Donor und Akzeptor ein starkes Dipolmoment. Im Onsager-Modell der Solvatation (s. Abschnitt 2p ist dieser Dipol in ein Lösungsmittel eingebettet, dargestellt durch ein dielektrisches Kontinuum. Das Lösungsmittel wird polarisiert und führt somit zu einer Solvatationsstabilisierung nach Gleichung 2.1. Je höher die freie Gibbsenergie der Solvatation $\Delta G_{\text {Solv }}$ ist, desto stärker wird der CT-Komplex im Vergleich zu den getrennten Fragmenten stabilisiert und das Gleichgewicht verschiebt sich auf dessen Seite. Die einzige dichteabhängige Größe in Gleichung 2.1 zur Berechnung von $\Delta G_{\text {Solv }}$ ist dabei der sogenannte Clausius-Mosotti-Faktor $f\left(\epsilon_{r}\right)$ :

$$
f(\epsilon)=\frac{\epsilon_{r}-1}{\epsilon_{r}+2}
$$

Somit gilt:

$$
\frac{\delta \Delta G_{\text {Solv }}}{\delta \rho}=-N_{A} \frac{\mu_{G}^{2}}{4 \pi \epsilon_{0} r^{3}} \frac{\delta f\left(\epsilon_{r}\right)}{\delta \rho}
$$

Die Druckabhängigkeit der Dielektrizitätszahl $\epsilon_{r}$ in $\mathrm{CO}_{2}$ ist in Abbildung 6.3 dargestellt. Diese Daten lassen sich leicht in die Dichteabhängigkeit des Clausius-Mosotti-Faktors $f\left(\epsilon_{r}\right)$ übertragen und sind in Abbildung 6.12 dargestellt. Empirische Gleichungen zur Berechnung des Clausius-Mosotti-Faktors $f\left(\epsilon_{r}\right)$ in anderen überkritischen Lösungsmitteln finden sich bei Maroncelli et al. [204]. Der Clausius-Mosotti-Faktor zeigt dabei bei 


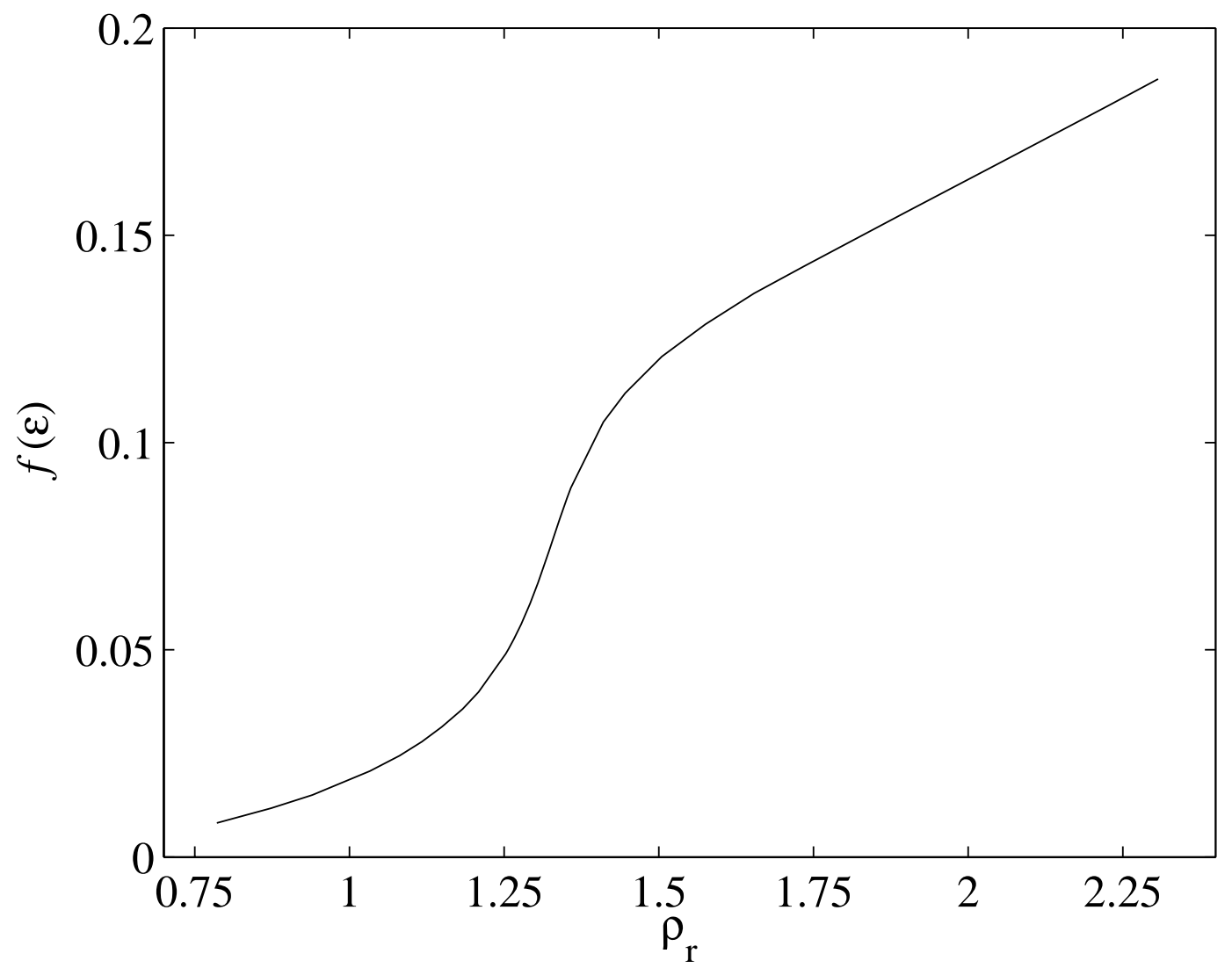

Abbildung 6.12: Abhängigkeit des Clausius-Mosotti-Faktors $f(\epsilon)$ von der reduzierten Dichte $\rho_{r}$ in sc. $\mathrm{CO}_{2}$ bei $50{ }^{\circ} \mathrm{C}[195]$

reduzierten Dichten $\rho_{r}>1,5$ eine nahezu lineare Dichteabhängigkeit. Die Gleichgewichtskonstante $K$ ist somit nicht nur eine Funktion der Schwingungstemperatur $T$ der Photofragmente, sondern auch von der Dichte des Lösungsmittels $\rho$ abhängig. Bei hohen Dichten wird das Gleichgewicht durch die bessere Solvensstabilisierung des CT-Komplexes auf dessen Seite verschoben.

\section{Diffusive Trennung der Photofragmente $k_{\text {Sep }}$}

Wenn die primären Photofragmente durch mindestens ein Solvensmolekül getrennt werden, besitzen sie keine CT-Wechselwirkung mehr. In diesem Fall können sie anfangen, sich durch einen diffusiv gesteuerten Prozeß voneinander zu separieren. Die mittlere quadratische Verschiebung $\left\langle x^{2}\right\rangle$ innerhalb einer Zeit $\tau$ wird dabei wie folgt berechnet [205]:

$$
\left\langle x^{2}\right\rangle=2 D \tau_{\text {Sep }} \quad \text { bzw. } \quad k_{\text {Sep }}=\frac{2 D}{\left\langle x^{2}\right\rangle}
$$




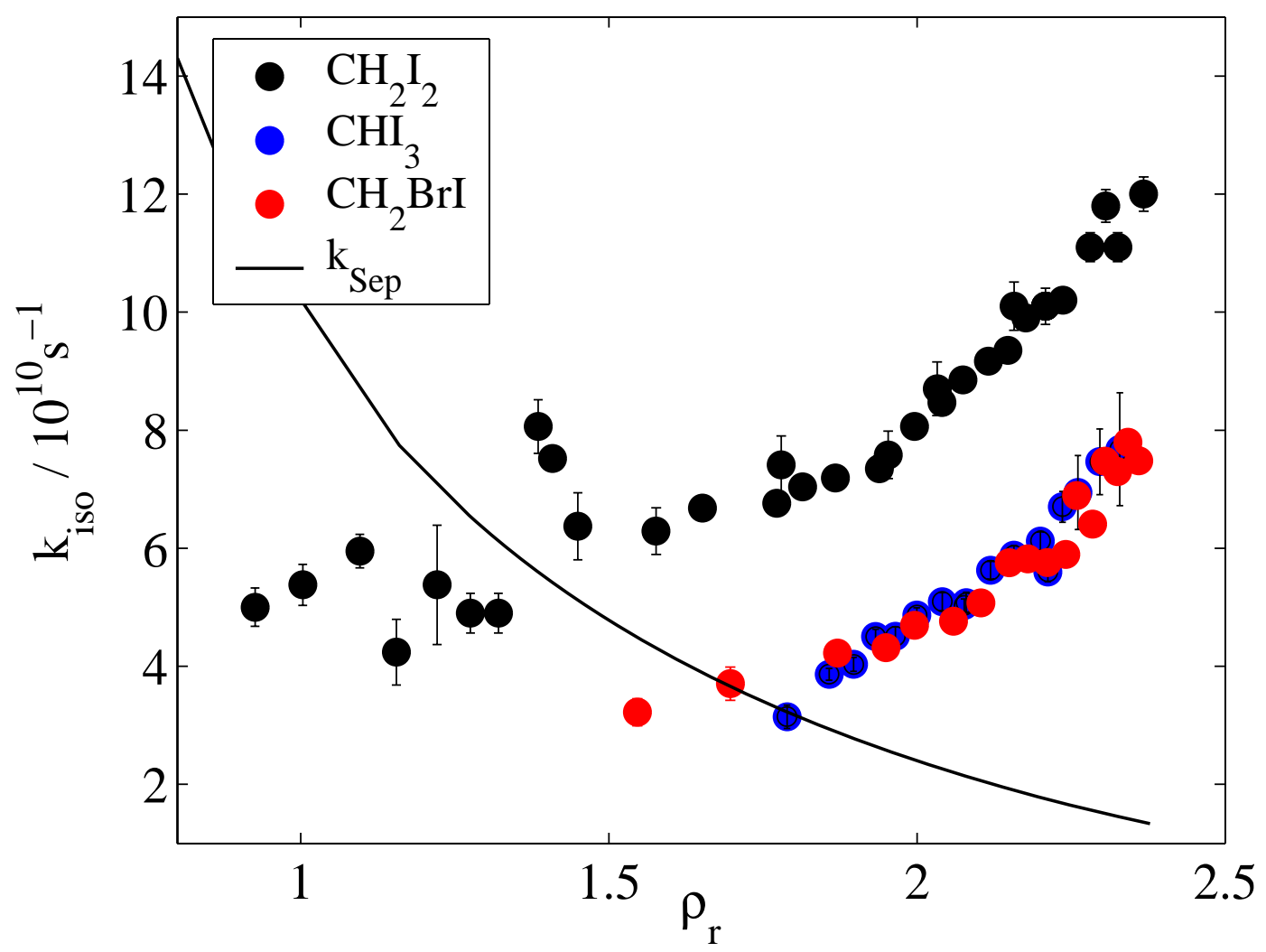

Abbildung 6.13: Vergleich von $k_{\text {iso }}$ von $\mathrm{CH}_{2} \mathrm{I}_{2}$ (300 nm Anregung und $405 \mathrm{~nm}$ Abfrage), $\mathrm{CHI}_{3}(295 / 460 \mathrm{~nm})$ und $\mathrm{CH}_{2} \mathrm{BrI}(288 / 400 \mathrm{~nm})$ in sc. $\mathrm{CO}_{2}\left(35^{\circ} \mathrm{C}\right)$ und der Abschätzung von $k_{\text {Sep }}$

Zur Bedeutung der diffusiven Separation soll diese am Beispiel $\mathrm{CH}_{2} \mathrm{I}_{2}$ in sc. $\mathrm{CO}_{2}$ abgeschätzt werden. Dazu wird als erstes der Diffusionskoeffizient $D$ benötigt, in einem viskosen Medium läßt dieser sich nach der Stokes-Einstein-Beziehung berechnen:

$$
D=\frac{k_{\mathrm{B}} T}{6 \pi \eta \sigma}
$$

Dabei ist $k_{\mathrm{B}}$ die Boltzmann-Konstante, $\eta$ die Viskosität des Lösungsmittels und $\sigma$ der Lennard-Jones-Durchmesser der diffundierenden Spezies. Es ist allerdings zu beachten, daß die Stokes-Einstein-Beziehung nur bei ausreichend hohen Dichten, insbesondere in Flüssigkeiten, zu guten Ergebnissen führt. Bei niedrigen Dichten ist es zweckmäßiger eine empirische Gleichung für den Diffusionskoeffizienten $D \mathrm{zu}$ verwenden, welche von Hippler et al. durch Interpolation von der kinetischen Theorie verdünnter Gase zur StokesEinstein-Gleichung für Flüssigkeiten gefunden wurde [206]:

$$
\frac{k_{\mathrm{B}} T}{\eta D}=2 \pi \sigma\left[1-\exp \left(-\frac{\rho}{\rho_{r}}\right)\right]
$$


Die Viskosität $\eta$ für den relevanten Dichtebereich $\rho_{r}=0,8-2,5$ wurde dazu dem NIST Chemistry Webbook entnommen 193 und für $\sigma$ wird der Lennard-Jones-Durchmesser eines Iodatoms mit $\sigma=4,32 \times 10^{-10} \mathrm{~m}$ [18 eingesetzt. Unter der Annahme, daß die Separation der primären Photoprodukte nach der 2. Lösungsmittelschale vollständig und irreversibel ist, kann als Kantenlänge $x$ des mittleren Verschiebequadrats $x^{2}$ laut den MDSimulationen von Zerbs und Kandratsenka [86] etwa $10 \times 10^{-10} \mathrm{~m}$ angenommen werden. Mit Hilfe der durch Gleichung 6.15 berechneten Diffusionskoeffizienten $D$ können nun mit Gleichung 6.13 die dichteabhängigen Geschwindigkeitskonstanten $k_{\text {Sep }}$ der diffusiven Separation der primären Photofragmente berechnet werden. Diese sind zusammen mit $k_{\text {iso }}$ von $\mathrm{CH}_{2} \mathrm{I}_{2}, \mathrm{CHI}_{3}$ und $\mathrm{CH}_{2} \mathrm{BrI}$ in sc. $\mathrm{CO}_{2}$ in Abbildung 6.13 dargestellt.

Dabei zeigt sich, daß die diffusive Separation der Photofragmente bei niedrigen Dichten durchaus eine Rolle spielen kann (insbesondere bei $\mathrm{CH}_{2} \mathrm{BrI}$ und $\mathrm{CHI}_{3}$ ). Bei höheren Dichten $\left(\mathrm{ab} \rho_{r} \geqslant 2\right)$ liegt $k_{\text {Sep }}$ allerdings signifikant unter der Geschwindigkeitskonstante der Isomerisierung $k_{\text {iso }}$ und der Prozeß der diffusiv Separation der Photofragmente ist zu vernachlässigen. Die Abschätzung von $k_{\text {Sep }}$ ist jedoch mit verschiedenen Unsicherheiten behaftet. Zum einen kann die makroskopischen Viskosität der Lösung nicht direkt auf die mikroskopische Separation zweier Teilchen innerhalb der ersten Lösungsmittelschalen übertragen werden, da sie die Lösungsmittelstruktur unbeachtet läßt. Desweiteren kann der Abstand, ab welchem die Separation der Photofragmente vollständig ist und diese nicht mehr zur Isomerbildung beitragen, nur geschätzt werden. Der dichteabhängige Verlauf von $k_{\text {Sep }}$ kann demnach nur als Hinweis gewertet werden, daß bei niedrigen Dichten einem diffusiven Käfigausbruch der Fragmente durchaus eine Bedeutung zukommt, während bei hohen Dichten die Diffusion im Vergleich zu anderen Käfigprozessen wie der Isomerisierung zu langsam ist.

Einen weiteren Hinweis auf eine diffusive Separation der Photofragmente vor Rekombination zum Isomer geben transiente Absorptionsmessungen von $\mathrm{CHI}_{3}$ bei niedrigen Drücken. In Abbildung 6.14 ist ein typisches Absorptionszeitprofil von $\mathrm{CHI}_{3}$ bei 180 bar dargestellt. Dabei zeigt sich nach dem Anfangspeak ein leichter Abfall innerhalb mehrerer Pikosekunden. Bei niedrigen Dichten ist somit die Separation der Photofragmente durch Diffusion schnell genug, um sie vor Ausbildung des Isomers zu trennen. Die Photofragmente, die endgültig separiert sind, können nicht mehr zum CT-Komplex zurückreagieren und somit fällt die Absorption zu frühen Zeiten ab. Anschließend schließt sich der langsame Anstieg der transienten Absorption an, welcher auf die Bildung des Isomers zurückzuführen ist. Bei höheren Dichten ist kein Abfall der Kurzzeitabsorption, welche der Absorption durch den CT-Komplex zuzuordnen ist, zu beobachten. Die Photofragmente, welche zwischenzeitlich durch das Lösungsmittel getrennt wurden und nicht in CT-Wechselwirkung stehen, können somit nicht schnell genug auseinander diffundieren und stehen weiterhin im schnellen Gleichgewicht mit dem CT-Komplex, der schlußendlich in das Isomer umgesetzt wird. Bei anderen Polyhalogenmethanen als $\mathrm{CHI}_{3}$ konnte kein Abfall der anfänglichen CT-Absorption beobachtet werden. Im Falle von $\mathrm{CH}_{2} \mathrm{I}_{2}$ ist die Isomerbildung wesentlich schneller und somit überdeckt der langsame Anstieg einen eventuellen Abfall. Für $\mathrm{CH}_{2} \mathrm{BrI}$ waren aufgrund der schlechten Löslichkeit der Verbindung in sc. $\mathrm{CO}_{2}$ keine Niederdruckmessungen möglich. 

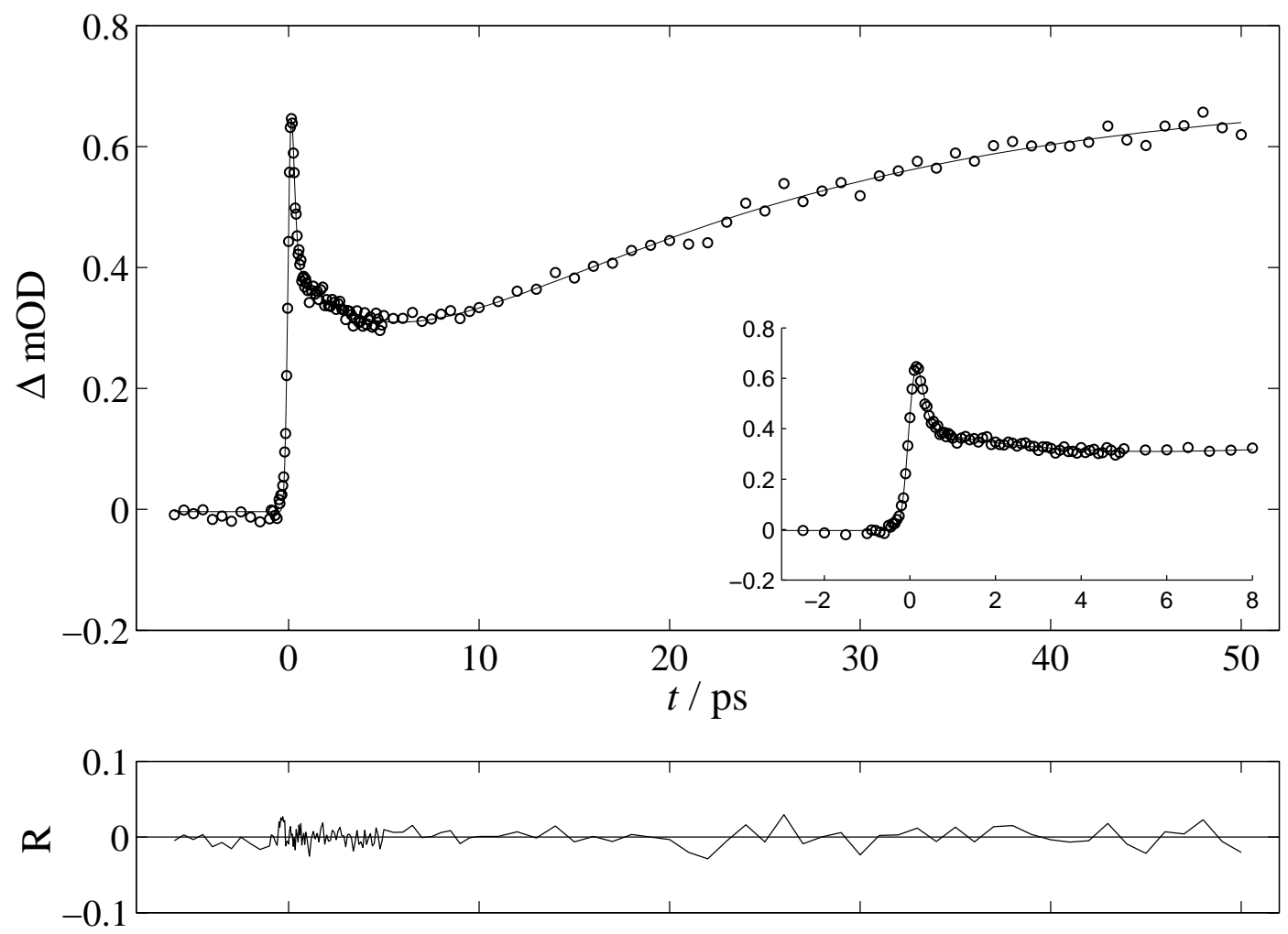

Abbildung 6.14: Absorptionszeitprofil von $\mathrm{CHI}_{3}$ in sc. $\mathrm{CO}_{2}\left(35^{\circ} \mathrm{C}, 180\right.$ bar $)$ nach $267 \mathrm{~nm}$ Anregung und $460 \mathrm{~nm}$ Abfrage

Abschließend läßt sich sagen, daß der nicht-lineare dichteabhängige Verlauf von $k_{\text {iso }}$ darauf zurückzuführen ist, daß $k_{\text {iso }}$ nach der Beziehung

$$
k_{\text {iso }}=k_{\mathrm{Sep}}(\rho)+K(\rho, T) \cdot k_{\mathrm{VER}}(\rho)
$$

von drei Größen abhängt, welche alle eine mehr oder weniger starke Dichteabhängigkeit zeigen. Eine genaue Voraussage auf der Grundlage des kinetischen Schemas und der abgeschätzten Dichteabhängigkeiten der einzelnen Geschwindigkeitskonstanten ist jedoch schwierig.

\subsection{Quantenausbeute}

Da die Berechnung von Quantenausbeuten für die Isomerisierungsreaktion aufgrund des unbekannten Extinktionskoeffizienten der Isomere leider nicht möglich ist, wird die in Abschnitt 5.2.2 eingeführte Größe der normierten Endamplitude $A_{\text {iso,n }}$ als Maß für die Quantenausbeute $\Phi_{\text {iso }}$ der Reaktion betrachtet. In Kapitel 5 ist die Dichteabhängigkeit von $A_{\text {iso,n }}$ in sc. $\mathrm{CO}_{2}$ bei verschiedenen Anregungswellenlängen für $\mathrm{CH}_{2} \mathrm{BrI}$ (s. Abbildung 5.11), $\mathrm{CH}_{2} \mathrm{I}_{2}$ (s. Abbildung 5.3) und $\mathrm{CHI}_{3}$ (s. Abbildung 5.22) dargestellt. Dabei zeigt 


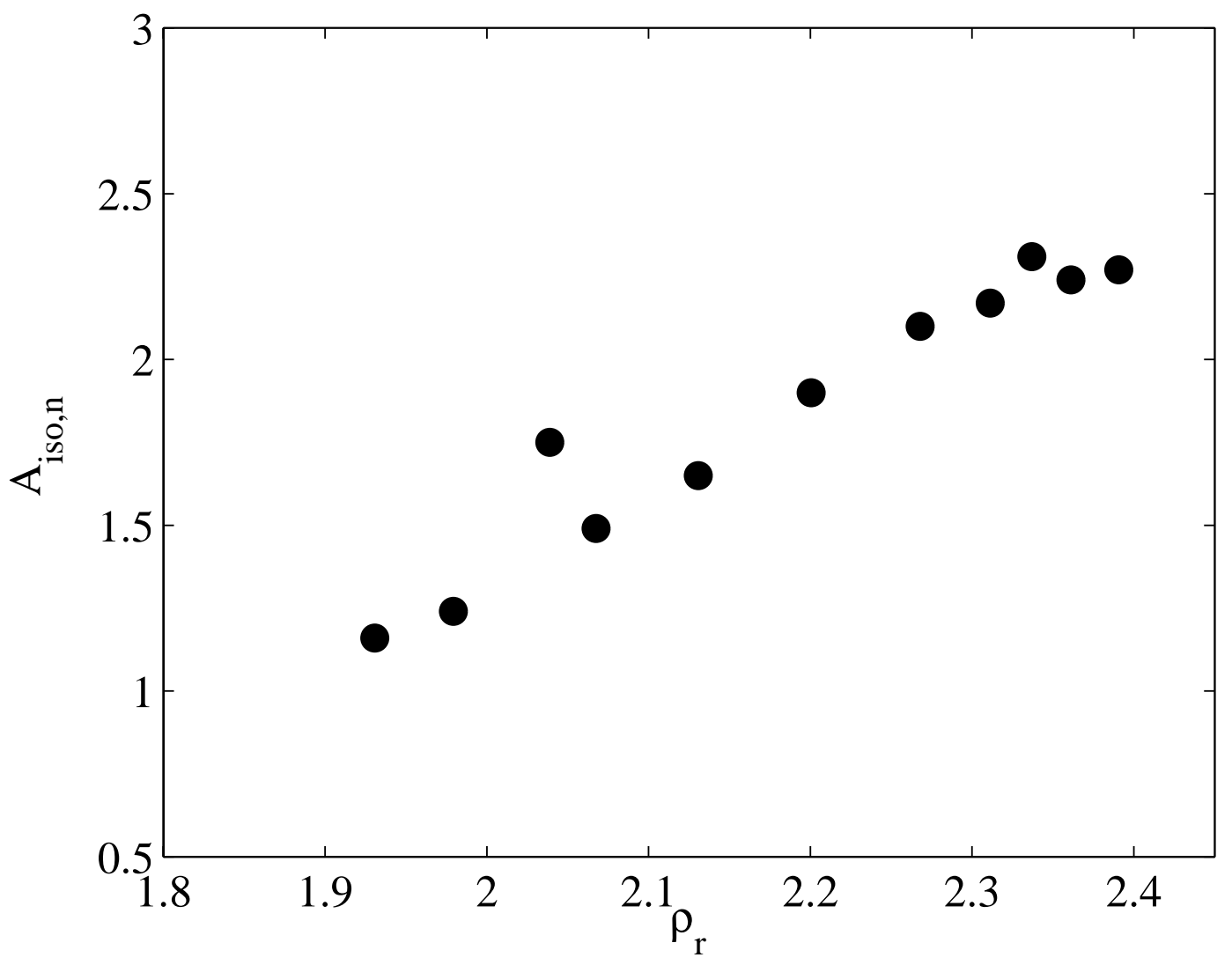

Abbildung 6.15: $A_{\text {iso,n }}$ von $\mathrm{CH}_{2} \mathrm{I}_{2}$ in sc. Xenon $\left(35^{\circ} \mathrm{C}\right)$ nach $300 \mathrm{~nm}$ Anregung und Abfrage bei $405 \mathrm{~nm}[103$ ]

sich für die untersuchten Polyhalogenmethane bei reduzierten Dichten $\rho_{r}>1,6$ eine annähernd lineare Dichteabhängigkeit. Vergleichbare Beobachtungen für die Dichteabhängigkeit von $A_{\text {iso,n }}$ von $\mathrm{CH}_{2} \mathrm{I}_{2}$ konnten in sc. Xe gemacht werden. Der lineare Zusammenhang von $A_{\text {iso,n }}$ und $\rho_{r}$ ist in Abbildung 6.15 dargestellt.

Dieser lineare Zusammenhang ist konsistent mit früheren Messungen von Schwarzer et al. [19] zum Käfigeffekt in überkritischen Lösungsmitteln (s. Abschnitt 2.1). Der Käfigeffekt ist in diesem Fall als ein rein kinematischer Effekt aufzufassen, nach der Dissoziation brechen die Photofragmente entweder direkt aus dem Lösungsmittelkäfig aus oder sie werden nach einem Stoß mit den umgebenden Lösungsmittelmolekülen darin gefangen gehalten. Die Wahrscheinlichkeit eines Käfigausbruchs ist direkt umgekehrt proportional zur Dichte $\rho$ des Lösungsmittels, die des Käfigeinfangs somit proportional zur Dichte $\rho$. Alle primären Photofragmente, welche im Käfig gefangen bleiben, werden im Laufe einiger Pikosekunden zum Isomer rekombinieren. Die Quantenausbeute $\Phi_{\text {iso }}$ bzw. $A_{\text {iso,n }}$ ist somit direkt proportional zur Dichte $\rho$ :

$$
\Phi_{\text {iso }} \propto A_{\text {iso }, \mathrm{n}} \propto \rho
$$




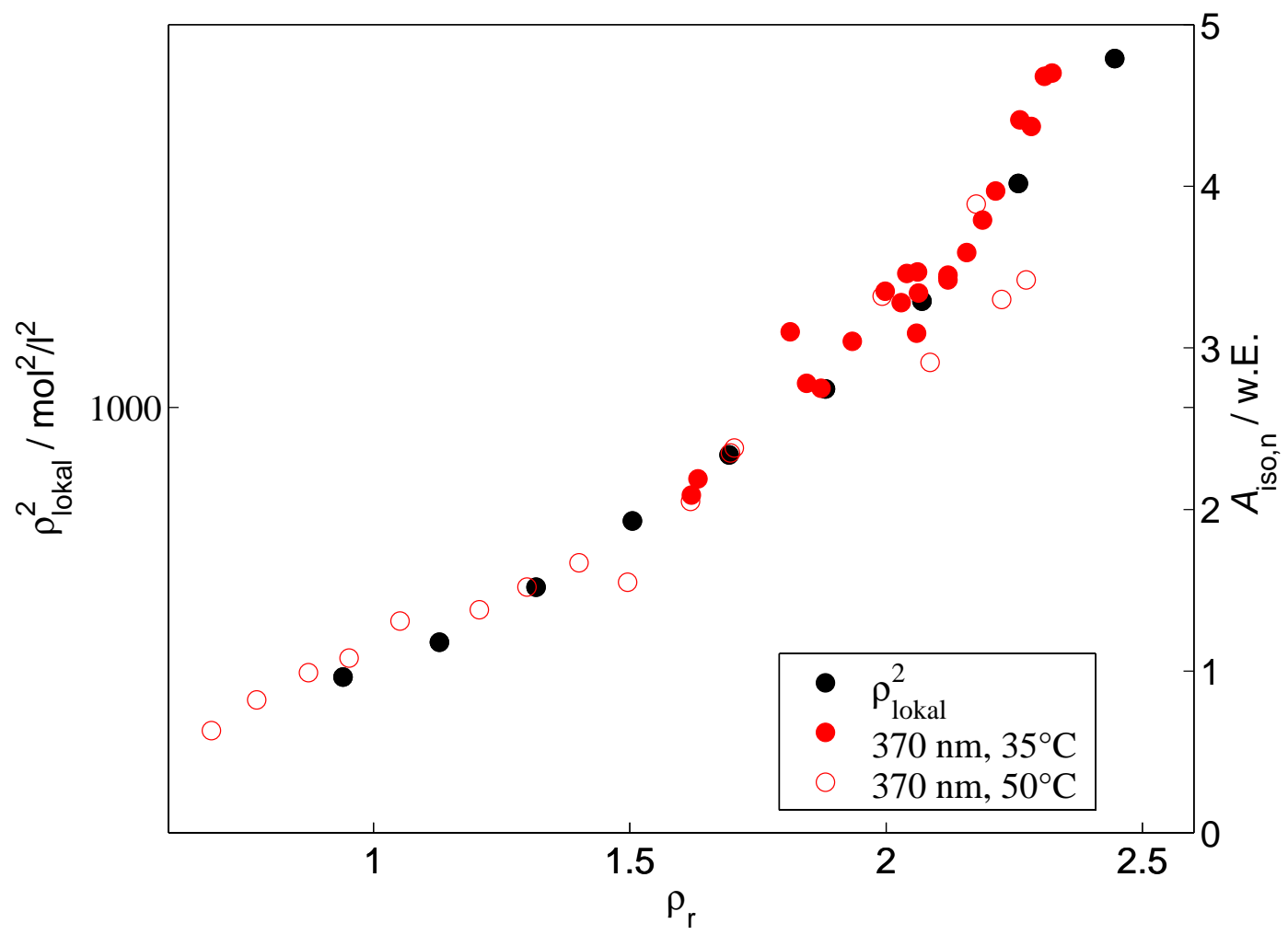

Abbildung 6.16: Vergleich $A_{\text {iso,n }}$ von $\mathrm{CH}_{2} \mathrm{I}_{2}$ in sc. $\mathrm{CO}_{2}\left(35^{\circ} \mathrm{C}\right)$ bei $370 \mathrm{~nm}$ Abfrage mit $\rho_{\text {lokal }}^{2}$

Der annähernd lineare Verlauf von $A_{\text {iso,n }}$ zeigt dabei, daß im gemessenen Dichtebereich von $\rho_{r} \sim 1,6-2,5$ keine lokalen Dichtevergrößerungseffekte eine Rolle spielen, da sonst die typische S-Form von dichteabhängigen Größen in überkritischen Lösungsmitteln zu beobachten sein sollte. Die MD-Simulationen zur radialen Verteilungsfunktion von $\mathrm{CH}_{2} \mathrm{I}_{2}$ in sc. $\mathrm{CO}_{2}$ [86] bestärken diese Beobachtung, da lokale Dichtevergößerungseffekte erst bei Dichten $\rho_{r}<1,5$ auftreten. Die Messungen bei höheren Dichten legen somit nahe, daß nur ein kinematischer Käfigausbruch direkt nach der Dissoziation zur Minderung der Quantenausbeute beiträgt.

Bei niedrigen Dichten $\left(\rho_{r}<1,6\right)$ ist keine lineare Dichteabhängigkeit von $A_{\text {iso,n }}$ zu beobachten. In diesem Dichtebereich spielt die bereits angesprochene diffusive Separation der Photofragmente zunehmend eine Rolle. In früheren Untersuchungen unserer Arbeitsgruppe zur Quantenausbeute der Isomerisierung bei niedrigen Dichten konnte eine nichtlineare Abhängigkeit nachgewiesen werden [86, 103, 196].

In Abbildung 6.16 ist die Dichteabhängigkeit der normierten Endamplitude $A_{\text {iso,n }}$ von $\mathrm{CH}_{2} \mathrm{I}_{2}$ in sc. $\mathrm{CO}_{2}$ (300 nm Anregung und $370 \mathrm{~nm}$ Abfrage) im Vergleich zum Quadrat der lokalen Dichte $\rho_{\text {lokal }}$ aufgetragen. Als Maß für die lokale Dichte wird dabei die aus den MD-Simulationen [86] erhaltene radiale Verteilungsfunktion $g(r)$ herangezogen (s.

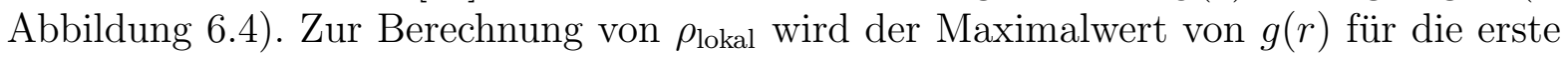
Lösungsmittelschale bestimmt und mit der homogenen Dichte $\rho$ multipliziert. Dabei zeigt 
sich, daß die über einen weiten Dichtebereich von $\rho_{r}=0,7-2,5$ gemessenen normierten Endamplituden $A_{\text {iso,n }}$ direkt proportional zum Quadrat der lokalen Dichte $\rho_{\text {lokal }}$ sind. Dieses Verhalten kann als das Ergebnis zweier konsekutiver Käfigausbrüche verstanden werden, nach dem ersten, rein kinematischen Käfigausbruch, welcher innerhalb einiger hundert Femtosekunden stattfindet, gewinnt bei niedrigen Dichte eine weitere Separation der Photofragmente vor der Isomerbildung zunehmend an Bedeutung. Beide konsekutive Käfigausbrüche mindern die Quantenausbeute:

$$
\Phi_{\text {iso }} \propto A_{\text {iso,n }} \propto \Phi_{1} \cdot \Phi_{2}
$$

Vom ersten, kinematischen Käfigausbruch ist bekannt, daß dieser proportional zur Dichte $\rho_{\text {lokal }}$ stattfindet [19]. Um die Proportionalität von $A_{\text {iso,n }}$ zum Quadrat der lokalen Dichte $\rho_{\text {lokal }}^{2}$ zu erklären, muß der zweite Käfigausbruch demnach auch proportional zur Dichte sein. Zwar deutet eine direkte Proportionalität zur lokalen Dichte auf einen kinematischen Käfigausbruch hin, jedoch ist thermische Äquilibrierung der Translationsenergie bereits nach wenigen oder auch nur einem Stoß mit dem Lösungsmittel abgeschlossen. Ein rein kinematischer Ausbruch kommt für die Photofragmente daher auf der Pikosekundenzeitskala des zweiten Ausbruchs nicht mehr in Frage. In diesem Falle muß ein eher diffusiver Charakter der Separation der Photofragmente angenommen werden. Eine Versuch der Beschreibung dieses diffusiven Prozesses insbesondere seiner Dichteabhängigkeit wurde im vorherigen Abschnitt 6.2.3 vorgenommen. Weiterhin ist die starke CTWechselwirkung der Photofragmente zu berücksichtigen, welche einer diffusiv gesteuerten Separation entgegenwirkt.

\subsection{Spezielle Fälle}

Neben vielen Gemeinsamkeiten der Photoisomerisierung in Lösung konnten bei der photoinduzierten Dynamik verschiedener Polyhalogenmethane auch einige Unterschiede beobachtet werden. Diese Eigenheiten sollen hier in Kürze diskutiert werden.

\subsubsection{Isomerisierung und Zerfall von Chloriodmethan}

Die nach Abspaltung eines Iodatoms innerhalb des Käfigs rekombinierten Isomere von $\mathrm{CH}_{2} \mathrm{I}_{2}, \mathrm{CHI}_{3}$ und $\mathrm{CH}_{2} \mathrm{BrI}$ sind auf der beobachteten Pikosekundenzeitskala stabil. Ihr Zerfall findet innerhalb von einigen Nanosekunden statt und kann im Rahmen des Experiments nicht beobachtet werden. Im Gegensatz dazu ist das aus $\mathrm{CH}_{2} \mathrm{ClI}$ gebildete $\mathrm{CH}_{2} \mathrm{Cl}$-I-Isomer instabil, es zerfällt im Laufe einiger hundert Pikosekunden.

Die Absorptionszeitprofile von $\mathrm{CH}_{2} \mathrm{ClI}$ in Acetonitril nach $258 \mathrm{~nm}$ Anregung, welche in Abbildung 5.27 dargestellt sind, zeigen das gewohnte Bild der Photoisomerisierung mit schnellem Anstieg, Abfall und einen wellenlängenabhängigen Anstieg von 4,5-12,5 ps. 


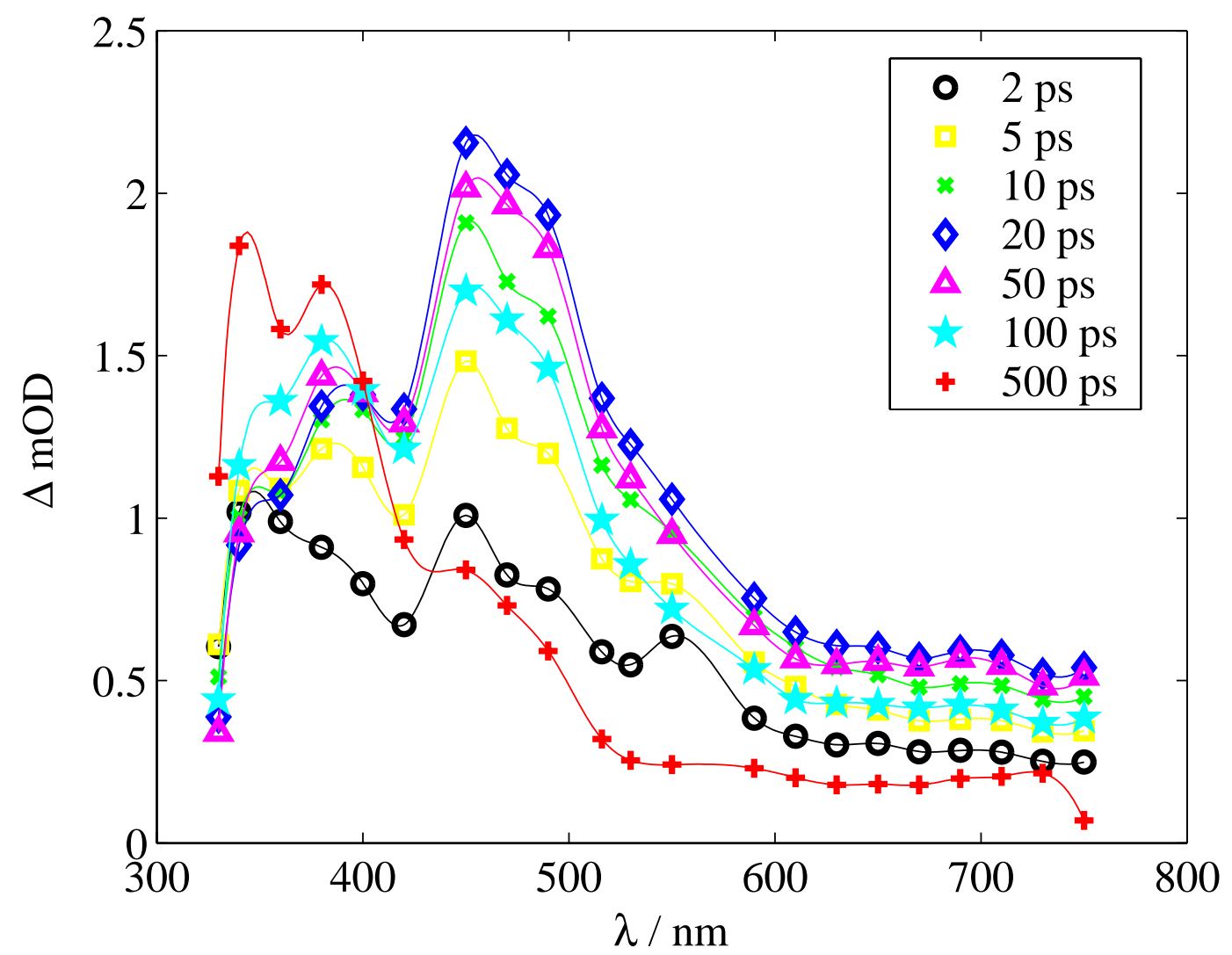

Abbildung 6.17: Rekonstruiertes, zeitaufgelöstes Spektrum von $\mathrm{CH}_{2} \mathrm{ClI}$ in Acetonitril nach $258 \mathrm{~nm}$ Anregung

Daran schließt sich ein Abfall mit einigen hundert Pikosekunden an. Anhand der Absorptionszeitprofile wurde ein zeitaufgelöstes Spektrum rekonstruiert, welches in Abbildung 6.17 wiedergegeben ist. Eine Populationsnormierung wie sie für andere Polyhalogenmethane vorgenommen wurde, ist in diesem Fall nicht sinnvoll, da das Isomer wieder zerfällt und somit nach absehbarer Zeit auch andere Spezies zur transienten Absorption beitragen.

Im zeitaufgelösten Spektrum in Acetonitril wächst innerhalb einiger Pikosekunden die Isomerbande bei $470 \mathrm{~nm}$ heran (vergleichbar zum Matrixspektrum in Abbildung 3.12), um danach im Laufe von etwa 150-200 ps wieder abzufallen. Zu langen Verzögerungszeiten (500 ps) wächst eine starke Absorption bei etwa $360 \mathrm{~nm}$ an, wahrscheinlich das primäre Zerfallsprodukt des $\mathrm{CH}_{2} \mathrm{Cl}$-I-Isomers. Somit verläuft der Bildungsmechanismus analog zu den anderen bisher behandelten Polyhalogenmethanen, nach der schnellen Photodissoziation findet innerhalb des Lösungsmittelkäfigs zuerst die Bildung des CT-Komplexes statt. Diesem kann bei jeder gemessenen Wellenlänge von 330-750 nm die Kurzzeitabsorption zu frühen Reaktionszeiten (2ps) zugeordnet werden. Anschließend findet die Schwingungsenergierelaxation (VER) des heißen $\mathrm{CH}_{2} \mathrm{Cl}$-Radikals statt, bis schlußendlich das $\mathrm{CH}_{2} \mathrm{Cl}$-I-Isomer gebildet werden kann. Messungen der transienten Absorption in unpolaren und überkritischen Lösungsmitteln stützen diese Aussage. Die in Abbildung 5.28 


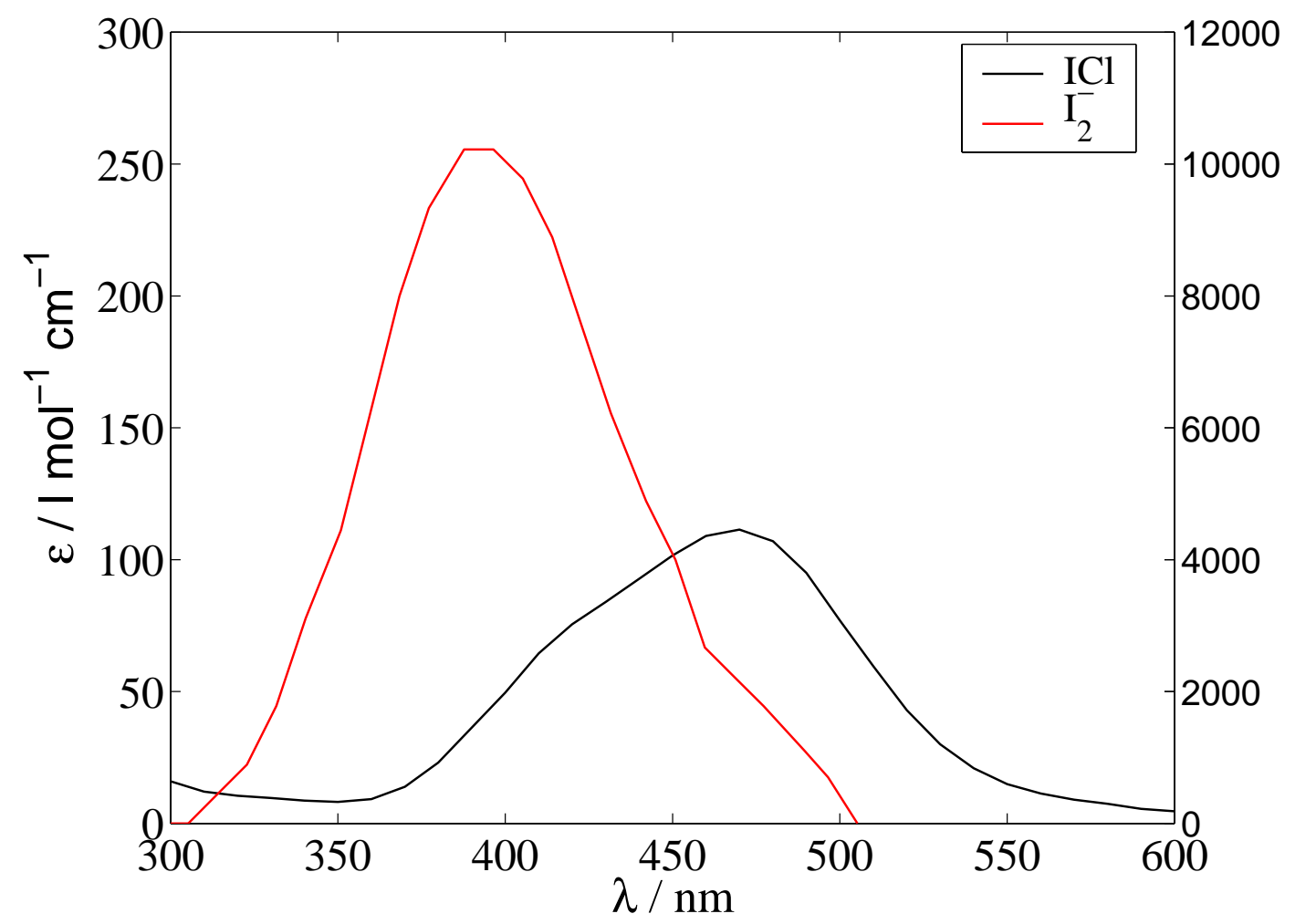

Abbildung 6.18: Absorptionsspektrum von ICl (rechte Skala) in der Gasphase und $\mathrm{I}_{2}^{-}$ (linke Skala) in Acetonitril

und 5.29 dargestellten Absorptionszeitprofile für $\mathrm{CCl}_{4}$ und $n$-Hexan sind sich sehr ähnlich, sie unterscheiden sich vornehmlich in der Zeitkonstanten des langsamen Anstiegs, welcher der durch Schwingungsenergierelaxation begrenzten Isomerbildung zugeordnet wird. Die Anstiegszeit beträgt für $\mathrm{CCl}_{4} 5,1 \mathrm{ps}$, während sie in $n$-Hexan als einem für die Schwingungsenergierelaxation effizienteren Lösungsmittel [207] lediglich 3,5 ps beträgt. Einen ähnlichen Trend zeigt der Vergleich der beiden überkritischen Lösungsmittel $\mathrm{CHF}_{3}$ und Ethan, bei vergleichbarer Dichte ist die Anstiegszeit mit 11,9 ps in $\mathrm{CHF}_{3}$ wesentlich langsamer als in Ethan mit 5,5 ps. Die bei niedrigen Dichten beobachtete diffusive Separation der im Käfig gefangenen Photofragmente vor der Rekombination, ist bei den hohen Dichten der gemessenen überkritischen Lösungsmittel sowie in flüssiger Lösung auszuschließen.

Tarnovsky et al. ordnen der Absorptionsbande bei $360 \mathrm{~nm}$, welche durch den Zerfall des $\mathrm{CH}_{2} \mathrm{Cl}-\mathrm{I}$ entsteht, $\mathrm{ICl}^{-}$als primäres Zerfallsprodukt zu. Ein Absorptionsspektrum von $\mathrm{ICl}^{-}$ist in der Literatur leider nicht bekannt, lediglich die Absorptionsmaxima wurden von Shida et al. [208] durch $\gamma$-Bestrahlung von ICl zu 385, 737 und $1030 \mathrm{~nm}$ bestimmt. Allerdings ist anzunehmen, daß das Absorptionsspektrum von $\mathrm{ICl}^{-}$dem von $\mathrm{I}_{2}^{-}$(s. Abbildung 6.18) sehr ähnlich ist. Dieses zeigt ebenfalls bei $385 \mathrm{~nm}$ eine sehr starke Absorptionsbande. 
Die Zuordnung der neu entstehenden Bande bei $360 \mathrm{~nm}$ nach Tarnovsky erscheint schlüssig. Wie in Abbildung 6.18 zu sehen, besitzt $\mathrm{ICl}^{-}$einen sehr großen Extinktionskoeffizienten, selbst wenn dieser Zerfallskanal nicht der dominierende ist, kann auch wenig gebildetes $\mathrm{ICl}^{-}$einen signifikanten Anteil zur transienten Absorption leisten. Die von Tarnovsky diskutierte mögliche Bildung von $\mathrm{I}_{2}^{-}$erscheint auf einer Zeitskala von etwa 200 ps eher unwahrscheinlich. Ein Blick auf die Absorptionszeitprofile in polaren und überkritischen Lösungsmitteln zeigt jedoch, daß neben $\mathrm{ICl}^{-}$noch andere Zerfalls- und Photoprodukte eine Rolle spielen können. So bleibt in $\mathrm{CCl}_{4}$ und $n$-Hexan bei einer Probewellenlänge von $460 \mathrm{~nm}$ nach dem langsamen Pikosekundenabfall ein signifikanter off-set stehen (s. Abbildung 5.28 und 5.29). Beim Zerfall anderer Isomere der Polyhalogenmethane konnte beobachtet werden, daß in unpolaren Lösungsmitteln keine geladenen Zerfallsprodukte gebildet werden (s. Abschnitt 3.5.2). Somit kann auch eine neutrale Spezies für diese Absorption zu langen Verzögerungszeiten bei dieser Wellenlänge verantwortlich sein. Ein denkbares Photoprodukt ist hierbei ICl, welches durch Zwei-Photonen-Absorption bei der Photoanregung vom $\mathrm{CH}_{2} \mathrm{ClI}$ abgespalten wird. Im Gegensatz zu $\mathrm{CH}_{2} \mathrm{I}_{2}$ ist die ZweiPhotonen-Absorption und Dissoziation in ein Carben-Radikal und ein ICl bzw. $\mathrm{I}_{2}$ für $\mathrm{CH}_{2} \mathrm{ClI}$ nicht symmetrieverboten (s. Abschnitt 3.2.5), somit kann ein Teil der Moleküle nach Photoanregung ICl abspalten. Dessen Absorptionsspektrum ist in Abbildung 6.18 dargestellt, das Absorptionsmaximum liegt bei $475 \mathrm{~nm}$ und kann somit zum konstanten end off-set in $\mathrm{CCl}_{4}, \mathrm{CHF}_{3}, n$-Hexan und Ethan beitragen. Eine genaue Betrachtung des zeitaufgelösten Spektrums in Acetonitril (s. Abbildung 6.17) zeigt zudem, daß das Spektrum zu langen Verzögerungszeiten (500 ps) auf der roten Flanke der Bande bei $360 \mathrm{~nm}$ im Bereich von 420-500 nm eine leichte Schulter besitzt. Dieser Wellenlängenbereich entspricht ziemlich genau dem Absorptionsspektrum von ICl, somit ist ein Zwei-PhotonenProzeß denkbar, welcher zur Abspaltung von ICl parallel zur Photodissoziation in ein $\mathrm{CH}_{2} \mathrm{Cl}$-Radikal und ein Iodatom führt.

\subsubsection{Photodynamik von Bromiodmethan nach $258 \mathrm{~nm}$ Anregung}

Der Vergleich der Absorptionszeitprofile von $\mathrm{CH}_{2} \mathrm{BrI}$ nach $258 \mathrm{~nm}$ (s. Abbildung 5.6) und $288 \mathrm{~nm}$ Anregung (s. Abbildung 5.7) zeigt, daß die Absorption nach $258 \mathrm{~nm}$ Anregung nach Erreichen der Endamplitude im Laufe einiger Hundert Pikosekunden wieder abfällt. Nach $288 \mathrm{~nm}$ Anregung ist dieser Abfall nicht zu beobachten, die Absorption bleibt bis zu einer Verzögerung von 400 ps konstant. Die Beobachtung des Abfalls nach $258 \mathrm{~nm}$ Anregung ist auch nach mehrfacher Justage der Verschiebebühne reproduzierbar, so daß nicht von einem Artefakt durch ungenügende Bühnenjustage auszugehen ist.

Für die Photodissoziation von $\mathrm{CH}_{2} \mathrm{BrI}$ ist bekannt, daß bei $258 \mathrm{~nm}$ neben der Anregung des C-I-Chromophor auch zu etwa 14 v. H. Bromatome abgespalten werden (s. Abschnitt 3.2.3). Bei $288 \mathrm{~nm}$ Anregung werden wie bei den anderen untersuchten Polyhalogenmethanen nur Iodatome abgespalten. Somit kann sich bei $258 \mathrm{~nm}$ Anregung sowohl das $\mathrm{CH}_{2} \mathrm{Br}-\mathrm{I}$ - und als auch das $\mathrm{CH}_{2} \mathrm{I}-\mathrm{Br}$-Isomer bilden, das $\mathrm{CH}_{2} \mathrm{Br}-\mathrm{I}$ ist dabei thermodynamisch stabiler. Der Abfall der Absorption kann daher dem Zerfall des $\mathrm{CH}_{2} \mathrm{I}-\mathrm{Br}$ 
zugeordnet werden, nach $258 \mathrm{~nm}$ Anregung werden beide Isomere innerhalb des Lösungsmittelkäfigs gebildet, anschließend zerfällt das $\mathrm{CH}_{2} \mathrm{I}-\mathrm{Br}$ innerhalb etwa $150 \mathrm{ps}$, während die Absorption des $\mathrm{CH}_{2} \mathrm{Br}-\mathrm{I}$ mit einer Lebensdauer von etwa 2,5 ns auf der beobachteten Pikosekundenzeitskala konstant bleibt.

Im Vergleich zu den anderen Polyhalogenmethanen ist das rekonstruierte, zeitaufgelöste Spektrum des $\mathrm{CH}_{2} \mathrm{BrI}$ (s. Abbildung 5.9) auch relativ breit und zeigt keinen eindeutigen Peak, sondern zwei lokale Maxima bei 380 und $420 \mathrm{~nm}$. Diese lokalen Maxima können den Absorptionsmaxima der beiden Isomere zugeordnet werden. Das Absorptionsspektrum des instabileren $\mathrm{CH}_{2} \mathrm{I}-\mathrm{Br}$ ist dabei etwas rotverschoben, im zeitaufgelösten Spektrum fällt die Absorption bei Wellenlängen $<430 \mathrm{~nm}$ im Laufe einiger Hundert Pikosekunden ab. Die Bildung der beiden Photoisomere kann anhand der gemessenen Absorptionszeitprofile nicht unterschieden werden. Der langsame Anstieg, welcher mit der Isomerbildung verknüpft wird, läßt sich monoexponentiell anpassen. Somit findet die Bildung der beiden Isomere mit zumindest sehr ähnlichen Zeitkonstanten statt.

\subsubsection{Photoinduzierte Heterolyse von lodoform in Lösung}

Transiente Absorptionsmessungen von $\mathrm{CHI}_{3}$ in Acetonitril bei $267 \mathrm{~nm}$ zeigen einige interessante Besonderheiten, welche bei anderen Polyhalogenmethanen in dieser Form nicht nachgewiesen werden konnten. So ist in Abbildung 5.23 ein typisches Absorptionszeitprofil von $\mathrm{CHI}_{3}$ in Acetonitril dargestellt. Der Pumppuls lag dabei mit $267 \mathrm{~nm}$ in der ersten Absorptionsbande des $\mathrm{CHI}_{3}$, während der Probepuls auf der roten Flanke des Absorptionsspektrums des Muttermoleküls bei $370 \mathrm{~nm}$ Probewellenlänge eingestrahlt wurde. Dabei zeigt sich ein typisches transientes Signal der Isomerisierungsreaktion mit den drei wohlbekannten Zeitkomponenten (Anfangspeak und anschließender langsamer Anstieg). Ein Ausbleichen, welches Rückschlüsse auf eine mögliche geminale Rekombination erlaubt, konnte nicht beobachtet werden. Durch Verschieben der Kollimatorlinse des Kepler-Teleskops der Frequenzverdopplung kann nun der Fokusdurchmesser in der Meßzelle variiert werden. Eine Verkleinerung des Fokusdurchmessers in der Meßzelle bewirkte ein gänzlich anderes transientes Signal, dieses ist in Abbildung 5.24 dargestellt.

Dieses Absorptionszeitprofil setzt sich aus einem von der Zeitauflösung begrenzten Abfall in den negativen Bereich und anschließender Relaxation dieses negativen Signals innerhalb einiger Pikosekunden zusammen. Das Verhältnis der negativen Anfangsabsorption und der Amplitude des Pikosekundenanstiegs hängt dabei vom Fokusdurchmesser ab. Je kleiner der Fokus gewählt wird, desto stärker ist die anfängliche negative Absorption und je geringer der langsame Anstieg. Der langsame Anstieg weist mit etwa 4 ps eine sehr ähnliche Zeitkonstante wie die der Isomerbildung in Acetonitril auf. Wahrscheinlich handelt es sich hierbei um denselben Prozeß. Das Signal setzt sich somit aus dem bekannten Isomersignal mit seinem charakteristischen Pikosekundenanstieg sowie einem anfänglichen negativen Signal in der Art eines Ausbleichens zusammen. In Abbildung 6.19 ist das Verhältnis der Amplitude des Pikosekundenanstiegs $A_{\text {iso }}$ und der Amplitude des schnellen Abfalls $A_{\text {Ausbleich }}$ in Abhängigkeit vom anfänglichen negativen Signal $\triangle \mathrm{m} O D_{\text {neg }}$ aufgetragen. Dabei zeigt sich, daß der Anteil der Amplitude des langsamen Anstiegs mit 


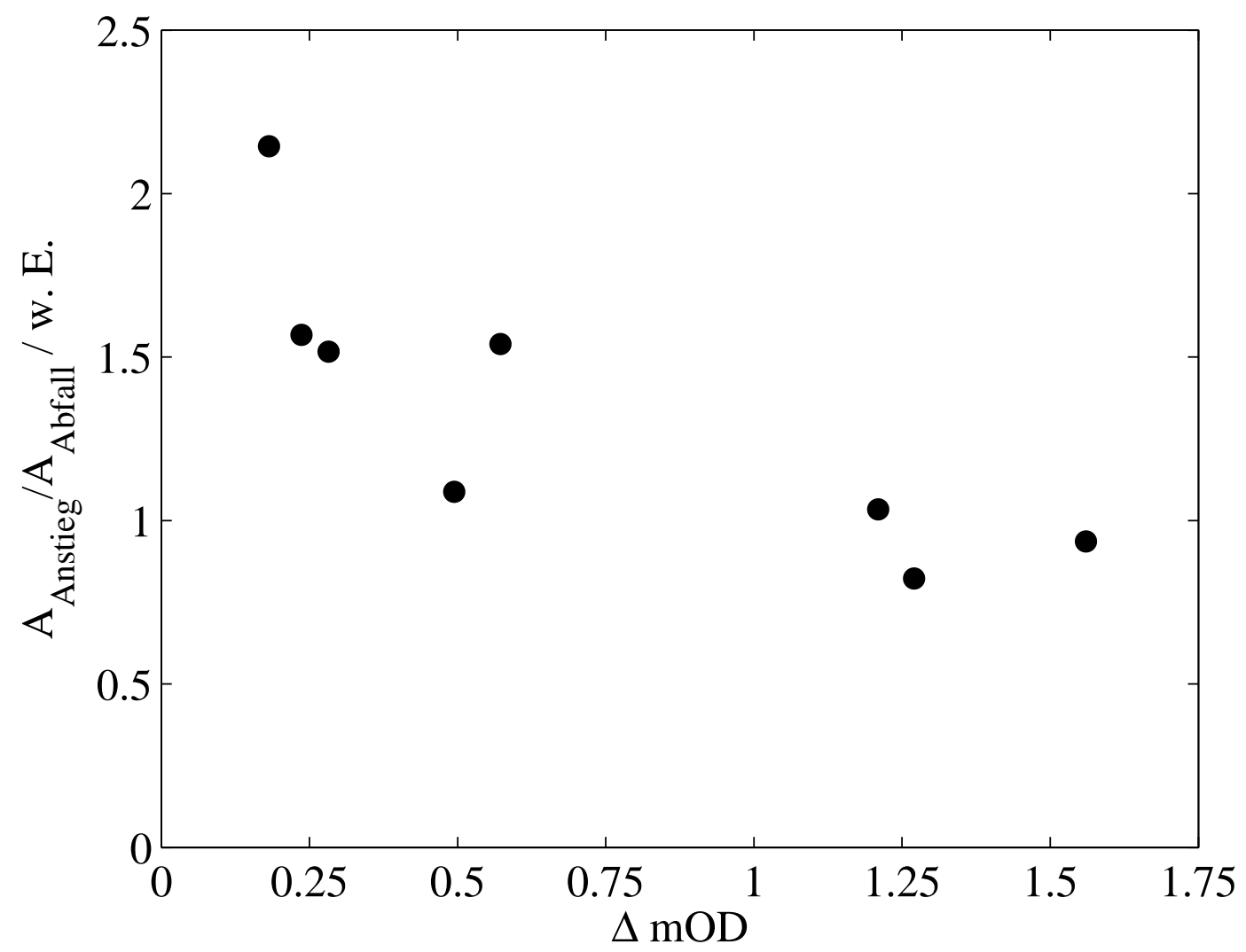

Abbildung 6.19: Amplitudenverhältnis des langsamen Anstiegs $A_{\text {Anstieg }}$ und des schnellen Abfalls $A_{\text {Abfall }}$ in Abhängigkeit vom anfänglichen Ausbleichsignal

zunehmender negativer Anfangsabsorption abnimmt. Das anfängliche Ausbleichen ist somit ein Konkurrenzprozeß zur Isomerbildung, welcher durch eine Verkleinerung des Fokusdurchmessers initiiert wird.

Weiterhin ändert sich das Absorptionsspektrum der mit kleinem Fokusdurchmesser bestrahlten Lösung stark. Nach UV-Bestrahlung mit größerem Fokusdurchmesser $(1 \mathrm{~h}$ bei $36 \mu \mathrm{W})$ und Detektion des bekanten Isomersignals wie in Abbildung 5.23, zeigt das Absorptionsspektrum weiterhin die Banden von $\mathrm{CHI}_{3}$ und die intensive Bande von $\mathrm{I}_{2}$ bei $475 \mathrm{~nm}$ als Zersetzungsprodukt des Muttermoleküls, welches in Lösung $\mathrm{I}_{2}$ abspaltet (s. Abbildung 6.20). Bei Bestrahlung mit kleinem Fokusdurchmesser (1h bei $42 \mu \mathrm{W})$ ändert sich das Absorptionsspektrum der bestrahlten Lösung weitaus stärker (s. Abbildung 6.20). Die $\mathrm{I}_{2}$-Bande erscheint nicht im Spektrum, während die Bande des Muttermoleküls bei $300 \mathrm{~nm}$ stark anwächst und die Bande bei $350 \mathrm{~nm}$ auf der roten Flanke sehr viel breiter wird. Diese signifikante Änderung des Absorptionsspektrums und somit der Photoprodukte nur durch Variation des Fokusdurchmessers deutet auf zwei unterschiedliche photoinduzierte Prozesse hin. Somit kommen insbesondere Zwei-Photonen-Prozesse in Frage, da eine Verkleinerung des Fokusdurchmessers zugleich die Intensität und die Wahrscheinlichkeit eines nicht-linearen Prozesses erhöht. Das Fehlen der $\mathrm{I}_{2}$-Bande zeigt weiterhin, daß die Photoprodukte mit dem $\mathrm{I}_{2}$ reagieren müssen, weil $\mathrm{CHI}_{3}$ in Lösung 


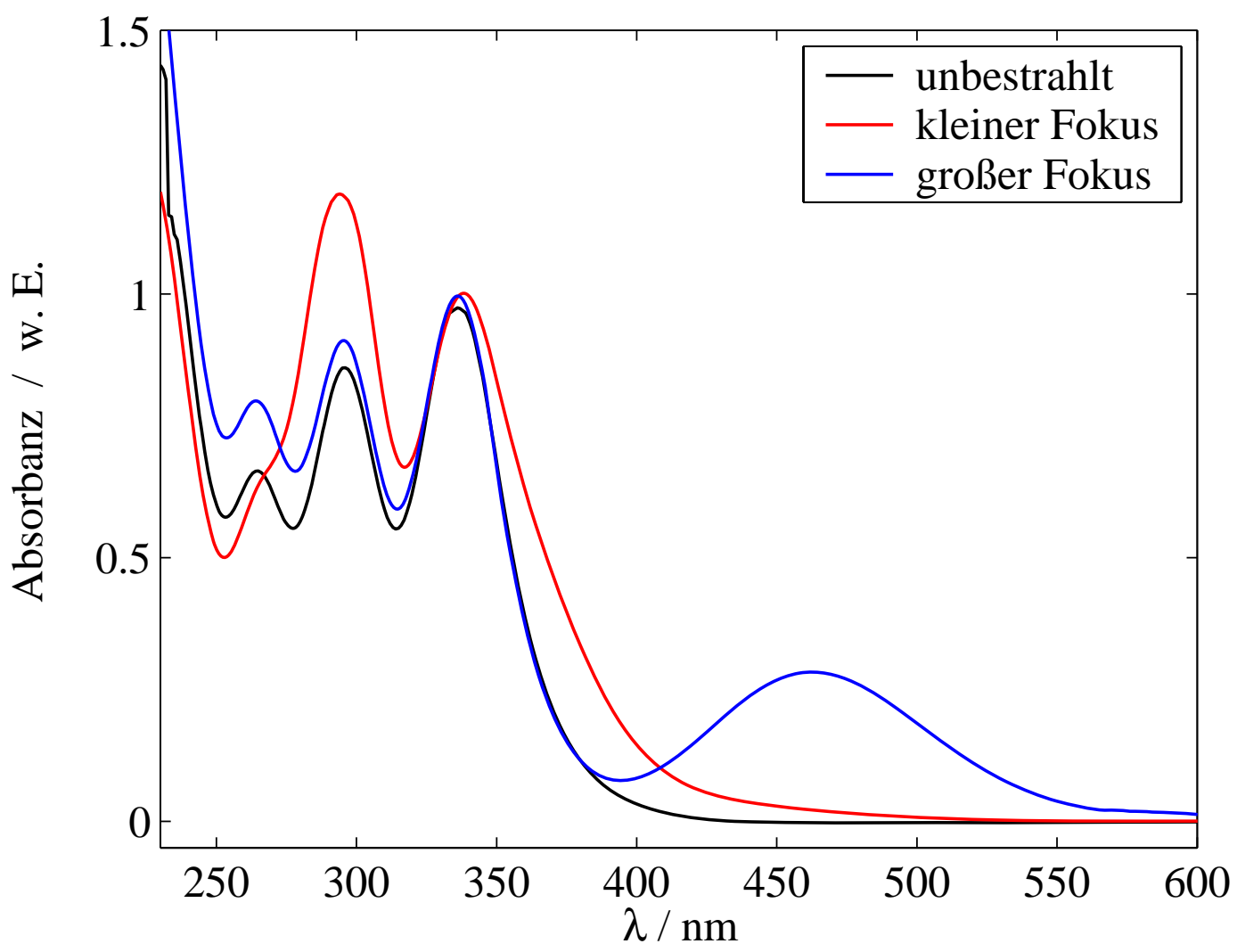

Abbildung 6.20: Absorptionsspektren von $\mathrm{CHI}_{3}$ vor und nach Photolyse bei $267 \mathrm{~nm}$ bei kleinem und großen Fokusdurchmesser in Acetonitril

auch ohne Bestrahlung immer etwas molekulares Iod abspaltet. Wie bereits in Abschnitt 3.2.5 erläutert, ist die Wahrscheinlichkeit einer Zwei-Photonen-Absorption von $\mathrm{CHI}_{3}$ aus Symmetriegründen wesentlich größer als die von $\mathrm{CH}_{2} \mathrm{I}_{2}$. Allerdings zeigt das Produktspektrum keine $\mathrm{I}_{2}$-Bande, so daß nicht von einer Fragmentierung in $\mathrm{CHI}$ und $\mathrm{I}_{2}$ ausgegangen werden kann.

Über die höheren Zustände, welche durch Zwei-Photonen-Absorption beim Iodoform besetzt werden können, ist nicht viel bekannt (s. Abschnitt 3.2.5). Eine Zwei-PhotonenAbsorption bei Anregung von $267 \mathrm{~nm}$ entspricht einer Anregungsenergie von 9,29 eV, damit sind entweder hohe Rydbergzustände oder ionische Zustände erreichbar. Im Falle eines neutralen Kanals könnte ein Rydbergzustand besetzt werden, infolge dessen das $\mathrm{CHI}_{3}$ wie gehabt in ein $\mathrm{CHI}_{2}$-Radikal und ein Iodatom dissoziert, die Photofragmente aber so viel Überschußenergie besitzen, daß sie nicht zum Isomer rekombinieren, sondern vollständig aus dem Lösungsmittelkäfig ausbrechen:

$$
\mathrm{CHI}_{3} \stackrel{2 \mathrm{~h} \nu}{\longrightarrow} \mathrm{CHI}_{2}+\mathrm{I}
$$

Anschließend reagieren die Photofragmente mit anderen Reaktionspartnern in der Lösung. Dazu wurden für mögliche Reaktionen mit Hilfe der von Marshall et al. [94] be- 
rechneten thermodynamischen Daten halogenierter Methane die Reaktionsenthalpien berechnet.

Das $\mathrm{CHI}_{2}$-Radikal kann z. B. mit dem nicht zerfallenen $\mathrm{CHI}_{3}$ reagieren:

$$
\mathrm{CHI}_{2}+\mathrm{CHI}_{3} \longrightarrow \mathrm{CH}_{2} \mathrm{I}_{2}+\mathrm{CI}_{3} \quad \Delta \mathrm{H}_{\mathrm{R}}=-8 \mathrm{~kJ} \mathrm{~mol}^{-1}
$$

Das dabei entstehende $\mathrm{CI}_{3}$ reagiert anschließend mit den anderen Photoprodukten in Lösung:

$$
\begin{array}{ll}
\mathrm{CI}_{3}+\mathrm{I} \longrightarrow \mathrm{CI}_{4} & \Delta \mathrm{H}_{\mathrm{R}}=-154,4 \mathrm{~kJ} \mathrm{~mol}^{-1} \\
\mathrm{CI}_{3}+\mathrm{CHI}_{3} \longrightarrow \mathrm{CI}_{4}+\mathrm{CHI}_{2} & \Delta \mathrm{H}_{\mathrm{R}}=+34,1 \mathrm{~kJ} \mathrm{~mol}^{-1} \\
2 \mathrm{CI}_{3} \longrightarrow \mathrm{I}_{3} \mathrm{C}-\mathrm{CI}_{3} &
\end{array}
$$

Nach Reaktionsgleichung R20 entsteht dabei $\mathrm{CH}_{2} \mathrm{I}_{2}$, dieses besitzt eine Absorptionsbande bei $300 \mathrm{~nm}$ (s. Abbildung 3.2), welche für das Ansteigen der Bande bei $300 \mathrm{~nm}$ im Produktspektrum (s. Abbildung 6.20 nach Photolyse unter Zwei-Photonen-Bedingungen (kleiner Pumpfokus) verantwortlich sein kann. Allerdings kann keine der im Reaktionsschema vorgeschlagenen Spezies die Verbreiterung der Bande bei $350 \mathrm{~nm}$ und das Verschwinden der $\mathrm{I}_{2}$-Bande bei $475 \mathrm{~nm}$ erklären.

Anstelle des neutralen Kanals ist wohl eher mit einer Zwei-Photonen-Ionisation des $\mathrm{CHI}_{3}$ zu rechnen, z. B. als Heterolyse:

$$
\mathrm{CHI}_{3} \stackrel{2 \mathrm{~h} \nu}{\longrightarrow} \mathrm{CHI}_{2}^{+}+\mathrm{I}^{-}
$$

Mit der Bildungsenthalpie von 9,77 eV für $\mathrm{CHI}_{2}^{+}$nach der Reaktion [209]

$$
\mathrm{CHI}_{3} \stackrel{\mathrm{h} \nu}{\longrightarrow} \mathrm{CHI}_{2}^{+}+\mathrm{e}^{-}+\mathrm{I}
$$

und der Elektronenaffinität eines Iodatoms (3,06 eV [193]) kann die Enthalpie für die Gasphasenheterolyse zu 6,71 eV berechnet werden. Dies entspricht einer Anregungswellenlänge von $185 \mathrm{~nm}$ bzw. einem Zwei-Photonen-Prozeß bei $370 \mathrm{~nm}$, ist also bei einer Anregung von $267 \mathrm{~nm}$ möglich. Dazu wird in Lösung noch die Solvatationenergie $\Delta G_{\text {Solv }}$ nach Abschluß der Solvatation frei. Das durch die Heterolyse gebildete $\mathrm{I}^{-}$kann nun mit dem vom $\mathrm{CHI}_{3}$ abgespaltenen $\mathrm{I}_{2}$ reagieren:

$$
\mathrm{I}^{-}+\mathrm{I}_{2} \longrightarrow \mathrm{I}_{3}^{-}
$$

Das Triiodid-Ion $\mathrm{I}_{3}^{-}$besitzt zwei intensive Absorptionsbanden bei 300 und $375 \mathrm{~nm}$. Diese können für das starke Anwachsen der Bande bei $300 \mathrm{~nm}$ und die Verbreiterung der Bande bei $350 \mathrm{~nm}$ verantwortlich gemacht werden. Desweiteren erklärt Reaktionsgleichung R26 die Abwesenheit der $\mathrm{I}_{2}$-Bande im Produktspektrum, da das molekulare Iod aus dem $\mathrm{CHI}_{3}$-Zerfall direkt mit dem $\mathrm{I}^{-}$aus der Heterolyse reagiert. Die Extinktionskoeffizienten von $\mathrm{CHI}_{3}$ und $\mathrm{I}_{3}^{-}$in Acetonitril können der Literatur entnommen werden [99], somit kann das gemessene Produktspektrum aus den Einzelspektren zusammengesetzt und deren Konzentrationsverhältnis bestimmt werden. In Abbildung 6.21 sind die Absorptionsspektren der bestrahlten Lösung, von $\mathrm{CHI}_{3}$ und $\mathrm{I}_{3}^{-}$sowie eine Kombination aus 


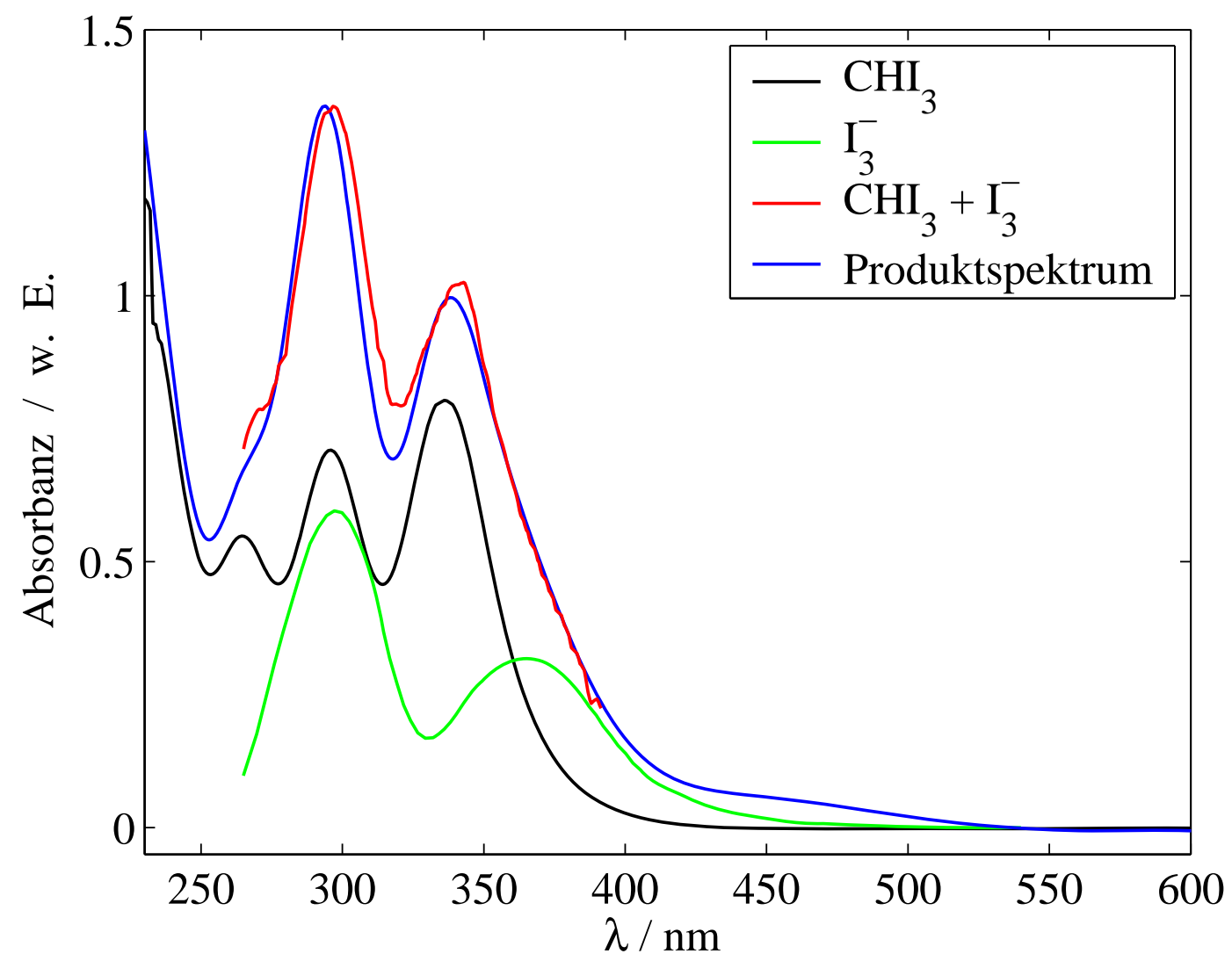

Abbildung 6.21: Absorptionsspektren von $\mathrm{CHI}_{3}$ vor und nach Photolyse bei $267 \mathrm{~nm}, \mathrm{I}_{3}^{-}$ und simuliertes Produktspektrum aus $\mathrm{CHI}_{3}$ und $\mathrm{I}_{3}^{-}$in Acetonitril

$\mathrm{CHI}_{3}$ und $\mathrm{I}_{3}^{-}$im Konzentrationsverhältnis 38:1 dargestellt. Dabei zeigt das aus $\mathrm{CHI}_{3}$ und $\mathrm{I}_{3}^{-}$zusammengesetzte Spektrum eine sehr gute Übereinstimmung mit dem nach Photolyse unter Zwei-Photonen-Bedingungen gemessenen Produktspektrum. Nach $1 \mathrm{~h}$ Photolyse bei $267 \mathrm{~nm}\left(E_{\mathrm{Puls}}=40 \mathrm{~nJ}\right)$ sind somit $2,5 \mathrm{v}$. H. des $\mathrm{CHI}_{3}$ zerfallen.

Die Messungen von $\mathrm{CHI}_{3}$ in Acetonitril unter Zwei-Photonen-Absorptionsbedingungen (kleiner Pumpfokus) lassen sich demnach wie folgt interpretieren:

- Durch Zwei-Photonen-Absorption wird eine Heterolyse des $\mathrm{CHI}_{3}$ bewirkt (s. Reaktionsgleichung R24

- Das dadurch bewirkte Ausbleichen ist in den Absorptionszeitprofilen als schneller Abfall und anschließendes negatives Signal zu beobachten

- Parallel dazu findet eine Isomerisierung nach Ein-Photonen-Absorption statt, welche als der langsame Anstieg in den Absorptionszeitprofilen erkennbar ist

- Das nach Heterolyse gebildete $\mathrm{I}^{-}$reagiert nach R26 zu I $\mathrm{I}_{3}^{-}$und bewirkt die Änderungen des beobachteten Produktspektrums 


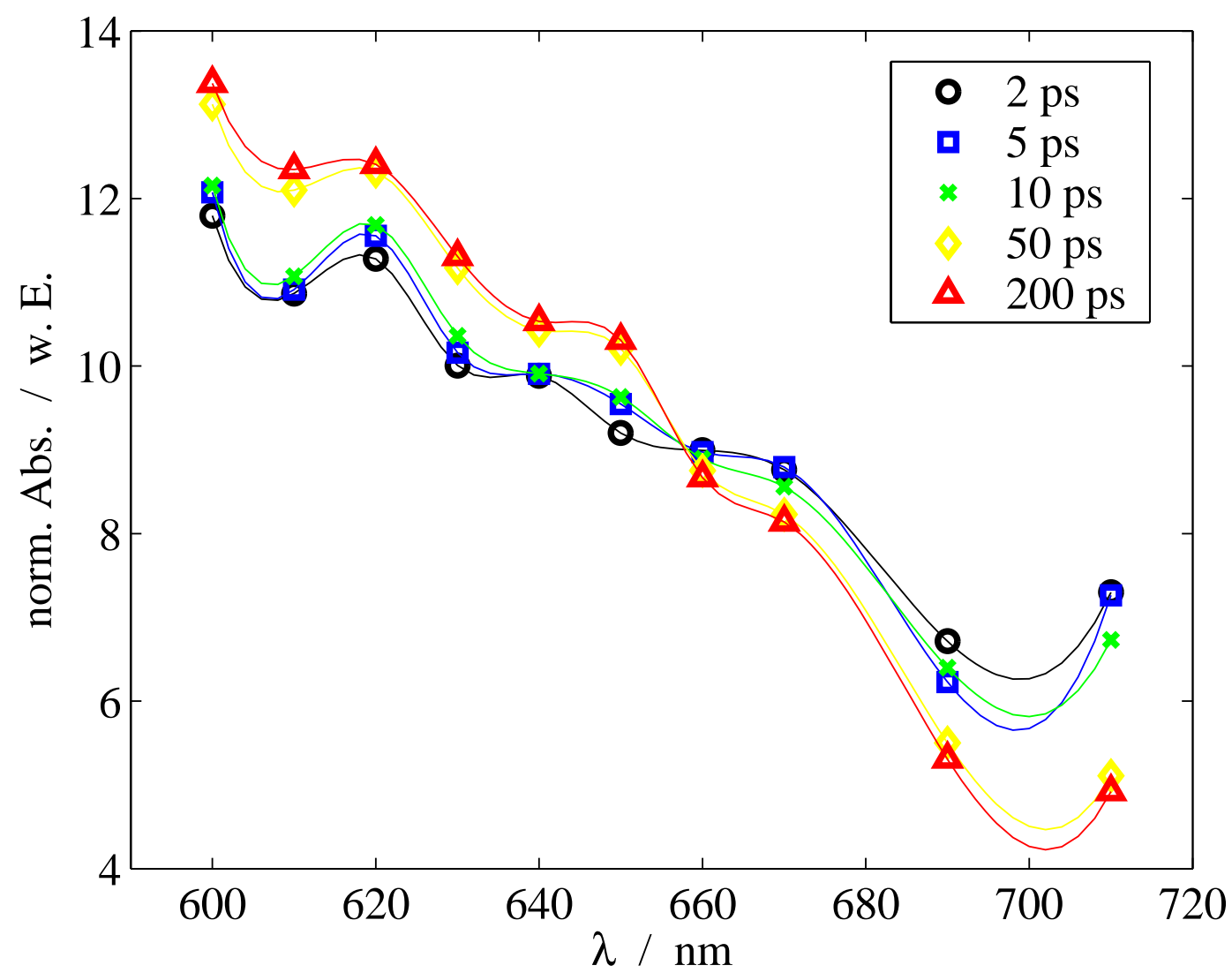

Abbildung 6.22: Normiertes zeitaufgelöstes Spektrum von $\mathrm{CHI}_{3}$ in sc. $\mathrm{CO}_{2}\left(35^{\circ} \mathrm{C}\right.$ 505 bar) nach $267 \mathrm{~nm}$ Anregung

\subsubsection{Niederdruckmessungen von lodoform in überkritischem Kohlenstoffdioxid}

Zur Untersuchung der Photoisomerisierung bei niedrigen Dichten wurden zeitaufgelöste Spektren von $\mathrm{CHI}_{3}$ in sc. $\mathrm{CO}_{2}\left(35^{\circ} \mathrm{C}\right)$ nach einer Anregung von $267 \mathrm{~nm}$ aufgenommen. Diese wurden aus den gemessenen Absorptionszeitprofilen (s. Abbildung 5.15 und Abbildung 5.16) rekonstruiert und sind in Abbildung 5.18 (505 bar, $\rho_{r}=2,15$ ), Abbildung 5.19 (192 bar, $\left.\rho_{r}=1,84\right)$ und Abbildung 5.20 (142 bar, $\left.\rho_{r}=1,72\right)$ dargestellt. Zur Populationsnormierung wurde das in Abschnitt 6.2.1 vorgestellte Verfahren verwendet, die populationsnormierten Spektren von $\mathrm{CHI}_{3}$ in sc. $\mathrm{CO}_{2}$ bei 505 und 192 bar sind in Abbildung 6.22 und 6.23 dargestellt.

Dabei zeigt sich, daß das zeitaufgelöste Spektrum bei 505 bar nach $267 \mathrm{~nm}$ Anregung, dem bei gleichen Druck und 365 nm Anregung gemessenen Spektrum (s. Abbildung 5.17 und 6.8) sehr ähnlich ist. Die populationsnormierten Spektren zeigen beide das typische Bild einer durch Schwingungsenergietransfer abkühlenden Spezies mit einem isosbestischen Punkt bei etwa $655 \mathrm{~nm}$, der durch die unterschiedliche Anregungswellenlänge nicht verändert wird. Ein vergleichbares Verhalten konnte bereits beim $\mathrm{CH}_{2} \mathrm{I}_{2}$ gezeigt werden, 


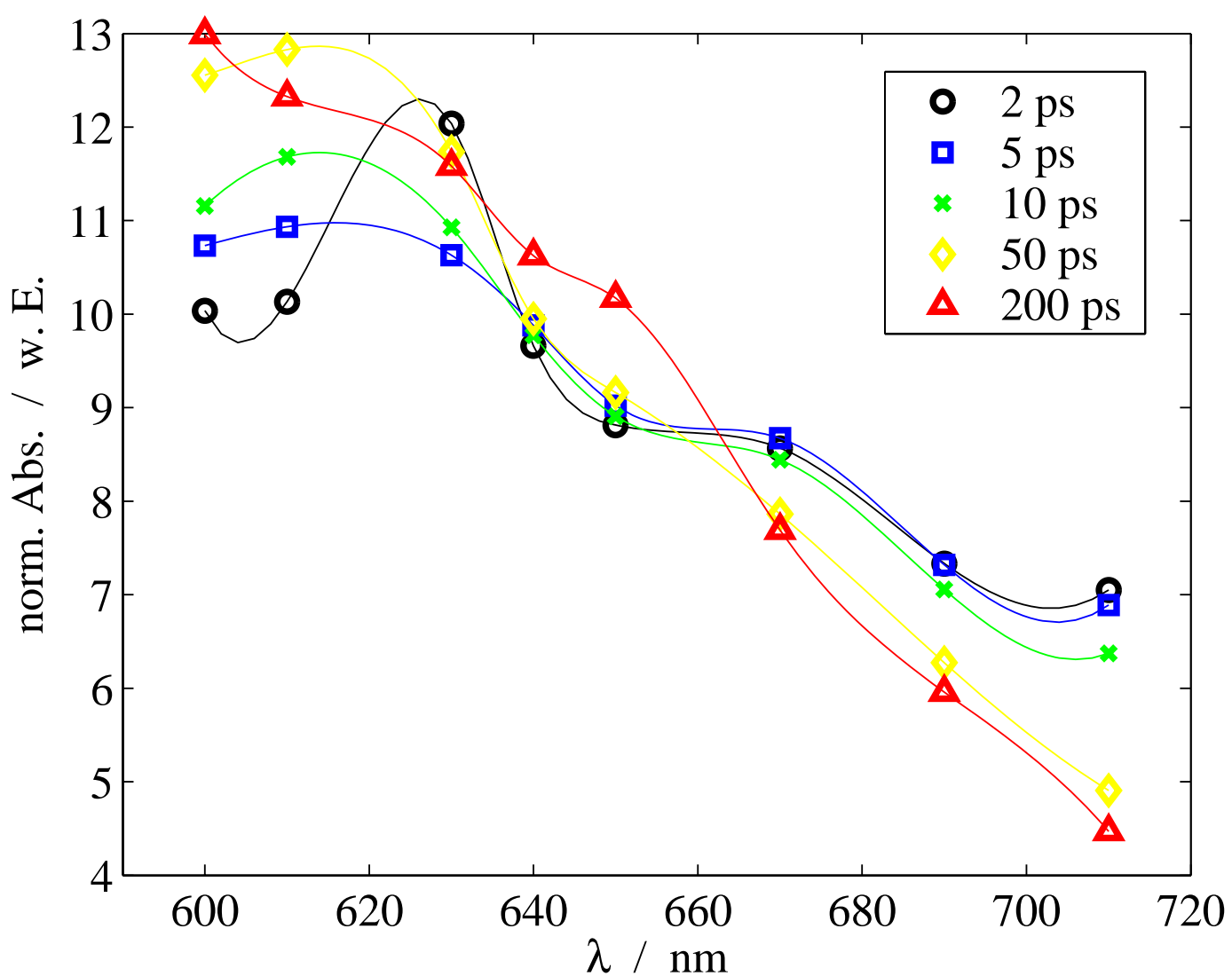

Abbildung 6.23: Normiertes zeitaufgelöstes Spektrum von $\mathrm{CHI}_{3}$ in sc. $\mathrm{CO}_{2}\left(35^{\circ} \mathrm{C}\right.$, 192 bar) nach $267 \mathrm{~nm}$ Anregung

eine Änderung der Anregungswellenlänge beeinflußt zwar die Geschwindigkeitskonstante $k_{\text {iso }}$, hat aber sonst keine signifikante Auswirkung auf die Gestalt des Spektrums.

Das zeitaufgelöste Spektrum von $\mathrm{CHI}_{3}$ nach $267 \mathrm{~nm}$ Anregung und einem Druck von 192 bar (s. Abbildung 6.23) ist dem bei höherem Druck (505 bar, Abbildung 6.22) ebenfalls sehr ähnlich. Das Absorptionsmaximum bei etwa $600 \mathrm{~nm}$ bleibt ebenso unverändert wie der isosbestische Punkt bei etwa $655 \mathrm{~nm}$. Die spektrale Dynamik ist erwartungsgemäß etwas langsamer, da der Schwingungsenergietransfer bei niedrigen Dichten weniger effizient ist. Das Spektrum zeigt somit keine Dichteabhängigkeit in Bezug auf die stattfindenden photochemischen Produkte und Prozesse. Allerdings wird die Menge an gebildetem Isomer immer weniger, wie es bereits in Abschnitt 6.3 anhand der Druckabhängigkeit der Quantenausbeute gezeigt werden konnte. Bei Probewellenlänge > $660 \mathrm{~nm}$ findet nahezu kein Anstieg mehr statt, die transiente Kurzzeitabsorption und somit die Menge an CT-Komplexes bleibt im Laufe der Reaktionszeit nahezu konstant.

Bei noch niedrigeren Dichten (s. Abbildung 5.20, 142 bar, $\rho_{r}=1,72$ ) wird nahezu kein Isomer mehr gebildet. Die Amplitude des langsamen Anstiegs ist im Vergleich zur Kurzzeitabsorption, welche der CT-Absorption zugeordnet wird, sehr gering. Es wird somit nur noch wenig Isomer gebildet, da bei solch geringen Dichten der kinematische Käfig- 

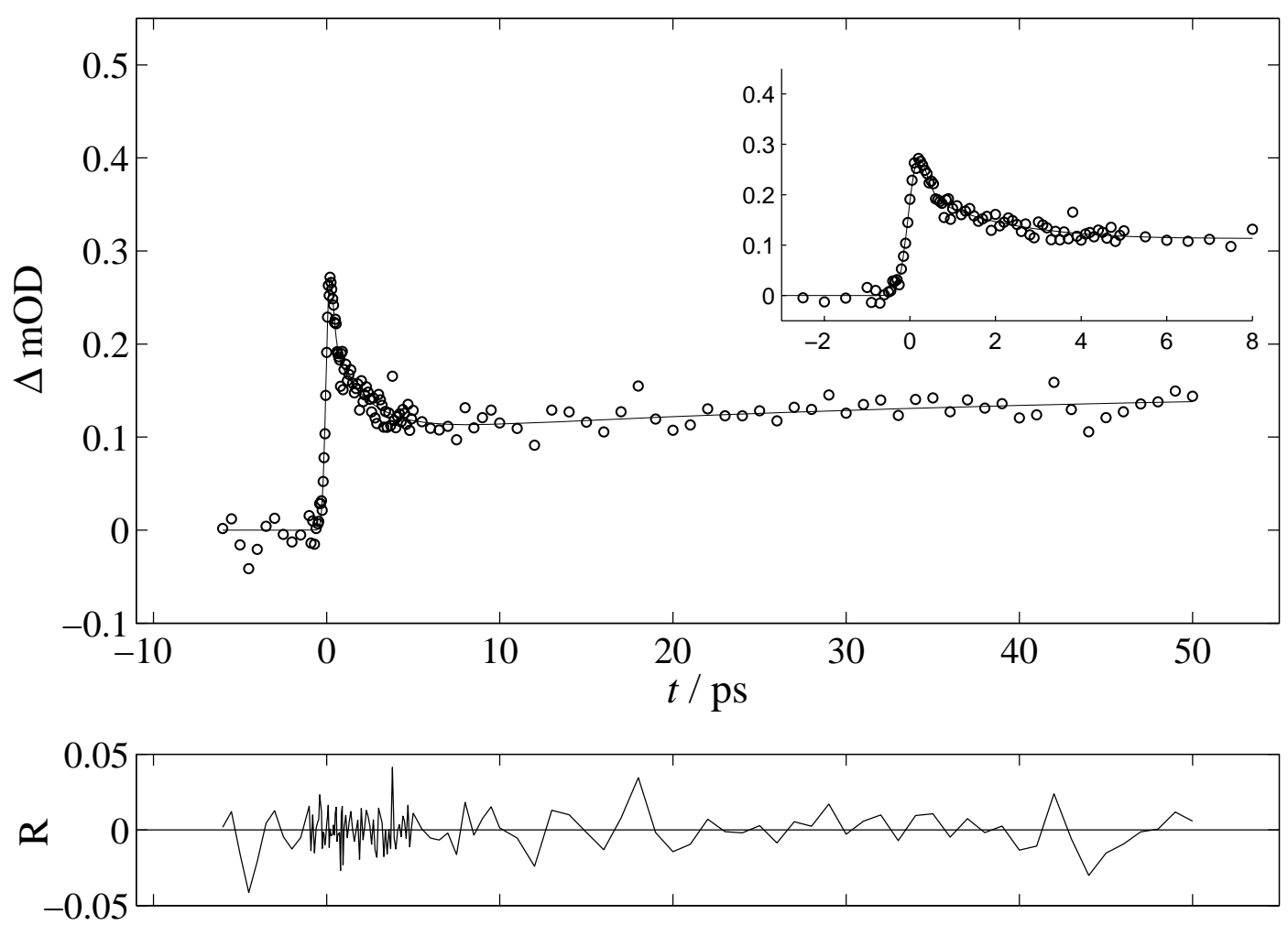

Abbildung 6.24: Absorptionszeitprofil von $\mathrm{CHI}_{3}$ in sc. $\mathrm{CO}_{2}\left(35^{\circ} \mathrm{C}, 139\right.$ bar $)$ nach $267 \mathrm{~nm}$ Anregung und $655 \mathrm{~nm}$ Abfrage

ausbruch eine immer größere Rolle spielt. Im zeitaufgelösten Spektrum ist dies dadurch erkenntlich, daß die Absorption auf der roten Flanke des Spektrums im Laufe der Reaktionszeit fast gar nicht anwächst und der Anstieg auf dem Bandenmaximum bei $600 \mathrm{~nm}$ nur gering ist. Eine Populationsnormierung wie sie für höhere Dichten durchgeführt wird, ist in diesem Fall nicht sinnvoll, weil das Isomer nicht mehr die dominierende Spezies in der transienten Absorption ist. Stattdessen ist bereits zu frühen Reaktionszeiten (2 ps) eine Absorptionsbande erkennbar, welche bei etwa $700 \mathrm{~nm}$ beginnt und zu kürzeren Wellenlängen hin ansteigt (s. Abbildung 5.20). Da $600 \mathrm{~nm}$ die kürzeste gemessenen Wellenlänge war, kann keine Aussage darüber getroffen werden, ob diese Absorptionsbande dort bereits ihr Maximum erreicht hat und wie weit sie sich zu kürzeren Wellenlängen erstreckt. Eine wie von Tarnovsky et al. postulierte schnelle Isomerbildung konnte bereits in Abschnitt 6.1.1 ausgeschlossen werden. Für diese Absorption zu frühen Reaktionszeiten kann somit der in Abschnitt 6.1.2 behandelte CT-Komplex verantwortlich gemacht werden. Das transiente Absorptionspektrum zu Verzögerungszeiten von wenigen Pikosekunden steht dabei in Einklang mit den spektralen Informationen, welche über den CT-Komplex gesammelt werden konnten. Das CT-Spektrum besteht dabei aus einer sehr breiten Absorptionsbande (s. Abbildung 6.5), welche der Mittlung über alle möglichen CT-Konfigurationen im Käfig entspricht und ein Maximum bei etwa $500 \mathrm{~nm}$ besitzt.

Eine Ausdehnung der Messungen zu noch geringeren Lösungsmitteldichten war leider aufgrund der schlechten Löslichkeit von $\mathrm{CHI}_{3}$ nicht möglich. Eine Erhöhung der Temperatur 
brachte dabei keine Vorteile, da das $\mathrm{CHI}_{3}$ sich in Lösung bei höheren Temperaturen als $35^{\circ} \mathrm{C}$ sehr schnell zu Abbauprodukten, insbesondere Iod, zersetzte. Der niedrigste Druck, bei dem noch eine transiente Absorption gemessen werden konnte, lag bei 139 bar, was einer reduzierten Dichte von $\rho_{r}=1,71$ entspricht. Diese Messung ist in Abbildung 6.24 abgebildet, dabei zeigt sich nach dem schnellen Abfall ein weiterer Abfall der transienten Absorption innerhalb von etwa 2 ps. Der anschließende Pikosekundenanstieg, welcher die Bildung des Isomers anzeigt, ist bei diesem niedrigen Druck sehr schwach ausgeprägt und nahezu nicht vorhanden.

Diese Daten zeigen an, daß bei niedrigen Drücken kaum noch Isomer gebildet wird, durch den Käfigeffekt jedoch die Photofragmente soweit zusammengehalten werden, daß sich bei allen gemessenen Lösungsmitteldichten noch ein CT-Komplex ausbilden kann. Die CTWechselwirkung spielt demnach eine Schlüsselrolle bei der Isomerisierung, sie ist in den ersten Pikosekunden nach der Photodissoziation die vorherrschende Wechselwirkung, wie man anhand der Spektren und Absorptionszeitprofile zu frühen Zeiten belegen kann. Da das bindende Potential des Isomers nur sehr flach ist, müssen die Photofragmente durch die starke CT-Wechselwirkung solange zusammengehalten werden, bis sich eine günstige Konformation zur Isomerbildung eingestellt hat und die Überschußenergie weitestgehend relaxiert ist. Dadurch unterscheidet sich der Käfigeffekt der Photoisomerisierung von Polyhalogenmethanen in Lösung grundlegend von der Photodissoziation und Rekombination von Iod. Im Falle der Ioddissoziation in Lösung ist eine solche stabilisierende CT-Wechselwirkung nicht gegeben, aber zur Rekombination der primären Photoprodukte auch nicht notwendig. Diese besitzen im Vergleich zu den Polyhalogenmethanen wesentlich weniger Überschußenergie und können direkt im Anschluß an die Photodissoziation wieder rekombinieren. Desweiteren ist der Potentialtopf des $X$-Grundzustands oder der A-Zustände (s. Abbildung 2.2), auf welchem die Rekombination der Iodatome stattfinden kann, wesentlich tiefer als das Grundzustandpotential des Isomers.

\subsection{Geminale Rekombination der Polyhalogenmethane}

Um die Bedeutung der geminalen Rekombination als Konkurrenzreaktion zur Photoisomerisierung innerhalb des Lösungsmittelkäfigs abzuschätzen, wurden für $\mathrm{CH}_{2} \mathrm{I}_{2}$ Ausbleichexperimente durchgeführt. Dazu wurde bei $320 \mathrm{~nm}$ angeregt und ein Probepuls auf der roten Flanke des Absorptionsspektrums von $\mathrm{CH}_{2} \mathrm{I}_{2}$ (s. Abschnitt 3.2) bei $360 \mathrm{~nm}$ eingestrahlt, um geminal rekombinierte Moleküle zu detektieren. Bei noch kürzeren Wellenlängen ist die Absorption des Probestrahls durch das Muttermolekül bereits zu groß. Dabei konnte kein Ausbleichen, sondern nur die Bildung des in diesem Bereich ebenfalls absorbierenden Isomers beobachtet werden. Im Falle eines Probepulses auf der blauen Flanke des Spektrums des Muttermoleküls $(\lambda<300 \mathrm{~nm})$ ist das Signal/Rausch-Verhältnis zu schlecht, um auswertbare Messungen durchzuführen. Dieses Problem stellte sich auch bei anderen Dihalogenmethanen $\left(\mathrm{CH}_{2} \mathrm{BrI}\right.$ und $\left.\mathrm{CH}_{2} \mathrm{ClI}\right)$ ein, entweder wurde das Ausbleichsignal der geminalen Rekombination von der Isomerbildung überdeckt oder ein sehr geringes Signal/Rauschverhältnis führte zu keinem auswertbaren Ergebnis. 


\begin{tabular}{|lll|}
\hline Normalmode & $\mathrm{CH}_{2} \mathrm{I}_{2}$ & $\mathrm{CH}_{2} \mathrm{I}-\mathrm{I}$ \\
\hline$\nu_{1}$ sym. C-H-Streckschwingung & 2968 & 3119 \\
$\nu_{2} \mathrm{CH}_{2}$-Deformationsschwingung & 1351 & 1409 \\
\hline & $\mathrm{CH}_{2} \mathrm{ClI}$ & $\mathrm{CH}_{2} \mathrm{Cl}-\mathrm{I}$ \\
$\nu_{1}$ sym. C-H-Streckschwingung & 2978 & 3131 \\
$\nu_{2} \mathrm{CH}_{2}$-Deformationsschwingung & 1392 & 1340 \\
\hline & $\mathrm{CH}_{2} \mathrm{BrI}$ & $\mathrm{CH}_{2} \mathrm{Br}-\mathrm{I}$ \\
$\nu_{1}$ sym. C-H-Streckschwingung & 2978 & 3115 \\
$\nu_{2} \mathrm{CH}_{2}$-Deformationsschwingung & 1374 & 1340 \\
\hline
\end{tabular}

Tabelle 6.3: Ausgewählte Normalmoden der Dihalogenmethane und ihrer Isomere (in $\mathrm{cm}^{-1}$ ) [166, 175, 177]

Dabei bleibt die Frage einer möglichen geminalen Rekombination unbeantwortet, entweder sie findet tatsächlich nicht statt, oder sie läßt sich nur nicht mit dem gewählten Experiment beobachten. Zur Klärung dieser Frage wurde ein alternative Experimente mit UV-Pump/IR-Probemessungen herangezogen. Dazu sind in Tabelle 6.3 einige ausgewählte Normalmoden der untersuchten Dihalogenmethane aufgelistet. Dabei fällt auf, daß die Frequenz der symmetrischen $\mathrm{C}-\mathrm{H}$-Streckschwingung vom Isomer im Vergleich zum Muttermolekül um über $100 \mathrm{~cm}^{-1}$ verschoben ist. Dadurch sollte es nach UV-Anregung möglich sein, ein Ausbleichen dieser Mode und eine anschließende geminale Rekombination zu beobachten. Die Frequenzverschiebung der $\mathrm{CH}_{2}$-Deformationsschwingung ist hierzu nicht ausgeprägt genug. Trotz ausgiebiger Messungen bei $266 \mathrm{~nm}$ Anregung und einem IR-Probepuls zentriert bei $3100 \mathrm{~cm}^{-1}$ war es jedoch nicht möglich, für die verwendeten Dihalogenmethane in $\mathrm{CD}_{3} \mathrm{CN}$ ein Signal zu finden. Möglicherweise ist die Intensität der beobachteten Bande zu schwach. Die Frage einer möglichen geminalen Rekombination bleibt demnach ungeklärt, da keines der durchgeführten Experimente auswertbare Ergebnisse hervorbrachte. Zu einer Abschätzung der Bedeutung der geminalen Rekombination ist es deshalb nützlich, $\mathrm{CH}_{3} \mathrm{I}$ als nächstähnliches Modellsystem zu verwenden (s. Abschnitt 6.6). Da bei diesem Molekül keine Isomerisierung als Konkurrenz zur Rekombination stattfindet, kann auf die Dissoziation neben dem Käfigausbruch nur geminale Rekombination folgen. 


\subsection{Photodynamik von lodmethan}

Zur Photodynamik von Iodmethan wurden Pump/Probeexperimente in verschiedenen Lösungsmitteln durchgeführt. Dabei wurde $\mathrm{CH}_{3} \mathrm{I}$ in der Absorptionsbande bei $260 \mathrm{~nm}$ angeregt und mit dem Probepuls ein breites Spektrum vom UV bis in den IR-Bereich abgedeckt.

\subsubsection{Bildung von CTTS-Komplexen}

Für Messungen von $\mathrm{CH}_{3} \mathrm{I}$ nach Anregung bei etwa $260 \mathrm{~nm}$ in Methanol, Acetonitril, Methylcyclohexan und $n$-Perfluorhexan als Lösungsmittel wurde der Probepuls im Bereich von 280-380 nm variiert. Dabei zeigt sich für die meisten Messungen ein sehr ähnliches Absorptionszeitprofil (s. Abbildung 5.33), nach einem von der Zeitauflösung begrenzten Anfangspeak fällt die Absorption auf ein Plateau ab und ändert sich bis zu einer Verzögerungszeit von 200 ps (maximal gemessene Verzögerungszeit) nicht mehr.

Der intensive Anfangspeak ist dabei zum größten Teil einem Lösungsmittelsignal zuzuschreiben. Messungen des reinen Lösungsmittels (s. Abbildung 5.40 zeigen eine starke von der Zeitauflösung begrenzte Absorption, welche zu kürzeren Wellenlägen hin zunimmt und auf nichtlineare kohärente Prozesse (wie z. B. Zwei-Photonen-Absorption, Kerr-Effekt u.ä.) im Lösungsmittel zurückzuführen ist.

Wie in Abschnitt 3.2 beschrieben, dissoziiert $\mathrm{CH}_{3} \mathrm{I}$ nach Anregung bei $260 \mathrm{~nm}$ in ein $\mathrm{CH}_{3}$-Radikal und ein Iodatom:

$$
\mathrm{CH}_{3} \mathrm{I} \stackrel{\mathrm{h} \nu}{\longrightarrow} \mathrm{CH}_{3}+\mathrm{I}
$$

Die Absorptionsbande von $\mathrm{CH}_{3}$ als primären Photoprodukt liegt bei etwa $215 \mathrm{~nm}$ (s. Abbildung 3.10). Für die im Bereich von 280-380 nm gemessene bis zu 200 ps konstante Absorption muß demnach eine andere Spezies verantwortlich sein. Eine denkbare Möglichkeit ist dabei die schnelle Bildung eines CT-Komplexes $(<200 \mathrm{fs})$ aus einem Iodatom und einem Lösungsmittelmolekül:

$$
\mathrm{CH}_{3} \mathrm{I} \stackrel{\mathrm{h} \nu}{\longrightarrow} \mathrm{CH}_{3}+\mathrm{I} \longrightarrow \mathrm{I} \cdots \text { Lösungsmittel } \quad \text { CT-Komplex }
$$

Die Bildung und die Absorptionsspektren solcher CT-Komplexe (s. Abschnitt 6.1.2) wurden bereits für viele Lösungsmittel-Iod CT-Komplexe in der Literatur beschrieben [190,191]. Diese Komplexe werden häufig als charge-transfer to solvent-Komplexe (CTTS) 


\begin{tabular}{|lc|}
\hline Molekül & Ionisierungspotential / eV \\
Acetonitril & 12,2 \\
Methanol & 10,84 \\
Methylcyclohexan & 9,62 \\
$n$-Perfluorhexan & k. A. \\
\hline
\end{tabular}

Tabelle 6.4: Ionisierungspotentiale der verwendeten Lösungsmittel nach NIST Chemistry Webbook [193]

bezeichnet. Die entsprechenden Spektren der CT-Komplexe aus Iod mit Methanol, Acetonitril und Methylcyclohexan sind in Abbildung 6.25 dargestellt Dabei zeigt sich, daß das Maximum des CT-Übergangs mit höherem Ionisierungspotential (s. Tabelle 6.4) zu kürzeren Wellenlängen verschoben ist (s. Gleichung 6.2). Die genaue Struktur der Absorptionsspektren läßt sich anhand der vorgenommenen Messungen (s. Spektren in Methanol (Abbildung 5.34), Methylcyclohexan (Abbildung 5.34) und Acetonitril (Abbildung 5.37)) nicht auflösen, dafür ist der gemessene Wellenlängenbereich von 280-380 nm zu klein und die spektrale Auflösung zu gering. Weiterhin ist eine Rekonstruktion von (zeitaufgelösten) Spektren anhand der gemessenen Absorptionszeitprofile fehlerbehaftet, da keine Normierung zum Ausgleich justageabhängiger Parameter vorgenommen werden kann. Eine Normierung auf den Anfangspeak wie es im Falle der Isomersignale (s. Abschnitt 5.2.2 durchgeführt wird, ist hier nicht zulässig, da der Anfangspeak zum größten Teil auf ein von der eigentlichen Reaktion unabhängiges Lösungsmittelsignal zurückzuführen ist. Deswegen wurde viel Wert auf eine gute Reproduktion der Versuchsparameter (Pumpenergie, Justage) gelegt, um die Vergleichbarkeit der Meßdaten zu gewährleisten.

Es zeigt sich anhand der Spektren der CTTS-Komplexe (s. Abbildung 6.25), daß diese im Wellenlängenbereich von 280-380 nm alle eine starke Absorption besitzen und somit der im UV-Bereich gemessenen transienten Absorption zugeordnet werden können. Die CT-Komplexe besitzen dabei eine hohe Stabilität, ihre charakteritische Absorption läßt sich noch auf einer Nanosekundenzeitskala beobachten [210].

Im Falle des $n$-Perfluorhexan als Lösungsmittel ist keine konstante Absorption nach dem Anfangspeak meßbar (s. Abbildung 5.38), welche einem CTTS-Komplex zugeordnet werden kann. Dieses Verhalten kann auf das hohe Ionisierungspotential des perfluorierten Lösungsmittels zurückgeführt werden. Zwar konnte kein Literaturwert für das Ionisierungspotential von $n$-Perfluorhexan gefunden werden, allerdings ist dieses Potential von fluorsubtituierten Molekülen im Vergleich zu unsubstituierten stark erhöht. So steigt das Ionisierungspotential von $\mathrm{CH}_{3} \mathrm{CN}$ durch Perfluorierung zu $\mathrm{CF}_{3} \mathrm{CN}$ von $12,2 \mathrm{eV}$ auf $13,93 \mathrm{eV}$ an. In der Reihe $\mathrm{CH}_{3} \mathrm{~F}(12,50 \mathrm{eV}), \mathrm{CH}_{2} \mathrm{~F}_{2}(12,71 \mathrm{eV}), \mathrm{CHF}_{3}(13,86 \mathrm{eV})$ und $\mathrm{CF}_{4}(16,2 \mathrm{eV})$ zeigt sich ebenfalls ein deutlicher Anstieg, für ein kurzkettigeres Perfluoralkan wie $n$-Perfluorbutan liegt das Ionisierungspotential bei $15,4 \mathrm{eV}$, so daß für $n$-Perfluorhexan mit einem Wert von etwas weniger als $15 \mathrm{eV}$ zu rechnen ist.

Durch dieses hohe Ionisierungspotential wird ein Ladungstransfer und somit eine CTWechselwirkung zwischen $n$-Perfluorhexan und Iodatom verhindert. Anstelle der CTAbsorption tritt in den Absorptionszeitprofilen (s. Abbildung 5.39) ein negatives Signal 


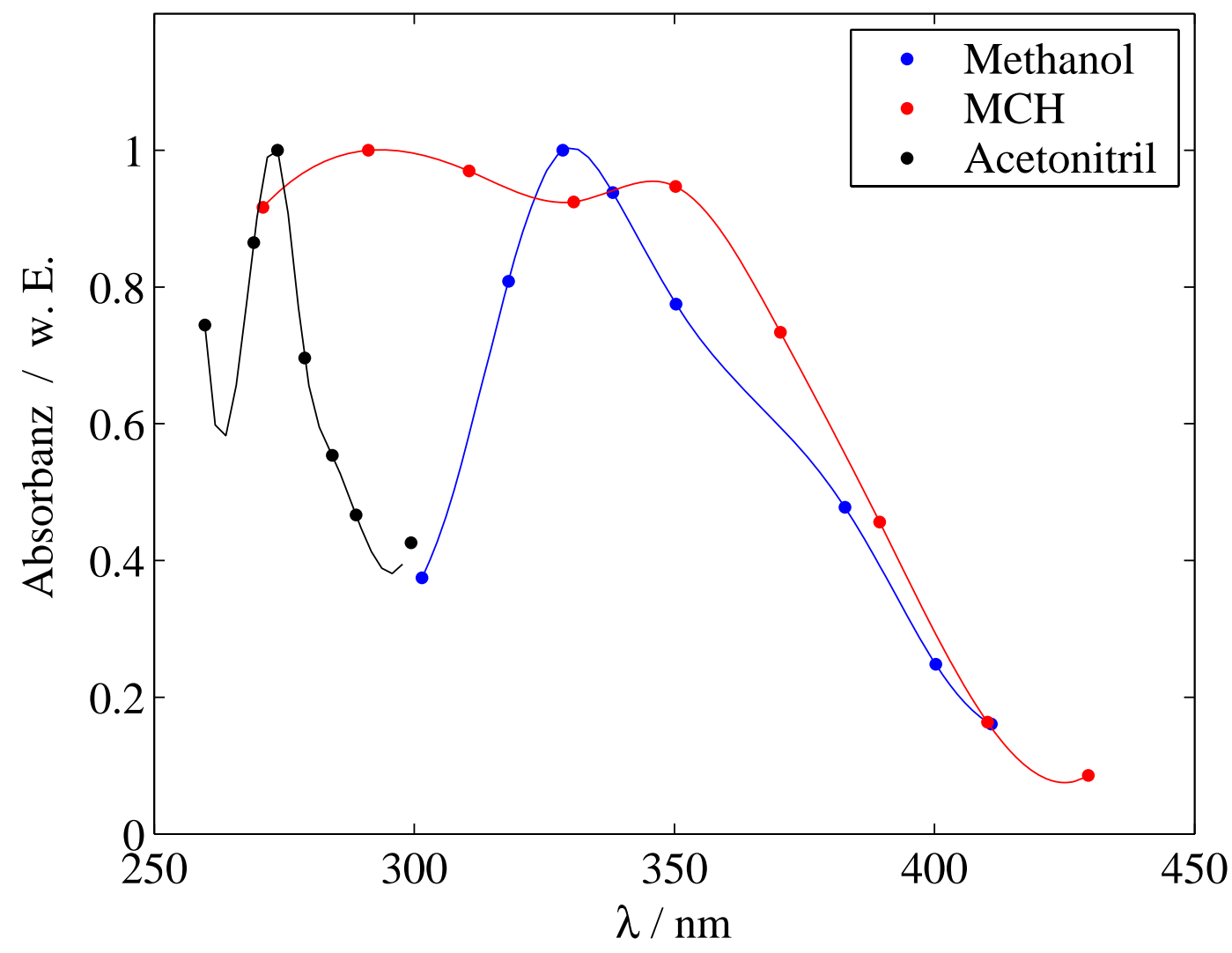

Abbildung 6.25: Normierte Absorptionsspektren von CTTS-Komplexen aus Iodatom und Lösungsmittel (Meßpunkte mit Interpolation der Literatur entnommen (Methanol [211], Methylcyclohexan [210], Acetonitril [212])

auf, möglicherweise ein Ausbleichen des Muttermoleküls nach Dissoziation. Allerdings nimmt dieses negative Signal zu längeren Wellenlängen hin zu und der Abfall verläuft innerhalb 6-10 ps. Da die Dissoziation des $\mathrm{CH}_{3} \mathrm{I}$ sehr schnell $\left(\tau_{\text {diss }}<200 \mathrm{fs}\right)$ ist, sollte ein Abfall, welcher auf eine Dissoziation zurückzuführen ist, innerhalb der Zeitauflösung von etwa 200 fs erfolgen. Desweiteren müßte ein Ausbleichsignal in Richtung Bande des Muttermoleküls größer werden.

Wenn der Ursprung des negativen Signales nicht auf das Ausbleichen infolge geminaler Rekombination zurückgeführt werden kann, dann bleibt noch die Möglichkeit der Emission aus einem langlebigen, besetzten Zustand. Allerdings sind die erreichbaren Zustände des $\mathrm{CH}_{3} \mathrm{I}$ alle zu kurzlebig, um eine Emission über einen Zeitraum von bis zu 50 ps zu beobachten. Die A-Zustände, welche durch Ein-Photonen-Absorption besetzt werden, sind alle repulsiv mit einer Lebensdauer von $<200$ fs. Die höheren Rydbergzustände $6 s$ und $10 s$, welche durch Zwei-Photonen-Absorption zugänglich sind, besitzen ebenfalls Lebensdauern von wenigen hundert Femtosekunden (s. Abschnitt 3.3) und kommen somit für die beobachtete Emission nicht in Betracht. Die Zeitkonstante des schnellen Abfalls (610 ps) liegt in der Größenordnung einer Käfigreaktion, was darauf hindeuten könnte, daß durch Photolyse generierte Radikale innerhalb des Käfigs zu neuen Produkten reagie- 
ren, welche die Emission erklären könnten. Allerdings sind Reaktionen des $\mathrm{CH}_{3}$-Radikals als primären Photoprodukt mit den nahezu inerten Lösungsmittel $n$-Perfluorhexan sehr unwahrscheinlich. Neben der Reaktion von Iodmethan und seiner primären Photoprodukte kann weiterhin das verwendete Lösungsmittel für die beobachtete Dynamik verantwortlich sein. Um diese Möglichkeit zu überprüfen und einen Beitrag des Iodmethan auszuschließen, müssen weitere Pump/Probe-Experimente mit dem reinen Lösungmittel $n$-Perfluorhexan durchgeführt werden.

\subsubsection{Quantenausbeuten der CTTS-Komplexe}

Da für einige CTTS-Komplexe (z. B. für Cyclohexan) die Extinktionskoeffizienten aus der Literatur bekannt sind, können die Quantenausbeuten der Komplexbildung abgeschätzt werden. Die Quantenausbeute $\Phi_{\text {CTTS }}$ berechnet sich wie folgt:

$$
\Phi_{\mathrm{CTTS}}=\frac{n_{\mathrm{CTTS}}}{n_{\mathrm{Ph}}}
$$

Wobei $n_{\mathrm{CTTS}}$ die Menge an gebildeten CTTS-Komplexen und $n_{\mathrm{Ph}}$ die Anzahl, der in der Probe absorbierten Photonen ist. $n_{\text {СтTS }}$ wird über das Lambert-Beer-Gesetz berechnet, während $n_{\mathrm{Ph}}$ über die Pulsenergie abgeschätzt wird. Für $\Phi_{\mathrm{CTTS}}$ gilt somit folgender Zusammenhang:

$$
\Phi_{\mathrm{CTTS}}=\frac{4 \Delta O D \pi r^{2} N_{A}}{3 n_{\mathrm{Ph}} \epsilon}
$$

$\triangle O D$ ist dabei die konstante Absorptionsstufe nach UV-Anregung, $N_{A}$ die AvogadroZahl, $\epsilon$ der Extinktionskoeffizient bei der entsprechenden Wellenlänge und $r$ der Fokusradius, welcher auf etwa $40 \mu \mathrm{m}$ abgeschätzt wurde. Auf diese Weise lassen sich die Quantenausbeuten in Methylcyclohexan berechnen, für die anderen Lösungsmitteln wurden leider keine Extinktionskoeffizienten der CTTS-Komplexe gefunden. Die Ergebnisse sind in Tabelle 6.5 zusammengestellt.

Die Quantenausbeuten $\Phi_{\text {CTTS }}$ liegen wellenlängenunabhängig bei etwa 0,1. Dabei ist jedoch zu beachten, daß dieser Wert anhand der relativ ungenauen Abschätzung des Fokusradius $r_{f}$ fehlerbehaftet ist (s. Abschnitt 6.6.6) Die relativ geringe Quantenausbeute von 0,1 zeigt, daß nach Photodissoziation ein großer Teil der Photofragmente direkt aus dem Käfig ausbricht. Dafür spricht die im Vergleich zu anderen Polyhalogenmethanen relativ hohe kinetische Energie, welche die Photofragmente nach der Dissoziation besitzen (s. Abschnitt 3.4). Weitere relevante Konkurrenzreaktionen, welche die Quantenausbeute der CTTS-Komplexbildung mindern, sind die Zwei-Photonen-Absorption (s. Abschnitt 6.6.5) und die geminale Rekombination der primären Photofragmente nach Käfigeinfang (s. Abschnitt 6.6.7). 


\begin{tabular}{|l|l|}
\hline$\lambda / \mathrm{nm}$ & $\Phi_{\mathrm{CTTS}}$ \\
\hline 320 & 0,093 \\
330 & 0,115 \\
340 & 0,106 \\
350 & 0,088 \\
360 & 0,099 \\
370 & 0,106 \\
380 & 0,115 \\
$\bar{M}_{\Phi_{\text {CTTS }}}$ & 0,103 \\
\hline
\end{tabular}

Tabelle 6.5: Quantenausbeuten der CTTS-Komplexbildung von $\mathrm{CH}_{3} \mathrm{I}$ in Methylcyclohexan bei verschiedenen Probewellenlängen

\subsubsection{Dynamik nach Zwei-Photonen-Absorption}

Bei Verwendung eines Probestrahls im Wellenlängenbereich von 400-600 nm zeigen sich in verschiedenen Lösungsmitteln unterschiedliche Absorptionszeitprofile (s. Abbildung 5.41, 5.42 und 5.43). Deren Verlauf zeigt zu längeren Wellenlängen (> $450 \mathrm{~nm}$ ) einen Abfall in der Größenordnung von 30-50 ps (polare Lösungsmittel) bzw. 100-150 ps (Methylcyclohexan). In polaren Lösungsmitteln wie $\mathrm{CH}_{3} \mathrm{CN}$ und $\mathrm{CH}_{3} \mathrm{OH}$ bleibt zudem ein konstanter off-set stehen, während in Methylcyclohexan die Absorption wieder komplett abfällt. Bei kürzeren Wellenlängen (um $400 \mathrm{~nm}$ ) zeigt sich in polaren Lösungsmittel nach dem kohärenten Anfangspeak ein negatives Signal, welches mit einer Zeitkonstanten von etwa 2 ps wieder relaxiert. Diese Emissionbande konnte in Acetonitril relativ gut aufgelöst werden, in Methanol konnten die Messungen nicht weit genug in den UV-Bereich ausgedehnt werden, um die komplette Bande abzubilden. Aus den gemessenen Absorptionszeitprofilen wurden zeitaufgelöste Spektren nach der bereits beschriebenen Methode rekonstruiert, diese sind in folgenden Abbildungen für Acetonitril (Abbildung 6.26), Methanol (Abbildung 6.27) und Methylcyclohexan (Abbildung 6.28) dargestellt. Dabei zeigt sich folgendes Bild: In Methanol und Acetonitril tritt deutlich eine Emissionsbande im Bereich 450-380 nm hervor, welche mit einer Zeitkonstanten von etwa 2 ps relaxiert. In Methylcyclohexan konnte eine negative Absorption und somit das Ausbilden einer Emissionsbande im Spektrum nicht beobachtet werden. Das zeitaufgelöste Absorptionsspektrum von Methylcyclohexan besteht aus zwei Banden, einer intensiveren bei $560 \mathrm{~nm}$ und einer schwachen bei $430 \mathrm{~nm}$, diese fallen im Laufe von 200-250 ps wieder bis auf Null ab. Eine konstant bleibende Absorption zu späten Reaktionszeiten , wie sie in den polaren Lösungsmitteln oder nach Ausbildung von CTTS-Komplexen (s. Abschnitt 6.6.1) beobachtet werden konnte, fehlt in Methylcyclohexan. Der intensive Anfangspeak bei kurzwelligen Messungen $(<450 \mathrm{~nm})$ ist auf ein kohärentes Lösungsmittelsignal zurückzuführen.

Die Dynamik und Bandenstruktur, insbesondere im sichtbaren Probewellenlängenbereich, von $\mathrm{CH}_{3} \mathrm{I}$ in Lösung ist auf den ersten Blick sehr ungewöhnlich. Vergleichbare Messungen aus der Literatur sind nicht bekannt, zudem sich die Arbeiten zu $\mathrm{CH}_{3} \mathrm{I}$ auf die Gasphase bzw. das isolierte Molekül konzentrieren. Lediglich die Arbeiten von Poth 


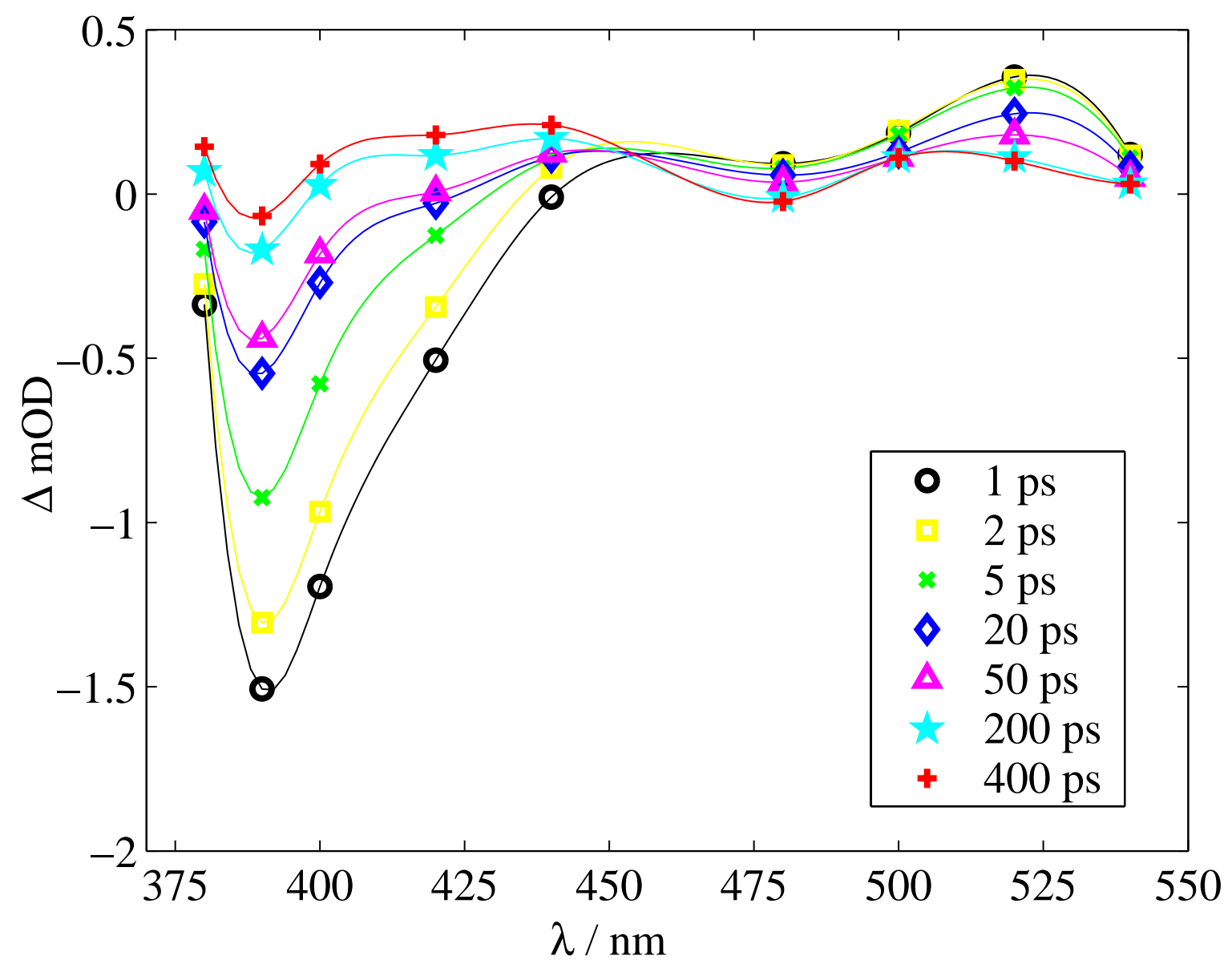

Abbildung 6.26: Rekonstruiertes zeitaufgelöstes Spektrum von $\mathrm{CH}_{3} \mathrm{I}$ in Acetonitril bei $265 \mathrm{~nm}$ Anregung

et al. 213] und Hopkins [163] sind insofern vergleichbar, da sie die Photodissoziationsdynamik von $\mathrm{CH}_{3} \mathrm{I}$ in Clustern bzw. in Lösung betrachten. Dabei sind die Messungen von Hopkins allerdings auf eine Probewellenlänge von $213 \mathrm{~nm}$ beschränkt, welches der Absorptionsbande des $\mathrm{CH}_{3}$-Radikals als primärem Photoprodukt entspricht (s. Abschnitt 3.4). Für die Dynamiken bei Probewellenlängen > $300 \mathrm{~nm}$ kann das $\mathrm{CH}_{3}$ ebensowenig wie das Iodatom verantwortlich gemacht werden. Eine einfache Dissoziation in Iodatom und $\mathrm{CH}_{3}$-Radikal kann demnach nicht für die beobachtete Dynamik verantwortlich sein, es müssen weitere Prozesse eine Rolle spielen.

Poth etal. 213] untersuchten die Photodissoziationsdynamik von $\mathrm{CH}_{3}$ I-Clustern bei $270 \mathrm{~nm}$ Anregung und einem intensiven Probestrahl bei $405 \mathrm{~nm}$ zur Erzeugung von Ionen, welche mit einem Massenspektrometer detektiert wurden. In ihrem Modell gehen sie von einer Zwei-Photonen-Anregung in den 10s-Rydberg-Zustand aus (s. Energiezustandsdiagramm 3.8), was zu einer schnellen Dissoziation des Moleküls führt. Innerhalb des Clusters ist es jedoch möglich daß die Fragmente durch den Käfigeffekt auf dem Potential des $6 s$-Rydberg-Zustands wieder rekombinieren. Das schwingungsheiße Rekombinationsprodukt relaxiert anschließend auf einer Pikosekundenzeitskala und geht danach in den disso- 


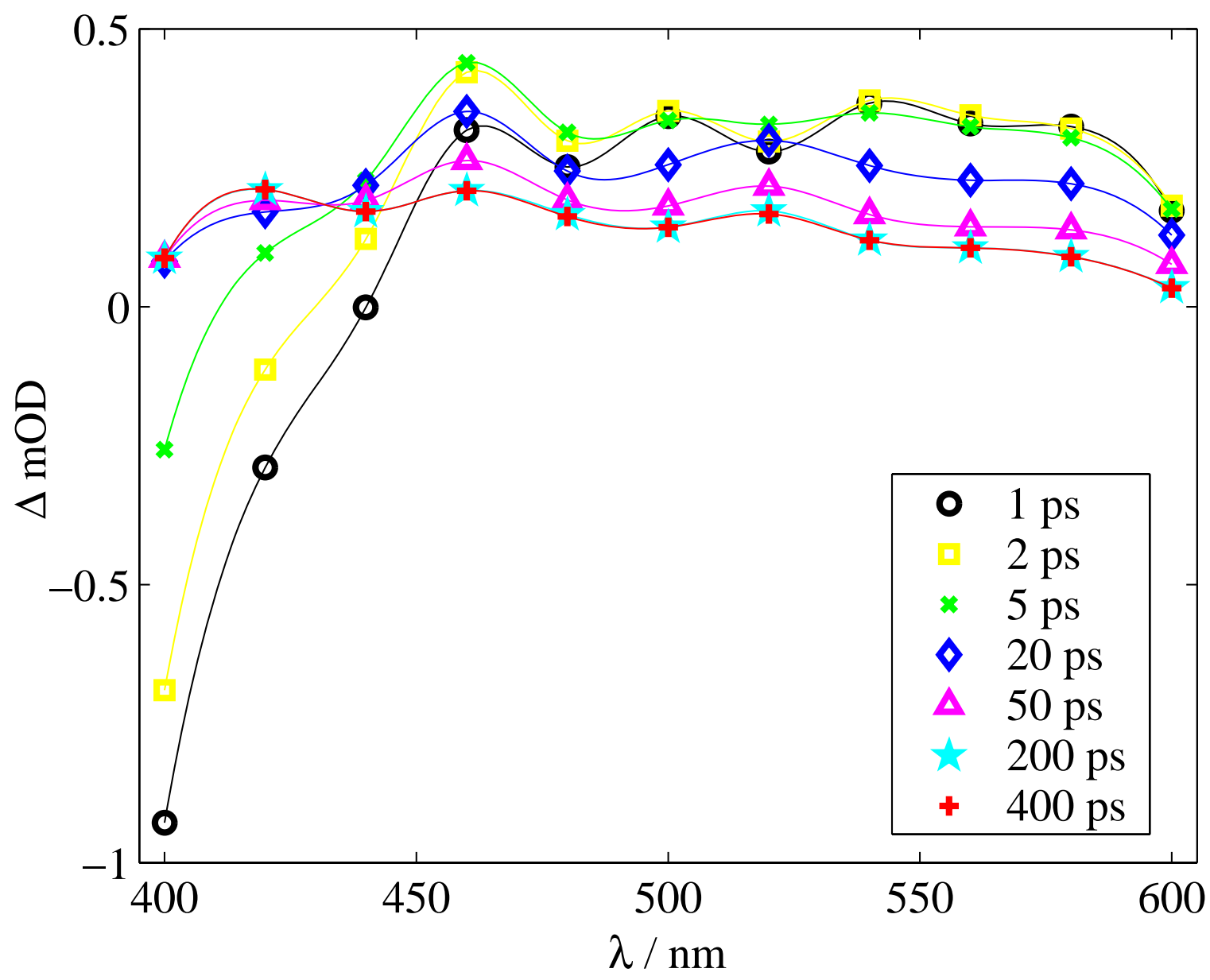

Abbildung 6.27: Rekonstruiertes zeitaufgelöstes Spektrum von $\mathrm{CH}_{3} \mathrm{I}$ in Methanol bei $255 \mathrm{~nm}$ Anregung

ziativen $A$-Zustand über, aus welchem die endültige Dissoziation nach etwa 60 ps erfolgt. Dieser Vorschlag eines Modells zur Photodissoziationsdynamik von $\mathrm{CH}_{3} \mathrm{I}$ in Clusterumgebung ließe sich auf die Interpretation der transienten Absorptionssignale in Lösungsmittel übertragen. Im Falle einer Zwei-Photonenanregung in den 10s-Rydbergzustand wird dieser schnell dissozieren (vergl. Lebenszeiten der Rydbergzustände). Die innerhalb des Käfigs auf dem 6s-Zustand rekombinierten Moleküle können durch den Probestrahl in ein höheres $S_{n}$-Niveau geprobt werden, dadurch steigt das Signal erst ein wenig innerhalb 1 ps an (Rekombination auf dem 6s-Potential) und fällt danach exponentiell ab. Dieses entspricht der Schwingungsenergierelaxation der heißen Moleküle, welche am Boden des Potentialtopfes vom $6 s$-Zustand in den $A$-Zustand übergehen und dissozieren.

Dabei weist das Modell von Poth et al. jedoch einige Unstimmigkeiten bei der Erklärung der Dynamik von $\mathrm{CH}_{3} \mathrm{I}$ in Lösung auf. So fällt die Absorption im unpolaren Methylcyclohexan zwar wieder komplett ab, ist jedoch mit 100-150 ps um einiges langsamer als die von Poth beobachteten 60 ps. Desweiteren kann diese Relaxationszeit in Lösung wohl kaum auf Schwingungsenergietransfer zurückgeführt werden, da dieser im Vergleich zur 


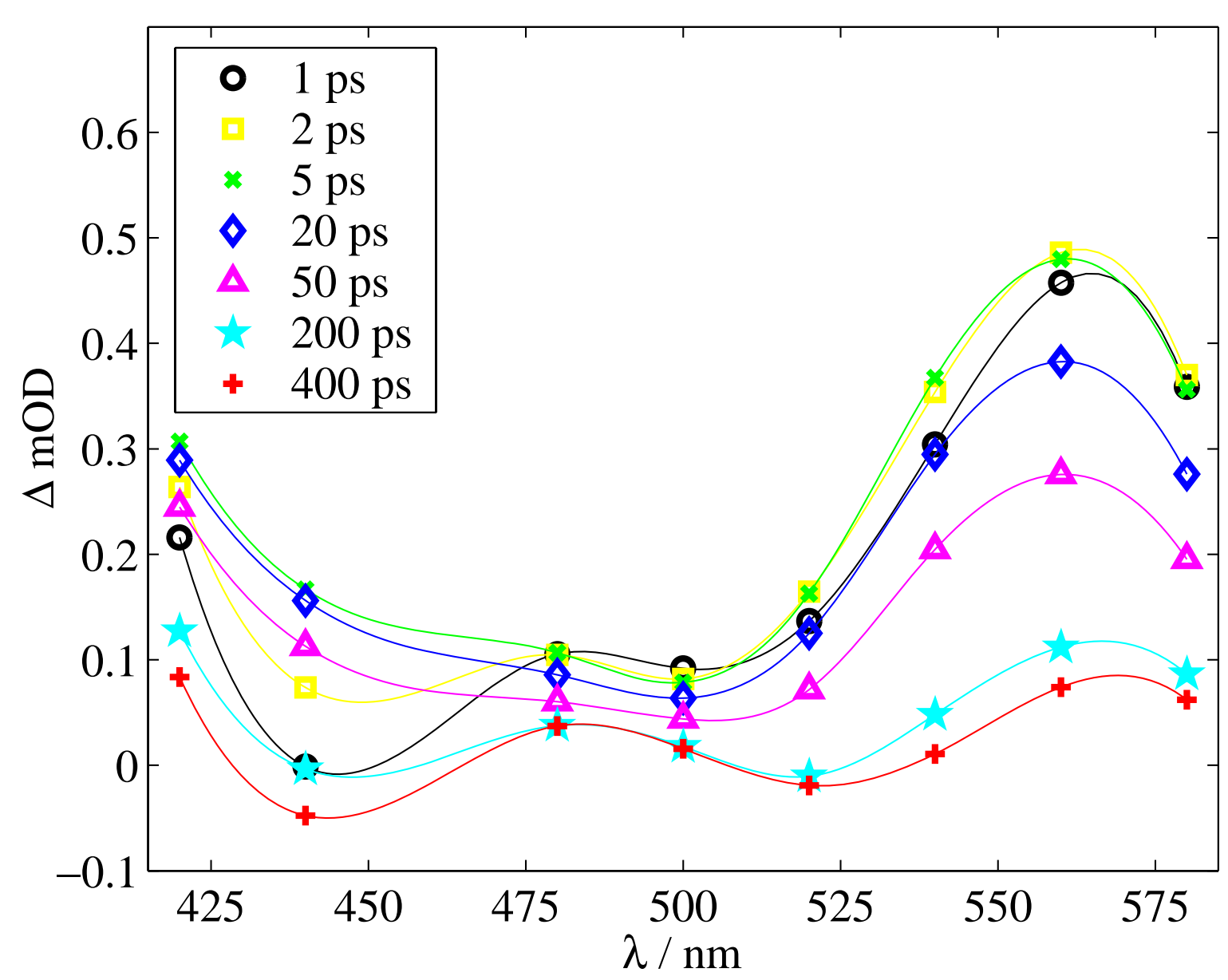

Abbildung 6.28: Rekonstruiertes zeitaufgelöstes Spektrum von $\mathrm{CH}_{3} \mathrm{I}$ in Methylcyclohexan bei $265 \mathrm{~nm}$ Anregung

Clusterumgebung wesentlich schneller vonstatten gehen sollte. Für polare Lösungsmittel wie Methanol und Acetonitril kann das Modell von Poth ebenfalls keine zufriedenstellende Erklärung für das experimentell beobachte Verhalten geben. So erklärt es in keiner Form die Anwesenheit einer Emissionsbande, desweiteren fällt die Absorption in diesen Lösungsmitteln zu langen Reaktionszeiten (> 100 ps) nie ganz auf Null ab. Dies würde bedeuten, daß der $6 s$-Rydbergzustand nach Schwingungsenergierelaxation nicht entleert wird und damit eine sehr lange Lebenszeit besitzt ( $>400 \mathrm{ps}$ ). Messungen der Lebenszeiten der Rydbergzustände von $\mathrm{CH}_{3} \mathrm{I}$ zeigen jedoch, daß diese im Bereich von wenigen hundert Femtosekunden liegen (s. Abschnitt 3.3). Neben den bereits aufgezählten Rydbergzuständen spielen demnach andere Zustände möglicherweise eine Rolle.

Zur Klärung der Frage welche Zustände beteiligt sind, ist zuerst die Möglichkeit einer Mehr-Photonen-Absorption zu überprüfen, wie sie für $\mathrm{CH}_{3} \mathrm{I}$ bereits oft beobachtet wurde. Dazu wurden intensitätsabhängige Messungen von $\mathrm{CH}_{3} \mathrm{I}$ bei $255 \mathrm{~nm}$ Anregung und $400 \mathrm{~nm}$ Abfrage in Methanol durchgeführt. In diesem Wellenlängenbereich zeigt sich im zeitaufgelösten Spektrum des $\mathrm{CH}_{3} \mathrm{I}$ die bereits angesprochende Emissionsbande. Die Ab- 

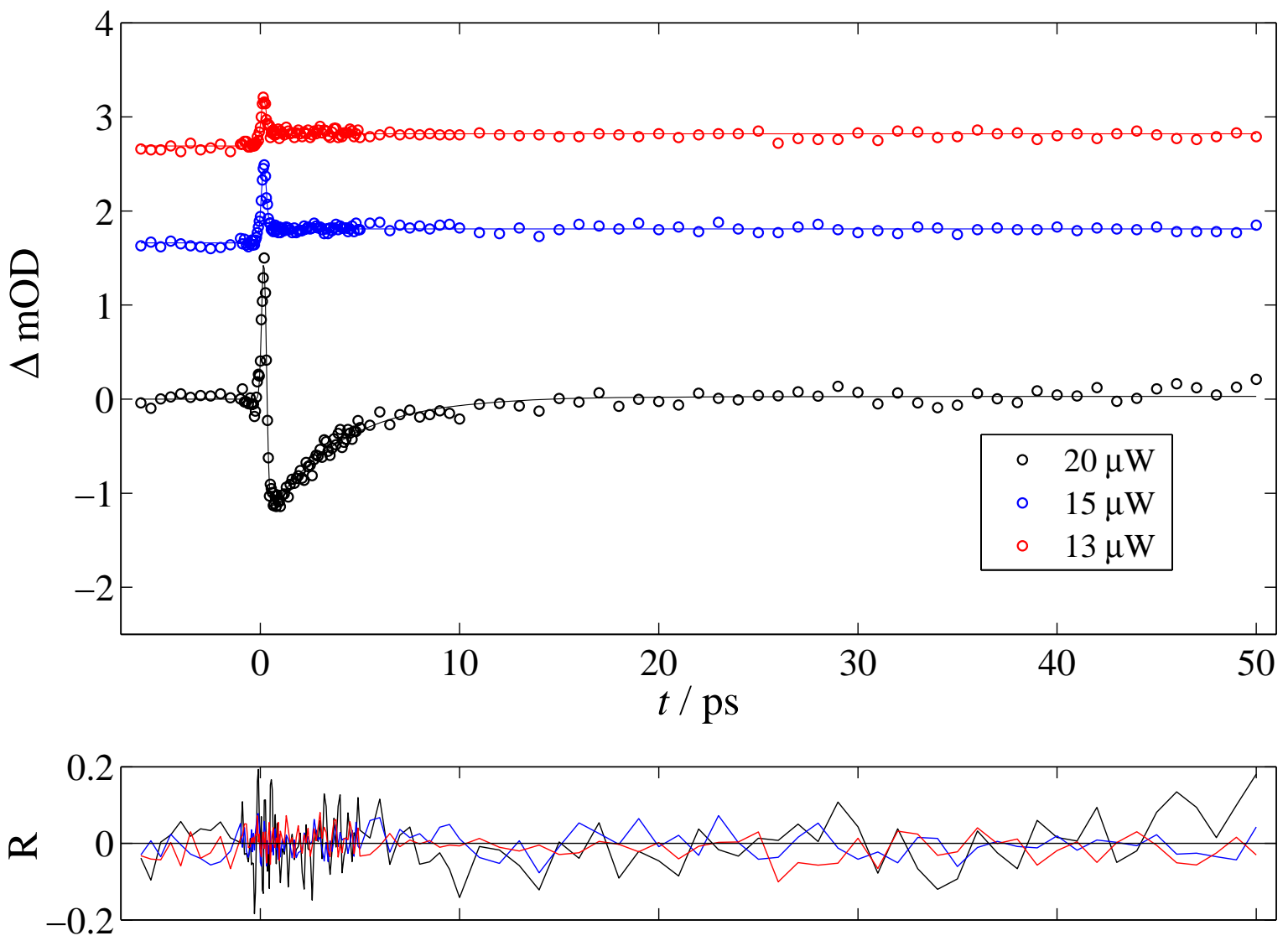

Abbildung 6.29: Intensitätsabhängigkeit der Absorptionszeitprofile von $\mathrm{CH}_{3} \mathrm{I}$ in Methanol bei $260 \mathrm{~nm}$ Anregung und $400 \mathrm{~nm}$ Abfrage. Die Meßdaten bei 15 und $13 \mu \mathrm{W}$ Pumppulsintensität sind zur besseren Übersicht verschoben dargestellt

sorptionszeitprofile bei einer Pumppulsenergie von 40, 30 und $26 \mathrm{~nJ}$ sind in Abbildung 6.29 dargestellt. Dabei zeigt sich bei voller Anregungsintensität die bereits beobachtete negative Absorption, geringfügige Abschwächung (15nJ) führt zu einem völlig anderen Bild. Anstelle der Emission tritt die bereits aus den UV-Spektren wohl bekannte Absorptionsstufe, die der Bildung eines CT-Komplexes aus Iodatom und Lösungsmittel zugeordnet werden konnte (s. Abschnitt 6.6.1). Weitere Abschwächung der Pulsenergie auf $13 \mathrm{~nJ}$ zeigt ebenfalls eine Absorptionsstufe mit etwas geringerer Intensität.

Diese Messungen sind ein relativ eindeutiger Beleg für das Vorhandensein eines ZweiPhotonen-Prozesse bei hohen Anregungsintensität (um $20 \mu \mathrm{W}$ ). Bei geringen Intensitäten überwiegt der Ein-Photonen-Prozess, welcher die schnelle Dissoziation in $\mathrm{CH}_{3}$ Radikal und Iodatom zur Folge hat und zu einer schnellen Bildung des bereits erwähnten CTTS-Komplexes führt. Bei hohen Intensitäten kommt offenbar ein anderer Prozeß zum Tragen, da sich das Bild der Absorptionszeitprofile vollkommen ändert. Ein Blick auf das Energiezustandsdiagramm (s. Abbildung 3.8) identifiziert mögliche durch MehrfachPhotonen-Absorption anregbare Zustände. Eine Anregungsphoton von $255 \mathrm{~nm}$ besitzt 


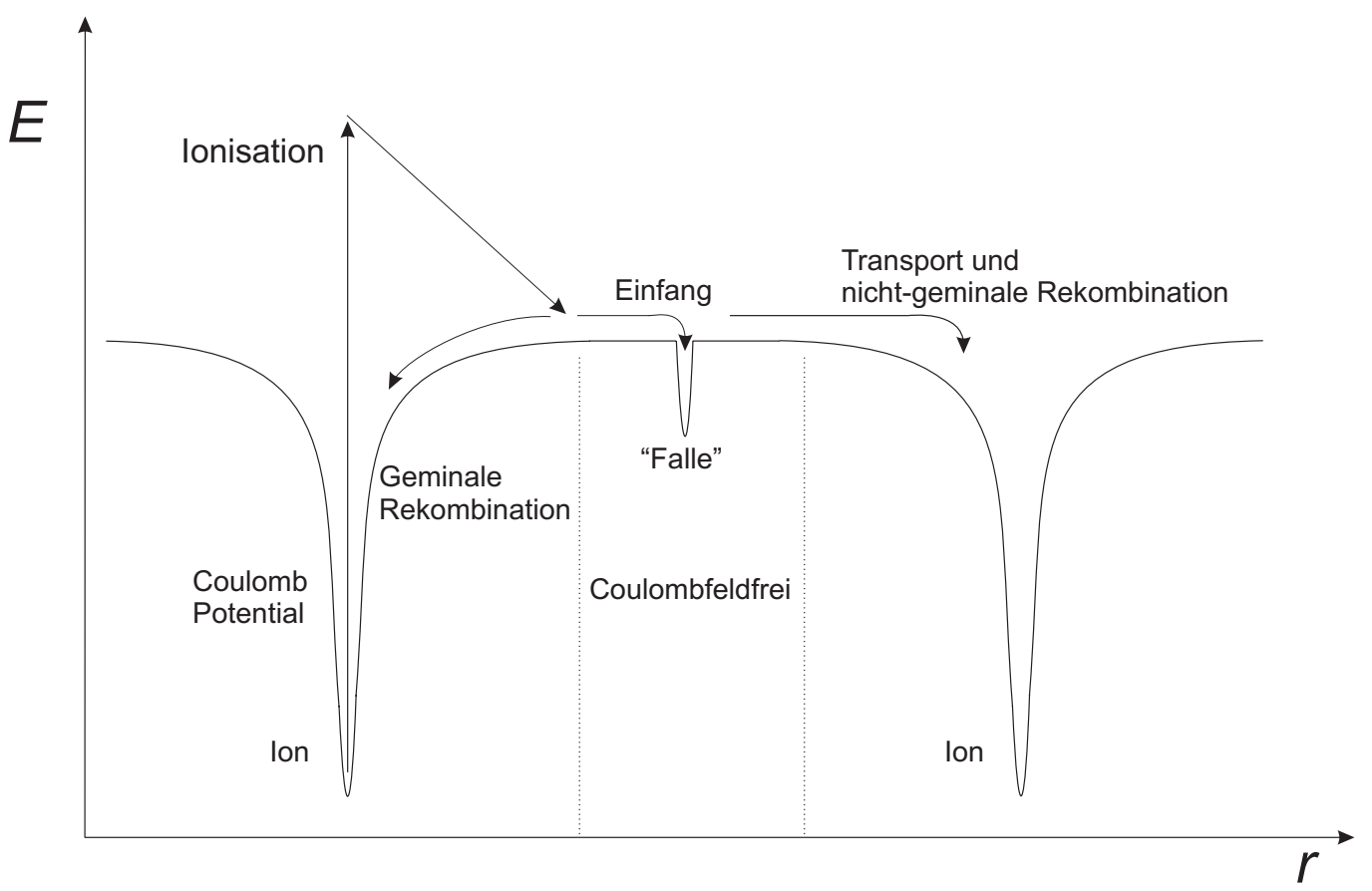

Abbildung 6.30: Dynamik des photogenerierten Elektrons in Lösung (nach Knoesel et al. [221])

eine Energie von $39215 \mathrm{~cm}^{-1}$, bei einer Zwei-Photonenanregung sind somit Zustände bis $78430 \mathrm{~cm}^{-1}$ zugänglich. Hierfür kommt der bereits von Poth etal. [213] diskutierte $10 s$-Rydbergzustand als auch der Grundzustand des $\mathrm{CH}_{3} \mathrm{I}^{+}$-Ions in Frage. In polaren Lösungsmitteln ist der ionische Grundzustand durch Solvatationseffekte energetisch abgesenkt und somit noch leichter zugänglich. Das $\mathrm{CH}_{3} \mathrm{I}$ kann demnach durch ZweiPhotonen-Absorption direkt $\mathrm{zu} \mathrm{CH}_{3} \mathrm{I}^{+}$ionisiert werden und ein freies Elektron an das Lösungsmittel abgeben.

\subsubsection{Dynamik des solvatisierten Elektrons}

Erstmalig nachgewiesen wurde die Existenz von freien Elektronen in Lösung bereits im Jahre 1864 durch Beobachtung gelöster Elektronen in flüssigen Ammoniak [214]. Die Abgabe eines freien Elektrons nach Ionisation zieht eine weitere Dynamik durch Solvatation des Elektrons nach sich. Untersuchungen dieser Dynamik und der daran beteiligten Zustände wurden unter anderem von Barbara et al. [215], Turi et al. [216], Pepin et al. [217] oder Laenen et al. 218 220] vorgenommen.

Der generelle Verlauf der Photoionisation und Dynamik des freien Elektrons in Lösung ist in Abbildung 6.30 wiedergegeben. Nach der Ionisation (z. B. durch Zwei-PhotonenAnregung) ist das Elektron im Kontinuumszustand des Lösungsmittelleitungsbands delokalisiert, dieses „freie“ Elektron besitzt eine entsprechende kinetische Überschußenergie. Anschließend gibt es einen Großteil seiner kinetischen Energie sehr schnell (etwa 100$200 \mathrm{fs}$ ) an das Lösungsmittel ab. Wenn sich das freie Elektron nach der thermischen Äqui- 


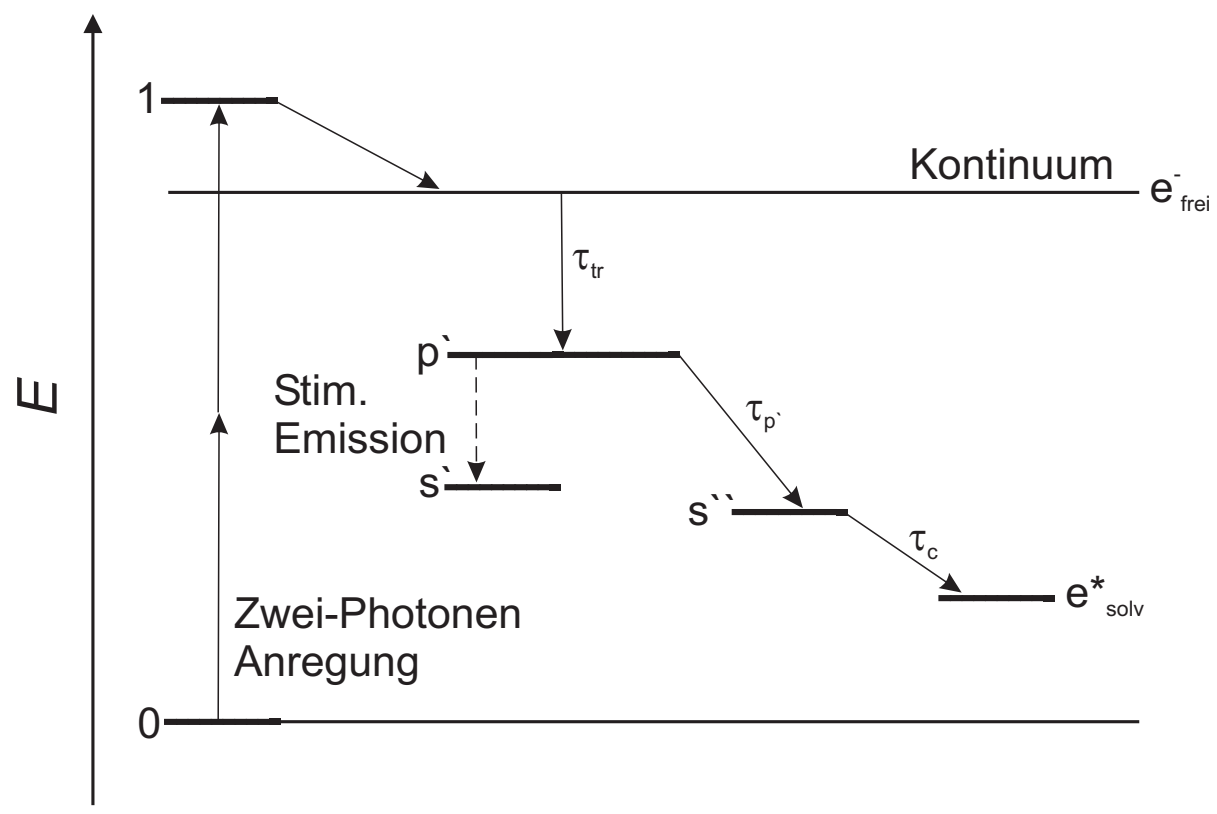

Abbildung 6.31: Energielevelschema des solvatisierten Elektrons nach Scheidt und Laenen 218

librierung noch im Einflußbereich des Coulombpotential des ionisierten Muttermoleküls befindet, erfolgt geminale Rekombination. Ansonsten kann es in einem geeigneten Zustand des Lösungsmittels, in welchem die Lösungsmittelmoleküle zufällig elektrostatisch günstig für das Elektron angeordnet sind (in Abbildung 6.30 als „Falle“ gekennzeichnet), lokalisiert und damit "gefangen" werden. Dort wird es vollständig solvatisiert und nach Diffusion im Lösungsmittel kann mit anderen Ionen eine nicht-geminale Rekombination erfolgen. Die Solvatisierung des Elektrons nach dem Einfang in Falle des Lösungsmittels verläuft über unterschiedliche Zustände, in der Literatur werden hierzu verschiedene Modelle diskutiert. Ein einfaches aus zwei Reaktionsschritten bestehendes Modell nach Eisenthal et al. 222] beschreibt die Solvatisierung wie folgt:

$$
e_{\mathrm{frei}}^{-} \longrightarrow e_{*}^{-} \longrightarrow e_{\mathrm{solv}}^{-}
$$

Dabei verläuft die Solvatation vom freien Elektron $e_{\text {frei }}^{-}$zum solvatisierten Elektron $e_{\text {solv }}^{-}$ über einen Zwischenzustand $e_{*}^{-}$, welcher in der Form eines „nassen“ oder teilgelösten Elektrons aufgefaßt wird. Trotz erfolgreicher Anwendung des Zwei-Zustands-Modell auf viele Messungen zur Solvatation von freien Elektronen, lassen sich nicht alle experimentellen Daten mit diesem einfachen Modell aus zwei Zuständen erklären. Deswegen wird anstelle des schrittweisen Übergangs zwischen verschiedenen, unterscheidbaren Zuständen in der Literatur zudem die Existenz einer lokalisierten Spezies diskutiert [215], welche einer kontinuierlichen Verschiebung des Absorptionsspektrum zu kürzeren Wellenlänge im Laufe der Solvatation vollzieht. Im einem einfachen kinetischen Schema läßt sich dieses Verhalten wie folgt ausdrücken: 


$$
e_{\text {frei }}^{-} \longrightarrow e_{p}^{-} \longrightarrow e_{*}^{-} \underset{\text { Verschiebung }}{\stackrel{\text { kont. }}{\longrightarrow}} e_{\text {solv }}^{-}
$$

Dabei handelt es sich bei $e_{p}^{-}$um einen angeregten elektronischen Zustand (nach der allgemeinen Konvention als $p$-Zustand bezeichnet), welcher in den noch nicht vollständig solvatisierten elektronischen Grundzustand $e_{*}^{-}$(s-Zustand) relaxiert. Durch Abgabe der Überschußenergie erfolgt eine Aufheizung der ersten Lösungsmittelschale. Die Relaxation des $e_{*}^{-}$-Zustands wird durch Umorientierung der Lösungsmittelmoleküle und somit der schrittweisen Solvatation erreicht. Diese Solvatation im elektronischen Grundzustand wird durch eine kontinuierliche spektrale Verschiebung an die experimentellen Daten angepaßt. Ein anderer Vorschlag zur Verbesserung des einfachen Zwei-Zustand-Modells ist seine Ergänzung um weitere transiente Zustände [218 220]. Dieser Ansatz mit verschiedenen diskreten Zuständen hat sich zur Interpretation der gesammelten Meßergebnisse als sinnvoll erwiesen. Das entsprechende Modell von Laenen et al. (s. Abbildung6.31) soll deswegen hier kurz näher vorgestellt werden. Nach der Photoanregung befindet sich das Elektron im Kontinuumszustand, relaxiert und wird anschließend mit der Geschwindigkeitskonstante $\tau_{\text {tr }}(\sim 100 \mathrm{fs})$ in einem geeigneten Zustand des Lösungsmittels „gefangen“. Dabei handelt es sich um den angeregten elektronischen Zustand $p^{\prime}$ mit dem korrespondierenden Grundzustand $s^{\prime}$. Da der Grundzustand nicht populiert wird, besteht eine Besetzungsinversion und stimulierte Emission ist prinzipiell möglich. Nach der Relaxation des angeregten Zustands $p^{\prime}$ mit $\tau_{p^{\prime}}$ in den modifizierten Grundzustand $s^{\prime \prime}$ muß noch eventuell vorhandene Überschußenergie der ersten Lösungsmittelschale abgeführt werden. Danach liegt das Elektron $e^{-}$im gelösten Zustand vor. Anschließend ist durch Diffusion der Elektronen im Lösungsmittel eine nicht-geminale Relaxation möglich.

\subsubsection{Kurzzeitdynamik von lodmethan nach Photoionisation in Lösung}

Ein Blick auf die Kurzzeitdynamik (im Bereich 0-15 ps) von $\mathrm{CH}_{3} \mathrm{I}$ in Methanol nach UVAnregung mit Abfrage im VIS-Bereich (s. Abbildung 6.32) zeigt je nach Probewellenlänge ein unterschiedliches Verhalten. Im Bereich von 400-440 nm beobachtet man nach dem kohärenten Anfangspeak eine monoexponentiell abklingende Emission, die Relaxationszeiten liegen um 1,7 ps und sind in Tabelle 6.6 zusammengestellt. Lediglich die Messung bei $400 \mathrm{~nm}$ Probewellenlänge weicht mit einer Zeitkonstanten von 2,48 ps von den anderen ab. Bei Wellenlängen > $440 \mathrm{~nm}$ ist keine negative Absorption zu beobachten, nach dem kohärenten Anfangspeak steigt die Absorption monoexponentiell an, um danach wieder abzuklingen. Der Abfall findet im Laufe von etwa 30 ps statt und ist für den Kurzzeitbereich nicht weiter relevant. Die Anstiegszeiten liegen bei etwa 1,6 ps (s. Tabelle 6.6) und sind im Rahmen der Zeitauflösung von etwa 250 fs nicht von der Probewellenlänge abhängig, zumindest ist kein eindeutiger Trend in Bezug auf die Wellenlänge festzustellen. Der Vergleich der Zeitkonstanten des monoexponentiellen Anstiegs bei Wellenlängen > $460 \mathrm{~nm}$ mit der Zeitkonstante des Abklingens der Emission bei Messungen kürzerer Wellenlänge zeigt, daß diese mit 1,6 ps und 1,7 ps annähernd identisch sind. Dadurch liegt der 


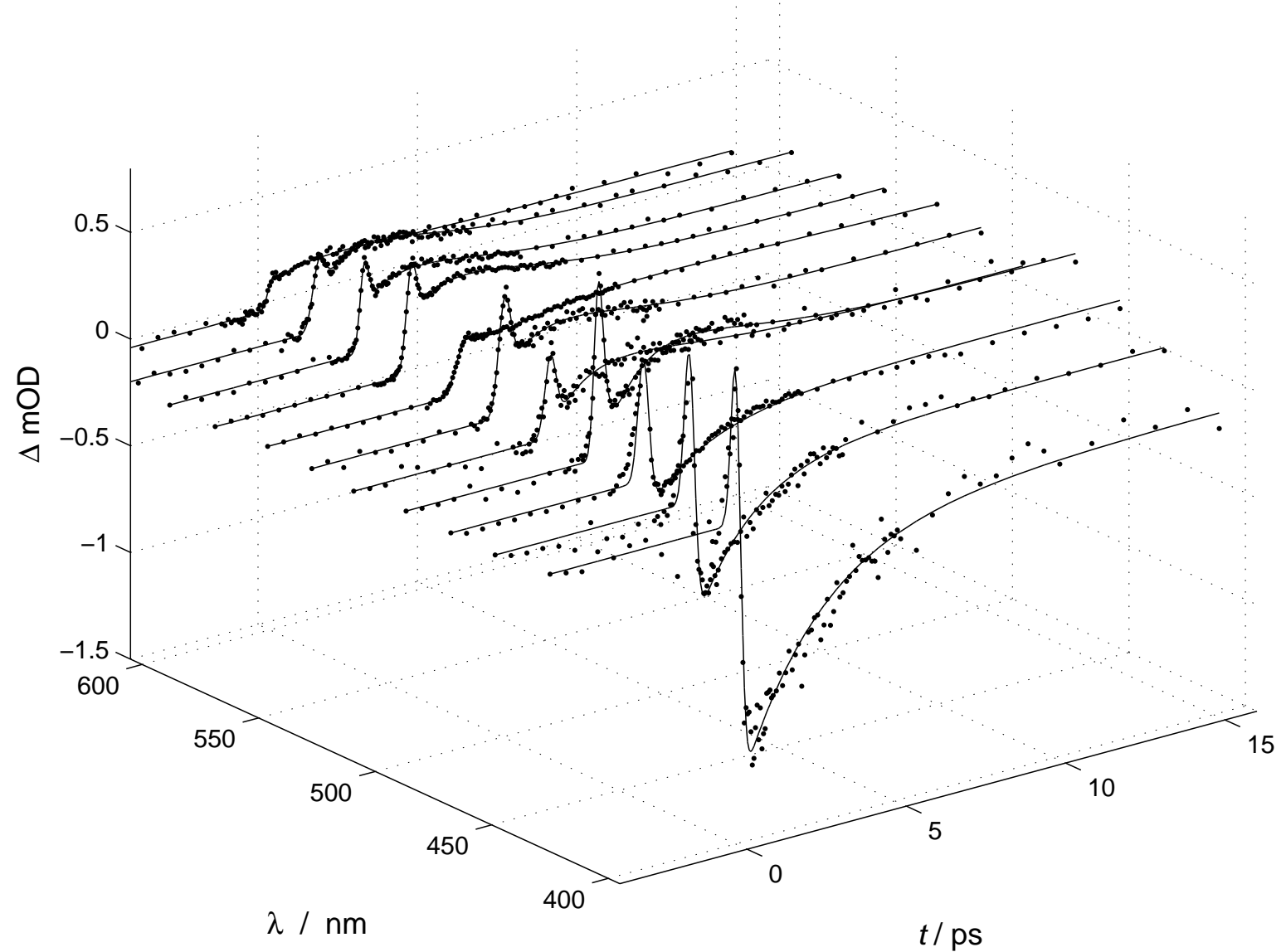

Abbildung 6.32: Kurzzeitdynamik von $\mathrm{CH}_{3} \mathrm{I}$ in Methanol bei unterschiedlichen Probewellenlängen nach $260 \mathrm{~nm}$ Anregung

Verdacht nahe, daß sowohl die Zeitkonstanten des monoexponentiellen Anstiegs als auch die des Abklingens der Emission der Lebensdauer desselben Zustands zuzuordnen sind. In Acetonitril und Methylcyclohexan als Lösungsmittel zeigt sich ein sehr ähnliches Bild beim Vergleich der Zeitkonstanten. In Tabelle 6.6 sind die Anstiegszeiten für Acetonitril dargestellt, die Emission um $400 \mathrm{~nm}$ klingt dabei mit einer Lebenszeit von etwa 2,9 ps ab, während der exponentielle Anstieg im Bereich von 440-560 nm eine Zeitkonstante von etwa 2,5 ps aufweist. In Methylcyclohexan konnte keine Emissionsbande gemessen werden, da keine Messungen bei einer Probewellenlänge $<420 \mathrm{~nm}$ aufgrund eines sehr kleinen Signal/Rausch-Verhältnisses möglich waren. Die Absorptionszeitprofile bei einer Probewellenlänge $>420 \mathrm{~nm}$ weisen alle nach einem mehr oder weniger intensiven kohärenten Anfangspeak einen monoexponentiellen Anstieg mit einem anschließenden Abfall auf. Die Anstiegszeiten liegen wie in Tabelle 6.6 dargestellt um 2,0 ps, ein den Zeiten in Methanol und Acetonitril sehr ähnlicher Wert.

Dieses sehr ähnliche Verhalten aller drei Lösungsmittel legt den Schluß nahe, daß es sich überall um denselben Prozeß handelt. In allen Lösungsmitteln kann somit nach Photoanregung derselbe Produktzustand beobachtet werden. Dieser Zustand besitzt demnach eine Lebensdauer von etwa 1,6 ps in Methanol, 2,5 ps in Acetonitril und 2,0 ps in Me- 


\begin{tabular}{|c|c|c|c|c|c|c|c|c|}
\hline \multicolumn{3}{|c|}{ Methanol } & \multicolumn{3}{|c|}{ Acetonitril } & \multicolumn{3}{|c|}{ Methylcyclohexan } \\
\hline$\lambda / \mathrm{nm}$ & $\tau_{\text {Auf }} / \mathrm{ps}$ & $\tau_{c c} / \mathrm{fS}$ & $\lambda / \mathrm{nm}$ & $\tau_{\text {Auf }}$ & $\tau_{c c} / \mathrm{fs}$ & $\lambda / \mathrm{nm}$ & $\tau_{\text {Auf }} / \mathrm{ps}$ & $\tau_{c c} / \mathrm{fS}$ \\
\hline 600 & 1,67 & 269 & 540 & 2,36 & 291 & 580 & 1,88 & 161 \\
\hline 580 & 1,48 & 359 & 520 & 2,57 & 253 & 560 & 2,37 & 413 \\
\hline 560 & 1,59 & 260 & 500 & 2,28 & 462 & 540 & 2,04 & 423 \\
\hline 540 & 1,70 & 300 & 480 & 2,46 & 435 & 520 & 2,09 & 401 \\
\hline 520 & 1,87 & 429 & $\bar{M}_{\tau_{\mathrm{Auf}}}$ & 2,41 & & 440 & 1,52 & 358 \\
\hline 500 & 1,58 & 369 & 420 & 2,71 & 220 & 420 & 1,74 & 382 \\
\hline 480 & 1,60 & 345 & 410 & 2,95 & 130 & $\bar{M}_{\tau_{\mathrm{Auf}}}$ & 1,94 & \\
\hline 460 & 1,66 & 244 & 400 & 3,23 & 174 & & & \\
\hline $\bar{M}_{\tau_{\text {Auf }}}$ & 1,72 & & 390 & 3,08 & 181 & & & \\
\hline 440 & 1,51 & 328 & 380 & 2,86 & 417 & & & \\
\hline 420 & 1,77 & 313 & $\bar{M}_{\tau_{\text {Emis }}}$ & 2,97 & & & & \\
\hline 400 & 2,48 & 293 & & & & & & \\
\hline $\bar{M}_{\tau_{\text {Emis }}}$ & 1,64 & & & & & & & \\
\hline
\end{tabular}

Tabelle 6.6: Zeitkonstanten des schnellen exponentiellen Anstiegs von $\mathrm{CH}_{3} \mathrm{I}$ in Methanol, Acetonitril und Methylcyclohexan. Die Abklingzeiten der Emission sind durch die vertikale Linie abgetrennt

thylcyclohexan. In den polaren Lösungsmitteln kann zudem eine stimulierte Emission mit fast derselben Lebensdauer beobachtet werden. Was bedeutet, daß der besetzte Produktzustand energetisch über einem anderen schwächer besetzten Zustand liegen muß, um stimulierte Emission bei etwa $400 \mathrm{~nm}$ zu ermöglichen. Anschließend relaxiert er in einen anderen Zustand, welcher eine Absorption bei 460-600 nm zeigt. Anhand dieser experimentellen Befunde kann ausgeschlossen werden, daß vorrangig eine einfache Dissoziation in ein $\mathrm{CH}_{3}$-Radikal und ein Iodatom stattfindet. Diese primären Photofragmente besitzen keinerlei Absorptionsbanden im gemessenen Probewellenlängenbereich. Wie in Abschnitt 6.6.1 bereits diskutiert, ist im Fall der Messungen von $\mathrm{CH}_{3} \mathrm{I}$ mit sichtbaren Probewellenlängen von einer Zwei-Photonen-Absorption auszugehen. Im Falle einer möglichen Zwei-Photonen-Ionisation entsteht neben dem $\mathrm{CH}_{3} \mathrm{I}^{+}$-Kation ein freies Elektron. Dieses freie Elektron kann als hauptverantwortliche Spezies für die Kurzzeitdynamik der beobachteten Absorptionszeitprofile identifiziert werden.

Wie in Abschnitt 6.6.4 beschrieben, liegt das Elektron nach Photoionisation zuerst als freies Elektron vor, bevor es innerhalb $100 \mathrm{fs}$ in einem geeigneten Zustand des Lösungsmittels gefangen wird. In Abbildung 6.33 sind die transienten Absorptionsspektren der verschiedenen elektronischen Zustände während der Solvatation in Methanol dargestellt. Das freie Elektron besitzt ein sehr breites Absorptionsspektrum, welches für nahezu alle gemessenen Probewellenlängen seinen Teil zur Absorption beiträgt. Somit setzt sich der Anfangspeak aus der Absorption des freien Elektrons vor Einfang in eine „Falle“ des Lösungsmittels und einem kohärenten Lösungsmittelsignal zusammen. Nach dem Einfang des Elektrons befindet es sich in einem angeregten Zustand $p^{\prime}$, dieser liegt über einem unbesetzten Grundzustand $s^{\prime}$. Dadurch kann stimulierte Emission in der Probe angeregt werden, die Emissionsbande liegt bei etwa $400 \mathrm{~nm}$ in Methanol und Acetonitril. In 


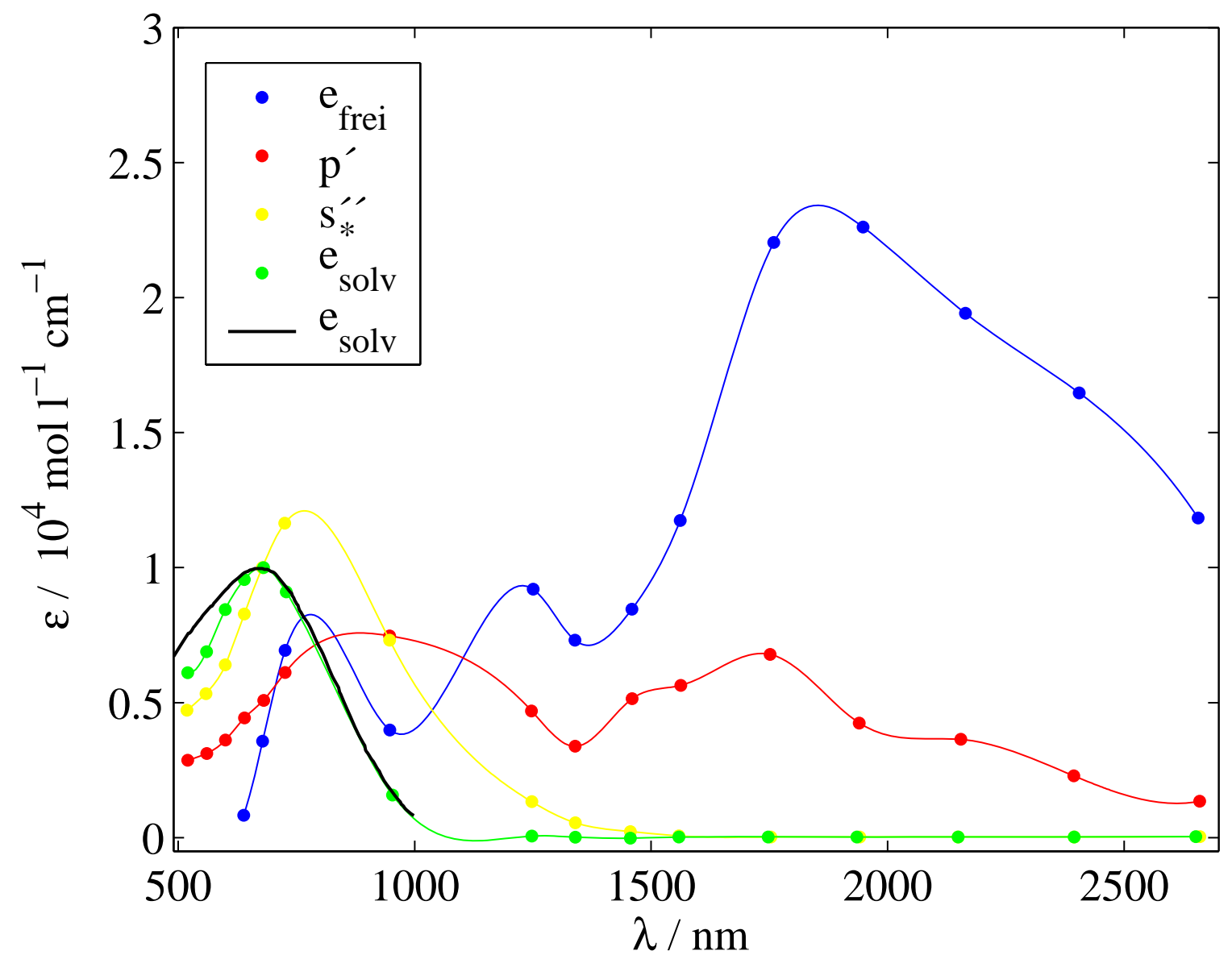

Abbildung 6.33: Absorptionsspektrum des solvatisierten Elektrons und seiner transienten Zustände in Methanol [218]

Methylcyclohexan wird die stimulierte Emission nicht beobachtet, dieses könnte darauf zurückgeführt werden, daß die Messungen aufgrund des geringen Signal/Rauschverhältnis nicht weit genug in den UV-Bereich ausgedehnt wurden. Die andere Möglichkeit ist, daß die in Methylcyclohexan hervorgerufene Besetzungsinversion zwischen $p^{\prime}$ und $s^{\prime}$-Zustand nicht ausreicht. Nach Photoionisation muß das Elektron zuerst aus dem Bereich des Coulombpotential des Kations ausbrechen (s. Abbildung 6.30) bevor es zum freien Elektron werden kann. Methylcyclohexan besitzt eine um den Faktor 15 kleinere relative Dielektrizitätskonstante $\epsilon_{r}$ (Methylcyclohexan: $\epsilon_{r}=2,07$, Methanol: $\epsilon_{r}=32,63$, Acetonitril: $\epsilon_{r}=$ 37,5), die Coulombwechselwirkung zwischen Elektron und dem Gegenion sind somit auch um diesen Faktor weitreichender. Ein Maß für die Reichweite des Coulombpotential ist der sogenannte Onsager-Radius $r_{\text {Ons }}$. Dieser beschreibt die Entfernung $r_{\text {Ons }}$, bei welcher die separierende, diffusionsbedingte Kraft und die rücktreibende, durch Coulombanziehung des Gegenion bedingte Kraft gleich groß sind. Der Onsager-Radius berechnet sich nach: 


$$
r_{\mathrm{Ons}}=\frac{e^{2}}{4 \pi \epsilon_{0} \epsilon_{r} k_{\mathrm{B}} T}
$$

Dabei beträgt $r_{\text {Ons }}$ für Methanol 1,7 nm, für Acetonitril 1,5 nm und der entsprechende Wert für Methylcyclohexan ist 27,4 nm. Die Separation der Ionen nach Photoionisation hängt in erster Linie von der Überschußenergie des freien Elektrons ab, eine typische Größenordnung sind 4-6 nm wie Messungen unter vergleichbaren Bedingungen für Hexan als Lösungsmittel ergeben haben [223 225]. Die anfängliche Separation ist somit zumindest in Methylcyclohexan geringer als $r_{\text {Ons }}$, was die Wahrscheinlichkeit der direkten geminalen Rekombination erhöht. Dadurch ist der Anteil der Elektronen, die als freie Ladungsträger in Lösung gehen, wesentlich kleiner und die daraus resultierende Besetzungsinversion zwischen $p^{\prime}$ und $s^{\prime}$-Zustand somit möglicherweise so gering, daß keine stimulierte Emission gemessen werden kann.

Der nach dem Einfang des Elektrons besetzte $p^{\prime}$-Zustand relaxiert mit der Zeitkonstanten $\tau_{p^{\prime}}$. Diese Zeitkonstante kann sowohl für das Abklingen der stimulierten Emission in Methanol und Acetonitril als auch für den monoexponentiellen Anstieg bei längeren Wellenlängen in allen drei Lösungsmitteln gemessen werden (s. Tabelle 6.6). Wie in Abbildung 6.33 ersichtlich, ist das transiente Absorptionsspektrum des $s^{\prime \prime}$-Zustands im Vergleich zu dem vom $p^{\prime}$-Zustand zu kürzeren Wellenlängen verschoben, woraus der monoexpentielle Anstieg im Wellenlängenbereich 440-600 nm resultiert. Ein Vergleich der experimentell bestimmten Zeitkonstante von 1,6-2,5 ps mit bereits in der Literatur veröffentlichten Werten zeigt eine gute Übereinstimmung. So berechneten Rossky et al. die Lebenszeitverteilung des $p^{\prime}$-Zustands in Methanol mit Hilfe von nicht-adiabatischen MD-Simulationen zu 0,4-4,3 ps, wobei die durchschnittliche Lebenszeit bei etwa 2,05 ps lag [226]. Experimentelle Untersuchungen von Laenen et al. 218] ergaben eine Relaxationszeit des $p^{\prime}$-Zustands in Methanol von 4,3 ps, diese Messungen waren allerdings auf Probewellenlängen $>500 \mathrm{~nm}$ beschränkt und konnten nicht die stimulierte Emission aus dem $p^{\prime}$ - in den $s^{\prime}$-Zustand beobachten. Weitere experimentelle Daten in Methanol von Pepin et al. [217] zeigen eine wellenlängenabhängige Relaxationszeit von 0,4-4 ps, Eisenthal et al. bestimmten $\tau_{p^{\prime}}$ zu 7,1 ps [222]. Für Acetonitril und Methylcyclohexan konnten keine vergleichbaren Daten gefunden werden, für diese Lösungsmittel existieren nur wenige Untersuchungen zur Solvatationsdynamik von freien Elektronen.

Die meßbare stimulierte Emission aus dem $p^{\prime}$-Zustand zeigt, daß die Relaxation von $p^{\prime}$ Zustand nicht in seinen korrespondierenden Grundzustand $s^{\prime}$ erfolgt, da in diesem Fall die Besetzungsinversion nicht aufrecht erhalten werden könnte. Der $p^{\prime}$-Zustand relaxiert somit in einen anderen Zustand, den modifizierten Grundzustand $s^{\prime \prime}$. Der Unterschied zwischen $s^{\prime \prime}$-Zustand und $s^{\prime}$-Zustand liegt in erster Linie in ihrer Solvensumgebung. Nach Kimura et al. [215] erfolgt eine schrittweise Solvatation durch Ausrichtung der Lösungsmittelmoleküle. Pepin et al. [217] unterscheidet die beiden Zustände als einen „schwach gebundenen“ und einen „stark gebundenen“ Zustand. Generell wird dabei dem ersten Zustand $s^{\prime}$ ein nur teilweise gelöstes Elektron zugeordnet, während im $s^{\prime \prime}$-Zustand die Solvatation durch Ausrichtung der Lösungsmittelmoleküle weitgehend abgeschlossen ist. 
Die vom Elektron abgeführte Überschußenergie trägt zur Aufheizung der ersten Lösungsmittelschale bei, Laenen et al. 218] unterscheiden die beiden Zustände $s^{\prime}$ und $s^{\prime \prime}$ anhand der lokalen Temperatur innerhalb der ersten Lösungsmittelschalen. Das Abführen dieser Überschußenergie durch Relaxation des Lösungsmittels hat eine weitere Dynamik zur Folge. Im vollständig relaxierten $s^{\prime \prime}$-Zustand liegt das Elektron schlußendlich in seiner gelösten Form $e_{\text {solv }}^{-}$vor. Dabei erfolgt eine weitere spektrale Blauverschiebung von $s^{\prime \prime}$ zu $e_{\text {solv }}^{-}$ wie in den Spektren von Abbildung 6.33 ersichtlich ist. Diese Langzeitdynamik erfolgt auf einer Pikosekundenzeitskala und wird im folgenden Abschnitt 6.6.6 diskutiert.

\subsubsection{Diffusive und nicht-diffusive Rekombination solvatisierter Elektronen und Kationen}

Die Langzeitdynamik (Piko- bis Nanosekundenbereich) von Iodmethan nach Photoionisation ist in erster Linie durch Schwingungsenergierelaxation und geminale Rekombination von freien Elektronen und Kationen geprägt. Nach Besetzung des $s^{\prime \prime}$-Zustands muß dieser noch die vorhandene Überschußenergie des Elektrons durch Relaxation ans Lösungsmittel abführen. Dabei vollzieht das Elektron auf dem Weg zum vollständig solvatisierten

Zustand $e_{\text {solv }}^{-}$eine weitere spektrale Blauverschiebung, wie anhand von Abbildung 6.33 zu sehen ist. Im sichtbaren Bereich von 460-700 nm steigt die Absorption im Laufe der Solvatisierung des Elektron demnach an. Allerdings ist bei den im sichtbaren Bereich gemessenen Absorptionszeitprofilen (s. Abbildung 5.41, 5.42 und 5.43) kein Anstieg der Absorption zu beobachten. Nach dem monoexponentiellen Anstieg mit einer Zeitkonstante um 2 ps (s. Abschnitt 6.6.5) fällt die Absorption bei allen Wellenlängen > $460 \mathrm{~nm}$ in Methylcyclohexan innerhalb mehrerer hundert Pikosekunden ab, in Methanol und Acetonitril erfolgt dieser Abfall schneller (innerhalb einiger zehn Pikosekunden). Dabei bleibt in Methanol und auch in Acetonitril ein konstanter end off-set stehen, während in Methylcyclohexan die Absorption wieder nahezu auf Null abfällt. Das Absorptionsspektrum des gelösten Elektron zeigt in diesem Wellenlängenbereich (460-600 nm) allerdings eine ausgeprägte Absorption (s. Abbildung 6.33), dem Abfall der Absorption muß demnach ein Abklingen der Konzentration der solvatisierten Elektronen zugrunde liegen. Ein möglicher Grund für den Abfall der Absorption könnte die diffusionsgesteuerte Rekombination der solvatisierten Elektronen mit entsprechenden Kationen sein. Dabei findet diese Rekombination in Methylcyclohexan nahezu vollständig statt, da die Absorption im Laufe der Zeit komplett auf Null abfällt, während der konstante end off-set in Methanol und Acetonitril dafür spricht, daß ein signifikanter Teil der gelösten Elektronen nicht auf der beobachteten Zeitskala rekombiniert. Eine nicht-geminale Rekombination der Ladungsträger nach Photoionisation in Hexan und Cyclohexan auf einer Pikosekundenzeitskala wurde beispielsweise von Knoesel et al. [221] durch zeitaufgelöste Terahertzspektroskopie gemessen. 


\section{Diffusive Rekombination in unpolaren Lösungsmitteln}

Die Modellierung der nicht-geminalen Rekombination erfolgt durch eine homogene, diffusionskontrollierte Reaktion von zwei verschieden geladenen Ionen. Dabei rekombiniert das freie Elektron mit einem Kation:

$$
\mathrm{CH}_{3} \mathrm{I}^{+}+\mathrm{e}_{\text {solv }}^{-} \stackrel{k}{\longrightarrow} \mathrm{CH}_{3} \mathrm{I}
$$

Bei der Reaktion handelt es sich um eine Kinetik 2. Ordnung, welche dem allgemeinen Geschwindigkeitsgesetz

$$
\frac{\delta n(t)}{\delta t}=-k n^{2}(t)
$$

gehorcht. Dabei ist $n(t)$ die Menge an solvatisierten Elektronen in Abhängigkeit von der Zeit, $k$ die Geschwindigkeitskonstante. Auf der rechten Seite der Gleichung wird das Produkt aus Kation- und gelöster Elektronendichte aufgrund der Ladungserhaltung mit dem Quadrat der Elektronendichte ersetzt. Diese Differentialgleichung kann folgendermaßen gelöst werden:

$$
\frac{n(t)}{n(0)}=\frac{1}{1+k n(0) t}
$$

Dabei ist $n(0)$ die anfängliche Dichte an freien Elektronen nach der Photoionisation. Die Geschwindigkeitskonstante $k$ ergibt sich aus der Lösung der Diffusiongleichung unter bestimmten Randbedingungen, eine exemplarische Lösung findet sich bei Hummel 227]. Findet zusätzlich zur Diffusion eine elektrostatische Wechselwirkung statt, muß man die Diffusiongleichung um das entsprechende Potential $V(r)$ der Wechselwirkung ergänzen. Ausgehend von den Fick'schen Gesetzen der Diffusion ergibt sich somit für den stationären Zustand einer solchen Reaktion:

$$
\frac{d c_{i}}{d t}=D_{i}\left(\frac{d c_{i}}{d r}+\frac{c_{i}}{k_{\mathrm{B}} T} \frac{d V(r)}{d r}\right)
$$

Mit der Startbedingung $c_{i}(r=\infty)=c_{0}$ und der Smoluchowski-Randbedingung, daß jeder Stoß reaktiv ist, läßt sich die Differentialgleichung folgendermaßen lösen:

$$
k=4 \pi D_{\mathrm{AB}} \beta
$$

Dabei ist $\beta$ : 


$$
\beta=\left[\int_{R}^{\infty} e^{V(r) / k_{\mathrm{B}} T} r^{-2} d r\right]^{-1}
$$

Für zwei jeweils mit der Elementarladung $e$ unterschiedlich geladene Spezies $A$ und $B$ beträgt $V(r)=-e^{2} / 4 \pi \epsilon_{0} \epsilon_{r} r$. Durch Integration der Gleichung 6.39 erhält man für $\beta$ :

$$
\beta=\frac{r_{\mathrm{Ons}}}{1-e^{-r_{\mathrm{Ons}} / R}}
$$

$r_{\text {Ons }}$ ist dabei der Onsager-Radius (s. Gleichung 6.33). Durch Einsetzen von Gleichung 6.40 in Gleichung 6.38 ergibt sich für die Geschwindigkeitskonstante $k$ :

$$
k=\frac{4 \pi D_{\mathrm{AB}} r_{\mathrm{Ons}}}{1-e^{-r_{\mathrm{Ons}} / R}}
$$

In einem unpolaren Lösungsmittel wie Methylcyclohexan ist $r_{\text {Ons }}$ wesentlich größer als der Kontaktabstand $R$ (s. Abschnitt 6.6.5), die Gleichung kann somit noch weiter zu

$$
k=4 \pi D_{\mathrm{AB}} r_{\mathrm{Ons}}
$$

vereinfacht werden. $D_{\mathrm{AB}}$ ist der wechselseitige Diffusionskoeffizient von solvatisierten Elektronen und Kation in Lösung. Da das Kation im Vergleich zum Elektron wesentlich unbeweglicher ist, kann man $D_{\mathrm{AB}}$ allein aus der Beweglichkeit des Elektrons $\mu_{e}$ berechnen:

$$
D_{\mathrm{AB}}=\frac{\mu_{e} k_{\mathrm{B}} T}{e}
$$

Durch Einsetzen von Gleichung 6.33 und Gleichung 6.43 in Gleichung 6.42 vereinfacht sich die Berechnung von $k \mathrm{zu}$ :

$$
k=\frac{e}{\epsilon_{0} \epsilon_{r}} \mu_{e}
$$

Die Geschwindigkeitskonstante $k$ hängt somit nur von der Elektronenbeweglichkeit $\mu_{e}$ und $\epsilon_{r}$ als Lösungsmittelparameter ab.

Messungen zur durchschnittlichen Beweglichkeit $\mu_{e}$ von Elektronen in Lösung (z. B. durch Radiolyse, Radikalfängermethoden oder Leitfähigkeitsmessungen) finden sich in der Literatur. Da allerdings kein Wert für Methylcyclohexan und Acetonitril gefunden werden konnte, mußte $\mu_{e}$ für Methylcyclohexan in erster Näherung durch den entsprechenden Wert von Cyclohexan ersetzt werden. Für die durchgeführte kinetische Modellierung wurde dabei nun mit $\mu_{e}$ in Cyclohexan $\mu_{\text {Cyc.Hex. }}=0,24 \mathrm{~cm}^{2} \mathrm{~V}^{-1} \mathrm{~s}^{-1}$ und $\mu_{\mathrm{MeOH}}=$ 

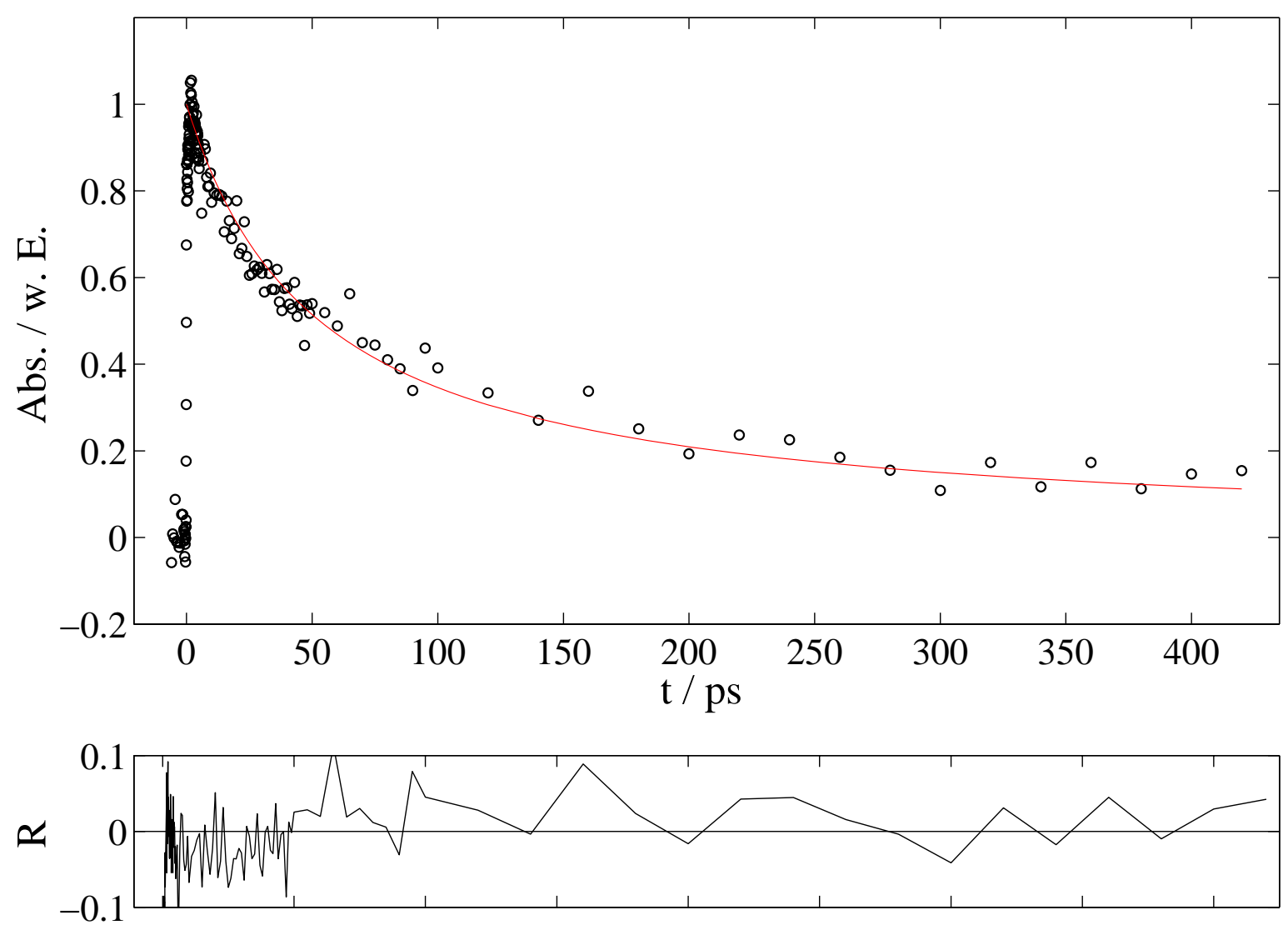

Abbildung 6.34: Kinetische Modellierung eines Absorptionszeitprofils von $\mathrm{CH}_{3} \mathrm{I}$ in Methylcyclohexan bei $265 \mathrm{~nm}$ Anregung und $580 \mathrm{~nm}$ Abfrage

$5,9 \times 10^{-4} \mathrm{~cm}^{2} \mathrm{~V}^{-1} \mathrm{~s}^{-1}$ in Methanol gerechnet [228]. Wie aus Gleichung 6.36 ersichtlich hängt die zeitabhängige Rekombination in hohem Maße von der Anfangskonzentration $n(0)$ der freien Elektronen ab. Da diese experimentell nicht zugänglich war, muß sie für die durchgeführten Messungen abgeschätzt werden. Die Pulsenergie bei $265 \mathrm{~nm}$ Anregung betrug etwa $E_{\text {Puls }}=40 \mathrm{~nJ}$. Die Anfangskonzentration der freien Elektronen berechnet sich nun aus der Anzahl Photonen pro Puls und dem Fokusvolumen, in denen die Photoelektronen generiert werden. Dabei erzeugen zwei Photonen ein Elektron, für diesen nicht-linearen Prozeß wird in erster Näherung eine Effizienz von etwa $\Phi=0,25$ angenommen

$$
n(0)=\frac{3 E_{\mathrm{Puls}} \Phi}{h \nu 4 \pi r^{3} 2}
$$

Der Durchmesser des Pumpfokus wird zu etwa $50 \mu \mathrm{m}$ abgeschätzt, damit ergibt sich eine Photonendichte von etwa $8 \times 10^{23}$ Photonen $\mathrm{m}^{-3}$ was einer Anfangselektronenkonzentration $n(0)$ von etwa $1 \times 10^{23}$ e $\mathrm{m}^{-3}$ entspricht.

Mit diesem Wert kann nun die die diffusiv gesteuerte Rekombination von solvatisierten Elektronen mit den entsprechenden Kation im relevanten Zeitbereich von 0-500 ps simu- 
liert werden. Zum Vergleich wurde eine typische Messung von $\mathrm{CH}_{3} \mathrm{I}$ in in Methylcyclohexan bei $580 \mathrm{~nm}$, also zentriert auf der Bande des solvatisierten Elektrons, herangezogen. Diese Messung wurde dazu um den off-set korrigiert und auf ihren Peak normiert. Simulation und Messung sind in Abbildung 6.34 dargestellt und zeigen eine relativ gute Übereinstimmung. Dabei ist allerdings zu beachten, daß einige der gemachten Näherungen mit großen Unsicherheiten versehen sind. So kann der Fokusradius $r_{f}$ nur grob über Fokussierung durch verschiedene Blenden mit unterschiedlichen Radien eingegrenzt werden, desweiteren sind keine näheren Informationen über die Effizienz der Zwei-PhotonenIonisierung verfügbar. Die abgeschätzten 25 v. H. stellen dabei nur einen Erfahrungswert über nicht-lineare optische Prozesse dar. Weiterhin läßt diese Abschätzung keinen Raum für die primäre geminale Rekombination der Ladungsträger vor Abschluß der Solvatation. Dadurch wird die Anfangskonzentration $n(0)$ verringert und der Abfall verläuft langsamer.

Nichtsdestotrotz läßt sich mit der Modellvorstellung einer nicht-geminalen diffusiven Rekombination der Abfall der Absorption in Methylcyclohexan zu späten Reaktionszeiten sehr gut simulieren. Die Energierelaxation der aufgeheizten Lösungsmittelschale, wie sie von anderen Arbeitsgruppen beobachtet wurde [218], ist möglicherweise von dieser Dynamik überdeckt und deswegen nicht beobachtbar. Trotz dieser Einschränkungen ist die Übereinstimmung zwischen simulierter Kurve und Meßdaten relativ gut, daß von einer diffusiv gesteuerten Rekombination als vorherrschenden Prozeß im Langzeitbereich auszugehen ist.

\section{Rekombination in polaren Lösungsmitteln}

Im Falle der polaren Lösungsmittel Methanol und Acetonitril zeigt sich ein etwas anderer Verlauf der transiente Absorption. So fällt das Signal im Spektralbereich des Absorptionsspektrum des solvatisierten Elektrons nicht auf Null ab (s. Abbildung 6.35 (Acetonitril) und 6.36 (Methanol)), ein signifikanter Anteil bleibt demnach erhalten. Desweiteren ist der Abfall in Methanol und Acetonitril wesentlich schneller als in Methylcyclohexan, er läßt sich monoexponentiell mit einer Zeitkonstante von etwa 20-30 ps anpassen während das Abklingen der Absorption in Methylcyclohexan nach 2. Ordnung in mehreren hundert Pikosekunden verläuft. Für eine kinetische Modellierung nach 2. Ordnung wie sie für Methylcyclohexan vorgenommen wurde, zeigt sich daß der Koeffizient für die Beweglichkeit der Elektronen in Methanol mit $\mu_{\mathrm{MeOH}}=5,9 \times 10^{-4} \mathrm{~cm}^{2} \mathrm{~V}^{-1}$ um drei Größenordnungen kleiner ist als der in Cyclohexan mit $\mu_{\text {Cyc.Hex. }}=0,24 \mathrm{~cm}^{2} \mathrm{~V}^{-1} \mathrm{~s}^{-1}$. Das bedeutet, daß nach Gleichung 6.44 die Geschwindigkeitskonstante $k$ ebenfalls drei Größenordnungen kleiner ist, die größere relative Dielektrizitätskonstante von Methanol $\left(\epsilon_{r(\mathrm{MeOH})}=32,63, \epsilon_{r(\mathrm{MCH})}\right.$ $=2,07)$ kann diesen Unterschied nicht kompensieren. Dies bedeutet, daß die Rekombination dementsprechend langsam verläuft. Mit der gleichen Anfangskonzentration $n(0)$ findet die Rekombination somit auf einer Nano-/Mikrosekundenzeitskala statt. Dieser relativ langsame Abfall erklärt den konstanten end off-set, die Rekombination und somit der Abfall der Absorption ist so langsam, daß er auf der beobachteten Pikosekundenzeitskala als konstant erscheint. Für den exponentiellen Abfall von etwa 20-30 ps, welcher in 

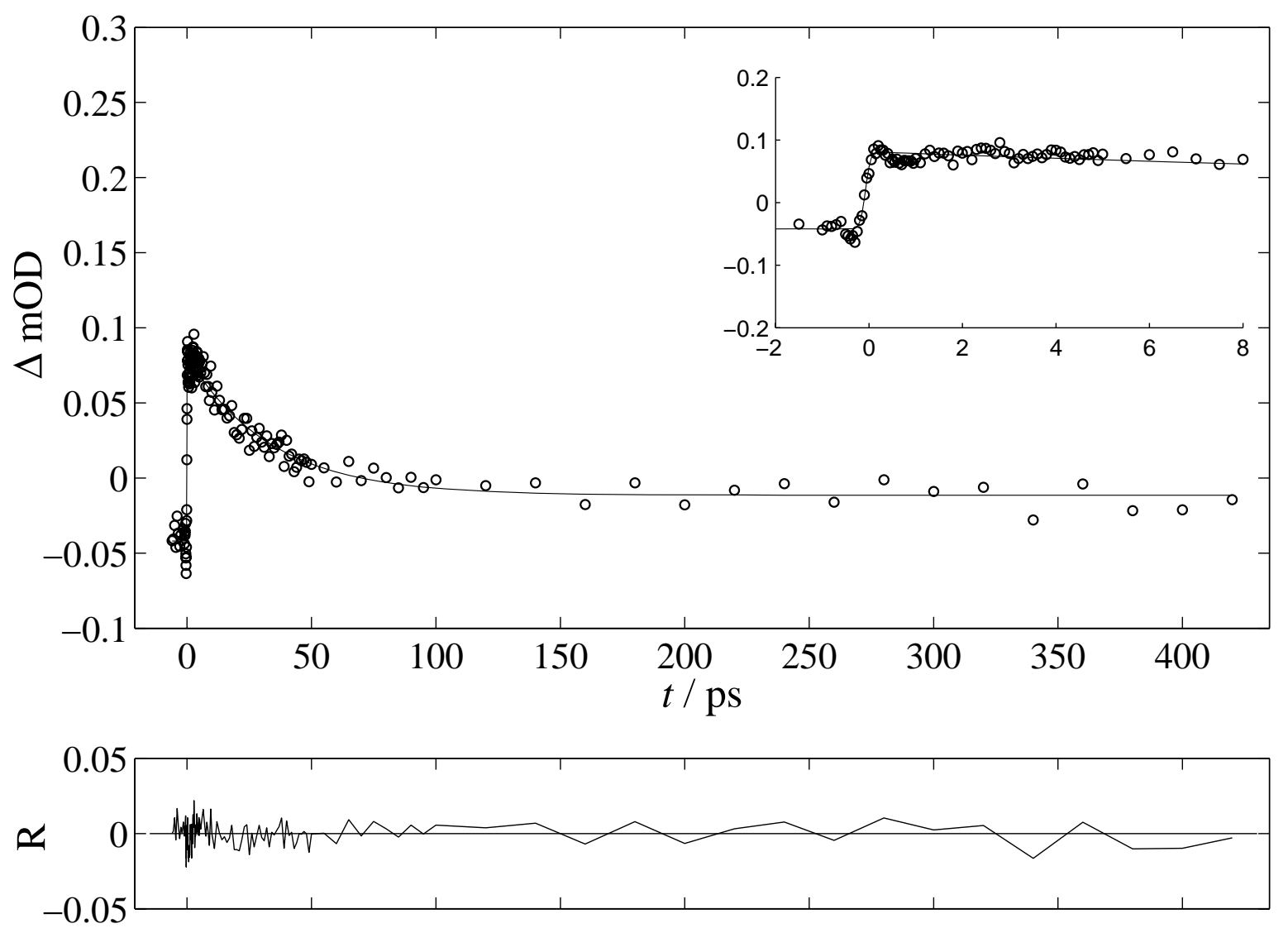

Abbildung 6.35: Absorptionszeitprofil von $\mathrm{CH}_{3} \mathrm{I}$ in Acetonitril bei $260 \mathrm{~nm}$ Anregung und $540 \mathrm{~nm}$ Abfrage

Methanol und Acetonitril beobachtet wird, kann demnach keine diffusiv gesteuerte Rekombination verantwortlich sein.

Jedoch wurde in der bisherigen Betrachtungsweise der Diffusion dabei immer von einer zeitunabhängigen Geschwindigkeitskonstante $k_{\infty}$ ausgegangen. Neben dem konstanten $k_{\infty}$ kommt insbesondere im Kurzzeitbereich der Diffusion einem zeitabhängigen, transienten Term $k(t)$ eine besondere Bedeutung zu. Ausgehend vom 2. Fick'sche Gesetz kann die Diffusionsgleichung unter den bekannten Startbedingungen $\left(c(r, 0)=c_{0}\right.$ und $c(\infty, t)=c_{0}$ ) sowie der Collins-Kimball-Randbedingung (s. Abschnitt 2.2) so gelöst werden, daß die Zeitabhängigkeit der Geschwindigkeitskonstante $k(t)$ in Form eines transienten Terms berücksichtigt wird. Eine exemplarische Lösung findet sich z. B. bei Nemzek und Ware [229] oder Ware et al. [230]. Dabei ergibt sich für $k(t)$ :

$$
k(t)=4 \pi R D_{\mathrm{AB}}\left[1+\frac{R}{\sqrt{\pi D_{\mathrm{AB}} t}}\right]
$$

Neben dem bekannten Smoluchowski-Ausdruck $k_{\infty}=4 \pi r D_{\mathrm{AB}}$ gilt es nun, den zeitabhängigen Teil zu beachten. Dabei kann für die betrachtete Rekombination von La- 

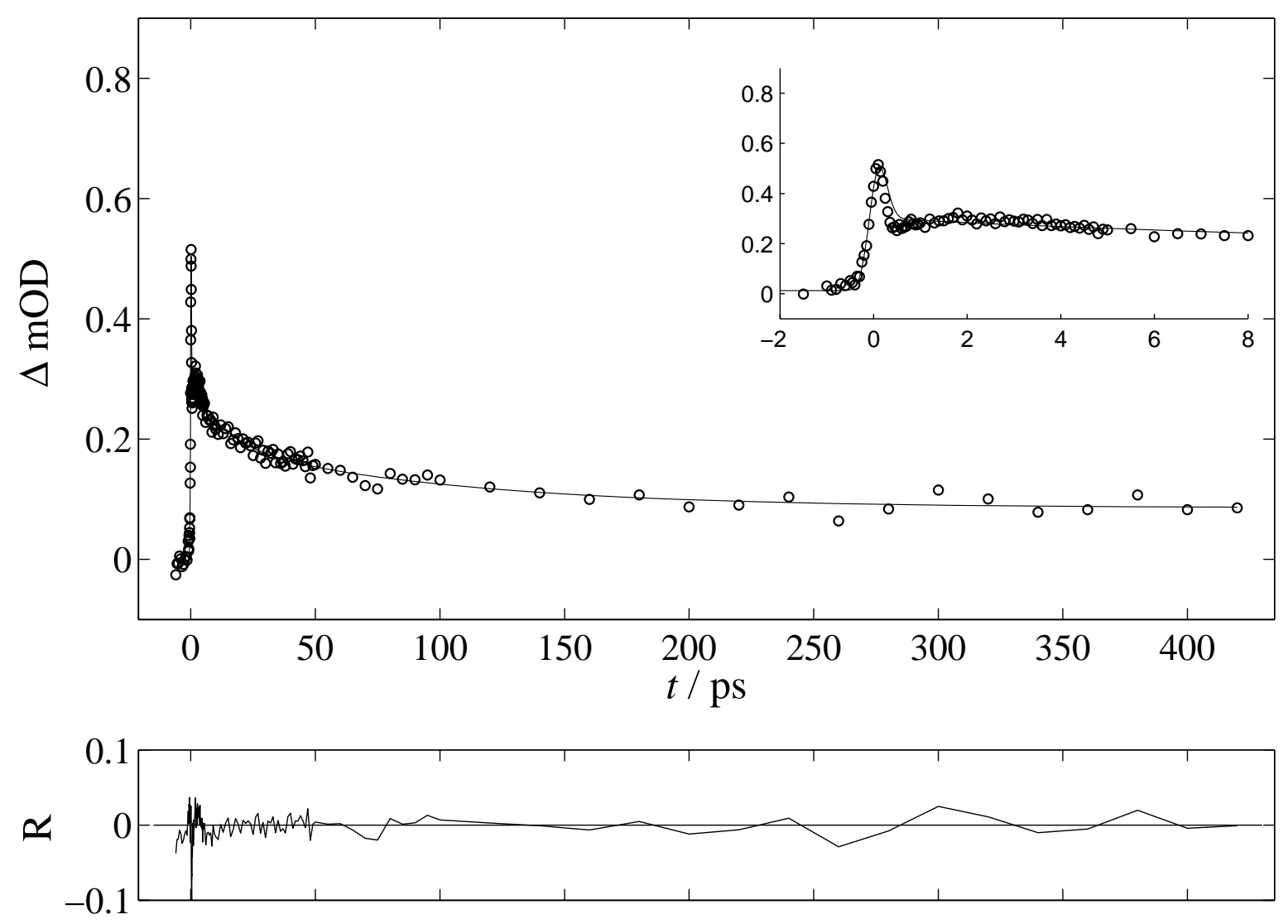

Abbildung 6.36: Absorptionszeitprofil von $\mathrm{CH}_{3} \mathrm{I}$ in Methanol bei $260 \mathrm{~nm}$ Anregung und $520 \mathrm{~nm}$ Abfrage

dungsträgern in Lösung für den Kollisionsabstand $R$, der zur Reaktion führt, wieder der Onsager-Radius $r_{\text {Ons }}$ eingesetzt werden. Somit gilt:

$$
k(t)=k_{\infty}+4 \pi D_{\mathrm{AB}} r_{\mathrm{Ons}} \frac{r_{\mathrm{Ons}}}{\sqrt{\pi D_{\mathrm{AB}} t}}
$$

Der Diffusionskoeffizient $D_{\mathrm{AB}}$ für die Bewegung von freien Elektronen in Lösung wird nach Gleichung 6.43 ausgerechnet. Der Einfluß des transienten Terms auf die diffusive Rekombination kann nun am Beispiel der Photoionisation von $\mathrm{CH}_{3} \mathrm{I}$ in Methanol ausgerechnet werden. Dazu werden $D_{\mathrm{AB}}$ und $r_{\text {Ons }}$ nach Gleichung 6.43 und 6.33 berechnet und in Gleichung 6.47 eingesetzt. Die so bestimmte zeitabhängige Geschwindigkeitskonstante $k(t)$ wird in die Lösung der Differentialgleichung zur Rekombination der Ladungsträger nach 2. Ordnung eingesetzt (Gleichung 6.36). Dazu wird als freie Elektronenkonzentration $n_{0}$ der nach Gleichung 6.45 abgeschätzte Wert von $1 \times 10^{23} e^{-3}$ verwendet. Dabei zeigt sich, daß der zeitabhängige Geschwindigkeitskoeffizient $k(t)$ immer noch 2-3 Größenordnungen zu klein ist, um für die beobachtete Dynamik auf der Pikosekundenzeitskala verantwortlich zu sein. Ein diffusiver Prozeß als Grundlage der experimentellen Beobachtungen in polaren Lösungsmitteln kann somit ausgeschlossen werden. 
Eine andere Möglichkeit für den Abfall der Absorption ist eine nicht-diffusiv gesteuerte Rekombination der geminalen Ladungsträger. Nach der Photoionisation werden das freie Elektron und das Kation separiert, in Methanol und Acetonitril ist der Onsager-Radius $r_{\text {Ons }}$ relativ klein ( um 1,5 nm), die Wahrscheinlichkeit einer vollständigen Separation somit relativ hoch. Nach Einfang des freien Elektrons in einer Lösungsmittelfalle und abgeschlossener Solvatation sind die gelösten Ladungsträger noch in relativ naher Entfernung. In polaren Lösungsmitteln wie Methanol ist die Potentialtopftiefe der „Falle“ des Lösungsmittel größer als unpolaren wie in Methylcyclohexan. Daraus resultiert eine viel kleinere Beweglichkeit der Elektronen, da die Aktivierungsenergie zum Verlasen der Lösungsmittelfalle viel größer ist. Somit können auf der beobachteten Piko/Nanosekundenzeitskala keine nicht-geminalen Ladungsträger rekombinieren, sondern nur die geminalen, welche sich nach der Solvatation noch in relativer Nähe befinden. Das gelöste Elektron kann die Falle nach Solvatation zwischenzeitlich verlassen und sich durch das Lösungsmittel bewegen bevor es von einen anderen Lösungsmittelzustand eingefangen wird oder auf das geminale Kation trifft. Die nachfolgende geminale Rekombination ist wahrscheinlich für den Abfall der Absorption des solvatisierten Elektrons innerhalb der beobachteten 2030 ps verantwortlich zu machen. Dabei handelt es sich um einen aktivierten Prozeß, da zuerst eine Aktivierungsenergie zum Auslösen der Elektronen aus der Lösungsmittelfalle notwendig ist.

Zeitaufgelöste Pump/Probe-Messungen zur Solvatation überschüssiger Elektronen in Acetonitril von Xia et al. 231] zeigen ebenfalls einen Abfall der Absorption des gelösten Elektrons mit einer Zeitkonstante von etwa 30 ps. Dieser Prozeß wird von Xia et al. ebenfalls auf die geminale Rekombination der primären Photoprodukte zurückgeführt. Ein ähnliches Verhalten konnten Vilchiz et al. [232] bei der Photoanregung von $\mathrm{I}^{-}$mit anschließender Abgabe des Elektrons an verschiedene polare Lösungsmittel beobachten. Die anschließende geminale Rekombination fand mit Zeitkonstanten zwischen 30 ps (Wasser) und 700 ps ( $n$-Butanol) statt. Von dieser sekundären geminalen Rekombination ist die primäre geminale Rekombination zu unterscheiden, welche zwischen den Photofragmenten stattfindet bevor das freie Elektron den Einflußbereich des Coulombpotentials des Kations verlassen hat. Diese Rekombination ist wesentlich schneller da es nicht zur einem Einfang des Elektrons in Lösungsmittelfallen mit anschließender Solvatation kommt. Entsprechende Zeitkonstanten für die primäre geminale Rekombination finden sich z. B. bei Lukin etal. 2224 mit 0,2 ps in Methylcyclohexan oder bei Reuther et al. [233. mit 2,7 ps für Wasser. Bei den gemessenen Absorptionszeitprofilen konnte jedoch kein Abfall der Absorption mit Zeitkonstanten in der Größenordnung von 1-2 ps beobachtet werden. Da im Falle des Methylcyclohexan als Lösungsmittel eine primäre geminale Rekombination aufgrund des großen Onsager-Radius $\left(r_{\text {Ons }}=27,4 \mathrm{~nm}\right)$ jedoch sehr wahrscheinlich (s. Abschnitt 6.6.5) ist, wird diese, wie von Lukin et al. mit $200 \mathrm{fs}$ vorrausgesagt, sehr schnell innerhalb der Zeitauflösung des Experimentes stattfinden und somit innerhalb des Anfangspeak nicht auflösbar sein. Für Methanol und Acetonitril können keine abfallenden Zeitkomponenten in der Größenordnung von 1-2 ps beobachtet werden, die primäre geminale Rekombination findet somit aufgrund des kleinen Onsager-Radius $\left(r_{\text {Ons }}\right.$ $\sim 1,5 \mathrm{~nm}$ ) nicht statt. Stattdessen entkommen die freien Elektronen aus der Reichweite des Coloumbpotentials des Kations, werden solvatisiert und können erst nach Abschluß 

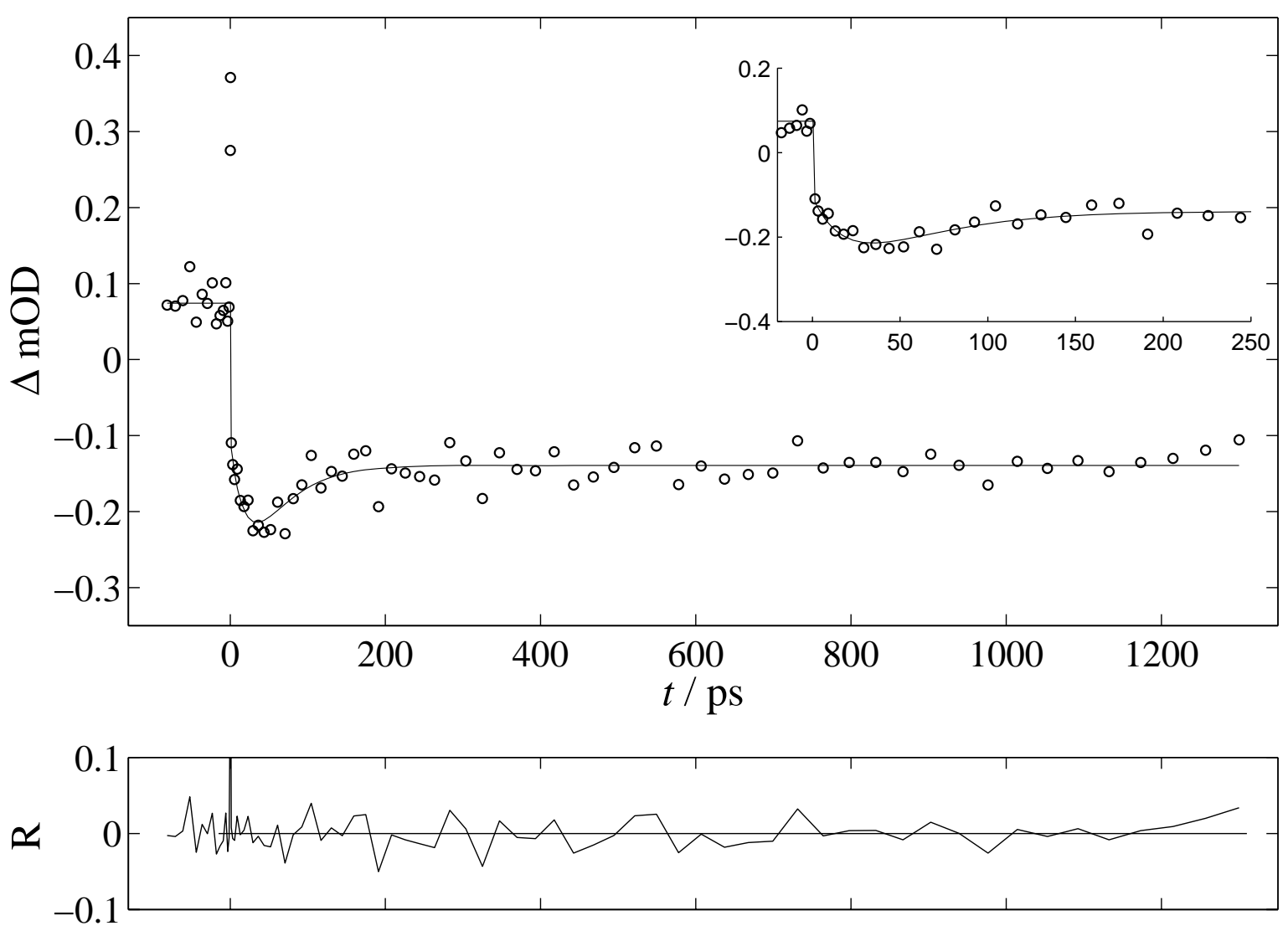

Abbildung 6.37: Zeitaufgelöste Integration über die Schwingungsbande bei $1250 \mathrm{~cm}^{-1}$ von $\mathrm{CH}_{3} \mathrm{I}$ in $\mathrm{CD}_{3} \mathrm{CN}$ nach $266 \mathrm{~nm}$ Anregung $\left(E_{\mathrm{Puls}}=17 \mu \mathrm{W}\right)$

dieser Dynamik mit ihrem geminalen Kation innerhalb der im Experiment beobachteten 20-30 ps rekombinieren.

\subsubsection{Geminale Rekombination von lodmethan in Lösung}

Die Fragestellung nach einer geminale Rekombination innerhalb des Lösungsmittelkäfigs konnte bisher für alle betrachteten Modellsysteme nicht zufriedenstellend beantwortet werden, weswegen weitergehende Messungen vorgenommen wurden. Da die in Abschnitt 6.6.1 bereits diskutierten Ausbleichexperimente keine Hinweise zur geminalen Rekombination geben konnten, sondern lediglich die Bildung von CTTS-Komplexen nachweisen konnten, wurden Pump/Probeexperimente mit IR-Probestrahl durchgeführt. Dazu wurde mit dem IR-Lasersystem (s. Abschnitt 4.2) eine Lösung von $\mathrm{CH}_{3} \mathrm{I}$ in $\mathrm{CD}_{3} \mathrm{CN}$ bei $266 \mathrm{~nm}$ angeregt und das IR-Spektrum zeitaufgelöst im Bereich 1220-1280 nm detektiert. Dabei wurde der Pumppuls durch einen ca. $10 \mathrm{~cm}$ dicken Quarzblock geführt, um ihn zeitlich zu verbreitern. Auf diese Weise konnte die Spitzenintensität des Pumppulses gesenkt werden, um das kohärente Anfangssignal des Lösungsmittels zu verkleinern und eine Zwei-Photonen-Absorption des $\mathrm{CH}_{3} \mathrm{I}$ zu vermeiden. Die Messungen wurden bei unterschiedlich Anregungsintensitäten vorgenommen (s. Abbildung 5.44 und 5.45). 

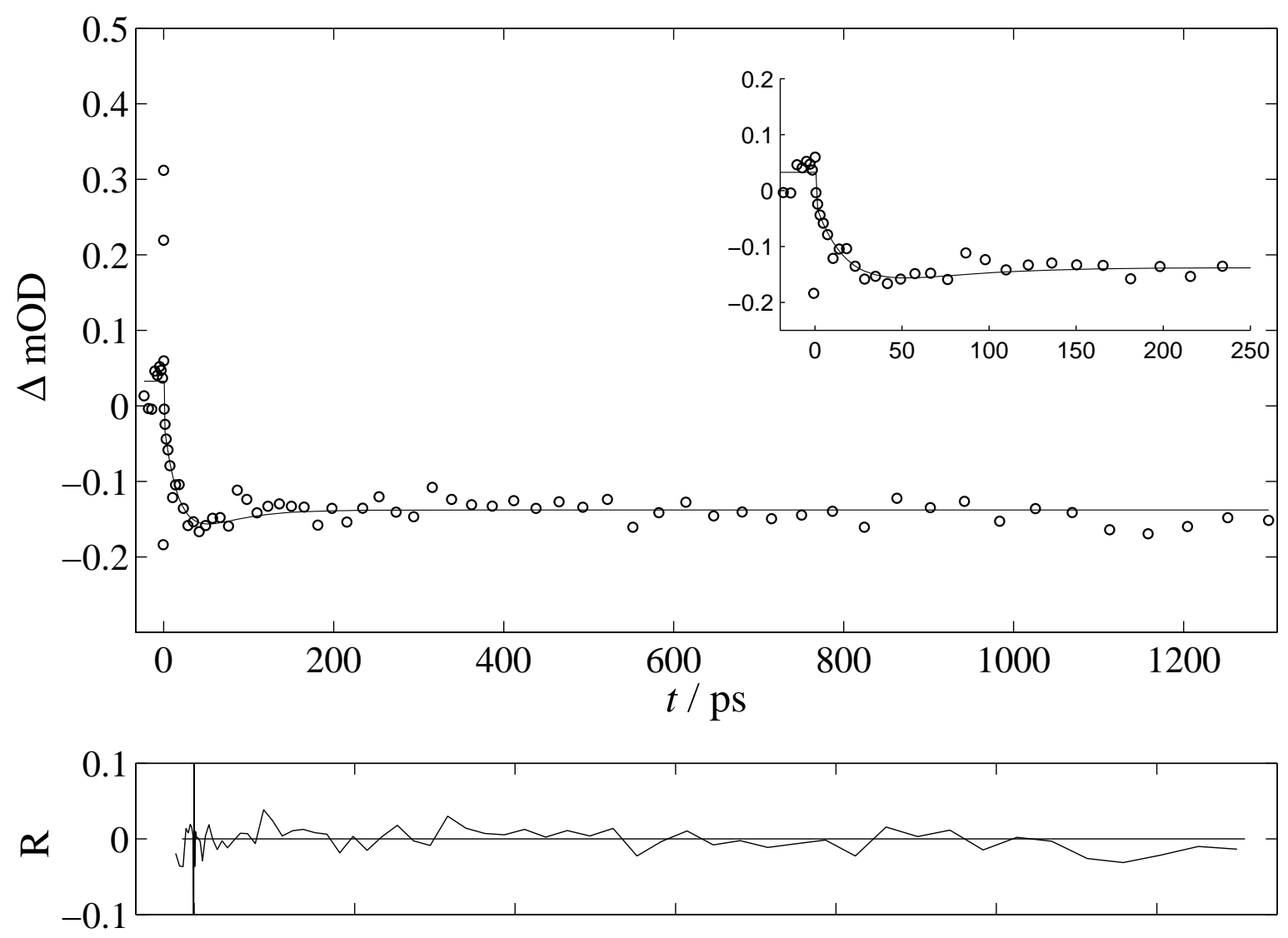

Abbildung 6.38: Zeitaufgelöste Integration über die Schwingungsbande bei $1250 \mathrm{~cm}^{-1}$ von $\mathrm{CH}_{3} \mathrm{I}$ in $\mathrm{CD}_{3} \mathrm{CN}$ nach $266 \mathrm{~nm}$ Anregung $\left(E_{\mathrm{Puls}}=5,5 \mu \mathrm{W}\right)$

Anhand der zeitaufgelösten Spektren läßt sich ein Ausbleichen der Schwingungsbande der symmetrischen $\mathrm{CH}_{3}$-Deformationsschwingung bei $1250 \mathrm{~cm}^{-1}$ innerhalb der Zeitauflösung beobachten. Die Integration über die komplette Schwingungsbande zeigt, daß die Absorption nach dem anfänglichen Ausbleichen innerhalb 40 ps wieder ansteigt (s. Abbildung 6.37 und Abbildung 6.38). Im Falle der Messung mit hoher Anregungsintensität $\left(E_{\mathrm{Puls}}=17 \mu \mathrm{W}\right)$ liegt die Zeitkonstante bei $42,7 \mathrm{ps}$, während bei geringerer Intensität $\left(E_{\text {Puls }}=5,5 \mu \mathrm{W}\right)$ die Amplitude wesentlich kleiner ist und die Anstiegszeit bei 39,8 ps liegt. Der Wiederanstieg der Absorption kann auf die zu erwartende geminale Rekombination der Photofragmente zurückgeführt werden. Nach der Photodissoziation (ZweiPhotonen-Photoionisation wird durch die zeitliche Streckung des Pumppulses verhindert) können die Photofragmente entweder kinematisch aus dem Löungsmittelkäfig ausbrechen oder bleiben darin gefangen. Die im Käfig gefangenen Fragmente können wieder rekombinieren, danach gibt das schwingungsheiße $\mathrm{CH}_{3} \mathrm{I}$ seine Überschußenergie aus der Photodissoziation durch Schwingungsenergietransfer (VER) an das Lösungsmittel abgeben.

Relaxationszeiten von schwingungsangeregtem $\mathrm{CH}_{3} \mathrm{I}$ in Lösung wurden von Crim et al. mit Hilfe von IR-Pump/UV-Probe-Experimenten gemessen [203, 234]. Dazu regten sie einen Oberton der C-H-Streckschwingung bei etwa 1,7 $\mu \mathrm{m}$ an und fragten mit einem UV- 
Probepuls die Absorption auf der roten Flanke des Absorptionsspektrums des $\mathrm{CH}_{3} \mathrm{I}$ ab. Dabei steigt die Absorption zuerst innerhalb weniger Pikosekunden an, um danach innerhalb etwa 50 ps wieder abzufallen. Die schnelle Zeitkomponente ist dabei der intramolekularen Schwingungsenergieumverteilung von der C-H-Streckschwingung in die anderen Schwingungsmoden des Moleküls (engl. intramolecular vibrational redistribution, IVR) zuzuordnen, während die langsame Zeitkomponente die Schwingungsenergierelaxation des $\mathrm{CH}_{3} \mathrm{I}$ durch Energieabgabe an das Lösungsmittel beschreibt. Diese Relaxationszeiten wurden von Crim et al. zu 50, 44 und 16 ps für $\mathrm{CCl}_{4}, \mathrm{CDCl}_{3}$ und $\left(\mathrm{CD}_{3}\right)_{2} \mathrm{CO}$ gemessen. Die Zeitkonstanten, insbesondere des Schwingungsenergietransfers in $\mathrm{CCl}_{4}$ und $\mathrm{CDCl}_{3}$ als vergleichbare Lösungsmittel, liegen sehr nah an den gemessenen Rekombinationszeiten des $\mathrm{CH}_{3} \mathrm{I}$ in $\mathrm{CD}_{3} \mathrm{CN}$. Somit ist naheliegend, daß die Schwingungsenergierelaxation bestimmend für die gemessenen Zeitkonstanten bei der geminalen Rekombination von $\mathrm{CH}_{3} \mathrm{I}$ in Lösung ist. Die relativ kleine Amplitude des Anstiegs des Bandenintegrals (auch bei hohen Anregungsintensitäten) zeigt, daß die geminale Rekombination nur eine untergeordnete Rolle nach der Photodissoziation von $\mathrm{CH}_{3} \mathrm{I}$ in Lösung spielt. Eine exakte Berechnung ist jedoch aufgrund fehlender Extinktionskoeffizienten nicht möglich. Anhand der Untersuchungen von Parker et al. [132, 133] ist bekannt, daß die primären Photoprodukte von $\mathrm{CH}_{3} \mathrm{I}$ im Vergleich zu anderen Polyhalogenmethanen wie $\mathrm{CH}_{2} \mathrm{I}_{2}$ einen hohen Anteil kinetischer Energie nach Photodissoziation besitzen (s. Abschnitt 3.2). Dadurch ist der Käfigausbruch besonders effizient, so daß wenige Moleküle im Käfig gefangen bleiben und nach Schwingungsenergierelaxation rekombinieren können. In Konkurrenzreaktion zur geminalen Rekombination steht desweiteren die Bildung eines CTTS-Komplexes aus Ioadtom und einem Lösungsmittelmolekül (s. Abschnitt 6.6.1)

\subsubsection{Zusammenfassung der photoinduzierten Dynamik von lodmethan in Lösung}

Ausgehend von den in den vorherigen Abschnitten diskutierten Verhalten von $\mathrm{CH}_{3} \mathrm{I}$ in Lösung soll ein einfaches Modell zur Photodissoziation und -dynamik aufgestellt werden. Dabei ist in erster Linie das Verhalten nach Ein- bzw. nach Zwei-Photonen-Absorption zu unterscheiden. Ein Schema der photoinduzierten Prozesse von $\mathrm{CH}_{3} \mathrm{I}$ in Lösung ist in Abbildung 6.39 dargestellt.

\section{Ein-Photonen-Absorption}

Im Falle einer Ein-Photonen-Absorption dissoziiert das $\mathrm{CH}_{3} \mathrm{I}$ sehr schnell $\left(\tau_{\text {diss }}<100 \mathrm{fs}\right)$ in ein $\mathrm{CH}_{3}$-Radikal und ein Iodatom:

$$
\mathrm{CH}_{3} \mathrm{I} \stackrel{\mathrm{h} \nu}{\longrightarrow} \mathrm{CH}_{3}+\mathrm{I}
$$




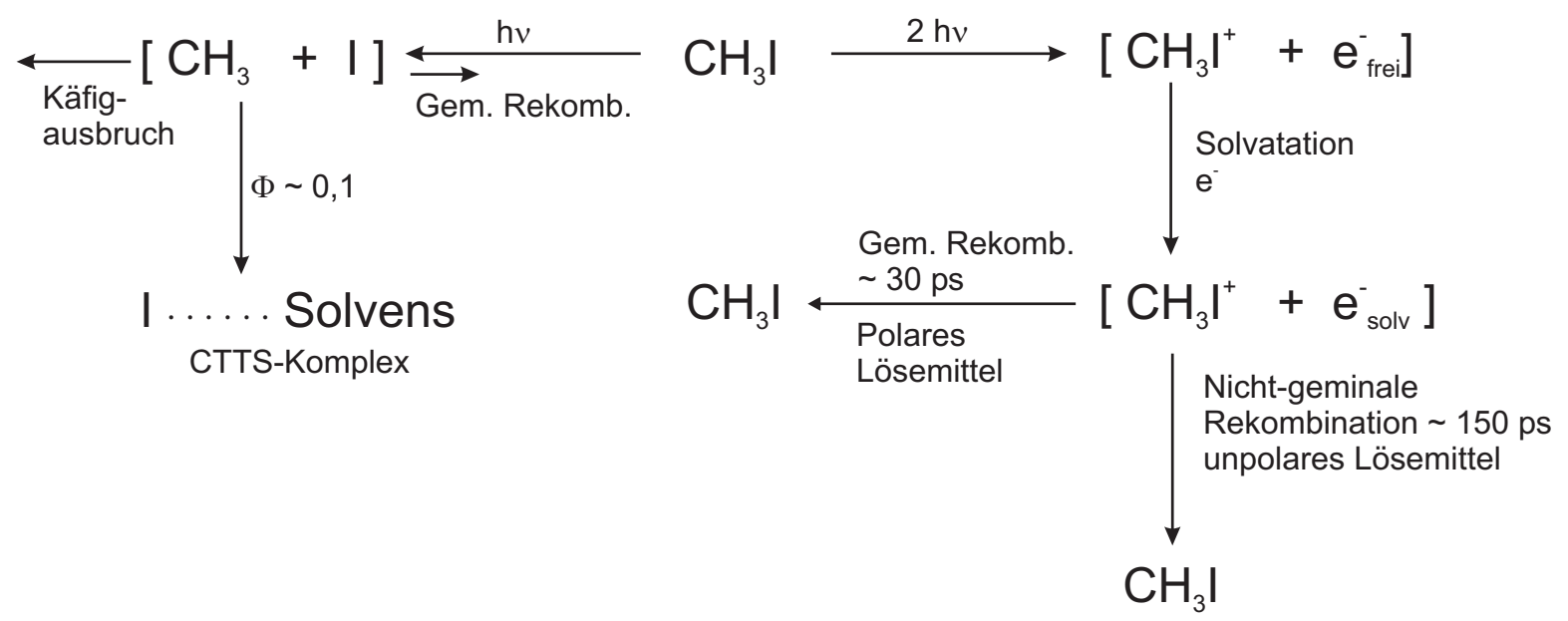

Abbildung 6.39: Modell zur Photoionisation von Iodmethan in Lösung mit anschließender Rekombination der erzeugten Ladungsträger

Die primären Photofragmente können nun entweder aus dem Lösungsmittelkäfig ausbrechen oder bleiben nach einem Stoß mit der Lösungsmittelwand im Käfig gefangen. Die im Käfig gefangenen Photofragmente können rekombinieren, danach gibt das schwingungsangeregte Rekombinationsprodukt seine Überschußenergie durch Schwingungsenergierelaxation innerhalb von etwa $40 \mathrm{ps}$ an das Lösungsmittel ab. Vor Abschluß der geminalen Rekombination können die im Käfig gefangenen sowie die ausgebrochenen Iodatome innerhalb weniger Femtosekunden mit einem Lösungsmittelmolekül einen CT-Komplex (CTTS-Komplex) bilden:

$$
\mathrm{CH}_{3} \mathrm{I} \stackrel{\mathrm{h} \nu}{\longrightarrow} \mathrm{CH}_{3}+\mathrm{I} \longrightarrow \mathrm{I} \cdots \text { Lösungsmittel CT-Komplex }
$$

Dem Absorptionsspektrum dieser CTTS-Komplexe liegt ihr CT-Übergang im UVBereich zugrunde, dieser CT-Übergang hängt in erster Linie vom Ionisierungspotential des verwendeten Lösungsmittels ab. Lösungsmittel mit sehr hohem Ionisierungspotentialen (wie z. B. perfluorierte Kohlenwasserstoffe) bilden keine CTTS-Komplexe. Die Quantenausbeute für die Bildung des CTTS-Komplexes wurde in Methylcyclohexan zu etwa 0,1 bestimmt.

\section{Zwei-Photonen-Absorption}

Die Dynamik nach Zwei-Photonen-Absorption unterscheidet sich stark von der EinPhotonen-Absorption. Durch die gleichzeitige Absorption von zwei Photonen wird das $\mathrm{CH}_{3} \mathrm{I}$ direkt ionisiert, also in den $\mathrm{CH}_{3} \mathrm{I}^{+}$-Grundzustand unter Abgabe eines Elektrons angeregt. Das freie Elektron kann entweder mit dem $\mathrm{CH}_{3} \mathrm{I}^{+}$-Kation geminal rekombinieren (vorzugsweise in unpolaren Lösungsmitteln) oder es wird durch das Lösungsmittel 


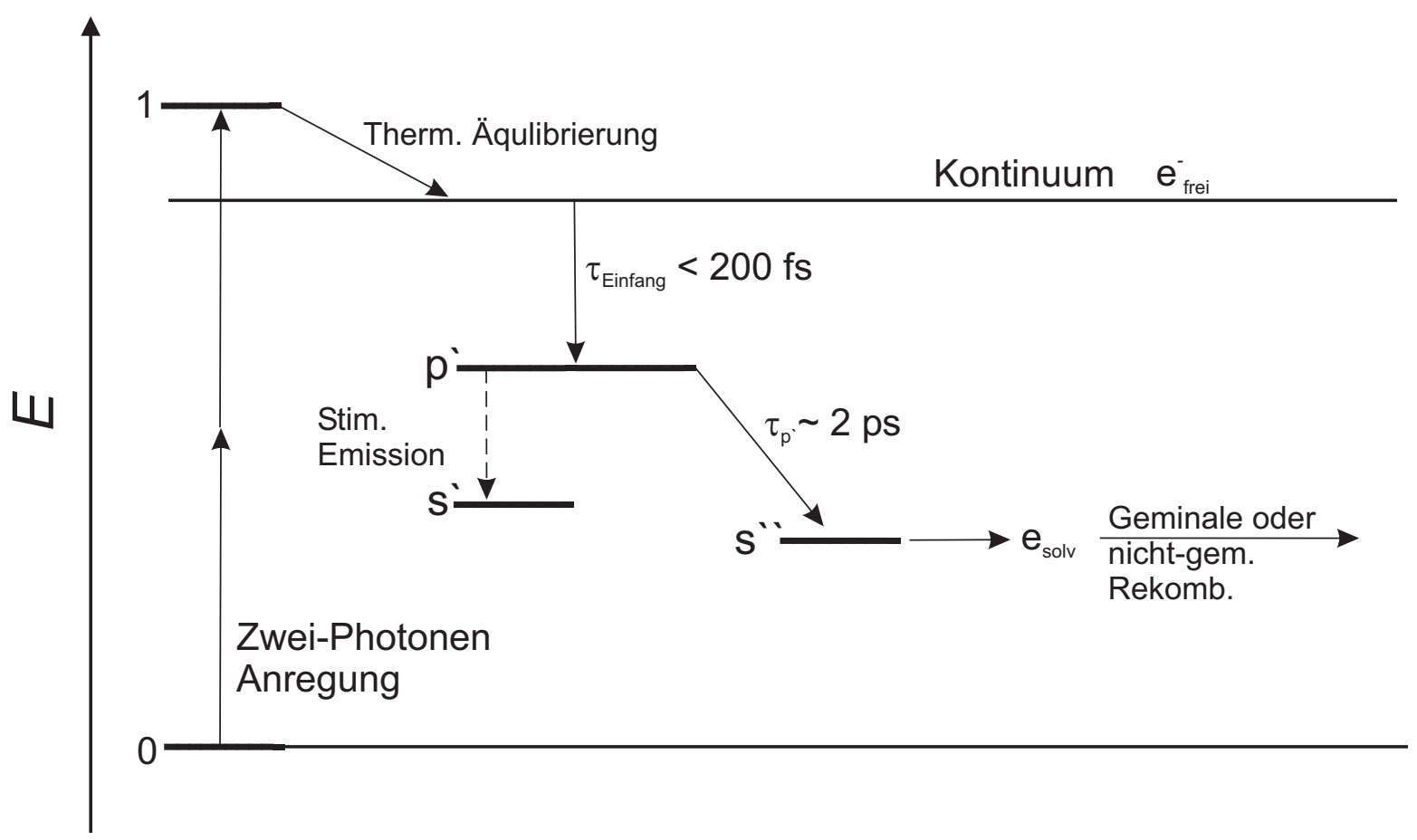

Abbildung 6.40: Modell der Solvatation des freien Elektrons in Lösung

solvatisiert. Nach Abschluß der Solvatation liegen freie Ionen in Lösung vor. Die Beweglichkeit des solvatisierten Elektrons entscheidet nun über die weitere Dynamik. In unpolaren Lösungsmitteln wie Methylcyclohexan ist die Beweglichkeit $\mu_{e}$ und somit die Diffusionsgeschwindigkeit groß genug, daß es zu einer nicht-geminalen diffusionsgesteuerten Rekombination der Ladungsträger kommt. Die Konzentration an freien Elektronen nimmt nach 2. Ordnung im Laufe von 150-200 ps ab.

Ein anderes Verhalten zeigt sich in polaren Lösungsmitteln wie Acetonitril oder Methanol. Aufgrund der wesentlich besseren Solvatation ist das Elektron in einer tieferen Potentialmulde des Lösungsmittels gefangen und kann sich erst nach Aufbringen einer Aktivierungsenergie frei im Lösungsmittel bewegen. Die durschnittliche Beweglichkeit des gelösten Elektrons und somit die Diffusionsgeschwindigkeit ist in polaren Lösungsmitteln dadurch viel geringer. Die nicht-geminale Rekombination findet auf einer Nano/Mikrosekundenzeitskala statt, im Bereich der in dieser Arbeit gemessenen Ultrakurzzeitdynamik ist der diffusionsgesteuerten Rekombination kein nennenswerter Beitrag zuzuschreiben. Aufgrund der eingeschränkten Beweglichkeit können nur geminale Elektronen und Kationen rekombinieren, diese Rekombination findet in etwa 20-30 ps statt.

Für die Solvatation des freien Elektrons in Lösung läßt sich anhand der aufgenommen Meßdaten ein einfaches kinetisches Schema (s. Abbildung 6.40), ausgehend vom Modell von Laenen et al. [218] aufstellen. Das Elektron wird durch die Zwei-Photonen-Absorption vom Muttermolekül abgelöst und geht als freies Elektron in Lösung. Nach der thermi- 
schen Äquilibrierung ist es entweder aus der Reichweite des Coloumbpotential des Kation ausgebrochen oder rekombiniert geminal. Das nicht rekombinierte freie Elektron wird anschließend innerhalb $200 \mathrm{fs}$ in einer Falle, also zufällig elektrostatisch günstig ausgerichteten Lösungsmittelmolekülen, im $p^{\prime}$-Zustand gefangen. Der entsprechende elektronische Grundzustand $s^{\prime}$ ist unbesetzt, so daß aufgrund der Besetzungsinversion stimulierte Emission im Experiment bei $400 \mathrm{~nm}$ beobachtet werden kann. Der $p^{\prime}$-Zustand relaxiert in den $s^{\prime \prime}$-Zustand mit einer lösungsmittelabhängigen Zeitkonstanten von etwa 2 ps. Der relaxierte $s^{\prime \prime}$-Zustand entspricht dabei dem Grundzustand des vollständig solvatisierten Elektrons $e_{\text {Solv }}^{-}$. Das solvatisierte Elektron wird anschließend wie im vorherigen Absatz beschrieben in polaren Lösungsmitteln zum Teil geminal und im unpolaren Methylcyclohexan nicht-geminal rekombinieren. 


\section{Ausblick}

Aufgrund der zur Photoisomerisierung von Polyhalogenmethanen in Lösung gesammelten Daten ergibt sich ein detailliertes Bild für den Reaktionsmechanismus. Die Möglichkeiten der transienten Absorptionsmessungen wurden dabei weitestgehend ausgeschöpft, es gilt lediglich noch in einigen Bereichen Detailfragen zu klären, wie z. B. den Zerfall des $\mathrm{CH}_{2} \mathrm{Cl}-\mathrm{I}$ in unpolaren Lösungsmitteln oder der Beitrag des $\mathrm{CHI}_{2}$-Radikals zur Photoisomerisierung von $\mathrm{CHI}_{3}$. Für diese Spezies ist das Absorptionsspektrum im Gegensatz zu den entsprechenden Radikalen $\mathrm{CH}_{2} \mathrm{I}, \mathrm{CH}_{2} \mathrm{Br}$ und $\mathrm{CH}_{2} \mathrm{Cl}$ unbekannt. Zur die Interpretation der zeitaufgelösten Spektren kann daher ein eventueller Beitrag des $\mathrm{CHI}_{2}$-Radikals zur spektralen Dynamik im Gegensatz zu den anderen primären Radikalen nicht berücksichtigt oder ausgeschlossen werden. Aus diesem Grund werden in unserer Arbeitsgruppe gerade entsprechende Messung zum Spektrum des $\mathrm{CHI}_{2}$ mit Hilfe von Laserblitzlichtphotolyse in Nanosekundenzeitauflösung durchgeführt.

Im Großen und Ganzen sind die Arbeiten im Rahmen der Durchführung von transienten Absorptionsmessungen jedoch abgeschlossen und weitergehende Untersuchungen sollten auf anderen theoretischen und experimentellen Gebieten durchgeführt werden. Das Hauptaugenmerk liegt dabei in erster Linie auf quantenchemischen Rechnungen und zeitaufgelöster Ramanspektroskopie.

\subsection{Theoretische Modellierung durch quantenchemische Rechnungen}

Da vielfältige experimentelle Daten zur Photoisomerisierung der Polyhalogenmethane vorliegen und aufgrund diesen ein möglicher Mechanismus postuliert werden konnte, ist es erstrebenswert, sich diesem Thema auch von der theoretischen Seite zu nähern.

Dazu können quantenchemische Rechnungen für die Potentialhyperfläche des $\mathrm{CH}_{2} \mathrm{I}+$ I-System durchgeführt werden. Ausgehend von der Gleichgewichtsgeometrie des $\mathrm{CH}_{2} \mathrm{I}-\mathrm{I}$ kann die Potentialfläche in Bezug auf Koordinaten wie Bindungslängen und -winkel berechnet werden. Nach Erstellung einer analytischen Potentialfläche können klassische molekulardynamische Simulationen zur Dynamik des Systems vorgenommen werden und deren Ergebnisse mit den experimentellen Befunden verglichen werden. Aufgrund der zwei im $\mathrm{CH}_{2} \mathrm{I}_{2}$ enthaltenen Iodatome sind solche quantenmechanischen Rechnungen zur Potentialfläche jedoch relativ anspruchsvoll und können nur in Zusammenarbeit mit einer Arbeitsgruppe aus dem Bereich der theoretischen Chemie durchgeführt werden. 


\subsection{Zeitaufgelöste Raman-Spektroskopie}

Quantenchemische Rechnungen von Phillips et al. zu den Schwingungsmoden der Isomere der Polyhalogenmethane haben gezeigt, daß diese insbesondere im niederfrequenten Bereich einige charakteristische Normalmoden besitzen. Zeitaufgelöste Schwingungsspektroskopie in Ergänzung zu den durchgeführten transienten Absorptionsmessungen kann somit weitere Details zum Mechanismus der Photoisomerisierung aufklären. Die im Rahmen dieser Arbeit durchgeführten zeitaufgelösten Infrarotabsorptionsmessungen sind dabei nur begrenzt einsetzbar, da sie auf die Untersuchung von Schwingungsmoden > $1200 \mathrm{~cm}^{-1}$ beschränkt sind. Das Mittel der Wahl zur Untersuchungen von niederfrequenten Moden $<1000 \mathrm{~cm}^{-1}$ ist die zeitaufgelöste Raman-Spektroskopie.

Entsprechende zeitaufgelöste Resonanz-Raman-Spektroskopie wurde von Phillips et al. durchgeführt (s. Abschnitt 3.5.3), so konnte beispielsweise für das $\mathrm{CH}_{2} \mathrm{I}-\mathrm{I}$-Isomer die Bildung der I-I-Streckschwingung bei $120 \mathrm{~cm}^{-1}$ innerhalb einiger Pikosekunden nachgewiesen werden. Aufgrund der Bandbreite der ultrakurzen Laserpulse ist (Resonanz)Ramanspektroskopie in Piko-/Femtosekundenauflösung jedoch in ihrer spektralen Auflösung stark eingeschränkt. Mit den von Phillips et al. verwendeten Methoden sind niederfrequente Moden $<100 \mathrm{~cm}^{-1}$ nur sehr schwer spektral aufzulösen. In diesem Frequenzbereich besitzen die Isomere jedoch einige charakteristische Moden wie z. B. die $\mathrm{C}-\mathrm{X}-\mathrm{I}-\mathrm{Knickschwingung.}$

Zur Raman-Spektroskopie mit hoher spektraler Auflösung in Femtosekundenauflösung müssen daher spezielle Techniken verwendet werden. Eine Möglichkeit hierzu bietet ein Drei-Puls-Raman-Experiment wie es von Yoshizawa und Kurosawa [235] beschrieben wurde.

Dazu werden nach Photoanregung mit dem Pumppuls mit einer Verzögerungszeit zwei Pulse eingestrahlt, ein spektral schmaler und zeitlich breiter Raman-Pumppuls sowie ein ultrakurzer Probepuls mit hoher spektraler Bandbreite, vorzugsweise ein Kontinuum. Der Probepuls wird anschließend über ein Spektrometer detektiert und das Ramansignal als Änderung der Transmission beobachtet. Die Zeitauflösung wird dabei durch die Kreuzkorrelation von Pump- und Probepuls begrenzt. Dadurch daß die Probe durch das Einstrahlen des schmalbandigen Raman-Pumppulses in den emittierenden Ramanzustand präpariert wird, erfolgt keine Verbreiterung des beobachteten Ramanspektrums durch den breitbandigen Probepuls. So konnten Yoshizawa und Kurosawa mit ihrem Versuchsaufbau eine Zeitauflösung von $250 \mathrm{fs}$ bei einer spektralen Auflösung von $25 \mathrm{~cm}^{-1}$ erreichen.

Messungen mit solch hoher zeitlicher und spektraler Auflösung wären eine gute Möglichkeit, das Anschwingen der niederfrequenten Normalmoden der Isomere nach photoinduzierter Isomerisierung der Polyhalogenmethane mit hoher Zeitauflösung zu betrachten. Dazu müßte das verwendete fs-Lasersystem allerdings vollständig umgebaut werden. So müssen drei Laserpulse erzeugt und räumlich und zeitlich in der Meßzelle zum Überlapp gebracht werden. Pump- und Probepuls könnten dabei direkt aus der Fundamentalen oder mit den bereits verwendeten optischen Komponenten erzeugt werden. Der zusätzlich 
benötigte schmalbandige Raman-Pumppuls kann mit einem Strahlteiler von der Fundamentalen oder dem Pumppuls abgetrennt werden und mit Hilfe von Interferenzfiltern auf die notwendige spektrale Breite gebracht werden. Zur Detektion des Probepulses ist ein Spektrometer mit hoher spektraler Auflösung notwendig.

Neben der experimentellen Herausforderung dieses Unterfangens, bleibt allerdings die Frage offen, ob die niederfrequenten Moden der Isomere ausreichend intensive Ramanlinien besitzen. Ein genügend große Signalintensität ist insbesondere für die Durchführung von Messungen in überkritischen Lösungsmitteln notwendig. 



\section{A Anpassungsparameter der Meßdaten}

\begin{tabular}{|lccccc|}
\hline Dateinummer & $p /$ bar & $\rho / \mathrm{mol} \mathrm{l}^{-1}$ & $\tau_{c c} / \mathrm{fs}$ & $\tau_{\text {Anstieg }} / \mathrm{ps}$ & $A_{\text {iso }} / 10^{-3}$ \\
\hline 6021 & 190 & 19,506 & 502 & 7,78 & 0,796 \\
6025 & 246 & 20,449 & 343 & 7,61 & 0,893 \\
6026 & 302 & 21,132 & 224 & 7,32 & 0,978 \\
6027 & 357 & 21,682 & 241 & 6,92 & 1,053 \\
6028 & 404 & 22,095 & 292 & 6,69 & 1,126 \\
6029 & 451 & 22,470 & 197 & 6,89 & 1,122 \\
6031 & 504 & 22,858 & 240 & 6,87 & 1,191 \\
6032 & 556 & 23,205 & 183 & 6,70 & 1,238 \\
6033 & 603 & 23,495 & 199 & 6,51 & 1,254 \\
6034 & 652 & 23,774 & 202 & 6,50 & 1,297 \\
6035 & 703 & 24,042 & 141 & 6,08 & 1,335 \\
6036 & 760 & 24,317 & 209 & 6,12 & 1,320 \\
6037 & 799 & 24,491 & 112 & 6,08 & 1,331 \\
6038 & 877 & 24,808 & 115 & 5,78 & 1,389 \\
6039 & 957 & 25,094 & 34 & 5,70 & 1,394 \\
\hline
\end{tabular}

Tabelle A.1: Anpassungsparameter der druckabhängigen Messungen von $\mathrm{CH}_{2} \mathrm{I}_{2}$ in sc. $\mathrm{CO}_{2}\left(35^{\circ} \mathrm{C}\right)$ nach $340 \mathrm{~nm}$ Anregung und $405 \mathrm{~nm}$ Abfrage

\begin{tabular}{|lcccccccc|}
\hline Datein. & $\lambda / \mathrm{nm}$ & $\tau_{c c} / \mathrm{fs}$ & $\tau_{1, \mathrm{ab}} / \mathrm{ps}$ & $\tau_{2, \text { auf }} / \mathrm{ps}$ & $\tau_{3, \text { auf }} / \mathrm{ps}$ & $A_{1}$ & $A_{2}$ & $A_{3}$ \\
\hline 6065 & 395 & 303 & 0,065 & 10,27 & 100,0 & 5,9 & 87,8 & 6,3 \\
6069 & 405 & 276 & 0,070 & 7,58 & 140,3 & 10,7 & 71,8 & 17,5 \\
6072 & 415 & 264 & 0,100 & 5,69 & 207,2 & 21,8 & 63,8 & 14,4 \\
6074 & 425 & 327 & 0,115 & 3,85 & 200,1 & 21,5 & 68,7 & 9,9 \\
6078 & 435 & 369 & 4,159 & 9,90 & 100,0 & 70,0 & 26,8 & 3,2 \\
6080 & 445 & 506 & 1,573 & 0,200 & 59,8 & 56,5 & 39,6 & 3,9 \\
6087 & 375 & 313 & 0,109 & 13,36 & 99,8 & 16,5 & 72,7 & 10,8 \\
6090 & 365 & 259 & 0,188 & 13,15 & 99,8 & 17,1 & 70,2 & 12,7 \\
6095 & 355 & 35 & 0,146 & 11,91 & 99,7 & 20,6 & 66,3 & 13,1 \\
\hline
\end{tabular}

Tabelle A.2: Anpassungsparameter (Zeitkonstanten und relative Amplituden) für das zeitaufgelöste Spektrum von $\mathrm{CH}_{2} \mathrm{I}_{2}$ in sc. $\mathrm{CO}_{2}\left(35^{\circ} \mathrm{C}, 400\right.$ bar $)$ nach $340 \mathrm{~nm}$ Anregung 


\begin{tabular}{|lcccccccccc|}
\hline Dateinummer & $\lambda / \mathrm{nm}$ & $\tau_{c c} / \mathrm{fs}$ & $\tau_{1, \mathrm{ab}}$ & $\tau_{2, \mathrm{ab}}$ & $\tau_{3, \text { auf }}$ & $\tau_{4, \mathrm{auf}}$ & $A_{1}$ & $A_{2}$ & $A_{3}$ & $A_{4}$ \\
\hline 51 & 450 & 566 & 0,061 & 150,0 & 7,27 & 80,0 & 0,1 & 26,5 & 33,2 & 40,4 \\
52 & 440 & 539 & 0,054 & 150,0 & 7,15 & 80,0 & 0,1 & 26,4 & 31,2 & 42,4 \\
54 & 430 & 561 & 0,055 & 150,0 & 7,89 & 80,0 & 0,1 & 26,0 & 31,5 & 42,5 \\
55 & 420 & 621 & 0,066 & 150,0 & 7,30 & 80,0 & 0,1 & 39,0 & 14,6 & 46,4 \\
56 & 410 & 624 & 0,050 & 150,0 & 7,80 & 80,0 & 0,1 & 35,0 & 20,5 & 44,5 \\
58 & 400 & 596 & 0,051 & 150,0 & 8,74 & 80,0 & 0,1 & 34,1 & 23,1 & 42,7 \\
59 & 390 & 620 & 0,049 & 150,0 & 8,74 & 80,0 & 0,1 & 38,6 & 16,6 & 44,7 \\
61 & 380 & 597 & 0,048 & 150,0 & 9,23 & 80,1 & 0,1 & 32,8 & 25,0 & 42,2 \\
63 & 370 & 572 & 0,047 & 130,5 & 9,14 & 81,3 & 0,1 & 38,7 & 15,9 & 45,5 \\
64 & 360 & 570 & 0,048 & 130,5 & 9,29 & 81,4 & 0,1 & 40,0 & 14,9 & 45,0 \\
66 & 350 & 532 & 0,047 & 139,5 & 9,09 & 99,2 & 0,1 & 44,9 & 8,0 & 47,1 \\
\hline
\end{tabular}

Tabelle A.3: Anpassungsparameter (Zeitkonstanten (in ps) und relative Amplituden) für das zeitaufgelöste Spektrum von $\mathrm{CH}_{2} \mathrm{BrI}$ in sc. $\mathrm{CO}_{2}\left(35^{\circ} \mathrm{C}, 600 \mathrm{bar}\right)$ nach $258 \mathrm{~nm}$ Anregung

\begin{tabular}{|lcccccc|}
\hline Dateinummer & $p /$ bar & $\rho / \mathrm{mol} \mathrm{l}^{-1}$ & $\tau_{c c} /$ fs & $\tau_{\text {Abfall }} /$ fs & $\tau_{\text {Anstieg }} / \mathrm{ps}$ & $A_{\text {iso, } \mathrm{n}}$ \\
\hline 226 & 210 & 19,888 & 266 & 200 & 23,67 & 2,93 \\
227 & 267 & 20,726 & 248 & 200 & 23,18 & 3,11 \\
228 & 310 & 21,218 & 236 & 200 & 21,30 & 3,37 \\
229 & 380 & 21,889 & 244 & 200 & 20,99 & 3,42 \\
230 & 437 & 22,362 & 247 & 200 & 19,72 & 3,51 \\
231 & 503 & 22,851 & 252 & 200 & 17,38 & 3,64 \\
232 & 551 & 23,173 & 244 & 200 & 17,18 & 3,74 \\
233 & 605 & 23,507 & 247 & 200 & 17,37 & 3,94 \\
234 & 662 & 23,823 & 246 & 200 & 16,97 & 4,02 \\
235 & 697 & 24,012 & 257 & 200 & 14,51 & 3,89 \\
236 & 754 & 24,289 & 243 & 200 & 15,60 & 4,37 \\
237 & 803 & 24,508 & 260 & 200 & 13,38 & 4,14 \\
238 & 854 & 24,718 & 261 & 200 & 13,70 & 4,38 \\
239 & 902 & 24,901 & 278 & 200 & 12,83 & 4,41 \\
240 & 954 & 25,084 & 270 & 200 & 13,37 & 4,87 \\
\hline
\end{tabular}

Tabelle A.4: Anpassungsparameter der druckabhängigen Messungen von $\mathrm{CH}_{2} \mathrm{BrI}$ in sc. $\mathrm{CO}_{2}\left(35^{\circ} \mathrm{C}\right)$ nach $288 \mathrm{~nm}$ Anregung und $400 \mathrm{~nm}$ Abfrage 


\begin{tabular}{|lcccccccc|}
\hline Dateinummer & $\lambda / \mathrm{nm}$ & $\tau_{c c} / \mathrm{fs}$ & $\tau_{1, \text { Abfall }}$ & $\tau_{2, \text { Anstieg }}$ & $\tau_{3, \text { Abfall }}$ & $A_{1}$ & $A_{2}$ & $A_{3}$ \\
\hline $138 \_9$ & 600 & 147 & 0,296 & 9,47 & & 13,8 & 86,2 & \\
140 & 610 & 482 & 0,193 & 9,35 & & 18,9 & 81,1 & \\
142 & 620 & 475 & 0,171 & 8,82 & & 24,4 & 75,6 & \\
143 & 630 & 555 & 0,122 & 9,08 & & 5,6 & 94,4 & \\
144 & 640 & 659 & 0,208 & 7,63 & & 23,0 & 77,0 & \\
145 & 650 & 241 & 1,061 & 5,91 & & 22,9 & 77,1 & \\
146 & 660 & 324 & 1,116 & 4,37 & & 32,8 & 67,2 & \\
147 & 670 & 700 & 0,773 & 3,87 & & 40,2 & 59,8 & \\
148 & 680 & 571 & 0,285 & 4,81 & & 44,3 & 55,7 & \\
149 & 700 & 585 & 0,311 & 3,96 & 138,4 & 47,0 & 19,8 & 33,2 \\
150 & 720 & 466 & 0,445 & 3,97 & 188,9 & 27,1 & 31,4 & 41,5 \\
\hline
\end{tabular}

Tabelle A.5: Anpassungsparameter (Zeitkonstanten (in ps) und relative Amplituden) für das zeitaufgelöste Spektrum von $\mathrm{CHI}_{3}$ in sc. $\mathrm{CO}_{2}\left(35^{\circ} \mathrm{C}, 505\right.$ bar $)$ nach $365 \mathrm{~nm}$ Anregung

\begin{tabular}{|lcccccccc|}
\hline Dateinummer & $\lambda / \mathrm{nm}$ & $\tau_{c c} / \mathrm{fS}$ & $\tau_{1, \text { Abfall }}$ & $\tau_{2, \text { Anstieg }}$ & $\tau_{3, \text { Abfall }}$ & $A_{1}$ & $A_{2}$ & $A_{3}$ \\
\hline 269 & 600 & 244 & 0,383 & 19,28 & 48,2 & 51,8 & \\
$270 \_1$ & 610 & 283 & 0,532 & 19,34 & 42,3 & 57,7 & \\
279 & 620 & 246 & 0,441 & 17,72 & 47,7 & 52,3 & \\
263 & 630 & 251 & 0,511 & 18,31 & 44,4 & 55,6 & \\
265 & 640 & 256 & 0,484 & 18,30 & 45,2 & 54,8 & \\
$248 \_9$ & 650 & 278 & 0,322 & 18,13 & 48,1 & 51,9 & \\
266 & 660 & 286 & 0,491 & 15,56 & 50,5 & 49,5 \\
251_2_3_4 & 670 & 371 & 0,291 & 15,55 & 54,3 & 45,7 \\
257_8_9 & 690 & 293 & 1,050 & 9,18 & 47,2 & 52,8 \\
275_6_7 & 710 & 260 & 0,328 & 8,05 & & 68,6 & 31,4 & \\
\hline
\end{tabular}

Tabelle A.6: Anpassungsparameter (Zeitkonstanten (in ps) und relative Amplituden) für das zeitaufgelöste Spektrum von $\mathrm{CHI}_{3}$ in sc. $\mathrm{CO}_{2}\left(35^{\circ} \mathrm{C}, 505\right.$ bar $)$ nach $267 \mathrm{~nm}$ Anregung

\begin{tabular}{|lcccccccc|}
\hline Dateinummer & $\lambda / \mathrm{nm}$ & $\tau_{c c} / \mathrm{fs}$ & $\tau_{1, \text { Abfall }}$ & $\tau_{2, \text { Anstieg }}$ & $\tau_{3, \text { Abfall }}$ & $A_{1}$ & $A_{2}$ & $A_{3}$ \\
\hline $475 \_6$ & 600 & 240 & 0,245 & 34,11 & & 74,1 & 25,9 & \\
473 & 610 & 309 & 0,404 & 23,02 & 71,6 & 28,4 & \\
470 & 630 & 237 & 0,919 & 28,09 & & 65,1 & 34,9 & \\
$468 \_9$ & 640 & 302 & 0,297 & 45,74 & 75,3 & 24,7 & \\
$477 \_8$ & 650 & 188 & 0,226 & 53,14 & 74,7 & 25,3 & \\
$480 \_1$ & 670 & 234 & 0,292 & 33,52 & 83,9 & 16,1 & \\
482 & 690 & 257 & 0,368 & 29,35 & 86,0 & 14,0 & \\
485 & 710 & 268 & 0,274 & 29,35 & & 97,0 & 3,0 & \\
\hline
\end{tabular}

Tabelle A.7: Anpassungsparameter (Zeitkonst. und rel. Amplituden) für das zeitaufgelöste Spektrum von $\mathrm{CHI}_{3}$ in sc. $\mathrm{CO}_{2}\left(35^{\circ} \mathrm{C}, 192\right.$ bar $)$ nach $267 \mathrm{~nm}$ Anregung 


\begin{tabular}{|lcccccccc|}
\hline Dateinummer & $\lambda / \mathrm{nm}$ & $\tau_{c c} / \mathrm{fs}$ & $\tau_{1, \text { Abfall }}$ & $\tau_{2, \text { Anstieg }}$ & $\tau_{3, \text { Abfall }}$ & $A_{1}$ & $A_{2}$ & $A_{3}$ \\
\hline 529 & 605 & 202 & 0,413 & 280,6 & & 62,5 & 37,5 & \\
$531 \_2 \_3$ & 615 & 225 & 0,513 & 131,2 & 78,3 & 21,7 & \\
$521 \_2$ & 625 & 176 & 0,508 & 49,3 & 77,9 & 22,1 & \\
$519 \_20$ & 635 & 301 & 1,228 & 66,8 & 63,1 & 36,9 & \\
518 & 645 & 235 & 0,339 & 72,9 & 82,8 & 17,2 & \\
517 & 655 & 159 & 0,741 & 57,3 & 73,0 & 27,0 \\
507 & 665 & 220 & 0,602 & 85,8 & 82,5 & 17,5 \\
508 & 675 & 207 & 0,470 & 49,5 & 98,6 & 1,4 \\
509 & 695 & 167 & 0,376 & 49,5 & 96,7 & 3,3 \\
510 & 715 & 203 & 0,383 & 49,5 & & 95,1 & 4,9 & \\
\hline
\end{tabular}

Tabelle A.8: Anpassungsparameter (Zeitkonstanten und relative Amplituden) für das zeitaufgelöste Spektrum von $\mathrm{CHI}_{3}$ in sc. $\mathrm{CO}_{2}\left(35^{\circ} \mathrm{C}, 142\right.$ bar $)$ nach $267 \mathrm{~nm}$ Anregung

\begin{tabular}{|lcccccc|}
\hline Dateinummer & $p /$ bar & $\rho / \mathrm{mol} \mathrm{l}^{-1}$ & $\tau_{c c} / \mathrm{fs}$ & $\tau_{\text {Abfall }} / \mathrm{fs}$ & $\tau_{\text {Anstieg }} / \mathrm{ps}$ & $A_{\text {iso,n }}$ \\
\hline $1010 \_1$ & 243 & 20,378 & 559 & 67 & 20,45 & 4,372 \\
$1012 \_3$ & 304 & 21,157 & 630 & 69 & 18,29 & 4,563 \\
$1014 \_5$ & 355 & 21,686 & 466 & 49 & 18,26 & 4,517 \\
1020 & 403 & 22,117 & 588 & 47 & 16,09 & 4,636 \\
1021 & 513 & 22,938 & 535 & 42 & 14,78 & 5,066 \\
1042 & 324 & 21,375 & 400 & 32 & 17,62 & 4,171 \\
1043 & 461 & 22,574 & 479 & 37 & 14,93 & 4,494 \\
1044 & 563 & 23,257 & 422 & 33 & 13,57 & 4,734 \\
1045 & 623 & 23,606 & 423 & 32 & 13,78 & 5,411 \\
1046 & 680 & 23,909 & 440 & 34 & 13,01 & 5,631 \\
1047 & 750 & 24,252 & 434 & 34 & 12,14 & 5,235 \\
1048 & 855 & 24,716 & 429 & 33 & 11,84 & 5,556 \\
1049 & 955 & 25,112 & 529 & 43 & 10,25 & 5,981 \\
$1055 \_6$ & 170 & 19,043 & 340 & 28 & 23,57 & 2,892 \\
$1064 \_5$ & 206 & 19,781 & 377 & 30 & 22,34 & 3,444 \\
\hline
\end{tabular}

Tabelle A.9: Anpassungsparameter der druckabhängigen Messungen von $\mathrm{CHI}_{3}$ in sc. $\mathrm{CO}_{2}$ $\left(35^{\circ} \mathrm{C}\right)$ nach $340 \mathrm{~nm}$ Anregung und $460 \mathrm{~nm}$ Abfrage 


\begin{tabular}{|lcccccc|}
\hline Dateinummer & $p /$ bar & $\rho / \mathrm{mol} \mathrm{l}^{-1}$ & $\tau_{c c} / \mathrm{fs}$ & $\tau_{\text {Abfall }} /$ fs & $\tau_{\text {Anstieg }} / \mathrm{ps}$ & $A_{\text {iso,n }}$ \\
\hline 1110 & 398 & 22,075 & 229 & 640 & 19,88 & 3,306 \\
1112 & 512 & 22,932 & 197 & 669 & 17,00 & 3,589 \\
1128 & 281 & 20,886 & 246 & 613 & 22,17 & 2,594 \\
1121 & 313 & 21,257 & 205 & 400 & 20,53 & 2,569 \\
1122 & 455 & 22,530 & 249 & 400 & 17,78 & 2,989 \\
1129 & 402 & 22,109 & 251 & 561 & 19,69 & 2,970 \\
1131 & 653 & 23,769 & 231 & 302 & 14,92 & 4,038 \\
$1134 \_5$ & 229 & 20,16 & 256 & 355 & 24,81 & 2,399 \\
1138 & 705 & 24,035 & 221 & 400 & 14,40 & 4,041 \\
1139 & 785 & 24,413 & 265 & 400 & 13,40 & 4,263 \\
1141 & 865 & 24,757 & 263 & 400 & 13,03 & 4,186 \\
$1143 \_4$ & 204 & 19,745 & 254 & 371 & 25,89 & 2,239 \\
$1145 \_6$ & 255 & 20,548 & 227 & 649 & 22,21 & 2,644 \\
1147 & 356 & 21,696 & 286 & 444 & 19,63 & 2,970 \\
$1150 \_1 \_2$ & 585 & 23,389 & 178 & 633 & 16,34 & 3,245 \\
$1158 \_9 \_60 \_1$ & 169 & 19,019 & 167 & 1997 & 31,78 & 2,942 \\
\hline
\end{tabular}

Tabelle A.10: Anpassungsparameter der druckabhängigen Messungen von $\mathrm{CHI}_{3}$ in sc. $\mathrm{CO}_{2}\left(35^{\circ} \mathrm{C}\right)$ nach $295 \mathrm{~nm}$ Anregung und $460 \mathrm{~nm}$ Abfrage

\begin{tabular}{|lcccccc|}
\hline Dateinummer & $p / \mathrm{bar}$ & $\rho / \mathrm{mol} \mathrm{l}^{-1}$ & $\tau_{c c} / \mathrm{fs}$ & $\tau_{\text {Abfall }} / \mathrm{fs}$ & $\tau_{\text {Anstieg }} / \mathrm{ps}$ & $A_{\text {iso,n }}$ \\
\hline 872 & 180 & 19,269 & 206 & 34 & 30,06 & 1,162 \\
874 & 400 & 22,092 & 308 & 63 & 22,01 & 1,760 \\
875 & 500 & 22,851 & 319 & 45 & 21,53 & 1,914 \\
876 & 600 & 23,476 & 324 & 964 & 19,37 & 2,388 \\
877 & 700 & 24,010 & 324 & 1240 & 17,31 & 2,508 \\
878 & 800 & 24,480 & 315 & 1155 & 15,05 & 2,594 \\
$917 \_8 \_20 \_1$ & 165 & 18,922 & 289 & 37 & 34,41 & 1,179 \\
$923 \_4$ & 254 & 20,534 & 280 & 38 & 30,94 & 1,524 \\
$925 \_6 \_7$ & 346 & 21,599 & 293 & 44 & 24,18 & 1,734 \\
$928 \_9$ & 900 & 24,899 & 324 & 43 & 15,17 & 2,682 \\
947_8 & 215 & 19,938 & 247 & 65 & 28,97 & 1,510 \\
949_50_1 & 288 & 20,971 & 263 & 40 & 27,97 & 1,820 \\
973_4_5 & 477 & 22,690 & 290 & 906 & 22,00 & 2,489 \\
976_7_8 & 556 & 23,214 & 264 & 81 & 19,19 & 2,287 \\
\hline
\end{tabular}

Tabelle A.11: Anpassungsparameter der druckabhängigen Messungen von $\mathrm{CHI}_{3}$ in sc. $\mathrm{CO}_{2}\left(35^{\circ} \mathrm{C}\right)$ nach $267 \mathrm{~nm}$ Anregung und $460 \mathrm{~nm}$ Abfrage 


\begin{tabular}{|lcccccccccc|}
\hline Datein. & $\lambda / \mathrm{nm}$ & $\tau_{c c} / \mathrm{fs}$ & $\tau_{1, \mathrm{ab}}$ & $\tau_{2, \mathrm{ab}}$ & $\tau_{3, \text { auf }}$ & $\tau_{4, \text { auf }}$ & $A_{1}$ & $A_{2}$ & $A_{3}$ & $A_{4}$ \\
\hline 346 & 330 & 316 & 0,121 & 13,52 & 1,43 & 741,3 & 28,2 & 14,4 & 11,8 & 45,6 \\
342 & 340 & 299 & 0,156 & 14,33 & 1,05 & 197,7 & 47,8 & 10,6 & 18,3 & 23,3 \\
337 & 360 & 337 & 0,152 & 17,81 & 1,26 & 76,0 & 37,9 & 11,6 & 26,9 & 23,6 \\
228 & 380 & 333 & 0,113 & & 1,88 & 107,1 & 32,7 & & 47,0 & 20,3 \\
223 & 400 & 312 & 0,150 & 300 & 3,13 & & 19,9 & 3,8 & 76,3 & \\
214 & 420 & 279 & 0,150 & 238,8 & 4,55 & & 26,4 & 23,5 & 50,1 & \\
209 & 440 & 361 & 0,150 & 219,8 & 7,03 & & 6,2 & 46,7 & 47,1 & \\
241 & 450 & 388 & 0,150 & 174,8 & 6,80 & & 7,8 & 42,2 & 50,0 & \\
246 & 470 & 280 & 0,081 & 156,6 & 8,40 & & 9,3 & 42,5 & 48,2 & \\
249 & 490 & 362 & 0,081 & 148,6 & 8,70 & & 4,7 & 46,0 & 49,3 & \\
269 & 516 & 481 & 0,176 & 150,8 & 8,62 & & 9,8 & 46,0 & 44,2 & \\
282 & 530 & 448 & 0,219 & 143,9 & 8,33 & & 12,7 & 45,3 & 42,0 & \\
$285 \_6$ & 550 & 407 & 0,154 & 131,9 & 8,78 & & 19,4 & 46,1 & 34,5 & \\
315 & 590 & 401 & 0,107 & 144,4 & 5,87 & & 10,1 & 44,7 & 45,2 & \\
313 & 610 & 366 & 0,092 & 124,6 & 5,86 & & 10,4 & 43,9 & 45,7 & \\
314 & 630 & 335 & 0,113 & 136,4 & 7,44 & & 16,4 & 42,5 & 41,1 & \\
317 & 650 & 305 & 0,126 & 117,0 & 9,96 & & 18,9 & 42,0 & 39,1 & \\
318 & 670 & 326 & 0,148 & 117,1 & 10,98 & & 21,2 & 40,4 & 38,4 & \\
319 & 690 & 339 & 0,128 & 97,1 & 12,51 & & 19,5 & 40,5 & 40,0 & \\
321 & 710 & 342 & 0,177 & 94,3 & 11,67 & & 23,9 & 37,8 & 38,2 & \\
322 & 730 & 286 & 0,315 & 88,5 & 10,45 & & 24,3 & 36,2 & 39,5 & \\
325 & 750 & 353 & 0,161 & 150,3 & 10,72 & & 22,0 & 42,8 & 35,2 & \\
\hline
\end{tabular}

Tabelle A.12: Anpassungsparameter (Zeitkonstanten (in ps) und relative Amplituden) für das zeitaufgelöste Spektrum von $\mathrm{CH}_{2} \mathrm{ClI}$ in Acetonitril nach $258 \mathrm{~nm}$ Anregung

\begin{tabular}{|c|c|c|c|c|c|c|c|c|c|}
\hline Dateinummer & $\lambda / \mathrm{nm}$ & $\tau_{c c} / \mathrm{fs}$ & $\tau_{1, \mathrm{ab}}$ & $\tau_{2, \mathrm{ab}}$ & $\tau_{3, \text { auf }}$ & $\tau_{4, \text { auf }}$ & $A_{1}$ & $A_{2}$ & $A_{3}$ \\
\hline 447 & 420 & 382 & 0,079 & 132,1 & 1,74 & & 78,0 & 12,1 & 9,9 \\
\hline 448 & 440 & 358 & 0,157 & 134,6 & 1,52 & & 90,2 & 3,6 & 6,3 \\
\hline 460_1 & 480 & 673 & 0,143 & 40,0 & 1,47 & & 33,4 & 47,1 & 19,4 \\
\hline 462_3 & 500 & 489 & 0,237 & 56,4 & & & 75,3 & 24,7 & \\
\hline $467 \_8$ & 520 & 401 & 0,051 & 64,9 & 2,09 & & 48,1 & 51,9 & 0,1 \\
\hline $474 \_5$ & 540 & 423 & 20,0 & 100,0 & 2,04 & & 12,3 & 50,1 & 37,6 \\
\hline 476_7 & 560 & 413 & 19,8 & 100,0 & 2,37 & & 21,5 & 52,3 & 26,2 \\
\hline 489_90_1_2 & 580 & 161 & 19,8 & 100,1 & 1,88 & & 29,9 & 48,8 & 21,2 \\
\hline
\end{tabular}

Tabelle A.13: Anpassungsparameter (Zeitkonstanten (in ps) und relative Amplituden) für das zeitaufgelöste Spektrum von $\mathrm{CH}_{3} \mathrm{I}$ in Methylcyclohexan nach $265 \mathrm{~nm}$ Anregung 


\begin{tabular}{|c|c|c|c|c|c|c|c|c|c|}
\hline Dateinummer & $\lambda / \mathrm{nm}$ & $\tau_{c c} / \mathrm{fs}$ & $\tau_{1, \mathrm{ab}}$ & $\tau_{2, \mathrm{ab}}$ & $\tau_{3, \text { auf }}$ & $\tau_{4, \text { auf }}$ & $A_{1}$ & $A_{2}$ & $A_{3} \quad A_{4}$ \\
\hline 348_9 & 400 & 323 & 0,129 & & 3,68 & & 61,1 & & 38,9 \\
\hline $346 \_7$ & 420 & 310 & 0,033 & 41,9 & 1,95 & & 0,1 & 8,1 & 91,8 \\
\hline $341 \_2 \_3$ & 440 & 333 & 0,082 & 34,9 & 1,45 & & 52,4 & 6,8 & 40,8 \\
\hline 222_3_4_5_6 & 460 & 208 & 0,117 & 31,2 & 3,9 & & 65,5 & 19,8 & 14,7 \\
\hline 318_9_20_1 & 480 & 136 & 19,0 & 100,0 & 1,15 & & 38,4 & 7,2 & 54,3 \\
\hline 234_5_6_7_8 & 500 & 307 & 0,091 & 134,6 & 19,7 & & 54,3 & 18,7 & 26,9 \\
\hline 296_7_8_9 & 520 & 363 & 12,4 & 149,4 & 6,2 & & 46,0 & 9,0 & 45,0 \\
\hline 244_5_6 & 540 & 273 & 0,03 & 30,0 & 1,70 & & 0,2 & 96,0 & 3,8 \\
\hline 247_8_9_50 & 560 & 303 & 0,024 & 25,6 & 0,62 & & 0,1 & 68,8 & 31,2 \\
\hline 254_5_6 & 580 & 274 & 2,2 & 30,0 & & & 6,1 & 93,9 & \\
\hline 279_80_1_2_3 & 600 & 197 & 37,5 & & 0,81 & & 70,0 & 30,0 & \\
\hline
\end{tabular}

Tabelle A.14: Anpassungsparameter (Zeitkonstanten (in ps) und relative Amplituden) für das zeitaufgelöste Spektrum von $\mathrm{CH}_{3} \mathrm{I}$ in Methanol nach $255 \mathrm{~nm}$ Anregung (Langzeitbereich bis 420 ps)

\begin{tabular}{|c|c|c|c|c|c|c|c|c|c|}
\hline Dateinummer & $\lambda / \mathrm{nm}$ & $\tau_{c c} / \mathrm{fs}$ & $\tau_{1, \mathrm{ab}}$ & $\tau_{2, \mathrm{ab}}$ & $\tau_{3, \text { auf }}$ & $\tau_{4, \mathrm{auf}}$ & $A_{1}$ & $A_{2}$ & $A_{3}$ \\
\hline 348_9 & 400 & 293 & 0,036 & & 2,48 & & 0,3 & 99,6 & \\
\hline 346_7 & 420 & 313 & 0,34 & & 1,77 & & 0,1 & 99,9 & \\
\hline 341_2_3 & 440 & 328 & 0,042 & & 1,51 & & 1,0 & 99,0 & \\
\hline 222_3_4_5_6 & 460 & 244 & 0,133 & 1,90 & 1,66 & & 16,1 & 39,7 & 44,1 \\
\hline 318_9_20_1 & 480 & 345 & 0,038 & 2,0 & 1,60 & & 0,1 & 47,1 & 52,9 \\
\hline 234_5_6_7_8 & 500 & 369 & 0,047 & 1,85 & 1,58 & & 0,1 & 48,8 & 51,1 \\
\hline 296_7_8_9 & 520 & 429 & 1,072 & 9,0 & 1,87 & & 38,4 & 13,0 & 48,5 \\
\hline 244_5_6 & 540 & 300 & 0,034 & 1,96 & 1,69 & & 0,1 & 49,9 & 50,1 \\
\hline 247_8_9_50 & 560 & 260 & 0,028 & 1,86 & 1,59 & & 0,1 & 49,7 & 50,2 \\
\hline 254_5_6 & 580 & 359 & 0,043 & 1,63 & 1,48 & & 0,1 & 49,3 & 50,7 \\
\hline 279_80_1_2_3 & 600 & 269 & 0,037 & 1,70 & 1,66 & & 0,1 & 49,7 & 50,3 \\
\hline
\end{tabular}

Tabelle A.15: Anpassungsparameter (Zeitkonstanten (in ps) und relative Amplituden) für das zeitaufgelöste Spektrum von $\mathrm{CH}_{3} \mathrm{I}$ in Methanol nach $255 \mathrm{~nm}$ Anregung (Kurzzeitbereich bis 15 ps) 


\begin{tabular}{|lcccccccccc|}
\hline Datein. & $\lambda / \mathrm{nm}$ & $\tau_{c c} / \mathrm{fs}$ & $\tau_{1, \mathrm{ab}}$ & $\tau_{2, \mathrm{ab}}$ & $\tau_{3, \text { auf }}$ & $\tau_{4, \text { auf }}$ & $A_{1}$ & $A_{2}$ & $A_{3}$ & $A_{4}$ \\
\hline 651_2_3 & 380 & 413 & 3,25 & & 3,25 & 233,3 & & & & \\
694_5_6_7_8 & 390 & 97 & 0,115 & & 3,97 & 136,2 & 30,2 & & 46,5 & 23,3 \\
571_2_3 & 400 & 198 & & & 3,32 & 115,3 & & & 72,8 & 27,2 \\
728_9_30 & 420 & 250 & 0,024 & & 2,29 & 199,9 & 0,1 & & 72,2 & 27,8 \\
742_3_4_5 & 440 & 263 & 0,071 & & 0,70 & 11,7 & 58,0 & & 30,3 & 11,7 \\
778_9_80_1 & 480 & 444 & 0,050 & 5,03 & 100,0 & & 0,1 & 18,6 & 81,3 & \\
838_9_40_1 & 500 & 328 & 1,40 & 10,2 & & & 34,9 & 65,1 & & \\
824_5_6 & 520 & 253 & 0,026 & 2,08 & 2,56 & & 0,1 & 50,4 & 49,6 & \\
877_8_9_80 & 540 & 178 & 33,6 & & & & 100 & & & \\
\hline
\end{tabular}

Tabelle A.16: Anpassungsparameter (Zeitkonstanten (in ps) und relative Amplituden) für das zeitaufgelöste Spektrum von $\mathrm{CH}_{3} \mathrm{I}$ in Acetonitril nach $255 \mathrm{~nm}$ Anregung (Langzeitbereich bis 420 ps). Messung 824_5_6 und 779_9_80_1 sind mit einem triexponentiellen Abfall angepaßt

\begin{tabular}{|c|c|c|c|c|c|c|c|c|c|}
\hline Dateinummer & $\lambda / \mathrm{nm}$ & $\tau_{c c} / \mathrm{fS}$ & $\tau_{1, \mathrm{ab}}$ & $\tau_{2, \mathrm{ab}}$ & $\tau_{3, \text { auf }}$ & $\tau_{4, \mathrm{auf}}$ & $A_{1}$ & $A_{2}$ & $A_{3} \quad A_{4}$ \\
\hline 651_2_3 & 380 & 417 & 1,28 & & 2,86 & & 10,5 & & 89,5 \\
\hline 694_5_6_7_8 & 390 & 181 & 0,149 & & 3,08 & & 68,6 & & 31,4 \\
\hline $571 \_2 \_3$ & 400 & 174 & 0,121 & & 3,22 & & 17,6 & & 82,4 \\
\hline 728_9_30 & 420 & 220 & 0,054 & & 2,71 & & 46,1 & & 53,9 \\
\hline 742_3_4_5 & 440 & 252 & 0,137 & & 0,40 & & 67,7 & & 32,3 \\
\hline 778_9_80_1 & 480 & 435 & 0,116 & 2,46 & 2,97 & & 6,4 & 46,8 & 46,7 \\
\hline 838_9_40_1 & 500 & 462 & 0,048 & 2,28 & 4,80 & & 0,1 & 47,8 & 52,2 \\
\hline $824 \_5 \_6$ & 520 & 253 & 0,026 & 2,57 & 2,08 & & 0,1 & 50,4 & 49,6 \\
\hline 877_8_9_80 & 540 & 291 & 0,050 & 2,36 & 4,97 & & 3,0 & 45,8 & 51,2 \\
\hline
\end{tabular}

Tabelle A.17: Anpassungsparameter (Zeitkonstanten (in ps) und relative Amplituden) für das zeitaufgelöste Spektrum von $\mathrm{CH}_{3} \mathrm{I}$ in Acetonitril nach $265 \mathrm{~nm}$ Anregung (Kurzzeitbereich bis 15 ps)

\begin{tabular}{|lc|cc|lc|lc|}
\hline \multicolumn{2}{|c|}{ Methanol } & \multicolumn{2}{c|}{ Acetonitril } & \multicolumn{2}{c|}{ Methylcyclohexan } & \multicolumn{2}{c|}{$n$-Perfluorhexan } \\
Datei & $\lambda / \mathrm{nm}$ & Datei & $\lambda / \mathrm{nm}$ & Datei & $\lambda / \mathrm{nm}$ & Datei & $\lambda / \mathrm{nm}$ \\
\hline 23 & 290 & 941 & 320 & 161 & 310 & 1127 & 310 \\
22 & 300 & 944 & 330 & 156 & 320 & 1110 & 320 \\
18 & 310 & 963 & 340 & 107 & 330 & 1099 & 330 \\
43 & 320 & 965 & 350 & 110 & 340 & 1081 & 340 \\
47 & 330 & & & 136 & 350 & 1071 & 350 \\
49 & 340 & & & 140 & 360 & & \\
50 & 350 & & & 143 & 370 & & \\
& & & & 146 & 380 & & \\
\hline
\end{tabular}

Tabelle A.18: Dateinummern der Messungen von $\mathrm{CH}_{3} \mathrm{I}$ in verschiedenen Lösungsmitteln mit UV-Probepuls 


\section{B Physikalische Eigenschaften der verwendeten Chemikalien}

\begin{tabular}{|llllll|}
\hline Formel & $\rho / \mathrm{g} \mathrm{ml}^{-1}$ & $M / \mathrm{g} \mathrm{mol}^{-1}$ & $T_{\text {Sdp }} /{ }^{\circ} \mathrm{C}$ & R-Sätze & S-Sätze \\
\hline $\mathrm{CH}_{2} \mathrm{I}_{2}$ & 3,325 & 267,84 & $67-69(11 \mathrm{mmHg})$ & $36 / 37 / 38$ & 26 \\
$\mathrm{CH}_{2} \mathrm{ClI}$ & 2,422 & 176,38 & $108-109$ & $36 / 37 / 38$ & $26-36$ \\
$\mathrm{CH}_{2} \mathrm{BrI}$ & 2,93 & 220,84 & $138-141$ & $37 / 38-41$ & $26-36 / 39$ \\
$\mathrm{CHI}_{3}$ & 4,008 & 393,73 & $118-121$ (Smp.) & $20 / 21 / 22-36 / 37 / 38$ & $26-36 / 37$ \\
$\mathrm{CH}_{3} \mathrm{I}$ & 2,28 & 141,94 & $41-43$ & $21-23 / 25-37 / 38-40$ & $36 / 37-38-45$ \\
\hline
\end{tabular}

Tabelle B.1: Physikalische Eigenschaften und R- und S-Sätze der untersuchten Polyhalogenmethane [236]

\begin{tabular}{|lllll|}
\hline Gas & Summenformel & $T_{k} /{ }^{\circ} \mathrm{C}$ & $p_{k} /$ bar & $\rho_{k} / \mathrm{mol} \mathrm{l}^{-1}$ \\
\hline Kohlenstoffdioxid & $\mathrm{CO}_{2}$ & 30,98 & 73,8 & 10,63 \\
Ethan & $\mathrm{C}_{2} \mathrm{H}_{6}$ & 32,18 & 48,72 & 6,87 \\
Fluoroform & $\mathrm{CHF}_{3}$ & 26,2 & 48,6 & 7,60 \\
\hline
\end{tabular}

Tabelle B.2: Kritische Daten der verwendeten Gase $\left(T_{k}=\right.$ kritische Temperatur, $p_{k}=$ kritischer Druck und $\rho_{k}=$ kritische Dichte) [193] 



\section{Abbildungsverzeichnis}

2.1 Iodatome im Lösungsmittelkäfig . . . . . . . . . . . . . . . . . . . . . . 7

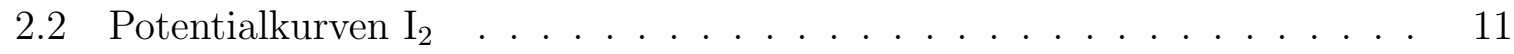

2.3 Lokale Dichtevergrößerung . . . . . . . . . . . . . . . . . . . . . . . . . . . . 13

3.1 Strukturformeln Polyiodmethane . . . . . . . . . . . . . . . . . . . . . . 16

3.2 Spektrum Diiodmethan . . . . . . . . . . . . . . . . . . . . . . . . 17

3.3 Spektrum Chloriodmethan . . . . . . . . . . . . . . . . . . . . . . . . 19

3.4 Spektrum Bromiodmethan . . . . . . . . . . . . . . . . . . . . . . . 20

3.5 Spektrum Iodoform . . . . . . . . . . . . . . . . . . . . . . . . . . 21

3.6 Spektrum Iodmethan . . . . . . . . . . . . . . . . . . . . . . . . . . . . . 23

3.7 Korrelationsdiagramm Iodmethan . . . . . . . . . . . . . . . . . . 24

3.8 Energiezustandsdiagramm Iodmethan . . . . . . . . . . . . . . . . . . . 25

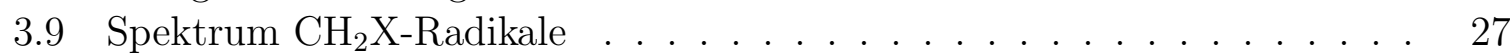

3.10 Spektrum $\mathrm{CH}_{3}$-Radikale . . . . . . . . . . . . . . . . . . . . . . . . . . . 28

3.11 Isomerisierung $\mathrm{CHI}_{3} \ldots \ldots \ldots \ldots \ldots \ldots$

3.12 Matrixspektren Isomere . . . . . . . . . . . . . . . . . . . . 31

4.1 Aufbau UV/VIS-Lasersystem . . . . . . . . . . . . . . . . . . . . . . . 38

$4.2 \quad$ Autokorrelation UV/VIS-Lasersystem . . . . . . . . . . . . . . . . . . . . . . . . . . 39

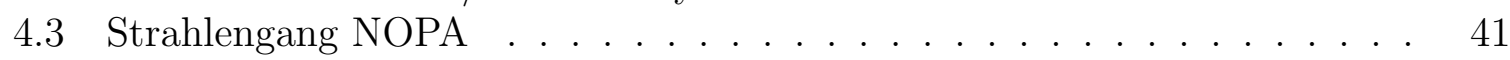

4.4 Elektronische Steuerung und Datenaufnahme. . . . . . . . . . . . . . . . 44

4.5 Hochdruckzelle für überkritische Fluide . . . . . . . . . . . . . . . . . . . 45

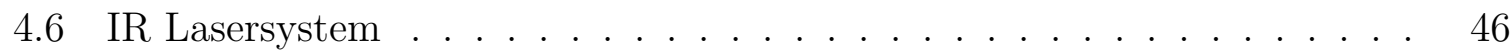

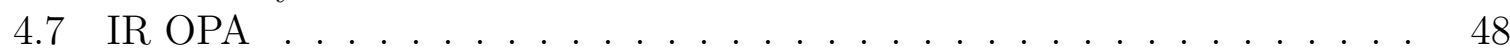

4.8 IR Detektion und Spektrometer . . . . . . . . . . . . . . . . . . . . . 49

4.9 Absorptionszeitprofil von $\mathrm{CH}_{3} \mathrm{I}$ in Methylcyclohexan . . . . . . . . . . . . 53

4.10 Absorptionszeitprofil von $\mathrm{CHI}_{3}$ in sc. $\mathrm{CO}_{2} \ldots \ldots \ldots \ldots$. . . . . . . 54

5.1 Absorptionszeitprofil $\mathrm{CH}_{2} \mathrm{I}_{2}$ in sc. $\mathrm{CO}_{2} 340 / 405 \mathrm{~nm} \ldots \ldots . . . . . . \quad 57$

5.2 Dichteabhängigkeit $k_{\text {iso }} \mathrm{CH}_{2} \mathrm{I}_{2}$ bei variabler Anregungswellenlänge . . . . $\quad 58$

5.3 Dichteabhängigkeit $A_{\text {iso }} \mathrm{CH}_{2} \mathrm{I}_{2}$ bei $340 \mathrm{~nm}$ Anregung und $405 \mathrm{~nm}$ Abfrage 59

5.4 Transiente Absorption $\mathrm{CH}_{2} \mathrm{I}_{2}$ in sc. $\mathrm{CO}_{2}$ bei $340 \mathrm{~nm}$ Anregung . . . . . . 60

5.5 Zeitaufgelöstes Spektrum $\mathrm{CH}_{2} \mathrm{I}_{2}$ in sc. $\mathrm{CO}_{2}$ bei $340 \mathrm{~nm}$ Anregung. . . . . 61

5.6 Absorptionszeitprofil $\mathrm{CH}_{2} \mathrm{BrI}$ in sc. $\mathrm{CO}_{2}$ bei $258 \mathrm{~nm}$ Anregung. . . . . . . . 62

5.7 Absorptionszeitprofil $\mathrm{CH}_{2} \mathrm{BrI}$ in sc. $\mathrm{CO}_{2}$ bei $288 \mathrm{~nm}$ Anregung. . . . . . . . 62

5.8 Transiente Absorption $\mathrm{CH}_{2} \mathrm{BrI}$ in sc. $\mathrm{CO}_{2}$ bei $258 \mathrm{~nm}$ Anregung . . . . . $\quad 63$

5.9 Zeitaufgelöstes Spektrum $\mathrm{CH}_{2} \mathrm{BrI}$ in sc. $\mathrm{CO}_{2}$ bei $258 \mathrm{~nm}$ Anregung. . . . . 64 
5.10 Dichteabhängigkeit $k_{\text {iso }}$ von $\mathrm{CH}_{2} \mathrm{BrI}$ bei $288 \mathrm{~nm}$ Anregung . . . . . . . . . 65

5.11 Dichteabhängigkeit $A_{\text {iso,n }} \mathrm{CH}_{2} \mathrm{BrI}$ bei $288 \mathrm{~nm}$ Anregung . . . . . . . . . . 66

5.12 Absorptionszeitprofil $\mathrm{CHI}_{3}$ in $\mathrm{CH}_{3} \mathrm{CN}$ bei $267 \mathrm{~nm}$ Anregung. . . . . . . . . 67

5.13 Absorptionszeitprofil $\mathrm{CHI}_{3}$ in sc. $\mathrm{CO}_{2}$ bei $267 \mathrm{~nm}$ Anregung. . . . . . . . . 67

5.14 Transiente Absorption $\mathrm{CHI}_{3}$ in sc. $\mathrm{CO}_{2}$ bei $365 \mathrm{~nm}$ Anregung . . . . . . . 68

5.15 Transiente Absorption $\mathrm{CHI}_{3}$ in sc. $\mathrm{CO}_{2}$ bei $267 \mathrm{~nm}$ Anregung . . . . . . . 69

5.16 Transiente Absorption $\mathrm{CHI}_{3}$ in sc. $\mathrm{CO}_{2}$ bei $267 \mathrm{~nm}$ Anregung . . . . . . . 69

5.17 Zeitaufgelöstes Spektrum $\mathrm{CHI}_{3}$ in sc. $\mathrm{CO}_{2}$ bei $365 \mathrm{~nm}$ Anregung . . . . . 70

5.18 Zeitaufgelöstes Spektrum $\mathrm{CHI}_{3}$ in sc. $\mathrm{CO}_{2}$ (505 bar) bei $267 \mathrm{~nm}$ Anregung 71

5.19 Zeitaufgelöstes Spektrum $\mathrm{CHI}_{3}$ in sc. $\mathrm{CO}_{2}$ (192 bar) bei $267 \mathrm{~nm}$ Anregung 71

5.20 Zeitaufgelöstes Spektrum $\mathrm{CHI}_{3}$ in sc. $\mathrm{CO}_{2}$ (142 bar) bei $267 \mathrm{~nm}$ Anregung 72

5.21 Dichteabhängigkeit $k_{\text {iso }}$ von $\mathrm{CHI}_{3}$ bei var. Anregung . . . . . . . . . . . . 73

5.22 Dichteabhängigkeit $A_{\text {iso }, \mathrm{n}}$ von $\mathrm{CHI}_{3}$ bei var. Anregung . . . . . . . . . . . 73

5.23 Absorptionszeitprofil $\mathrm{CHI}_{3}$ in $\mathrm{CH}_{3} \mathrm{CN}$ bei $267 \mathrm{~nm}$ Anregung. . . . . . . . . 74

5.24 Absorptionszeitprofil $\mathrm{CHI}_{3}$ in $\mathrm{CH}_{3} \mathrm{CN}$ bei $267 \mathrm{~nm}$ Anregung. . . . . . . . 75

5.25 Absorptionszeitprofil $\mathrm{CHI}_{3}$ in $\mathrm{CH}_{3} \mathrm{CN}$ bei $267 \mathrm{~nm}$ Anregung. . . . . . . . . 75

5.26 Absorptionszeitprofil $\mathrm{CH}_{2} \mathrm{ClI}$ in $\mathrm{CH}_{3} \mathrm{CN}$ nach $258 \mathrm{~nm}$ Anregung . . . . . $\quad 76$

5.27 Transiente Absorption $\mathrm{CH}_{2} \mathrm{ClI}$ in $\mathrm{CH}_{3} \mathrm{CN}$ nach $258 \mathrm{~nm}$ Anregung. . . . . . 77

5.28 Absorptionszeitprofil $\mathrm{CH}_{2} \mathrm{ClI}$ in $\mathrm{CCl}_{4}$ nach $258 \mathrm{~nm}$ Anregung . . . . . . . $\quad 78$

5.29 Absorptionszeitprofil $\mathrm{CH}_{2}$ ClI in $n$-Hexan nach $258 \mathrm{~nm}$ Anregung . . . . . 78

5.30 Absorptionszeitprofil $\mathrm{CH}_{2} \mathrm{ClI}$ in sc. $\mathrm{CHF}_{3}$ nach $258 \mathrm{~nm}$ Anregung. . . . . . 79

5.31 Absorptionszeitprofil $\mathrm{CH}_{2}$ ClI in sc. Ethan nach $258 \mathrm{~nm}$ Anregung . . . . 79

5.32 Absorptionsspektren $\mathrm{CH}_{3} \mathrm{I}$ in verwendeten Lösungsmitteln . . . . . . . . 80

5.33 Absorptionszeitprofil $\mathrm{CH}_{3} \mathrm{I}$ in $\mathrm{CH}_{3} \mathrm{OH} 255 / 320 \mathrm{~nm} \ldots \ldots \ldots$. . . . . . . 82

5.34 Transiente Absorption $\mathrm{CH}_{3} \mathrm{I}$ in $\mathrm{CH}_{3} \mathrm{OH}$ UV-Abfrage . . . . . . . . . . . . 82

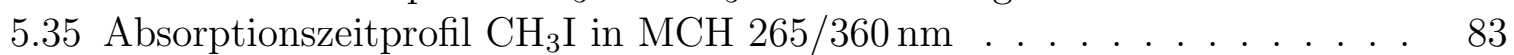

5.36 Transiente Absorption $\mathrm{CH}_{3} \mathrm{I}$ in MCH UV Abfrage . . . . . . . . . . . . . 83

5.37 Transiente Absorption $\mathrm{CH}_{3} \mathrm{I}$ in $\mathrm{CH}_{3} \mathrm{CN}$ UV-Abfrage . . . . . . . . . . . . 84

5.38 Absorptionszeitprofil $\mathrm{CH}_{3} \mathrm{I}$ in Perfluorhexan $265 / 330 \mathrm{~nm}$. . . . . . . . . 84

5.39 Transiente Absorption $\mathrm{CH}_{3} \mathrm{I}$ in Perfluorhexan UV-Abfrage . . . . . . . . 85

5.40 Lösungsmittelsignal Methanol . . . . . . . . . . . . . . . . . . 85

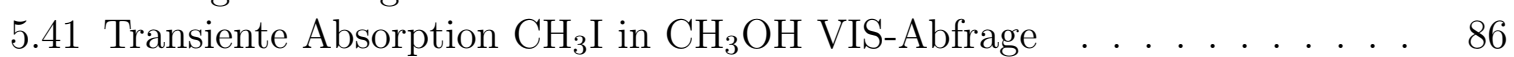

5.42 Transiente Absorption $\mathrm{CH}_{3} \mathrm{I}$ in $\mathrm{CH}_{3} \mathrm{CN}$ VIS-Abfrage. . . . . . . . . . . . 87

5.43 Transiente Absorption $\mathrm{CH}_{3} \mathrm{I}$ in Methylcyclohexan VIS-Abfrage . . . . . . 88

5.44 Absorptionszeitprofil $\mathrm{CH}_{3} \mathrm{I}$ in $\mathrm{CD}_{3} \mathrm{CN}$ bei $1250 \mathrm{~cm}^{-1}$ Abfrage . . . . . . . 89

5.45 Absorptionszeitprofil $\mathrm{CH}_{3} \mathrm{I}$ in $\mathrm{CD}_{3} \mathrm{CN}$ bei $1250 \mathrm{~cm}^{-1}$ Abfrage . . . . . . . 90

6.1 Absorptionszeitprofil $\mathrm{CHI}_{3}$ in sc. $\mathrm{CO}_{2} \ldots \ldots \ldots \ldots$. . . . . . . . . . . 92

6.2 Potentialdiagramm eines CT-Komplexes . . . . . . . . . . . . . . . . . 94

6.3 Druckabhängigkeit $\epsilon_{r} \mathrm{CO}_{2} \quad \ldots \ldots \ldots \ldots \ldots$. . . . . . . . . . . . . . 96

6.4 Radialverteilungsdichte $\mathrm{CH}_{2} \mathrm{I}_{2}$ in sc. $\mathrm{CO}_{2} \ldots \ldots \ldots$. . . . . . . . . . 97

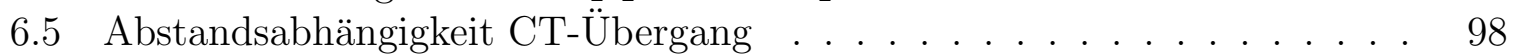

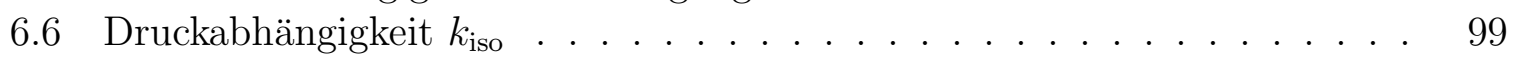

6.7 Normiertes zeitaufgelöstes Spektrum $\mathrm{CH}_{2} \mathrm{I}_{2}$ in sc. $\mathrm{CO}_{2}$. . . . . . . . . . 101

6.8 Normiertes zeitaufgelöstes Spektrum $\mathrm{CHI}_{3}$ in sc. $\mathrm{CO}_{2} \ldots \ldots$. . . . . . . . 102 
6.9 Zeitkonstanten spek. Entwicklung $\mathrm{CHI}_{3}$. . . . . . . . . . . . . . . . . . . 103

6.10 Vorläufiges kinetisches Schema zur Isomerbildung . . . . . . . . . . . . . 105

6.11 Erweitertes kinetisches Schema zur Isomerbildung . . . . . . . . . . . . . 106

6.12 Dichteabhängigkeit Clausius-Mosotti-Faktor $f(\epsilon)$. . . . . . . . . . . . . . 109

6.13 Vergleich $k_{\text {iso }}$ und $k_{\text {Sep }} \ldots \ldots \ldots \ldots \ldots \ldots$. . . . . . . . . . . . 110

6.14 Absorptionszeitprofil $\mathrm{CHI}_{3}$ in sc. $\mathrm{CO}_{2}$ bei $180 \mathrm{bar}$. . . . . . . . . . . . . 112

$6.15 A_{\text {iso,n }} \mathrm{CH}_{2} \mathrm{I}_{2}$ in sc. Xe . . . . . . . . . . . . . . . . . . 113

6.16 Vergleich $A_{\text {iso,n }} \mathrm{CH}_{2} \mathrm{I}_{2}$ in sc. $\mathrm{CO}_{2}$ mit $\rho_{\text {lokal }}^{2} \ldots \ldots \ldots \ldots$. . . . . . . 114

6.17 Zeitaufgelöstes Spektrum $\mathrm{CH}_{2} \mathrm{ClI}$ in $\mathrm{CH} 3_{\mathrm{C}} \mathrm{N}$. . . . . . . . . . . . . . . 116

6.18 Absorptionsspektrum von $\mathrm{ICl}$ und $\mathrm{I}_{2}^{-} \ldots \ldots \ldots \ldots$. . . . . . . . . . 117

6.19 Amplitudenverhältnis Ausbleichen $\mathrm{CHI}_{3}$ in $\mathrm{CH}_{3} \mathrm{CN}$. . . . . . . . . . . 120

6.20 Produktspektren $\mathrm{CHI}_{3}$ nach Photolyse in $\mathrm{CH}_{3} \mathrm{CN}$. . . . . . . . . . . . . 121

6.21 Produktspektren $\mathrm{CHI}_{3}$ und $\mathrm{I}_{3}^{-}$nach Photolyse in $\mathrm{CH}_{3} \mathrm{CN}$. . . . . . . . . 123

6.22 Normiertes zeitaufgelöstes Spektrum $\mathrm{CHI}_{3}$ in sc. $\mathrm{CO}_{2}$. . . . . . . . . . . 124

6.23 Normiertes zeitaufgelöstes Spektrum $\mathrm{CHI}_{3}$ in sc. $\mathrm{CO}_{2}$. . . . . . . . . . . 125

6.24 Absorptionszeitprofil $\mathrm{CHI}_{3}$ in sc. $\mathrm{CO}_{2}\left(35^{\circ} \mathrm{C} 139 \mathrm{bar} . \ldots \ldots\right.$. . . . 126

6.25 Absorptionsspektren CT-Komplexe . . . . . . . . . . . . . . . . . 131

6.26 Zeitaufgelöstes Spektrum $\mathrm{CH}_{3} \mathrm{I}$ in $\mathrm{CH}_{3} \mathrm{CN}$. . . . . . . . . . . . . . . . . 134

6.27 Zeitaufgelöstes Spektrum $\mathrm{CH}_{3} \mathrm{I}$ in $\mathrm{CH}_{3} \mathrm{OH}$. . . . . . . . . . . . . . . . . 135

6.28 Zeitaufgelöstes Spektrum $\mathrm{CH}_{3} \mathrm{I}$ in Methylcyclohexan . . . . . . . . . . . . 136

6.29 Intensitätsabhängigkeit Absorptionszeitprofil von $\mathrm{CH}_{3} \mathrm{I}$ in $\mathrm{CH}_{3} \mathrm{OH}$. . . . 137

6.30 Dynamik photogenerierter Elektronen . . . . . . . . . . . . . . 138

6.31 Energielevelschema solvatisiertes Elektrons . . . . . . . . . . . . . . . . . 139

6.32 Kurzzeitdynamik von $\mathrm{CH}_{3} \mathrm{I}$ in $\mathrm{CH}_{3} \mathrm{OH}$ bei unterschiedlichen Probewellen-

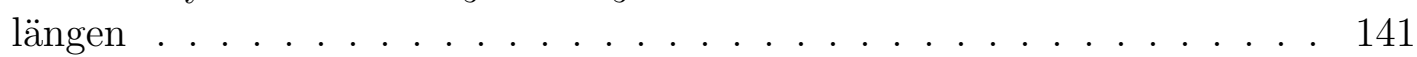

6.33 Spektrum transienter Zuständer des solvatisierten Elektrons . . . . . . . 143

6.34 Kinetische Modellierung eines Absorptionszeitprofils von $\mathrm{CH}_{3} \mathrm{I}$ in $\mathrm{MCH}$. 148

6.35 Absorptionszeitprofil von $\mathrm{CH}_{3} \mathrm{I}$ in $\mathrm{CH}_{3} \mathrm{CN}$ bei $260 / 540 \mathrm{~nm}$. . . . . . . . 150

6.36 Absorptionszeitprofil von $\mathrm{CH}_{3} \mathrm{I}$ in $\mathrm{CH}_{3} \mathrm{OH}$ bei $260 / 520 \mathrm{~nm}$. . . . . . . . . 151

6.37 Zeitaufgelöstes Bandenintegral von $\mathrm{CH}_{3} \mathrm{I}$ in $\mathrm{CD}_{3} \mathrm{CN}$ bei $1250 \mathrm{~cm}^{-1}\left(E_{\mathrm{Puls}}\right.$

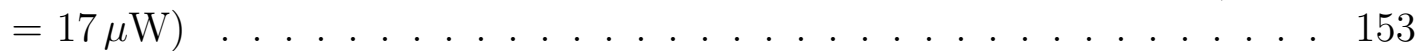

6.38 Zeitaufgelöstes Bandenintegral von $\mathrm{CH}_{3} \mathrm{I}$ in $\mathrm{CD}_{3} \mathrm{CN}$ bei $1250 \mathrm{~cm}^{-1}\left(E_{\text {Puls }}\right.$

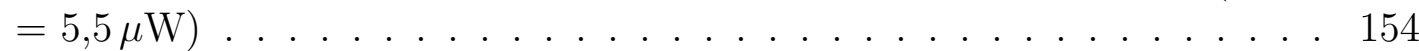

6.39 Modell der Photoionisation und Rekombination von $\mathrm{CH}_{3} \mathrm{I}$. . . . . . . . . 156

6.40 Modell der Solvatation des freien Elektrons in Lösung . . . . . . . . . . . 157 



\section{Tabellenverzeichnis}

3.1 Strukturparameter Polyhalogenmethane . . . . . . . . . . . . . . . 15

3.2 Strukturparameter $\mathrm{CH}_{\mathrm{x}} \mathrm{I}_{\mathrm{z}} \mathrm{X}$-Radikale . . . . . . . . . . . . . . . . . 29

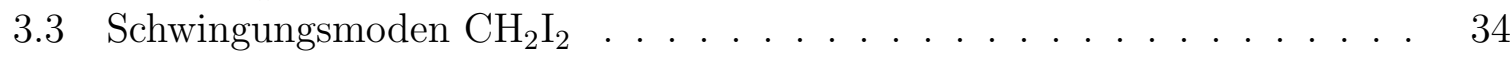

3.4 Schwingungsmoden Polyhalogenmethane . . . . . . . . . . . . . . . . . 35

4.1 Verwendete Chemikalien . . . . . . . . . . . . . . . . . . . . . . . . 55

5.1 Lösungsmittelabhängiges Absorptionsmaximum $\mathrm{CH}_{3} \mathrm{I}$. . . . . . . . . . . 81

$6.1 \quad$ Ionisierungspotentiale der primären Radikale und Lösungsmittel . . . . . 95

6.2 Schwingungsenergierelaxationszeiten . . . . . . . . . . . . . . . . 104

6.3 Normalmoden Dihalogenmethane und Isomere . . . . . . . . . . . . . . . 128

6.4 Ionisierungspotentiale der Lösungsmittel . . . . . . . . . . . . . . . . . . 130

6.5 Quantenausbeuten CTTS-Komplexbildung in MCH . . . . . . . . . . . . 133

6.6 Anstiegszeiten $\mathrm{CH}_{3} \mathrm{I}$ in $\mathrm{CH}_{3} \mathrm{OH}, \mathrm{CH}_{3} \mathrm{CN}$ und $\mathrm{MCH}$. . . . . . . . . . . . 142

A.1 Anpassungsparameter $\mathrm{CH}_{2} \mathrm{I}_{2}$ in sc. $\mathrm{CO}_{2} 340 \mathrm{~nm} \ldots \ldots \ldots$. . . . . . . 163

A.2 Anpassungsparameter Spektrum $\mathrm{CH}_{2} \mathrm{I}_{2}$ in sc. $\mathrm{CO}_{2}$ (400 bar) $340 \mathrm{~nm}$ An-

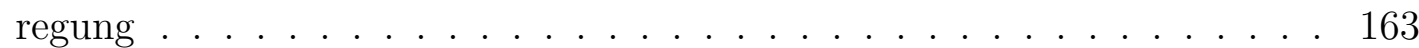

A.3 Anpassungsparameter Spektrum $\mathrm{CH}_{2} \mathrm{BrI}$ in sc. $\mathrm{CO}_{2}$ (600 bar) $258 \mathrm{~nm}$ An-

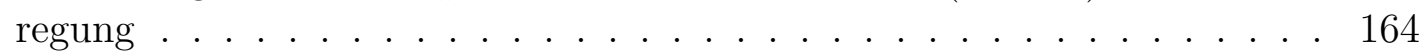

A.4 Anpassungsparameter $\mathrm{CH}_{2} \mathrm{BrI}$ in sc. $\mathrm{CO}_{2} 288 \mathrm{~nm} \ldots \ldots \ldots$. . . . . . . 164

A.5 Anpassungsparameter Spektrum $\mathrm{CHI}_{3}$ in sc. $\mathrm{CO}_{2}$ (505 bar) $365 \mathrm{~nm}$ Anregung 165

A.6 Anpassungsparameter Spektrum $\mathrm{CHI}_{3}$ in sc. $\mathrm{CO}_{2}$ (505 bar) $267 \mathrm{~nm}$ Anregung 165

A.7 Anpassungsparameter Spektrum $\mathrm{CHI}_{3}$ in sc. $\mathrm{CO}_{2}$ (192 bar) $267 \mathrm{~nm}$ Anregung 165

A.8 Anpassungsparameter Spektrum $\mathrm{CHI}_{3}$ in sc. $\mathrm{CO}_{2}$ (142 bar) $267 \mathrm{~nm}$ Anregung 166

A.9 Anpassungsparameter $\mathrm{CHI}_{3}$ in sc. $\mathrm{CO}_{2} 340 \mathrm{~nm} \ldots \ldots \ldots$. . . . . . . 166

A.10 Anpassungsparameter $\mathrm{CHI}_{3}$ in sc. $\mathrm{CO}_{2} 295 \mathrm{~nm}$. . . . . . . . . . . . . . . 167

A.11 Anpassungsparameter $\mathrm{CHI}_{3}$ in sc. $\mathrm{CO}_{2} 267 \mathrm{~nm} \ldots \ldots \ldots . \ldots . . \ldots 167$

A.12 Anpassungsparameter Spektrum $\mathrm{CH}_{2} \mathrm{ClI}$ in $\mathrm{CH}_{3} \mathrm{CN} 258 \mathrm{~nm}$ Anregung . . 168

A.13 Anpassungsparameter Spektrum $\mathrm{CH}_{3} \mathrm{I}$ in MCH $265 \mathrm{~nm}$ Anregung . . . . 168

A.14 Anpassungsparameter Spektrum $\mathrm{CH}_{3} \mathrm{I}$ in $\mathrm{CH}_{3} \mathrm{OH} 255 \mathrm{~nm}$ Anregung . . . 169

A.15 Anpassungsparameter Spektrum $\mathrm{CH}_{3} \mathrm{I}$ in $\mathrm{CH}_{3} \mathrm{OH} 255 \mathrm{~nm}$ Anregung . . . 169

A.16 Anpassungsparameter Spektrum $\mathrm{CH}_{3} \mathrm{I}$ in $\mathrm{CH}_{3} \mathrm{CN} 265 \mathrm{~nm}$ Anregung . . . 170

A.17 Anpassungsparameter Spektrum $\mathrm{CH}_{3} \mathrm{I}$ in $\mathrm{CH}_{3} \mathrm{OH} 255 \mathrm{~nm}$ Anregung . . . 170

A.18 Dateinummern der $\mathrm{CH}_{3} \mathrm{I}$-Messungen mit UV-Probe . . . . . . . . . . . . 170

B.1 Physikalische Eigenschaften verwendeter Chemikalien . . . . . . . . . . . 171 
B.2 Kritische Daten der verwendeten Gase . . . . . . . . . . . . . . . . . . . 171 


\section{Literaturverzeichnis}

[1] J. Schroeder, in: J. H. Moore, N. D. Spencer, Hgs., Encyclopedia of Chemical Physics and Physical Chemistry, vol. 1, Institute of Physics Publishing, 2001 711-743.

[2] J. T. Hynes, Ann. Rev. Phys. Chem., 1985, 36, 573.

[3] A. L. Harris, J. Brown, C. B. Harris, Ann. Rev. Phys. Chem., 1988, 39, 341.

[4] H. Kramers, Physica, 1940, 7, 284.

[5] J. Schroeder, J. Troe, Ann. Rev. Phys. Chem., 1987, 38, 163.

[6] L. Onsager, J. Am. Chem. Soc., 1936, 58, 1486.

[7] J. G. Kirkwood, J. Chem. Phys., 1939, 7, 911.

[8] M. Born, Z. Physik, 1920, 45, 1.

[9] C. J. Cramer, D. G. Truhlar, Chem. Rev., 1999, 99, 2161.

[10] T. A. Litovitz, E. H. Carnevale, P. A. Kendall, J. Chem. Phys., 1957, 26, 465.

[11] W. M. Madigosky, T. A. Litovitz, J. Chem. Phys., 1961, 34, 489.

[12] C. Delalande, G. M. Gale, Chem. Phys. Lett., 1977, 50, 339.

[13] D. Schwarzer, J. Troe, M. Zerezke, J. Chem. Phys., 1997, 107, 8380.

[14] S. A. Adelman, J. Phys. Chem., 1985, 89, 2213.

[15] J. Franck, E. Rabinowitsch, Trans. Faraday Soc., 1934, 30, 120.

[16] E. Rabinowitsch, W. C. Wood, Trans. Faraday Soc., 1935, 32, 547.

[17] E. Rabinowitsch, W. C. Wood, Trans. Faraday Soc., 1936, 32, 1381.

[18] B. Otto, J. Schroeder, J. Troe, J. Chem. Phys., 1984, 81, 202.

[19] D. Schwarzer, J. Schroeder, C. Schröder, Z. Phys. Chem., 2001, 215, 183.

[20] C. Lienau, A. H. Zewail, J. Phys. Chem., 1996, 100, 18629.

[21] H. Hippler, V. Schubert, J. Troe, J. Chem. Phys., 1984, 81, 3931.

[22] T. Kühne, P. Vöhringer, J. Chem. Phys., 1996, 105, 10788.

[23] T. Kühne, P. Vöhringer, J. Phys. Chem. A, 1998, 102, 4177.

[24] S. Hess, H. Bürsing, P. Vöhringer, J. Chem. Phys., 1999, 111, 5461. 
[25] C. L. Thomsen, D. Madsen, J. Thøgersen, J. R. Byberg, S. R. Keiding, J. Chem. Phys., 1999, 111, 703.

[26] C. L. Thomsen, J. Thøgersen, S. R. Keiding, J. Chem. Phys., 2001, 114, 4099.

[27] C. C. Cooksey, P. J. Reid, Photochem. Photobiol., 2004, 80, 386.

[28] J. Thøgersen, C. L. Thomsen, J. A. Poulsen, S. R. Keiding, J. Phys. Chem. A, 1998, 102, 4186.

[29] C. L. Thomsen, P. J. Reid, S. R. Keiding, J. Am. Chem. Soc., 2000, 122, 12795.

[30] C. L. Thomsen, D. Madsen, J. A. Poulsen, J. Thøgersen, S. J. Knak Jensen, J. Chem. Phys., 2001, 115, 9361.

[31] D. Madsen, C. L. Thomsen, J. A. Poulsen, S. J. Knak Jensen, J. Thøgersen, S. R. Keiding, E. B. Krissinel, J. Phys. Chem. A, 2003, $107,3606$.

[32] J. Larsen, D. Madsen, J.-A. Poulsen, T. D. Poulsen, S. R. Keiding, J. Thøgersen, J. Chem. Phys., 2002, 116, 7997.

[33] N. Winter, I. Benjamin, J. Chem. Phys., 2004, 121, 2253.

[34] C. D. Garr, R. G. Finke, J. Am. Chem. Soc., 1992, 114, 10440.

[35] R. J. Sension, D. A. Harris, A. G. Cole, J. Phys. Chem. B, 2005, 109, 21954.

[36] T. G. Traylor, D. Magde, J. Marsters, K. Jongeward, G. Z. Wu, K. Walda, J. Am. Chem. Soc., 1993, 115, 4808.

[37] T. G. Grogan, N. Bag, T. G. Traylor, D. Magde, J. Phys. Chem., 1994, 98, 13791.

[38] P. Bosch, J. L. Mateo, J. Serrano, J. Photochem. Photobiol. A, 1997, 103, 177.

[39] C. J. Jenks, A. Paul, L. A. Smoliar, B. E. Bent, J. Phys. Chem., 1994, 98, 572.

[40] J. M. Tanko, R. Pacut, J. Am. Chem. Soc., 2001, 123, 5703.

[41] J. D. Harris, A. B. Oelkers, D. R. Tyler, J. Organometallic Chem., 2007, 692, 3261 .

[42] J. F. Cahoon, M. F. Kling, S. Schmatz, C. B. Harris, J. Am. Chem. Soc., 2005, $127,12555$.

[43] H. K. van Dijk, J. van der Haar, D. J. Stufkens, A. Oskam, Inorg. Chem., 1989, 28,75 .

[44] M. Smoluchowskyi, Ann. Phys., 1915, 48, 1103.

[45] M. Smoluchowskyi, Z. Physik. Chem., 1918, 92, 129.

[46] F. C. Collins, G. E. Kimball, J. Colloid Sci., 1949, 4, 425.

[47] S. A. Rice, in: C. H. Bamford, C. F. H. Tipper, R. G. Compton, Hgs., Comprehensive Chemical Kinetics, vol. 25, Elsevier, 1985. 
[48] A. Szabo, J. Phys. Chem., 1989, 93, 6929.

[49] G. Weiss, J. Stat. Phys., 1986, 42, 3.

[50] R. M. Noyes, J. Am. Chem. Soc., 1955, 77, 2042.

[51] J. Zimmerman, R. Noyes, J. Chem. Phys., 1950, 18, 658.

[52] F. W. Lampe, R. M. Noyes, J. Am. Chem. Soc., 1954, 76, 2140.

[53] D. Booth, R. M. Noyes, J. Am. Chem. Soc., 1960, 82, 1868.

[54] L. F. Meadows, R. M. Noyes, J. Am. Chem. Soc., 1960, 82, 1872.

[55] R. M. Noyes, Z. Elektrochm., 1960, 64, 153.

[56] R. M. Noyes, J. Chem. Phys., 1954, 22, 1349.

[57] H. van Beijeren, W. Dong, L. Bocquet, J. Chem. Phys., 2001, 114, 6265.

[58] K. Luther, J. Troe, Chem. Phys. Lett., 1974, $24,85$.

[59] J. J. Valentini, J. B. Cross, J. Chem. Phys., 1982, 77, 572.

[60] K. Saenger, G. McClelland, D. Herschbach, J. Phys. Chem., 1981, 85, 3333.

[61] F. G. Amar, B. J. Berne, J. Phys. Chem., 1984, 88, 6720.

[62] T. J. Chuang, G. W. Hoffman, K. B. Eisenthal, Chem. Phys. Lett., 1974, 25, 201.

[63] S. H. Northrup, J. T. Hynes, J. Chem. Phys., 1979, 71, 871.

[64] S. H. Northrup, J. T. Hynes, J. Chem. Phys., 1979, 71, 884.

[65] D. L. Bunker, B. S. Jacobson, J. Am. Chem. Soc., 1972, 94, 1843.

[66] W. Balk, C. Brook, S. Adelman, J. Chem. Phys., 1983, 79, 804.

[67] D. J. Nesbitt, J. T. Hynes, J. Chem. Phys., 1982, 77, 2130.

[68] J. K. Brown, C. B. Harris, J. C. Tully, J. Chem. Phys., 1988, 89, 6687.

[69] J. N. Murrel, A. J. Stace, R. Dammel, J. Chem. Soc. Faraday Trans. 2, 1978, 74, 1532.

[70] V. S. Batista, D. Coker, J. Chem. Phys., 1996, 105, 4033.

[71] J. P. Bergsma, J. R. Reimers, K. R. Wilson, J. Chem. Phys., 1986, 85, 5625.

[72] A. H. Zewail, M. Dantus, R. M. Bowman, A. J. Mokthari, Photochem. Photobiol. A, 1992, 62, 301 .

[73] Q. Liu, J.-K. Wang, A. H. Zewail, Nature, 1993, 364, 427.

[74] C. Wan, M. Gupta, J. S. Baskin, Z. H. Kim, A. H. Zewail, J. Chem. Phys., 1997, $106,4353$.

[75] J.-K. Wang, Q. Liu, A. H. Zewail, J. Phys. Chem., 1995, 99, 11309. 
[76] N. F. Scherer, L. D. Ziegler, G. R. Fleming, J. Chem. Phys., 1992, 96, 5544.

[77] N. F. Scherer, M. D. Jonas, G. R. Fleming, J. Chem. Phys., 1993, 99, 153.

[78] R. Zadoyan, Z. Li, P. Ashjian, C. C. Martens, V. A. Apkarian, Chem. Phys. Lett., 1994, 218,504 .

[79] P. G. Debennedetti, R. S. Mohamed, J. Chem. Phys., 1988, 90, 4528.

[80] W. Song, R. Biswas, M. Maroncelli, J. Phys. Chem. A, 2000, 104, 6924.

[81] O. Kajimoto, Chem. Rev., 1999, 99, 355.

[82] R. S. Urdahl, K. D. Rector, D. J. Myers, P. H. Davis, M. D. Fayer, J. Chem. Phys., 1996, 105, 8973 .

[83] R. S. Urdahl, K. D. Rector, D. J. Myers, P. H. Davis, B. J. Cherayil, M. D. Fayer, J. Chem. Phys., 1997, 107, 3747.

[84] D. Schwarzer, J. Troe, M. Votsmeier, M. Zerezke, J. Chem. Phys., 1996, 105, 3121.

[85] J. F. Brennecke, J. E. Chateauneuf, Chem. Rev., 1999, 99, 433.

[86] J. Zerbs, Photolytischer Käfigeffekt von Dihalomethanen in überkritischen Lösungsmitteln, Dissertation, Georg-August-Universität Göttingen, 2005, http:// webdoc.sub.gwdg.de/diss/2005/zerbs/zerbs.pdf

[87] O. Hassel, H. Viervoll, Acta Chem. Scand., 1947, 1, 149.

[88] I. Ohkoshi, Y. Niide, M. Takano, J. Mol. Spec., 1987, 124, 118.

[89] D. Prystupa, B. H. Torrie, B. M. Powell, P. N. Gerlach, Molec. Phys., 1989, 68, 835.

[90] T. Kawaguchi, A. Wakabayashi, M. Matsumoto, T. Takeuchi, T. Watanabe, Bull. Chem. Soc. Jpn., 1973, 46, 57.

[91] H. Takeuchi, T. Ozaki, T. Takeshima, T. Egawa, S. Konaka, J. Mol. Struct., 2003, $657,381$.

[92] I. J. McNaught, J. Chem. Educ., 1982, 59, 879.

[93] P. D. Mallinson, J. Mol. Spec., 1975, 55, 94.

[94] P. Marshall, G. N. Srinivas, M. Schwartz, J. Phys. Chem. A, 2005, 109, 6371.

[95] R. Vogt, R. Sander, R. von Glasow, P. J. Crutzen, J. Atmos. Chem., 1999, 32, 375 .

[96] J. C. Mössinger, D. E. Shallcross, R. A. Cox, J. Chem. Soc. Faraday Trans., 1998, 94, 1391.

[97] H. E. Simmons, R. D. Smith, J. Am. Chem. Soc., 1959, 81, 4256.

[98] D. C. Blomstrom, K. Herbig, H. E. Simmons, J. Org. Chem., 1965, 30, 959. 
[99] A. N. Tarnovsky, V. Sundström, E. Åkesson, T. Pascher, J. Phys. Chem. A, 2004, $108,237$.

[100] R. S. Mulliken, J. Chem. Phys., 1940, 8, 382.

[101] K. Kimura, S. Nagakura, Spectrochim. Acta, 1961, 17, 166.

[102] H. Xu, Y. Guo, S. Liu, X. Ma, D. Dai, G. Sha, J. Chem. Phys., 2002, 117, 5722.

[103] P. Wagener, Käfigdynamik der Photodissoziation von Diiodmethan: Druck- und Temperaturabhängigkeit in überkritischen Lösungsmitteln, Diplomarbeit, GeorgAugust-Universität Göttingen, 2004.

[104] C. Grimm, Femtosekunden Photolyse von Diiodmethan in überkritischen Fluiden: Konkurrenz zwischen Photodissoziation und Photoisomerisierung, Dissertation, Georg-August-Universität Göttingen, 2003, http://webdoc.sub.gwdg.de/ diss/2004/grimm/grimm.pdf.

[105] M. Kawasaki, S. Lee, R. Bersohn, J. Chem. Phys., 1975, 63, 809.

[106] Y.-J. Liu, L. De Vico, R. Lindh, W.-H. Fang, Chem. Phys. Chem., 2007, 8, 890.

[107] T. F. Hunter, K. S. Kristjansson, Chem. Phys. Lett., 1982, 90, 35.

[108] J. B. Koffend, S. R. Leone, Chem. Phys. Lett., 1981, 81, 136.

[109] G. Schmitt, F. J. Comes, J. Photochm. and Photobiol. A, 1987, 41, 13.

[110] D. Senapati, K. Kavita, K. D. Puspendu, J. Phys. Chem. A, 2002, 106, 8479.

[111] D. Senapati, P. K. Das, Chem. Phys. Lett., 2004, 393, 535.

[112] S.-Q. Man, W. M. Kwok, D. L. Phillips, J. Chem. Phys., 1996, 105, 5842.

[113] S. J. Lee, R. Bersohn, J. Phys. Chem., 1982, 86, 728.

[114] L. Butler, E. Hintsa, S. Shane, Y. T. Lee, J. Chem. Phys., 1987, 86, 2051.

[115] D. G. Abrashkevich, M. Shapiro, P. Brumer, J. Chem. Phys., 2002, 116, 5584.

[116] M. Wall, A. N. Tarnovsky, T. Pascher, V. Sundström, E. Åkesson, J. Phys. Chem. A, 2003, 107, 211.

[117] P. J. Dyne, D. W. G. Style, J. Chem. Soc., 1952, 2122.

[118] D. W. G. Style, J. C. Ward, J. Chem. Soc., 1952, 2125.

[119] H. Okabe, M. Kawasaki, Y. Tanaka, J. Chem. Phys., 1980, 73, 6162.

[120] C. Fotakis, M. Martin, R. J. Donovan, J. Chem. Soc. Faraday Trans. 2, 1982, 78, 1363.

[121] U. Marvet, M. Dantus, Chem. Phys. Lett., 1996, 256, 57.

[122] S. R. Cain, R. Hoffmann, E. R. Grant, J. Phys. Chem., 1981, 85, 4046.

[123] U. Marvet, Q. Zhang, E. J. Brown, M. Dantus, J. Chem. Phys., 1998, 109, 4415. 
[124] U. Marvet, E. J. Brown, M. Dantus, Phys. Chem. Chem. Phys., 2000, 2, 885.

[125] E. D. Tweeten, B. J. Petro, R. W. Quandt, J. Phys. Chem. A, 2003, 107, 19.

[126] O. V. Rattigan, D. E. Shallcross, R. A. Cox, J. Chem. Soc. Faraday Trans., 1997, 93, 2839.

[127] S. Yabushita, K. Morokuma, Chem. Phys. Lett., 1988, 153, 517.

[128] A. van den Brom, Nonadiabatic dynamics in photodissociation of state-selected OCS and $\mathrm{CH}_{3} \mathrm{I}$, Dissertation, Vrije Universiteit Amsterdam, 2003.

[129] A. Gedanken, M. D. Rowe, Chem. Phys. Lett., 1975, 34, 39.

[130] T. F. Hunter, K. S. Kristjansson, Chem. Phys. Lett., 1978, 58, 291.

[131] M. Shapiro, J. Phys. Chem., 1986, 90, 3644.

[132] A. T. J. B. Eppink, D. H. Parker, J. Chem. Phys., 1998, 109, 4758.

[133] A. T. J. B. Eppink, D. H. Parker, J. Chem. Phys., 1999, 110, 832.

[134] R. S. Mulliken, E. Teller, Phys. Rev., 1942, 61, 283.

[135] M. R. Dobber, W. J. Buma, C. A. de Lange, J. Chem. Phys., 1993, 99, 836.

[136] K. Tsukiyama, B. Katz, R. Bersohn, Chem. Phys. Lett., 1986, 124, 309.

[137] J. A. Syage, Chem. Phys. Lett., 1993, 212, 124.

[138] D. Zhong, P. Y. Cheng, A. H. Zewail, J. Chem. Phys., 1996, $105,7864$.

[139] J. A. Syage, Chem. Phys. Lett., 1988, 143, 19.

[140] P. B. Roussel, P. D. Lightfoot, F. Caralp, V. Catoire, R. Lesclaux, J. Chem. Soc. Faraday Trans., 1991, 87, 2376.

[141] E. Villenave, R. Lesclaux, Chem. Phys. Lett., 1995, 236, 376.

[142] J. Sehested, T. Ellermann, O. J. Nielsen, Int. J. Chem. Kinet., 1994, 26, 259.

[143] J. Zhang, D. G. Imre, J. Chem. Phys., 1988, 89, 309.

[144] P. Kroger, P. Demou, S. Riley, J. Chem. Phys., 1976, 65, 1823.

[145] B. Hickel, J. Phys. Chem., 1975, 79, 1054.

[146] R. de Nalda, J. G. Izquierdo, J. Durá, L. Bañares, J. Chem. Phys., 2007, 126, 21101.

[147] J. P. Simons, P. E. R. Tatham, J. Am. Chem. Soc., 1966, A, 854.

[148] H. Mohan, K. Rao, R. Iyer, Radiat. Phys. Chem., 1984, 23, 505.

[149] G. Maier, H. P. Reisenauer, Angew. Chem., 1986, 98, 829.

[150] G. Maier, H. P. Reisenauer, J. Hu, L. J. Schaad, B. A. J. Hess, J. Am. Chem. Soc., 1990, 112, 5117. 
[151] B. J. Schwartz, J. C. King, J. Z. Zhang, C. B. Harris, Chem. Phys. Lett., 1993, $203,503$.

[152] K.-i. Saitow, Y. Naitoh, K. Tominaga, K. Yoshihara, Chem. Phys. Lett., 1996, 262, 621.

[153] M. N. Glukhovtsev, R. D. Bach, Chem. Phys. Lett., 1997, 269, 145.

[154] A. E. Orel, O. Kühn, Chem. Phys. Lett., 1999, 304, 285.

[155] A. N. Tarnovsky, J.-L. Alvarez, A. P. Yartsev, V. Sundström, E. Åkesson, Chem. Phys. Lett., 1999, 312, 121.

[156] J. Davidsson, J. Poulsen, M. Cammarata, P. Georgiuo, R. Wouts, G. Katona, F. Jacobson, A. Plech, M. Wulff, G. Nyman, R. Neutze, Phys. Rev. Lett., 2005, 94, 245503.

[157] M. Odelius, M. Kadi, J. Davidsson, A. N. Tarnovsky, J. Chem. Phys., 2004, 121, 2208.

[158] A. N. Tarnovsky, M. Wall, M. Gustafsson, N. Lascoux, V. Sundström, E. Åkesson, J. Phys. Chem. A, 2002, 106, 5999.

[159] A. N. Tarnovsky, M. Wall, M. Rasmusson, T. Pascher, E. Åkesson, J. Chin. Chem. Soc.-Taip., 2000, 47, 769.

[160] W. H. Hamill, R. H. Schuler, J. Am. Chem. Soc., 1951, 73, 3466.

[161] R. F. Pottie, W. H. Hamill, R. R. Williams, J. Am. Chem. Soc., 1958, 80, 4224.

[162] C. D. Bass, G. C. Pimentel, J. Am. Chem. Soc., 1961, 83, 3754.

[163] H. Zhu, J. Chen, J. B. Hopkins, in: Ultrafast reaction dynamics and solvent effects, vol. 298, AIP conference proceedings, 1994 34-49.

[164] W. M. Kwok, D. L. Phillips, J. Chem. Phys., 1996, 104, 2529.

[165] W. M. Kwok, D. L. Phillips, Chem. Phys. Lett., 1995, 235, 260.

[166] W. M. Kwok, C. Ma, A. W. Parker, D. Phillips, M. Towrie, P. Matousek, D. L. Phillips, J. Chem. Phys., 2000, 113, 7471.

[167] C. Grimm, M. Kling, J. Schroeder, J. Troe, J. Zerbs, Isr. J. Chem., 2003, 43, 305.

[168] X. Zheng, D. L. Phillips, Chem. Phys. Lett., 2000, 324, 175.

[169] V. Sablinskas, P. Klaeboe, C. J. Nielsen, D. Sülzle, Analyst, 1992, 117, 365.

[170] W. Bacher, J. Wagner, Z. Phys. Chem. B, 1939, 43, 191.

[171] H. Bakker, J. Chem. Phys., 1993, 98, 8496.

[172] W. M. Kwok, D. L. Phillips, J. Chem. Phys., 1996, 104, 9816.

[173] W. M. Kwok, D. L. Phillips, Chem. Phys. Lett., 1997, $270,506$. 
[174] W. M. Kwok, D. L. Phillips, Molec. Phys., 1997, 90, 315.

[175] W. M. Kwok, C. Ma, A. W. Parker, D. Phillips, M. Towrie, P. Matousek, X. Zheng, D. L. Phillips, J. Chem. Phys., 2001, 114, 7536.

[176] X. Zheng, D. L. Phillips, J. Chem. Phys., 2000, 113, 3194.

[177] W. M. Kwok, C. Ma, D. Phillips, A. W. Parker, M. Towrie, P. Matousek, D. L. Phillips, Chem. Phys. Lett., 2001, 341, 292.

[178] Y.-L. Li, D. L. Phillips, Chem. Phys. Lett., 2001, 349, 291.

[179] M. Kling, Experimentelle und Theoretische Untersuchungen der FemtosekundenKinetik des Photoinduzierten Zerfalls Aromatischer Peroxidverbindungen, Dissertation, Georg-August-Universität Göttingen, 2002.

[180] R. Mohrschladt, Dynamik von Umlagerungsprozessen über kleine Energiebarrieren in komprimierter Lösung: Tetraphenylethylen und 1,1'-Binaphthyl im $S_{1}$-Zustand, Dissertation, Georg-August-Universität Göttingen, 1993.

[181] P. Maine, D. Strickland, P. Bado, M. Pesset, G. Mouru, IEEE J. Quantum Electron., 1988, 24, 398.

[182] J. Piel, E. Riedle, Optics Letters, 1997, 22, 1494.

[183] J. Piel, M. Beutter, E. Riedle, Optics Letters, 2000, 25, 180.

[184] W. Lauterborn, T. Kurz, Coherent Optics, 2 edn., Springer, 2003.

[185] Jobin Yvon GmbH, Manual: NOPA Nichtkollinear gepumptes OPA-System, 2001.

[186] B. Valeur, Molecular Fluorescence. Principles and Applications, Wiley-VCH, 2001.

[187] Light Conversion, TOPAS: User's Manual, 2001.

[188] C. Reichardt, Aufklärung des photoinduzierten Zerfallsmechanismus von aromatischen Peroxoestern mittels Femtosekunden-IR-Spektroskopie, Dissertation, GeorgAugust-Universität Göttingen, 2007.

[189] T. Lenzer, K. Oum, J. Schroeder, K. Sekiguchi, J. Phys. Chem. A, 2005, 109, 10824.

[190] M. Tamres, in: R. Foster, Hg., Molecular Complexes, vol. 1, Paul Elek (Scientific Books) Ltd., London, 1973 49-116.

[191] M. Tamres, in: R. Foster, Hg., Molecular Associations, vol. 2, Academic Press Inc. (London) Ltd., London, 1979 331-456.

[192] L. E. Orgel, R. S. Mulliken, J. Am. Chem. Soc., 1957, 79, 4830.

[193] National Institute of Standards and Technology, NIST Chemistry WebBook, NIST Standard Reference Database, 69 edn., 2005, http://webbook.nist.gov/ chmistry/. 
[194] V. A. Brosseau, J. R. Basilia, J. F. Smalley, R. L. Strong, J. Am. Chem. Soc., 1971, 94, 716 .

[195] A. Michels, L. Kleerekoper, Physica, 1939, 7, 586.

[196] C. Grimm, A. Kandratsenka, J. Schroeder, P. Wagener, J. Zerbs, J. Phys. Chem. A, 2006, 110, 3320 .

[197] L. Brouwer, J. Troe, Chem. Phys. Lett., 1981, 82, 1.

[198] P. Sulzer, K. Wieland, Helv. Phys. Acta, 1951, 25, 653.

[199] H. Hippler, J. Troe, H. J. Wendelken, J. Chem. Phys., 1983, 78, 5351.

[200] L. Nikowa, D. Schwarzer, J. Troe, Chem. Phys. Lett., 1995, $233,303$.

[201] L. Brouwer, H. Hippler, L. Lindemann, J. Troe, J. Phys. Chem., 1985, 89, 4608.

[202] A. Charvat, J. Aßmann, B. Abel, D. Schwarzer, K. Henning, K. Luther, J. Troe, Phys. Chem. Chem. Phys., 2001, 3, 2230.

[203] M. M. Heckscher, L. Sheps, D. Bingemann, F. F. Crim, J. Chem. Phys., 2002, $117,8917$.

[204] J. Lewis, R. Biswas, A. Robinson, M. Maroncelli, J. Phys. Chem. B, 2001, 105, 3306.

[205] A. Einstein, Ann. Phys., 1905, 17, 549.

[206] H. Hippler, V. Schubert, J. Troe, Ber. Bunsenges. Phys. Chem., 1985, 89, 760.

[207] U. Sukowski, A. Seilmeier, T. Elsaesser, S. F. Fischer, J. Chem. Phys., 1990, 93, 491.

[208] T. Shida, Y. Takahashi, H. Hatano, M. Imamura, Chem. Phys. Lett., 1975, 33, 491.

[209] B. P. Tsai, T. Baer, A. S. Werner, S. F. Lin, J. Phys. Chem., 1975, 79, 570.

[210] S. R. Logan, R. Bonneau, J. Joussot-Dubien, P. Fornier de Violet, J. Chem. Soc. Faraday Trans., 1975, 71, 2148.

[211] P. Fornier de Violet, R. Bonneau, J. Joussot-Dubien, Chem. Phys. Lett., 1973, 19, 251.

[212] A. Treinin, E. Hayon, Int. J. Radiat. Phys. Chem., 1974, 7, 387.

[213] L. Poth, Q. Zhong, J. V. Ford, A. W. Castleman, J. Chem. Phys., 1998, 109, 4791.

[214] W. Weyl, Poyg. Ann., 1864, 123, 350.

[215] Y. Kimura, J. C. Alfano, P. K. Walhout, P. F. Barbara, J. Phys. Chem., 1994, 98, 3450 .

[216] L. Turi, P. Holpar, E. Keszei, J. Phys. Chem. A, 1997, 101, 5469. 
[217] C. Pepin, T. Goulet, D. Houde, J.-P. Jay-Gerin, J. Phys. Chem., 1994, 98, 7009.

[218] T. Scheidt, R. Laenen, Chem. Phys. Lett., 2003, 371, 445.

[219] A. Thaller, R. Laenen, A. Laubereau, J. Chem. Phys., 2006, 124, 24515.

[220] R. Laenen, T. Roth, A. Laubereau, Phys. Rev. Lett., 2000, 85, 50.

[221] E. Knoesel, M. Bonn, J. Shan, F. Wang, T. F. Heinz, J. Chem. Phys., 2004, 121, 394.

[222] X. Shi, F. H. Long, H. Lu, K. B. Eisenthal, J. Phys. Chem., 1995, 99, 6917.

[223] L. D. A. Siebbeles, U. Emmerichs, A. Hummel, H. J. Bakker, J. Chem. Phys., 1997, 107, 9339 .

[224] L. V. Lukin, A. A. Balakin, Chem. Phys., 2001, 265, 87.

[225] A. Mozumder, J. Phys. Chem. A, 2002, 106, 7062.

[226] P. Minary, L. Turi, P. J. Rossky, J. Chem. Phys., 1999, 110, 10953.

[227] A. Hummel, in: Farhataziz, M. A. J. Rodgers, Hgs., Radiation Chemistry: Principles and Applications, VCH Publishers, Inc., New York, 1987 97-136.

[228] J. A. Delaire, M. O. Delcourt, J. Belloni, J. Phys. Chem., 1980, 84, 1186.

[229] T. L. Nemzek, W. R. Ware, J. Chem. Phys., 1975, 62, 477.

[230] J. C. Andre, M. Niclause, W. R. Ware, Chem. Phys., 1978, 28, 371.

[231] C. Xia, J. Peon, B. Kohler, J. Chem. Phys., 2002, 117, 8855.

[232] V. H. Vilchiz, X. Chen, J. A. Kloepfer, S. E. Bradforth, Radiat. Phys. Chem., 2005, 72, 159 .

[233] A. Reuther, A. Laubereau, D. N. Nikogosyan, J. Phys. Chem., 1996, 100, 16796.

[234] C. G. Elles, M. J. Cox, F. F. Crim, J. Chem. Phys., 2004, 120, 6973.

[235] M. Yoshizawa, M. Kurosawa, Phys. Rev. A, 1999, 61, 13808.

[236] Sigma-Aldrich Chemie GmbH, Sigma-Aldrich online Chemikalienkatalog, 2008, http://www.sigma-aldrich.com. 


\section{Danksagung}

Herrn Prof. Dr. J. Troe danke ich für die fortwährende Unterstützung beim Anfertigen der Arbeit. Die angenehme Arbeitsatmosphäre in seiner Arbeitsgruppe habe ich stets sehr genossen.

Herrn Prof. Dr. J. Schroeder danke ich für sein stetiges Interesse am Fortgang der Arbeit und seine Diskussionsbereitschaft zu allen auftretenden Fragestellung.

Herrn Prof. Dr. D. Schwarzer danke ich für die freundliche Übernahme des Korreferats und seine tatkräftige Unterstützung beim Durchführen von Messungen.

Weiterhin danke ich allen Kollegen, die mit mir in meiner Zeit das Femtoland bevölkert haben. Da fällt mir als erster Dr. Jochen Zerbs ein, der mich in die hohe Kunst der Femtologie eingewiesen hat und mir schließlich sein Femtoland vererbt hat. Ich habe Monate gebraucht, um den Geist von NC auszutreiben. Dann danke ich Dr. Christian Müller für seine tägliche Anekdotenstunde (sei es nun über Fritze Kausch, Gerhard Herzberg oder barocke Cembalomusik) und sein professionell geheucheltes Interesse an Fußball oder Heavy Metal. In dieser Aufzählung fehlt noch Dr. Christian Grimm, dank ihm und Dr. Jörg Hahn laufen unsere Computer auch nach Jahren der Nichtbeachtung immer noch.

Damit auch in Zukunft neues Leben aus den Ruinen blüht, möchte ich mich auch bei Arne Walter, Sebastian Stalke, René Moré und Lars Sölter für gute Zusammenarbeit bedanken und Ihnen alles Gute an der Femtofront wünschen. Ansonsten möchte ich mich bei allen anderen Mitgliedern meiner Arbeitsgruppe bedanken, insbesondere bei Reinhard, Thomas und Kawon, Olli und meinen vielen fleißigen PC-F Praktikanten. Weiterhin danke ich auch dem an das MPI ausgelagerten Teil unserer Arbeitsgruppe, insbesondere Dr. Christian Reichardt und Tim Schäfer dafür, daß sie mir so geduldig beim Messen der zeitaufgelösten IR-Spektren geholfen haben. Wenn auch am Anfang eine Menge taube Nüsse dabei waren, haben wir am Ende doch noch ein Signal gefunden.

Daß es an bestimmten Tagen überhaupt noch vorwärts ging, war in erster Linie der schnellen und kompetenten Hilfe der Institutswerkstätten zu verdanken. Dabei möchte ich mich stellvertretend bei den Werkstattleitern Herrn Volker Meyer und Herrn Andreas Knorr bedanken.

Neben der Uni gab es natürlich auch jede Menge Menschen, die mir mit allerlei Spaß und Heiterkeit den Rücken gestärkt haben. Dabei seien in erster Linie meine Eltern erwähnt, die durch ihren unerschütterlichen Rückhalt dafür gesorgt haben, daß ich überhaupt erst soweit gekommen bin. 
Auch danke ich Gesine, die meine Promotionszeit entscheidend geprägt, verschönert und am Ende durch fleißiges Korrekturlesen unterstützt hat.

Besonders danke ich denen, die mit mir das Löwenbanner hochgehalten haben: meinen unermüdlichen Berater Constantin (Paul), meinem Vetter Ailrik (Lupus) meinem Knappen Alonzo (Hans), den Heilern Frey und Tyria (Olli und Anna), dem Barden (Lennard), der Löwensteiner Landwehr und allen anderen. Dann sag ich noch der wöchentlichen Weltvernichtungsrunde insbesondere Paul, Thomas, Olli, Lupus sowie Moritz $1+2$ Danke für viele angenehme Abende.

Desweiteren danke ich dem Göttinger Metal-Stammtisch im Trou für viele heitere Donnerstagabende, dabei besonders hervorzuheben sind Markus die Gurke, Steffen der Killer, Gesine of Death, Eibe of Doom, die Fototante, Leutnant Werner und natürlich Tim der Thekenschläger. Wo wir grad beim Metal sind, geht auch ein Gruß in den Harz und zwar an Sandy \& Andy, Arne, Harti und die üblichen Verdächtigen des FestivalEinsatzkommandos, die gerne eine dicke Lippe riskieren und die Nase immer voll haben. Nicht das Stäbele vergessen, hauptsache bunt und leuchtet. Dann danke ich noch meinem langjährigen Kommilitonen Tobias Bender für ungezählte Fußballabende und -diskussionen, wir wären die besten Bundestrainer geworden.

Und niemals würd ich die Mitmenschen aus dem schönen Weserbergland vergessen. Da danke ich insbesondere Wiebke und Tino, König Tobi, Dezi und Jenni stellvertretend für alle TV-Leute, Nico \& Melli, dem großen Manitou, Eichhoff, Fabian, Lupus und meinem Chemielehrer Herrn StD. Horst Scheppelmann für seine wunderbare Einführung in die faszinierende Welt der Chemie. 


\section{Lebenslauf}

Am 13.10.1979 wurde ich als Sohn von Gerhard Wagener und Ehefrau Jutta, geb. Weber, in Hameln geboren, ich besitze die deutsche Staatsangehörigkeit.

Von 1986 bis 1990 besuchte ich die Grundschule Groß Berkel. Nach dem Besuch der Orientierungsstufe West in Hameln von 1990 bis 1992 wechselte ich auf das SchillerGymnasium Hameln, wo ich die Allgemeine Hochschulreife im Juni 1999 erhielt.

Von Juli 1999 bis April 2000 leistete ich meinen Wehrdienst bei der 1./Panzerbataillon 33 in Neustadt am Rübenberge.

Im Sommersemester 2000 immatrikulierte ich mich an der Georg-August Universität Göttingen für den Diplomstudiengang Chemie und bestand im April 2002 die Diplomvorprüfung. Die Zeit von März bis Mai 2003 verbrachte ich im Rahmen des ErasmusAustauschprogramm an der Università degli Studi di Firenze (Florenz, Italien) im Arbeitskreis von Prof. Alberto Brandi. Im Januar 2004 begann ich unter Anleitung von Prof. Jürgen Troe am Institut für Physikalische Chemie meine Diplomarbeit mit dem Titel „Käfigdynamik der Photodissoziation von Diiodmethan: Druck- und Temperaturabhängigkeit in überkritischen Lösungsmitteln" an und schloss das Studium im November 2004 mit der Diplomprüfung ab. Vom Wintersemester 2005/2006 bis zum Sommersemester 2007 absolvierte ich das Fernstudium Lasertechnik an der Friedrich-SchillerUniversität Jena.

Ab Januar 2005 begann ich meine Doktorarbeit am Institut für Physikalische Chemie der Universität Göttingen als Stipendiat der „Stiftung Stipendien-Fonds des Verbandes der Chemischen Industrie" und beschäftigte mich mit Femtosekundenspektroskopie von unimolekularen und ultraschnellen Reaktionen in Lösung. Dabei erforschte ich die Photoisomerisierung von all-trans-1,4-Diphenylbutadien, ultraschnellen elektronischen Energietransfer in bichromophorischen Molekülen (in Zusammenarbeit mit Prof. Vlastimil Fidler, Prag) und die Photodynamik und -isomerisierung von Polyhalogenmethanen in flüssigen und überkritischen Fluiden als Hauptthema. Als Ergebnis dieser Arbeiten entstand die vorliegende Dissertation. 